

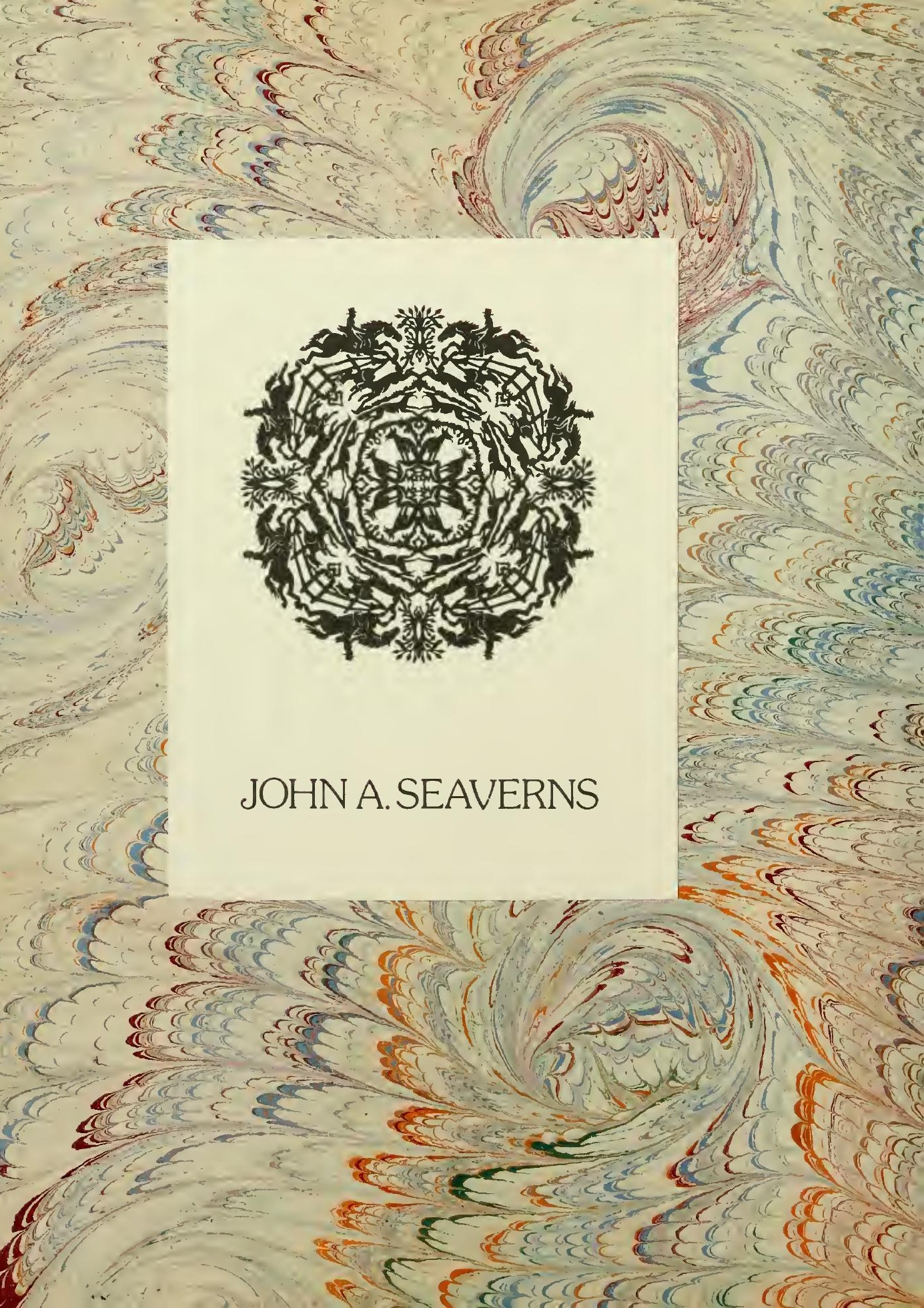



Lw. Pann 



\section{WORKS ON}

\section{HORSES AND EQUITATION.}

A

BIBLIOGRAPHICAL RECORD

OF

H I P P O LOGY,

BY

F. H. HUTH.

$1844-1918$

LON DON :

BERNARD QUARITCH,

I 5 PICCADILLY.

I 887 . 

TO

\section{HIS ROYAL HIGHNESS}

\section{THE DUKE OF CAMBRIDGE,}

K.G., K.T., K.P., F.M., G.C.B., G.C.H., G.C.M.G., G.C.S.I., P.C., I.C.L., L.L.D.,

THIS WORK IS

WITH PERMISSION,

MOST RESPECTFULLY DEDICATED. 



\section{PREFACE.}

LTHOUGH there is no doubt that an enormous
write upon any given subject in searching for
their materials, and although it is quite certain that
in many cases the wrong books are looked over, and the
right books overlooked, simply because it is impossible
to know what has been written on the subject, yet, among
the many branches of literature, there is perhaps none so
much neglected, or when accomplished so lightly esteemed
by the general public, as that dealing with bibliography and
the cataloguing and indexing of works already in existence.

To those however engaged in any literary work of a responsible character, to students, authors, and librarians, the value of works of reference of this class is manifest and incontestable, as being in a manner the tools with which they work. To these a general subject index has long been felt to be a crying want, a want that is impossible to be supplied by any one worker. 
General works like those of Watts, Kayser, Lorenz, or Brunet, wonderful as they are in their gigantic undertaking, are yet necessarily very imperfect and altogether inadequate to the requirements of the age. Had Brunet indeed confined himself exclusively to recording the works written before the seventeenth century, it would have been more satisfactory to succeeding bibliographers, inasmuch as his researches would have acquired additional value, in proportion to their concentration on a period now too distant to be easily retraced.

In view therefore of the utter incapability of General Bibliography to cope with the ever increasing mass of special and technical information, it becomes every day more of a necessity to index the various branches of literature separately and fully.

Much has been done already as may be seen by Petzholdt's Bibliotheca Bibliographica; much has been done so well, that it may be said to have been done for all time, such as Baker's Biographia Dramatica, and the Guide to the Literature of Botany by Mr. Jackson, published by the Index Society; much however remains to be done before the reader will be in a position to see at a glance what treasures of research are at his command.

This object, to be properly and thoroughly attained, should be carried out by a Society. Such a Society already exists in the above named Index Society, and it is much to be regretted that its labours should be restricted for 


\section{Preface.}

vii.

want of adequate support. What it has already published, however, demonstrates the completeness attainable, when the work is undertaken by those, who having a special knowledge of the subject, devote their time and attention to it.

I have endeavoured in the following pages to supply the subject of Hippology, and it is believed that in them will be found a fuller and more trustworthy reference on the subject of the Genus Equus and everything appertaining to it than has before been published.

Some years ago, being then a collector of curious and scarce books on the Horse, I was induced to compile for my own satisfaction and use, a catalogue containing very full bibliographical details of the works on that subject in my own possession. It was afterwards suggested to me to extend the scope of my catalogue, and form an index on the bibliography of the Horse. Acting on this, I increased by long-continued and troublesome research, the number of books tenfold, and as the work grew in size, I expunged all superfluous remarks; details which however interesting to myself, would not have compensated for the increased bulk entailed in their retention. So at least I judged at the time, and though I have since sometimes regretted having done so, it is now too late again to change the character of the work. But although I have endeavoured to condense this volume to the utmost limit of its utility, by the excision of all superfluous matter, I have but rarely curtailed the title of any work that has come under my 
viii.

Preface.

notice, for I consider that the meagre and abridged form so often found in catalogues, either from want of space or information, often does not suffice to acquaint the searcher with the contents of the book, and the similarity which must obviously occur in the abbreviated headings of some thousands of works on one subject, bears me out in this view, and points to the necessity of the fullest description of the title page.

In the present Index I have adopted the chronological system of arrangement generally, and when there are several works published in the same year, they are arranged alphabetically in order of the authors names. Later editions and translations are put under the same heading as the first edition, with their size, place of publication and date, while a general list of authors, with the dates of their works will be found at the end of the book, and also the names of those who have written on each subdivision of the subject, such as Anatomy, Natural History, the Veterinary Art, Cavalry, Equitation, Driving, Shoeing, Bitting, etc., etc., etc., and which serves as an Index to the body of the work,

By far the greater number of books collected in these pages are monographs on the Horse; but I have not thought it wise to exclude other works on Natural History dealing with the Horse, Ass, or Mule in any very distinct degree. Magazine articles or articles from Sporting Journals are however not included, excepting in one case. Works written before the invention of printing, are placed under the year in which it may be presumed they were 
written. It would be absurd, for instance, to place Xenophon after Juliana Barnes, or Gwyllame Twici after Bracy Clark. The same work is only again mentioned when it has been entirely recast, or a new compilation is made, such as the Rei rustica scriptores. In the alphabetical arrangernent of names, I have had to meet the usual difficulty of foreign double names, such as :-Esprit Paul de la Font Pouloti, Franz Max Freiherr von Bouwinghausen von Wallmerode, Francisco de Cespedes y Velasco, etc. In such cases I have endeavoured to follow the custom as near as may be, of the double-named one's country.

Under "Horses and Equitation," I have included everything appertaining to the Horse. Fiction is excluded, and generally all books of which only a part relates to horses; but here again are exceptions, such as Montaigne's Essays, Beckmann's Inventions, and Reynold's Medicine. Indeed the nearer any class of book comes to the boundary line, the less perfect does the Index naturally become, and the more numerous the exceptions to the general rules I have endeavoured to observe. Books on Farming, Natural History and the like are therefore far from being completely represented; it is the same with Sporting papers; while fiction is allowed occasionally to creep in, as in the Dialogue betwixt a Warre Horse and a Mill Horse, which though really a Civil War Tract, yet the characters talk as horses, there is a cut of horses on the title, and we are informed (Sig. A 3) that a horse's skin was then worth ten groats. There is a little better excuse for the Coach and 
Sedan pleasantly disputing, in which Peacham mentions that coaches do not exceed four miles an hour, describes how they are built, are laced, fringed, and gilded, and have leather curtains, and says among other things that the English were the most backward nation in horse-breeding. A few other fictions, by men well acquainted with horses, have also been admitted.

In conclusion I must confess with regret that the Index is far from perfect, and I probably could go on adding to it for the rest of my natural life; in that case however it would possibly never see the light at all, and perhaps it is better therefore to publish it as it is. I do not think many important works have escaped me, but the smaller catchpenny fry are legion; they appear, live their short life, and disappear leaving scarcely a trace behind them. I hope at least that others will be saved some of the time this Index has taken me in preparation.

FRED H. HUTH.

Beckford House,

Lansdozen Crescent,

Bath. 
A N I N D EX

To

WORKS ON

HORSES \& EQUITATION. 



\section{AN INDEX}

TO

\section{WORKS ON}

\section{HORSES AND EQUITATION.}

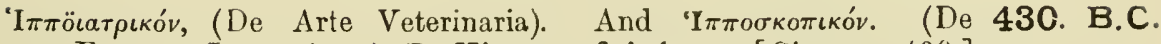
Equorum Inspectione.) By Kimon of Athens. [Circ:a B.c. 430.] So far as known, they are mercly portions of une work, $\pi \epsilon \rho i$ i $\iota \pi \iota \kappa \hat{\eta} s$.

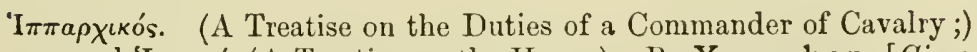
380. and 'I $\pi \pi \iota \kappa \eta$ ', (A Treatise on the Horse.) By Xenophon. [Circa B.c. 380 .] First minted at Florence, folio. 1516.

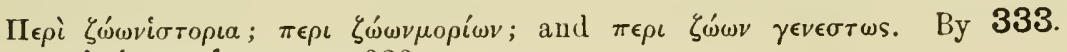

Aristoteles. B.c. 333 . First printed at Basle, 1531.

A Treatise on Agriculture and the Veterinary Art. By Mago 200. Carthaginiensis. Date unknown. His work was in xxvı1. books, translated into Latin by Dionysius Silanus; and into Greek with omissions and additions by Cassius Dionysius, of which an epitone was made by Diophanes of Bithynia.

The fragments still extant were published in the collections of Ruellius, folio. Paris, 1530.

De Re Rustica. By M. T. Varro. B. c. 37.

First printed in the Scriptores rei rustica, A.D. 1472.

37.

De Re Rustica, Libr. XII. By Columella. [Circ 20 A.D.]

First printed in the Scriptores rei rustica, folio. Venice, 1472.

20. A:D.

In French, 4to. Paris, 1555 ; 1556 ; 8vo. 3 vols, 1845-6.

In Italian, 8vo. Venice, $1554 ; 1557 ; 1559 ; 2$ vols, 4 to. Verona, 1808.

In English, 4to. London, 1745.

In Latin alone, 8vo. Dublin, 1732.

Historia Naturalis, Book VIII. By C. Plinius Secundus. [Circa 50. A.D. 50.]

The first time printed at Venice, 1469. For other editions see Brunet. 
120. Epitedroma. A work treating of the Combat of Infantry against Cavalry. By the Emperor Hadrian. A. D. 120.

225. Kєбтоi. By Sextus Julius Africanus. [Circa A.D. 225.] First published in the collection of Ruellius, folio. Paris, 1530. Some only of the books still exist, in MS.

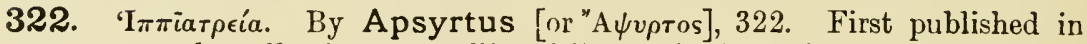
the collection of Ruellius, folio. Paris, 1530, in Latin.

In Greek, in the same, 4to. Basle, 1537.

Sprengel published 'Programma de Apsyrto Bithynio,' 4to. Halle, 1832.

350. A Treatise on the Veterinary Art. By Hippocrates. [Circa 350.] First published in the collection of Ruellius, folio. Paris, 1530, in Latin translation.

In Greek, in the same, 4to. Basle, 1537. Also, 8vo. Leyden. 1665 ; 4to. Naples, 1757. And in Greek, Latin, and Italian, 8 vo. Rome, 1814.

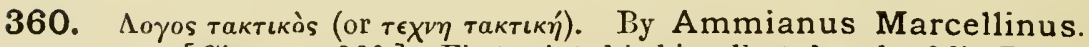
[Circa A.D. 360.] First printed in his collected works, folio. Rome, 1474.

Ayain in Bonon, folio. 1515; and Basel, folio. 1518. These three editions however are incorrect and only contain thirteen books. Augsburg, folio. contains five more. Other editions:Basel, folio. 1533 ; folio. 1544 ; 8vo 1546 ; Lugd. Bat., $12 \mathrm{mo.}$ 1552 ; 4to. Hamburg, 1609.

400. A Treatise on Veterinary Surgery. By Litorius. [Circa 400.] Only a few fragments remain. First printed in the collection of Ruellius, folio. Paris, 1530.

A Treatise on Veterinary Surgery. By Theomnestus. [Circa 400.] The only fragments extant first published in the collection of Ruellius, folio. Paris 1530, in Latin.

In Freek, 4to. Basle, 1537.

A Treatise on Veterinary Surgery. By Tiberius [Circa 400.]

The only fragments extunt first published in the collection of Ruellius, folio. Paris, 1530.

950. A Treatise on Veterinary Surgery. By Hierocles. [Circa 950.] The only fragments extant first published in the collection of Ruellius, folio. Paris, 1530.

1300. Opera di mascalcla By Pier de Crescenzi. [Circa 1300.] 
I çi cómence le Art de Venerie, le quel Mestre Guyllame Twici 1307. Venonr le Roy d'Engletere fist en son temps pur apprendre antres. MS. [Circa 1307.]

First printed at Middle Hill Press, 8vo. 1840.

Livro de Alveitaria dividido em duas partes. No primiero trata das cousas que convem ao Cavallo desde que nace, até que the poem a Sella, e o freyo. A segunda trata de toda as infermidades dos Cavallos, e suas curas. By Mestre Giraldes. MIS. Lisbon, 1318.

Livre de la Chasse. By Gaston III. comte de Foix. MS. [Circa 1387. 1387.]

A Persian MS. treatise on Farriery, translated from the Sanscrit work 1407. known as Salihota. By Abd Ullah Ben Safi. small 4to. written in the city of Kulburgah. A.H. 810. [A.D. 1407.]

Contents:-A Preface and table of Chapters. Legendary account of the Creation of the Horse which is said to have been originally endowed with wings; Defects of the Horse in 52 chapters; good points of the Horse in 12 chapters; signs of the age of Horses; Diseases of the Horse with their treatment, and management of the Horse.

Este livro fez Affonso Esteves morador em santarem Ferrador 1425. del-Rey, o qual escreveo Jaoã de Aveiro morador na Certaà criado que foy do Prior D. Fr. Alvaro camelo, que Deos perdoe, e foy acabado no anno de N. Senhor Jezus Christo. 1425.

$M S$. in the Bibliotheca Severiann.

Da Arte de domar os Cavallos. By Duarte Rei de Portugul e do 1434. Algarve, e Senhor de Ceuta. MS. circa 1434.

Preserved in the Bibliotheque Nationale de Paris. Printed 1842, 4to, Paris, under the title :-Leal conselheiro, o qual fez Dom Duurte Pela graça de Deos Rei de Portugal. . . A requerimento da muito excellente Ruinha Dona Leonor sua mulher; sequirlo do libro da ensinança de bein cavalgar toda sella . .

Liber Marescalciæ Equorum. By Laurentius Ruffus. MIS. 4to. 1462. Rome, 1462.

This is probably the original manuscript and shows by the following colophon that Zurdon Russo or Rusto the generally accepterl name of the author is wrong; "Hic ergo explicitur Liber Muriscalcice Equorum compositus per Magistrum Laurentium, dictum Ruphum de Urbe familiarem et marescalcum Reverendissimi in Christo Patris. Domini Napoleonis de Ursinis Sancti Andriani Diaconi Cardinalis." Anno Domini 1462. 
1462. The following are the printed editions:-Liber Marescalciæ, 4 to. Rome, 1490. Another edition, 4to. printed at Speyer, about the same date. Another, Hippiatria, sive Marescalia Laur. Rusii. . . in qua praeter variorum morborum plurima, ac saluberrima remedia, quadraginta tres commodissimæ frænorum formæ excusæ sunt, ut nullam tam novo oris vitio laborantem equum invenias, cui non hinc occurrere possis. folio. Paris, 1531. Another edition, of the same by the same printer, but differing fiom the above, 4to. Paris, 1532 ; 8vo. Parlua, 1818.

Italian editions:-Arte de cognoscere la natura de' cauael, \&c. .. in vulgare, per Fratre Gabriele Bruno. 4to. Venice, 1492 ; 8 vo. Venice, 1554; 4to. Bologna, I561; 8vo. Venice, 1563; $12 \mathrm{mo.}$ Venice, 1559.

French entitions:-La Mareschalerie de Laurens Ruse, translatée de Latin en françoys, avec les figures des Mors, folio. Paris, 1533. Another by the same printer bnt different edition and title, folio. Paris, 1541. Other editions:-4to. Paris, 1558; 1563; 4 to. Paris, 1583; 4to. Paris, 1610.

1472. Rei Rusticæ Scriptores, (scilicet: Marcus Porcius Cato, Marcus Terentius Varro, et Lucius Junius Moderatus Columella, ex recens Georgii Alexandrini, Palladius Rutilius Taurus Emilianus, studio Franc. Coluciæ verzinenses). folio. Venice, 1472. Also 1482; Bologna,1494; Ibirl.,1496; Ibil.,1499; 4to, Venice, 1514 ; Florence, 1515 ; 1521 ; Venice, 1533 ; 8vo. Lyons, 1541 ; 1548-9 ; Paris, 1543 ; 4to. Leipsic, 1735 ; 2 vols, 4to. Leipsic, $1773-4$; 5 vols, 8 vo. Mannheim, 1781 ; 4 vols, 8 vo. Deux Ponts, 1787 ; 5 vols, Venice, 1783-5; 7 vols, 8vo. Leipsic, 1794-1797; Ibid., 2 vols, 4 to. 1827.

In French:-Traduction d'anciens ouvrages latins relatifs à l'agriculture ęt à la médecine vétérinaire avec des notes par Saboureux de La Bonnetrie, 6 vols. Svo. Paris, 1771-75.

In Schneider's edition, 1794, there is a full account of the various MSS.

1478. A Persian MS. in the British Museum entitled Kurrat ul-Mulk. Date A.H. 833. [A.D. 1478].

The work is divided into 12 chapters, subdiviled into sections. and numbered at the beginning as follows: 1.-Breeds of various countries. 2.-Modes of choosing Horses. 3.-Omens derived from the movement of Horses. 4.-Colours of Horses. 5.-Their blemishes. 6.--Their limbs. 7.-Diseases and remedies. 8.-Bleeding. 9.-Diet and food. 10.-Fattening, and treatment of sores. 11.-How to know the age of Horses by their Teeth; and, 12.-On lucky and unlucky marks in Horses. 
The Bokys of Haukyng and Huntyng, and also of Cootarmuris. By 1486. Juliana Barnes. [or Bernes, or Berners] folio. St. Albans, 1486, For Biblingrophical particulars see Hazlitt. There was also an edition published in facsinile, 4to. 1881.

Libro de albeyteria, es a saher de los Cavallos y de las Mulas. By 1495. Manuel Diaz. [or 1liez, or Dias,] folio. Saragossa, 1495.

Other editions, Ibid, 1499 ; 4 to. Valladolid, 1500.4 to. Barcelona, 1505. 4to. Toledo, 1507 and 1571. 4to. Burgos, 1530; and 4 to. Saragossa, 1545.

Tratta della Pittura. By Leonardo di Vinci. Embodying subject of 1499. work on Horses, entitled "Notomai dei Cavalli," uritten about 1499.

First printed, Rome, 1651.

French translations:-1651; 12mo. 1716; 8vo. 1796; 1803. The most complete, 4 to. Rome, 1817.

All Leonardo di Vinci's works are now being reprinted (1885, etc.)

La Mlédecine des chevaux et des bestes chevalines. Anon. 4to. 1506. Paris, [Circa 1506.]

Libro de la natura di cavalli. Anon. 4to. Milan, 1517.

1517.

Incomincia il Libro de Maistro Augustino Culombre maneschal- 1518. cho de Sancto Seuero Dedicato al Re Ferdinãdo de Ragona. 4to. Venice, 1518. 8vo. $1536 ; 1547 ; 1561$; and 4 to. 1622 .

Artis Veterinariae, sive Mulomedicinæ Libri quatuor. From the 1524. works of Vegetius Renatus. 4to. Basil, 1524. Ibid., 4to. 1574 , also 8vo. 1748 and 1781 .

French translations : 4to. Paris, 1563 ; also in the 6th vol. of the Anciens ouvrages latines relutifes ì l'agriculture.

In English: "Of the Distempers of Horses" "4to. London, 1572 ; 8vo. 1748 ; 8 vo. 1756 .

Dises buchlein zeyget an und lernet ein masz oder proporcio der Ross, 1528 . nutzlich iungen gesellen, malern vnd goltschmidē. Ky Hans Sebald Beham. 4to. Nuremberg, 15.28.

Libro aureo de Marco Aurelio, emperador y eloquētissimo orador. 1529 [By Antonio de Guevary.] [Circa 1529.]

Hallam says, on the anthority of Dr. West, that the manuscript was lent by the author to the emperor, and uas pirated lefore the first known editions of 1529 were printed. (Literature of Europe, London, 1843, vol. i. p. $\$ 90$, note.) 
1529. First printed in Italian, Venice, 1606 ; In French, 4 to. Paris, 1531 ; In English, 8vo. London, 1534; In Latin, Turgau, folio. 1611.

Fur Bibliogr, see Branet; Antonio, Bill. Hisp. Script. I. 126; De Salva, II. 150 .

1530. Ex Apsyrto et aliis collecti veterinariæ medecinæ libri duo. By Jean Ruel. folio. Paris, 1530.

An edition in the original Greek was published at Basle, 4 to. 1537.

This is a collection of fragments compiled by Ruel (or, us he is more commonly called, Ruellius), from fragments of Apsyrtus, Anutolius, Hippocrates, Himerius, Pamphilus, Hierocles, Tiberius, Emitius Hispanus, Africanus, Mago Carthageniensis, Theomnestus, Eumelus, Didymus, Pelagonius, Archedemus, Litorius: Beneventanus, and Diophanes; mostly existing only in MS.

1531. Libri duo Philippicorum sive de Equorum natura electione, educatione, disciplina et curatione. By Petrus A. Naaldwyck. 4to. Leyden, 1531.

1539. Iтпоко́ $е к о$, sive de equis tractandis et historiola rei nummariæ. By Joachim Camerarius. 8vo. Tubingen, 1539.

1546. Libro de albeyteria. En el qual se veran todas quantas enfermedades y desastres sucten aedescer a todo genero de bestias y la cura de dellas. Assim ismo se verā las colores y faciones para conoscer vn buen cauallo $\mathrm{y}$ vna buena mula. El mas copioso $\bar{q}$ hasta agora se ha visto. By Francisco de la Reina. 4to. Mondonedo, 1546 and 1552. 4to. Burgos, 1564; 4to. Salamanca, 1580 ; and Alcala, $1582-83 ; 1602 ; 1623 ; 1647$.

1547. A volume containing a series of 370 original coloured drawings of ornamental Bits for Horses, done upon 186 sheets of fine vellum, with descriptions to each in old French. This MS. was executed in 1547 by Jacques de Genoillac, Governor of Languedoc, Privy Councillor and Master of the Horse to Henry II. of France. Roy. folio, RM. with arms and monogram in gold on the sides and back and clasps. At the beginning is a declaration, dated 1564, made by the Marquis of Voysy, Master of the Horse to Charles IX., with his signature in full, to the effect that he leaves this MS. to his male descendants.

1551. De la Cavalleria de la Gineta. By Ferdinando Chacon. 4ti. Seville, 1551. 
Historiæ animalium, Liber I. de quadrupedibus viviparis. By 1551. Conrad Gesner. folio. Murich, 1551.

The other parts were published, ii., 1554; iii., 1555; iv., 1558 ; and $v$., 1587.

Later editions of Book I., 1585 ; $1603 ; 1620$.

Ordini di Caualcare, et modi di conoscere le nature de' caualli, emen- 1552. dare i vitii loro, et ammaestrargli per l'uso della guerra et commodità de gli huomini. By Federico Grisoni. svo. Venice, 1552 and 1553.

The edition of Venice, 4to. 1571, bears on the title:-Aggiangeuisi una scielta di notabili aumertimenti, perfare eccelenti razze, e per rimediare alle infermità de' Caualli. It is from this edition that others of more recent date (viz: 1584, 1590, 1610, etc.) have been printed.

Grison's Ordini di Caualcare has also been translated into Spanish under the title:- "Reglas de la Caualleria de la brida," etc, by Ant. Flores de Benauides. 4to. Baeça, 1568. And into German under that of "Kunstlicher Bericht," etc., by Jean Fayser. folio. Augsburg, 1570.

The French editions are as follows:-L'Ecurie du S. Federico Grison, gentilhomme napolitain. En laquelle est monstre l'ordre et l'art de choysir domter, piquer, qu'autres commodités de l'homme. Auec figures de diuerses dresser et manier les cheuaux tant pour l'usage de la guerre sortes de mors de bride. 4to. Paris, 1559 ; 4to. 1563 ; 4to. 1565 ; 4to. 1568 ; 4to. 1575 ; 4to. 1579 . Paris and Lyons, 4to. 1584 and 4 to. 1585 . Tournon. 4 to. 1599, and Paris, 4to. 1610.

There was a translation (never printed) into Portuguese:Ordens da Cavallaria . . . traduzido de Italiano em Portuguez por Leonel da Costa. folio.

Trattato di Mascalcia. By Filippo Scacco. 4to. Venice, 1553, 1553. 4 to. Padua, 1628.

Trattato dell' imbrigliare, attegiare, e ferrare cavalli, etc. By Cæsar 1556. Fiaschi. In three parts, 4 to. Bologna, 1556. Also 4to. Venice, 1598 and 1604 ; 4to. Padua, 1626 ; and 8vo. Venice, 1561 and 1565 .

Translated into French, with the title "Traité de la manière de bicn embrider, manier, et ferrer les chevaux. By Fr. de Prouane. 4to. Paris, $1564 ; 1567 ; 1578 ; 1579$ and 1611 .

Delle razze, disciplina del cavalcare et altre cose pertinenti ad esser- 1560 . citio cosi fatto. By Gio. Bat. Ferraro. 8vo. Naples, 1560; Paira, 1564 ; Venice, 1653. 
1564. Recopilaciō de los mas famosos autores Griegros y Latinos que trałarō de la excelēcia y generaciō de los cauallos. Y asimismo como se han de doctrinar y curar sus enfermedades. $\mathrm{Y}$ tambien do las Mulas y su generacion. By Alonso Suarez. folio. Toledo, 1564.

1565. The fowre chiefyst offices belongyng to Hursemanshippe. That is to saye. The office of the Breeler, of the Rider, of the Keper, and of the Ferrer. In the firste parte whereof is declared the order of breding of horses. In the seconde howe to breake them, and to make theym horses of seruyce. Conteyninge the whole arte of Ridynge lately set forth, and nowe newly corrected and amended of manye faultes escaped in the fyrste printynge, as well touchyng the bittes as otherwyse. Thirdly, howe to dyet them, as well when they reste as when they trauell by the way. Fourthly to what diseases they be subiect, together with the causes of such diseases the sygnes howe to knowe them, and finally howe to cure the same. Whyche bookes are not onely prynfully collected out of a number of anethours, but also orderly dysposed and applyed to the vse of thys our couttrie. By Thomas Blundevile. 4to. London, 1565-6. 4to. $1580 ; 4$ to. 1597 ; 4 to. 1609.

The last three parts have distinct title payes.

1566. La Gloria del Cauallo, opera dell' illustre Pasquali Carraciolo, diuisa in dieci libri : né i quali oltra gli ordini pertinenti alla Cauelleria, si descrivono tutti i particolari che son necessarii nel' alleuare, custodiro, maneggiate, et curar di Caualli, etc. Venice, 4to. 1566-1567. With additions, 4to. Venice, 1585; 1586; 1589 ; 1608 .

To the lust entition was added Giovani Antunio Cito's 'Del conoscere le infirmite che avvengono al cavallo.'

1568. L'Art Vétérinaire ou Grande Marechaleric. By Jean Massé. 4to. Paris, 1568.

Del Can, y del Cavallo, y de sus calidades: dos animales de gran instincto y sentido, fidelissimos amigos de los hombres. By Luyz Perez. 8vo. Valladolid, 1568.

1569. Ein neuw Thierbuch, Eigentliche vnd auch grïndliche beschreibung allerley vier vnd zweyfüssigen Thieren, vom grossen biss zum kleinsten, sampt derer Art, Wesen, Natur vnd Eigenschafft: Erstlich durch den weitberhümbten Hansen Bocksperger den juingern von Saltzburg in visirung gestellt, folgendts gerissen dureh den kunstreichen Josz Amman von Zürich : nun jetzt durch Georgium Schallerum von München gantz fleissig beschrieben, vnd in Teutsche Reimen gefasset, etc. 4to. Frankfurton-Maine, $1569 ; 1.592$. 
Libro de Marchi de' Cavalli, con li nomi di tutti li principi, et privati 1569. signori che hanno razza di Caualli. Anon. 8vo. Venice, 1569 ; 12 mo. Venice, 1593 ; 18 mo. Venice, 1626.

Trattato grande dell 'arte cavalleresca. By Tommaso Campanella. 1570. [Cirec 1570.]

Libro de Enfrenamientos de la Gineta. By Eugeno Manzánas, 4 to. Toledo, 1570. And folio. Toledo, 1583.

Discorsi cavallerechi, ne' quali oltre gli eserciti equestri, si ragiona di 1571 . tutte le disciplini che si ricercano in un compito cavaliero. By Gasparo Toralto. 4to. Naples, 1571.

Tractado de la Cavalleria de la Gineta cōpuesto y ordonado, por el Capitã Pedro de Aguilar . . . Contiene diversos avisos y documētos, y otras muchas reglas vtiles y necessarias, assi para lo que toca a la doctrina y enfrenamiento de los caullos, como para la perfection y destreza que en esta facultad conniene $\tilde{y}$ tengan, en cosas de paz yde guerra los cauaileros. 4to. Seville, $1572 ; 4$ to. Nalaga, 1600.

Il Cavalerizzo, nel quali si tratta della natura de' Cavalli, del modo di $\mathbf{1 5 7 3 .}$ domargli e frenargli. By Claudio Corte. 4to. Marseilles, 1573. 4to. Venice, 1573.

An English translation:- -The Art of Riding, containing divers necessarie instructions, demonstrations, helps and corrections appertaining to horsemanship. Written at large in the Italian toong by Maister Claudio Corte, a man most excellent in this Art. Here briefly reduced into certeine English Discourses to the benefit of Gentlemen desirous of such knowledge. [Translated by Thomas Bedingfield, under John Astley.] 4to. London, 1584.

The Noble Arte of Venerie or Hvnting. Wherein is handled and set out, the Vertues, Nature and Properties of fiuetene sundrie Chaces together with the order and maner how to Hunte and Kill euery one of them. Translated and collected for the Pleasure of all Noblemen and Gentlemen out of the best approned Authors which haue writtell any thing concerning the same; and reduced into such Order and proper Termes as are vsed here in this noble Realme of England. By Christopher Barker. 4to. London, 1575.

Another edition, 4 to. Thomas Purfoot, London, 1611 , angmented, with many new additions by another hand.

Thiswork is generallybound up with Turbervile's Bookeof Falconrie.

A plaine and easie way to remedy a Horse that is foundered in his feete. By which vsing this remedy (within xxiiii. howres after his instant foundering) you may within xxiiii. hours after the cure vsed, trauell your horse and iourney him at your pleasure, as if he had not beene foundered at all. Set out by Nicholas Malbie. 4to. London, $1576 ; 1583 ; 1594$. 
1576. Remedies for the Dyseases in Horses, Approoued and allowed by diuers very auncient learned Mareschalls. By Nicholas Malbie. 4to. London, [1576]; 1583 : 1594.

No copy known of the edition of 1576. Only one copy, and that imperfect of the edition of 1583, and only one of the edition of 1594 .

1577. The Lamentable historie of the death of ij. horses, sometime seruantes to Nicholas Sinbor Hackeneyman. Anon. [1577].

Diversarum gentium armatura equestris, ubi fere Europæ, Asiæ atque Africæ equitandi ratio propria expressa est. By Abraham de Bruyn. 4to. Coloniæ, [1577.]; 4to. Amsterdam, 1617.

1578. Wie vnd wa man ein gestuit von gutten edlen Kriegssrossen auffrichten, vnderhalten, die jungen. . erziehen soll, ete. By Max. Fugger. 4to. [Frankfurt], 1578. Folio. Frankfurt 1584; folio. Frankfurt, 1611 .

A new edition of the above, edited by Joh. Gli. Wolstein, 8vo. Vienna, 1786, 1788; 8vo. Brunswick, 1796 ; 8vo. Hamburg, 1800 ; 8vo. Innsbruck, 2 vols. 1805.

Equile Joannis Austriaci, Caroli V, imper. F., in quo omnis generis equorum ex variis orbis partibus insignis delectus. Ad vivun omnes delineati. By Johan Stradan. Plates (41) chiefly engraved by Phil. Galleus. Obl. fol. Antwerp, 1578.

In the 2nd edition the title differs slightly, heginning ' Equile seri speculum equnum,' and at the bottom of the frontispiece is added Antwerpice, apud Joan Galleum.

There is also an edition by Mark Sadeler.

1580. De la natvraleza del cavallo: En que estan recopiladas todas sus grandezas: juntamenti con el orden que se a de quardar enel hazer delas castas, y crier delos Potros: y como se an de domar, y enseñar buenas costumbres: y el modo de enfrenarlos y castigarlos de sus vicios y siniestros. By Pedro Fernandez de Andrada. 4to. Seville. $1580 ; 1598 ; 1616$.

Les Essais de messire Michel (-Eyquem). . . de Montaigne Parts I. and II., Bordeaux. 1580.

Part I., ch. 48, contains, "Des Destriers" Part III, ch. 6, contains "Des Coches."

Editions of parts I. and II. also: 1 vol, 8 vo. Borleaux, 1582 ; with III. part, now first published, 5th edition, 4to. Paris, 1588.

This was the last pullished in the life time of the author. For other editions, and translations into Italian and English, see Brunet. 
Del andar a caballo en ambas tillas de gineta y brida. By Juan de 1580 Peralta. 4to. Seville, 1580.

Escurie de De Pavari vénitien. [In Italian and French,] folio. 1581. Lyons, 1581.

Libro de Albeyteria, en el qual se trata del cavallo, y mulo y Iumēto, 1582.

$\mathrm{y}$ de sus miembros y calidades y de todas sus enfermedades, con las causas, senales y remedios de cada una de ellas. . . y un nenvo arte de herrar. By Fernando Calvo. folio. Plasencia, 1582 ; Salamanca, 1587 ; Madrid, 1675.

Artliche vnnd Kunstreiche Figurn zu der Reutterey, sampt jrem 1584. musterhafitem Geschmuck, Dergleichen nie aussgegangen. Jetzt erst durch den Kunstreichen Jost Amon wohuhafít zu Nörn berg gerissen. Obl. 4to. Francfurt-on-Maine, 1584.

Ritterlich Reutter kunst, Darinen ordentlich begriffen Wie ma zuvorderst die Ritterliche vnd adeliche Ubung der Reutterey, bevorab in Teutschland, mit musterhafftigem Geschmuck, Ritterspiel, Mumerey, Kleidung, vnd allem andern, so dero beides in schimptf vnd ernst anhängig, gebrauchen vnd vnderscheiden möge, etc. Anon. folio. Frankfurt, 1584.

The Art of Riding, set foorthe in a breefe treatise, with a due interpretation of certeine places alledged out of Xenophon and Gryson, verie expert and excellent Horssemen: Wherein also the true vse of the hand by the said Grysons rules and precepts is speciallie touched: and how the Author of this present worke hath put the same in practice, also what profit men maie reape thereby: without the knowledge whereof all the residue of the order of Riding is but vaine. Lastlie, is added a short discourse of the Chaine or Cauezzan, the Trench, and the Martingale. [By John Astley.] 4to. London, 1584.

Trattato dell' arte della pittura, divso in sette libri. By Geo. Paolo Lomazza. 4to. Milan, 1584.

In Bk.I.cap.xxi.is, "Delle misure de Cavallo da memlnea membre."

Other editions: Ibid, 1585 ; 3 vols, Rome, 8vo. 184t; In English, 2 vols, folio. Oxford, 1598; In French, the first book only, folio. Toulouse, 1649.

Schoole of Horsemanship, wherein is discovered what skill and 1585. knowledge is required in a good horseman, and also how to reform any restive Horse; briefly touching the knowledge of the Breeder, Sadler, Smith, and the horse-leach, with a strange and rare invention how to make a new Racke, and how to teach a Horse to lie upon his belly nntill the Rider take his Backe. By Christopher Clifford. 4to. London, 1585. 
1587. The first Booke of Cattell, wherein is shewed the gonernement of Oxen, Kine, Calves, and how to use Bulls and other Cattell to the Yuake and Fell; the seconde Booke intreating of the gonernement of Horses with the approned remerlies against most diseases, verie protitable for all men, having a charge and gouernement thereof and chietly for Husbandmen, with diuers other remedies practised in this land. Gathered by Leonard Mascall. 4to. London, $1587 ; 1591 ; 1596 ; 1605 ; 1627 ; 1662$.

De procreandis, eligendis, alendis, frænandis, et tractandis Equis, experientia. By Richard Sadler. 4to. London, 1587.

1538. I neri disegni di marchii di tutte le più famose razzi di Caualli che sono in regno. By G. B. Cappello. Svo. Naples, 1588.

Libro de Albeyteria que trata del principio y generacion de los cavallos hasta su vejez. Y asimismo los remedios para curar sus enfermedades, y de las mulas y otros animales, muy útil y provechoso para todos los albeytares y cirujanos, y para otras cualesquier personas que tuvierenó criaren los dichos animales. By Pedro Lopez Zamora. fulio. Logroño, 1588.

1590. Discurso para estar a la gineta, con gracia y hermosura. By Juan Arias de Avila. 8vo. Madrid, 1590.

Del conoscere le infirmità che avvengono al cavallo e al buove, co' i rimedi à chiaschedunas di essa. By Giovani Antonio Cito. 4to. Venice, 1590.

Another edition, 4to. Venice, 1608, with P. Carraciolo's " $L a$ gloria de Cavallo."

1591. De Arte Equestri Germanice. By Joh. Creutsberger.Vienna, 1591.

Opera di mescalzia, doue si contiene tutte l'infermità de' caualli, cosi interiori, come esteriori, et gli segni da cognosceri, et le cure con potioni, et untioni, et sanguigne per esse caualli. By M. Filippo Scaccho da Tagliacozzo. 4to. Rome, 1591.

1593. Le Cavalerice François. Contenant les precepts principaux qu'il faut observer exactement pour bien dresser les Chevaux anx exercises de la carriere et de la campagne. Le tout divisé en trois livres. Le premier traicte de l'ordre générale et plus facile des susdits exercises, et de la proprieté du Cavalier, le seconde des modernes et plus juste proportions de tous les plus beaux airs et maneges, le troisiesme des qualitez de toutes les parties de la bouche du Cheval et des divers effets de plusiers brides differentes pourtraites et representées par leurs juste mesures aux lieux necessaires. By Solomon de la Brove. folio. Paris, 1593-4; 1602; 1610; 1646. 
A Discovrse of Horsemanshippe. Wherein the breeding and ryding of 1593. Horses for sernice, in a breefe manner is more methodically sette downe then hath been heretofore. With a mole easie and direct course for the ignorant, to attaine to the same arte or knowledge. Also the manner to chuse, trayne, ryde and dyet, both Huntinghorses, and Running-horses: with all the secretes thereto belonging liscouered. An arte neuer lieretofore written by any Authour. Printed by I. C. for Richard Smith. [By Gervase Markham]. 4to. London, 1593.

Hazlitt, never having seen a cop!y of this work, suggests that it is Astley's work of 1584 with a new title, on the ground that the running headings of the latter are the same as Marlihain's title, and the collation is the same. The works however are entirely distinct. Moreover Marhliam's is printed in black: letter, while Astley's is in roman character's.

Maroccus Extaticus. Or, Banke's Bay Horse in a Trance A Dis- 1595. course set downe in a merry Dialogue between Lankes and his Beast: Anatomizing some abuses and bad trickes of this age. Written and intituled to mine Host of the Belsauage, and all his honest Guests. By Iohn Dando the wierdrawer of Hadley, and Harrie Funt, head Ostler of Bosomes Inne. Printed for Cuthbert Burby. 4to. 1595 .

This celelnated trick horse is mentioned by several Elizabethan writers : Ben Jonson, "Every Man out of his Humour," iv. 6; Shakespere, "Alls Well," ii. 8; "Jack Drum's Entertainment," Sig. Bz.; Deliker's "Wonderful Year," 1603; Digly "Hist. of Bodies," c. 37, p. 393 ; Sir W. Rawleigh, "Hist. of the World," 1st Part, p. 178. See also L'Ane D'or, trad. de J. de Montlyard, Paris, 16.3, pp. 250-254.

How to chuse, ride, traine, and dyet both hunting and running 1596. Horses. [By Gervase Markham]. 4to. London, 1596; 4to. London, 1599 ; 4to. London, 1606. Published first in the Discourse of Horsemanshippe, 4to. London, 1593.

Dell' anatomia e dell' infermità del cavallo. By Carlo Ruini. 21598. vols. folio. Bologna, 1598 .

Vol. 2 has a separate title page, reading "Dell' Infermetic del Cavallo."

Another edition, Anatomia del Cavallo, infermità, et suoi remedii del Signor Carlo Ruini, senator Bolognese. Venice, 1618.

Hippostologie; c'est à dire Discours des Os du Cheval. By Jean 1599 Hervard. 4to. Paris, 1599. 
1599. Libro de la gineta de España, eñ el qual se trata el modo de hacer las castas y criar los potros y como se han de enfrenar y castigar los cavallos; y como los cavelleros mozos se han de poner a cavallo, guardando el orden antiquo de la gineta de Españar; y ultimamente, como se han de pensar y engordar los cavallos. By Pedro Fernandez de Andrada. 4to. Seville, 1599.

How to chuse, ride, traine, and diet, both Hunting-horses and rumning Horses. With all the Secrets theretu belonging discovered : an Arte neuer heere-to-fore written by any authour. Also a dis. course of horsemanship, etc. By Gervase Markham. 4to. London, 1599.

This embodies the two previous works of 1593 and 1596.

Raccolta breve d'alcune cose più segnalate e hebbero gli antichi, e d'alcune altre trovate da modcrni. Con l'aggiunta d'alcune considerationi curiose e utili di F. Gualtieri. By Guido Panciroli. 4 to. Venice, 1599 and 1612 .

A Latin translation by Henry Salmuth, 2 vols, 8vo. Hamburg, 1599 ; 8vo.1607; 8vo. 1612; 8vo. Frankfurt. 1622 ; 4to. [1629]; 4to. 2 vols, 1660 ; entitled 'Rerum memorabilium, jam ulin deperditarum et recens inventarum. Libri II.

A French translation by Pierre de la Nuve. 12mo. Lyon, 1617.

An English translation "The History of many memorable things lost," 2 vols. $12 \mathrm{mo}$. London, 1715 . The same with new title, 2 vols. $12 \mathrm{mo}$. London, 1727 , in which Chap. VI., Sect. 1v. treats of Horses, Bridles, etc., and Chap. XVI., Vol. II. is entitled "Of Saddles, Stirrups and Horse Shoes," giving an account of their origin.

1600. Libro di exercicios de la gineta. By Bernardo de Várgas Machuca. Svo. Madrid, $1600 ; 1619$.

Compendio dell' arte di Cavalleria. By Alex. Massarius Malatesta. 4 to. Venice, 1600.

A later edition:-Compendio dell' heroica arte di cavelleria, precetti, quatro. . . corretto dal medesino autore et agiuntoui il quinto del modo di ordinar vn squadron di caualleria, etc. folio. Dantzig, 1610.

Livro de Cavallarias, que consta de dous Cavalleiros chamados Nanferleste, e Bictapor. By Fernando Telles de Menezes. [MS. Circa 1600].

Nanferleste and Bictapor, represent Telles and the Bishop of Oporto.

1602. Cavallo frenato, con discorsi notabili sopra briglie, etc. By Pietro. Ant. Ferraro. folio. Naples, 1602 ; Venice, 1620 ; 1653.

Delle infirmita di cavalli. By Filippo Scacco. 4to. Rome, 1602. 
How to trayne and teach Horses to amble. By Gervase Markham. 1605. 4to. London, 1605.

Modo de pelear a la ginta. By Simon de Villalóbos. 8vo. Valladolid, 1605.

Tractatus de modo equos frænandi. . . cum diversorum frænorum $160 \%$. variis figuris. By Alex. Massario Malatesta. folio. Venice, 1607.

An Italian edition of the same:-Della Ragione e modi d'imbrigliar cavalli. folio. Rome, 1613.

Cavelarie, or the English Horseman. By Gervase Markham. 4to. London, 1607.

Another edition, newly imprinted, corrected and augmented, with many secrets not before known. Divided into eight books with separate titles. The 2 nd and 3 rd books bear the date of 1616. With the title:-Cavelarice, Or the English Horseman ; Contayning all the Art of Horse-manship, as much as is necessary for any man to vnderstancl, whether hee be Horse-breeder, Horse-ryder, Horse-hunter, Horse-runner, Horse-ambler, Horse-farrier, Horsekeeper, Coachman, Smith, or Sadler. Together with the discovery of the subtil trade or mystery of Horse-coursers, and an explanation of the excellency of a horses vnderstanding: or how to teach the to do trickes like Banke's bis Curtall. And that Horses may be made to draw dry-foot like a Hound. Secrets before vnpublished, now carefully set downe, for the profit of this whole Nation. By Gervase Markham. 4to. London, 1617.

The Historie of Foure-footed Beastes. Describing the true and liuely figure of every beast, with a discourse of their seuerall Names, Conditions, Kindes, Vertues, (both naturall and medicinall). Countries of their breed, their loue and hate to mankinde, and the wonderfull worke of God in their Creation, etc. By Edward Topsell. 2 vols. folio. London, $1607 ; 1658$.

Tratado de la Gineta. By Franciscus de Cespedes y Velasco. 1609. 8 vo. Olisipone, 1609.

Della Cavalleria, Grūndtlicher Bericht von allem was zu der Reutterei gehörig vnd einem cauallier davon zu wissen gebürt. By G. E. Loehneysen. folio. 1609-10.

The Perfection of Horsemanship, drawn from Nature, Arte, and Practise. By Nicholas Morgan. 4to. London, 1609. Another edition, 1692. 
1610. A very perfect discourse and order, how to know the age of a Horse, and the discases which breede in him, with the Remedies to cure the same; as also the description of every veyne, and how and when to let him Blood, according to the diversity of the Disease, as hath been proved by the Author, L.W.C. 4to. $1610 ; 1620 ; 1630$.

A Cure for all Diseases in Horses. By Gervase Markham. 4to. London, 1610.

Republished under the title of:-Methode or Epitome : wherein is shewed his approved Remedies for all Diseases whatever, incident to Horses, etc. 8 vo. London, 1616; 12mo. 1623; 12mo. 1641.

Le Cavesson François, recherché et mis en usage. By Balthasar Prevost, sieur de La Tigerie. 4to. Poitiers, 1610.

1611. Country Contentments; or, the Husbandman's Recreations. [By Gervase Markham]. 4to. London, 1611.

This is only the first book, but inclurles the "Treatise on Horses," etc. In 1615 was published the "English Huswife," which formed the second book; often, according to Hazitt, reprinted. In 1623 appeared a new editim, called "Cheape and Good Husbandry." Ibid, 1631. In 1648 it was published under the title of "A Way to get Wealth. . . . The first five bookes gathered by G. M. The last by Master W. L. . . The seventh time corrected, and augmented by the Author." 4to. London, of which there were other editions, $1653 ; 1656 ; 1676$; etc.

Regole Militari, sopra il Governo e sevitio, particolare della Cavalleria. By Fr. Lodovico Melzo. folio. Milan, 1611.

A French translation by Varroy:-Reigles Militaires touchant la cavalerie. folio Antwerp, 1615 .

A Spanish translation by Galderico Gali:-Reglas Militaires sobre el govierno, y servicio particular de la Cavalleria. folio. Milan, 1619.

1612. Il governo della cavalleria leggiera. By George Basta. 4to. Venice, 1612; Oppenheim, 1616.

Translated into Spanish under the title of, 'El Gobierno de la cabellaria legera,' 4 to. Brussels, 1624.

Translated into German by J. Theod. de Bry. Frankfurt, 1641.

In French as:-Le gounernement de la cavallerie legiere: traicté qui comprend mesme ce qui concerne la graue, pourl 'intelligence des capitaines. . . par George Basta; mis en lumiere en sa forme originalle en langue italienne par Jerosme Sirtori. . . traduit a present en langue françoise par Jehan Theodore de Bry. fulio. Hanau, 1614 ; Rouen, 1627. 
Pratique du Cavalier, ou l'exercise de monter à cheval. By René de 1612.

Menou. 8vo. Paris, 1612 ; 1629. In 4to. Paris ; $1643 ; 1650$;

and three other editions.

Philippica, on Haras de Chevaux. By Jean Tacquet. 4to. Ant- 1614. werp, 1614.

Markhams Maister-Peece, containing all knowledge belonging to the 1615 .

Smith, Farrier, or Horse-leech, touching the Curing of all Diseases of Horses. By Gervase Markham. 4to. London, 1615.

Other editions:- 1636 ; 1675 ; 1683 ; 11th impression, 1710 ; 1723.

De Quadrupedibus solidepedibus volumen integrum. By Ulysses 1616.

Aldrovandus. folio. Bononiæ. 1616.

Several times reprinterl in the collected works.

Nuevos discursos de la gineta de España sobre el uso del cabezon. By Pedro Fernandez de Andrada. 4to. Seville, 1616.

Methode or Epitome, wherein is shewed his approuved Remedies for all Diseases whatever, incident to Horses, etc. By Gervase Markham. 8vo. London, $1616 ; 12 \mathrm{mo} .1623 ; 12$ mo. 1641.

Kriegskunst zu Pferde. By Jean Jacques de Wallhausen. folio. Frankfurt, $1616 ; 1641$.

The above wus printed by Paul Jacques, who also published a French translation, with the title:-Art de Chevalerie comprenant apres un advertissement necessaire touchant l'estat doulereux de la Chrestienté l'instruction de tonts avantages et dexteritez necessaire a chascun chevalier. Jamais publié par cy-devant mais maintenant pour le bien des Chevaliers, et de la noblesse, et touts amateurs de la milice. 4to. Frankfurt, 1616. Another French translation appeared later, as follows:-Art Militaire a Cheval, instruction des principes et fondements de la cavalerie et de ses quatres espèces, a scavoir: lances, corrasses, arquebus, et üragons. . . avec quelques nouvelles inventions de battaills, ordonées de Cavalerie. . . experimenté, descripte et représenté par plusieurs belles figures entaillées en cuivre. By André d'Aelst. folio. Zutphen, 1621.

Diversarum Gentium Armatura Equestris. Anon. 1617.

An Hipponomie or Vineyarl of Horsemanship, with the Art of breed- 1618. ing and dieting Horses. By Michael Baret 4to. London, 1618. 
1619. Arte di maneggiar la spada a piedi e a cavallo. By G. Batista Gajani. 4 to. Loano, 1619.

Las Reglas militares sobre el gobierno y servicio particular de la Cavellaria. By Galdericus Gali. folio. Milan, 1619.

1620. The Horsemans Honour, or the Beautie of Horsemanship as the Choise, Natures, Breeding, Breaking, Riding, and Dieting, whether outlandish or English Horses. With the true, easie, cheape, and most approved manner, how to know and cure all diseases in any Horse whatsoever. Not invented and drawne from foraigne Nations, but by long experience and knowledge of many years practice, and now published at the request of divers honorable and worthy persons for the generale good of this noble nation of Great Britain. (Anon, probably by Gervase Markham). $12 \mathrm{mo}$. London, 1620.

La Cavalerie Françoise et Italienne; ou l'art de bien dresser les Chevaux, selon les preceptes des bonnes écoles des deux nations. Tant pour le plaisir de la carriere et des Carousels, que pour la service de la guerre. By Pierre de la Noue. folio. Strasbourg, 1620 .

1621. Doctrina nueva de la gineta. By Bernardo de Vargas Machuca. 8vo. Madrid, 1621.

1622. Del modo, di conoscere la natura de' cavalli e le medecina appartenenti a loro. By Agost. Colombri. 4to. Venice, 1622.

1623. Maneige royal, où l'on peut remarquer le défaut et la perfection du chevalier en tous les exercises de cette art. . . fait et pratiqué en l'instruction du roy, par Antoine Pluvinel, son ecuyer principal. . . obl. folio. Paris, $1623 ; 1624 ; 1625 ; 1671$.

In German, A la ville de Brunvic, au dépens de Gotfreid Miuller, folio, 1626. The plates of this last are inferior copies of the original, by Crispin De Pas.

1624. Fiftie Years Practice: or an exact Discourse concerning SnaffleRiding. By T. Browne. 4to. London, 1624.

1625. L'essercito della Cavalleria, et d'altre materie. By Flaminio della Croce. 4to. Antwerp, 1625 ; folio. 1629.

The Souldiers Accidence, or an Introduction to Military Discipline; also the Cavalry, or Forms of framing Horse Troops. By Gervase Markham. 4to. London, $1625 ; 1626$.

Perfette regole et modi di cavalcare. By L. Palmieri. 4to. Venice, 1625 . 
Arte de enfrenar. By Francisco Perez de Navarrete. 4to, 1626. Madrid, 1626.

Delle Caccie. By Eugenio Roimonda, 4to. Naples, 1626.

Tratado da Gineta ordenado de vinte e quatro preguntas que hum 1629. Curioso lhe mandou preguntar. By Fr. Pedro Gallego. 8vo. Lisbon, 1629.

Discurso de albeyteria. By Baltasar Francisco Ramirez. 4to, Madrid, 1629.

De Militia Equestri, antiqua et nova, ad Regem Philippun IV. Libri 1630. quinque. By Hermann Hugo. folio. Antwerp, 1630.

Militarie Instructions for the Cavallrie, or Rules and Directions for the service of Horse, collected out of divers Forrain Authors, ancient and modern, and rectifled and supplied according to the practise of the Low Countrey Warres. By J. C. [Probably John Crusoe]. folio. Cambridge, 1632 ; and 1644.

Del arte de andar a caballo. By Gaspar Bonifaz. 1635.

1635.

The Faithful Farier, discovering some secrets not in print before. By Gervase Markham. 8vo. London, 1635; 1638 ; 4 to. London, 1649 .

La disciplina del cavallo con l'uso del piliero. By Gio. Paolo d' 1636. Aquino. 4to. Udine, 1636.

Coach and Sedan, Pleasantly disputing for place and precedence: the Brewer's Cart being Moderator. [By Henry Peacham]. 1636.

The Compleat Horseman and expert Farrier, in two Bookes. 1639. The first shewing the best manner of breeding good horses, with their choyce, nature, riding and dieting, as well for running as for hunting, and how the rider ought to behave himself in the breaking and riding of colts, as also teaching the groome and keeper his true office touching the Horses and Colts committed to his charge. and prescribing the best manner how a stable ought to be scituated and made; not hitherto so fully described by any; the second directing the most exact and improved manner how to know and cure all maladies and diseases of the horse, a work containing the secrets and best skill belonging either to Ferrier or Horse-leech: the cures placed alphabetically, with many hundreds of medicines never before imprinted in any Author, etc., etc. By Thomas de Grey. folio. London, $1639 ; 1651$; $1656 ; 1670$. 
1639. Del Arte de andar a cavallo. By Joannes de Valencia. 1639. La perfettione del cavallo. By F. Liberati. 4to. Rome, 1639.

1640. Reglas militares para el servicio de la Cavalleria. By Joannes Muñoz de Peral. 8vo. Saragossa, 1640.

1642. Direction and Order for the exercising of Horse and Foot. By General Lesley. 4to. London, 1642

Aphorismorum militarum disp. iv. de equitatu. By Georg Marschalk. 4 to. Soroe, 1642.

1643. A Dialogve betwixt a Horse of Warre and a Mill-Horse; wherein the content and safety of an humble and painfull life is preferred above all the Noyse, the Tumults, and Trophies of the Warre. Full of harmless Mirth and variety. Anon. 4to. London, 1643.

Exercicios de la Gineta. By Gregorio de Tapia y Salzédo. 4 to. Madrid, 1643.

1647. La vraye Cognoissance du Cheval, ses maladies et remedes, avec l'anatomie du Ruini. By T.T.D.E.M. folio. Paris, 1647.

1648. Act anent the Levies of Horse and Foot. 12mo. Edinburgh, 1648.

1650. A Persian treatise on Farriery, [Brit. Mus., ] translated from the Sanscrit by Abd Ullah Khan Behadoor Firüz Jang. Written in the 17 th century, 1650 .

Contents:-Introduction treating of the creation of the Horse and its colours, partly abridged from a Persian Faras Nîmeh, written in the time of Mahmud Ghaznavi. Knovledge of Horses and of their gond and bad signs, in 12 chapters. Diseases of the Horse and their treatment in 38 chapters.

An English translation of Abd Ullah Khân's version, has teen published by Joseph Earles. Calcutta, 1788.

Il Cavallo de Maneggio Libro, dove si tratta della nobilissima virtù del Cavalcare, etc. By G. B. di Galiberto. folio. Tienna, $1650 ; 1658 ; 1659$.

Manuale per l'ufficiale di Fanteria e Cavalleria. By Pietro della Valle. Firenze, 1650.

Animadversions on War, with notes on the Drilling of Horse Troops By Ward. London, Circa 1650. 
Traicté (de la cognoissance et maladies) des Chevaulx desdiè à la 1651. noblesse françoise. By R. Baret, sieur de Rouvray. 4to. Paris, 1651.

A Broadside prohibiting Horse Races. By Oliver Cromwell. 1654. folio. 1654 .

The Perfect Horseman, or the experienced secrets of Mr. Markham's 1656. Fifty years Practice, shewing how a man may come to be a general horseman by the knowledge of these seven offices, viz :Breeder, Feeder, Ambler, Rider, Keeper, Bnyer, Farrier. Pubu. by Launcelot Thetford, practitioner in the same art for the space of 40 years. Svo. London, $1656 ; 1671 ; 1673 ; 1680$.

A Discourse of the Knowledge of Beasts. By Le Chambre. 12mo. $165 \%$. London, 1657.

Méthode et invention nouvelle de dresser les chevaux, par Guillaume .

Marquis et comte de Newcastle, etc; œurre auquel on apprend à travailler les chevaux selon la nature, et parfaire la nature par la subtilité de l'art; traduit de l'anglais de l'auteur. folio. Anvers, 1657 ; folio. London, 1737.

Though in French and translated from the English, the edition of 1657 is the first printed edition.

Translated into English as:-The manner of feeding, dressing, and training of horses for the service of the Field in time of War, or for the exercise and improvement of gentlemen in the Academy at home; a science peculiarly necessary throughout all Europe and which has hitherto been so much neglected or discouraged in England, that young gentlemen have been obliged to liave recourse to Foreign Nations for this part of their education. folio. London, 1658.

Also translated into Portuguese under the title:-Arte de Cavallaria composto pelo Duque de Neucastel. By Manoel Telles da Sylva. But it only exists in MS.

Libro de albeyteria en el cual se cotienen muchas cosas curiosas y provechosas para los albeitares y otras que hasta hoy no se se han escrito. By Miguel de Paracuellos. 8vo. Sarragossa, 1658; 1702.

Il cavallo di razza reconoscinto dal segno de' marchi delle piu perfette razze del Veneziano. Lombardia, e parte dell Romagna. By Anania Zen. 16 mo. Venice, 1658.

A Proclamation to restrain the Abvses of Hackney Coaches in London and Westminster. 1660.

A Broadsicle. Another, Whitehall, 1687.

1658. 
1660. Compendio dell' eroica arte di cavalleria. By Aless. Malatesta Massari. Venice, 1660.

A Persian MS. on Farriery. Contents:-Mukaddimah. Creation and domestication of the Horse. Marhalah $I$. Its good and bad qualities, and other things relating to the knowledge of Horses in nine chapters. Marhalah II. Rules concerning the rearing of Horses and the running of races, in nine chapters. Marhalah III. Treatment of the diseases of the Horse, in nine chapters. Khre timah, on the Horses of the Shah and on Amulets. By Nizam Ud-Din Ahmad. written A.H. 1071. [A D. 1660].

1661. Il Mondo festeggiante, balletto a cavallo, fatto. . per le reali nozze di Cosimo terzo di Toscana et Maria Luisa d'Orleans. 4 to. Firenze, 1661.

Obras de Albeiteria. By Martin Arredondo. 4to. Madrid, 1661 ; Folio. Madrid, $1669 ; 1705 ; 1725$.

1662. Tesoro militar de cavalleria ; antiquo y moderno modo de armar cavalleros, y professar, segun las ceremonias de qualquier orden militar. By Jos. Micheli y Marques. folio. Madrid, 1662.

Norma seu regula armentorum equinorum rectè ac perfectè instituendorum ex optimis tum antiquis tum recentioribus auctoribus . . By Iohann Wilhelm v. Strubenberg. 4to. Vienna, 1662.

1663. Traité des emboucheures qui descouvre la methode de bien brider et emboucher les chevaux. By Jean Fouquet. 4to. Paris, 1663.

1664. L'art de monter à cheval, qui monstre la belle et facille methode de se rendre bonne homme de cheval. By le Sieur Delcampe. 8vo. Paris, 1664 ; 12mo. Paris, 1691.

Le Parfait Mareschal, qui enseigne a connoistre la beauté et les defauts des Chevaux, les signes et les causes des Maladies; les moyens de les prévenir, leur guerison et le bon ou mauvais usage de la purgation, de la saignée, etc. Ensemble un Traité du Haras, pour elever de beaux et de bons Poulains et les Préceptes pour bien emboucher les Chevaux, etc. By Jacques de Solleysel. 4to. $1664 ; 1685$. Other editioms $1746 ; 1754 ; 1775$. Augmented in 1746 by d'Eisenberg's Art de Monter a cheval.

An English translation of the above as follows: Compleat Horseman; discovering the surest marks of the Beauty, Goodness, Faults and Imperfections of Horses ; the signs and cures of their diseases; the true method both of their preservation and cure; with reflections on the regular and preposterous use of Bleeding and Purging. By Sir William Hope. folio. London, 1696; 1717. The sume abriaged by Sir William Hope. 8vo. 1711. 
Advis, on peut en France eslever des chevaux aussi beaux, aussi grands, 1666. et aussi bons, qu'en Allemagne, et royaumes voisins, etc. By Querbrat Calloet. 4to. Paris, 1666.

There is a later edition, s.d. with the title:-Beaux chevaux, qu'on peut avoir en France aussi beaux qu'en Espagne, Angleterre, etc. 4 to. Paris.

A new method and extraordinary invention to dress Horses, and work 1667. them according to nature; as also to perfect nature by the subtlety of Art. By William Cavendish, Duke of Newcastle. folio. London, $1667 ; 1677$.

This, as the Duke says himself, is not a translation of the "Methode et invention nouvelle" of 1657, but a different work which may be regarded as a supplement to it. It has been translated into French under the same title as the 'Methode et Invention,' folio. London, 1671, without plates, and re-translated by Solleysel with anotations, 4to. Paris, 1667. In German with the French by Pernauer, Baron de Pernay, folio. Nuremberg, 1700, with plates ; reprinted, folio. Nuremberg, 1674.

An edition of the whole works was printed, 2 vols, folio. London, 1743. There are some copies of this edition with a newo title dated 1738. It has also been printed at Nuremberg and at Paris, with additional plates. There is also a selection from the works entitled: "The Cavalier and the Lady," 8vo. Oxford, 1872.

Observations concerning a Blemish in a Horse's Eye, not hitherto 1668. discovered. By Richard Lower M.D., Phil. Trans. Abr. 1, 216. London, 1668.

La perfettione del cavallo. By Fr. Liberati. 4to. Rome, 1669.

Introduction de la Grace et belle Posture que le Cavalier doit avoir à Cheval tres utile aussi aux Femmes. Traité de l'Instruction du Cavalier pour le rendre Capable de dresser et emboucher toutes sortes de Chevaux. By le Sieur Du Breuil Pompee, Gentilhomme Poictevin. 12mo. Arnheim, 1669.

The Shepheard's Legacy ; or John Clearidge, his forty years experience 1670. . . . with some certain and assured cures for the Horse, Cow and Sheep. By John Claridge. 8vo. London, 1670.

Probably went through a great number of editions, afterwards re-appeared as the Shepherd of Banbury's rules, 1744, 1748, 1827 , etc.

Das Alleredelste Pferd der gantzen Welt. By E. Francisci. 8vo. Frankfort, 1670. 
1670. Tratado da Cavallaria da Gineta com a doutrina dos melhores Authores.

By Francisco Pinto Pacheco. 4to. Lisbon, 1670.

Much of this was tulien from a MS. of Jour de Ataide Azevedo.

Courses de Testes et de Bague Faittes par le Roy et par les Princes et Seigneurs de sa Cour en l'Année, 1662. By C. Perrault and E. Fletchier. folio. Paris, 1670.

This was afterwards issued in the series, entitled: "Cabinet du Roi."

1671. Le modelle du Parfait Cavalier. By Jean Fouquet. 8vo. Paris, 1671.

Practica et Arte di Cavalleria. By C. Lieb. folio. Utrecht, 1671.

Espejo del Cavallero de ambas sillas. By Antonius Ludovicus Ribero. 4to. Madrid, 1671.

De Re Vehiculari. By Jean Scheffer. 4to. Amsterdam, 1671.

1672. Reasons for Suppressing such Stage Coaches and Caravans as are unnecessary. By John Cresset. 1672.

The same pamphlet was re-published in the following year under the title of: "The Giand Concern of England Explained."

The Gentleman's Jockey, with Divers Curiosities, collected by J. H. 8vo. London, 1672.

Military Observations, or the Tacticks put into Practice, Collected and Composed for the Exercise both of Horse and Foot, to our present mode of Discipline, with the original of Ensignes and their duties and Postures of their Colours, hereunto is adder Sir Francis Veares's notes of direction for Officers. By Captain Thomas Venn. folio. London, 1672.

De Re Equaria Tractatio nova. By George Simon de Winter. folio. Nuremberg, 1672 and 1678.

The text in Latin, German, French and Italian.

1673. English Horseman and Complete Farrier. By Robert Almond. 8vo. London, 1673.

1674. The Gentleman's Recreation in four parts, viz:-Hunting, Hawking, Fowling and Fishing, wherein these exercises are treated of, etc. By Nicholas Cox. 8vo. London, $1674 ; 1677 ; 1686 ; 1697$; $1706 ; 1721$.

Palestra particular de los exercicios del Cauallo; sus propiedades, y estilo de Torear, y jugar las cañas; con otras diferentes demnstraciones de la Caualleria Politica. By Don Andres Davila y Heredia. 8vo. Valencia, 1674. 
Les devoirs militaires des officiers rle la cavalerie, contenant l'exercise des gens de guerre, selon la pratique de cet temps. By De la Fontaine. 12 mo. Paris, 1675.

In English as:-The Military duties of the Officers of Cavalry. Translated by A. Lovell. $12 \mathrm{mo}$. London, 1678.

Nouvelle Methode pour dresser les Chevaux en suivant la nature et mesme la perfectionnant par la subtilité de l'art. By Jacques de Solleysel. 4to. Paris, 1677.

Arte de Cavalleria de Gineta, e Estardiota ; bom primor de ferrar ; e Alveitaria, dividada em tres tratados, que contem varios discursos, e experiencias desta Arte. By Antonio Galvao de Andrade. folio. Lisbon, 1678.

Description of the Nature of Four-Footed Beasts. By John Bryce. folio. Amsterdam, 1678.

Bellerophon sive eques peritus, hoc est Artis Equestris, accuratissima institutio, opere bipartito seu duobus Libris, absoluta : etc., etc. By George Simon de Winter. folio. Nuremberg, 1678.

In Latin and German.

Hippiater Expertus, seu medicina equorum absolutissima, tribus libris comprehensa. By George Simon de Winter. 2 vols, folio. Nuremberg, 1678.

In Latin and German.

L'écuyer françois, qui enseigne à monter à cheval, à voltiger, et à bien 1679 . dresser les chevaux. [By Imbotti de Beaumont]. 8vo. Paris, $1679 ; 1682$ or 1685 .

There should be at the end of the volume some extra sheets, viz: $A-F 3$, containing 35 plates and explanation; originally $p u b$. lished separately in 1679.

Instruçaō da Cavallaria da Brida com hum copioso tratado da Alveitaria. By Antonio Peregra Rego. 4to. Coimbra, 1679; 1712.

Practica y observaciones pertencientes al Arte de albeytaria, en que se manifiesta el modo particular con que se deben curar las màs graves causas, que se pueden oferecer en esta arte. By Juan Alvarez Borges. 4to. Madrid, 1680.

Les Arts de l'homme d'epée, ou le Dictionnaire du gentilhomme, divisé en trois parties, dont la première contient: l'Art de monter à cheval; la seconde: l'Art militaire; et la troisième : l'Art de la navigation. By La Sieur de Guillet. 12mo. La Haye, 1680; 1682-83. 
1680. The Countryman's Companion, or a new method of orlering Horses and sheep, so as to preserve them both from disease and casualties and to recover them if fallen ill. By Thomas Tryon. $12 \mathrm{mo}$. London, $1680 ; 1700$.

1681. The Gentleman's Jockey, and approved Farrier, instructing in the natures, causes and cures of all diseases incident to Horses. [By Gervase Markham]. 12mo. London, $1681 ; 1687 ; 1717 ; 1722$.

1683. Le parfait chasseur. By de Selincourt. 12mo. Paris, 1683.

The Anatomy of an Horse, containing an exact and full description of the Frame, situation and connexion of all his parts, (with their actions and uses,) exprest in 49 copper plates. To which is added an appendix containing two discourses, the one, of the generation of animals, and the other of the motion of the chyle and the circulation of the blood. By Andrew Snape, Junr., Farrier to his Majesty [Charles II.] folio. Lonclon, 1683.

Translated into French as: Anatomie général du cheval. By F. A. de Garsault. 4to. Paris, 1732 ; $1734 ; 1737$.

1685 Verdadera albeyteria dividida en cuatro libros juntos en una magnitud. Lleva diferentes estampas, donde van delineadas las enfermedales que sobrevienen en el cuerpo, brazos y piernas del caballo; y van enumeradas y los numeros corresponden con mucha claridad a los capitulos que cada uno pertenece. By Pedro Garcia Conde. folio. Madrid, 1685 ; Barcelona, 1734.

1686. Breve paràfrasis de albeyteria sacado de varios autores. By Miguel Nicolas Ambrùs. 4to. Saragosa, 1686.

The Gentleman's Recreation. In Two Parts. The first being an Encyclopedy of the Arts and Sciences. . . . The second part, Treats of Horsemanship, Hawking, Hunting, Fowling, Fishing and Agriculture, with a short Treatise of Cock Fighting. . . all which are collected from the most authentick Authors. . The whole illustrated with about an Hundred Ornamental and useful Sculptures, Engraven in Copper, etc. By Richard Blome. folio. London, 1686. With additions, folio. $1710 ; 1735$.

1688 The Compleat Jockey,or the most exactrules and methods to be observed for the training up of Race Horses. An on. 4to. London, 1688.

Les Fonctions du Capitaine de Cavalerie et principales de ses officiers, subalterns. By le Sieur de Birac. $12 \mathrm{mo}$. La Haye, 1688.

A later edition, with title as follows:-Les Functions d'un Capitaine de Cavalerie etd'Infanterie. Les Devoirs de l'homme de guerre et aussi la pratique de la guerre. $12 \mathrm{mo}$. La Haye, 1693. 4 parts. 
Also a translation in English, entitled: The Duties of Officers of 1688. Horse. The Art of War, etc. Trans. by an English Officer. $12 \mathrm{mo}$. London, 1707.

Experienc'd Farrier, or a compleat 'Treatise of Horsemanship. By 1691. E.R. 4 to. 1691-92.

L'arti di ben conoscere e distinguere le qualita de' cavalli, d'introdurre 1692. e conservare una razza nobile e di risanare il cavallo da mali à quali soggiace, etc. By Marino Garzoni. 4to. Venice, 1692; $1697 ; 1713 ; 1733 ; 1750 ; 8$ th edition, 1784 .

Arte de herrar caballos, Aora nuevamente compuesto. By Bartolome 1694. Guerrero Ludeña. folio. 1694; Madrid, 1735.

Account of an Extraordinary Cure of a Horse, which was staked in 1695. the stomach. By Dr. John Wallis. Phil. Trans. Abr. iv., p. 65. Londun, 1695 .

L'arte del cavallo ovvero l'arte di ridurre a tutta perfettione il cavallo 1696. ed il modo di usarlo in guerra ed in festa. By Nicolo and Luigi Santa Paulina. folio. Padua, 1696.

Novello giardino della pratica ed esperienza medica intorno a' morbi 1699. de' cavalli. By Gio. Battista Trutta. 4to. Naples, $1699 ; 1770$.

The Countryman's Companion, or a new method of Urdering Horses 1700. and Sheep so as to preserve them both from Diseases and Casual ties, etc. By Philotheos Physiologus. Anon. 12mo. London, [N.D. Circa 1700.]

Avizos para Novatos da Cavalleria. By Manoel Barradas Soria. [MS. Circa 1700.]

New Drawing Book of Horses, designed by Chevalier le Clerk, Vandermulin. etc., proper for Painters, and adapted for Youth to draw after. Obl. 12mo. London, [N.D. Circa 1700.] Chevalier le Clerk died 1714, and Vandermulin died 1690.

Grand Mareschal expert et Français. Anon. Toulouse, 1701.

1701.

Gentleman's Dictionary in the Art of Riding the Great Horse, Military 1705. Art and Navigation. Anon. 8vo. London, $1705 ; 1707$.

Doctrina del cavallo, Y Arte de enfrenar. By Gregorio de Zuñiga Y Arista. 4to. Lisbon, 1705.

The Gentleman's New Jockey, or Farrier's Approved Guide, with a 1708. second part, containing many rare and new secrets. By L.G.

12 mo. London, 1708. 
1709. Racing Calendar, containing an account of the Plates, Matches and Sweepstakes run for in Great Britain and Ireland from the year 1709. Anon.

1710. Practica de Cavallaria onde se contem Arte de ensinar, conhecer e criar os Cavallos. Arte de enfrear os Cavallos à brida com estampas de todos os freyos, e arte de ferrar os Cavallos. By Joaó Teyxeira de Sampayo. folio. (MS., Circa 1710.)

1711. Pietra paragone de' cavallieri, divisa in cinque libri. By Giuseppe Allessandro, duca di Peschiolanciano. Folio. Naples, 1711; 1723 .

1712. La Connoissance parfaite des Chevaux, contenant la manière de les gouverner, nourrir et entretenir en bon corps et de les conserver en santé dans les voyages, etc. Et l'art de monter à Cheval et de dresser les Chevaux de manége; Tiré non seulement des meilleurs auteurs qui en ont ecrit, mais encore des memoires manuscrits de feu Monsieur Delcampe. Edited by Louis Liger. 8vo. Paris, 1712 ; 1730.

1714. Premier des magnifiques carosses de Monseigneur le I) uc d' Ossuna Ambassadeur extraordinaire et premier plenepotentiaire de Sa Majesté Catolique Philippe V. pour la Paix faits pour l'entrée publique de son excellence à Utrecht, 1713. By B. Picart. folio. Amsterdam, 1714.

1716. A Persian MS. entitled Risalah Faras Nameh. By Muhammad Ali Hazin. Written in Ispahan, A.H. 1127. [A.D. 1716.]

1717. Compendio de albeyteria. By Fernando Sande y Lago. 4to. Madrid, 1717, and 1729.

1718. The Compleat Sportsman, containing Hunting, Shooting and Fishing. By Giles Jacob. 8vo. 1718.

1719. L'Art de toute sorte de chasse et de pêche, avec celuy de guerir les chevaux, les chiens et les oiseaux. Et un Dictionnaire de la chasse, et de la pêche, avec une explication des termes de la fauconnerie mis en dialogue. Anon. 2 vols. 8vo. Lyon, 1719.

1720. The Farrier's New Guide, containing, First, the anatomy of a Horse, being an exact and compendious description of all his parts, with their actions and uses, illustrated with figures curiously engraved on copper plates ; Secondly, an account of all the diseases incident to horses, with their signs, causes and methods of cure, wherein 
many defects of the Farriers' practice are now carefully supplied, their errors exposed and amended, and the Art greatly improved and advanced, according to the latest discoveries. The whole interspersed with many curious and useful observations concerning feeding and exercise. By William Gibson. 8vo. London, $1720 ; 1721 ; 1725 ; 1720 ; 1727 ; 1729$.

True Method of Dieting Horses. By William Gibson. 8vo. 1721. London, $1721 ; 1726$.

The Farrier's Dispensatory. By William Gibson. In 3 parts. 8vo. London, $1721 ; 1729$.

Trattato sopra le qualità del buon cavallo. By Aluise Dandolo. 1722. 4to. Padua, 1722.

L'Art de monter à cheval. By Joh. Elias Ridinger. folio. Augsburg, $1722 ; 1744$.

The original designs for the engravings of the above work were brought from Germany 80 or 90 ye ars ago, by an Earl of Kintyre, and given by him to the late Sir Robert. D. H. Eilphinstone, of Logie Elphinstone, Aberdeenshire, where they now are, in possession of his son, Sir James D. H. Elphinstone, Bart., M.P. The drawings, 22 in number, are excutes in Indian ink, signed and dated.

Acconnt of a Stone taken ont of a Horse. By Paul Dudley. 1724. Philosophical Transactions, vol. vii., p. 187. London, 1724.

Detail instructif du haras. By A. Guerini. 12mo. Cambray, 1724.

Manuel des Ecuyers, ou reciieil des differens remèdes pour la guérison des maladies qui arrivent aux Chevaux et autres animaux servant à l'utilité de l'homme. By Carbon de Begrieres. Paris, 1725.

The Farrier's and Horseman's Dictionary : being a Complete System 1726. of Horsemanship. Anon. 8vo. London, 1726.

Cartilla, en que se proponen las Reglas, para Torear à Caballo, y practicar este Valeroso, Noble Exercicio, con toda destreza By Nicolas Rodrigo Noveli. 8vo. Madrid, $172 b$.

Templador veterinario de la furia vulgar, en defensa de la facultad veterinaria, ó medicina de bestias. Y de los albeitares peritos y doctos. Asimesmo contra el desprecio que de todos hace el doctor D. Francisco Suarez de Rivera en su Templador medico. Y manifesto de qe la albeiteria medicina y cirujia es toda una ciencia 0 arte. By Francisco Garcia Cavero. 4to. Madrid, 1727. 
1727 Description du Manége Moderne dans sa perfection. By the Baron d'Eisenberg. obl. 4to. Paris, 1727.

Reprinted at La Haye, unter the title of "L'Art de monter " Cheval on Description du Manege Moderne." 4to. 1733; 1737 ; 1740. Another edition, Amsterdum and Leipsic 4to. 1749, to which is added the "Dictionnaire des Termes du Manege," and sometimes the "Anti-Maquignonage." "L'Art de monter" has 59 plates by Picart, and an engraved title page. The "AntiMaquignonage" has 9 plates.

Dend vel indrettede og proberede Heste-Cuur eller Lœge-Bog, som indeholder adskillige forsogte Raad og Midler for Heste, etc. By Peter Esbach. Copenhagen, 1727.

1728. Curacion racional de irracionales y conclusiones veterinaras deducidas de diferentes principios filosoficos, conque se prueba ser la medicina cirujia y albeiteria una misma ciencia ó arte. By Francisco Garcia Cavero. 4to. Madrid, 1728.

1729. Veterinaria apologética curacion racional de irracionales. Organo donde se tocan las inconsecuencias con sus altos y bajos, destemplados mas que armonicos, del doctor D. Antonio Monrava y Roco. By Francisco Garcia Cavero. 4to. Madrid, 1729.

Historical List of all Horse Matches run in England and Wales in 1727 and 1728. 2 vols. By J. Cheny. $12 \mathrm{mo}$. London, 1729. Continued at various dates in 24 vols. up to 1750 .

1730. The Gentleman's Pocket Farrier, shewing how to use your Horse on a journey and what remedies are proper for common misfortunes that may befall him on the road. By Capt. William Burdon. 8 vo. London, 1730 ; 1732; 1735; 1737; 1748; 12mo. Armagh, 1806.

1731. Apéndice dogmatico al Templador. By Francisco Garcia Cavero. 4to. Madrid, 1731.

Specimen of a work on Horse Hoeing Husbandry. By Jethro Tull. 4to. London, 1731 ; fol. 1733 ; 1739 ; 8vo. 1772 .

This has been translated into French.

1732. Coloquios de Albeiteria. By Francisco Benavides. 4to. Madrid, 1732.

Adicion racional y metodica à la curacion de la lupia tumorosa y repuesta a los coloquios de albeiteria. By Francisco Garcia Cavero. 4to. Madrid, 1732.

Consulta contra la que hizo Cavero sobre la curacion de una lupia tumorosa. By Jose Andres de Morabda. 4to. Madrid, 1732. 
Essay on Hunting, by a Country Squire. Anon. 8vo, 1733. Reprinted 8vo. and 4to. 1820.

1733.

Fonctions et devoirs d'un officier de cavalerie. By Jean Charles de Folard. 12mo. Paris, 1733.

Ecole de cavalerie, contenant la connoissance, l'instruction, et la conservation du cheval. By $\mathrm{Fr}$. Robichon De La Guerinière. folio. Paris, 1733.

Other editions:-Paris, Guerin, 2 vols., 8vo. 1736 ; Paris, 2 vols., 8vo. $1751 ; 1754 ; 1756 ; 1769$; Metz, 8vo. 1802.

Manejo real, en que se propone lo que deben saber los Cavalleros en esta facultad, para llenar con practica este grand nombre; y lo que si gustare en aplicacion, podràn adelantur para saber por si hacer un cavallo, como qualquiera Picador. By Manuel Alvarez Osorio y Vega. Madrid, 1733 ; 4to. Valladolid, 1741 ; 4to. Madrid, 1769.

Adicion á los coloquios. By Francisco Benavides. 4to. Madrid, 1r34. 1734.

Le nouveau manége, représentant l'homme de cheval parfait dans tous ses exercises, inventé et exposé en 26 planches. Expliquées en franç, en allemand et en latin. 2 parts. By John Elias Ridinger. folio. Augsburg, $1734 ; 1736$.

Llave de albeyteria, Primera, y Segunda Parte, en que se trata de todos los Principios asi antiguos como, modernos de la Facultad, declarando muchos Phenomenos y Arcanos, que nuestros Authores no los alcançaron, deseando con ellos hazer un Albeytar consnmado. By Domingo Royo. folio. Saragosa, 1734.

La parfaite connoissance des chevanx, leur anatomie, leur bonnes et mauvaise qualitez, leurs maladies et les remèdes qui y conviennent. By Jean and Gaspard de Saunier. folio. La Haye, 1734.

An Fnglish translation of the above, entitled: "Guide to the perfect knowledge of Horses, wherein everything necessary for the choice, management and preservation of that noble animal is clearly laid down." 8vo. London, 1769.

In German: Vollstandige Erkenntniss von Pferden, ihren Eigenschaften, Krankheiten und Mitteln dariber. Translated by C. H. Wilken. folio. Glogau, 1767.

Sportsman's Dictionary, or Gentleman's Companion, containing full instructions for Angling, Hunting, Cocking, Hawking, Racing, etc. By H. J. Pye. 8vo. 2 vols. London, 1735 ; 4to. 1778 ; 4to. 1782 ; 8vo. 1800 ; 4to. 1807. 
1735. Principios compendiosos de albeiteria. By Jose Perez Zamora. 8vo. Madrid, 1735.

1736. Deleyte de cavalleros, y placer de los cavallos. By Maestre de San Juan Lucas. folio. Madrid, 1736.

1737. Farrier's Assistant. By Allen. 8vo. London, 1737.

1738. Farriery improved, or a compleat treatise upon the art of Farriery, wherein is fully explained the nature, structure and mechanism of that noble and useful creature, a Horse; the diseases and accidents he is liable to and methods of cure set down in as clear and intelligible manner as the subject will admit of; the use and abnse of the science discovered, whereby any gentleman may be able to judge for himself whether or no he is imposed upon by ignorant grooms and other pretenders to this Art. Together with many necessary and useful observations and remarks concerning the choice and management of horses, likewise an account of drugs and merlicines used in Farriery, with some remarks on their genuineness and adulteration, and their several prices, set down alphabetically at the end of the work. By Henry Bracken. 8 vo. London, 1738 ; 12mo. 2 vols. $1739 ; 12$ mo. 2 vols. 1743 . 5th edition, 12 mo. 2 vols. 1745 . 6th edition, 12mo. 1749. 9th edition, $12 \mathrm{mo} .1756$.

In German as:-Verbesserte Rossarzneikunst. 8vo. Altenburg, 1758 .

Libro nuevo, bueltas de escaramiza, de Gala, a la Gineta. By Bruno José de Morla Melgarejo. 4to. Madrid, [1738.]

1739. Supplement to the New Horse Hoeing Husbandry. By J. Tull. London, 1739.

1740. Instituciones de albeyteria, y examen de praticantes di ella; divididas in seis tratados. By Francisco Garcia Cavero. 4to. Madrid, 1740 ; 4to. Alcala de Henares, 1789.

Another erition with the "Adiciones" of 1756, 4to. Madrid, 1792.

Eléments de Cavalerie. By Fr. Robichon de La Gueriniere. 12mo. Paris, $1740 ; 1741 ; 1754 ; 1768$.

Another edition of this work, under the title of Manuel du Cavalier, 8vo. La Haye, 1742.

1741. Le Nouveau Parfait Maréchal, ou la Connoissance générale et universelle du Cheval, divisé en sept traités. By F. A. de Garsault. La Haye, 1741 ; 4to. 1770 ; 1778 ; 4to. Paris, 1805 ; 1843. 
The Traveller's Pocket Farrier, or a Treatise upon the distempers and 1742. common incidents happening to horses upon a journey, being very useful to all gentlemen and tradesmen who are obliged to travel the countries. By Henry Bracken. $12 \mathrm{mo}$. London, $1742 ; 1743$. 5th edition, 1750 .

Sanidad del caballo y otros animales sujetos al arte de albeiteria, ilustrada con el de herrar. By Salvador Monto y Roca. 4 to. Valencia, 1742 .

Le nouveau Newcastle, ou nouveau traité de cavalerie géometrique et pratique. [By Claude Bourgelat.] 8vo. Lausanne and Geneva, 1744. $12 \mathrm{mo}$. Lyons, 1771 .

Nachrichter's nuitzliches und aufrichtiges Pferd-und-Ross-Arzneibuch, in welchem die innerlichen Krankheiten und ausserlichen Zustärde der Rosse auf das deutlichste erklärt werden, sammt Beifiigung der dazu gehorigen approbirten Recepte. Nebst einem Anhang von Rindvieh Arzneien. By Johann Deigendesch. $16 \mathrm{mo}$. Tiibingen, 1744 . A new edition, $16 \mathrm{mo}$. Stuttgart, 1857.

Observaciones practicas de albeiteria. By Sebastian Robredo y Villaroya. 4to. Valencia, 1744.

An account of a large stone found in the colon of a Horse, several 1746. stones taken from the intestines of a Mare. Phil. Trans., 1746, Abr. ix, p. 278. By Edward Bailey.

Dictionnaire des Termes du Manége moderne, pour servir de supple- 1747. ment à L'Art de monter à Cheval. By Baron d' Eisenberg. obl. 4to. Amsterdam and Leipzig, 1747.

This Dictionnry, which was published separately in 1747, was afterwards added to the 1759 edition of "L'Art de Monter a Cheval," by the same Author.

Histoire Naturelle, générale et particulière. By George Louis 1749. Leclerc, Count de Buffon. 4to. 44 vols. Paris, 1749 ; 1804. For the bibliography of the French editions of this work, see Brunet. Among the English translations is one from his Natural History of the Horse, to which is added that of the Ass, Bull, Cow, Ox, Sheep, Goat and Swine, with accurate descriptions and full directions for Breeding, Chusing, Feeding and Improving these useful creatures. 8vo. London, 1762.

Traité sur le véritable siége de la morve des chevaux, et les moyens d'y remédier. By Etienne Guillaume Lafosse. 8vo. Paris, 1749. 
1749. A translation of the above into English, with notes, by $H$. Bracken, as: "A treatise upon the true seat of the Glanders in Horses, together with the Method of Cure." 12mo. London, 1751.

In German:-Abhandlung von dem wahren Sitze des Rotzes bei den Pferden. Translated by D. G. Schreiber. 8vo. Halle, 1752. Another translation:-Gedanken iber den Rotz der Pferde. Vom Wurm der Pferde. Translated by F. C. M. Scheibeler. 8vo. Hannover, 1795 ; in the "Sammlung merkwurdiger Abhandlungen über Thierkranliheiten." Also Abhandlung uiber den Rotz der Pferde. Translated by Jh. Ant. Meier. 8vo. Vienna, 1871.

Les vrais principes de la Cavalerie. By Gaspard de Saunier. 12mo. Amsterdam, 1749.

1750. Elémens d' hippiatrique, ou nouveaux principes sur la connoissance des Chevaux. By Claude Bourgelat. 2 vols. in 3, 8vo. Lyons, 1750-53.

In English as :-A New System of Horsemanship. Translated hy Richard Berenger. 4to. London, 1754.

A new Treatise on the diseases of Horses, wherein what is necessary to the knowledge of a horse, the cure of his diseases and other matters relating to that subject, are fully discussed in a plain and easy manner, from many years' practice and experience, with the cheapest and most efficacious remedies. By William Gibson. 4to. London, 1750 ; 2 vols. 8vo. London, 1754.

In German as :-Abhandlung von den Krankheiten der Pforden und ihrer Heilung, etc. Translated by J. C. Ch. Koch. 8vo. Göttingen, 1780.

Manêge et équitation. By Harguinez. folio. Paris, [Circa 1750].

Account of a Horse bitten by a mad Dog. By John Starr, M.D. Phil Trans., Abr. x, p. 43, 1750.

1751. Arte de enfrear Cavallos. By Pedro da Sylva. MS. N.D.

This is probably the same work that was translated into Spanish as:-Cartilla de enfrenar para cavalleros, aficionados al arte de montar a cavallo. Translated by Santiago Romero. 4to. Madrid, 1751.

1752. No Foot, no Horse, an Essay on the Anatomy of the Foot of that noble animal, a Horse, wherein each part is accurately described, their structure, use and conformation considered; the disorders each part is liable to are treated of and proper remedies for the cure of each case are offered, together with particular directions for the cure of some of the chief internal diseases which Horses are subject to. By Jeremiah Bridges. 8vo. London, $1752 ; 1759$. 
In German as:-Versuch einer genauen Zergliederung der Pferdefuisse, Behandlung ihrer Zufälle und die nöthigen Mittel dagegen. 1752.

Historical List of Horse Matches run and of Plates and Prizes run for in Great Britain and Ireland in 1751. By Reginald Heber. 12 mo. London, 1752.

Türkischer Pferdesaufputz sammt e. d. nöthigen Anmerkungen hierzu enthalten. By John Elias Ridinger. folio. Augsburg, 1752.

Sammlung von Nationen Pferden. By John Elias Ridinger. obl. fol. Augsburg, 1752.

Instructions sur l'exercise de la Cavalerie du 29 Juin, 1753. Anon. 1753 4to. Paris, 1753.

Instructions sur le service que les regimens de cavalerie devront faire dans les camps . . . 29 Juin, 1753. Anon. 4to. Paris, 1753.

La Perfezione ed i Jifetti del Cavallo. By Baron d' Eisenberg. folio. Florence, 1753. In Italian and French.

The Sporting Kalendar, containing a distinct account of what Plates and Natches have been run for in 1752. By John Pond. $12 \mathrm{mo}$. London, [1753] etc.

Kurzer und deutlicher Unterricht zur Anweisung eines jungen Cavaliers im Reiten, etc. By Jos. Eph. Zehentner. 8vo. Frankfort-onOder, 1753. 3rd ed. 1769 ; 4th ed. 1785 .

Instruction sur le service que les regimens de Cavalerie devront faire 1754 dans les camps ... 14 Mai, 1754. Anon. 4to. Paris, 1754.

Instruction sur l'exercice de la cavalerie, du 14 Mai, 1754. Anon. 4to. Paris, 1754 .

The Gentleman's Farriery, or a Practical Treatise on the Diseases of Horses : wherein the best writers on that subject have been consulted, and M. La. Fosse's method of Trepanning Glandered Horses is particularly considered and improved: also a new invented method of Nicking horses is recommended. By J. Bartlett. 8vo. London, $1754 ; 1756 ; 1777$.

In German as :-Pharmacopie oder Apotheke eines Rossarztes ; nach der dritten Ausgabe mit einer Vorrede vom Rotze. Translated by W. H. Seb. Bucholz. 8vo. Weimar, 1778. 
1754. Observations et decouvertes faites sur des chevaux, avec une nouvelle pratique ser la ferrure . . By Etienne Guillaume Lafosse. 8vo. Paris, $1754-56$.

The 'Nouvelle Pratique' has a separate title, but the pagination is continuous.

An English translation as follows:-Observations and Discoveries made upon Horses, with a new method of Shoeing. By the Sieur La Fosse. 8vo. London, 1755.

In German:-Neue Entdeckungen an Pferden, zum Behuf der Armeen, Landwirthe, Kurschmiede, etc. Translated by D. G. Schreber. 8vo. Halle, 1759.

In Spanish :-Nueva practica de herrar los caballos de monte $\mathrm{y}$ de coche á fin de precaverlos de muchas desgracias, $\mathrm{y}$ hacerlos firmes en todo tiempo sobre el empedrado aunque sea de losas: con algunas observaciones y descubrimientos sobre los caballos, y con un tratado pequeno sobre el verdadero sitio del muermo, y los medios para remediarlo: y un reniedio muy seguro para detener la saugre, sin ligadura, de las gruesas arterias cortadas. Translated by Pedro Pablo Pomar. 4to. Madrid, 1760.

Institutions Militaires pour la Cavalerie et les Dragons. By Major de La Porterie. 8vo. Paris, 1754.

Les Chevaux, les Mulets, le Asnes. By John Elias Ridinger folio. 1754-55.

Kurzer und gründlicher Unterricht von der Pferdezucht. By Jos. Eph. Zehentner. 8vo. Berlin, 1754; 8vo. Berlin, 1770.

1755. A short practical method of cure for Horses. By William Gibson. 8vo. London, 1755.

Extracted from his "New Treatise on the diseases of Horses," (1750) with notes of reference to the original; to which is added an Appendix on the right method of Fireing and Shoeing.

1756. Rules and Directions for all such persons who are to find Horse or Foot in the Militia. Anon. London, 1756.

Instructions sur le service que les regiments de Dragons devront faire dans les camps qui s'assembleront pendant la present année 1756. Anon. 4to. Paris, 1756.

Essai sur la Cavalerie, tant ancienne que moderne. By Charles Louis d'Autheville des Amourettes. 4to. Paris, 1756.

Adiciones á las instituciones de albeiteria. By Francisco Garcia Cavero. 4to. Madrid, 1756.

A posthumous work. Republished with the "Instituciones." 4to. Madrid, 1792. 
Traité des voitures, pour servir de supplément au nouveau parfait maréchal, avec la construction d'une berline nouvelle nommée inversable. By F. A. de Garsault. 4to. Paris, 1756.

A Dissertation on Horses; wherein it is determined by matters of fact, as well as from the principles of philosophy, that Innate qualities do not exist, and that the excellence of this Animal is altogether Mechanical, and not in the blood. By William Osmer. 8vo. London, $1756 ; 1757$.

L'art de la Cavalerie, ou la manière de devenir Bon Ecuyer, par des règles aisées et propres à dresser les Chevaux à tous les usages, qui l'utilité et le plaisir de l'homme exigent, etc. By Gaspard de Saunier. folio. Amsterdam and Berlin, 1756; Paris, 1756.

Doctor Daniel Dove and his Horse Nobs: Nonpareil or the quint- 1757. essence of Wit and Humour, to which is added an Index to Mankind. 12mo. London, 1757.

Arte de andar a cavallo, dividido en tres partes. La primera ; contiene las reglas, y methodo de poner al Cavallero Discipulo à cavallo, con la enseñanza que necessita para saberlo mandar en todos los manejos, puesto en Dialogo. La Segunda : el methodo de criar, y enseñar el potro hasta el estado de perfeccion, con las reglas mas essenciales para embridar los Cavallos. La tercera : el methodo de manejar la cuerda, con todas las operaciones que se deben hacer con ella, el efecto que causan, assi para la crianza de Potros, como para los resabios, ó defensas, que generalmente se hallan en los Cavallos. By Francisco Pasqual Bernad. 4to. Madrid, 1757.

Il dilettante de' cavalli istruito. By Francesco Bonsi. Venice, 1757 ; Florence, N.D.

The Art of Farriery both in Theory and Practice; containing the Causes, Symptoms, and Cure of all Diseases incidental to Horses, ete. By John Reeves. 8vo. London, $1757 ; 1763$.

A Compendious Treatise of Farriery, wherein are set forth in a plain familiar manner the Disorders incident to Horses and their respective cures. By John Wood. 8vo. London, 1757.

Griindliche Abhandlung von der Kunst Pferde zu kennen. By Jos. Eph. Zehentner. 8vo. Frankfurt-on-Oder, 1757; 1766; 1775. 
1758. Treatise on the Diseases of Horses. By J. Bartlett. 8 vo. London, 1758 ; London, 1764.

1759. Every man his own Farrier; or the best methods of preventing and curing the injuries and diseases of that truly valuable creature, a Horse; laid down in a different manner from which has hitherto appeared on this subject, chiefly from cases and facts, and performed by the cheapest ingredients. By William Ellis. 8vo. London, 1759.

A Treatise on the Diseases and Lameness of Horses, in which is laid down a proper method of shoeing (in general) and treating the different kinds of feet. To which are added some new observations in the Art of Farriery, and on the nature and difference of Horses, shewing on what principles their perfection depends, and by what method their Breed may be greatly improved and amended. By William Osmer. 8vo. London, 1759. 5thed. 8vo. London, 1830.

1760. On the Stony concretion taken from the colon of a Horse. By Henry Baker. Phil. Trans. 1760, xi. p. 484.

Del Moto a Cavallo. By G. Benvenuti. Lucca, 1760 .

Der sichere und wohlerfahrne Huf-und Reitschmied. By Jh. Adam Kersting. 8vo. Göttingen, 1760, 1777.

Unterricht Pferde zn beschlagen und die an den Füszen der Pferde vorgefallene Gebrechen zu heilen. By Joh. Adam Kersting. 8vo. Göttingen, 1760. 3rd edition, 1794 .

Vorstellnng und Beschreibung der Schul und Campagne Pferden nach ihren Lectionem, und in was für Gelegenheiten solche können gebraucht werden. By John Elias Ridinger. folio. Augsburg, in German and French, 1760; reprinted, 1766.

Sportsman's Companion, containing the Portraits and Pedigrees and Performances of Forty of the most eminent Horses, drawn and engraved by J. and H. Roberts. 8 vo. London, 1760 ; and 8 ro. London, 1820.

1761. A Military Essay on the Raising, Arming, Clothing and Discipline of the British Infantry and Cavalry. By Campbell Dalrymple. 8vo. Londen, 1761.

Militair Handbock voor de Kavalery. By L. W. F. van Oebschelwitz. 's Hage, 8vo. 1761. 
Military Equitation, or a method of Breaking Horses and teaching 1761. soldiers to ride, designed for the use of the Army. By Henry, Earl of Pembroke. $12 \mathrm{mo}$. London, 1761 ; sm. 4 to. London, 1778 ; sm. 4to. London, 1793.

A French translation, by Bergeret de Trouville, 8vo. Paris, 1784.

In Italian as :-Equitazione Militare, o sia metodo di scozzonare i Cavalli. 4 to. Naples, 1788.

Description du Cheval selon ses poils principaux et leurs diverses divisions. By John Elias Ridinger. German and French, 4to. Augsburg, 1761.

Remarques de Carousels. By John Elias Ridinger. 4 to. 16 plates, Augsburg, 1761.

Generally found in the same volume as 'Représentation et description de toutes les leçons, etc.' 4to. Augsburg, 1760.

Dissertazioni storiche e eritiche sopra la cavalleria antica e moderna, secolare e regolare, con note, e molte figure in rame. By Onorato Santa Maria da Carmel. 4to. Brescia, 1761.

Rules for Bad Horsemen, addressed to the Society for the Encourage1763. ment of Arts "Nescit equo rudis Hœrere" Hor. By Charles Thompson. 8vo. London, $1763 ; 1765$.

A new edition, with additions by J. Hinds, 12mo. London, 1830.

Anecdotes relating to the antiquity and progress of Horse Races for $\mathbf{1 7 6 4 .}$ above Two Thousand years. Anon. 8vo. London, 1764.

A Philosophical Enquiry into the Origin of the Distemper among Horses, with the method of Cure, and also into Epidemics in general. By a Physician. Anon. 8vo. London, 1764.

Pharmacopœia Hippiatrica, or the Gentleman's Farrier's Repository of elegant and approved Remedies for the Diseases of Horses, in two Books. By J. Bartlett. 8vo. Eton, 1764. 174 ₹n.

Anti-Maquignonage, pour éviter la surprise dans l'emplette des Chevaux. By Baron d'Eisenberg. obl. 4to. Amsterdam, 1764.

Generally found bound with "L'art de monter is Cheval" by the sume author.

In German:-Entdeckte Rosstäuscherkunste zur Vermeidung der bei den Pferdekaufen vorfallenden Betrügungen, worinnen von der Vervollkommenheit und den Mängeln der Pferde behandelt, auch alles durch nöthige Kupfer erlautert wird. By Rosenzweig. 8vo. Leipzig, 1766. 
1764. Recherches sur l'époque de l'équitation, et de l'usage des Chars équestres chez lez anciens. By Gabriel Fabricy. 2 vols. 8 vo. Rome, $1764 ; 1765$.

A Description of the Virtues and Uses of the Preservative Electuary against the Glanders in Horses. By the Baron de Sind. 8vo. London, 1764.

Der vollkommene Pferdekenner, oder vollständiger Unterricht von der Pferdezucht, dem Pferdehandel und der Rossarzneikunst. By Frhr Wolf Chrfr. Reitzenstein. 8vo. Anspach, 1764. 3rd edition. 1806.

Die beste und bewahrteste Erfindung wider das Koppen der Pferde. By Frhr Wolf Chrfr. Reitzenstein. 4to. Uffenheim, 1764.

Lettera critica, ed istruttiva sopra il vero metodo di addestrare il cavallo. By Niccolo Rosselmini. 4to. Livorno, 1764.

Del' obbedienza de' cavalli. By Niccolo Rosselmini. 4to. Livorno, 1764.

En sikker og grundig Anviisning om Hæste-Creaturemes forefaldende Sygdomme, Egenskaber, Aarsager og Ceuer etc., Indeholdende adskillige og tilforladeligen forsogte Hielpe-Midler udvortes og indvortes for Hæste. By Ole Olsen Smidt. Christiania, 1764.

The Farrier's and Horseman's Complete Dictionary : containing the Art of Farriery in all its branches, with whatsoever relates to the manege and to the knowledge of breeding, feeding and dieting of Horses, as delivered by the best writers upon this subject. By Thomas Wallis. 8vo. London, 1764.

2nd edition, without pagination. 3rd edition, 8vo. London, 1775 .

1765. Matière médicale raisonnée à l'usage de l'école vétérinaire. By Claude Bourgelat. 8 vo. Lyons, 1765 . 4th edition, 8vo. 2 vols. Paris, 1805.

Translated into Italian by Jac. Odardi, 8vo. Belluno, 1776-79.

In Spanish as:-Elementos de vétérinaria que se han de enseñar a los alumnos del real colegio de vétérinaria de Madrid, (Anatomia). 4 vols. 4 to. Madrid, 1793-94. Translated by Sigismundo Malats.

In German as :-Anfangsgründe der Vieharzneikunst, oder kurzer Begriff von der Zergliederung des Pferdes. 8vo. Dantzig, 1782. 
The Art of Riding; or Horsemanship made easy, exemplified by Rules 1765. drawn from Nature and Experience. By J. L. Jackson. $12 \mathrm{mo}$. London, 1765.

Bewährte Horn-Schaaf-Pferde-und Federvieharzneikunst. By F.J. v. Tamm. 8vo. Vienna, 1765.

Ordonnance du Roi, pour régler l'exercise de la cavalerie du ler. Juin, 1766. 1766. folio. Paris, 1766.

Instructions concernant les soldats, cavaliers, et dragons, qui se rejoignent par leurs régimens . . . du 16 Août 1766. folio. Paris, 1766.

Ten Minutes Advice to every Gentleman going to purchase a Horse out of a Dealer, Jockey or Groom's Stable. Anon. $12 \mathrm{mo}$. London, 1766.

Hippographia; Beschreibung eines Pferdes von einem Englischen Rossartzt. Anon. 8vo. Leipzig, 1766.

Guide du maréchal ; ouvrage contennant une connoissance exacte du cheval, et la manière de distinguer et de guérir des Maladies; ensemble un Traité de la Ferrure. By Etienne Guillaume Lafosse. 4to. Paris, 1766.

There are many later editions.

In English:-The Veterinarian's Pocket Manual; a Treatise on the Diseases of Horses, particularly the glanders. 1804.

Neederlansche Stalmeester, behelzende al het geen betrekking heeft tot de ziektens en gebreken der Paarden en hoe dezelben te genezen. By L. W. F. van Oebschelwitz. 8vo. Amsterdam, $1766 ; 1777 ; 1803$.

In German as :-Der Holländische Stallmeister, oder Unterweisung was zum Kennen, Zäumen, Satteln, Beschlagen, und Unterhalten der Pferde und zur Reitkunst gehört. 8vo. Leipzig, 1766.

Sicher und geschwind heilender Pferde-Arzt, oder gründliches Unterricht iiber die Erkenntniss, Ursachen und Heilung der Krankheiten der Pferde. Handbuch fiir angehende Pferdeärzte; auch für Oekonomen und Pferdeliebhaber nützlich zu gebrauchen. Von Carl Wilh. Ammon. Mit Anmerkungen und Zusatzen versehen von Seyfurt v. Tennecker. By J. B. v. Sind. 8 vo. Frankfurt, $1766 ; 1791$.

Die Kunst Pferde zu zaumen und zu beschlagen. By Baron J. B. v. Sind. 8vo. Frankfort, 1766 ; and 8vo. 1782.

L'Art du Manége pris dans ses vrais principes. By J. B. de Sind. 4to. Bonn, 1766 ; 8vo. Vienna, $1772 ; 1774$. 
1766. The Anatomy of the Horse, including a particular description of the Bones, Cartilages, Muscles, Fascias, Ligaments, Nerves, Arteries, Veins and Glands, in eighteen tables all done from nature. By George Stubbs, painter. Oblong folio. London, 1766.

The complete book contains only fifteen tables.

1767. Zootomie ou anatomie comparée. By Claude Bourgelat. 8vo. Lyons, 1767-69.

Afterwards published under the title of "Eléments de l'Art vétérinaire précis anatomique du corps de cheval comparé avec celui du boouf et du mouton." 4 th edition, 8vo. Paris, 2 vols. 1807 .

Instruccion metodica y elemental para la Tactica, Manejo, y Disciplina de la Cavellaria y Dragones. By Don Garcia Ramirez de Arellano. 2 vols. Madrid, 1767.

Vernunftiges-und erfahrungsmässiger Versuch, wie die giftigansteckenden Viehseuchen unter dem Hornvieh und den Pferden wohl erkannt, präservirt und curirt werden können. By Joh. Nik. Textor. 8vo. Frankfort, 1767.

1768. De la conformation extérieure des Animaux. By Claude Bourgelat. 1768 ; 4 to. 1776.

The title was afterwards changed to "Traité de la conformation extérieure du Cheval," 6th edition, 8vo. Paris, 1808 ; 8th edition, 1832.

Translated into Italian by Jac. Odardi, Belluno, 8vo. 1776-79. And into Spanish as:-Exterior del caballo. By Hipolito Estevez. 2 vols. 4 to. Madrid, 1793.

British Zoology. By Thomas Pennant. 4 vols., 8vo. London, $1768 ; 1776 ; 1812$.

Vollständige Abhandlung von der Rehkrankheit der Pferde, mit anatomischer Beschreibung des Vorderschenkels. By J. B. v. Sind. 8vo. Frankfort, $1768 ; 1780$.

Neue und sichere Lehrart, die Pferde in kurzer Zeit zu dressiren. By Baron J. B. v. Sind. 8vo. Frankfurt, 1768.

A Dissertation on Breeding Horses, upon Philosophical and Experimental Principles, being an attempt to promote thereby the Breed of Racers and Horses in general; with some observations on Foreign Horses. By Richard Wall. 8vo. London, 1768.

L'utile à tout le monde, ou le parfait écuyer militaire et de campagne. By A. de Weyrother. 2 vols. 8vo. Brussels, 1768 ; 3rd.ed., 1804. 
Unterricht von Erziehung der Pferde, nebst Mitteln wider alle innern 1768. und äusserlichen Krankheiten derselben. By Chr. Zorn. 8vo. Erfurt, 1768.

Essai sur les Haras. By the Comte de Brézé. 4to. Turin, 1769. Translated into Italian under the title "Saggio sopra le razze." 12 mo. Turin, 1770 and 1780 . Also as "Il dilletante de' Cavalli," 12 mo. Venice, $1782 ; 1795$.

Pratique de l'équitation. By Dupaty de Clam. 8vo. Paris, 1769.

Kurze Anweisung über die Wartung der Pferde, um sie auf Reisen und in Lagern gesund $z u$ erhalten; nebst einer Anweisung uiber die Mittel, das Einreissen des Rotzes zu verhuten. By Eberhart. 8vo. Cassel, $1769 ; 1800$.

La Guide du Cavalier. By F. A. de Garsault. 12mo. Paris, 1769 ; 1770.

Discours sur le farcin, maladie qui attaque communement les chevaux. By Hurel. Paris [Amsterdam]. 12mo. 1769; 1775.

Recopilacion de la sauidad de albeiteria. By Manuel Perez Sandoval. 8vo. Madrid, 1769.

Abhandlung der Pferdezucht und Anlegung der gestiite. By Baron J. B. v. Sind. 8vo. Frankfurt, $1769 ; 1777$.

Dansk Heste-og Qvœglœge, eller Haandbog for Bondestanden, till 1770. Veiledning ved de almindeligste Sygdomme hos Heste, Hornqveg Far og Svine. By Peter Christian Abildgaard. 8vo. Copenhagen, 1770. Another edition, 1848.

In Ferman as :-Unterricht von Pferden, Kuhen, Schaafen und Schweinen, wie man dieselben warten und ausziehen muss, ingleichen von ihren Krankheiten und Heilmitteln. 8vo. Copenhagen, $1771 ; 1784$; 5th edition, 1805.

Essai sur les appareils et sur les bandages propres aux quadrupèdes, By Claude Bourgelat. 8vo. Paris, $1770 ; 1813$.

Another edition, followed by the "Essai théorique, etc. sur la ferrure." $12 \mathrm{mo}$. Lyons, 1776.

Translated into Italian by Jac. Odardi. 8vo. Belluno, 1776-79.

In German as :-Versuch uiber den Bandagen und die bei den äusserlichen Krankheiten der Pferde und den vierfussigen Thiere iberhaupt schicklichsten chirurgische Verrichtungen. 8vo. Leipzig, 1801. 
1770. An anatomical description of the Bones in the Foot of a Horse. By James Clark. 8vo. Edinburgh, $1770 ; 1772$.

Observations on the shoeing of Horses, and Diseases of the feet of Horses, in two parts. By James Clark. 8vo. Edinburgh, $1770 ; 1775 ; 1782$.

In German as:-Anmerkungen von der Hufbeschlage der Pferde und von den Krankheiten an der Fiissen der Pferde. Trans. from 2nd edition, 8vo. Leipzig, 1777.

Praktische Pferdearzneikunde, oder der durch lange Erfahrung sicher kurirende Pferdearzt mit der Anweisung zum Wallachen Englisiren, etc. By J. Gaab. 8vo. Erlangen, 1770; 3rd erlition, 1809.

Churcollnischen Oberst an eines Cavallerie-regiments und crsten Stallmeisters Voltstandiger Unterricht in den Wissenschaften eines Stallmeisters. By Baron J. B. von Sind. folio. Göttingen and Gotha, 1770.

Vollständiger Unterricht in den Wissenscharten eines Stallmeisters; mit einem Sehrbegriff der Pferdearzneikunst. By J. B. v. Sind. 2 vols. 8vo. Gotha, 1770 ; 2nd edition, Göttingen, 1775.

Le Manuel du Cavalier qui renforme les connaissances nécessaires pour conserver le cheval en santé, etc. By Baron J. B. v. Sind. 12mo. Cologne, [Circa 1770].

The Sporting Calendar. By William Tuting and Thomas Fawconer. 8vo. London, 1770, etc.

An historical list of Horse Matches, Plates and Prizes run for in Great Britain and Ireland, in the year 1769-(70). By B. Walker. 2 vols. 12 mo. London, $1770 ; 1771$.

1771. Newmarket, or, an Essay on the Turf, containing amongst other grave and weighty matters, a Parallel (though not after the manner of Plutarch and Mr. Spence) between Newmarket Races and the Olympic Games; very proper to be had in all pockets at the next Newmarket Meeting. Anon. 2 vols. $12 \mathrm{mo}$. London, 1771 ; 1775 .

Essai théorique et pratique sur la ferrure. By Claude Bourgelat. 8 vo. Paris, $1771 ; 1804 ; 1813$.

Another edition, preceded by the "Essai sur les appareils, etc.' 12mo. Lyons, 1776. Translated into Italian by Jac. Odardi, Belluno, 8vo. 1776-79. 
The History and Art of Horsemanship. By Richard Berenger. 1771. 2 vols. 4 to. London, 1771.

This work is a compound of Xenophon's Treatise on Horsemanship; Bourgelats' Elemens, 1750-53 ; and contains a Dissertation on the Ancient Chariot; the Exercise of it in the Race; and the Application of it to real Service in War. By $T$. Pownall.

Traite de l'équitation, avec une traduction du traité de la cavalerie de Xenophon. By Dupaty de Clam. 8vo. Deux-Ponts and Paris, 1771 or 72 .

Entwurf eines Verzeichnisses veterinarischer Biicher und einzelner Abhandlungen die zur theoretisches und praktisches Kenntniss von Pferden, Esel, Mauleseln, Rindvieh, Schaafen, Schweinen und Ziegen, Vieharzneikundigen, niitzen können. By Joh. Carl. Gli. Henze. 8vo. Stendal, $1771 ; 1781 ; 1785$.

Mosaisches Recht. By Johann David Michaelis. 8vo. Frankfurt, 1771-75; 8vo. Biehl, 1777.

Hus a chapter on the History and Breeding of Horses in Palestine.

In English as :-Commentaries on the Law of Moses. Translated from the German by Alexander Smith. 4 vols. 8vo. 1814.

Synopsis of Quadrupeds. By Thomas Pennant. 8vo. London, 1771.

Beschreibung des so bekannten Sennergestuites in der Graffschaft Lippe. By Joh. Gfr. Prizelius. 8vo. Lemgo, 1771.

Pferdearzneikunst, oder Unterricht die Gebrechen der Pferde aus der Grunde zu heilen. By D. Robertson. 8vo. Frankfort, 1771. 5th edition, 8vo. Vienna, 1789.

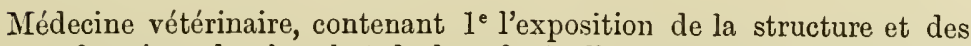
fonctions du cheval et du boeuf; $2^{\mathrm{e}}$, l'exposition des maladies du cheval, du boeuf, et de la brebis etc. ; $3^{\mathbf{e}}$ l'exposition des médica-

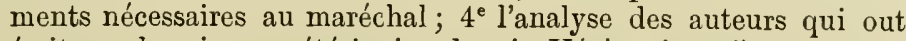
écrit sur la science vétérinaire depuis Végèce jusqu'à nos jours. By Louis Vitet. 3 vols. 8vo. Lyons, 1771 and 1783.

Kurze Beschreibung wie man flüchtige Pferde von einer Kutsche mit einem Riemen losspannen Kann. By Jh. Geo. W. Wiehe. 4to. Hildesheim, 1771.

Von den Krankheiten der Pferde und deren Heilung. By Joh. Cr. Zeiher. 8vo. Berlin, 1771. 
1772. Die edle Reitkunst, und von der Rossarzneikunde. Anon. 8vo. Eisenach, 1772 .

Observations historiques et critiques sur les commentaires de Folard et sur la cavalerie. By the Count De Brézé. 8vo. 2 vols. Turin, 1772.

Dictionnaire Vétérinaire et des animaux domestiques, contenant leurs moeurs, leur caractères leurs descriptions anatomiques, la manière de les nourir, de les éléver, et de les gouverner, les alimens qui leur sont propres, les maladies aux quelles ils sont sujet, et leurs propriétés, tant pour le médicine et la nourriture de l'homme que pour tous les differens usages de la vie civile, au quel on a joint une Tauna Gallica. By Pierre Joseph Buchoz. 8vo. 2 vols. Paris, 1772. 8ro. 6 vols. Paris, 1790, 1795.

The Compleat Horseman, or the art of Riding made easy. Illustrated by Rules drawn from nature, and confirmed by experience; with directions to the Ladies to sit gracefully, and ride with safety. By Charles Hughes. $12 \mathrm{mo}$. London, 1772.

Cours d'Hippiatrique, ou Traité Complet de la Médicine des Chevaux. By Etienne Guillaume Lafosse. folio. Paris, 1772.

In German as:-Lehrbegriff der Pferdearzneikunst. Translated by Th. Knoblauch. 8vo. Prague, $1787 ; 1788$.

1773. Essai sur l'équitation, ou principes raisonnés sur l'art de monter les chevaux. By Mottin de la Balme. 12mo. Amsterdam and Paris, 1773.

Practical Farriery, or the Complete Directory in whatever relates to the Food, Management and Cure of Diseases, incident to Horses, the whole alphabetically digested and illustrated with copper plates. By John Blunt. 12mo. London, 1773.

The structure and draught of Wheel-Carriages. By Jacob. 4to. London, 1773.

Modo facile, o sieno alcune brevi e principali regole per domare cavalli, etc. By Vincenzo Lombardi. Naples, 1773.

1774. The Modern Riding Master, or a key to the knowledge of the Horse and Horsemanship, with several necessary rules for young Horsemen. By Philip Astley. 8vo. London, 1774; 1775.

La mascalcia ossia la medecina veterinaria ridotta ai suoi veri principii. By Gio Antonio Brugnone. 8vo. Turin, 1774. 
Anfangsgriinde zur Anatomie der Pferde. By Mart. A. Tögl. 8vo. 1774. Vienna, 1774 and 1818.

Der, nach medicinische Lehrsätzen, sicher und geschwind curirende $\mathbf{1 7 7 5 .}$ Pferdeartzt. Anon. 8vo. Leipzig, 1775.

Handbuch der Pferdewissenschaft zu Vorlesungen. By Joh. Gfr. Prizelius. 8vo. Lemgo, 1775.

Anstellung der Pferde nach ihren Hauptfarben und verschiedene Abtheillungen. By John Elias Ridinger. 4to. Augsburg, 1775 .

Éléments de tactique pour la cavalerie. By Mottin de la Balme. 1776. 8 vo. Paris, 1776.

La science et l'art de l'équitation démontrés d'après nature. By Dupaty de Clam. 4to. Paris, 1776.

Counterfeit edition, 8vo. Yverdon, 1777.

Traité sur la cavalerie. By Count Drummond de Melfort. 2 vols. folio. Paris, 1776.

Also reprinted at Dresden, 2 vols. 4to. 1786. The engravings are reduced and inferior to those of the original edition.

Dictionnaire raisonné d' Hippiatrique, Cavalerie, Manége, et Maréchalerie. By Etienne Guillaume Lafosse. 2 vols. 8vo. Paris, 1776.

In German as :-Handbuch zum Gebrauch der Pferdeärzte bei Regimenten. 8vo. Hanover, 1785.

A Treatise on Cattle, shewing the most approved methods of Breeding, Rearing and Fitting for Use, Horses, Asses, Mules, Horned Cattle, Sheep, Goats, Swine, with directions for the further treatment of them, in their several disorders; to which is added a dissertation on Contagious Diseases. By John Mills, F R.S. 8vo. London, 1776 .

A new Treatise on the method of Breeding, Breaking and Training Horses. By William Ward. 8vo. Edinburgh, 1776.

Abhandlung von den Unterschied zwischen den Strengel und der Druse der Pferde. By Frz. Max. Fr. v. Bouwinghausen v. Wallmerode. 8vo. Stuttgart, 1777 .

Abhandlung von einer allgemeine Krankheit der Pferde, und den taglichen bei der Heilung sich ausserndern Fehlern. By Baron Leopold v. Hartmann. 4to. Burghausen, 1777. 
1777. Vollständige Pferdewissensehaft. By Joh. Gfr. Prizelius. 4 to. Leipzig, 1777.

Dictionnaire d'hippiatrique pratique. By Jos. Robinet. 4to. Brussels and Naney, Versailles and Paris, 1777.

1778. Von den Pferde Krankheiten, besonders von Kropfe und Rotze und deren Heilung. Anon. 8vo. Brandenberg, 1778.

Praktische Versuche in der Darmgicht der Pferde. By Joh. Chr. Ehrmann. 8vo. Frankfurt, 1778.

Discipline of the Light Horse. By Captain Hinde. 8vo. London, 1778.

The New Complete Horse Doctor, or Horseman's Sure Guide and every man his own Farrier. By John Maples. 12mo. London, [1778].

Another edition in the following year, with the title "Horseman's Sure Guide, or every man his own Farrier," 12 mo. London, 1779.

Traité d'équitation. By Montfaucon de Rogles. 4to. Paris, 1778 and Svo. Paris, 1810.

1779. Anweisung die Pferde nuitzlicher und besser, als bisher geschehen, zu beschlagen; nebst den Krankheiten des Hufes. By Frz. Max. Fr. v. Bouwinghausen v. Wallmerode. 8vo. Stuttgart, 1779 and 1781 .

Praktische Versuche in der Maulsperre oder Hirschkrankheiten der Pferde. By Joh. Chr. Ehrmann. 8vo. Frankfurt, 1779.

Sammlung praktische Abhandlungen von verschiedenen Krankheiten der Pferde, Schaafe, wie auch der Hornviehseuche, nebst Fiirsehungs und Heilungs-Mitteln. By Baron Leopold von Hartmann. 8vo. Stein, 1779 ; and 8vo. Nuremberg, 1785.

Veterinarius, oder theoretisch-praktische Unterricht von der Behandlung Kur und Wartung der Pferde und des Hornviehes, etc. By J. [A.] v. W[efeld.] 2 parts, 8vo. Gotha, 1779-80.

Unterricht für Fahnenschmiede über die Verletzungen die den Pferden durch Waffen zugefugt werden. 2nd part:-Bruchstucke iber wilde, halbwilde, militair, und Landgestuite. By Jh. Gli. Wolstein. 8vo. Vienna, 1779; 1786; $1788 ; 1796 ; 1797$.

In Latin:-Tractatus de vulnerationibus equorum per arma inflictis pro veterenariis belli tempore; auct. et ex idiomate germanico in latinum vers. ab G.S.T.A. de Högelmuiller. 8vo. Vienna, [1800]; 1803. 
Traité de l'éducation des Animaux qui servent d'amusment a l'Homme. 1780. Anon. 12mo. Paris, 1780.

Beyträge zur Geschichte der Erfindungen. By Johann Beckmann. 8 vo. 5 vols, Leipsig, 1780-1805.

In English:-A History of Inventions and Discoveries, translated by William Johnston. 8vo. London, 3 vols, 1797; 8vo. London, 4 vols, 1815 ; 8vo. London, 2 vols, 1846. A fourth vol. was added to the first edition in 1814 .

This work contains an account of the origin of saddles, stirrups, and horse-shoes.

Praktisches Versuch in der Dampfe der Pferde. By Joh. Chr. Ehrmann. 8vo. Frankfurt, 1780.

Préjugés Militaires sur la cavalerie; De ce qu'elle est aujourd'hui-du coup de sabre-sur le choc-du poids de la cavalerie-de la formation-du pausement-de la nourriture-des maladies des chevaux--des accidents-du manége et du haras-de l'exercise. By Charles Joseph, Prince de Ligne. 8vo. Kralovelhota, $1780 ; 1783$.

Der aufrichtige Leipziger Rossarzt. By Bernhard Naumann 8 vo. Leipzig, 1780 .

Vollständiger Rossarzt oder Unterricht die Krankheiten der Pferde zu kennen und zu kuriren. By Wilhelm G. Ploucquet. 8vo. Tubingen, 1780. 3rd edition, 1803.

Etwas von der Pferdearzney-Wissenschaft. By Joh. Nic. Rohlwes. 8vo. Göttingen, 1780.

Neuröffnete Rossapotheke, oder Mittel die Krankheiten der Pferde aus dem Grunde sicher zu heilen; nebst sicherer Cur für den Rindvieh, Schaafe, Schweine, Gänse, etc. By P. F. Roserus. 8vo. Quedlinburg, 1780.

Examen critique du militaire François. By [Baron Loubat de 1781 Bohan.] 3 vols. 8 vo. Geneva, 1781.

The third vol., including some extracts from the other two, was reprinted, 8vo. Paris, 1821, under the title of: "Principes pour monter et dresser les chevaux de guerre."

Trattato delle razze de Cavalli. By Gian. Brugnone. 8vo. Turin, 1781.

In French :-Traité sur les haras, extrait de l'ouvrage de G. Brugnone. Translated and condensed by C. Barentin de Montchal. 8vo. Paris, 1807. 
1781. In German as:-Von der zucht der Pferde Esel und Maulthiere, und von den gewöhnlichen, Gestutekrankheiten. Aus dem Ital. ibersetzt mit einem Anhang und Anmerkungen, von J. Gf. Fechner. Mit ein Vorrede von M. S. Stumpf. 8vo. Prague, 1790.

Ilustracion veterinaria y tratado de afectos y modo de febricitar el animal. By Miguel Pedro Lapuerta y Chaquet. 4to. La Almunia, 1781.

First volume only published.

1782. Gedanken uiber die Reitkunst und was dahin einschläglich, nebst einem Anhang von Recepten gegen die Pferdekrankheiten. By Frhr. Karl. v. Zyllnhardt. 8vo. Mannheim, 1782.

1783. Every man his own Farrier, or the whole art of Farriery laid open, containing the causes, symptoms and most approved methods of cure of the Diseases of Horses and Dogs. By Francis Clater. 8vo. Newark, $1783 ; 1787$. 21st edition, 1812. 30th edition, London, 1854.

In French as:-Médecine Vétérinaire. By Duvome. $12 \mathrm{mo.}$ Nevers, 1842. And:-Le Nouveau vétérinaire domestique, ou L'art de guerir soi-même ses chevaux et autres animaux domestiques. By P. L. Prétot. 8vo. Troyes, 1847.

In German as:-Der Hauspferdearzt oder die Kunst seine Pferde selbst zu heilen. By S. v. Tennecker. 8vo. Ilmenau, 1823. And:- I'ferde Hausarzt, nach den 25 Original Ausgabe herausgegeben von J. C. F. Lentin. Nebst ein Veterinär Pharmacopie als Anhang, 8vo. Weimar, 1834.

Englische Zucht und Behandlung der Pferde. By Joseph Hiver, 8vo. Vienna, 1783.

Abhandlung von den äusserlichen Krankheiten der Pferde. By Joh. Nic. Rohlwes. 8vo. Luneberg, 1783.

1784. Life of a Racehorse, a set of 6 large oval tinted plates. By F. Jukes. after C. Ansell. 1784.

Anweisung iiber die Behandlung und Abrichtung der Pferde etc. By Th. Geo. C. Koch. 8vo. Hanover, 1784.

Historia de Abiponibus, equestri bellicosaque Paraquariæ natione. By Martin Dobrizhoffer. 3 vols. 8vo. Vienna, 1784.

In German :-By A. Kreil. 3 vols. 8vo. Vienna, 1785.

In English:-An account of the Abiponians, an Equestrian and Warlike people of Paraguay. [Translated by Miss Southey]. 3 vols. 8 vo. London, 1822. 
A Practical Treatise on Farriery, deduced from the experience of 1784. above forty years in the services of the late Sir Watkin Williams Wynn, the present Earl Grosvenor, and the present Sir Watkin Williams Wynn, Bart. By William Griffiths, groom at Wynnstay. 4 to. Wrexham, 1784 .

Récherches sur les carrousels anciens et Modernes. By le chevalier Adrien Marie François de Verdy du Vernois. 8vo. Cassel, 1784.

Abhandlung von Bau Alter und Farbe der Pferde. Anon. 8vo. 1785. Vienna, 1785.

Considerations on the Breed and Management of Horses; interspersed with some remarks and calculations on the Exportation and Importation of Corn and the importance of an improved cultivation. Addressed to the King. Anon. 12mo. London, 1785.

On the Qualities of Horses, a Hindustani work. By Sa'ádatáyr Khán. Lucknow, A.H. 1269, [A.D. 1785].

Thoughts relative to the Medicine and Horse Acts. By Francis Spilsbury. 4to. London, 1785.

Authentic Racing Calendar of all Races run at York from 1709 to 1785, with an Account of the Cock Matches, etc. By WiAliam Pick. 8vo. York, [1785].

Novello giardino della prattica ed esperienza [del cavallo]. By G. A. Trutta. 4to. Naples, 1785.

Du Cheval et des proportions et à plombs des nembres du taureau. 1786. 8vo. Paris, 1786.

Anleitung zur Vorbauung und Heilung des Zungenkrehses unter dem Hornvieh und bei Pferden. Anon. 4to. Duisberg, 1786.

Istituzione di Mascalcia, conducente . . . ad escercitare, con solidi fondimenti la medicina de' Cavalli. By Franc. Bonsi. 2 vols. 8vo. Venice, $1786-87 ; 1801$.

Anleitung zur Pferde-und Maulthierzucht nebst einer historische Nachricht von den Herzoglichen Würtembergischen Stutereien. By Johann George Hartmann. 8vo. Tuibingen, 1786.

In French as:-Traité des Haras. Translated by J. B. Huzard. 8vo. Paris, 1788.

The Laws concerning Horses, or every Horsekeeper his own Lawyer; containing all the Acts of Parliament, and the cases adjudged in the different Courts at Westminster, respecting Horses, etc. By William Lucas. 8vo. London, 1786. 
1786. A Treatise on the Strangles and Fevers of Horses: with a Plate representing a Horse in the Staggers clung. By Thomas Prosser. 8 vo. London, $1786 ; 1790$.

Von dem innerlichen Krankheiten der Pferde. By Joh. Riem.8vo. Leipzig, 1786.

Unterricht für die Churf. Sächs. Fahnenschmiede, vom vernunftigen und zweckmässigen Beschlage der Pferde, sowohl bei gesunden als fehlerhaften und kranken Füssen. By Geo. Ludw. Rumpelt. 8vo. Leipzig, 1786. Another edition, 1813.

Guia Veterinaria original. By Alonso and Francisco Rus y Garcia. 4 vols, 8vo, Madrid, 1786-1788.

Trattato delle malattie esterne del cavallo. By Fr. Toggia. 2 vols. 8vo. Vercelli, 1786.

In German :- Von der Ursachen der Blindheit bei den Pferden und den mitteln. sie zu verhuten. Translated from a part of the above, by Ludis Cerutti. 8vo. Leipzig, 1821.

1787. Vollständiges Unterricht für den Landmann, wie er sich bei herrschenden Viehseuchen sowohl als auch bei anderen Krankheiten des Pferd, Horn, Schwein und Schaafviehes mit Vorbau-und Heilungsmitteln zu verhalten habe. By Mar. Geo. Blumenschein. 8vo. Munich, 1787.

An Academy for grown horsemen, containing the completest instructions for walking, trotting, cantering, galloping, stumbling, and tumbling; 12 plates and portrait of the Author, Geoffery Gambado, Esq., Riding Master, Master of the Horse, and Grand Equerry to the Doge of Venice.

Also published with and generally bound with the above, though with separate title page:-Annals of Horsemanship, containing accounts of accidental experiments, and experimental accidents both successful and unsuccessful, together with most instructive marks thereon and answers thereto, etc. By Geoffery Gambado. [H. Bunbury.] fol. London, 1787 ; 4to. London, 1787 and 1791 ; 8vo. Dublin, 1792 ; 4to. London, 1796 ; 8vo. London, 1809 ; 8vo. London, 1811 ; 4to. London, 1822.

Der vollkommene Pferdekenner. By J. A. F. Brück. 8vo. Hanover, 1787.

Von den Hornkliuften der Pferde und ihrer Heilung. By Heinrich Daum. 8vo. Marburg, 1787.

Anleitung zur Pferdekunde. By Joseph Hiver. 8vo. Kempten, 1787. 
Unterricht für Bauersleute von Krankheiten der Pferde, des Horn- 1787. viehes, der Schaafe und Schweine. By Erdmann Hülfreich. 8vo. Leipzig, $1787 ; 1820$.

Anweisung zur Kenntniss und Heilung der innern Pferdekrankheiten. By Joh. Adam Kersting. 8vo. Marburg, [1787]; 1788; 1817.

The Rural Economy of Norfolk. By John Marshall. 2 vols. 1787.

Nouveau régime pour les Haras, ou Exposé des moyens propres à propager et à ameliorer les Races de Chevaux; avec la notice de tous les ouvrages écrits ou traduits en François relatifs à cet objet. By Esprit Paul de la Font Pouloti. 8vo. Turin and Paris, 1787.

Das Buch von innerlichen Krankheiten der Füllen der Kriegs-und Biirgerpferde. By Jh. Gli. Wolstein. 8vo. Vienna, 1787; 3rd edition, 1808.

First Lines of Veterinary Physiology and Pathology. By James 1788. Clark. 8vo. Edinburgh, $1788 ; 1806$.

A Treatise on the Prevention of Diseases incidental to Horses, from bad management in regard to stables, food, water, air and exercise; with some surgical and medical branches of farriery. By James Clark. Svo. Edinburgh, 1788; 1790 ; 4th edition, 8vo. London, 1805.

In German as :-Abhandlung von Verhutung der Pferdekrankheiten, welche ihren Grund in fehlerhafter Beschaffenheit der Stalle, des Futters, des Wassers, der Luft und der Bewegung haben; nebst einige Bemerkungen iiber dem chirurgischen und medicinischen Theilen des Rossarzneikunde. 8vo. Vienna, 1790.

Beiträge zur Pferdearzneikunde, mit nöthigen Recepten. By Heinrich Daum. 8vo. Marburg, 1788.

Traité de l'éducation du cheval en Europe, contenant le développement des vrais principes des haras, du vice radical de l'éducation actuelle, et des moyens de perfectionner les individus, en perfectionnant les espèces, avec un plan d'exécution pour la France. By M. de Preseau de Dompierre. 8vo. Paris, 1788.

A Treatise of Horses, entitled Saloter, or a Complete System of Indian Farriery, in two parts, the first containing a description of all the disorders they are subject to, etc. Compiled originally by a Society of Learned Pundits in Sanscrit, and now translated from the Persian by Joseph Earles. 8vo. Calcutta, 1788. 
1788. The Gentleman's Experieneed Farrier; containing the method of Diet, Exercise, Bleeding, Purging, etc., of Horses; the anatomical parts described ; the Disorders incidental to Horses and their various cures. By William Forrester. 8vo. London, 1788.

Zeichenlehre oder Anweisung zur Kenntniss und Beurtheilung der vorzüglichsten Beschaffenheiten des Pferdes. By Jh. Adam Kersting. [Circa 1788] ; 8vo. Marburg, 1804.

Anweisung zur Kenntniss und Heilung der äussern Pferdekrankheiten. By Jh. A. Kersting. [Circa 1788]; 8vo. Marburg, 1796. 6th edition, 1819.

Von den Ursachen der Viehseuche und den nöthigen Vorbauungsmitteln nebst einem Anhang vom Beschlagen der Pferde und den Folgen welche daraus entstehen können. By Andr. Lorenz. 8vo. Salzburg, 1788.

The Classical Farrier, exhibiting the whole anatomy of the Horse, etc. By William Merrick. 8vo. London, 1788.

The Gentleman's Stable Directory, or Modern System of Farriery; comprehending the present entire improved mode of Practice, and containing all the most valuable prescriptions and approved remedies: Vol. 2 containing Experimental Remarks upon Breeding, Breaking, Shoeing, Stabling, Exercise and Roweling : to which is added particular instructions for the general manayement of Hunters and Road Horses, with concluding observations on the present state of the Turf. By William Taplin, 2 vols. 8 vo. London, $1788 ; 1793$.

The same work entitled 'A compendium of Practical and Explanatory Farriery, equally adapted to the convenience of the Gentleman, the Farrier, the Groom, and the Smith.' 2 vols. 8 vo. - London, 1796.

In German as:-Stallmeister, oder neuere Rossarzneikunde. nebst einem Anhang iiber die Hundseuche. Translated by S Hahnemann 8vo. Leipzig, 1797-98. New edition, 1804.

1789. Disertaciones sobre caballos, bueyes, mulas asnos, ganado lanar, cabris y de cerda ; peste de marsella y brutos maquinas de cartesio. By Juan Franciscn Calvo y Cavero. Saragossa, 1789.

Instructions et observations sur les maladies des animaux domestiques. By Chabert, Flandrin, and Huzard. 6 vols. 8vo. Paris, $1789 ; 1791-95$; and 1806-9. 
In German as:-Abhandlung von den Wurm-Krankheiten der europaischen Hansthiere, besonders der Pferde, des Hornwiehes der Schaafe und Hunde. Aus dem Französisch übersetzt und mit Anmerkungen und Zusatzen vermehrt von F. Albert Ant. Meyer. 8vo. Göttingen, 1789 ,

Sammlung gemeinniitzlicher Recepte, bei den Fehlern der Pferde zu gebrauchen. By Fr. Kahn. 8vo. Liibeck, 1789.

Nachgelassene manuscripte iiber die Pferdearzneiwissenschaft; herausgegeben von $\mathrm{O}$. Sothen. By Joh. Adam Kersting. 8vo. Braunschwieg, 1789 ; 1801 ; 1803; 1817 ; 1818. Berlin, 1789 ; 1792. Marburg, 1796.

Antangsgründe in der Reitkunst. By Hnr. Aug. Kollner. 8vo. Greiz, 1789.

Tratado de las enfermedades, endémieas, epidémicas y contagiosas de toda especie de ganados. By Juan Antonio Montes. Aranjuez, 1789. Reprinted a few years after.

Versuch eines chirurgisches Handbuchs fiir neuangehende Hufschmiede und Pferdeärzte. 2 parts. By Karl Aug. Oehlmann. 8vo. Leipsic, 1789.

Memoria en quel se trata de los Caballos de España, escribiata en 1784. By Don Pedro Pablo Pomar. 4to. Madrid, 1789.

Practische Abhandlung iiber die Darmgicht der Pferde. By Johann Conrad Heinrich Sander. 8vo. Hildersheim, 1789; 1799.

Recueil d'opuscles sur les différentes parties de l'équitation. Auxquelles on a joint le meilleur régime que l'on doit faire suivre aux différentes espèces de chevaux, pour en tirer le parti le plus avantageux et les conserver le plus long temps qu'il est possible. By Le Vaillant de St. Denis. 8vo. Paris, 1789 and 1790.

Description de la cavalcade, accompagnée de chars de triomphe, qui sera exécutée par les Écoliers du Collège de la compagnie de Jésus à l'occasion de Jubilé de quatre cent ans ... le 16 et le 29 Juillet 1790. Anon. 4to. Brussels. 1790.

Nogle anmærkninger om Cavallarieh æf en Dansk Subaltern Officer. Anon. Copenhagen, 1790.

Lehrbuch des Pferdekenntniss, oder volständiger Unterricht von den Schönheiten und Fehlern dieser Thiere. Anon. 8vo. Leipzig, 1790.

1789. 
1790. Luz da liberal e nobre arte da cavalleria, offercida as senhor D. João, principe de Brazil. By Manoel Carlos de Andrade. sm. folio. 2 vols. Lisboa, 1790.

The General History of Quadrupeds. By Thomas Bewick. 8vo. Newcastle, $1790 ; 1791 ; 1792 ; 1800 ; 1807 ; 1811 ; 1820 ; 1824$; 1886.

Principes d'Equitation et de Cavalerie. By le chevalier de Boisdeffre. Paris, 1790.

Belehrung für den Landmann bei den unter Pferden und Rindvieh urngehenden todlichen Seuch. By Frz. Max. Fr. v. Bouwinghausen $\mathrm{v}$. Wallmerode. 8vo. Tuibingen, 1790 .

Anweisung zum Satteln und Packen, dass kein Pferd gedriickt wird. By Joh. Karl Frae Rex. 8vo. Berlin, 1790, and 1791.

Anweisung zum Kutschfuhrwesen. By Heinrich Aug. Köllner. 8vo. Greiz, 1790.

Theoretisches-praktisches Lehrbuch uber die Reitkunst. By Heinrich Aug. K"llner. 8vo. Greiz, 1790.

Anleitung zur äussern Pferdekenntniss. By Jh. Chr. Plon. 8vo. Berlin, $1790 ; 1820$.

Ueber die Hauptmängel der Pferde, sowohl für Pferdeliebhaber und Handler, als fürnehmlich für Rechtsgelehrte, etc. By Wilhelm G. Ploucquet. 8vo. Stuttgart, 1790 .

Leichte und neue Art Pferde zu englisiren, nebst auch verschiedenen anderen Operationen an Pferden. By D. Robertson. 8vo. Wesel, 1790. ; 8vo. Arnheim, 1792.

Neu edirt von Ph. Mogalla. 8vo. Breslau, $1793 ; 1796 ; 1801$.

Catalogo de algunos autores espagñoles que han escrito de veterinaria, de equitacion, y de agricultura, contiene por orden cronologico el año y lugar de su impresion, las ediciones que se han hecho, y un juicio imparcial del mérito de cada obra. By Bernardo Rodriguez. 4to. Madrid, 1790.

Der Vollkommene Pferdearzt, oder praktisches Pferdearzneibuch, mit einem Anhang von Rindvieharzneien. By Joh. Nicl. G. Schmidt. 8vo. Leipzig, $1790 ; 1800$.

Irish Racing Calendar; an account of the Plates, Matches, and Sweepstakes run for in Ireland. Begun by Pat Sharkey. $12 \mathrm{mo}$. 1790 ; subsequently carried on by $R$. and $J$. Hunter. 
Der deutsche Rossartzt, zur Heilung der Krankheiten der Pferde. 1791. Anon. 8vo. Halle, 1791.

Anweisung wie die gewöhnlichste Krankheiten der Pferde und der Hornviehs, etc. . . . zu heilen sind. Anon. 8vo. Lingen, 1791.

Taschenbuch für Pferdeliebhaber, Reiter, Pferdezuchter und vorgesetzte grosse Marställe f. 1792-1802. By Frz. Max. Fr.v. Bouwinghausen v. Wallmerode. 16mo. Tuibingen, 17911801.

A Treatise on the Inoculation of Horses for the Strangles; in which is clearly laid down the manner and time of the operation, the preparation necessary and the mode of treatment durng the continuance of the Disorder. The whole being the result of long experience. By Richard Ford. 12mo. Birmingham, 1791.

Remarks on Forest Scenery, and other Woodland Views. By William Gilpin. 2 vols. 8vo. London, $1791 ; 1794 ; 1808$.

At the end of the second vol. is:- "Observations intended to expose the practice of Docking, Nicking, and Cropping Horses."

Introduction to a General Stud Book. By T. Gosden. 8vo. 1791.

Anleitung zu den natiirlichsten und leichtesten Art Pferde abzurichten. By Ludwig Hünersdorf. 8vo. Marburg, 1791; 4th edition, 1819 ; 5th edition, 1826.

In French :-Equitation allemande, méthode la plus facile et la plus naturelle pour dresser le cheval d'officier et d'amateur. Translated by A. de Brochowski. 8vo. Brussels, 1843.

Versuch uiber die Farbe und Eigenschaften deutscher und einiger ausländischen Pferde. By Heinrich Aug. Kollner. 8vo. Greiz, 1791.

Das Rosstäuscher Recht. By Joh. Carl. C. Münter. 8vo. Hanover, 1791 ; Brd edition, 1810.

Memoire sur les courses de chevaux et de chars en France, envisagées sous un point de vue d'utilité publique. By Esprit Paul de La Font Pouloti. 8vo. Paris, 1791.

Of the Proportions of "Eclipse." By Charles Vial de Saint Bel. 4 to. London, 1791 ; 1797. In French and English.

Die Pferdelust, ein Beitrage zur Unterhaltung der Pferde-liebhaber. 1792. Anon. 8vo. Nuremberg, 1792; 1793. 8 
1792. Der Deutsche Rossartz, für Verwalter, Schmiedemeister, und für Bauersleute. Anon. 8vo. Grätz, 1792.

Anleitung zur Beurtheilung des aussern Pferdes, in Beziehung auf dessen Gesundheit und Tuchtigkeit zu verschiedenen Diensten. By August Conrad Haveman. 8vo. Hannover, 1792 ; 1805 , and 1822 .

Every man his own Judge; or Grandeur and Utility pointed out in the formation of the Horse. By Thos. Henry Morland. Ipswich, 1792.

The British Sportsman, or Noblemen, Gentlemen and Farmers' Dictionary of Recreation and Amusement, including a most improved system of Modern Farriery and Anatomical Dissection of a Horse. By William A. Osbaldiston. 4to. London, 1792 ; 1795.

The Sporting Magazine, or Monthly Calendar of the Transactions of the Turf, the Chase, and every other Diversion interesting to the Man of Pleasure and Enterprize. 8vo. London, 1792 to 1870. In 1846 the Sporting Magazine was united with the New Sporting Magazine, the Sporting Review and the Sportsman. Each of these was published under its respective title, although othervise precisely the same as the Sporting Magazine.

Ueber das Verhalten der Kriegspferde in Winterquatiren nach schweren Sommer-und Herbst Campagnen. By Jh. Gli. Wolstein. 4to. Vienna, 1792.

1793. Unterricht für Kutscher, Stall-und-Reitknechte. Anon. 8vo. Vienna, 1793.

Der erfahrene Pferdeartzt. Anon. 8vo. Grätz, 1793.

Der Pferdearzt, oder gründliche Anweisung zur richtigen Kenntniss der gemeinsten Pferdekrankheiten nebst bewährten Heilmitteln. By C. D. Meyer. 8vo. Leipzig, 1793.

Causas de la escasez y deterioro de los caballos de España y medios de mejorarlos demostrades en dos informes dados á S. M. y por su Real órden sobre el estado de las castas de Andalucia. By Pedro Pablo Pomar. folio. Madrid, 1793.

Lectures on the Elements of Farriery, or the Art of Horse-Shoeing and on the Diseases of the Foot. Designed chiefly for the use of the Pupils of the Veterinary College, London. By Charles Vial de Saint Bel. 4to. London, 1793. 
Handbuch fur Oekonomen und Landleute, von den Seuchen und 1793. anderen gewohnlichen Krankeiten des Hornviehs, der Schafe, Pferde, etc. By Jh. Werner. 8vo. Breslau, 1793 ; 3rd edition, 8vo. Breslau, 1804.

Instruction to Young Dragoon Officers. Anon. London, 1794. 3rd 1794. edition, 1803.

Anweisung wie die gewohnlichen Krankheiten der Pferde und des Hornviehs, der Schweine und Schafe sicher und wohlfeil zu heilen sind. Anon. 8vo. Lingen, 1794.

Abhandlung iiber die Zergliederung der Schönheiten und Mangel der Pferde. By Joh. Marc. Beyer. 8vo. Meissen, 1794.

Six Letters on the Subject of the Armed Yeomanry. By Major Francis Percival Eliot. 8vo. Stafford, 1794 ; 8vo. London, 1797.

A Treatise on Carriages, comprehending Coaches, Chariots, Phaethons, etc., with their proper harness. By William Felton. 2 vols. 8vo. London, 1794-95.

Supplement, 8vo. London, 1796; another edition, 3 vols, London, 1805.

Treatise on the Horses of India. By Lieut. Piggott. 8vo. London, 1794.

Beitrag zur Erkenntniss der Natur und Heilart des Kollers der Pferde. By Fr. Aug. Rober. 4to. Leipzig, 1794.

The Law respecting Horses. By Aistroppe Stovin. 12mo. Hull, 1794.

Bruchstiicke zur Kenntniss von Pferden, vorzuglich den Krankheiten dieser Thiere betreffend. By S. von Tennecker. 8vo. Freiburg, $1794 ; 1797$.

Memorandums of Field Exercise for the Troops of Gentleman and 1795: Yeomanry Cavalry. By an Officer of Light Dragoons. Anon. 8vo. London, 1795.

Dizionario ragionato di veterinaria teoretico practica. By Fr. Bonsi. 5 vols, 8vo. Venice, $1795 ; 1803$. 
1795. Observations Anatomical, Physiological and Pathological, on the Pulmonary System; with remarks on some of the diseases of the Lings, viz : on hæmorrhage, wounds, asthma, catarrh, croup and consumption, tending to establish a new Pathology of the Lungs, founded on the anatomy and physiology of the parts; also some remarks on the Broken Wind of Horses. By William Davidson. 8vo. London, 1795.

Complete Sportsman, or, Country Gentleman's Recreation. By J. Fairfax. 12 mo. London, 1795.

Charakteristik und Geschichte der vorzuglichste Hengste und Zuchtstuten der Kön. preuss. Hauptgestiite, nebst ihrer Abbildung nach den Leben, als Beitrag zur Gestuitkunde. By F. M. Helmbrecht and Johann George Naumann. 8vo. Berlin, $1795-97 ; 1813$.

The New England Farrier. By Paul Jewett. 18mo. Newburyport, 1795.

The Practice of Farriery, calculated for the East Indies. By Antoine de Letang. 8vo. Pondicherry, 1795; 8vo. Madras, 1839.

Directions for using the contents of the portable horse-medicine chest adapted for India. By William Moorcroft. 8vo. London, 1795 .

Bewahrte Mittel gegen den Milzbrand bei dem Rindvieh und den Pferden, desgleichen gegen die Bräune bei den Schweinen und das Aufblähen des Rindviehes, so wie von den Ursachen und der Natur dieser Viehkrankheiten. By Joh. Geo. Schäfer. 8vo. Frankfurt, 1795.

Beantwortung der Anfrage iiber die Kenntniss und Heilung der sogenannten Salzkrankheit bei den Pferden. By Th. Eph. Steinhoff. 4to. Schwerin, 1795.

An Address to the Yeomanry of England. By Walter. London, 1795.

1796. Anweisung über die Behandlung der Pferde auf Reisen und in Lägern.

Anon. In German and French. 8vo. Strasburg, 1796.

Praktische Bemerkungen iiber die Zucht-Krankheiten von Pferde des Rindviehes, der Schaafe, Ziegen, Schweine, des Federviehes, der Fische, Bienen und Seidenwürmer. Anon. 12 mo. Leipzig, 1796 ; 1828 . 
Taplin Improved, or a Compendium of Farricry by an experienced 1796. Farrier. Anon. 12mo. London, 1796.

Praktisches Handbuch für Pferdliebhaber uud Pferdehändler, der äussere Bildung, Schönheit, Giite, Fehler, etc., der Pfercle selbst beurtheilen zu können. Anon. 8vo. Leipsic, 1796

Filantropie für Pferde, in einem Plane vorgetragen. By Hippofilos. [Gottlob August Beaumgarten-Crusius]. 8vo. Marburg, 1796.

An Essay on the Bots. By Bracy Clark. Linneean Society's Transactions, vol. 3, 1796 .

Observations on the Mechanism of the Horse's Foot; its natural spring explained and a mode of shoeing recommended by which the foot is defended from external injury with the least impediment to its spring. By Strickland Freeman. 4to. London, 1796.

In German as :-Abhandlung über den Ban und Mechanismus des Pferdefusses, nebst Beschreibung einem neuen, der Natur und Construction des Pferdefusses angemesseneren methode des Beschlagens. Translated by R. F. Buschendorf. 4to. Leipsic, 1797.

A Complete Dictionary of Farriery and Horsemanship, containing the Art of Farriery in all its branches, with an explanation of the Terms and a Lescription of the varions particulars relating to the Manege and to the knowledge of Horses. By James Hunter. 8 vo. London, 1796.

The Sportsman, Farrier, and Shueing Smith's New Guide, being the substance of the works of the late Charles Vial de St. Bel. By John Lawrence. 8vo London, 1796.

A Philosophical and Practical Treatise on Horses, and on the moral duties of Man towards the Brute creation, comprehending the choice, management, purchase and sale of every description of the Horse, the improved method of Shoeing, medical prescriptions and surgical treatment in all known diseases. By John Lawrence. 8vo. London, $1796 ; 1798 ; 1810$. - 2 n.

An Essay on the Comparative advantage of Oxen for Tillage in competition with Horses, being the result of Practical Husbandry. By the Chevalier de Monray. 8vo. London, 1796.

A Treatise on the Discipline of Light Cavalry, with annexed plates. By L. Neville. 8vo. London, 1796. 
1796. Kleine Schriften vermischten Inhalts, insbesondre in Beziehung auf Pferde und Pferdezucht. Bemerkungen auf einer Reise von Hannover durch Mecklenburg und die Priegnitz nach den Preus. Gestiitsanstalten bei Neustadt an der Dosse. By Geo. Fr. Petersen. 8vo. Hannover, 1796.

Anleitung dem ferneren einreissen der Rindviehseuche zu steuern. By M. G. Thilenius. 8vo. Lauterbach, 1796.

Gründliche Abhandlung der Fechtkunst auf den Hieb zu Fuss und zu Pferde zum Gebrauch der Cavallerie. By C. Timlich. 4to. Vienna, 1790.

Anleitung zur Heilung der Pferdekrankheiten. By E. A. Trautenberg. 8vo. Erlangen, 1796.

1797. Anleitung zur Erkenntniss und Heilung der Krankheiten Darmgicht und Entzuindung der Lunge bei Pferden. zum Besten der Landleute herausgegeben. Anon. 8vo. Heilbronn, 1797.

Der gliicklich geschwind und wohlfeil heilende Pferdeartzt. Anon. 8vo. Berlin, 1797.

Pferlezucht in Amerika; nach Englischen Grundsätzen verbessert. Anon. 8vo. Leipzig; 1797.

Kleine veterinärische Schriften. By J. D. Busch. 8vo. Marburg, 1797.

Dictionäre für Pferdeliebhaber, Pferdehändler, Bereiter, Cur und Hufschmiede, oder vollständiges Handwörterbuch der sämmtlichen Rosskunde, fortgesetzt von v. Arnim. By Carl Friedr. Buschendorf. 8vo. Leipzig, 1797-1806.

Instructions sur les Moyens de s'assurer de l'existence de la morve; sur ceux propres à prevenir l'invasion de cette maladie et en préserver les chevaux etc. By Ph. Chabert and J. B. Huzard. 8vo Paris, 1797.

In German as :-Anweisung den Rotz der Pferde zu erkennen. 8vo. Leipzig, 1797.

Treatise on the Disorders incident to Horned Cattle. . . . To which are added Receipts for curing the Gripes, Staggers, and Worms in Horses, and an appendix .. . By Joseph Downing. 8vo. London, 1797.

Anleitung zur vollständigen Pferdekenntniss der Landwirthe, nebst einem Vorbericht von dem Thierreich. Bv Frz. Fuss. 8vo. Prague, 1797. 
Essay on the use of mixed and compressed cattle fodder for feeding 1797. and fattening horses, oxen, cows, sheep, hogs and pigs ; particularly adapted for young stock and for horses and cattle on shipboard, in camps, or in garrisons, with useful tables. By J. Lawson. 8 vo. 1797.

Der wohlfeile, geschwind-und sicher heilende Pferde-und Vieharzt. By Fr. Ant. Mayr. 8vo. Pesth, $1797 ; 1821$.

Lehrbuch der populären Thierheilkunde fiir aufgeklärte Oekonomen. (Erste Theil, die Krankheiten des Horuviehes und des Pferdes.) By Bernh. Nath. Glo. Schreger. 8vo. Nuremberg, 1797.

Vereinigte Wissenschaften der Pferdezucht. By S. v. Tennecker. 8vo. Manheim, 1797 ; 1799 ; and 4to. 1831 .

Der Fahnenschmidt im Kriege ; oder Unterricht uiber die Heilung der Wunden die den Pferden durch Waffen zugefingt werden. By S. v. Tennecker. fol. Leipzig, 1797.

The Light Horse Drill, describing the several evolutions in a progres- 1798. sive series, from the first rudiments to the Manœuvres of a Squadron, illustrated with 10 copper plates. Designed for the use of the Privates and Officers of the Volunteer Corps of Great Britain. Anon. 4to. London, 1798.

Minutes and Observations for the use of gentlemen yeomanry. By William Allen. 8vo. London, 1798.

A Digest of the Acts of Parliament for raising a provisional force of Cavalry, to the end of 37 th George III. By Edward Boswell. 12 mo. London, 1798.

Observations on the Structure, Economy, and Diseases of the Foot of the Horse, and on the Principles and Practice of Shoeing. By Edward Coleman. 4to. London, vol. 1, 1798; vol. 2, 1802.

In German as:-Grundsätze des Hufbeschlages der Pferde. Translated by L. Bojanus. 8vo. Giessen, 1805 .

Hinsicht auf die Arzneiwissenschaft in Beziehung auf VeterinärEinrichtung. By Jh. Hnr. Schmidt. 8vo. Brunswick, 1798.

Ueber die bösartige Druse der Pferde. By J. H. Schmidt. 8vo. Brunswick, 1798.

Unterhaltung fur angehende cavalerie Officiere. By S. v. Tennecker. 8vo. Freiburg, 1798. 
1798. Messgeschenk zur belehrter Unterhaltung fiir Liebhaber der Pferde und der Reitkunst. By S. v. Tennecker. 12mo. Leipzig, 1798; 1799 .

1799. Instructions for Hussars and Cavalry ; a Treatise written by an Officer. Anon. London, about 1799.

Pocket Volunteer Cavalry Instructor in the Sword Exercise; in Five Parts. Anon. 24mo. London, 1799.

Analysis of Horsemanship, teaching the whole Art of Riding in the Manege, Military, Hunting, Racing, or Travelling System, together with the Method of Breaking Horses and Dressing them to all kinds of Manege. By John Adams. 8vo. London, 1799; 3 vols, 8vo. London, $1805 ; 1812$.

Anatomy of the Horse, accompanied with Remarks, Physiological, Pathological, Chirurgical, and Natural. By Delabere Pritchett Blaine. fol. London, $1799 ; 1802$.

Magazin für Thierarzneikunde, oder Beobachtungen, Versuche und Erfahrungen iiber die innerlichen und auserlichen Krankheiten der Pferde und des Hornviehes. Edited by Joh. H. Rohlwes. 8vo. Berlin, 1799, etc.

Handbuch der practische Heilmittlelehre, zum Gebrauch fiir angehende Pferdeärzte und Freunde der Arzneikunde. By S. v. Tennecker. 8 vo. 2 vols. Leipzig, 1799 ; 1801 . Another edition, 1824.

Equitation. Oeuvres complètes de Charles Thiroux. 2 vols. 4 to. Versailles, an VII, [1799.]

Grundliche Abhandlung von der Pferdekenntniss, zum nutzlichen Gebrauch fuir die Cavallerie-Officiere bei Remontirung und sonstigem Ankaufe eines Pferdes. By Th. Wopperer.8vo. Pesth, 1799

Kurze Abhandlung von den in und äusserlichen Krankheiten und sonstigen Beschädigung, welche den Pferden bei der armee im Felde und Quartieren am gewohnlichsten zustossen. By Th. Wopperer. 8vo. Pesth, 1799.

1800. An Address to the Yeomanry of England. By a Field Officer of Cavalry. Anon. London, [Circa 1800].

Der vollkommene Kutscher, oder die Kunst zu fahren, Kutschpferde $\mathrm{zu}$ behandeln und sie zu heilen.

Anon. 8vo. Leipzig, [1800.] 
Instructions and Regulations for the movements and formations of 1800 the Cavalry, comprising the additional instruction for 1799. London, [1800.]

Elucidation of several parts of His Majesty's Regulations for the Formations and Movements of Cavalry. Anon. 8vo. London, $1800 ; 1803$.

Neueste Englische Pferdegeschirre und Zäumung. Anon. Fol. Leipzig, 1800 .

Rossarzneibuch für Bereiter, Kutscher, Fuhrleuter und Reisender. Anon. 12mo. Leipzig, [Circa 1800].

System of Eqnestrian Education, exhibiting the Beauties and Defects of the Horse, with serious and important observations on his general excellence, preserving him in Health, grooming, etc. By Philip Astley. 4to. London, 1800 ; 1801.-8th edition, 1802. Another edition:-Projects in the mallagement of the Horse, rendering it calm on the Road, in Harness, etc. Such acquirements may prevent dreadful accidents. By Philip Astley; being an abridgement of his popular Book of Equestrian Education. To which is prefixed many excellent remedies for the diseases in Horses, etc. 12 mo. London, 1804.

The Narrative or Address of Samuel Chifney . . . to the Public in general, but more particularly to such of them as . . . are... connected with the Turf. 8vo. London, 1800.

Observations on the Formation and uses of the Natural Frog of the Horse, with a Description of a patent artificial frog, to prevent and cure Contracted Hoofs, Thrushes, Cankers, and Sandcracks. By Edward Coleman. 8vo. London, 1800.

Das Ganze der Pferdezucht, oder Vollständiger Unterricht in der Wartung. Pflege und Behandlung der Pferde, ihrer Verwendung, Kenntniss und Heilung ihr Krankheiten. 2 volumes. By Joh. Chr. Gotthard. 8vo. Erfurt, 1800.

Unterricht in der Zucht, Wartung, und Pflege der Esel und Maulthiere ihre Benutzung, Kenntniss und Heilung ihrer Krankheiten. By Joh. Chr. Gotthard. 8vo. Erfurt, 1800.

Vollstandiges praktisches Handbuch für Pferdeliebhaber und Rossärzte, etc. By Hugues. 8vo. Hamburg, 1800.

Journal der practische Rossarznei und Reitkunst. Edited by S. v. Tennecker. 8 vo. Leipzig, 1800. 
1800. Das fehlerhafte Pferd, an welchem alle Fehler, welche ein Pferd nur haben kann (einige 50) bemerkbar sind. By Major von Klatte. fol. Prague, [Circa 1800]; folio, Magdeburg, 1821.

Light Horse Drill. By Richard Lawrence. [Circa 1800].

Cursory Account of the various methods of shoeing horses, hitherto practised, with incidental observations. By William Moorcroft. 8vo. London, 1800.

In German as:-Beschreibung aller bisher gebrauchlichen Methoden des Hufbeschlags der Pferde. Translated by F. Beck. 8vo. Hannover, 1802.

Beschreibung des Condé eines alten Liebreitpferdes S. Maj. Frederick I. Bildliche Darstellung. By Johann George Naumann. fol. Berlin, [Circa 1800].

Ueber die Vorziiglichsten Theile der Pferdewissenschaft. Ein Handbuch fiir Offiziere Bereiter und Oekonomen. By Joh. Geo. Naumann. 8vo. Berlin, $1800 ; 1815 ; 1828$.

Beobachtungen und Erfahrungen wie auch versuchte Kuren und Heilungsarten, nebst verschiedenen Zergliederungen an Kühen, Ochsen, Schaafen, Ziegen und Schweinen, zum Nutzen der deutschen Hausväter und Hausmiitter, etc. By Karl Aug. Oehlmann. 8vo. Leipsic, 1800.

Das Buch von der Kehlsucht und Rotzkrankheit der Pferde für die Einwohner auf dem Lande. By Ignatius Schanzel. 8vo. Linz, [Circa 1800.]

Versuch uiber den Koller der Pferde; nebst ein allgemeiner Bemerkung iiber die Thierarzneikunde. By Fr. Uden. 8vo. St. Petersburg, 1800.

Danske Benœvnelser til Hestens Anatomie, Bygning og Behandling Vesternte til Brug ved Underviisningen i. d. Kgl. d. Dyrlægeskole. By E. N. Viborg and J. W. Neergard. Copenhagen, 1800.

Praktisches handbuch für Thierärzte und Curschmiede; nebst einer besondere Vorschrift fuir Viehbeschauer. By Er. Jac. Vierordt. 8vo. Carlsruhe, 1800.

A Compendium of the Veterinary Art, containing plain and concise rules for the treatment of all Disorders and Accidents to which the Horse is liable, with observations on Grooming, Feeding, Exercise and the Construction of Stables. Also a brief description of the structure, economy and diseases of the Horse's Foot, etc. By James White. 8vo. London, 1800; 12 mo. Canterbury, 1802. 
6th edition, 12mo. London, 1810 ; 12th edition, 1820 ; 13 th 1800. edition, 1822; 18 th edition, 1851, by W. C. Spooner; $19 t h$ edition, 1856 ; 20th edition, 1861.

In Spanish as:-Manual del Albeitar. Translated by Nicolas Casas de Mendoza. 8vo. Madrid, 1829.

Nützlicher Unterricht fiir den Landmann zur Erziegelung [sic] einer guten Gattung Pferde. By Th. Wopperer. 8vo. Vienna, [1800].

Der Erfahrener Rathgeber für Pferdebesitzer. Anon.8vo. Berlin, 1801. 1801.

Der vollkommene Kutscher, oder Anweisung wie man Pferde erziehen kann. Anon. Svo. Leipzig, 1801.

The Adjutant General's Instructions and Regulations for the Formations and Movements of the Cavalry. Anon. 8vo. London, 1801.

Veterinary Transactions, containing observations on the effects and treatment of wounds of Joints and other circumscribed cavities. Anon. 8vo. London, 1801.

Taschenbuch fur Pferdefründe, Pferdeärzte und Beschlagschmiede, Eine gründliche Anweisung zur richtigen Kenntniss, Behandlung. etc. By K.F.B. 8vo. Halle, 1801.

Veterinary Transactions. By Edward Coleman. 8vo. London, 1801 , etc.

Rural Sports, Hunting, Hawking, Fowling, Shooting, etc. By William B. Daniel. 2 vols, 4 to. London, 1801-3. 4 vols, 8 vo. 1810-13. Supplement, 4to. London, 1813.

Anviisning til Omgang med Heste og deres Behandling. Edited by D. Didrichsen. Copenhagen, $1801 ; 1802$.

An Inquiry into the Structure and Animal œconomy of the Horse, comprehending the diseases to which his limbs and feet are subject, with proper directions for shoeing and pointing out a method for ascertaining his age until the twelfth year. To which is added an attempt to explain the laws of his progressive motion on mechanical and anatomical principles. By Richard Lawrence. 4to. London, 1801 ; 1804.

Taschenbuch fiir Pferdeliebhaber, Rossärzte und Huffschmiede. By Lehmann. 8vo. Pforzheim, 1801.

Veterinärische und ökonomische Mittheillungen von meiner Reise durch Deutschland, England, Frankreich, Holland, der Schweiz, etc. mit Anmerkungen. By George Ludwig Rumpelt. 8vo. Dresden, 1801. 
1801 Veterinary Pathologos; or a Treatise on the Cause and Progress of the Diseases of the Horse with the most approved methods of Prevention and Cure. By William Ryding. 8vo. York, 1801 ; London, $1802 ; 1804 ; 1814$.

Taschenbuch belehrte Unterhaltung fuir Pferdeliebhaber. By S. v. Tennecker. 3 vols. 8 vo. Leipzig, $1801 ; 1802 ; 1803$.

Treatise on Military Equitation. By W.Tyndale. 8vo. London, 1801. Anviisning til at kjende ag vehandle Quærke, Hestekopper og Snive. By Erik Nissen Viborg. Copenhagen, 1801.

The Anatomy and Physiology of the Horse's Foot concisely described, with practicable observations on Shoeing. By James White. 18 mo. London, 1801.

Instructions for the Armed Yeomanry. By Sir W. Young. London, 1801.

1802. A Manual for the use of Coachmen, Grooms, Ostlers and all Persons concerned in the management of Horses, being a collection of near three hundred Receipts for the several Disorders to which that noble animal is subject. Anon. $12 \mathrm{mo}$, London, 1802.

Der practische Pferdearzt, sowohl im Hause als auf Reisen, etc. Anon. 8vo. Erfurt, 1802.

Auserlesne rar und bewährt gesundene arzneimittel, eines alten erfahrenen-Husaren-Obristen, fiir alle Krankheiten der Pferde mit einem Anhang 'Der neue wohlerfahrene turkische Rossarzt.' Anon. 8vo Niimberg. 1802.

Der Bauer als Pferdearzt, oder Arzneibuch für die Krankheiten der Pferde. Anon. 8vo. Strasburg, 1802.

La Connaissance parfaite des Chevaux, l'art de monter à cheval, augmentée d'un nouveau dictionnaire de manége. Anon. 8vo. Paris, 1802.

Praktische Abhandlung über den Krankheiten der Pferde und des Rindviehes, auf Brownische Grundsätze und Erfahrungen gesrundet. By Carl Wilhelm Ammon. 8vo. Nürnberg, 1802.

The outlines of the Veterinary Art, or the Principles of Medicine, as applied to a knowledge of the Structure, Functions, and Economy of the Horse, the Ox, the Sheep, and the Dog, and to a more scientific and successful manner of treating their various diseases. By Delabere Prichett Blaine. 2 vols. 8vo. London, 1802. Ibid, 1816 ; 5th edition, $1826 ; 1841 ; 1854 ; 1865$.

In French :-Notions fondamentales de l'art vétérinaire 3 vols. 8vo. Paris, 1803. 
The Dictionary of the Veterinary Art. By Thomas Boardman. 1802 4to. London, 1802-3. Another edition, 1805-1815.

Ein ganz neues Biichlein von wohlprobirten hossarzneien. By M. Böhmen. 8vo. Prenzl, 1802.

Regole per conoscere perfettamente le belezze e i difetti de' cavalli. By Franc. Bonsi. 8vo. Rimini, 1802.

A Treatise on the Diseases of Horses, in which various causes are plainly and accurately delineated, and a method of cure recommended conformable to practicable observations and experience. By John Denny. 8vo London, 1802.

Elements of the Veterinary Science. By Louis Grellier. 1802.

Beschreibung einer Maschine die das Durchgehen der Reit-und Wagenpferde verhindert. By J. Geo. Herklotz. 8vo. Dresden, 1802 .

Heniastik, oder die Kunst diffieile Pferde und die Vortheile angenehmen Führung zu erhalten und zu zäumen. By Eph. Klatte. 8 vo. Breslau, 1802.

Elementi di cavallerizza. By Federigo Mazzucchelli.4to. Milan, 1802 ; Forli, 1841.

In German as:-Handbuch des Thierheilkunde, oder von dem Baue, Verrichtungen und Krankheiten des Pferdes Rindviehes und der Schaafe. Trans. by L. Ceruitti. 2 vols. 8vo. Leipzig, 1820-21. And:-Ueber der Heilung der Krankheiten der Pferde, Kühe, Schaafe, und Hunde. Translated by W. F. Domeier. 8vo. Leipzig, 1805.

Der praktischer Pferdearzt, sowohl im Hause als auf Reisen. By Karl Aug. Oehlmann. 8vo. Erfurt, 1802.

Oekonomischer veterinärische Unterricht, iiber die Zucht, Wartung und Stallung der Pferde. By Joh. Riem. 4to. Liepzig, 1802.

Das Pferde in sienen Verrichtungen, ein Lesebuch zur lehrreichen Unterhaltung für Knaben. By S. v. Tennecker. 8vo. Leipzig, 1802.

Der pracktische Rossartzt oder Handbuch über die Erkenntniss und Cur der gewöhnlichen Pferdekrankheiten. By S. v. Tennecker. 2 vols. 8 vo. Stuttgart, $1802 ; 1804$. 
1803. Der Geschwind curirende Pferdeartzt. Ein Taschenbuch vorzüglich für zu Pferde Reisende, etc. Anon. 8vo. Leipzig, 1803.

Hints to the gentlemen of the Corps of Mounted Yeomanry. Anon. London, 1803.

Kurze Beschreibung zur genauen Kentniss des Pferdes, etc. Anon. 8 vo. Erfurt, 1803.

A Manual for Volunteer Corps of Cavalry. Anon. 8ro. 1803.

Sword Exercise for Cavalry, Rules and Regulations by H.M.'s Command. 8vo. London, 1803.

Vieharzneikunde, oder theoretische praktische Behandlung der Pferde und des Hornviehes im gesunden und kranken Zustande, etc. Anon. 8vo. Gotha, 1803.

A Domestic Treatise on the Diseases of Horses and Dogs, so conducted as to enable persons to practise with ease and success on their own animals without the assistance of a Farrier, including likewise the Natural Management, as stabling, feeding, exercise, etc., together with the outlines of a plan for the establishment of genuine medicines for these animals throughout the kingdom. By Delabere Pritchett Blaine. $12 \mathrm{mo}$. London, 1803. Another, 1805 ; 4 th edition, 1810 ; 5th edition, 1841.

Castor und Erichton Zeitschrift fur Reiter, Oekonomen und Pferdeliebhaber. Edited by Eph. Klatte. 8 vo. Breslau, 1803 ; etc.

Handbuch zur Kenntniss der Pferde in Riicksicht ihres Körperbaues, Farbe, Vaterlands, Nahrungsmittel, etc. By Jh. Engelhardt. 8 vo. Leipzig, 1803.

A New System of Farriery, containing a systematic arrangement of the internal structure of the Horse. Illustrated with copperplates representing the exact proportions of a blood horse, and a description of all the defects which tend to impede velocity. Rules for diseases of the Horse, etc. By John Feron. 4 to. London, 1803.

A subsequent edition entitled "A Complete Treatise on Farrier"y, comprising the modern practise of the Veterinary Art." 8vo. London, 1809.

In German as:-Geometrische Ausmessungen des Pferdes. Translated by Kr. L. Schwab. 8vo. Munich, 1814.

Anleitung zu den nöthigsten Kenntnissen bei der Pferdezucht in Fragen und Antworten. By Fr. v. Gmeiner. 8vo. Munich, 1803. 
Die Kunst, die Lebens-und Dienstdauer der Pferde zu verlängern, nach 1803. den Vorschriften der besten Lehrer. By Carl Hoffman. 8vo. Coburg, 1803.

Theoretisches-praktisches Handbuch der Thierheilkunde oder Beschreibung aller Krankheiten und Heilmethoden der sämmtlichen Hausthiere etc. By Bernard Laubender. 4 vols. 8 vo. Erfurt, 1803 ; 1807 ; 8vo. Munich, 1812.

Réflexions sur la réorganisation des haras, l'amélioration des chevaux et le rétablissement des manèges. By $\mathrm{L}$. de Maleden. 8vo. Versailles, 1803.

Die Kunst ohne alle Anweisung regelmässig Reiten zu leınen und seine Pferde selbst zu heilen. By G. Meyer. 8vo. Gotha, 1803; 1808.

A Treatise on the Cavalry and Saddle Horse, with Remarks on Pacing and Leaping, Observations on Breeding, etc. Dedicated by permission to His Royal Highness the Duke of York. By G. Orr. $12 \mathrm{mo}$. London, 1803.

Turf Register. Sportsman and Breeder's Stud Book, containing the Pedigrees and Performances of all Racers. By William Pick. 2 vols. 8 vo. York, 1803-5.

Systematisches Handbuch der theoretisch-praktischen Veterinär-Wissenschaft. By Friedrich Pilger. 1803.

Anweisungen zur Reiten nebst Bemerkungen uiber die Wahl eines Reitpferdes und iiber Fehler der Pferde. By Andr. Politz. 8vo. Magdeburg, 1803.

Zeichenlehre, oder Anweisung zur Kenntniss und Beurtheil vorzüglichste Beschaffenheit eines Pferdes. By Ernst Schwabe. 8vo. Marburg, 1803.

The General Stud Book, 8vo. London, 1803. Vols. 1, 2, 3, 2nd edition, published $1820 ; 1827$.

Sporting Dictionary and Rural Repository of Information relating to the Sports of the Field. By William Taplin. 2 vols. 8vo. London, 1803.

Geschichte eines Racenpferdes von seiner Geburt an bis zu seinem Tode. By S. v. Tennecker. Svo. Leipzig, 1803.

Anecdotes, Original and Select, including characteristic Sketches of Eminent Persons on the Turf, and the most Extraordinary Events in the Sporting World, etc, etc. By an Amateur Sportsman. Anon. 8vo. London, 1804. 
1804. Beiträge zur Kenntniss und Heilung der Kolik oder Darmgicht der Pferde. Anon. 8vo. Halle, 1804.

Der Geschwind und gründlich heilende Pferdeartzt, ein Hiulfsbuch fur Landbewohner, etc. Anon. 8vo. Halle, 1804.

Vollstandiges Handbuch der praktischer Pferdearztneikunst, nebst einem Anhange, der wichtigsten Krankheiten des rindviehes und der Schafe enthaltend. By Carl Wilhelm Ammon. 8vo. Heilbronn, 1804-7 ; 1827.

Genius Genuine. By Samuel Chifney. of Newmarket. A Fine Part in riding a race, known only to the author. Why there are so few good runners, or why the Turf Horses degenerate. A guide to recover them to their strength and speed as well as to train horses for hard running and hunters and hacks for hard riding. To preserve their sinews from being so often destroyed, with reasons for horses changing in their running, likewise a full account of the Prince's Horse, "Escape," running at Newmarket on the 20th and 21st days of October, 1791 ; with other interesting particulars, 8vo. London, $1804 ; 1871$,

On the Form of Animals. By Henry Cline. 4to. London, 1804 ; 8vo. Dublin, 1806 ; 8vo. London, 1829.

Instructions and Regulations for Yeomanry and Volunteer Cavalry. By Col. John Charles Herries. 8vo. Philadelphia, 1804; 1811.

Theoretische systematische Vorlesungen üher die Bearbeitung und Zahmung des Soldaten Pferdes nach Organograph, und psychischen grundsätzen. By Eph. Klatte. 8vo. Berlin, 1804.

Praktische Pferdearzneikunst. By Frz. Ludw. Konigstadter. 8 vo. Eisenach, 1804.

Kenntniss und Heilung der Engbrïstigkeit und des Dampfs bei Pferden. By Bernhard Laubender. 8vo. Nüremberg, 1804. Another edition, 8vo. Leipzig, 1811.

Anleitung zu dem Unterricht der Rekruten der Kavallerie auf der Reitbahn, für Unteroffiziere. By Ritter A. Fr. Aug. Lindenau. 8vo. Leipzig, 1804.

Der Taschenpferdearzt. Ein handbuch für alle Stände, vorziiglich zum Gebrauch der cavallerie. By Joh. N. Rohlwes. 8vo. Berlin, $1804 ; 1809 ; 1819$.

Kurzgefasstes Handbuch der Pferde Arzneikunst für Layen. Als ein Anhang zu dessen neuem militärischen Handbüche. By Fr. Wihl. Leop. v. Saint Paul. 8vo. Breslall, 1804. 
Beschreibung und Heilung der jetzt grassirenden Pferdeseuche, der 1804. sogenannten Druse. By Geo. Fr. Tscheulin. 8vo. Carlsruhe, 1804.

An Address to Yeomanry Cavalry, respecting the Management of their Horses. By James White. 8vo. London, 1804.

System of Veterinary Medicine; containing the Materia Medica and Pharmacopœia. By James White. 8vo. London, 1804.

Das Pferd fuir Liebhaber zur Belehrung und Unterhaltung. Anon. 1805. 8vo. Breslau, 1805.

Taschenbuch für Herrschaften und Kutscher, oder Unterricht von dem was bei dem Einkauf der Kutschpferde und bei ihrer Wartung, Pflege und Fitterung zu beobachten ist, etc. By Carl Wilhelm Ammon. 8ro. Sulzbach, 1805.

Griindliche Anleitung zur Reitkunst. By J. Ch. H. Andre. 8vo. Breslau, 1805 .

Section of the head of the Horse, as large as life, with a description and remarks. By Bracy Clark. London, 1805.

Praktischer Rossheilkunde, oder Anleitung zur Kenntniss und Heilung der innern und äussern, der ortlichen und allgemeinen Krankheiten der Pferde nach den Grïndsatzen der geläuterten Erregungstheorie für Thierarzte, Stallmeister u. s. w. Nebst Vorredung von S. v. Tennecker. By Carl Hoffman. 2 vols. 8vo. Erfurt, 1805.

Anweisung zur Verbesserung der Pferde, vorziiglich für Landwirthe. By J. B. Huzard. 8vo. Konigsberg, 1805.

The duties of Light Cavalry in the Field; compiled for the use of the Yeomanry of the United Kingdom of Great Britain. By Capt. John Kirke. $12 \mathrm{mo} .1805$.

Patriotisches Versuch iber der Pferdezucht in Schlesien und ihre Verbesserung. By Eph. Klatte. 8vo. Züllichau, 1805.

Ideen zur Organisirung einer selbstständigen Veterinär-Polizei, etc. By Bernhard Laubender. 8vo. Niirnberg, 1805.

Scuola equestre. Elementi di cavallerizza, lezioni equestri, il cavallo, ammalato, catechismo, avvertimenti ed istruzione. By Fed. Mazzuchelli. 2 vols. 4to. Milan, 1805.

Zwei untriigliche hereits erprobte Mittel, sich beim Durchgehen der Pferde gegen alle Gefahr zu schuitzen. By J. Riem. 4to. Liepzig, 1805 .

Io 
1805. Farriery. By Edward Snape. 4to. London, 1805.

Handbuch der niedern und höhern Reitkunst, etc. By S. von Tennecker. 2 vols. 8vo. Leipzig, 1805.

Ueber Krankheiten an Pferden und ihre Heilung. By Hieron Waldinger. 12mo. Vienna, [1805]; 1806.

Wahrnehmungen an Pferden um über ihren Zustand urtheilen zu können. By Hieron Waldinger. 8vo. Vienna, 1805; 1810 ; $12 \mathrm{mo} .1818$.

Anweisung für die Einwohner der gegenden in welchen die Pferde von der jetzt grassirenden Zeuche ergriffen werden. By Jh. Gli. Wolstein. 8vo. Altona, 1805.

Die Kunst ohne Anleitung Pferde Rindvieh ... selbst zu erziehen. By Jh. Gli. Wolstein. 8vo. Erfurt, 1805.

1806. Anweisung bei den Krankheiten der Pferde die Haarseile oder Eiterbänder und Fontanellen nützlich abzuwenden. Anon. 8vo. Marburg, 1806.

Belehrungen eines erfahrenen Pferdekenners uber den Strengel die Druse den Rotz den Wurm und der Lungensucht der Pferde. Anon. 8vo. Marburg, 1806.

Der erfahrenen Rathgeber beim Einkauf der Pferde. Anon. 8vo. Marburg, 1806.

Versuch eine Anweisung zur Geburtshülfe der Pferde. Anon. 8vo. Marburg, 1806.

Essays on the Anatomy of Expression in Painting. By Charles Bell. 4to. London, $1806 ; 1824$.

Pp. 73 and 75. Plate V. with explanations, pp. 78, 79, 87, and 89.

A Compendiwn of the Anatomy, Physiology and Pathology of the Horse. By B. W. Burke. 12 mo. London, 1806.

System der theoretische und praktische Thierheilkunde. By J. D. Busch. 4 vols. 8vo. Marburg, 1806.

Dictionnaire de médecine, de chirurgie, de pharmacie, des sciences accessoires et de l'art vétérinaire. By Joseph Capuron. 8vo. Paris, 1805 ; 1810 ; 1814 ; 1824 ; 1833 ; 1835 ; 1839 ; 1841 ; $1845 ; 1854$.

It has also been translated into Spanish, 8vo. Madrid, 1856. 
A Military Catechism for the use of Young Officers and Non-Com- 1806. missioned Officers of Cavalry. By James Charles Dalbiac. London, 1806.

Laws of Gaming, Wagers, Horse Racing, etc. By John Disney. 8vo. London, $1806 ; 1809$.

Ausführliche Beschreibung der im Frihjahr, 1805, herrschend gewesenen sogenannten Brustseuche der Pferde, ihrer Ursachen, Kennzeichen und Heilmittel; nebst einigen besonders merkwürdigen und sehr seltenen Beobachtungen und Erfahrungen. By Jos. C. Fehr. Svo. Göttingen, 1806.

Tabelle nach welcher Cavalleristen, Kutcher Reit-und Stallknechte etc. ihre Pferde striegeln und warten miissen etc. By Fd. Aug. Fiedler. folio. Hamburg, 1806.

The Art of Horsemanship, altered and abreviated according to the principles of the late Sir Sydney Medows. By Strickland Freeman. 4to. London, 1806.

Entwurf zu einer Anweisung wie das Benehmen eines Cavalleristen bei Gefechten in zerstreuter Ordnung in Friedenszeiten zu lehren sey. By Ritter A. Fr. Aug. von Lindenau. 8vo. Leipzig, 1806.

L'Ecuyer des dames, ou lettres sur l'equitation, contenant des principes et des exemples sur l'art de monter à cheval Dédié au beau sexe. By L. H. Pons-D'Hostun. 8vo. Paris, 1806.

Die Pferdezucht, oder Veredlung der Pferde in den Preuss. Staaten, in einer Darstellung des Königl. Preuss. Friedrich Wilhelm Gestiits bei Neustadt an der Dosse. By Joh. N. Rohlwes. 4to. Berlin, 1806.

Das Ganze der niedern Reitkunst nebst Anleitung zur Voltigiren. By Geo. Fr. Sebald. 8vo. Ulm, $1806 ; 1810$.

Cases in Farriery, in which the Diseases of Horses are treated on the principles of the Veterinary School of Medicine. By John Shipp. 4 to. London, 1806.

Der erfahrene und berathende Thierarzt, oder die bewährtesten Heil mittel in den gewohnlichen Krankheiten des Rindviehes, der Pferde, Schafe und Schweine. Anon. 8vo. Chemnitz, 1807; 1827.

Untrügliche Anweisung ein Thierarztes, wie die Lösidorre und Rindviehpest zu kuriren, Pferde zu englisiren und zu wallachen, etc. Anon. 8vo. Marburg, 1807. 
1807. Abhandlung iiber die Natur und Heilung der Augenentziindung bei Pferlen und ihre Folgen, etc. By Carl Wilhelm Ammon. 8vo. Ansbach, 1807.

Hinterlassener Unterricht über den Hufbeschlag der Pferde. By Gervas Binz. 8vo. Vienna, 1807.

Anatomie des Animaux Domestiques. By J. Girard. 2 vols. 8vo. Paris, 1807. 4th edition, 1841 .

In German :-Lehrbuch der Anatomie der Hausthiere. Translated by K. L. Schwab. 8vo. Munich, 1811 and 1821.

Translated into Arabic by Mustapha Hassan Kassàt. 4to. Boulak, 1833.

Taschenbuch für Freunde der Reitkunst. By Eph. Klatte. $16 \mathrm{mo}$. Breslau, 1807.

The Country Gentleman's Architect, or Designs for Farm-yards and Houses, Cottages and Stables. By Lugar. 4to. London, 1807.

Der dentsche Rossarzt, oder griindliche Anweisung auch ohne Vorkenntnisse Pferde aufzuziehen und abzurichten, ihre Krankheiten zu erkennen und zu heilen; nebst einer Anleitung durch Selbstunterricht Reiten zu lernen. By Karl Aug. Oehlmann. 8vo. Gotha, 1807.

Osservazioni ed esperienzi pratiche sulla morva de' cavalli, etc. $\mathrm{By}$ Francis Toggia. Turin, 1807.

Bemerkungen uiber die Enstehung und Verbreitung des Rotzes unter den Pferden Maulthieren und Eseln und über die Entwicklung andre Seuchen und Pesten bei Menschen und Thieren. By Jh. Gli. Wolstein. 8vo. Hamburg, 1807.

Lehrbuch der Krankheiten der Thiere, und besonders der Pferde. By Stephen Zipf. 8vo. Mannheim, 1807.

1808 Die Kunst, die Lebens-und Dienstdaner der Pferde zu verlängern, ein nuitzliches Taschenbuch fuir Pferdeeigenthümer and Pferdeliebhaber. By Carl Wilhelm Ammon. 8vo. Sulzbach, 1808.

Also under the following title:-Taschenbuch für Pferdeeigenthiimer und Pferdeliebhaber, oder Unterricht von der besten Wartung Pflege und Behandlung der Pferde.

Ueber die Unzulänglichkeit der gesetzlichen Hauptmängel bei Pferden, Rindvieh, Schaafen und Schweinen, um Betriggereien im Viehhandel zu verhiiten. By Carl Wilhelm Ammon. 8vo. Nurenberg, 1808. 
Unterricht fiir Thier-Aerzte, Oekonomen und Landleute iiber die unter 1808. dem Rindviehe, den Pferden und Schweinen häufig herrschende Seuche, der Milzbrand gennant, nebst einem Anhange über die Lungenseuche des Rindviehes. By Carl Wilhelm Ammon. 8vo. Ansbach, 1808.

Manœuvres d'artillerie à Cheval. By Tadeusz Kosciuszko. [Circa, 1808].

In English:-Manœuvres of Horse Artillery, translated with notes and descriptive plates. By Jonathan Williams. 8vo. New York, 1808.

Military Instructions for forming young men and horses in the Rudiments of the Cavalry Service. By Captain Skeene. 8vo. 1808.

The Wonders of the Horse, recorded in anecdotes and interspersed with poetry, selected by Joseph Taylor. 12 mo. London, 1808.

Praktische Handbuch der Erkenntniss und Heilung der Seuchen, Contagionen und der vorzüglichsten sporadischen Krankheiten der Rinder, Pferde, Schaafe und Schweine, wie auch der Hundswuth. By Alex. Tolnay. 8vo. Leipzig, 1808.

Ueber die Nahrungs-und Heilmittel der Pferde. By Hieron Waldinger. Svo. Vienna, 1808; 12 mo. 1811 ; 12 mo. 1816.

Manuel de l'Ecuyer et du Vétérinaire ou nouveau traité sur les maladies des Chevaux avec la manière de les élever. Anon. 8vo. Lyons, 1809 .

Woran kann der Hauptmann die des Rotzes und des Wurmes verdachtigen Pferde erkennen. Anon. 4to. Breslau, 1809.

Allgemeines Hausvieh-Arzneibuch oder vollstandiger Unterricht, wie man die Krankheiten der Pferde, des Rindviehes, der Schaafe, etc., auf die leichteste und wohlfeilste Art heilen kann. By Carl Wilhelm Ammon. 8vo. Ansbach, 1809; 3rd edition, 1830.

Aperçu Générale sur la Perfectibilité de la Médecine Vétérinaire. By Aygaleng. 8vo. Paris, 1809.

Memoirs of British Quadrupeds, illustrative principally of their Habits of life, Instincts, Sagacity, and Use to Mankind. By W. Bingley. 8vo. London, 1809.

A Series of original Experiments on the Foot of the living Horse, exhibiting the changes produced by shoeing, and the causes of the apparent mystery of this Art. By Bracy Clark. 4to. London, Part I, 1809. Part II, 1812. 
1809. Observations on Carriage Wheels. By Cumming. 4to. London, 1809.

Guide to the Turf. By Gard. 12mo. London, 1809.

Arehiv von durch vieljahrige Erfahrung gesammelten richtigen Kentnissen von Pferden und deren bei denselben vorkommenden innerlichen und äusserlichen Krankheiten und geprüften Heilungsmitteln. By C. W. Jacobi. 2 vols. 8vo. Breslau, 1809-12; 1822 .

The History and delineation of the Horse, in all its varieties, comprehending the appropriate uses, management and progressive improvement of each, with a particular investigation of the character of the Race Horse and the business of the Turf. Illustrated by aneedotes and biographical notices of distinguished sportsmen. The engravings from original paintings with instructions for breeding, breaking, training, and the general management of the Horse both in a state of health and disease. By John Lawrence. 4to. London, 1809.

Observations on the Causes which constitute unsoundness in Horses, considered in regard to the sale and purchase of those animals. By Richard Lawrence. 8vo. London, 1809.

Treatise on the Breeding and Management of Live Stock ; comprising, Cattle, Sheep, Horses, Asses, Pigs, etc. By Richard Parkinson. 2 vols. 8 vo. London, 1809.

Einege Bermerkungen über die Lehre vom Exterieur der Pferde. By Konrad Ludwig Schwab. 8vo. Munich, 1809.

Om Hesterkiods Spiisning. Skreveh efter d. Kgl. d. Cancellies Befaling isœer for Norge. By Erik Viborg. Copenhagen, 1809.

Abhandlıng iiber die Kohle, als Heilmittel der verdächtigen Drüsen bei Pferden. By Hieron Waldinger. 8vo. Vienna, 1809.

The Gentlemen's Veterinary Monitor and Stable Guide. A concise treatise on the various diseases of Horses, their symptoms and most humane methods of cure. Calculated to enable persons to form an accurate judgment of the diseases of their own horses, and apply the proper remedies without the assistance of a Farrier, etc., etc. By Yorick Wilson. 18mo. London, 1809.

1810. Naturgeschichte des Pferdes. By E. D'Alton. 2 vols. folio. Weimar, $1810: 1819$.

The anatomical engravings in the 2 volumes are after the English work on that subject by Stubbs. 
Précis historique de l'art vétérinaire, pour servir d'introduction à une 1810. bibliographie vétérinaire générale. By P. J. Amoreux. 8vo. Montpellier, 1810.

A Treatise on the Foot and Shoeing, in which the obscurities of this art are explained, and many new parts shewn; a Defect demonstrated in the principle itself of Modern Shoeing, more mischievous to the Feet than the abuses complained of. To the above is added an important narrative of the consequences of turning horses to grass without shoes, in order to remedy contracted feet. Also an Essay on the knowledge of the Ancients respecting shoeing, originally published in 2 parts. By Bracy Clark. 4to. London, [Circa 1810.]

Part III. of the above vas published subsequently entitled "Stereoplea;" and is confined to the actual practice of shoeing. 4to. London, Circa 1812; another edition, 1832.

Directions to Farriers respecting cutting the Frog. By Bracy Clark. 4to. London, 1810.

Le parfait chasseur, traité général de toutes les chasses. By Auguste Desgraviers. 8ro. Paris, 1810.

Unterricht für Bauersleute ïber die Zucht und Wartung, wie anch über die Erkenntniss und Heilung der Krankheiten der Pferde, des Hornviehes der Schaafe und der Schweine. By Erdmann Hulfreich. 8vo. Vienna, 1810.

Neues Wiener Taschenbuch fiir Freunde der Pferde, etc. By Eph. Klatte. 16mo. Vienna, 1810.

The Danger of Travelling in Stage Coaches and a remedy proposed. By William Milton. 8vo. Reading, 1810.

Histoire des Chevaux Célèbres. Contenant un recueil des anecdotes relative à ce noble animal: les mentions les plus interessantes qu'en ont faites les auteurs ancien et modernes, en vers et en prose : ainsi que les productions des beaux-arts, qui ont rapport aux Chevaux : et generalement tout ce qui peut amuser et instruire dans un pareil sujet, les amateurs des Chevalux, et tous ceux qui en font usage. By P. J. B. N. 12mo. Paris, 1810.

Rathgeber für die welche rohe Pferde zum Cavalleriendienst zurichten. By G. L. v. Pollnitz. 8vo. Neustadt, 1810.

Vermischte Beiträge zur praktischen und gerichtlichen Thierheilkunde, etc. By Jh. K. H. Sander. 8vo. Berlin, 1810. 
1810. Handbuch zur Kenntniss und Heilung der Krankheiten unserer vorziiglichsten Hausthiere, By Geo. Fr. Tscheulin. 8vo. Karlsruhe, 1810.

1811. Theoretische-praktische Darstellung der Pferde und Maulthierzucht und der Pferdearznei-Wissenschaft, nebst einer Anweisung zur genauen Kenntniss der Fehler und Mängel der Pferde, und der Kunstgriffe und Betriigereien der Rosshändler. By M. Blumhoser. 8vo. Grätz, 1811.

Anleitung zur Kenntniss und Behandlung der wichtigsten Seuchen unter den Rindvieh und den Pferden. By Ldw. H. Bojanus. 8vo. Riga, 1811 ; 8vo. Wilna, 1820 ; 16mo. Leipzig, 1830.

Proposals for the formation of a Corps of Lancers. By J. B. Drouville. 4 to. 1811.

Explanation of the various manœuvres of a Regiment of Cavalry. By Captain William Hackett. 8vo. London, 1811 ; 1819.

Anleitung zur Erkenntniss des Pferdealters aus den natürlichen veränderungen der Zähne. By Geo. Hogelmuller. 8vo. Vienna, 1811 ; 8vo. Munich, 1819.

Anleitung zur Pferdezucht fiir den österreichischen Staaten. By Jos. Kail. 8vo. Vienna, 1811.

Unterricht iiber dem Beschlag und der Behandlung gesunder und kranke Hüfe der Pferde. J. Langenbacher. 8vo. Vienna, $1811 ; 1813$.

Die Seuchen der landwirthschatichen Hausthiere nebst Geschichte, derselben. By Bernhard Laubender. 8vo. Munich, 1811.

The genealogy of the English Racer from the earliest times, to the Period of the Introduction of Foreign Blood into England, with Remarks on the Improvement of Blood Horses and a view of the present system of Breeding for the Turf, etc. By George Hanby Morland. 8vo. London, $1811 ; 1812$.

Lehrbuch der Pferdekenntniss. By Joh. Geo. Naumann. 8vo. Berlin, 1811 ; 1822.

Every Man his own Horse, Cattle, and Sheep Doctor. By Robert Pearson. 8vo. Leicester, 1811.

Thierärtzliche Feldapotheke, oder Noth-und Hülfsbuchlein für Offiziere der Cavallerie. By G. L. v. Pollnitz. 8vo. Altenburg, 1811.

Die Druse der Pferde und deren Heilung. By Heinr. Simon. Svo. Erlangen, 1811. 
Abhandlung iiber den Rotz der Pferde und die mit gutem Erfolge 1811. dagegen angewandten Mittel, nebst mehreren Beobachtungen iiber dessen Heilung. By Geo. Fr. Tscheulin. 8vo. Carlsruhe, $1811 ; 1812$.

Beschreibung einer durch Erfahrung erprobten Erfindung, das Durchgehen der Pferde beim Fahren für die im Wagen befindlichen Personen gänzlich gefahrlos zu machen. By K. Mldr. Barth. 4to. Dresden, 1812.

Handbuch der Zergliederungstunde des Pferdes, als Lcitfaden zu Vorlesungen am k. k. Thierarznei-Institute in Wien. By Joh. R. Jos. Brosche. 8 vo. Vienna, 1812.

Grundsätzer der rationellen Reitkunst für Privat-und Cavallerie-Officiere By Eph. Klatte. 8vo. Vienna, 1812.

Prodromus eine polizeigerichtliche Thierarzneikunde. By Bernhard Laubender. 8vo. Munich, 1812; 1827.

Vorschläge zur Verbesserung der Pferdezucht und Organisation des Landgestütes. By Konrad Ludwig Schwab. 8vo. Munich, 1812.

Die Geschichte des Pferdes, naturgeschischte, physikalisch, ökonomisch, technisch, historisch, etc, dargestellt und herausgeg. von K. W. Ammon. By Geo. Fr. Sebald. 8vo. Nuremberg, 1812.

Sulla epizoozia de' cavalli comparsa in Piemonte nel settembre 1811. By F. Toggia. Turin, 1812.

Allgemeine Pathologie der grösseren Hausthiere für angehende Thierarzte. By Hieron Waldinger. 12 mo. Vienna, 1812.

Lezioni di cavallerizza. By Stefano Arcellazzi.4to. Modena, 1813. 1813.

Beurtheilung und Erkenntniss der Beschaffenheit des äussern lebenden Pferdes oder das sogenannte Exterieur desselben. By Joh. Rep. Jos. Brosche. 8vo. Vienna, 1813.

A Description of the Structure of the Horse, as it appears by making a section through the body, with the diseases of the parts exposed, and also a new account of the framing or general composition of the Horse. By Bracy Clark. 4to. London, 1813.

The 2nd edition was published under another title, namely: "Original Remarks on the general framing of the Horse," 4 to. London, 1842. In both these editions there were plates; and also a large sectional figure of the Horse, which could be bought separately.

I I 
1813. On the Nature and cure of Rumning Frushes and Corns. By Bracy Clark. 4to. London, 1813.

Gedanken und Meinungen über Reitkunst. By Ludwig Daum. 8vo. Marburg, 1813.

Uber die Erkenntniss des Pferdealters aus den Zähnen. By Ign. Jos. Pessina. 8vo. Vienna, $1813 ; 1824$.

Therapie oder praktische Heilverfahren bei fieberharten Krankheiten der grösseren nützbaren Hausthiere, für angehende Thierärzte und Landwirthe. By Hieron Waldinger. 8vo. Vienna, 1813; $12 \mathrm{mo}$. Vienna, 1821.

1814. Sulla regenerazione delle razze de' cavalli, e sulla equitazione. By General Campagnola. Mantua, 1814.

A Description of a New Apparatus for Casting Horses, when it is necessary to secure them for performing painful operations. By Bracy Clark. 4to. London, 1814.

A Translation of this work: into French was minted at Paris, 1814 .

Ueber Drüsenkrankheiten der Pferde. By Mich. v. Erdelyi. 8vo. Vienna, 1814.

Die Darmgicht der Pferde und deren Heilung, oder Anweisung diese seither nnheilbare Krankheit mit wenigen Kosten zn beseitigen. By L. A. F. 8vo. Leipzig, 1814; 1824.

Anleitungzum zweckmässigen Beschlagen und Behandlung der gesunden und kranken Hufe der landwirthschaftliche Thiere aus dem Pferdegeschlechte. By Bern. Ant. Greve. 8vo. Hannover, 1814.

To all Sportsmen and particularly to Farmers and Gamekeepers. Above 'Thirty Years' Practice in Horses and Dogs ; how to feed and take care of them and also to cure them of all common disorders. Effectually to allure and catch all Vermin. The Ratcatching Secret; to catch every Rat on the premises alive. witlout using poison ... of Running Horses, training and breeding young Colts ... To which is added a plan for training and disciplining a Corps, etc. By Colonel George Hanger. 8vo. London, 1814.

Plan zu einer vergleichenden Zoologie, mit einer Linleitung in die vergleichende Anatomie des Pferdes. By Carl Fr. v. Kielmeyer. 8 vo. Tülingen, 1814 . 
Almanach für Freunde der Pferde und der Reitkunst. By Eph. 1814.

Klatte. 12mo. Vienna, 1814.

Aphorismen über die Pferdezucht, nebst einer Abhandlung über der natürlicher Bestimmung und Bevollkommnung der ungarischen Raceu. By Eph. Klatte. 12mo. Pesth, 1814.

Vergleichende Anatomie und Physiologie der Zähne der Säugethiere überhaupt, und besonders des Menschen und der Pferde. By Jens Weibel Neergaard. 4to. Copenhagen, 1814; 1816; 1823.

Observations, chiefly practical, on some of the more common Diseases of the Horse, together with remarks upon the general article of Diet and the ordinary Stable Management of that Animal. By Thomas Peall. 4to. Cork, 1814; 4to. London, 1815.

Veterinary Medicine and Therapeutics; containing the Effects of Medicines in various Animals. By William Peck. 8vo. London, 1814.

An Examination of the different systems of Shoeing Horses. By Richard Powis. 8vo. London, 1814.

In German :-Der neuste Englischer Hufschmidt,oder Prüfungen und Erfahrungen über die Zweckmässigkeit der neuesten und besten in England erfundenen und daselbst üblichen Hufeisen, nach Maasgabe der verschiedene Beschaffenheit der Pferdehufe, nebst Bescreibung der vorzuglichsten Krankheiten und Fehler der Pferdehufe und Angabe der Heilmittel. By L.L.H., 8vo. Pesth, 1817. From the 3rd English edition of the original.

Katechismus der Hufbeschlagkunst. Oder Theoretisch-Praktischer Unterricht iber den Beschlag der Hufe und Klauen im gesunden und kranken Zustande. By Konrad Ludwig Schwab. 8vo. Stuttgart, 1814; 8vo. Munich, 1815; 6th edition, 8vo. Stuttgart, 1834 ; 10th edition, 1851 ; 11th edition, 1855.

Yorkshire Farriery. By John Tindall. 8vo. London, 1814; 1834 ,

Ueber Gestiite. By Hieron Waldinger. 8vo. Pesth, 1814.

Anleitung wie marı nach bestimmten Verhältnissen die passendste Stangenzäumung finden kann. Nebst einer einfachen Ansicht der Grundsätze der Zäumung. By Maximilian v. Weyrother. 8 vo. Vienna, $1814 ; 1826 ; 1855$. 
1814. In French:-De l'embouchure du Cheval, ou Méthode pour trouver la meilleure forme de Mors; manière d'emboucher le Cheval, et principes sur l'art de le brider; suivie de la description d'une bride qui empêche le cheval de se cabrer. 8vo. 1828. Translated from the 2nd edition of the German by a French Officer.

1815. System of Cavalry Manœurres in Line. Anon. 8vo. London, 1815.

Des principales causes de la dégénération des races des chevaux et des règles à suivre pour les relever. By Ldw. H. Bojanus. 8vo. Wilna, 1815.

History of the Bots of Horses, and of other Animals. Giving an account of their species, habits and propogation; correcting some mistakes of Linnæus, Vallisneri, and Reaumur. Two new species are added, and some account of the skin flies of North America, forming a new genus (Cuterebra), since confirmed by Latreille. By Bracy Clark. 4to. London, 1815. 2 Plates.

An E'ssay on this subject was in 1796 communicated to the Linncean Society by the same author and republished in the above more extended form, 1815.

A Treatise on the Breeding, Training and Managing of Horses. By William Flint. 8vo. Hull, 1815; 1846.

Der Rathgeber für Reisende, ihre Pferde gehörig zu satteln, zu zaumen, etc., und von den ersten gewöhnlichen Krankheitsanfällen selbst zu heilen, etc. By Eph. Klatte. 16mo. Berlin, 1815.

Gesammelte Schriften von der Pferdewissenschaft. By Eph. Klatte. 8vo. Breslau, 1815.

The Improved Art of Riding. By G. Lloyd and R. Symes. 8vo. London, 1815.

Neue Entdeckung, den Fluss-Spat und Steingalle, so wie die Dampf, Husten, Darmgicht, etc. der Pferde zu kuriren nebst Anleitung wie man sich beim Einkauf der Pferde zu verhalten hat, auch dieselb bei starken strapazen immer gesund zu erhalten. By Wilh. Fr. Mahlbeck. 8vo. Elberfeld, 1815.

Geschichtliche Darstellung der Behandlung eines vollkommen geheilten Pferdebeinbruches, für Thierärzte und Pferdeliebhaber. By Theodore Merk. 8vo. Munich, 1815.

Kleine Reitschule zur Selbstbelehrung. By G. L. v. Pollnitz. 8vo. Erfurt, 1815. 
Katechismus fur Beschlag-schmiede, oder kurzgefasster Unterricht 1815. uiber dem Huf beschlag. By Konrad Ludwig Schwab. 8vo. Nürnberg, 1815 ; Munich, 1817 ; $1820 ; 1822 ; 1828$.

The 3rd and following editions have besides:- "Praktische Unterricht ïber die gewohnlichen Krankheiten des Pferdes."

Materialien zu einer Pathologische Anatomie der Hausthiere. By Konrad Ludwig Schwab. 8vo. Munich, 1815.

Vollständiges Naturgeschichte des Pferdes, mit der Literatur iiber diesen Gegenstand, herausgegeben von K. W. Ammon. By Geo. Fr. Sebald. 8vo. Ansbach, 1815.

Die Sicherste und Einfachste Heilmethode der Pferdekrankheiten, etc. By S. v. Tennecker. 8vo. Dresden, 1815.

Kunst die Nervenkrankheiten der vorzuglichsten Hausthiere zu erkennen, ihnen vorzubeugen und sie zu heilen. By Geo. Fr. Tscheulin. 8vo. Karlsruhe, 1815.

Bemerkungen über Lösedürre. By Hieron Waldinger. Erlited by D. J. Lidl. 8vo. Vienna, 1815.

Treatise on Veterinary Medicine. By James White. 4 vols. 12 mo. London, 1815.

Archiv für Thierheilkunde Von der Gesellschaft Schweizerischer Thierärzte. Anon. 8vo. Zurich, 1816.

Handbuch der Thierarzneikunde, oder Unterricht wie jeder Landwirth seine Pferde, Schaafe, Schweine, Ziegen und Hunde aufziehen warten und füttern und ihre Krankheiten erkennen und heilen soll. Anon. 8vo. Leipsic, 1816.

The Beauties and Defects in the Figure of the Horse, comparatively delineated in a series of coloured plates. By H. Alken. Svo. London, [1816.]

In German as:-Das Schöne und Mangelhafte im Exterieur des Pferdes, nach dem Englisch von F. L. C. Steinhoff und $\mathrm{Ch}$. F. M. Peters bildlich dargestellt. folio. Hamburg, 1830.

A Practical Treatise on the Diseases of the Foot of the Horse, with Observations on Shoeing. By Richard Hayward Budd. 8vo. 1816.

An Essay on the Gripes of Horses, with an improved and successful mode of treating this Disorder; the Secret Remedy long used by the author in this complaint, is made public. By Bracy Clark. 4to. Londun, 1816. 
1816. The 2nd erlition of the above was published under the title of A Description of the Gripes of Horses and of a better mode of treating it ; also Human Cholera explained shewing its identity with the above, etc. 4to. London, 1837.

Le Règne animal distribné d'après son organisation, pour servir de base à l'histoire naturelle des animaux et d'introduction à l'anatomie comparée. By the Baron George Leopold Chrétien Frederic Dagobert Cuvier. 4 vols. 8vo. Paris, 1816.

There are many French editions, also an English translation; 16 vols. 8vo. London, 1827 , etc.

Erfahrungen und Bemerkungen iiber die in der neuesten Zeit am meisten geprissenen Heilmittel in der Rotzkrankheit der Pferde. By Fr. Karl Lappe. 8vo. Gottingen, 1816.

Lehrbuch der gerichtlichen Thierarzneikunde zun Gebrauch fiir angehende Gerichsthierärate. By Bernhard Laubender. 8vo. Munich, 1816.

The Complete Farrier and British Sportsman, containing a systematic enquiry into the structure and animal economy of the horse. The causes, symptoms, and most approved methods of prevention and cure for every disease to which he is liable. A detection and exposure of the erroneous and bad methods of treatment generally adopted, with numerous approved and original recipes for various diseases. The whole rendered easy and familiar with a view to general utility and founded on the latest discoveries and experimental facts, to which the progress of improvement for the last 20 years in the Veterinary Art has led. Including a faithful delineation of the various dogs used in the sports of the field, with canine pathology, interspersed with sporting anecdotes and an account of the most celebrated horses, dogs, etc., etc., equally important and interesting to the British sportsman as well as innkeepers, coachmasters, farmers, owners of stage wagons, etc., embellished with a series of engravings executed in the first style of art, from original drawings in the possession of noblemen and gentlemen of the Turf, and copied by permission, exclusively for this work. By Richard Lawrence. 4to. London, 1816.

Théorie de l'escrime à cheval, pour se défendre avec advantage contre toutes les espèces d'armes blanches. By Alexandre Muller. 8vo. Paris, 1816.

2nd edition contrining 60 new articles with an atlas of 54 figures, 8vo. Paris, 1828, Atlas, 4 to. 
Der Pferdearzt im Felde; Ein noth-und Huilfsbüchlein fiir die Caval- 1816. lerie. By Joh. Nic. Rohlwes. $12 \mathrm{mo}$. Berlin, 1816.

Vorschläge zu einem Zweckmässige Unterricht der Cur und Fahnenschmiede. By S. v. Tennecker. 8vo. Leipzig, 1816.

Erfahrungen von und für praktische Pferdeärzte, Cur und Fahnenschmiede. By Chr. Gfr. Wagner. 8vo. Berlin, 1816-17.

Das ganze der Pferdezucht. Ein Handbuch fuir Jedermann der Pferde 1817. nöthig hat und damit umgehen muss; vorziglich fiir Landleute, Kavallerie-Officiere, Bereiter, Pferdeverleiher und fuir Staadt-und Dorfschmiede, etc. Nebst Angabe verschiedener Betriigereien, deren sich die Rosshändler beim Verkaufe bedienen, etc. Mit einer Abbildung des Pfertes und aller seiner Theile. Anon. Leipsic, 1817.

Taschenbuch für Pferdebesitzer, oder Verhaltungsregelı, Recepte und Operationvorschriften, wonach Man seine Pferde gesund erhalten und ihnen, sowohl bei inneru Krankheiten als anch bei Verwundungen und andern äussern Schaden, in Ermangelung eines Rossarztes, ohne nachtheiligen Verzug Selbst helfen kann. Anon. 8vo. Berlin, 1817.

Des Troupes Légères, on refléxions sur l'Infanterie et la Cavalerie légère. By le Comte de la Roche Aymon. Svo. Paris, 1817.

Auflösung der Preisfrage, wie scheu gewordene Pferde von den in dem Wagen befindlichen Personen schnell abzulosen sind. By J. G. Bruggemann. folio. Hamburg, 1817.

Die Reitkunst auf dem Jagd, im Felde, im Militiir und auf der Academie. By Ludwig Daum. 8vo. Marburg, 1817.

De l'affection Tuberculeuse, Vulgairement appelée Morve. By Dupuy. 8vo. Paris, 1817.

Cour's théorique et pratique de maréchalerie vétérinaire. By $\mathrm{Fr}$. Jauze. 2 parts, 4to. Paris, 1817-18.

In German as:-Die Vollständige Huf beschlagkunst, oder Anweisung jede Gattung von Arbeitsthieren zu beschlagen, ihre Hufkrankheiten zu verhiiten, und dieselben zweckmässig zu behandeln und zu heilen. Ein fiir Thierärzte, Stallmeister, Posthalter, und Landwirthe, so wie für andere Besitzer von Thieren, bei denen der Hufbeschlag anwendbar ist, sehr nützliches Werk. Translated by J. G. Müller. 8vo. Berlin, 1834.

Sammlung der vorziiglichsten Schriften über höhere Reitkunst und Pferdekunde. By Eph. Klatte. 8ro. Breslau, 1817. 
1817. Mittel zu einer sparsamern und zugleich nuitzlichen Fiitterung der Pferde. By Ritter Jos. Arn. von Lewaneu. 8ro. Vienna, 1817.

Arztliche Gemälde der Lungenentziindung der Pferde. By Fr. H. v. Sallwurk. 8vo. Heidelberg, 1817.

Taschenbuch des Pferdekunde fïr Stallmeister, Offiziere, Oekonomen, und Thierärzte. By Konrad Ludwig Schwab and Ant. Will. 12 mo. Munich, $1817 ; 1823$.

Beschreibung und Heilung des Nervenfiebers, welches im Frihjahr und Sommer 1817, unter den Pferden hier und in der Gegend geherrscht hat. By Geo. Fr. Tscheulin. 8vo. Carlsruhe, 1817.

Handbuch des Veterinärkunde, in besonderer Beziehung auf den Seuchen der nuitzbarsten Haus-Säugethiere, etc. By Johann Elias Veith. 8vo. Vienna, 1817-18; $1822 ; 1831$.

1818. Ueber die zweckmässigste Pferde zur algemeinem Zucht zunächst in Dänemark und den Herzogsthiimern. By Ludwig Berghofer. 8vo. Hamburg, 1818.

The Sand-crack and Quittor. By Bracy Clark. 4to. London, 1818.

Das Gefechtslehre der beiden verbunden Waffen, Kavallerie und reitende Artillerie. By Karl v. Decker. 8vo. Berlin, 1818.

Das Pferd und die Pferdezucht, oder Beschreibung der merkwürdigsten Raçen, der Eigenschaften, Vollkommenheiten und Fehler, etc., der Pferde in verschiedenen Ländern. By Heinr. Gust. Florke. Svo. Berlin, 1818.

Der Hause-pferdeartzt. Ein Handbuch fiir Pferdebesitzer. By Eph. Klatte. 8vo. Berlin,1818.

Zeitgemässige Abhandlung uber den Pferdeschlachten den Fleischgenuss und den daraus hervorgehenden Letzten

Pferdes nach allen seinen Theilen. By Jh. Mart. Kreutzer. 8vo. Kempt, $1818 ; 1838$.

Gegenbemerkungen zu dem von Tennecherschen Taschenbuche für Pferdeliebhaber By G. Ludwig v. Pollnitz. 8vo. Erfurt and Gotha, 1818.

Das Pferd, oder vollstandige Anleitung zur Erkenntniss alles dessen was man bei einem Pferde zu wissen unumgänglich nöthig hat in Hinsicht rler Fütterung. Pflege und Wartung. By G. Ludwig von Pollnitz. 8vo. Erfurt and Gotha, 1818. 
British Field Sports, embracing practical instructions in Shooting, Hunting, Coursing, Racing, Cocking and Fishing, with observations on the breaking and training of Dogs and Horses, etc., etc. By William Henry Scott. 8vo. London, 1818.

The Horseowner's Guide, containing valuable information on the management and cure of the Diseases incident to Horses, more particularly that very fatal disease called Glanders, with many esteemed recipes. By Thomas Smith. Svo. London, 1818.

Der Pferdearzt, oder Anleitung fiir Landwirthe, die Pferde gesund zu erhalten und die gewohnlichsten Krankheiten derselben $\mathrm{zu}$ verhiiten und vernünftig zu behandeln. Neit ein. Vorr. von J. D. Busch. By Chr. Thielemann. 8vo. Marburg, 1818.

Wochenblatt der Viehzucht, Thierarzneikunde, Reitkunst und des Thierhandels. By Joh. Jac. Weidenkeller. 4to. Nuremburg, 1818 ; 8vo. Nuremburg, 1821.

A Treatise on the most important Diseases which attack the Horse. By William Wilkinson. 4to. London, 1818.

Kurze Anleitung zur Erkenntniss des Pferdealters aus den natürlichen 1819. Veranderungen der Zähne. Anon. 8vo. Munich, 1819.

Recreations in Natural History, or popular Sketches of British Quadrupeds. Anon. 8vo. London, 1819.

Regulations and Instructions for the Cavalry Sword Exercise. Anon. London, 1819.

Grundlinien der Eingeweidlehre der Haussäugethiere, insbesondere des Pferdes. By Mich. v. Erdelyi. 8vo. Viemna, 1819.

Grundlinien der Nerven-und Gefasslehre der Haussäugethiere, insbesondere des Pferdes. By Mich. von Erdelyi. 8vo. Vienna, 1819.

Krankheiten der Pferde der Strengel die Bräune, die Lungenentzündung, die Druse die Verdächtige oder falsche Druse, der Rotz, der Wurm die Brustwassersucht. By Ludwig Daum. 8vo. Marburg, 1819.

Anviisning for Kudske og Enhver, rom, har med Heste at bestille, tilligerned en Underretning om Malkekoers Pleie og Rogh. By Ant. Fr. Jush. 1819.

Ueber den Umgang mit Pferden und nenestes Art den wildesten und bei der Behandlung, besonders beim Beschlagen bösartigen, und beim Gebrauche zum ziehen gefährlichst widersetzlichen Pferde in möglichst kurzer Zeit zahm, gutartig und brauchbar zu machen. By Karl Kegel. 8vo. Bamberg, 1819. 
1819. Die Zahmungskunde, ein Handbuch fur Cavallerie Offiziere Bereiter, und Pferdeliebhaber. By Eph. Klatte. 8vo. Berlin, 1819.

Schutz in Gefahren bei Wagenfahrten, oder solche Einrichtung der Wägen, mittelst welcher die durch das Ausreissen der Pferde geschehende Ungliicksfälle beseitigt werden. [By C. B. von Machay. 8vo. Pesth, 1819.]

Sportsman's Directory on Shooting, Fishing, Breeding, Hunting, etc. By John Mayer. Svo. London, 1819; 1828.

Praktisches Handbuch fiir Stallmeister, Offiziere, Oekonomen, Thierärte und Pferleliebhaber. Nebst tabellarische Uebersicht der in verschiedenen Krankheiten behandelten Pferden. By Theodore Merk. Svo. Munich, 1819; 1826.

Militar Reitschuler, oder Anweisung alles dessen was ein Unteroffizier der Cavallerie wissen muss, um junge Soldaten anzuweisen. By G. L. v. Pollnitz. 8vo. Halberstadt, 1819.

Reitschule für Militär-und Civil-Personem. Enthalt das Wissenswiirdigste fiir Cavallerie-Unterofficiere zum Reitunterricht fiir junge Soldaten und zum Selbsterlernen der Reitkunst. By Gottlob Ludwig von Pollnitz. 8vo. Bautzen, 1819. 3rt erlition, 1849 .

Beschreibung einiger gefahrlichen Krankheiten unter Pferden Rindvich und Schaafen und deren Heilung, ingleichen iiber die Futterarten unter denselben und iiber Einrichtung der Schaafkrippen mit Raufen. The 2nd part of the work entitled-Rathgeber für Schäfereibesitzer und Land wirthe. 5 parts. By Joh. Nic. Rohlwes. 8vo. Berlin, 1819-23.

Bescreibung und Heilung der Wumkrankheiten und anderer gefahrlichen Krankheiten der Pferde; ingleichen Anleitung die nicht Milch gebenden Kuhe melkend zu machen, so wie einen Auszug aus Culley's Werk "iiber die Veredlung der Schaafe." By Joh. Nic. Rohlwes. 8vo. Berlin, 1819-23.

Thierärztliches Receptbuch, oder Auswahl der wirksamsten und Zuverlassigsten Arzneimittel und Operationen in den innerlichen und äusserlichen Krankheiten der Pferde, des Hornviches, der Schaafe, Schweine und Hunde, etc., nach vieljährige Beobachtungen aufgezeichnet. By J. G. Schmidt. 8vo. Pesth, $1819 ; 1829$.

Anatomische Abildungen der Pferdekorpers für bildende Kunstler, etc. By Konrad Ludwig Schwab. fol. Munich, 1820. 
Beobachtungen und Erfahrungen uiber die Erkentnniss und Heilung 1819. die jetzt unter den Pferden herschenden Druse. By S. von Tennecker. 8vo. Dresden, 1819.

Lehrbuch der Veterinär-Chirurgie oder Wundärtzneikunst. By S. von. Tennecker. 8vo. Prag., $1819 ; 1820$.

Su le cause le più perniciose della cecità de' cavalli. By Fr. Toggia. Turin, 1819.

Die Pferdezucht in einem Gespräch, etc. By Geo. Fr. Tscheulin. 8vo. Karlsruhe, 1819.

Auch eine Ansicht über Unterrichtsansstalten für Kavallerie in Bezug 1820 auf die Königliche preussiche Lehr-Eskadron. Anon. 1820.

Instructions in Military Equitation wherein the Principles and Practice of Horsemanship, the Proper Position on Horseback, and the Aids of the Bridle, Hand, and of the Legs are clearly defined; with Directions for the Breaking and Training of Horses. To these are added Instructions in the Elements of the Field Movements prescribed for the Cavalry by His Majesty's command. Anon. London, 1820.

Standing Orders for the Light Cavalry of the Army of Fort St. George. Anon. Madras, [1820.]

The Art of Riding and to Manage a Vicious Horse. Anon. 8vo. London, 1820.

The Review, Exercise and Evolutions of a Squadron (as published by authority) methodically arranged and illustrated by a series of Engravings, descriptive of the relative situations of the Commissioned Staff and non-commissioned Officers on Parade and in Manœuvre. Anon. 8vo. Gloucester, [Circa 1820].

Ueber Wartung und Abrichtung der Remontepferde. Anon. 1820.

Felddienst-Instruktion für die Kavallerie. By General Graf. v. Bismark. 1820.

In French as:-Tactique de la cavalerie, suivie d'élements de manœuvres. Translated by Max. F. de Schauenburg. 8vo. Strasbourg, 1821.

In English as:-Lectures on the Tactics of Cavalry 8 vo. London, 1827. Translated by N. Ludlow Beamish. 1855.

Schreiben an den Redacteur des Jahrbuchs der Preussischen Landwirthschaft der englische Pferdezucht betreffend. By.C. F. W von Burgsdorf. 8vo. Tilsit, 1820. 
1820. On the Treatment of Horses' Feet. By F. C. Cherry. 8vo. London, 1820 .

Von den Hornspalten, den Ochsenklaue und Rehkrankheit der Pferde. By Ludwig Daum. 8vo. Marburg, 1820.

Zaumkunst. By Ludwig Daum. 8vo. Wieshaden, 1820.

Traitement pour toute espèce de Maladies des Chevaux. By E. Desmarè. [Circa 1820].

Benennungen der einzelnen Regionen und Theile des äussern Pferdekörpers und Angabe des Sitzes der daran vorkommenden Fehler und Krankheiten. Mit Hinweisung auf das dazu gehorende Werk; Die Fehler und Gewährsmängel bei den Pferden zu erkennen. By J. F. C. Dieterichs. [1820].

The Natural History of British Quadrupeds. By E. Donovan. 3 vols. 8 vo. London, 1820 .

Grundlinien der Knochenlehre des Pferdes, mit Berucksichtigung der Abweichungen bey den übrigen Haussäugethieren. Nebst ein vollständiger Anweisung zur Beurtheilung des Alters aus den Zähnen. By Mich. v. Erdelyi. 8vo. Vienna, 1820.

Darstellung des Zahnalters des Pferdes, Rindes, Schaafes und Schweines nebst ein Pferde-skelette mit den äussern Umrissen auf 3 Folio Kpfn. sammt Erklärung, Texte und I. Tab. By Mich. von Erdelyi. 8vo. Vienna, 1820.

Versuch ein Zoophysiologie des Pferdes und den iibrigen Haussängethiere. Nebst ein Skizze und 2 Uebersichts-Tabelle der merkwiirdigste in d. österr. Monarchie befindliche gestiite, und ein Anlang iiber der Färbung der Haare bey den Pferden und den uibrigen Haussäugethieren. Als Handbuch fiir angehende Thierärzte und Oekonomen bearbeitet. By Mich. v. Erdelyi. 8vo. Vienna, $1820 ; 1830$.

New System of Shoeing horses, with an account of the various modes of different nations, and observations on the Diseases of the Feet. By J. Goodwin. 8vo. London, 1820 ; 1824.

Das Innere des Pferdes in seinen Muskeln bildlich dargestellt und erklärt. By August. Konrad Havemann. 8vo. Vienna, 1820.

Mittheilungen aus dem Umfange der Pferdezucht, Pferdekenntniss, Reitkunst, etc. By Karl Kegel. 8vo. Bamberg, 1820. 
Ueber die Pferdezucht in England. By Freikerr K. von 1820. Knobelsdorff. 8vo. Berlin, 1820.

Hülfsbuch zur Erlernuug der Anatomie für angehende Hufschmiede. By Jos. König. 8vo. Vienna, 1820.

Ueber die Zähne der Thiere und die Krankheiten derselben, vorziiglich bei Pferden, Hunden, Schweinen, Haasen, etc., Hans-und Waldthieren, nebst einer vollständig mit Kupfern versinnlichter Erklärung, das Alter dieser Thiere aus den Zähnen zu erkennen, etc. By Jos. König. 8vo. Vienna, 1820.

Modern Farriery, or Diseases of the Horse and their cure, with Sporting Aneedotes. By A. Lawson. 8vo. London, [1820.]

Der praktischer Pferdearzt. Ein Handbuch fiir Pferdliebhaber und Oekonomen. Mit deutschen Recepten. By Theod. Merk. 8vo. Munich, 1820. Another edition, 1826.

Exercise and Manœurres of the Lance. By Lieutenant-Colonel Montmorency. 4to. London, 1820.

Das fehlerhafte Pferd, oder Darstellung aller an einem Pferde äusserlich sichtbaren Mangel und Gebrechen, nebst kurzer Beschreibung und Heilung derselben. By G. L. von Pollnitz. 8vo. Halberstadt, 1820 .

In Swedish as:-Haistens yttre fel. Anwisning till deras botande. Med ett Bihang inuehällende konsten att kunna noga bestämma hästens älder, jemte fyra proberade recepter. En nyttig Handbok för alla Landtbrnkare, äfwensom för Hästegare i allmänhet. $16 \mathrm{mo}$. Stockholm, 1857.

Der Rathgeber für Pferdebesitzer beim Ankauf, und Zweckmässigen Behandlungen der Pferde, Kenntniss ihrer Schönheiten und Fehler, etc. By Rasemann. 8vo. Chemuitz, 1820.

Die unentdeckte gründliche Heilung der Drüsenkrankheiten der Pferde, und vorziiglich des bisher fuir unheilbar gehaltenen wirklichen Rotzes, etc. By A. Schlichting. 3 vols. 12 mo. Oppeln, 18:0.

Entwurf einem algemeinem Pathologie der Hausthiere. By Konrad Ludwig Schwab. 8vo. Munich, 1820.

Sportsman's Repository ; comprising a Series of Engravings representing the Horse and the Dog in all their varieties, by John Scott. from original paintings by Reinagle, Gilpin, Stubbs, Cooper, and Landseer, with Descriptions and Anecdotes. 4to. London, $1820 ; 1845$. 
1820. Zähmung und Abrichtung der Wildfänge, Ein Handbuch für den Reiter. By Max Swoboda. 12mo. Vienna, 1820.

Anleitung zu der Einrichtung einer Feld-und Hausapotheke für Dekonomen, Thierärzte, Cur und Fahnenschmiele, etc. By S. von Tennecker. 8vo. Leipzig, 1820.

Beobachtungen und Erfahrungen iiber die Erkenntniss und Cur der Darmentziundung bei Pferden. By S. von Tennecker. 8vo. Prag, 1820.

Die Sicherste und zuverlässigte methode stallböse und widerspenstige Pferde in der möglichst kurzesten Zeit mit Sicherheit und gefahrlössigkeit an den Hufbeschlag und an den $\mathrm{Zug} z \mathrm{u}$ gewohnen. By S. v. Tennecker. 8vo. Leipzig, 1820.

Lehrbuch der Gestutswissenschaft, Stallmeister, etc. By S. von Tennecker. 8vo. Prag, 1820.

Lehrbuch der Pferde ärtzlicher geburtshïlfe und Heilung der gewöhnliche Krankheiten der Mutterstiten und Fohlen. By S. von Tennecker. 8vo. Prag, 1820.

Ueber die englische Pferdezucht, mit Beziehung ihre Grundsätze auf der Veredlung des Pferdegeschlechts im übrigen Europa und besonders in Deutschland. By Count R. von Veltheim. 8vo. Brunswick, 1820.

Abhandlung über den Schwefel und seine Verbindungen mit metallen, Kalien, und Erden, wie sie am und im Thierischen Körper wirken, vorziiglich bei Pferden in Krankheiten der Sauggefässe um dem Rotze vorzubeugen. By Hieron Waldinger. $12 \mathrm{mo}$. Vienna, $18 \% 0$.

Das Pferd, seine verschiedenen Zuchten und Spierlaten, seine Erziehung, Geschichte, seine Verbreitung, Nutzen, Krankheiten, Fehler und Feinde. By Fr. Ludw. Walther. 8vo. Giessen, 1820.

1821. Anleitung den Starrkramf oder die Hirschkrankheit der Pferde zu erkennen und grundlich zu heilen. By Carl. Fried. Bohlmann. 8 vo. Leipzig, 1821.

Theoretische Unterricht uiber Feld-und Schul-Militär, und SchulparadeReiten. By Ludwig Daum. 4to. Coblenz and Hadamar, 1821.

Kurzer Unterricht fiir Beschlagschmiede über dem Hufbeschlag der Pferde. By G. L. Feuring. 8vo. Hamm, 1821. 
Theoretisches praktisches Handbuch der äussern Pferdekenntniss und 1821. der Wartung und Pflege der Pferde. By Conrad v. Hochstetter. 8vo. Bern, 18\%1-1824.

Die Kenntniss von dem Pferde, in Hinsicht auf dessen Natur, Körperschönheit, Eigenschaften u. s. w., nebst einem Unterricht zur Behandlumg der Rehe, der Druse und der Kolik, als die bei Liebhaberpferden am meisten vorkommenden Krankheiten, so wie auch einer Tabelle iiber die Körperlichen Verhältnisse eines idealisch-schönen Pferdes. By Joh. Chr. Ribbe. 8vo. Altenburg, 1821.

Kleine Beiträge zur praktische Veterinär-IVissenschaft. By Konrad Ludwig Schwab. 8vo. Munich, 1821.

A new and complete guide to the Art of Riding, teaching the whole System of Horsemanship, etc. By Charles Steward. 8vo. London, 1821.

Shoeing. By Teast. 1821.

Die Rieitschule, oder grundliche Anweisung zur Reitkunst, etc. By S. von Tennecker. 8vo. Leipzig, $1821 ; 1823 ; 1832$.

Pracktisches Lehrbuch der Hufbeschlagungskunst und der Erkenntniss und Heilung der Hufkrankheiten. By S. von Tennecker. 8vo. Altenburg, 1821.

Polizeiliche Thierarzneikunde für officielle Thierärzte und Fahnensclmmiede. By Geo. Fr. Tscheulin. 8vo. Karlsinhe, 1821.

Vollständige Uebersicht und Belehrung, iiber die gesetzliche bestimmte Hauptmängel rer Pferde und des Rindviehes. By J. Ueberacker. 8vo. Rothenburg, $182 \mathrm{l}$.

Hestens Ydrelœre. By Eric Nissen Viborg. Copenhagen, 1821.

Anweisung für jeden Pferde-und Schafbesitzer die Drusenkrankheit der Pferde die Faulegel-und Lungenwïrmer-Krankheit bei Schafen und Pferden zu erkennen und zu heilen. By Jh. Werner. 8vo. Breslau, 1821.

Annals of Sporting and Fancy Gazette, 8vo. London, 1822.ete. 1822.

System der Reiterei. By General Graf v. Bismark. 1822.

On Canker and Ring Bone. By Bracy Clark. 4to. London, 1822.

Patologia general y Terapéutica. By Cusac. 8vo. Valencia, 1822. 
1822. Die zweckmässigste Art, oriental Pferde zuzureiten, welche von der schnellen und stehenden Art sind, oder davon abstammen. By Ludwig Daum. 8vo. Coblenz, 1822.

Ueber das Koppen der Pferde den besten Mittel es zu verhuiten, die daher entstehende Kolik schnell zu heilen, und oder auf den Nachkommen erbe, od ob man einen Beschäler, welcher koppt, in einem Gestiite leiden soll. By Ludwig Daum. 8vo. Hadamar and Coblentz, 1822.

Anleitung das Alter des Pferdes, der Rinder und der Schaafe nach den naturlichen Zahnwechsel und den Veranderungen der Zähne zu erkennen, etc., Nebst Anmerkungen uiber das Koppen und uiber einige Krankheiten die mit der Durchbruche der Zähne vorkommen. By Joh. Fr. Chr. Dieterichs. 8vo. Berlin, 1822; 1837.

Handbuch der Veterinär-Chirurgie, oder das Kunst die äusseren Krankheiten der Pferde zu erkennen und zu heilen. By Jh. F. Chr. Dieterichs. 8vo. Berlin, $1822 ; 1825 ; 1829$.

Traité concernant la connaissance du cheval, la manière de le nourrir, tant en voyage qu à l'écurie, et de le préserver des accidents et maladies occasionnés par le mauvais traitement, suivi des Principes d'Equitation Moderne. By le Vicomte E. Dutoict. $12 \mathrm{mo}$. Brussels, 1822.

Abhandlung iiber die sichere Anwendung des Kalkes, als Vorbeugungsmittel wider die Krankheiten des Lymphsystems, besonders wider den Rotz und den Hautwurm bei dem Pferdegeschlechte. By Ch. Halberbach. 12mo. Berlin, 1822.

Unterricht über die Pferdehufbeschlagkunst, und die Behandlung der Kranken und fehlerhaften Hufe; nebst einer Abhandlung uiber die Castration der Pferde. By Sigm Hoerdt. 8vo. Stuttgart, $1822 ; 1829$.

Anleitung zur Beurtheilung der Hauptmängel der Hausthiere. By J. Dan. Hofacker. 8vo. Tübingen, $1822 ; 1825$.

Das Alter des Pferdes nach den Zähnen zu bestimmen. Zusammengestellt nach Ge. Kirtland u. Ign. Jos. Pessina. folio. Weimar, 1822.

Das Zäumungsmesser, oder die Variationsstange, eine neuerfundene Hulfsmaschine. By Eph. Klatte. 4to. Berlin, 1822.

Systematisches Lehrbuch der Campagne-Reitkunst und der rationellen Bearbeitung des Campagne-Pferdes. By Eph. Klatte. 8vo. Berlin, 1822. 
Exposé rles causes qui retardent les progrès de la médicine vétérinaire. 1822.

By F. 1.. Morel. 8vo. Paris, 1822.

Fassliches Unterricht zur campagne-Reiterei. By Andr. Politz. 8vo. Berlin, 1822.

Trattato in compendio sull' ugna organizzata e sulla ferratura del cavallo. By Raffaele Fusco. Naples, 1822.

Das Ganze der Thierheilkunde nebst allen damit verbundenen Wissenschaften, oder Bücher der Thierarzneiwissenschaft für Landwirthe, Cavalleristen, Pferdeziichter, Thierarzte und Pferdeliebhaber nach des Verfassers Tode vom 3n. Bande an fortgesetzt von Chr. Ehrf. Seyfert v. Tennecker. By Joh. Nic. Rohlwes. 8 vo. Leipzig, 1822 .

Grïndliche neue Heilung der Drusenkrankheiten, also auch vorziiglich des Rotzes der Pferde oder des nun zu nennenden kleinen und grossen Drusens. By Aloys. Schlichting. 12mo. Oppeln, 1822.

Lehrbuch des Pferdehandels und der Rosstäuscherkunste. By S. v. Tennecker. 8vo. Hannover, 1822; 8vo. Jimenau, 1824; 8vo. Hannover, 1829.

Pferdeärtzliche Praxis. By S. v. Tennecker. 3 vols. 8vo. Altenburg, 1822-1823.

Kriegspferdekunde für Officiere, Thierärzte und Fahnenschmiede. By Geo. Fr. Tscheulin. 8vo. Carlsruhe, 1822.

Die Russischen und Polnischen Pferde in den süddeutschen staaten rucksichtlich ihrer Lebensdauer, vorzuglichsten Krankheiten und Gebrechen, thierarztliche Behandlung und ihrer Brauchbarkeit zum Militair und Civildienste, mit einem Hinblick auf die inländische Pferdezucht. By Joh. Jac. Weidenkeller. 8vo. Nuremberg, 1822.

An Authentic history of the celebrated horse, American Eclipse. 1823. Anon. 8vo. New York, 1823.

The Modern Farrier. The best mode of preserving the Health and curing the Disorders of domestic animals, with practical instructions to Sportsmen. Anon. 8vo. Newcastle, 1823.

Archiv für Viehzucht, Pferdekenntniss, Reitkunst, Thierarzneikunde und Thierhandel. In verbindung mit Seyff. v. Tennecker herausgegeben von J. J. Weidenkeller. 8vo. Altenburg, 182327 , etc.

Continued as:-Archiv fuir Stallmeister, Pferdezuchter, Bereiter, etc. 8vo. Altenburg, 1827, etc. 
1823. Ueber die hitzige Kopfkrankheiten der Pferde, mit einige Anhänge By Chr. Fr. Authenrieth. 8vo. Tiibingen, 1823.

Elementos de hipotomia. By Antonio Bobadilla. 8vo. Madrid, 1823.

Vereinfachte Campagnen-Reiterei oder die Soldaten-Reiterei. Ein Leitfaden zum beliebigen Gebrauch. By Carl v. Braunschweig. 1823.

Bibliothek der Veterinärkunde fur Thierärzte und Oekonomen. By H. Burger. 8vo. Berlin, 1823.

Reformed Pharmacopœia for Horses, containing a general view of Horse medicine, a Materia Dietetica, Materia Medica, and Pharmacopœia with many new and valuable prescriptions. By Bracy Clark. 4to. London, $1823 ; 1824$.

3rd edition, under title of 'Pharmacnpoir Equina or New Pharmacopreia for Horses,' 4to. London, 1833.

L'Ippossiade o l'accademico equestre. By E. Conti. 8vo. Turin, 1823.

Die Hufbeschlagskunst oder Anleitung sowohl die gesunden, als auch fehlerhaften Hufe der Pferde zweckmässig zu behandeln und zu beschlagen. By Joh. Fr. Chr. Dieterichs. Svo. Berlin, 1823.

Handbuch der allgemeiner und besondern sowohl theoretische als praktische Arzneimittellehre fiir Thierärzte und Landwirthe. Oder allgemein verstandlicher Unterricht iiber die in der Thierheilkunde zu benutzenden Arzneimittel, ihre Kennzeichen, Bestandtheile, Wirkungen und Verheilungsart; mit Bestimmung der Gabe und Form, in welcher das Heilmittel, gegen den verschiedenen Krankheiten anzuwenden sind. By Joh. Fr. Chr. Dieterichs. 8vo. Berlin, 1823.

Traité des Haras, et de Médecine Vétérinaire Simplifiée, mis a la portée de tout le monde. By le Vicomte E. Dutoict. $12 \mathrm{mo}$. Brussels, 1823.

Lehrbuch uiber die gewöhnlichen allgemeinen Krankheiten des Pferdes Rindviehes, Schaafes, Schweines, Hundes und uiber der Heilung und Verhutung derselben nebst einer Anhange von recepten. Zum Gebrauch bei Vorlesungen und zum Selbstunterrichte, etc. By J. Dan Hofacker. Svo. Tuibingen, 1823.

Médecine Vétérinaire. Aperçu général sur l'inflammation. By F. L Morel. 8vo. Paris, 1823. 
Traité raisonnée de la Morve. By F. L. Morel. 8vo. Paris, 1823. 1823.

Valuable Recipes for Neat Stock, Horses, Sheep, Pigs, and Dogs. By Murrell. 18:3.

Essai Elémentaire sur l'art de l'équitation. By Louis Charles Pellier. 18 mo. Paris, 1823 ; $1834 ; 1858$.

A Series of Elementary Lectures on the Veterinary Act, wherein the Anatomy, Physiology, and Pathology of the Horse are essayed on the general principles of Medical Science. By William Percivall. 2 vols. 8vo. London, 1823 and 1824.

A Guide to Practical Farriery, containing hints on the diseases of Horses and Neat Cattle, with an appendix. By J. Pursglove. 8vo. London, 1823.

Jahrbuch für Pferdezucht, Pferdekenntniss, Pferdehandel, die Militair Campagne-schul und Kunstreiterei, und den Rossarzneikunst in Deutschland und den angränzenden Länden. By S. v. Tennecker. 12mo. Ilmenau, 1823, etc.

Practische Beobachtungen und Erfahrungen über die Erkenntniss und Heilung der im Herbst 1822 und noch jetzt fortdanernde chronisch Lungen-und Leberentzundung. By $\mathbf{S}$. von Tennecker. 8 vo. Ilmenau, 1823.

Katechismus der Thierarzneikunde oder Unterricht, etc. By Geo. Fr. Tscheulin. 8vo. Karlsruhe, 1823.

A set of six large and fine plates of celebrated horses, comprising Phantom, Monitor, Primrose and foal, Moses, (the property of the Duke of York), a Persian horse, and a Cossack horse. By James Ward. folio. London, 1823.

Der Praktische Pferdearzt. Ein nutzliches Handbuch fiir Pferdebesitzer. 1824. Anon. 8vo. Gotha, 1824.

Regulations and Instructions for the Cavalry Sword Exercise, with a large Target and another plate. 4to. Horse Guards, London, [1824.]

Regulations for the Carbine, Pistol, and Lance Exercises. Horse Guards, 8vo. London, [1824.]

Specimens of Riding near London. Drawn from life, by Henry Alken. obl. 4to. London, 1824.

Field Movements of Cavalry. By Bamford. Part I. [all published] folio. 1824 . 
1824. Ueber die Verschiedenen Knochenbrüche der Hausthiere, besonders der Pferde nebst einer neuen und sichern Heilmethode dagegen. Mit 5 Stntaf., die neu erfundene Stelz-und Rinn-maschine vorstellend. By Pantal. Binz. 8vo. Tuibingen, 1824.

A Short History of the Horse and progress of Horse Knowledge. By Bracy Clark. 4to. London, 1824.

Description of a New-invented Horse-shoe, which moves to the inpressions of the Foot. By Bracy Clark. 4to. London, 1824.

'Traité raisonné d'Equitation en harmonie avec l'ordonnance de Cavalerie mis en pratique à l'Ecole Royale de Cavalerie de Versailles, aujourdhui à Saumer. By Cordier. 8vo. Paris, 1824.

Cenni sulla conformazione esteriore del cavallo. By Giovanni Cros. Milan, 1824.

Ueber Gestuits-und Zuichtungskunde. Nebst einer Anleitung die Gestiits-Krankheiten vorzubengen, sie $z u$ erkennen und $z u$ heilen desgleichen der Geburtshiilfe bei den Pferden auszuiiben. ByJoh. Fr. Chr. Dieterichs. 8vo. Berlin, 1824.

New system of shoeing Horses, with observations on Diseases of the Foot. By J. Goodwin. 8vo, London, 1824.

Anatomische Abbildungen der Haus-Säuge Thiere. And Supplement. By Ernst Frederick Gurlt. obl. folio. and 8vo. Berlin, 1824 and 1848,4 vols.

The 2nd and $3 r d$ editions were published under the title of Handbuch der vergleichenden Anatomie der Haus-Sängethiere. 2 vols. 8vo. Berlin, 1833-34, and Berlin, 1843-1844.

The art of preserving and defending the Foot of the Horse, deduced mathematically from the structure and function of the hoof ; and observations on the different states of Horses Feet, without and under various artificial defences. By Thomas Hodgson. 8vo. London, 1824.

Grïndlich heilender Pferde-und Viehdoctor. By P. Hulfreich. 8vo. Marktbreit, 1824.

Memoirs of J. Decastro, with Analysis of the Life of Philip Astley. By R. Humphreys. 2 vols. 8vo. London, 1824.

Traités des maladies des yeux observées sur les principaux animaux domestiques, principalement le cheval. By Urbain Leblanc. 8 vo. Paris, 1824.

In German as :-Abhandlung iiber den Angenkrankheiten den wichtigsten Hausthiere, vorziiglich des Pferdes. Eine gekrontes Preischrift. By J. Radius. 8vo. Leipzig, 1825. 
Hauptmomente aus der Abrichtungskunst des polnischen respectiv 1824. moldaner Pferdes, für Cavallerie-Officiere und Pferdeliebhaber. By Fr. von Loweneck. 8vo. Augsburg, 1824.

Enthuillte Geheimnisse aller Handels vortheile und Pferdeverschönerungskünste der Pferdehändler. Auss den Papieren des verstorbenen israelitischen Pferdehändlers Abraham Mortgens, in Dessau, zum Nut zund Frommen aller Derer mitgetheilt, welche beim Ein und Verkanf von Pferden mit Vortheil handeln und Schaden und Betrug vermeiden wollen. Nebst einem Anhang uiber die leichteste und einfachste Art des Englisirens und die für den Händler daraus erwachsenden Vortheile. Edited by C. F. Lentin ; 1824.

A new edition of Abraham Mortgens work from his unpublished papers, 8vo. Weimar, 1856.

Trattato della conoscenza del cavallo. By Antonio Miglio. Naples, 1821.

Recueil de Médécine Vétérinaire. By F. J. J. Rigot Svo. Paris, 1824 , etc.

Manuale di Veterinaria, coronata dall'academie d'agricoltura, commercio ed arti di Verona. By Giulio Sandri. 16mo. Verona, 1824.

6th edition, 16mo. Verona, 18.54; 7the edition, $16 \mathrm{mo}$. Milan, 1857.

Katechismus iiber die Kenntniss und Behandlung des Pferdes und seiner gewöhnlicher Krankheiten. By Fr. L. C. Steinhoff. 8 vo. Rostock, 1824.

Der Landmann als Thierarzt bei Krankheiten der Pferde, des Rindviehes, Schweine, Schaafe, Ziegeu, Hunde, des Federviehes und der Stubenvôgel, etc. By Chr. Fr. G. Thon. 8vo. Ilmenau, 1824 ; Weimar, 1850.

Kunst die auschläge-und abzehrungs-Krankheiten der grössern Hausthiere zu erkennen, ihnen vorzubeugen und sie zu heilen. By Geo. Fr. Tscheulin. 8vo. Karlsruhe, 1824.

Kort Veiledning til Hingstens, Folhoppens, samt Follets og Folens rigtige Behandling indtil deh femte Aar. By Carl Viborg. Copenhagen, 1824.

German translation, Karlsruhe, 1826.

Die Skelette der Haussäugethiere und Hausvögel. Für Naturforscher, Aerzte, und zu den Vorlesungen auf Universitaten und Thierarzneischulen entworfen. By M. J. Weber. folio. Bonn, 1824; 1850 . 
1825. Instruction zum Reit-Unterricht für die Königlich Preussische Kavallerie. Anon. 8vo. Berlin, 1825; 1846; 1872.

Laws relating to Horses. London, 1825.

Some particulars relating to the History of Epsom, containing a succinct and interesting description of the Origin of Horse Racing and of Epsom Races, etc. 8vo. London, 1825.

Anecdotes on the Origin and Antiquity of Horse Racing from the Earliest Times. Anon. 8vo. London, 1825.

Principles of Modern Riding for Gentlemen, in which the late improvements of the manege and military systems are applied to practice on the promenade, the road, the tield, and the course. By John Allen. 8vo. London, 1825.

Principles of Modern Riding for Ladies, in which all improvements are applied to practice on the promenade and the road. By John Allen. 8vo. London, 1825.

Baily's Magazine of Sports and Pastimes. 8vo. London, Circa 1825, etc.

Instituzioni di mascalcia conducenti con brevità e chiarezza a esercitare con sodi fondamenti la medecina de' cavalli. By Fr. Bonsi. Ancona, 1825.

Versuch iiber die seit mehreren Jahren in Deutschland unter den Pferden herrschende Epizootie, und iiber dieselbe Krankheiten bei Schaafen und bei den Rindvieh. By J. Fr. Brauell. 8 vo. Weimar, 1825.

Praktische Hufeschlagskunde. By A. Fr. Brunn. 8vo. Neu Brandenburg, 1825.

Reflexions sur l'art de l'équitation en France, et conseils aux commandants des écoles royales. By A. C. 8vo. Paris, 1825.

Instructions and Regulations recommended to the Junior officers, N. C. officers and gentlemen, belonging to the ranks of the Roxburgshire Yeomanry Cavalry. By Carruthers. 8vo. 1825.

A Disclosure of the best method of making the expansion shoes and putting them on. By Bracy Clark 4to. London, 1825 ; 1827.

Some account of a New Instrument called the Clavidux, for boring the Hoof to any depth for the nails, by which individuals may shoe their own Horses. By Bracy Clark. 4to. London, 1825. 
Katechismus der Pferdezucht, oder vollständiger leichtfasslicher Unter- 1825. richt über die Zucht, Behandlung und Veredlung der Pferde. By Jh. F. Chr. Dieterichs. 8vo. Berlin, 1825.

Sporting Anecdotes, Original and Selected, including numerons Characteristic Portraits of Persons in Every Walk of Life, who have acquired Notoriety from their Achievements on the Turf, Table and Field, with an Account of noted Pedestrians, Trotting Natches, ete. By Pierce Egan. 8vo. London, 1825.

Bibliotheca Veterinaria oder Verzeichniss aller brauchbaren in älterer und neuerer Zeit bis zur Mitte des Jahres 1824 in Deutschland erschienenen Biicher iiber aller Theile der T'hierarzneikunde. By Theod. Chr. Fr. Enslin. 8vo. Berlin, 1815.

2nd edition to 1842, 8vo. Leipsic, 1843.

The Art of Manage, Riding and Breaking Horses systematically. By T. Gibbons. 8 vo. London, 1825 .

De qualitatibus parentum in sobolem transeuntibus, præsertim ratione rei equariac, Diss. inaug. med. defendet Fr. Notter By J. Dan. Hofacker. 4to. Tiibingen, [1825.]

Die Bearbeitung des Pferdes an der Hand und mit dem von mir erfundennem Spanischen Reiter. By Eph. Klatte. Sro. Berlin, 1825 ; 1827.

Vorschule der Soldaten-reiterei, oder wahres Reiter-Exercitium zu Fuss. By Eph. Klatte. 8vo. Berlin, 1825.

Hints to Purchasers of Horses. By Charles Knight. 12 mo. London, 1825 .

Il Perfetto Cavaliere, Opera corredata di 77 Stampe nere e miniate rappresentanti le diverse specie dei cavalli; colla storia naturale del cavallo, scritta da Buffon, e la scuola di cavallerizza di la Gueriniere. By Ant. Locatelli. 4to. Milan, 1825.

Anweisung und sichere Leitung zum Englisiren der Pferde ; ein Handbuch für diejenigen, welche diese Operation unternehmen, oder auch nur sich genau davon unterrichten wollen. By Andr. Politz. 8vo. Berlin, 1825.

Anatomie du Cheval. Osteologie et myologie. By Hector Revarchon. folio. Lyons, 1825.

Observations on Breeding Race-Horses for the Turf, etc. By Nicholas Hankey Smith. 8vo. London, 1825. 
1825. Lehrbuch der äussern algemeinen Pferdekenntniss. By S. v. Tennecker. 8vo. Altenburg, 1825.

Vorträge über die Wartung, Pflege, Behandlung und Benutzung des Pferdes. By Joh. Jac. Weidenkeller. 3 vols. 8vo. Dinkelsbiihl, 1825-1829.

1826. Anleitung zu Behandlung der Remonten. Als Anhang zum Reit Unterricht für die Kavallerie. Anon. 8vo. Berlin, 1826.

Unentbehrliches Schatzlein für Liebhaber der Pferde und deren Besitzer. Oder Unterricht üher die Krankheiten der Pferde und Mittel dagegen, etc. 8vo. Ulm, 1826.

Praktischer Unterricht uiber die Wartung der Pferde, das Catteln, Packen und Zäumen, so wie iiber die gemeinsten Regeln des Hufbeschlags und der Verpflegung und Abwartung kranker Pferde. Von einen alten Cavallerieoffizier d. Kön. Sächs. Armee. Anon. 8vo. Dresden, 1826.

Erzählungen, Anecdoten und Biographien fiir Freunde des Militairstandes und der Militairgeschichte, auch interessante Notizen und Belehrunger für Pferdeliebhaber. Nebst einem Anhang über das Beschläg der Pferde. Aus franzosiche Schriften gezogen von C. Ch. Beck. 8vo. Ludwigsburg, 1826.

Etudes anatomiques du Cheval. Utile à sa connaissance interieur et exterieur. By Brunot. folio. Paris, 1826.

The Knowledge of the age of the Horse by his Teeth, with remarks. By Bracy Clark. 4to. London, 1826.

Om Hestes, Qvœgs og Faars Fodring m. v. Af Niels Gjersing. Tiende-Commissair in Ny Holbeks Amt. Eier af Hesselberg og Appelsberg. 8vo. Copenhagen, $1826 ; 1848$.

Cours d'hippiatrique à l'usage des Officiers et sous Officiers de cavalerie. Comprenant: 1 . Un précis anatomique du cheval; 2 . Un résumé d'exterieur; 3 . Une notice sur l'hygiène; 4. Une petite thérapeutique vétérinaire. By François Maxime Jacquemin. 32mo. Strasburg, 1826.

4 th edition, 32mo. Strasburg, 1850, added to which was the 'Petite Monographie des boiteries du cheval,' by the same author, which was also published in a separate form in the same year, (1850.)

Katechismus des Pferde-Erhaltungskunde, oder Unterricht zur Wartung und Pflege der oriental, Englisch, nord-und süd-deutschen Pferderacen, etc. By Eph. Klatte. 8vo. Leipzig, 1826. 
Reiterkatechismus, oder theoretischer Reitunterricht für alle Stande, 1826. etc. By Eph. Klatte. 8vo. Leipzig, 1826.

Praktischer Unterricht in Kunstdarstellungen von Pferden, oder Anleitung den Pferden alle Kunstfertigkeiten zu lehren, etc. By Genl. Baptist Loiset. 8vo. Ilmenau, 1826.

Commentaria veterinaris medica de melanosi cum in hominibus tum in equis obveniente specimen pathologiæ comparate. By C. A. Noack. 4to. Leipzig, 1826.

Pelagonii Veterinaria. By Sarchiano. 8vo. Florence, 1826.

Lehrbuch der Veterinär-Physiologie. By Konrad Ludwig Schwab. 8vo. Munich, 1826.

Handbuch der gesammten gerichtlichen Thierarzneikunde für Aerzte, Thierärzte, Oekonomen und Rechtsgelehrte. By Johann Elias Veith. 8vo. Vienna, $1826 ; 1836 ; 2$ vols. $1840 ; 1850$.

Den ondartele Lungesyge hos Hesten beskreveh til nytte for Landmanden. By Erik Nissen Viborg. Copenhagen, 1826.

Reuter-Bibliotek. By Count v. Bismark. 16mo. Karlsruhe, 1827. 1827.

Versuche eines Beweises, dass das Pferderennen in England, so wie sio jetzt bestehen, Kein wesentliches Beförderungsmittel den bessern edlen Pferdezucht in Deutschland werden Können. By C. F. W. von Burgsdorf. 8vo. Königsberg, 1827.

Cours élémentaire et analytique d'Equitation, ou Résumé des principes de M. d'Auvergne suivi d'un essai sur les Haras. By Chabannes. 1827.

Beschriebung der einzelnen Gestiite des österr. Kaiserstaates, nebst Bemerkungen über Hornviehzucht, Schafzucht und Oekonomie Mit mehreren Uebersichstabellen und 2 lith. Taf. die GestiitsBrandzeichen vorstellend. By Mich. v. Erdelyi. 8vo. Vienna, 1827.

Traité des hernies inguinales dans le cheval et autres monodactyles. By J. Girard. 4to. Paris, 1827.

The Traveller's Oracle, with rules for Purchasing Horses and Carriages. By John Jervis (an old Coachman). Revised by Dr. Kitchener. $12 \mathrm{mo}$. London, 1827.

Anleitung iuber das Beschlagen der Pferdehüfe, ein kurzer leicht fasslicher Unterricht für Hufschmiede und Pferdebesitzer. By J. Kertitschka. 8vo. Grätz, 1827.

14 
1827. Anleitung zur Verbesserung und Veredlung der Pferde durch Landgestiite und gemeine Land-oder Hauszucht. Ein kurzgefasster popular Unterricht für die Pferdezüchter der österr. Monarchie, mit besonderen Beziehungen auf die Proving Steyermark. By J. Kertitschka. 8vo. Grätz, 1827.

Die Mecklenburgisch Pferdezucht. By Alex. v. Lengerke. 8vo. Berlin, 1827.

Delle arte della Ferratura del Cavallo. By Carlo Lessona. 8vo* Turin, 1827.

Gründliche und Kurzgefasste Darstellung der verschiedener Arten von Knochenbriichen Hufkrankheiten unserer landwirthschaftliche Hausthiere, um sie mittelst einer Schwebe-Maschine leicht zu heilen. By Joh. Peterka. 8vo. Prague, 1827.

Beschreibung des schönen und fehlerhaften Pferdes. By Gottl. Rienecker. 8vo. Hildburghausen, 1827.

Wohlerfahrener, sicherer und leicht heilender Wunderdoktor oder Anweisung fiir Landwirthe und Oekonomen, wie sie sich bei der Gesundheitspflege und Krankheitsbesorgung der Pferde, des Rindviehes, der Schaafe, Ziegen, und Schweine zu verhalten haben. By Fr. Silberschmidt. 8vo. Heilbronn, 1827.

The Young Horsewoman's Compendium of the modern art of riding, comprising a progressive course of lessons; designed to give ladies a secure and graceful seat on horseback; at the same time so effectually to form the hand, that they may in a short time acquire perfect command of their Horses. By Edward Stanley. 8vo. London, 1827.

Beobachtungen und Erfahrungen uiber die Erkenntniss und Kur der Kolik bei delı Pferden. By S. v. Tennecker. 8vo. Altenburg, 1827.

Naturlehre des Pferdes für alle diejenige welche den Organismus diesen Thiere genau kennen lernen wollen. By S. v. Tennecker. 8vo. Munich, 1827.

1828. Ueber die Eigenschaften des Soldatenpferdes und die Mittel, die Zucht desselben zu befordern. By Georg Gottlieb Ammon. 1828.

Annals of Sporting. 15 vols. 8vo. London, 1828.

De la Cavalerie ou des changements nécessaires dans la composition et l'instruction des troupes à cheval. By Comte de la RocheAymon. 2 vols. 8vo. Paris, 1828. 
Hufeschlag ohne Zwang. Nach rationellen, aus der Psychologie des 1828. Pferdes geschöpften Grundsätzen. By Constantin Balassa. 8 vo, Vienna, 1828.

In French:-Traité de la Ferrure sans contrainte, ou moyen de ferrer les chevaux les plus vicieux en moins d'une heure, et de les corriger pour tonjours de leurs défauts. Système puisé dans les principes de la physiologie du cheval. 8vo. Paris, 1828.

In Norwegiun as:-Kunsten at skoe Heste uden Tvang. Translated by Tydske. 8vo. Christiana, 1830 .

Bombay Sporting Magazine. 5 vols. 8vo. Bombay. Begun 1828 ; ceased 1833.

Taschenbuch für Pferdebesitzer und Liebhaber der Reitkunst. By Ludwig Daum. 16mo. Wiesbaden, 1828.

Handbuch der speziellen Pathologie und Therapie für Thierärzte und Landwirthe. Oder die Kunst die innern Krankheiten der Pferde, Rinder, Schafe, Schweine, und Hunde zu erkennen, zu verhiiten und zu heilen. By J. F. C. Dieterichs. 8vo. Berlin, 1828; $1835 ; 1851$.

Essay on Wheel Carriages, with a Concise View of their Origin. By Fuller. 8vo. London, 1828.

Ueber die Taktik der Cavallerie des Herm Major von Decker. By Thassile von Heydebrand und der Lasa. 8vo. Breslau, 1828.

Ueber die Eigenschaften, welche sich bei Menschen und Thieren von den Eltern auf die Nachkommen vererben, mit besonderer Rïcksicht auf die Pferdezucht; mit Beiträgen von Fr. Notter. By J. Dan. Hofacker. 8vo. Tübingen, 1828.

Vollständiger Vicharzneibuch oder Unterricht iiber die Erziehung, Wartung, Fiitterung, Krankheiten und deren Heilung von Pferden, Rindvieh, Schafen, Schweinen, Federvieh und Hunden. Nebst einem Anhang über die Krankheiten der Stubenvögel und deren Heilung. By J. B. Hoffmann. 8vo. Augsburg, 1828; 1850 .

Das verbesserte Cutiner Amts-und Stadtgestiit, und das allenhalten anwendbare Harmoniegestuit, von Pferdebesitzern, Cutinischer und Holsteinischer Dorfschaften erfunden, und verbessert, respectiv von Ersterm auch bevorredet, und herausgegeben, eingefuhrt aber von Letzterem. By G. C. M. Hofmeister. 8vo. Hannover, 1828. 
1828. Beleuchtungen und Bemerkungen über die vom Rittmeister C. Balassa erfundene neue psychologische methode, böse Pferde in einer Stunde zum Hufbeschlagen ohne Zwang abzurichten. By Eph. Klatte. 8vo. Berlin, 1828.

Berliner Almanach für Reiter Gestuitsbesitzer und Pferdeliebhaber. By Eph. Klatte. 8vo. Berlin, 1828.

Bemerkungen und Noten zu Hünersdorfs Anleitung zu der natürlichsten und leichtesten Art Pferde abzurichten als ein nöthiger Anhang zu diesem Werke der Reitkunst. By Gen. Baptist Loiset. 8vo. Marburg, 1828.

Handbuichlein der Hufbeschlags-Lehre, für Cavalleristen, Pferdebesitzer und Beschlags schmiede. Joh. Chr. Gottfr. Lupke. 12 mo. Quedlinberg, $1828 ; 1836$.

Remarks on the early use of carriages in England, and on the mode of Travelling adopted by our Ancestors. By James Heywood Markland. 4to. 1828.

Danske Benœvnelser de betegne samtlige enkelte Dele af Hestens ydre Bygning, dens Fuldkommen heder og Lyder. By J. W. Neergard. Copenhagen, 1828.

Beschlags-Katechismus, oder fassliches Unterricht in der beste Methode die Pferde $\mathrm{zu}$ beschlagen. By Friedrich Nüsken. $12 \mathrm{mo.}$ Minden, 1828.

Handbuch für Cavallerie-Offiziere, etc. so wie überhaupt für alle Pferdebesitzer. Enthalt. : das Ganze der Schmiedekunst und der Hufbeschlags, nebst einem Anhang über ein bewährter Stahlbeschlag, ein eigenthümliches Härtewasser, eines neuerfundene Hufmesser (Podometer) und ein neue zweckmassige Hufnagelart. By Fried. Nüsken. 8vo. Lemgo, 1828.

Ansichten über die Entstehung und Ausbildung des Edlen Pferdes, und die zur Verbesserung der Pferdezucht anzuwendenden Mittel. sowie iiber die Nothwendigkeit einer verändete Einrichtung der Landesherrlichen Gestiite in Deutschland, und Berichte, Urtheile und Vorschläge uiber die Mecklenburg. By F. J. C. Pogge. 8 vo. Güstrow, $1828 ; 1836$.

Der Hufschmied, oder die Pferde auf richtige Art zu beschlagen. By Gottl. Rienecker. 8vo. Suhl, 1828; 8vo. Leipzig, 1834.

De l'éducation des Chevaux en France, ou causes de l'abatardissement successif de leurs races, et des moyens à employer pour les regénérer et les améliorer. By $\mathbf{A}$. de Rochau. 8vo. Paris, 1828 ; and 8vo. Paris, 1832. 
Leitfaden zur Beurtheilung des Pferdes. By J. J Rychner. 8vo. 1828. Berne, 1828.

Bemerkungen und Zusätze zı Waldingers Therapie, oder practisehe Heilverfahren bei den gewöhnlichen innerliche Pferde Krankheiten, etc. By S. v. Tennecker. 8vo. Marburg, 1828.

Denkwiirdigkeiten meiner Zeit in Beziehung auf Pferdezucht, Pferdekenntniss, Pferdehandel, Pferdearznei, und Reitkunst, etc. By S. v. Tennecker. 8 vo. Munich, 1828.

Lehrbuch de speciallen Pferdekunst, oder Noten und Bemerkkungen zu A. E. Havemann's Beurtheilung des aussern Pferdes. By S. v. Tennecker. 8vo. Marburg, 1828 .

Elémens de Pathologie Vétérinaire, ou, Préeis de la Médicine et de la Chirurgie des principaux animaux. By P. Vatel. 2 vols. 8vo. Paris, 1828.

The Veterinarian, edited by Pereivall, Youatt, W. Dick, W. F. Harkeek, Morton and Simonds. 8vo. London, 1828, ete.

Wie und auf welche Weise kann in kuirzester Zeit und am zweekmässigsten die Pferdezucht in Baiern ohne vom Staate neue Aufopferung zu forden gehoben un vervollkommnet werden? By Jh. Jak. Weidenkeller. 8vo. Dinkelsbuihl, 1828.

Das sicherste Mittel nur grosse und gut ausgebildete Pferde zu erziehen nebst einer Anweisung zu ihrer Vorbereitung zu kunftigen Dienstleistungen. Ein auf lange Erfahrung gegriindeter giter Rath an Pferdeziiehter. By Georg Gottlieb Ammon. 8vo. Königsberg, 1829, and 1849.

In Russian as :-Върнбйщее средство къ разведенію рослыхъ I здоровыхь лошадей Нъсколько совбтовъ коннозаводчнкамq. Translated by N. Brief. 8vo. St. Petersburg, 1857.

Hufbeschlag ohne Zwang, oder die Abrichtung des Pferdes, sowoll die noch ganz rohen, als die wilderspenstigen. Nach den Gesetzen der Natur und den besonderen Eigenthumlichkeiten eines jeden Pferdes. By Chr. de Bach. 8vo. Dresden, 1829; 1834.

Conversations on Conditioning, The Groom's Oracle and Pocket Stable Directory, in which the management of Horses generally as to health, dieting, and exereise are considered in a series of familiar dialogues, between two grooms engaged in training horses to their work, with notes and an appendix, including Extracts from the receipt book of John Hinds [pseud. for John Badcock]. 8 vo. London, 1829. 
1829. Rules for Bad Horsemen. By John Hinds [pseud. for John Badcock]. 12mo. London, 1829.

Veterinary Surgery and Practice of Medicine. By John Hinds [pseud. for John Badcock]. 12mo. London, 1825.

The Turf Expositor, containing the origin of Horse Racing, Breeding for the Turf, Trainers, Jockeys and Cocktails. By C. F. Brown. 8vo. London, 1829.

Ueber dem homöopathischen System in Beziehung auf der Heilung der Pferde, orler Beweis, dass die geschiktesten und erfahtesten Pferdeärzte ohne dass sie es wissen und es wollen, ihre Kranken doch homöopathisch behandeln. By L. Brückner. 8vo. Dresden, 1829.

Hippodonomia, or the true structure, laws, and economy of the Horse's Foot. Also Podophthora, or a ruinous defect in the principle of the common shoe detected and demonstrated by experiments, with a proposition for a new principle of shoeing, which abundant practice has since confirmed. By Bracy Clark. 4to. London, 1829.

In German:-Hippodonomia, oder der wahre Bau, die Naturgesetze und Einrichtung des Pferdefusses; und Podophthora, oder die durch angestellte Versuche erwiesene schädliche Behandlungen der bisher gewöhnliche Hufeisen etc. 4to. Frankfurt, 1832. Another translation:-Der Bau und die Verrichtungen des Pferdes Hufes, nebst einer durch Erfahrung bewälirt gefundenen neuen Beschlags-Methode Translated by F. S. Rhode. 4to. Frankfurt, 1832.

Grundlinien der Muskellehre des Pferdes mit Beriicksichtigung der Abweichungen bey den ibrigen Haussängethieren. By Mich. v. Erdelyi. 8vo. Vienna, 1829.

A Treatise on Deportment, Fencing, etc., including the science of Horsemanship; being a complete manual of instruction for the use of young persons, in the acquirement of those accomplishments, as well as a self monitor in the exercise and duties of a cavalry soldier, for the use of gentlemen who are members of yeomanry corps, comprising likewise instructions for the lance and carbine exerciscs. Also the description of a Military game resembling the game of Chess ; calculated to initiate young gentlemen who are designed for the army, in the science of Tactics or Mlilitary Manœuvres. By Samuel Gribble. 8vo. Derby, 1829. 
Des Haras domestiques et des Haras de l'Etât en France. By J. B. 1829. Huzard. 8vo. Paris, $1829 ; 1842$.

Gemeinniitzlicher Unterricht über Kenntniss der Pferde und des Rindviehes, ihre Fiitterung, Wartung, Pflege, und Zucht. Für die Biirger und Landmänner Nebst dem Anhang: Die gewöhnliche Krankheiten des Pferdes und des Rindviehes, ihre Erkenutniss und Heilung; nach den bewährten Behandlungsarten gesammelt v. J. F. Alpin. By Joseph Ant. Ithen. 8vo. Chur, 1829. Ird edition, 1831.

Die Schnelldressur des Remontepferdes, nach psychologischen und tacktischen Grundsätzen ohne Zerstörung des materials fiir die Kriegszustand eingerichtet, etc. By Eph. Klatte. 8vo. Berlin, 1829 .

The Horse in all its varieties and uses, his breeding, rearing, and management, whether in labour or rest, with rules occasionally interspersed for his preservation from disease. By John Lawrence. 8 vo. London, 1829, and 1835 .

Storie d'Alcune Pericolose e difficile Maladie nel Polmone del Cavallo. By Vincenzo Massa. 1829.

Anatomy of the Horse, reduced to a systematic Form, embracing new and enlarged views of the structure of the Foot. By William Percivall. 8vo. London, Circa $1829 ; 1836 ; 1858$.

Diccionario de veterinaria y ciencias auxiliares. By Carlos Risueño. 5 vols. 4to. Madrid, 1829.

Praktische Heilverfahren bei den gewöhnlichen ausserlichen und innerlichen Krankheiten der Pferde nebst Angabe seine Methode des Englisirens und Kastrirens der Pferde. By J. G. Salzmann. 8vo. Dresden, 1829.

Die Behandlung des Pferdes. Enthaltend: Die Beschaffenheit der Pferde-Racen, die Charaktere der Pferde, die Pferdezucht und Gestiute-Einrichtung, Pflege der Pferde im Stalle, den Beschlag der Pferdehufe und die Behandlung der Pferde bei verschiedenen Ereignissen. By Frz. Xav. Jos. Schreiner. 8vo. Munich, $1829 ; 1837$.

Neue Gebisse und Methode ein Pferd gut zu zäumen mit einigen Noten herausgegeben vom Obristen v. Schepeler. By J. Segundo and Col. von Schepeler. 8vo. Aachen, 1829. 
1829. Das Landgestiite in d. Königl. Preuz. Provinz Schlesien, mit Hinsicht auf dessen innere Einrichtung und die dabei stattfindende Verwaltungs-Verhältnisse, so wie über die Ergebnisse seiner 10 jährice Wirksamkeit; nebst allgemeine Betrachtungen iiber der Pferdezucht, besonders in Schlesien. By Wilh. Sohr. 8vo. Breslau, 1829.

Aus Bunzlau in Schlesien, des alten schäfers, seine Kuren an Pferden, von ihm selbst in seincr Mund-und Schreibart beschrieben und zum Besten seiner Nebenmenschen herausgegeben von einem Sohne, dem Schäfer Thomas in Weissenborn. By Thomas. 8vo. Glogau, 1829. Second edition with a preface by Tenneker, 8vo. Glogau, 1834.

Istruzioni della scherma a cavallo. By Giuseppe Veiss. Naples, 1829.

1830. Every Man his Own Farrier, or The Pocket Farrier, a Treatise on the Veterinary Art, containing the Materia Medica and Pharmacopœia. By a Practical Farrier. Anon. 16mo. London, Dublin and Derby. [Circa 1830.]

Kleine Sattelhiilfe oder kurze Reitanweisung sowie praktische Regeln für die Behandlung der Pferde. Für alle Laien in der Kunst, die wohl Lust, aber keine Gelegenheit oder Mittel haben, eine Reitschule $\mathrm{zu}$ besuchen, vorzüglich aber für künftige Cavalleristen, Studirende, etc. Anon. 8vo. Halle, $1830 ; 1851$.

Pferdekunde, oder Anweisung das Alter der Pferde bis zu 16 Jahren trotz trügerischer Kunstgriffe an den Zähnen, so wie verschiedene wichtige Krankheiten derselben zu erkennen, und letztere zu behandeln und zu heilen für Landwirthe und Pferdeliebhaber. Aus der Handschrift eines erfahrenen praktischen Thierarztes. Anon. 8vo. Schweidnitz, [Cirea 1838.]

Ridekunsten, eller Veiledning til Ridehestens rigtige Behandling. By Peter Wilh. v. Balle. Copenhagen, 1830.

Recherches sur la Nature et les Causes de la Morve. By Barthelemy. Paris, 1830.

Einiges über edle Pferde. By Baron Gli. Wlh. Ldw. Biel. 8vo. Dresden, 1830.

Biographical Sketches and Authentic Anecdotes of Horses and the allied species. By Capt. Thomas Brown. 12mo. Edinburgh, 1830 . 
Die Veterinair-Diagnostik, oder die Kunst die innern und äussern 1830. Krankheiten unserer Hausthiere zu erkennen und von einander zu unterscheiden, etc. By H. Buerger. folio. Berlin, 1830.

Tratado de Veterinaria. By Nicolas Casas de Mendoza. 8vo. Madrid, $1830 ; 1844$.

A Treatise on the Bits of Horses, (Chalinologia). By Bracy Clark. 4to. London, [Cirea 1830.]

There is an article in Rees' Encyclopcedia on "Bits" by Bracy Clark, date about 1809, but this can hardly be the 1st edition of the above worl, as the author in the 2nd edition mentions the 1st, of which he says he published only a few copies.

2nd edition, 4to. London, 1835.

Tidende fur Hesteelskere og Landmœnd. Edited by P. D. and C. F. Faber. Aalborg, 1830-31.

The Farrier and Naturalist. Articles contributed by Eminent Writers. Edited by a Member of the Zoological Society, 1828, and 1829. Continued as:-The Hippiatrist, 8vo. London, 1830. 3 vols, all published.

Der Königliche privilegirte Gesundheits-Handschuh für der Behandlung das Pferdes. Oder arabische Bïrste, genannt Kaffah (die Haut des Pferdes zu glätten). By F. J. Götz. 8vo. Leipsic, 1830.

Lehrbuch der praktischen Veterinär-Geburtshülfe, nebst einem Anhang uiber den Wahl der Zuchtpferde. By J. H. F. Gunther. 8vo. Hanover, 1830.

Theoretisch-praktisches Lehrbuch der Entzuindung, ihre Ausgängs: Reaktion, Kongestion, Blutung, und ihrer Uebergänge: Fehler der Horngebilde, krankhafte Absonderung von Schleim, Serum, Ausschwitzung von Lymphe, Bildung von Verhärtungen, Tuberkeln, Wümern, Eiter, Jauche, Brand, als pathologische Grundprozesse aller sporadischen und seuchenartigen, äusserlichen und innerlichen Krankheiten der nutzbaren Hausthiere, in Bezug ihrer Erkenntniss, Ursachen, Schätzung, Heilung, Verhiithung, Vergleichung, veterinär polizeilichen, gerichtlichen Behandlung -Oder Ausmittelung der fünf diagnostischen und therapentischen, in der angehängten Fragtabelle angegebenen Punkte. Nebst einer ansführlichen Einleitung zur Begriindung des Vorgetragenen und Regelung der Bedingmingen des Lebens und seiner bildenden bewegenden und empfindenden Kräfte $\mathrm{zu}$ verschie. denen Heil-und Gebrauchszwecken. By Anton Hayne. 8vo. Vienna, $1830 ; 1849$.

I 5 
1830. Merkwurdige Verletzung eines Pferdes durch den Blitz auf der Solitiide bei Stuttgart, am 24 Juni, 1829. By Sigm. Hoerdt. 8vo. Stuttgart, 1830.

Corso di Chirurgia Veterinaria. By Vincenzo Massa. 4 vols. [Circa 1830.]

Tratado de Veterinaria. By Guillermo Sampedro. 8vo. Madrid, $1830 ; 1844$.

History of Veterinary Schools. By John Stewart. Edinburgh and London, [Circa 1830.]

Kurzgefasster Unterricht in der Pferdewissenschaft, etc., Nebst ein Anleitung den gesunden und kranken Hufen der Pferde, Esel, und des Rindviehes zweckmässig $z u$ behandeln und zu beschlagen. Ein Hülfsbuch für angehenden Hufschmiede und Oekonomen. By Fried. Aug. Teller. 8vo. Eisenberg, 1830; 1840.

Neu-eröffnete Reitschule gezeichnet von Aegidius Touchemolin. radirt von Joh. Chr. Erhard. 4to. Nuremberg, [Circa 1830.]

A Practical Treatise on Glanders and Farcy in the Horse, descriptive and explanatory of its origin, progress and termination, and the most effectual methods of treatment and cure. By Richard Vines. 8vo. London, 1830.

Speciellen Pathologie und Therapie oder Anleitung den einzelnen Krankheiten der nutzbarsten Hausthiere zu erkennen und zu heilen. By Hieron Waldinger. 8vo. Vienna, [1830]; 3rd edition, 1832-33.

Groom's Guide and Valet's Dictionary. By Jer. Weal. 12mo. [Circa 1830.]

Die besten, einfachsten, bewährtesten, und sichersten Mittel das Durchgehen der Wagenpferde zu verhindern. By Chr. Wohlehn. 8vo. Dresden, 1830.

1831 Die Pferdezucht oder vollständige Anweisung zur Erziehung und Wartung der Pferde. Nebst eine kurzen Anhang iiber der Eselund Maulthierzucht. Anon. 8vo. Leipzig, 1831.

Instruction à l'usage des Sons-officiers et canonniers du 1le Regl. d'artillerie, sur les soins à donner aux chevaux en route et en campàgne. Paris, 1831. 
Die gewöhnlichen Krankheiten der Pferde und des Rindriehes, ihre Erkenntniss und Heilung; nach den bewährtesten BehandlungsArten. By J. F. Alpin. 8vo. Chur, 1831.

Remarks on the Condition of Hunters, the Choice of Horses and their Management in a series of familiar letters, originally published in the Sporting Magazine, between 1822 and 1828. By Nimrod [pseud. for Charles James Apperley]. 8vo. London, 1831 ; 8vo. London, 1837 ; 4the edition, 8vo. London, 1855.

In French, translated by Guyton from the 4th edition:Remarques sur la Condition des Hunters, le Choix des Chevaux, et leur ménagement, dans une serie de lettres familières, par Nimrod. 8vo. Brussels, 1862.

Des Cosaques et de leur utilité à la guerre. Memoire rédigé et présenté, à S. M. l'Empereur de Russie en 1816. By General Benkendorff. 8vo. 1831.

The Village Farrier. By Ephraim Blaine. 1831.

Avant-postes de cavalerie legère. Souvenirs. By F. de Brack. 18mo. Dôle, 1831 ; Paris, 1844 ; 1863.

In English :-Advance Posts of Light Cavalry-Recollections. Translated by Maj. P. J. Begbie. 8vo. 1850.

Another:-Light Cavalry Outpost Recollections. Translated by Mrijor Hale and Captain Hobson. 8vo. London, 1876.

Anatomische Studien des Pferdes in Bezug auf Knochenbau, Muskulatur und Bewegung, als Hülfsmittel zur richtigen Beurtheilung der aussern conformation. By Brunot. folio. Carlsruhe, 1831.

Ueber der sichere Heilung der Kolik des Pferdes, eine Inauguralschrift. By Fr. Wilhelm Brunswig. 8vo. Rostock, 1831.

Remarks on the shoeing of the Ancients. By Bracy Clark. 4to. London, 1831.

The High-Mettled Racer (a Ballad), to which is added many anecdotes of the Race Horse. By Charles Dibdin (the elder). $12 \mathrm{mo}$. London, 1831.

Thierheilkunde oder Beschriebung und Behandlung sowohl der ausserlichen Krankheiten unsere Hausthiere, als auch den innern Krank heiten der Schafe, Rinder und der Pferde. By Joh. Fr. Chr. Dieterichs. 8vo. Leipsic, 1831.

Lehrbuch der Pathologischen Anatomie der Haus-Säugethiere Nebst einem Anhang. By Ernst Friederich Gurlt. 8vo and folio. Berlin, 1831-32. 
1831. Sportsman's Cyclopedia, containing Information for the Country Gentleman, the Farmer, and the Sportsman. By J. B. Johnson. 8vo. London, 1831.

Wirkung des Zügel, nebst einer Betrachtung iiber dem Pferd in Riicksicht auf Mechanik, etc. By C. F. Kayser. 4to. Neuwied, [1831]; 1832 .

Drei neue Zäumungsmaschinen für Pferde, welche bei der Bearbeitung mit der Trense die Zunge iber das Gebiss legen; für andere, welche den Kopf zu niedrig tragen, und endlich für solche, welche eine zu empfindliche Kinnkettengrube haben, und deren Maulspalte zum Durchfallen der Stange geneigt ist. By Eph. Klatte. 8vo. Berlin, 1831.

Neuer praktischer Reitunterricht oder 25 Anweisungen in kurzer Zeit ohne fremde Anleitung einer Pferdekenner und guter Reiter zu werden, etc. By Adolph Kruger. 12mo. Quedlinburg, 1831; 8 vo. 1857 ; 4 th edition, $12 \mathrm{mo} .1848$; and another in 1852, leaving out chapter on illnesses.

Bemerkungen eines Veteranen über edle Pterde. By Graf von Lindenau. 8vo. Brunswick, 1831.

New Sporting Magazine. 8vo. London, 1831 to 1844.

Afterwards incorporated with the Sporting Magazine, but still published with its distinctive title and cover.

Moyen pour empêcher les Chevaux de se blesser par la selle, avant de se mettre en route ou en campagne. By T. D'Outrepont. 8vo. Paris, 1831.

Verzeichniss der anatomische-pathalogische Präparate, welche sich in den Museum der Königl. baier. central-Veterinär-Schule zu München befinden. By Konrad Ludwig Schwab. 8vo. Munich, $1831 ; 1841$.

Anleitung zur äussern Pferde-Kenntniss. By Konrad Ludwig Schwab. 8vo. Munich, 1831; 3rd edition, 8vo. Zurich, 1836.

Advice to purchasers of Horses, being a short and familiar treatise on the external conformation of the horse, the nature of soundness and unsoundness, and the laws relating to the sale and warranty with copious directions for discovering unsoundness prior to purchasing. By John Stewart. 8vo. Glasgow, [1831]; 1836 $1837 ; 1840$; and 1844 .

In French:-Conseils aux acheteurs auxquels les chevaux sont sujet, avec de nombreuses observations de Chevaux; Exposé des maladies, et des vices de conformation destinées à les faire connaîtré avant l'achat, suivid'un traité de l'age du cheval et d'un vocabulaire 
des termes d'hippiatriques. Also another translation by Baron 1831. d'Hunens, 'Conseils aux acheteurs de chevaux, ou Traité de la Conformation extérieure du Cheval à l'état de santé ou de maladie. 8vo. Paris, 1865.

In German :-Rath fiir Pferdekäufer. Eine Kurze und genaue Abhandlung uber dem Bau des Pferdes, dessen Gesundheit und Kranklieit. Nebst vielen Anweisungen, die Krankheit vor dem Kaufe zu entdecken. Translated by Carl Hoyer. 12 mo. Hannover, 1837.

It has also been re-printed in America.

The Horseman's Manual, being a treatise on Soundness, the law of warranty, and generally on the laws relating to Horses. By $\mathbf{R}$. S. Surtees. 8vo. London, 1831.

Handbuch der Pferdezucht, Pflege und Heilart ihrer Krankheiten, etc. By C. Fd. Thomann. 8vo. Grätz, 1831.

Bemerkungen über die Rotzkrankheit des Pferdegeschlechts und Beobachtungen uiber das Pro. und Contra ihrer Ansteckbarkeit. By C. Walch. 8vo. Marburg, 1831-34.

Katechismus von der Pferdekenntniss, so wie von der Zucht, Warte, Pflege und Behandlung der Pferde. By Jh. Jak. Weidenkeller. 4to. Nurenberg, 1831.

The Horse. By William Youatt. Knight's Store of Knowledge. 8vo. London, Circa 1831.

Also published in Vol. 1 of the Farmer's Library. 8vo. London, [184i]. "Appendix to the Horse," by W. C. Spooner. 8vo. London, 1849. "The Horse, its History, Management, and Treatment," 8vo. London, 1853. A new edition, re-edited and revised, with observations on breeding Cavalry Horses, by Cecil. 8vo. London, 1855. Under the superintendence of the Society for the Diffusion of Useful Knowledge. Revised and enlarged by W. Watson. 8vo. London, 1866. Another under the same superintendence, by E. N. Gabriel. 8vo. London, 1859.

In German :-Das Pferd seine Zucht, Behandlung, Structur, Mangel und Krankheiten, mit einer Abhandlung über den Fuhrwesen und ein vollständiger-Register. Unter der Leitung der Londoner Gesellschaft zur verbreitung nutzlicher Kenntnisse. Translated by C. Hering. Also under the follmwing title, 'Die Englische Viehzucht,' etc. 8vo. Stuttgart, 1837; 1850.

In French:-Le Cheval. Traduit de l'ouvrage anglais 'The Horse' de William Youatt. Translated by Cluseret. $16 \mathrm{mo.}$ Paris, 1851. Anuther edition, "Nouvelle edition, augmentée du tableau synoptique des différentes races équestres du globe," 8vo. Brussels, 1852. 
1832. Book of Sports and Mirror of Life. Anon. 8vo. London, 1832 ; 1860.

Hints to Grown Sportsmen. Anon. 12mo. London, 1832.

Remarks on the proposed regulations for the Instruction, Formations and Movements of Cavalry, together with some Observations on the service of Cavalry generally and upon the Yeomanry Cavalry. Anon. 8vo. London, 1832.

A Pamphlet by the inventor of the military figures for the Elucidation of Cavalry Movements.

Rules and Regulations to be observed by the South Herts Yeomanry in the Formation of the Squadron, and other movements. Anon. St. Albans, 1832.

Leichtfasslicher Unterricht über Pferdekenntniss, Hufbeschlag und die Erkennung und Heilung der Krankheiten der Pferde. Ein Noth und Hülfsbüchlein für Pferdebesitzer, etc. Anon. $16 \mathrm{mo}$. Ludwigsburg, 1832.

Die Reitkunst auf der Grundlage des Pferdebaues und der Mechanik seiner Bewegungen, mit Beziehung auf den verschieden Diensten des Pferdes. By Fr. Autenrieth. 8vo. Tübingen, 1832.

Ueber Einfuhrung ein vortheilhaftiger Betrieb der Pferdezucht, gestutzt auf Erfahrungsgrundsätze und mit steter Berücksichtigung der übrigen zweige der Oekonomie. In einem zweigespräche nebst einem Anhang der gewöhnlichsten Fohlen-und Pferdekrankheiten überhaupt enthaltend. Bearbeitet zunächst für Bauern, aber auch für Gutsbesitzer, Thierärzte, etc. By J. R. Benfert. 8vo. Würzburg, 1832.

Bengal Sporting Magazine. Edited by Stocqueler.8vo. Calcutta, 1832 , etc.

Das Pferde von Innen und Aussen kennen zu lernen, hinsichtlich der Bewegung und der Darstellung in Bezug auf die Kunst. By Brunot. folio. Carlsruhe, 1832.

Esterior del caballo. By Nicolas Casas de Mendoza. 8vo. Madrid, 1832 ; 4to. 1843 ; 4to. 1850.

English Smith Menalcas, on French Shoeing. By Bracy Clark. 4to. London, 1832.

Abhandlung über der zuverlässichste und auf Erfahrung gegründete Heilung der akuten und chronischen Kollers der Pferde. By P. Eckert. 8vo. Freiburg; 1832. 
Der Pferdearzt. Ein nothwendigiges Huilfsbuch fiir Pferdebesitzer, nach 1832. dem MS ein erfahrenes Rossarztes. Mit Benutzung der nenesten und besten Hulfsmittel ausgearbeitet. By G. M. S. Fischer. 8vo. Halle, 1832 ; Leipzic, 1837 ; Quedlinburg, (with an altered title), 1848.

Anatomischer Atlas des Pferdes (Icones Anatomicae equi). By Fr. and Jos. Volmar Gerber. fol. Bern, 1832-34.

Traité de l'âge du Cheval. By Jean Girard. 8vo. [1832]; 3rd edition, 1834.

In Italiun as:-Ippodonteologia, accresciuta del trattato sull'eta del bove, della pecora, del cane e del maiale. Translated by Carlo Gros. Svo. Milan, 1853.

Die Anatomie des Pferdes in 70 lith. Taf. By Ernst Friederich Gurlt. folio. Berlin, 1832.

In English:-Anatomy of the Horse, etc. Translated by J. Willimott. 8vo and folio. London, 1833. Only one part pubTished.

Practische Instruktion, Handgriffe und Vortleile für Kutscher und Stallleute in fürstlichen Marställen und bei andern Herrschaften, oder deutliche Anweisung zur Stallpflege, zum Reiten und besonders zum Fahren mit 2, 4, und 6 Pferden und zum sonstigen richtigen und wohlanständigen Verhalten in und ausser dem Dienst. By Jh. Sam. Hafftendorn. 8vo. Ilmenau, 1832.

Ueber der Einrichtung, den Verhältnisse und Leistungen der $\mathrm{Kgl}$ Wiirtemberg. Thier-Arzney-Schule in den seit ihrer Errichtung verflossenen zehn Jahren. By Ed. Hering. 8vo. Stuttgart, 1832.

Hippologische Blätter; eine Zeitschrift für veredelte Pferdezucht. Edited by Count v. Holmer. 8vo. Keil, 1832, etc.

Der Ungarische Sattel so wie er sein soll. Zum Gebrauch der Kavallerie. By Fedor von Karacsay de Wallje-Szaka. 8vo. Vienna, $1832 ; 1850$.

The Central Stud-book Guide. By Antoine de Letang. 8vo. Calcutta, 1832.

Einleitung in die Organiatrik und insbesondere die Zoïatrik oder Thierarzneikunde; für Vorlesungen iiber Thierarzneikunde und als Vorbereitung zum Studium derselben. Nebst Angabe der wichtigeren allgemeinem, teutschen und ausländischen, thierarzneilichen Schriften. By Friedr. Siegm. Leuckart. 8vo. Heidelberg, 1832. 
1832. Einige Bemerkungen iber die Pferdezucht in Aegypten und insbesonderes iiber die Pferderace von Dongola. By Baron $\mathrm{H}$. von Minutoli. 8vo. Berlin, 1832.

Handbuch der Hufbeschlagkunst, in besonderer Beziehung auf den Regeln und Anweisungen zum Anfertigen der Hufeisen für die der Thierheilkunde sich widmenden, für Huffschmiede und Schmiedelehrlinge, überhaupt für alle diejenige welche Aufklarung iiber der richtige Anfertigung von Hufeisen wïnschen. By Joh. Gottlb. Muller. 8vo. Berlin, 1832.

Quelque Réflexions sur le haras contonal en opposition aux detracteurs de cet établissement. By J. B. Rossier. 8vo. Vevey, 1832.

The Veterinary Surgeon's Manual, a Guide to the cure of all Diseases. By John Rydge. 8vo. London, 1832.

Die Starrsucht der Fiillen und ihre symptomatische Gelenkenstzuindungen beobachtet und dargestellt fiir Thierärzte, Oekonomen und Gestiitsmänner. By G. Strauss. 8vo. Vienna, 1832.

The Gentleman's Pocket Farrier, showing how to use your horse on a journey. By F. Tuffnell. 18mo. Boston, 1832.

The Veterinary Examiner or monthly record of physiology, pathology and Natural History. 8vo. London, 1832-1833.

Allgemeines Vieharzneibuch, oder griindlicher und leicht fasslicher Unterricht, die Krankheiten der Hausthiere zu erkennen und sicher zu heilen. Mit nenn zum Theil neuen Folio-Tafeln in Stahlstich. By Johann Ludwig Wagenfeld. 8vo. Konigsberg, 1832. 7th edition, ibid. 1849 ; 8th edition, 1853 ; 9th edition, 1857.

Gründliche Anweisung die Krankheiten des Pferdes, sowohl die innern als die äussern zu erkennen und zu heilen, etc. By L. Wagenfeld. 8vo. Dantzig, 1832.

Vollstandiges, Receptbuch, für Thierärzte, Landwirthe, so wie iiberhaupt fiir Eigenthümer von Hausthieren jeder Art. Oder Auswahl von mehr als 2000 der bewahrtesten und wirksamsten Arzneiformeln aus dem Praxis der besten und erfahrensten Thierärzte für alle innern und äussern Krankheiten der Pferde, Rinder, etc., sowie auch des Federviehes. By A. P. Wilhelmi. 2 vols. 12 mo. Leipzig, 1832.

Handbuch der Hippologie oder die Lehre von der Schätzung des Pferdes auf dessen ökonomischen und pecuniären Werth, etc. By Ludw. Phil. Wüppermann. 8vo. Vienna, 1832. 
Veddelobs-Feiden eller Hesteyn-der foreningens og Sjugard Freidur- 1833. heims Opsatser, etc. Anon. 8vo. Christiania, 1833.

Handbuch der gesammiten Gestiits-kunde und Pferdezucht. By George Gottlieb Ammon. 8vo. Königsberg, 1833.

Histoire pittoresque de l'equitation, ancieune et moderne. By Charles Aubry. folio. Paris, 1833.

Dictionnaire raisonné d'équitation. By F. Baucher. 8vo. Paris, $1833 ; 1859$.

Anatomia Patologia. By Nicolas Casas, de Mendoza. 8vo. Madrid, $1833 ; 1848$.

A Dissertation on the Epidemic Cholera. By Bracy Clark. in 2 parts. 4to. London, 1833.

Zoologie Vétérinaire. By Grogniez. 8vo. Paris, 1833.

In Spanish:-Zoologia veterinaria. Translated by D. F. San Pedro y Guzman. 8vo. Madrid, 1852.

Naturalist's Library, Mammalia, vol. 1. By Sir W. Jardine. 8vo. Edinburgh, 1833-35; 16mo. 1843,

Katechetische Anleitung zur Kenntniss und Beurtheilung des Pferdes im gesunden und kranken Zustände, so wie zur zweckmässiger Behandlung der gewöhnlichen Pferdekrankheiten, Fï jeden Pferdebesitzer und Pferdeliebhaber, besonders aber für Cavallerieund Fuhrwesens Unteroffiziere und für Eskadrons-und Fuhrwesens-Abtheilungs-Schmiede bearbeitet. By Joh. Mart Kreutzer. 8vo. Augsburg, 1833.

Nouveau manuel complet du charron et du carrossier. By Lebrun. 2 vols. 18 mo. Paris, $1833 ; 1851$.

Istruzioni sulla morva ad uso delle truppe a cavallo. By Carlo Lessona. 8vo. Turin, 1833.

Regimental Instructions for the Formation and Movements of the West Somerset Regiment of Yeomanry Cavalry. By Captain Maher. Taunton, 1833.

Field Book of Sports and Pastimes of the United Kingdom. By Maxwell. 8vo. 1833. 
1833. Vollstandiges Handbuch des praktische Hausthier-Heilkunde, enthal tend aller innerlichen und ausserlichen Krankheiten der Pferde, des Rindviehes, der Schafe, Schweine, Ziegen, und Hunde. Mit einer Kurzen Anleitung zur Zucht und Wartung der Hausthiere. Also under the following title, Der Haus-Thierarzt als Hausfrennd, etc., Ein nutzliches Handbuch fiir Landwirthe. By Theodore Merk. 8vo. Munich, 1833; 3rd edition, 1840; 4 th edition, 1851.

Der Thierarzt als Rathgeber bei allen Krankheiten der Pferde, Rinder, Schafe, Ziegen, Schweine, Hunde, Katzen, und des Federviehes, ein Handbuch zur Belehrung für Landwirthe und Viehbesitzer jeder Art. By F. A. Schrader. 2 parts. 8vo. Meissen, 1833.

Veterinary Tablet, being a Concise Account of the Diseases of Horses, Cattle and Dogs, with their Cause, Symptoms, and Cure, to which are added numerous valuable recipes. By Matthew Small. London, N.D. [Circa 1833].

A large sheet folded in small case for the pocket.

In French:-Tableau indicatif des Maladies du Cheval et des Remèdes qu' ont pu appliquer Setons, des Maux et accidens, folio. [Circa 1834].

In German :-Uebersichts-Tafel der innern und äussern Krankheiten bei Pferden, Rindvieh, Schaafen und Hunden, ihrer Ursachen, Kennzeichen und Heilung. Translated by Lentin and Weissenborn. [Cirea 1834]; 6th edition, Folio. Weimar, 1840.

Another:-Thierarzneiliche Tabelle; eine Uebersicht der Krankheiten der Pferde, des Rindviehes, und der Hunde, mit ihrer Ursache, Symptomen und Heilung. Translated by Carl Hover. folio. Minden, 1841.

Stable Economy, a treatise on the management of Horses in relation to Stabling, Grooming, Feeding, Watering, and Working. By John Stewart. 12mo. Glasgow, 1833 ; 8vo. London, 1838; 1840 ; $12 \mathrm{mo}$. London, 1852 ; 6th ettition, 8vo. Edinburgh, 1854 ; 7 th edition, 8vo. London, 1860.

In French:-Economie de l'Ecurie, Manuel concernant les soins à donner aux chevaux la dispositions des écuries, les attributions des grooms, la nourriture, l'abreuvage et le travail. 8vo. Paris, 1859.

A Practical Treatise on the most important Diseases incidental to Horses, more particularly Glanders and Farcy. By Richard Vines. 8vo. London, 1833. 
In German :-Praktische Abhandlung uiber die Rotzkrankheit 1833. und dem Hautwurm des Pferdes. Translated by L. Wagenfeld. 8vo. Dantzig, 1833.

Cours théorique et pratique d'hippiatrique, à l'usage de M. M. les officiers des troupes à cheval: rédigé d'après le programme ministérielle envoyé à tous les corps, et divisé en trois parties. Premier partie; Anatomie et physiologie appliquée a l'équitation Deuxième partie: Extérieur du Cheval, haras jurisprudence vétérinaire militaire. Troisième partie: Hygiéne. By Felix Vogeli. 3 vols. $32 \mathrm{mo}$. Paris, 1833.

System of Cavalry Tactics. Anon. 8vo. Washington, 1834.

Yeomanry Regulations. Anon. 1834.

Revised and corrected in 1844 and 1851 .

Der vorsichtige Thierarzt, oder 119 erprobte Mittel wider die Krankheiten und Gebrechen der Thiere, besonders der Pferde des Rindviehes, der Schweine, und der Schafe. Herausgegeben von einem praktischen Oekonomen. Durchgesehen von K. F. W. F. Anon. 8vo. Leipzig, 1834.

Le nouveau Maréchal expert, ou le guide du Maréchal-ferrant, du Vétérinaire, de l'écuyer, etc., avec un précis de la connaissance des chevaux des anes et des mulets, de leur éducation et conservation. By Delacroix. [pseud.] Anon. 12mo. Limoges, 1834 ; $12 \mathrm{mo}$. Paris, $1853 ; 1855 ; 1856$.

Nachrichten von der Pferdezucht der Araber und von den Arabischen Pferden. By K. H. Ammon. 8vo. Nuremberg, 1834.

Grundziige der Lehre von der äussern Gestalt des Pferdes, nebst einer Anhange über Behandlung der Pferde. By Peter Wilhelm von Balle. 8 vo. Kiel, 1834.

Réponse aux observations de M. d'Aure sur la nouvelle méthode d'équitation et analyse de son traité d'équitation. By $\mathbf{F}$. Baucher. 8vo. Paris, $1834 ; 1842$.

Fisiologia Veterinaria. By Nicolas Casas de Mendoza. 8vo. Madrid, 1834 ; 4to. Madrid, 1855.

Pratique de l'élève des chevaux et de l'entrainement des chevaux de course; suivi d'observations sur l'état actuel de l'élève chevaline et sur les ameliorations qu'elle réclame. By Olivier Chuteau. 8 vo. Paris, 1834. 
1834. Zeitsclırift für die gesammte Thierheilkunde und Viehzucht. In verbindung mit mehreren der vorziiglichsten Thierärzte und Thierzüchter herausgegeben. By J. F. C. Dieterichs, Dr. Ernst Ludwig Nebel, and Dr. Karl Wilhelm Vix. 8vo. Berlin, 1834. 15th edition, 8vo. Giessen, 1848; 16th edition, 1849; 17th edition, 1851.

Kleines Handbuch für Pferdekaufer. By Ant. Engelhart. 8vo. Quedlinberg, $1834 ; 1835$.

Der Wohlunterrichteter Hufbeschlagschmied, nebst Belehrungen iiber den Castration oder dem Wallachen der Hengste. Ein nützliches Hülfsbuch für jeden Kur-und Huffschmied. By W. Friedr. Hartung. 8vo. Quedlinburgh, 1834.

Vorlesungen für Pferde-Liebhaber, mit 233 bildliche Darstellungen. By Ed. Hering. 4to Stuttgart, 1834.

Das Pferd, naturhistorisch und technisch dargestellt für Pferdezüchter, etc., und Thierärzte. By J. Hormann. Svo. Vienna, 1834.

Journal des haras, chasses, courses de chevaux et d'Agriculture appliquée à l'élève du cheval et des bestiaux en général. Recueil périodique Consacré à l'étude du cheval, à son éducation, à l'amélioration de ses différentes races en France, et à toutes les grandes réunions d'utilité publique ou privée, de luxe ou de plaisir, qui ont lieu en France, dans les Pays bas, en Angleterre, en Allemagne, en Hongrie, et dans les autres pays de l'Europe. Recueil aussi consacré à l'étude des maladies des animaux domestiques et aux moyens de les en préserver et de les enquérir Founded in 1834 by Rochau, and since 1847 edited by E. Houël.

Der Hausthierarzt oder der Rathgeber bei den Verletzungen und Krankheiten der Hunde, Katzen, Pferde, Gänse, etc. Nebst einer Anweisung der Viehseuche des Rindviehes vorzubeugen, und es im vorkommene Falle dawider zu schützen. By Carl Lenz. 8 vo. Leipzig, 1834.

Patologia del Cavallo. By Carlo Lessona. 8vo. Turin, 1834.

Veterinair-Receptirkunst nach neuern medicinischen Grundsätzen und Erfahrungen für Thierärzte und denkende Oeconomen. By Joh Chr. Gottfr. Lüpke. 8vo. Aschersleben, 1834.

Traité complet des Haras, et moyens d'améliorer et de multiplier les chevaux en France. By De Moussy. 8vo. Paris, 1834. 
Waarnemingen omtrent de horzel maskers welke in de maag van het 1834 . paard huisvesten. By A. Numan. 4to. Amsterdam, 1834.

In Germun:-Ueber die Bremsenlarven im Magen der Pferde. Aus der Holländische iibersetzt, mit Zusatzen und mit einem Anhang uiber die übrigen Bremsenlarven bei den Hausthieren. Von C. H. Hertwig. 8vo. Berlin, 1858.

Record of the Royal Regiment of Horse Guards, or Oxford Blues. By Edmund Packe. 8vo. London, 1834.

Hippopathology : A systematic treatise on the disorders and lameness of the horse, with their modern and most approved methods of cure, embracing the doctrines of the English and French Veterinary Schools, the opinions of the late Professor Coleman, Director Girard, Hurtrel, I)'Arboval, and other British and Foreign Veterinarians. By William Percivall. 4 vols. 8 vo. London, 1834, and 1849; 2nd edition, 1855, (only 2 vols. published.)

Patalogia Veterinaria. By Carlos Risueno. 8vo. Marlrid, 1834.

Anatomia Veterinaria. By Guillermo Sampedro. 8vo. Madrid, $1834 ; 1852$.

Nouveau manuel complet d'équitation à l'usage des deux sexes; con tenant le manége civil et militaire, le manége pour les dames, etc. By Amand Denis Vergnaud. 18mo. Paris, 1834; 1841; 1859 .

In German as:-Praktischer Unterricht in der Reitkunst für Herren und Damen. 8vo. Quedlinberg, 1840.

Praktische Beschlaglehre, oder die Lehre der Kunst, die Hüfe der Pferde, Maulthiere, Esel und die Klauen des Rindviehes durch Eisen am zweckmässigsten vor dem zu starken Abuutzen zu sichern, sie gesund zu erhalten und die krank und fehlerhaft gewordenen zu heilen oder durch dem Beschlag, so zu schützen, dass die Thiere baldmöglichst wieder zur Arbeit zu gebrauchen sind. By Karl Wilhm Vix. 8vo. Giessen, 1834.

British Manly Exercises : in which Riding, Driving, Racing, are now first described. By Donald Walker. 12mo. London, 1834.

3rd edition, with additions, $12 \mathrm{mo}$. London, 1835 ; others $1847 ; 1855 ; 1856$.

Nouveau traité d'Equitation, par un officier de cavalerie Polonais. 1835. Anon. Bordeaux, 1835. 
1835. Nimrod's Hunting Tours, interspersed with characteristic anecdotes ... of Sporting Men ... to which are added Nimrod's letters on Riding to Hounds. [By Charles James Apperley]. 8vo. London, 1835.

Die Zahmung des Pferdes, rationelle Behandlungsart der Remonten und jungen Pferde ïberhaupt und der bözen verdorbenen und reizbaren insbesonders, aus der innern und aussern Natur des Pferdes practisch entwickelt. By Constantin Balassa. folio. Vienna, $1835 ; 8$ vo. Vienna, 1844.

Treatise on Equitation. By Bates. 8vo. London, 1835.

Broken Wind-Pneumarox. By Bracy Clark. 4to. London, 1835. Appeared first as a contribution to Rees' Encyclopadia, Art. 'Broken Wind,' about 1809.

Allernyeste og fulstaendigste Dyrlaegebog, eller 140 paalidelige og letfallelige anvisninger til selv at kjende og behandle alle forekommende sygdomme hos hesten, oxen, faaret og svinet. By Jasper Dahl. 8vo. Odense, 1835; 3rd edition, 1854.

Handbuch der praktische Pferdekenntniss, oder, Beurtheilung des Pferdes seinem gesundem und fehlerhaften Baue nach, mit Angabe der Kennzeichen, der Fehler seiner Theile, des Alters, der gewöhnlichen Krankheiten bei denen schnelle Hiilfe nöthig ist, deren Verhuitung und Beseitigung, so wie der Fouragen, etc. Für Officiere der Kavalerie und Artillerie und für jeden Pferdeliebhaber. By Joh. Fr. Chr. Dieterich. 8vo. Berlin, 1835.

Typhus chez les Animaux domestiques. By Eléoute. 8vo. Paris, 1835 .

Gemeinnutziges Thierarzneibuch, für Stallmeister, Pferde-Zuichter, Gutsbesitzer, Oekonomen, Landwirthe und Pferde-Besitzer iiberhaupt. By H. J. Erkens. 12mo. Aachen, 1835; 1838 ,

Neues Vieharzneibuch für Jedermann. Ein grïndlicher Unterricht zur Behandlung der Krankheiten der Pferde, des Rindviehes, der Schafe, Ziegen, Schweine und Hunde. By H. J. Erkens. 8vo. 1835 ; 3rd edition, 8vo. Aachen, 1852,

A I)ictionary of Sports with Essays upon National Amusements. By H. Harewood. 8vo. London, 1835.

Abrége d'hippologie, ou précis sur la connaissance du Cheval, et sur les moyens de le conserver en santé, à l'usage des officiers, et des sous officiers des trumpes à cheval. By E. Laborde. Paris 1835 ; $12 \mathrm{mo}$. Paris, 1843. 
Magazin für die gesammte Thierheilkunde. Edited by 1835.

Ernst Frederick Gurlt. 8vo. Berlin, 1835, etc.

Essai sur l'exterieur du Cheval. By General Louis Michel Morris. 4to. Paris, 1835 ; 8vo. Paris, 1857.

Den Kloke och förståndige fårgubben, eller en korrt och sannfärdig berättelse, huru Sven Andersen i Ljungberga räddade sina får under den svåra fårdöden förliden höst och vinter, etc. By Sven Svensson [J. Th. Nalhorst]. $12 \mathrm{mo}$. Verlander, 1835.

In Norwegian:- Den Kloge og forstandige Faaregubbe. Overs. fra del Svenske af M. A. Boye. 8vo. Chrsand, 1835.

A Treatise on Equitation or the Art of Horsemanship simplified progressively for Amateurs; Forming complete lessons for Training Horses, and instructions for beginners. By J. G. Peters. Svo. London, 1835.

Terapeutica mecanica con obstetricia y arte de herrar. By Antonio Santos. 8vo. Madrid, $1835 ; 1852$.

The Sportsman, a magazine on Angling, Horse Racing, Fox Hunting, etc. 4 vols, 8vo. London, 1835-36.

The Sportsman and Veterinary Farrier. 8vo. London, 1835 to 1843 .

Afterwards united with the Sporting Review, the New Sporting, and the Sporting Magazine, but also published separately.

The Adventures of a gentleman in Search of a Horse. By Caveat Emptor [Sir George Stephen]. 8vo. London, 1835; 1836; 1837 ; 5th edition, 1841 ; another, 1861.

- Also reprinted in America, 8vo. Philadelphia, 1857.

100 jahrige Scharfrichter-Kuren an Pferden, in allen gewöhnlichen krankhaften Zustanden der Pferde, und in ausserordentlichen, deren Heilung bisher stets als ein Geheimniss betrachtet wurde. Aus dem Nachlasse des verstorbenen weitberïhmten Verfasser herausgegeben von F. W. Bruckbräu. By Hanns-Tob Velten. 8vo. Augsburg, 1835.

Des vétérinaires militaires en France, histoire critique de ce qu'ils sont et ce qu'ils ont été, avec un essai sur se qu'ils devraient être. By Felix Vogeli. 8vo. Paris, 1835.

A Comparative View of the Form and Character of the English Racer 1836. and Saddle-Horse during the last and present centuries. Anon. 4 to. London, 1836 ; 1855. 
1836. Allgemeines Thierarzneibuch, nach den besten Hülfsmitteln und eigenen Erfahrungen für Oekonomen, Landleute und Viehbesitzer überhaupt bearbeitet. Als Anhang, eine Anleitung zur Kenntniss des Pferdes und seiner Theile. 8vo. Nordhausen, 1836.

Praktische Mittheilung iiber die Zahnfistel der Pferde und deren Heilung, so wie auch hierbei iiber einige begangene Irrthümer, dem Verdacht der Rotzkrankheit der Pferde betreffend, nebst einem Vorwort vom Hrn. Geh. Med. Rathe von Vogel. By J. H. Ascheberg. 8vo. Rostock, 1836.

Traité raisonné d'Equitation. By P. A. Aubert. 2 vols. 4to. Paris, 1836.

Recueil de copies de lettres extraites de la correspondance de M. Aubert, avec les hommes que leurs connaissances en équitation, ont rangé parmi les maitres les plus capables dans cet art. 8vo. Paris, 1855.

A separate portion of 'Traité d'équitation,' by P. A. Aubert.

Ueber die Erkenntniss des Alters der nutzbarsten Hausthiere aus den natürlichen Veränderungen der Zähne. Mit einem Anhang uiber die Vorsichtsmassregeln sich beim Ankauf des Pferdes gegen Betrug zu sichern. By Eisele. 8vo. Sigmaringen, 1836.

Handbuch der speciellen Pathologie und Therapie der grosseren nutzbaren Haussäugethiere. Ein Hilfsbuch bei Vorträgen für Lehrer der praktischen Thierheilkunde, sowie zum Selbstunterrichte für Stabsärzte, Polizeibeante, Thierärzte und Gebildete Oeconomen. By Carl Friedrich Wilhelm Funke. 2 vols. 8vo. Leipzig, $1836-39 ; 1852$.

Manual de Remontista. By Jose Maria Giles. 8vo. Ecija, 1836.

Эаводская книга кровных' и скакавпіхъ лошадеп въ россіи изданная управленгемъ государственнаго коннозаводства. By Grajewski. 8vo. St. Petersburg, $1836 ; 1842$.

Die Krankheiten des Pferdes und ihre homöopathische Heilung. Ein Hülfsbuch für Cavallerie-officiere, Landwirthe und Pferdebesitzer. By Friedrich August Günther. 8vo. Sondershausen, 1836. 6th edition, 1850; 7th edition, 1852; 8th edition, 1855; 9th edition, 1857.

In French :-Nouveau manuel de médecine vétérinaire homœopathique. Translated by P. J. Martin. 8vo. Paris, 1846; $12 \mathrm{mo.}$ Paris, 1871. 
In Spanish:-Tratado completo de medicina veterinaria homeopatica o tratamiento homeopatico de los enfermedades del caballo, mulo y asno, del buey, oveja, cabra, cerdo, perro, gato, aves de corral, y enjauladas, seguido de una farmacopea homeopatico-veterinaria y de un memorandum terapeutico, para el uso le los profesores, de los ganaderos, labradores y simples particulares por Günther. Traducidos de aleman al frances por Martin y Sarrazin, y al castellano con muchas adiciones por D. Nicolas Casas, etc., etc. 8vo. Madrid, 1850.

In English :-New Manual of Homœopathic Veterinary Medicine or the Homœopathic Treatment of the Horse, the Ox, the Sheep, the Dog, and other Domestic Animals. 8vo. Boston, 1856.

In Hungarian:-Hasonszenvi allatorvos. Dr. Friedr. Aug. Giinther, 'Der homoopathische Thierarzt' ik bövittet, es javitott kiadasa utan irta s sajat Koltsegen kiadta Naray Imre, ügyed. 8vo. Arad, 1857.

Praktischer Unterricht uiber Pferdezucht. Zum Gebrauche fiir grössere und kleinere Landwirthe. By Johann August Herbst. 8vo. Nunich, $1836 ; 1854$.

Horses past and present state. A comparative view of the form and character of the English racer and saddle-horse during the past and present centuries. By Thomas Hookham. 4to. London, 1836 .

Encyclopädie der gesammten theoretische und praktische Pferde-und Rindvieh-Heilkunde, in Alphabetische Ordnung. By J. J. Rychner and Ed. Im-Thurn. 8vo. Berne, 1836-1838; 18401841 .

Flore fourragère, ou Traité complet des aliments du cheval. By Felix Vogeli. 8vo. Paris, 1836.

Neueste Entdeckung den schwarzen Staar der Pferde auf eine untrugliche und ausserordentlich leichte Weise zu erkennen. Nebst einige Bemerkungen über die Augen und Augenleiden der Pferde und einer kurzgefassten Naturgeschichte des Pferdes, besonders der arabischen raçe. Eine wichtige Schrift für jeden Pferdekaufer. Anon. $16 \mathrm{mo}$. Nordhausen, 1837.

Homöopathischer Taschen-Rossarzt, ein unentbehrliches Hand-und Hiilfsbuchlein für Diejenigen, welche die Krankheiten der Pferde leicht, sicher, einfach und wohlfeil heilen wollen. Anon. 8vo. Leipzig, 1837. 
1837. English Pleasure Carriages; their Origin, History, Varieties, Materials, Construction, Defects, Improvements, and Capabilities ; with an Analysis of the construction of Common Roads and the Public Vehicles used on them; together with Descriptions of New Inventions, illustrated with numerous designs for the use of Carriage purchasers and constructors. By William Bridges Adams. 8vo. London, 1837.

The Chase, the Turf, and the Road. By Nimrod [Charles James Apperley.] 8vo. London, 1837 ; 12mo. 1843; 8vo. 1852; 8vo. 1870 .

Another edition, 12mo. 1851, in which the Chase, the Turf, and the Road are separate.

The Life of John Mytton, Esq., of Halston, Shropshire, M.P. for Shrewsbury, etc., with his Hunting, Racing, Shooting, Driving, and extravagant exploits. By Nimrod [Charles James Apperley]. Illustrated by Alken and Rawlins. 8vo. London, 1837 ; 5th edition, 1870.

The edition of 1837 is the first separate edition. The work originally appeared in the Nero Sporting Magazine.

History of British Quadrupeds, including the Cetacea. With 200 woodeuts by Van Voorst. By Thomas Bell. 8vo. London, $1837 ; 1874$.

Undervisning i hästkännedom. By Johann Samuel Billing. $12 \mathrm{mo}$. Stockholm, 1837 ; 3rd edition, 12mo. Stockholm, 1850.

Nouveau Dictionnaire Pratique de Médicine, de Chirurgie et d'Hygiène Vétérinaire. Published by $\mathbf{H}$. Bouley and Reynall. 4to. Paris, 1837 ; 8vo. Paris, Toulouse, Lyons, 1855.

Bellezze, imperfezioni, malattie e conservazione del cavallo. By Ponsiglione Ferrero. 8vo. Cagliare, 1837.

Nyeste Dyrloegebog, indeholdende Underretning om, hvorledes Heste, Oxer, Koer, Faar og Sviin, bor behandles i frisk Tilstand, etc. By Jos. Fogg. 8vo. Christiana, 1837.

Untersuchungen und Erfahrungen im Gebiete der Anatomie, Physiologie und Thierarzneikunde. Erste Lieferung: Erection des Penis, nach Beobachtungen und Versuchen an Thieren, besonders an Pferden. By J. H. Fr. Günther. 8vo. Hannover, 1837.

Lehrbuch der vergleichende Physiologie der Haus-Säugethiere. By Ernst Friederich Gurlt. 8vo. Berlin, 1837. 
Handleiding voor de rijkunst, welke gevolgd wordt bij de kon. milit. $183 \%$. akad. By B. van Merlen. 8vo. Breda, 1837 ; $1846 ; 1850$.

A Manual of Pharmacy for the Student of Veterinary Medicine containing the substances employed at the Royal Veterinary College, with an attempt at their classification, and the Pharmacopoia of that Institution. By William John Thomas Morton. 8 vo. London, 1837 ; 5th edition, 1854.

Die Pferdewissenschaft in ihrem ganzen Umfange populär dargestellt. By Ignaz Reska. 8vo. Prague, 1837-40.

Tableau synoptique de la connaissance du cheval, offrant les notions les plus utiles sur son histoire naturelle, son anatomie, ses qualités et ses défauts, etc. By Ed. Saucerotte. Paris, 1837.

In German:-Uebersichts-Tafel der Pferdekenntniss, enhaltend die nuitzlichsten Punkte über die Naturgeschichte des Pferdes, seine Anatomie, seine Vollkommenheiten und Fehler, etc. folio. Weimar, 1839.

Die vervollkommnete Viehzucht, gründliche Anleitung zur richtigen Zucht, Wartung, Fütterung, Veredlung und Heilung des Rindviehes, der Pferde, Schaafe, Schweine, etc. By Paul v. Schmid. 8vo. Pesth, 1837.

Stud Book Remembrancer. By Thacker. 1837 ; 1855.

Grundriss einer speciellen Pathologie und Therapie des Pferdes. By L. Wagenfeld. 8vo. Dresden and Leipzig, $1837 ; 1839$.

The Racing Calendar from 1773 to 1837 . By E. and J. Weatherby. $12 \mathrm{mo}$. London, [1837].

Annali de Veterinari, de una Societa di veterinari. Anon. 1838-39. 1838.

Northern 'Tour, descriptive of the Principal Hunts in Scotland and England. By Nimrod [Charles James Apperley.] 8vo. London, 1838.

Sporting; embellished by large engravings ... from Gainsborough, Landseer ....etc. Edited by Nimrod [Charles James Apperley.] 4to. London, 1838.

Dictionnaire de Médécine, de Chirurgerie et de Hygèine Vétérinaire. By Hurtrel D'Arboval. 4to. Paris, $1838 ; 3$ vols. 8 vo. Paris, $1874 ; 1875$.

Bible Quadrupeds. By Thomas Bingley. London, [1838]; 3rd edition, 16mo. London, $1840 ; 1856 ; 1864$ 
1838. Praktisches Heilverfahren bei den gewöhnlichsten innerlichen Krankheiten des Pferdes nach den Grundsätzen der praktischen Thierarzneischule in Wien. By Johann Bleiweis. 8vo. Vienna, $1838 ; 1854$.

Du traitement de l'affection calcaire, vulgairement nommée morve des chevaux. By Bouley and Dupuy. Paris, 1838.

Hippiatria, or The Surgery and Medicine of Horses. By Bracy Clark. 4to. London, 1838.

A Treatise on the care, treatment, and training of the English Race Horse; in a series of rough notes. By Richard Darvill. 2 vols. 8vo. London, 1838-40.

Traité de la police sanitaire des Animaux Domestiques. By Onésime Delafond. 8vo. Paris, 1838.

Traité de pathologie générale comparée des animaux domestiques. By Onésime Delafond. 8vo. Paris, $1838 ; 1855$.

Der Pferde-Arzt. Ein treuer Rathgeber zur Erkenntniss und Heilung sowohl der inneren als äussern Krankheiten der Pferde. Вy $\mathrm{H}$. J. Erkens. $12 \mathrm{mo}$. Aachen, 1838.

Die inneren und äusseren Krankheiten der landwirthschaftlichen Haussäugethiere. Eiu Lehrbuch für höhere landwirthschaftliche Lehranstalten und zum Selbstunterrichte für angehende Thierärzte und Landwirthe. By Gottlieb Carl Haubner. Svo. Anclam, $1838 ; 1848 ; 1858$.

In Dutch :-Gesondheidsleer der landhuishoudelijke huisdieren. Translated by E. C. Enklar. 8vo. Zwolle, 1850.

A Mannal of British Vertebrate Animals. By the Rev. Leonard Jenyns. 8vo. London, 1838.

Ueber Kollerkrankheiten der Pferde in allen ihren Verschiedenheiten, deren mögliche Heilung, und Anfuhrung derjenigen Unstände, unter welchen der Verkauf eines innerhalb der gesetzliche Frist, als kollerkrank sich ausweisendes Pferdes giiltig zu beurtheilen ist. Für Behörden, Thierärzte Pferdebesitzer und Oekonomen; nach vieljährigen Erfahrungen bearbeitet. By Fried. Nüsken. 8vo. Minden, 1838.

The Noble Science of Fox-hunting, for the use of the Rising generation of Sportsmen. By F. P. D. Radcliffe. 8vo. London, $1838 ; 1839$. 
Lady's Practical Guide to Horsemanship. By George Reeves. 1838. $12 \mathrm{mo}$. Bath, 1838.

Analisi della materia purulenta proveniente dalle cavità nasali de' cavalli affetti da morva. By Fran. Rossi. 4to. Turin, 1838.

De l'emploi de la cavalerie à la guerre By Baron de Schauenburg. 8vo. Paris, 1838.

Grundriss der Vétérinär-Pharmakologie, oder tabellarische Uebersicht der Abstammung, äussern Kennzeichen, physisch-chemische Eigenschaften, Bestandtheile, Bereitungsart, Wirkung, Indication, Gabe, Art und Form bei der Anwendung aller in der Thierheilkunst Gebräuchliche Arzneimittel. Fïr angehende Thierärzte, Cavallerie-Officiere, Stallmeister und Oekonomen von einem Königl, preuss, Kreis-Thierarzt. Anon. 4to. Weimar, 1839.

Stallhandbuch, oder Unterricht, wie die Zeichen der haufigern innern und äussern Pferde-Krankheiten genau zu erkennen und die hauptsächlichen und leichtern Pferdekuren sicher und einfach auszufiihren sind, mit einem Anhang iber das zweckmässigste Beschlage fuir jeden gesunden und kranken Pferdehuf; fiir Pferdebesitzer, Kurschmiede, Land und Fuhrleute. Anon. 4 to. Freiburg, 1839.

Neuestes und gemeinnutzigstes Taschenbuch, fiir Pferdebesitzer und Pferdeliebhaber. Ein treuer Rathgeber bei Krankheiten, Dressur und gewöhnliche Behandlung des Pferdes. Nach Sind, v. Tennecker und andere guten Quellen bearbeitet und herausgegeben von einem alten Cavallerie offizier. Anon. 8vo. Leipzig, 1839.

Stories about Horses, compiled by the Editor of the British Workman [Thomas Bingley.] 16mo. London, 1839 ; 4th edition, 1858 ; a new edition, 1875 .

Traité de chirurgie vétérinaire ouvrage contenant le résumé du cours de siderotechnie vétérinaire. By A. J. Brogniez. 3 vols. With Atlas. 8vo. Paris, 1839-1845.

Furs Namu, of Mohammed Buksh. Lucknow, 1839.

A Hindustani work on Horses, composed in rhyme, at the suggestion of Mohammed Buksh.

On the Vices of Horses. By Bracy Clark. 4to. London, 1839.

Voyage en Syrie et dans le désert. By Louis Damoiseau. $12 \mathrm{mo}$. Brussels, 1839.

In German:-Hippologische Wanderungen in Syrien und der Wuiste. Translated by Theodore Heinze. 18mo. Leipzig, 1842. 
1839. Observations sur l'ordonnance sur l'exercise et les evolutions de la Cavalerie. By O. Dejean. Paris, 1839.

Hints on Horsemanship, to a Nephew and Niece; or, common sense and common errors in common riding. By an officer of the Household Brigade of Cavalry [Colonel G. Greenwood.] 8 vo. London, $1839 ; 1861$.

Die Venerische Krankheit der Pferde. Eine monographische Beilage zu den Handbiichern der Kameralisten, Medizinalbeamten, Thierärzte, Pferdezüchter und Oekonomen. By F. L. Haxthausen. 8vo. Breslau, 1839

Praktische Anleitung zur Kenntniss des Pferdes, oder Knochenmuskeln, und ihre Wirkung und Auslruck auf der belebten Oberfläche des Pferdekörpers. By Adolph Hess. folio. Vienna, 1839.

Specielle Pathologie und Therapie der Hausthiere. Fïr Thierärzte und Viehbesitzer. By F. X. Korber. 8vo. Quedlinburg, 1839.

Die Diätetik oder Gesundheitspflege des Pferdes, Schafes und Rindes. By F. A. Kuers. 8vo. Berlin, 1839.

The Equestrian, a Handbook of Horsemanship. By Captain $\mathbf{M} \longrightarrow$. With illustrations by Frank Howard. 12mo. London, [1839].

Der Marstall, Journal zur Unterhaltung und Belehrung für Pferdebesitzer und Pferdeliebhaber. Edited by Otto v. Corvin-Wiersbietzty, and S. v. Tennecker. 8vo. Leipzig, 1839, etc.

Traité de la folie des animaux et de ses rapports avec celles de l'hommes et des legislations actuelles. By Claude Charles Pierquin de Gembloux. 2 vols. 8vo. Paris, 1839.

Traité de pathologie et de thérapeutique générale vétérinaires. By J. Rainard. 2 vols. 8vo. Lyons and Paris, 1839-1840.

Die erkenntniss und Heilung der wichtigsten Krankheiten des Pferdes, nach homöopathischen Grundsatzen bearbeitet für Oekonomen und Pferdeliebhaber. By Ernst Ferd. Rückert. 8vo. Meissen, 1839.

Natural History of Horses. By Colonel Hamilton Smith. 12 mo. London, 1839.

Ueber das Vorkommen von Eiterknoten-Abscessen (vomicis) in den Lungen der Pferde, und deren bessere Würdigung in der gerichtlichen Thierheilkunde By Wern. Theod. Jos. Spinola. 8vo. Giessen, 1839. 
The Sporting Review, or Monthly Chronicle of the Turf, the 1839. Chase, and Rural Sports in all their varieties. Edited by Craven. 8vo. London, 1839 to 1843.

In 1843 it was incorporated with the Sportsman, but still publisher with its distinctive title and cover.

Das Buch für den Landmann, oder was Pferde und Rindviehbesitzer thun und lassen sollen um zu Nutz und Freud Hausthiere aufzuziehen, anzukaufen, zu fuittern und zu pflegen, in gesunden wie in kranken Tagen. By J. J. Rychner. 8vo. Berne, [1839]; 1840.

The obligation and extent of Humanity to Brutes, principally considered with reference to the Domesticated Animals. By William Youatt. 8vo. London, 1839.

Der wohlerfahrene Thierarzt, ein bewährtes Vieharzneibuch bei aller- 1840 . hand Krankheiten des Rindviehes der Pferde, Schafe und Schweine, von einem praktischen Thierarzt. Anon. 8vo. Reuttingen, 1840 and 1851.

A Persian MS. (in the British Museum) on the Diseases of Horses and their treatment, N.D.

Prefixed to the vol. is a letter written in English by Tirmal Rao, son of Rao Buhadoor Venlut Rao, principal Sudr Ameen of Dhurwar, presenting the work to the Bombay Branch of the Royal Asiatic Society. The letter is dated Dhurwar, August 20th, 1840 .

Equestrian Portraits. By a Walking Gentleman. Anon. London, 1840.

Philosophy of the Turf. Anon. 18mo. London, 1840.

American Turf Register and Sporting Magazine. 8vo. Baltimore, 1840 , etc.

Instructions for Cavalry Sword Exercise. By Henry Angelo. 12mo. London, 1840.

Passe-temps équestres suivi de notes explicatives. By F. Baucher. 8vo. Paris, 1840.

An Encyclopodia of Rural Sports, Hunting, Shooting, Fishing, Racing, etc., illustrated by Alken, Landseer, etc. By Delabere Pritchett Blaine. 8vo. London, 1840 ; $1852 ; 1858$; 1870.

Sammlung einiger durch Erfahrung erprobter zum Theil nicht bekannter Heilmittel gegen haufig vorkommene Krankheiten der Pferde. By Ritter v. Bultzingstoven. 8vo. Erfurt, 1840. 
1840. A short history of the celebrated Race-Horse, Eclipse. By Bracy Clark. 4to. London, [1840].

Tractatus de vulnerationibus Equorum. By S. R. J. Georgio. [Circa 1840].

Cavalry Sword Exercise. By Colonel George Greenwood. 12mo. London, 1840.

Zootomische Darstellung des Pferdes. By J. Hermann. 8vo. Vienna, 1840.

Groom and Coachman's Guide. By Knight. [Circa 1840].

Veterinär-medicinische Propädeutik und Hodegetik, oder Anleitung zum Studium und zur Ausübung der Veterinärmedizin. Zum Gebrauche bei Vorlesungen und für Thierärzte, Sanitäs-Beamte und Landwirthe. By Joh. Mart. Kreutzon. 8vo. Augsburg, 1840.

Résumé de quelques récherches relatives à l'étude des maladies du cour des principaux animaux domestiques. By U. Leblanc. 8vo. Paris, 1840.

Observations raisomées sur l'hygiène vétérinaire militaire. By Legros. 18 mo. Paris, 1840.

The Breeds of Domestic Animals of the British Islands. By David Low. 2 vols. folio. Edinburgh, 1840; London, 1842; 8vo. London, 1845.

Vol. 1 is in two, and Vol. 2 in three parts, each with separate indexes and pagination.

A Natural History of Quadrupeds and other Mammiferous Animals, comprising a Description of the Class Mammalia, including the principal varieties of the Human Race. By William Charles Linnæus Martin. London, 1840.

Anleitung zum praktischen Hufbeschlag der Pferde, so wie zur Kenntniss und Kur der vorzïglichsten Hufkrankheiten. Ein grundlicher, aber leichtfassliche Unterricht für Beschlagschmiede, Landwirthe, Cavallerie-offiziere und jeden Pferdebesitzer. By Theodore Merk. 8vo. Munich, 1840.

Lessen in de rijkunst en het voltigeeren. By B. van Merlen. 8vo. Breda, 1840.

De afrigtingskunst. By B. van Merlen. 12mo. Breda, 1840.

De hongere afrigtingskunst. By B. van Merlen. 12mo. Breda, 1840 . 
Des Institutions hippiques et de l'élève du cheval daus les principaux 1840.

Etats de l'Europe. By the Count Achille de Montendre. 3 vols. 8 vo. 1840-1844.

A Veterinary Toxicological Chart, containing those Agents which are known to cause Death in the Horse; with the symptoms, antidotes, action on the tissues, and tests. By W. J. T. Morton. London, 1840.

The New Sporting Almanack, a Manual of Instruction and Anusement. Edited by Wildrake. [George Tattersall.] 6 vols. 12 mo. London, 1840, etc.

De la cavalerie et du casernement des troupes à cheval. By Nicolas

Charles Victor d'Oudinot, Duke de Reggio. 8vo. Paris, 1840.

Question Chevaline. Lettre de M. le lieutenant général Marquis d'Oudinot, à M. le duc de Grammont, president du comité hippique. By Nicolas Charles Victor d'Oudinot, Duke de Reggio. 8vo. Paris, [Circa 1840].

Taschenbuch der pferdeärztlichen Praxis fuir Pferdeärte. Kur-und Fahnenschmiede und jeden Pferdebesitzer. By Joh. Friedr. Persch. 8vo. Quedlinburg, 1840.

De l'organisation et de l'etàt actuel de la cavalerie. By le Vicomte Claude Antoine Hippolyte de Préval. 8vo. Paris, 1840; 1842.

Gangrène traumatique. Mémoires et observations cliniques sur une de ses causes les plus fréquentes dans les animaux domestiques. By Eugéne Renault. 8vo. Paris, 1840.

Analyse du règlement d'exercise pour la cavalerie suédoise. By Rigault. 8vo. Paris, 1840.

Traité complet de l'Anatomie des animaux domestiques. By F. J. J. Rigot, and continued by A. Lavocat. 8vo. 4 vols. Paris, 1840-1848.

De la Ferrure sous le point de vue de l'hygiene. By J. B. C. Rodet. 8 vo. Paris, 1840.

Novisima Cavero. By Guillermo Sampedro. 4to. Madrid, 1840.

Anleitung zur möglichst genauer Erkenntniss des Pferdealters aus den Veränderungen der Zähne von der Geburt bis zum 30 Lebensjahre. Ein Handbuch zum Selbstunterricht und zum Gebrauch seiner Vorlesungen. By Joseph Schultes. 8vo. Salzburg, $1840 ; 1848$. 
1840. Die Oestraciden-Bremsen der Pferde Rinder und Schaafe. Eine naturgeschichtliche-therarztliche Abhandlung zur Erinnerung an das 50 jährige Bestehen der Kön. Central-Veterinär-Schule in München. By Konr. Ludw. Schwab. 4to. Munich, 1840.

A Treatise on the Structure, Functions, and Diseases of the Foot and Leg of the Horse. By W. C. Spooner. 8vo. London, 1840.

Handbuch der praktische Anatomie der Haussäugethiere, besonders des Pferdes, mit Beriicksichtigung der Physiologie. By Fr. L. C. Steinhoff. 8vo. Hamburg, 1840.

Considérations sur les chevaux limousins, sur les causes de la destruction presque totale de cette race, et sur les moyens de la reproduire. By Baron François Simon Marie Jules Gay de Vernon. 8vo. Paris, 1840.

De la morve considérée sous le rapport de sa transmission à l'espéce humaine. By J. B. Veyssière. 8vo. Paris, 1840.

Wie heilt der Bauer und schlichte Landmann seine kranken Pferde? Eine Belehrung in Fragen und Antworten. By Ludwig Wagen feld. 8vo. Leipzig, $1840 ; 1848$.

History of the British Turf from the Earliest Period to the Present Day. By James Christie Whyte. 2 vols. 8vo. London, 1840.

Om Hestekjods Afbenyttelse til Fode for Mennesker. By G. C. With. 8vo. Copenhagen, 1840.

1841. Anschauliche Anseinandersetzung der Pferde-Muskeln, des PferdeGerippes, der Pferde-Krankheiten, und des Pferde-Exterieurs. Anon. folio. Vienna, 1841.

How to buy a Horse. Choice, Shape, Appearance, Action, Soundness, or Defects, Exposition of Tricks, Feeding, Stable Management, Exercise, etc. Anon. 12mo. London, 1841.

Nouvelle école d'équitation, a l'usage des militaires et des particuliers suivie de la Diététique. Anon. 8vo. Paris, 1841.

United States Cavalry Tactics. Anon. Washington, 1841.

Viertes Verzeichniss der preussischen Vollblutpferde. Anon. 1841.

Veterinair-Pharmacopœ for militair Dyrlæger. Af en Commission. Anon. 8vo. Copenhagen, 1841. 
رسالغ في مناصل النر سي [نز نعة الهكا صل في معر نة المفاصل] 1841.

Translatcd by Mahommad Abd al-Fattah, revised by Mustafa Hasan Kassàb and Rafaâh Ráfi. A Treatise on the Joints of Horses. 8 vo. Boulak. 1257, 1841.

A French translation of the above:-Traité des articulations du cheval. By F. J. J. Rigot.

L'Argus des Haras et des Rémontes, Journal de la réforme des abus dans l'intérêt des Eléveurs de chevaux, de la Cavalerie et de L'Agriculture. Edited by X. de Nabat. Paris, 1841.

Calendrier des courses de chevaux, ou Racing Calendar français pour l'année 1841. By Thomas Bryon. 12mo. Paris, 1841.

De l'etablissement des troupes à cheval dans les grandes fermes. By Th. Rob. Bugeaud. Paris, 1841.

Nuovo metodo per curare il moccio o morve de' cavalli. By Francesco Caputo. Naples, 1841.

Observations d'un éleveur sur la mesure nouvellement adoptée par le ministre de la guerre d'entretenir des étalons dans lss dépôts de remonte de cavalerie. By Baron Louis de Curnieu. 8vo. Paris, 1841.

Observations d'un éleveur sur la brochure intitulée, Des remontes de l'armée, par le lieutenant-général marquis Oudinot, et sur le rapport présenté par M. le Comte de Morny au conseil général de l'agriculture par la commission des chevaux. By Baron Louis de Curnieu. 8vo. Paris, 1841.

De la Penurie des chevaux de cavalerie et des moyens à employer pour améliorer nos races propres aux remontes Militaires. By P. Daunay and Lignée. 8vo. Paris, 1841.

Traité de l'histoire naturelle et médicale des substances employées dans la médicine des animaux domestiques. By O. Delafond and J. L. Lassaigne. 8vo. Paris, $1841 ; 1853$.

Manual of Veterinary Science. By W. Dick. 8vo. Edinburgh, 1841; 1862.

Manuale d'ippiatrica militare, ossia ristretto metodico delle condizioni veterinarie indispensabili all'uffiziale di cavalleria. By Roberto Fauvet. 12 mo. Naples, 1841.

Fragments de recherches comparées sur la nature constitutive de différentes sortes de fibrine du cheval dans l'étàt normal et pathologique. By L. R. de Fellenberg. 8vo. Berne, 1841. 
1841. Notice sur le vomissement daus les principaux quadrupédes domestiques. By J. Girard. 8vo. Paris, 1841.

Essai sur la tactique des trois armes isolées et réunie. By Henri de Giustiniani. 8vo. Paris, 1841.

Des Différentes espèces de chevaux en France, depuis les temps les plus anciens jusqu’à nos jours. By Ephrem Houël. 8vo. Paris, 1841.

VollständigesHandbuch der Veterinärkunde für Thierarzte, Oekonomen, Pferdeliebhaber; zum Selbstunterricht und zu Vorlesungen auf Veterinar-Schulen. By Ed. Im-Thurn. 12mo. Schaffhausen 1841.

Journal vétérinaire et agricole de Belgique, consacré aux progrès des différentes branches de la médecine vétérinaire, de l'agriculture, et des sciences physiques et naturelles qui s'y rapportent. Edited by Brogniez, Delwart, Froidmont, Graux, Scheidweiler, and Thienesse. 8vo. Alost, 1841.

Uber Einrichtung und Leitung der Thierarzneischulen, zum Besten der Landwirthe und Thierärzte und über allgemeine Einführung des guten Hufbeschlages. By F. A. Kuers. 8vo. Berlin, 1841.

De l'amelioration de la race chevaline dans le departement de l'Aisne, du prix de revient des jeunes chevaux elevées dans les herbages et au sec, et quelques idées sur les rémontes de l'armée. By Count de La Tour-Du-Pin-Chambly. 8vo. Laon, 1841; 8 vo. Orleans, 1848.

Pharmacie vétérinairc, chimique, théorique et pratique. By J. Ph. Lebas. 8vo. Paris, [1841]; 6th edition revised by Lelong, 8 vo. Paris, 1845.

Récherches relatives à la determination de l'âge des lésions des plévres et des poumons du cheval au point de vue médico-legal. By Urbain Leblanc. 8vo. Paris, 1841.

Du Crapaud, ou Podoparenchydermite chronique du cheval ; suivie du Piétin, ou Podoparenchydermite du mouton. By Mercier de l'Eure. 8vo. Paris, 1841.

Abhandlung iiber die Pferde-Influenza, auch Brustfell-oder Lungenentziundung genaunt. Zur Belehrung für Oekonomen, Pferde, besitzer und angehende Thierärzte. By F. Meyer. Svo. Potsdam. 1841. 
Quelques réflexions sur la mécanique animale appliquée au cheval, 1841. By D. J. Mignon. 8vo. Paris, 1841.

Memoires et observations pratique de médecin vétérinaire. By $\mathbf{B}$. Mousis. 8vo. Paris, 1841.

Resultatet af en Reise til Jycland (Fuldblodshingsterne). By J. W. Neergard. 8vo. Copenhagen, 1841.

Les Chevaux français en 1840. By Felix Person. 8vo. Paris, 1841.

Veterinar-Memorabilien. Beiträge zur praktische Thierheilkunde. Der Stelzfuss der Pferde und der Sehnenschnitt zur Heilung desselben, etc. By Carl. Glob. Prinz. 8vo. Dresden, 1841.

Traité de l'âge du cheval. By Charles Goupy de Quabeck. 8vo. Paris, 1841.

Ueber die Ursachen der bei Pferden und Rindvieh als Folge des Aderlasses an der grossen Halsblutader (Drosselvene) häutig vorkommene Aderfistel und deren leichte Heilung ohne Operation. Fiir praktische Thierärzte. By C. F. Rath. Svo. Quedlinburg, 1841 .

Domesticated Animals. By Mary Roberts. 12mo. London, 1841 ; 5th edition, 8vo. London, 1854.

Handbok i Boskapsskötseln. Om Hästens, Hornboskapens, Farets, Svinets uppö̈dning och skötsel. By Lars Tiden. 8vo. Jonköping, 1841 ; 1850.

Notitia Venatica. A Treatise on Fox-hunting to which is added a compendious kennel stud-book. By R. T. Vyner. 8vo. London, $1841 ; 1847 ; 1874$.

Prolegomena til Veterinair propœedeutik. By G. C. With, 8vo. Copenhagen, 1841.

Der wohlunterrichtete Cur-und Hufschmied oder gründliche Anweisung zu einem Natur-und vernunftgemässen Beschlage gesunder und fehlerhafter Hufe, sowie zu den gewöhnlichsten, bei Pferden vorkommenden Operationen und den dabei anzuwendenden Heilmitteln. By Theodor Ferdinand Zerrener. 8vo. Weimar, 1841 ; 1854.

Bulletin officiel des courses de chevaux. Anon. 4to. Paris, 1842, etc. 1842

Handbook of Horsemanship. Anon. 12mo. London, 1842. 
1842. Handweiser fuir Alle, welche ohne thierärztliche Kenntnisse ihr krankes Vieh selbst heilen und die Krankheiten desselben schnell und sicher erkennen wollen. Anon. 8vo. Quedlinburg, 1842.

Жћурналь кониозаводства и охоты. Санстпетербургъ. Въ типогpaфiı И. Глазунова; вт французкои типографіи; въ тип. Кесневиля ; въ тип. министерства государствднныхъ имуществ' и в' Карла Метцига. Edited by Borodin. 8vo. St. Petersburg, 1842.

The Horse and the Hound, their various Uses and Treatment, including Practical Instructions in Horsemanship and a Treatise on Horse dealing. By Nimrod [Charles James Apperley.] 12 mo. Edinburgh, $1842 ; 8$ vo. 1843 ; 8vo. 1858.

The Life of a Sportsman. By Nimrod [Charles James Apperley.] 8 vo. London, 1842.

Equitation des Dames. By P. A. Aubert. 8vo. Paris, 1842.

Quelques observations sur le système de M. Baucher pour dresser les chevaux. By P. A. Aubert. 8vo. Paris, 1842.

Observations sur la nouvelle méthode d'équitation. By Antonio Henri Phillippe Comte d'Aure. 8vo. Paris, 1842.

Méthode d'équitation basée sur de nouveaux principes, augmentée de documents inédits de rapports officiels en faveur de l'application de la méthode aux chevaux de troupes. By F. Baucher. 8vo. Paris, 1842; 9th edition, 1850.

A German trans., 8vo. Berlin, 1843 and 1852. A Spanish trans., 4 to. Madrid, 1848. A Russian trans. from 10th edition, 8vo. St. Petersburg, 1857.

In English :-A method of Horsemanship founded upon new principles including the Breaking and Training of Horses, with Instructions for obtaining a good seat.

Third American edition from the ninth Paris, 12mo. Philadelphia, 1852.

Sopra tre casi di pretesa comunicazione della morva e del farcino dal cavallo all' uomi. By G. Bonino. 8vo. Turin, 1842.

Essai sur la situation de l'industrie chevaline, ses besoins et les nzoyens de régénération. By le vicomte A. de C. 8 vo. Paris, 1842.

Art of Shoeing Horses by the Sieur de Solleysel, with notes on his practice. By F. C. Cherry. 8vo. London, 1842. 
La Comédie à Cheval, ou Manières et Travers du monde équestre; 1842. jockey club, cavalier, maquignon, olympique, etc. By Albert Cler. 12mo. Paris, 1842.

Recherches sur la composition du sang de quelques animaux domestiques dans l'etât de santé et de maladie. By O. Delafond, and Andral. 8vo. Paris, 1842.

Die Zucht der Vollblut-und Landpferde, so wie unserer Hausthiere iiberhaupt, nebst Belehrungen iiber die Krankheiten der Pferdo und Füllen, und über die Geburtshulfe derselben. Fiir Landwirthe und jeden Pferdebesitzer. By Joh. Fr. Chr. Dieterichs. 8 vo. Berlin, 1842.

Handbuch der Veterinär-Chirurgie, oder die Lehre von den wichtigsten, an Hausthieren vorkommenden blutigen Operationen. Вy J. F. C. Dieterichs. 8 vo. Berlin, $1842 ; 1851$.

Les Haras et les remontes; la guerre et les brochures. By Adolphe Dittmer. 8vo. Paris, 1842.

Der Eskadrondienst. Ein Handbuch fur Kavallerie-Offiziere der Königlich-prussischen Armee. By Theod. v. Dolffs. 1842.

Der sicher heilende Pferde-und Rindvieharzt, oder wie kann der Städter und Landmann die vorkommenden äussern und innern Krankheiten bei Pferden und Rindvieh und die so wichtige Lungenseuche bei den Rindvieh nicht nur erkennen, sondern auch auf die einfachste Art selbst heilen (sowohl nach dem allöopathischen als auch nach dem homöopathischen Heilverfahren). Nebst Unterricht über die Zucht, Wartung und Fütterung dieser Thiere. By Friederich Fuhrmeister. 8vo. Quedlinburg, $1842 ; 1855$.

Nouveau traité des vices redhibitoires et de la garantie dans les ventes et échanges des animaux domestiques. By Ch. Galisset, and J. Mignon. 8vo. Paris, $1842 ; 1852$.

De l'entraînement des Chevaux et des luttes sur les hippodromes. By P. N. Hamont. 8vo. Paris, 1842.

Traité complet de l'élève du cheval en Bretagne. By E. Houel. 8 vo. Paris, 1842.

Des Haras et des Remontes de l'armée. By Lieut.-Col. Alex. Itier. 8vo. Paris, 1842. 
1842. Praktisch-thierärztlicher Rathgeber gegen die meisten, sowohl innerlichen, als äusserlichen Krankheiten der Pferde. Mit einer Abhandlung iiber einige allgemeine Krankheiten, dann einem Anhang von verschiedenen Gattungen der besten Clystiee, und einer Zugabe von dem kalten Fieber und Rotz; nebst den dagegen dienlichen Fürsehungs-und Heilungsmitteln. By Christian Nicolaus Kaestner. 16mo. Passau, 1842; 1853.

De l'Equitation et des Haras. By Count Savary de LancosmeBréves. 4to. Paris, $1842 ; 1843$.

De la Cavalerie française et de la necessité de lui adjoindre des irreguliers en temps de guerre. By George Nicholas Marc Baron de Létang. 8vo. Paris, 1842.

Principles d'hygiène vétérinaire, ou règles dans lesquels on doit entretenir et gouverner les animanx domestiqnes, cultiver les fourrages, etc. By J. H. Magne. 8vo. Lyon, 1842; 1844 ; 1858-59.

Handleiding tot de behandeling der wapens, en grondregels voor den kavallerist in het afzonderlijk gevecht. By B. van Merlen. 8vo. Breda, 1842.

Traité élémentaire de matière médicale ou de pharmacologie vétérinaire. By L. Moiroud. 8vo. Paris, [1842]; 1843.

Recensenten og Dyret. Neergard og mine Hingste. By M. L. Nathanson. 8vo. Odense, 1842.

Danmarks forqvaklede Hestevœsen. By J. W. Neergard. 8vo: Copenhagen, 1842.

Des remontes de l'armée, et de lenr rapports avec l'administration des haras. By Nicolas Charles Victor d'Oudinot. 8vo. Paris, 1842 .

Allgemeines Vieharzneibuch oder Unterricht wie der Landmann Pferde, Rindvieh, Schaafe, Schweine, Ziegen und Honde aufziehen, warten, füttern und deren Krankheiten erkennen und heilen soll. Nebst einem Anhang. By Johann Nicolaus Rohlwes. 8vo. Berlin, 1842.

Guide to the Turf. By William Ruff. 12mo. London, 1842. ete.

Hippiatrik, oder systematisches Handbuch der äusserlichen Krankheiten des Pferdes und ihrer Heilung. By J.J. Rychner. 8ro. Berne, 1842. 
Equitation Fashionable. By Saint-Ange-Saint-Paul. 8vo. Paris, 1842. 1842.

Ansichte über die Fortschritte der Pferdezucht in Preussen nebst einem Vorschlage ohne Kosten für den Staat und Landmann binnen wenigen Jahren im ganzen Lande den allgemeinen Anforderungen entsprechende Pferde in grösserer Zähl als bisher zu zuichten. By E. F. Seidler. 8vo. Berlin, 1842.

Remarks on the Transport of Cavalry, with suggestions for the management of Horses, before, during, and after a long sea voyage. By Lieutenant-Colonel Shirley. London, 1842.

Sporting Sketch Book, a Series of Characteristic Papers by the most Distinguished Sporting Writers of the Day. Edited by J. W. Carleton. Plates by Herving, Alken, etc. 8vo. London, 1842.

Sporting Architecture, Stud Farm, Stables, etc. By George Tattersall. 4to. London, $1842 ; 1850$.

Heste-og Fœbog eller om Hestens, Hornkvœgets, Faarets, etc. By Lars Tidén. 12mo. Christiania, 1842.

Mémoire sur l'amélioration des chevaux Normands. By le vicomte de Tocqueville. 8vo. Paris, 1842.

Myologie des Pferdes. Nach den anatomische Zeichnungen von E. F. Gurlt und Schwab's Handbuch über Anatomie dargestellt. By Louis Voetsch. 4to. Tubingen, 1842.

Gedanken eines deutschen Praktikers über die Baucherische Dressur- 1843. methode. Dargestellt in einem Dialog zweier Trakehner Stallnachbarn. Anon. 1843.

Historical Record of the Light Horse Volunteers of London and Westminster. Anon. London, 1843.

The Griffin's Aide-de-Camp. By Blunt Spurs, [pseud]. 8vo. Madras, 1843 ; 3rd edition, 1860.

Cavalry Manual. By Major Ainslie. London, 1843.

Des principes qui servent de bases à l'instruction et à la tactique de la cavalerie; précedés d'une revue historique des divers systèmes d'instruction et des ordonnances de cette arme, etc. By Flavin D'Aldéquier. 8vo. Paris, 1843.

Du Cheval en France. By Charles de Boigne. 8vo. Paris, 1843. Abhandlung über die Abrichtung des Kampagne-Pferdes im Frein. By Rudolph von Brudermann. 1843. 
1843. Farmacopea Veterinaria. By Nicolas Casas de Mendoza. 8vo. Madrid, $1843 ; 1848 ; 1856$.

Treatise on the Power of the Horse. By Thomas Clarendon. 8vo. London, 1843.

Traité de thérapeutique générale vétérinaire. By O. Delafond. 2 vols. 8vo. Paris, 1843-1844.

Blood Letting in Diseases of Horses. By H. Ferguson. 8vo. 1843.

Vingt pages à lire, ou la Question Chevaline simplifiée. By Alexandre Girardin and the Marquis de Torcy. 8vo. Paris, 1843.

Considérations sur les institutions hippiques, et sur les moyens de propager et d'ameliorer les races chevalines en France. By the duc de Grammont. 8vo. Paris, 1843.

Field Days of the 2nd Life Guards. By Colonel George Greenwood. 8vo. London, 1843.

Considérations générales sur l'amélioration des Chevaux en France. By P. N. Hamont. 8vo. Paris, 1843.

Traité des Courses au Trot. By Ephrem Hoüel. 8vo. Paris, 1843. Other editions, 1860 and 1864 .

Notice sur les chevaux comtois, et sur les moyens propres à ameliorer cette race. By E. Laborde. 8vo. Paris, 1843.

La vérité à cheval. By Connt Savary de Lancosme-Brèves. 8vo. Paris, 1843.

Plan d'établissement de haras et de fermes-modèles dans la Perche. By A. de Laulanheir. 8vo. Versailles, 1843.

Projet de Conservation de la Race Chevaline dans le Perche. By A. de Laulanheir. 8vo. Versailles, 1843.

Du Cheval de Montagne. By G. Bergasse de Laziroules. 8vo. Paris, 1843.

Traité de l'extérieur du Cheval et des principaux animaux domestiques. By Felix Lecoq. 8vo. Paris, 1843 ; 4th edition, 1869 ; 5 th edition, 1875 .

In Italian :-Trattado dell Esteriore del Cavallo del Signor Le Coq. Translated by Luigi Ghilardi. 8vo. Milan, 1852.

Examen du système Baucher, de son application à notre cavalerie. By L. Lecornué. 8vo. Paris, 1843. 
Refutation complète de la nouvelle méthode d'équitation proposée par 1843.

M. Baucher. By Legros. 8vo. Paris, 1843.

Nuovo trattato d'equitazione. By Carlo Le Maire. 8vo. Turin, $18+3$.

Memoranda relative to the Worcestershire Yeomanry Cavalry now the Queen's Own raised by Other Archer 6th Earl of Plymouth in 1831, now under command of Lieutenant-Colonel the Hon. Robert Henry Clive, M.P. By Martin. 8vo, 1843.

Privately printed.

The Horsekeeper's Guide. Practical Directions for keeping and managing Horses, with the Treatment of Diseased Horses. To which are prefixed plain directions for the choice and purchase of horses, etc. By James Mills. 12 mo. London, [1843].

4th edition, $12 \mathrm{mo} .1854 ; 1858 ; 1871$. 12th Extition under the title:-The Gentleman's Guide for the Choice, Treatment, and Management of Saddle, Carriage, Gig, and Cart Horses. How to ascertain their good qualities, detect their faults, and save the expense of a Veterinary Surgeon, with T. S. Rarey's instructions for the taming of Horses, and the Art of Horsemanship. 8vo. London, 1876.

Guide du Maréchal-ferrant. By Mathurin Papin. 12mo. Paris, 1843.

Traité d'équitation sur des bases géométriques, contenant 74 figures. By A. C. M. Parisot. 8vo. Paris, 1813.

Anleitung zum Fechten mit den Säble und dem Kürassierdegen, zuvörderst dem Unterricht in Kavallerie-Abtheilungen angeeignet, nebst Bemerkungen für den ernstlichen Kampf zu Fuss und Pferde. By E. F. Seidler. 8vo. Berlin, 1843.

A Rough Sketch of the Rise and Progress of the Bengal Irregular Horse. By Captain Carmichael Smythe. 8vo. Calcutta, 1843.

Neues und vollständiges Vieharzneibuch oder Universal-Handbuch aller unserer Hausthiere, und zwar das Pferd, Rindvieh, Schaf, die Ziege, das Schwein, der Hund, die Katze, das Hausfedervieh und die Stubenvögel, im gesunden und kranken Zustande betreffenden Kenntniss. By Heinrich Wilhelm Stephan. 8vo. Bielefeld, $1843 ; 1848$.

A New System of Broad and Small Sword Exercise with instructions in Horsemanship. By Stephens. 12mo. Philadelphia, 1843. 
1843. De la Morve et du Farcin Chroniques, chez l'homme et chez les Solipèdes. By Ambroise Tardieu. 4to. Paris, 1843.

Nimrod's Hunting Reminiscences. Illustrated by Wildrake LGeorge Tattersall]. 8vo. London, 1843.

Thierarztliche Zeitung. Edited by Christopher Joseph Fuchs. 4to. Carlsruhe, 1843.

Anatomisch-physiologiske Bemærkninger over Hestens Taa. By $\mathbf{H}$. C. Tscherning. 8vo. Copenhagen, 1843.

Haandbog i Veterinairpharmacologien. By H. C. Tscherning. 8vo. Copenhagen, 1843.

1844 Feltjenste, uddraget af Instructionen for Cavaleri-Exerceerskolert. Anon. Aarhans, 1844.

Udtog af Instructionen for Cavalleri-Exerceerskoler. Anon. 8vo. Copenhagen, 1844.

8 vigtige Hemmeligheder for Hesteeiere (Hestedressering og Hestekjob) af in gl. Cavaleri-Officeer. Anon. 8vo. Copenhagen, 1844.

Zusammenstellung einiger Zahlen fuir den Dienst der Kavallerie. Anon. 1844.

Traité d'équitation illustré. Précédé d'un aperçu des divers modifications et changements apportés dans l'équitation depuis XVIe siècle jusqu'à nos jours: suivi d'une appendice sur le jeune cheval, du trot Anglais, el d'une lettre sur l'équitation des Dames. By Antonio Henri Phillippe Léon Comte d'Aure. 8vo. Paris, [1854]. Srd edition, 8vo. Paris, 1846. 8vo. Paris, 1870.

Dialogues sur l'équitation. Premier dialogue entre le grand HippoTheo, dieu des quadrupèdes, par un Cavalier et un Cheval. By F. Baucher. 8vo. Paris, 1844.

Nouveau traité des robes ou nouances chez le cheval, l'âne et le mulet, chez l'espèce bovine et les petites espèces domestiques. By C. Brivet. 8vo. Paris, 1844.

Biblioteca del ganadero y agricultor, que comprende : $1^{\circ}$ Economia Rural : $2^{\circ}$ Cria del caballo: $3^{\circ}$ Cria de los ganados : $4^{\circ}$ Cria de las aves: $5^{\circ}$ Enfermedades de los ganados : $6^{\circ}$ Agricultura y : $7^{\circ}$ Higiene. By Nicolas Casas de Mendoza. 7 vols. 4 to. Madrid, 1844 to 1849. 
Cours d'hippologie, ou Exposé des connaissances hippiques. By 1844.

Dubroca. 8vo. Paris, 1844.

Field Movements for a Division of Cavalry. By Lord William Lennox Lascelles Fitzgerald, Baron de Ros. London, 1844.

Yeomaury regulations; being an abridgment of the Regulations for the Formations and Movements of the Cavalry, adapted to the use of the Yeomanry Corps, with suggestions on Discipline and rules for piquets. By Lord William Lennox Lascelles Fitzgerald, Baron de Ros. 12mo. London, 1844; 1853.

Cours d'hippiatrique à l'usage des sous-officiers de l'artillerie et de Cavalerie. By J. B. A. Goux and Merche. 8vo. Paris, 1844.

Sur l'étât de la population chevaline en France, et ses conséquences. By Lieutenant-General Comte de Girardin. 8vo. Paris, 1844.

Handbuch der Zoo-Pathologie und der Therapie oder: planmässige, methodische, mittelrechte Erkenntniss des Charakters, Sitzes, der Art, Ursache und Grösse, sowie kurative, präservative, veterinär-polizeiliche, gerichtliche und vergleichende Behandlung der sporadischen und seuchenartigen Krankheiten der Haussäugethiere. By Anton Hayne. 8vo. Vienna, 1844; 1852.

The Horseman. By Hersenberger. 12mo. New York, 1844.

Handbook of Farriery. By J. Joce. 12 mo. [Circa 1844].

On Calculous Concretions in the Horse, Ox, Sheep, and Dog, being the substance of two essays read before the Veterinary Medical Associations. By W. J. T. Morton. 8vo. London, 1844.

Annals of York and Doncaster. By Orton. 8vo. London, 1844.

Idiomologie des Animaux, ou recherches historiques, anatomiques, physiologiques et glossologiques sur le langage des bêtes. By Claude Charles Pierquin (de Gembloux). 8vo. Paris, 1814.

Hygiène vétérinaire militaire. By Léon J. B. Rochas. 8vo. Paris, 1844.

Nature and Management of the Horse. By William Roper. 8vo. London, 1844. 
1844. The Horse in health and disease, also the management of the Hunter; with Instructions for Stabling, Training, etc. By William Roper. 8vo. London, 1844 ; 1850.

In Dutch from the 2nd English edition:-Behandeling van het Paard in gezonden en zieken staat, met aanwijzing voor stalling, afrigting, etc. $8 \mathrm{vo}$. Utrecht, 1853.

Turf Betting Simplified. By Rollo Rouse. 18mo. London, 1844.

De la disparition en France du Cheval léger, de la necessité d'en avoir, et des moyens d'y faire prospérer cette espèce. By J. B. Sablon 8vo. Paris, 1814.

De la race chevaline en Algérie, et des moyens de l'accroître et de laméliorer. By J. B. Sablon. 8vo. Paris, 1844.

Ĉtre ou ne pas être. Du cheval de cavalerie légère, de la nécessité d'en mettre la production et tout ce qui en dépend dans les attributions du ministre de la guerre. By J. B. Sablon. 8vo. Paris, 1844.

Les animaux domestiques, considérés sous le rapport de leur conservation, de leur amélioration et de la guérison de leurs maladies, ou guide théorique et pratique du propriétaire, du fermier, du cultivateur, de l'éleveur, etc. By Maximilien de Saive. 8vo. Brussels, $1844 ; 1849$.

Unparteiische Ansichten iiber das Baucher'sche System der Pferdedressur nebst theilweisem Vergleich mit den bei uns im allgemeinen iblichen Prinzipien. By E. F. Seidler. 8vo. Berlin, 1844.

Die Influenze der Pferde, in ihren verschiedenen Modificationen dargestelt. By Werner Theodor Joseph Spinola. 8vo. Berlin, $1844 ; 1849$.

The Cracks of the Day. Edited by Wildrake [George Tattersal1]. 8vo. London, 1844.

Résumé de la question des haras et des remontes, suivi de quelques expérience et d'un nouveau système d'éducation des chevaux. By le comte Joseph de Turenne. 8vo. Paris, 1844.

1845. Cavalry Sword Exercise. 8vo. London, 1845.

Observations sur le moyen de simplifier le dressage des chevanx de troupe. By P. A. Aubert. 8vo. Paris, 1845.

Opuscule sur l'île et les chevaux de la Camárgue. By Auboyer. sgo. Paris, 1845 . 
De l'industrie chevaline en France et des moyens pratiques d'en 1845. assurer la prosperité. By Antoine Henri Phillippe Comte d'Aure. 8vo. Paris, 1845 ; 3rd edition, 1847.

Résuné de la question des haras et des remontes. Réponse à M. le comte de Turenne. By Antonio Henri Phillippe Comte d'Aure. 8 vo. Paris, 1845.

Reitschule fïr Liebhaber des Reitens aller stände, By P. W. Balle. 8vo. Kiel, $1845 ; 1849$.

In Danish as :-System til Ridehestens Dressur. 8vo. Copenhagen, 1847 ; from the Kiel edition of 1845 . Another transtation from the 2nd edition:-Kjorescole. 8 vo. Copenhagen, 1851.

Anleitung zum Betriebe der Pferdezucht für Thierärzte, Gestiitsbeamte und Landwirthe. By Johann Wilhelm Baumeister. 8 vo. Stuttgart, $1845 ; 1854$.

In Dutch as:- Handleiding tot de paardenkenniss voor veeartsen, paardenfokkers en paardenliefhebbers. Translated by J. A. Alers. 8vo. Gouda, 1857.

Anleitung zur Kenntniss des Aeusseru des Pferdes fiir Thierärzte, Gestutsbeamte und Pferdebesitzer jedel Standes. By Johann Wilhelm Baumeister. 8vo. Stuttgart, $1845 ; 1852$; 1857

In Dutch as:-De tandenkennis als middel tot zekere beordeling van den ouderdom des paards. 8vo. Almelo, 1854.

Stable Talk and Table Talk, or Spectacles for Young Sportsmen. By Harry Hieover [Charles Brindley]. 8vo. London, 1845-1846.

L'abili compratore di cavalli. By Alessandro Bucellati. Milan, 1845.

Dictionnaire d'hippiatrique, et d'équitation. By François Cardini. 2 vols. 8 vo. Paris, $1845 ; 1848$.

De l'Hydroémie anhémique ou cachexie aqueuse du cheval, et de la congestion sanguine apoplectique du mouton. By Pierre Charlier. 8vo. Paris, 1845.

The Steeple Chase Calendar; a consecutive Chronicle of the Sport of Great Britain, from 1826 to 1844 . By Henry Corbet. $12 \mathrm{mo}$. 1845 .

Aide-mémoire militaire, a l'usage des officiers d'infanterie et do cavalerie. By Charles de Fouan. 12 mo. Paris, 1845.

Die Pferde-Racen. By Robert Froriep. folio. Weimar, 1845 ; $3 \mathrm{rd}$ edition, 1852 ; 4 th edition, 1857 ; another edition, folio. Leipzig, 1874. 
1845. Nogle Bemœrkninger ved. Kommende Cavallerie-Baabenet i den Norske Armee. By Berthold. H. Hagemann. 8vo. Christiana, 1845 .

Ueber einiger Seuchenkrankheiten der Hansthiere in Sibirien und in Suidlichen-europaïschen Russland, namentlich über die (auch bei Menachen vorkommende) Sibirischen Beulenseuche, etc. By Wilhelm Haupt. 8vo. Berlin, 1845.

Handbook of Horsemanship. By H. R. Herschberger. 32mo. London, 1845.

Das Orientalisch Pferd und das Privat-Gestuite S. Maj. des Königs von Württemburg, ein hippologische Monographie, etc. By Aug. Jäger. 8vo. Stuttgart, 1845.

Journal de médecine vétérinaire. Published by Rainard, in conjunction with Rey, Tisserant, Tabourni, Lecoq. 8vo. Lyons, 1845 , etc.

Traité d'hygiène Vétérinaire appliquée. By J. H. Magne. 2 vols. 8 vo. Lyons, 1845.

Another edition in 1857 with extended title: Étude de nos races d'animaux domestiques et des moyens de les améliorer, suivie des règles relatives à I'entretien, à le multiplication, $\grave{a}$ l'élevage du cheval, de l'âne, etc.

The History of the Horse. Its Origin, physical and moral characteristics, its principal varieties and domestic allies. By W. C. L. Martin; with an Appendix on the Diseases of the Horse, by W. Yonatt. $12 \mathrm{mo}$. London, 1845.

The Horse's Foot and how to keep it sonnd, with illustrations. By William J. Miles. 8vo. London, 1845.

7th edition, 1850, with an appendix on shoeing generally, and Hunters in particular. 8th edition, London, 1856.

In French :-Le Pied du Cheval et la manière de la conserver sain. 8vo. Brussels, 1862. Translated by Guyot from the 8th edition.

In German:-Der Huf des Pferdes und dessen fehlerfreie Erhaltung. Nebst einem Anhang iiber den praktischen Hufbeschlag. Transla'ed by Guitard. 8vo. Frankfurt, 1852.

The Sportsman's Library, containing Hunting, Shooting, Conrsing, and Fishing. By John Mills. 8vo. [Edinburgh], 1845. 
Considérations sur l'élève du cheval de course et du cheval de chasse, 1845. puisées dans plusiers ouvrages anglais. By the Count Achille de Montendre. 8vo. Paris, 1845.

Trattato di patologia speciale e descrittiva del cavallo. By Fran. Papa. Turin, 1845.

Manuel équestre. By C. Raabe. 8vo. Paris, $184 \check{.}$

Der wohlunterrichtete Pferdearzt oder leichtfassliche Anweisung fiir jeden Pferdebesitzer alle Krankheiten und Gebrechen seiner Pferde selbst und sicher zu heilen, theils durch natiirliche Mittel, theils durch sympathetische Kuren. Nebst einem Anhang, welcher interessante Belehrungen iber das Alter, die Zähne, die Zucht, die Nahrung und die verschiedenen Rassen der Pferde enthält. By Carl Raimund. 8vo. Leipzig, 1845; 1849.

Traité complet de la parturition des principales femelles domestiques, suivi d'un traité des maladies propres aux femelles et aux jeunes animaux. By J. Rainard. 2 vols. 8vo. Paris, 1845.

Observations sur les ravages exercés par la morve chronique et le farcin sur les chevaux du 12e chasseurs, depuis 1840 jusqu'en 1845. By Ed. Rigau. 8vo. Tarbes, 1845.

Elémens d'équitation militaire; ouvrage utile aux jeunes gens qui veulent cultiver cet art, et particulièrement à ceux qui se destinent à remplir les fonctions d'instructeurs, By Roy. Paris, [Circa 1845].

The Sportsman's Magazine of Life in London and the Country. History of Boxing, Cricket, Angling, Papers on Hunting, Sporting Characters, Anecdotes, etc. Edited by Mile's Boy. 4 to. London, 1845.

Praktischer Reitunterricht nebst Anleitung zur Wartung, Pflege, Zäumung und Sattelung des Pferdes. By Wilhelm Stroh. 8 vo. Frankfort, $1845 ; 1856$.

Grundziige für den Gebrauch der reitenden Artillerie in ihrer Verbindung mit Kavallerie. By Taubert. 1845.

De l'administration des haras, de son système et de ses doctrines: Réponse à l'écrit anonyme distribué au congrès d'agriculture en Maï, 1845. By le comte Joseph de Turenne. 8vo. Paris, 1845 .

Cours d'hippiatrique militaire. By S. Verheyen, 12mo. Brussels, 1845 . 
1845. The Veterinary Record and Transactions of the Veterinary Medical Association. Edited by Spooner, Simonds, and Morton. 8 vo. London, 1845 , etc.

1846. Katechismus über das Aeussere, die Pflege, die Gänge, das Beschlagen, die Erkennung und Behandlıng der Krankheiten, die Zucht und die Stämme des Pferdes für Männer, welche auf, bei, neben und unter Pferden leben. Anon. 12mo. Straubing, 1846; 1853.

The Horse and his Rider. By Rollo Springfield [pseud.]. $12 \mathrm{mo}$. London, $1846 ; 1847$.

Pratique de l'art du Maréchal-ferrant. By Berthelot. 8vo. Paris, 1846.

System of Foot and Equitation Drills. By William Bishop. $12 \mathrm{mo}$. London, 1846.

Manual of Modern Farriery embracing the cure of diseases, incidental to Horses, Cattle, Sheep, Swine, and Dogs ; with Instructions in racing, hunting, coursing, shooting, fishing, and field sports generally, together with a summary of the game laws. By Thomas Brown. 8vo. London, 1846 and 1848.

Cartilla de Sanidad. By Nicolas Casas de Mendoza. 8vo. Madrid, 1846.

Manual de examinandos. By Nicolas Casas de Mendoza. 8vo. Madrid, 1846.

Tratado de epizootias. By Nicolas Casas de Mendoza, 4 to. Madrid, 1846.

Turfite's Computer. By G. J. Dow. 18mo. 1846.

Traité des chevaux ardennais. By Dubroca. 8vo. Paris, 1846.

Elémens abrégés d'un cours d'équitation Militaire. By A. Dupont. 18mo. Paris, 1846.

Die Wuthkrankheit der Thiere und des Menschen, mit Beniitzung der Akten des königlich wiirtembergischen Medicinal-Collegiums. By Dr. Wilhelm Eberhard Faber. 2 vols. 8vo. Mannheim, 1846 and 1850 .

Anleitung zur Kenntniss des Pferdes nach seiner äusseren Körperform. Als Leitfaden bei seinem Vorlesungen. By Leopold Graf. 8 vo. Vienna, $1846 ; 1851$.

Anviisning til den letteste og naturligste maade at dresere Heste, etc. By Ludw. Hunersdorff. Svo. Christiania, 1846. 
Rijkunstig handboek, bijzonder ingerigt voor burger-rijscholen en 1846.

liefhebbers van paarden. By B. van Merlen. 12 mo. Amsterdam, 1846.

Recueil de médecine vétérinaire pratique. Journal consacré à la médecine et à la chirurgie vétérinaires, à l'hygiène, au commerce des animaux domestiques, et à l'analyse des ouvrages et journaux traitant de l'art vétérinaire. Edited by $\mathrm{H}$. Bouley. 8 vo. Paris, 1846 , etc.

Corso completo di nosologia e terapie speciale veterinaria. By $\mathbf{S}$. Rigoni. 8vo. Florence, vol. 1, 1846; vol. 2, 1852.

De l'équitation Militaire, de l'ancienne et de la nouvelle école. By Clément Thomas. 8vo. Paris, 1846.

Antidossa, ovvero opinone contraria sulla trasmissione del contagio moccioso e farcinoso del cavallo all' nomo. By Toggia. Turin, 1846.

Der homöopathische Dorf-Thierarzt. Ein nützliches Handbuch zunächst fiir Oeconomen und Landleute zur Behandlung und Heilung erkrankter Pferde, Rinder, Schafe, Ziegen, Schweine und Hunde. Nebst einer Einleitung zur Homöopathie iberhaupt. By Theodor Traeger. 8vo. Leipzig, $1846 ; 1853$.

Allgemeines homöopathisches Thierarzneibuch. Ein zuverlässiger Rathgeber fuir jeden Viehbesitzer, welcher die vorkommenden Krankheiten der Hausthiere sicher, schnell und wohlfeil heilen will. By F. W. Tuczek. 8vo. Leipzig, $1846 ; 1856$.

Landwirthschaftiche Thierproduktion. By August von Weckherlin. 8vo. Stuttgart, $1846 ; 1851 ; 1857$.

In French:-Zootechnie générale. Réproduction. Amélioration et élevage des animaux domestiques. With a preface by $P$. S. J. Verheyen. $18 \mathrm{mo}$. Brussels, 1857.

The Horse in health and disease. By J. W. Winter. 8vo. London 1846.

Om faarenes Rogt eller den Kloge og forstandige Faarehyrde. Anon. 8vo. Christiania, 1847.

Recueil de mémoires et observations sur l'hygiène et la médicine vétérinaire militaires, redigé sous la surveillance de la commission d'hygiène hippique, publié par ordre du ministre secrétaire d'etat au département de la guerre. $8 v o$. Paris, 1847 , etc.

Tome II. avec les documents administratifs sur les rémontes de l'armée. 8vo. Paris, 1849. 
1847 Principes sommaires de l'élevage du cheval. By Colonel Paul Basserie. $18 \mathrm{mo}$. Paris, 1847.

Atlas de Anatomia y Cirujia. By Nicolas Casas de Mendoza. folio, Madrid, 1847.

The Horse's Foot. By Thomas Clarendon. 12mo. London, 1847.

Le Vétérinaire domestique, ou l'Art de soigner et de guérir les chevanx et bêtes de somme, etc. By Gervais. 8vo. Paris, 1847.

Handbuch der Zoophysiologie der nutzbaren Haussaiigethiere, als Leitfaden zu seinen Vorlesungen. By Leopold Graf. 8vo. Vienna, $1847 ; 1851$.

Landwirthschaftlich polizeiliches Handbuch. Eine Sammlung sämmtlicher Gesetze in Betreff der Viehkrankheiten, deren Abwehr und Heilung. By W. G. von der Heyde. 8vo. Magdeburg, 1847 ; 1850 .

Der kleine Stallmeister. Thenretisch-praktische Regeln der Reitkunst, nebst allen beim Umgange mit Pferden erforderlichen Wissenschaften. By Heinrich Klemm. 12mo. Leipzig, 1847 ; 1850; 12mo. Dresden, 1855.

Historique du Haras royal du Pin et considérations sur l'amélioration des chevaux en France. By A. de Lespinats. 8vo. Paris, 1847.

Nature et éducation des chevaux achetés par les dépôts de remonte; un mot sur ces différents établissements. By A. Louchard. Svo. Paris, 1847.

The Law concerning Horses, Racing, Wagers, and Gaming, with an Appendix containing recent cases, statutes, etc. By George Henry Hewitt Oliphant. 12mo. London, 1847 ; $12 \mathrm{mo}$. London, 1854 ; 1864 ; 8vo. London, 1865.

De la Conformation du Cheval, suivant les lois de la plysiologie et de la méchanique. Haras, Courses, Types, Reproducteurs, amélioration des Races, Vices redhibitoires. By A. Richard du Cautal. 8vo. Paris, 1847.

Another edition-Etude de la conformation du cheval, suivant les principes élémentaires des sciences naturelles et de la méchanique animale, 12mo. Paris, 1869.

Guide de l'acheteur de chevaux. By Captain Rivet. 12mo. Paris, 1847 . 
Allgemeines Gestüt-Buch. Ein Verzeichniss der Vollblut-Pferde nebst 1847.

ihrer Abstammung welche sich in allen deutschen Staaten, sowie den nicht zum deutschen Bunde gehörigen Landestheilerı der preussichen und dänischen Monarchie, desgleichen in Ungarn und seinen Nebenländern, in Siebengebiirgen und Galizien befindlichen. By Carl Heinrich Vogler. 8vo. Berlin, 1847 ; $18 \overline{2} 2$

Annuaire de la Sociéte nationale et centrale de médecine vétérinaire. 1848. 8vo. Paris, 1848.

Calendrier officiel des courses de chevanx 1847. Publié sous les auspices de la Société d'encouragement pour l'amélioration des Races de Chevaux en France, d'après les documents fournis par la dite Société et par l'Administration des haras. Par le Secrétaire de la Société d'encouragement (Jockey Club) Un Chapitre est spécialement consacrée aux Courses de Belgique et d'Angleterre. $12 \mathrm{mo}$. Paris, 1848.

Das Hausthier, in Hinsicht seiner Pflege und Benützung mit Angabe der zweckmässigsten Heilmittel bei den Krankheiten desselben, nebst einer Technologie, insofern sie sich auf landwirthschaftliche Gegenstände bezieht. Anon. 8vo. Breslau, 1848.

Den palitliga Djurläkaren. Anon. 8vo. Hamburg, 1848.

Der vollkommene Pferdearzt, oder Anweisung, wie der Landmann seine Pferde füttern, deren Krankheiten erkennen und heilen soll. Nebst einer Sammlung von Recepten. Herausgegeben von einem Schweizer. Anon. 8vo. Augsburg, 1848.

Exerceerreglement for Cavalleriet. Anon. 8vo. Christiana, 1848.

Histoire Critique des exploits et vicissitudes de la Cavalerie pendant les guerres de la Revolution et de l'Empire, jusqu'à l'armistice du 4 juin, 1813. Anon. 2 vols. 8vo. Paris, 1848.

Kurze Anleitung zur Aufucht und Verbesserung der Pferde. Ein Handbuch für den Landmann. Anon. 8vo. Münster, 1848.

Quelques mots sur l'administration des haras. Anon. 8vo. Paris, 1848.

Société d'agriculture de Morlaix. Question des haras. Pétition adressée à M. M. les représentants du Finistère à l'assemblée nationale. 4 to. Morlaix, 1848.

Vorschriften für die Zusammensetzung und Einübung der Bespannungen bei der Königl. Preussischen Artillerie. 8vo. Berlin, 1848. 
1848. Traitement homœopathique des Animaux. By Alexandre. 8vo. Paris, 1848.

History and Description of the Horse, Mule, Cattle, Sheep. etc., with directions for their Management, etc. By R. L. Allen. 8vc. New York, $1848 ; 1852$.

Des tares osseuses dans le cheval. By Louis Auzoux. 8vo. Paris, 1848.

Gemeinfassliches Handbuch der gesammten Thierheilkunde ir alphabetischen Ordnung, enthaltend die Beschreibung der Krankheiten des Pferdes, Rindes, Schafes, Schweines, Hundes u. s. w., ihre Pflegung und Haltung, nebst genauer Angabe der Arzneimittel, etc., für Thierärzte und zum selbstgebrauch für Landwirthe, etc. By Johann Wilhelm Baumeister and F. M. Duttenhofer. 8vo. Stuttgart, 1848.

The Pocket and the Stud, or practical hints on the management of the Stable. By Harry Hieover [Charles Brindley]. 8vo. London, 1848 ; 3rd edition, 1857.

Précis du squelette pour l'hippiatrique. By V. Brivet. A folio sheet. Paris, 1848.

Anatomie des Pferdes. In Beziehung auf Knochenbau, Muskulator, und Bewegung. In 20 litho-graphirten Platten, nebst Erläuterrungstabelle nach Bourgelat's Anatomie. By Brunot. folio. Carlsruhe, 1848.

Bulletin de la Société nationale et centrale de médecine vétérinaire, publié par les soins de son bureau. 8vo. Paris, 1848, etc.

Jahresbericht über die Fortschritte der Thierheilkunde im Jahre 1847. By Dr. Canstatt and Dr. Eisenmann. 4to. Erlangen, 1848, etc.

Central Archiv für die gesammte Veterinär-Medicin und fiir die veterinär-ärztlichen Unterrichts, Standes-und Vereins-Angelegenheiten. Unter Mitwirkung von mehreren Thierärzten. Edited by Johann Martin Kreutzer. 8vo. Augsburg, 1848, etc.

Notions sur le dressage des jeunes chevaux au trait et à la selle. By L. D. Cocher [Louis Baron de Curnieu]. 8vo. Paris, 1848.

Praktische Anweisung zur Bereitung des Rossfleisches. By Henriette Davidis. 8vo. Elberfeld and Iserlohn, 1848. 
Art: 'Chevaux.' By O. Delafond. 8vo. Paris, 1848. In Vol. II. 1848. pp. 2273, 2304 of Instruction pour le Peuple, Cent Traités, etc. In Spanish :- 'Caballos.' 8vo. Madrid, 1859. Translated by Fr. de Laiglesia y Darrae, in Vol. II. of Instruccion para el Paeblo.

Handbuch der gesammten Hausthierzucht für Landwirthe. By J. F. C. Dieterichs. 8vo. Leipzig, 1848.

Die veterinär-chirurgische Instrumenten-Verband-und Operationslehre. By Johann Ernst Ludwig Falke. 8vo. Leipzig, 1848.

Lehrbuch iiber den Hufbeschlag und die Hufkrankheiten. By Johann Ernst Ludwig Falke. 8vo. Leipzig, 1848.

Handbuch zum Betriebe der Viehzucht oder kurzgefasste Anleitung einer sachgemässen Zucht, Futterung, Wartung und Pflege unserer Landwirthschaftlichen Hausthiere nebst der Angabe, wie die bei denselben vorkommenden Krankheiten von jeden Viehbesitzer leicht erkannt, und auf wohlfeile Weise griindlich und schnell geheilt worden können zum allgemeinen Gebrauch fiir Landwirthe und Viehbesitzer. By C. A. Fritsch. 8vo, Altenburg, 1848.

La France Chevaline. 1st part: Institutions hippiques. 2nd part: Etndes hippologiques. By Eugène Gayot. 8 vols. 8vo. Paris, 1848-1852.

In German:-Die Zucht des Arabischen und Englischen Vollbluts in Frankreich und die Anglo Arabische VollblutFamilie. Translated by Carl Graefe. 8vo. MIagdeburg, 1854 .

Standing Orders for the 7th Dragoon Guards. By LieutenantColonel Hope Gibson. 8vo. London, 1848.

Higiene Veterinaria. By Jose Maria Giles. Svo. Ecija, 1848.

Die Muskellehre des Pferdes in 13 Blättern. By Leopold Graf. folio. Vienna, 1848.

Der geschickte Grob-und Hufschmied, oder vollständige Anweisung zur Verfertigung aller Arten regulärer und irregulärer Hufbeschläge der verschiedenen Wagen und Chaisenbeschlage, Radreifen, Kutschfedern, Ketten, Nägel und Ackergeräthschaften, sowie der an meisten vorkommenden Bau-ınd-Eisenbahnarbeiten, so weit sie der Schmied fertigt. Nebst einem Anhange uiber das Schmieden der Aexte, Beile, Ackerwerkzeuge, und andere gröbere Schmiedewerkzenge, sowie einem Wörterbuch des Schmiedes. By Friedrich Harzer [pseud. for Dr. Hartmann]. 8 vo. Weimar, 1848-1852. 
1848. Repertorium der Thierheilkunde nach homöopathischen Grundsätzen, enthaltend alle bis jetzt bekannten homôopathischen Krankheitsheilungen und Beobachtungen an Hunden, Katzen, Pferden, Rindern, etc. Ein Hiilfsbuch fur jeden Viehbesitzer. By J. M. Hellmund. 16mo. Gotha, 1848 .

Pferdefleisch und das Pferdefleisch-essen. By H. W. Henzte. 8vo. Elberfeld and Iserlohn, 1848.

Ueber die Verwendung der thierischen Ueberreste unserer Hausthiere das Pferdefleischessen und die Aufhebung der Kleemeistereien. Eine Abhandlung für Staatsbeamte, Amtsversammlungen, Landwirthe, Landwirthschaftliche Vereine, Thierbesitzer und Thierärzte, namentlich Württembergs. By C. J. F. Hoehning. 8vo. Stuttgart, 1848.

Histoire du cheval chez tous les peuples de la terre. By E. Hoüel. 2 vols. 8 vo. Paris, 1848-1852.

Bijdrage tot de Kennis en de Behandling von de Ziekte der Hoeven bij het Paard, bekend onder den Naam van Straal-of Hoefkanker. By Auguste Jacques Janné. 8vo. Amersfoort, 1848.

Die Aufstallung, Fiitterung, Wartung und Behandlung der Hausthiere. By Christian Friedrich John. 8vo. Zerbst, 1848.

Travellers' Oracle, or Maxims for Locomotion. By Dr. William Kitchener. 12mo. London, 1828.

Belehrungen iiber die für Menschen und Thiere höchst gefährlichen Folgen der Ansteckung durch die ansteckenden Krankheiten der Hausthiere nebst Angabe der geeigneten Mittel und Wege zur Vermeidung derselben besonclers den Viehbesitzern und Ortsvorstehern gewidmet. By F. X. Koerber. 8vo. Merseburg, 1848.

Der Reiter und sein Pferde. Ein Kavalleristisches Fragment. By Carl Kress von Kressenstein. 8vo. Vienna, 1848.

La Clinique Vétérinaire. By Urbain Leblanc. 8vo. Paris, 1848.

Hästens fot och bästa sättet att varda densamma med synnerligt afseende pa Skoningen. By Mathias Leyon. 8vo. Stockholm, 1848.

Magazin für die neuesten Beobachtungen und Erfahrungen im Gebiete der homoopathischen Thierheilkunde. Edited by Friedrich August Guinther. 8vo. Sonderhausen, 1848, etc.

Magazin voor vee-artsenijkunde, vorgelijkunde geneeskunde, en verteelt. Edited by Dr. Alexander Numan. 8vo. Utrecht, 1848, etc. 
Der wohlerfahrene thierärztliche Rathgeber oder die besten und 1848. zuverlässigsten Mittel gegen die Krankheiten der Pferde, des Rindviehes, der Schafe, Schweine, Ziegen, Hunde, des Federviehes und der Stubenvögel. By George Marquard. 8vo. Eisenberg, 1848; $1848 ; 1851$.

Zur Morphologie der Harn-und Geschlechtswerkzeuge der Wirbelthiere in ihrer normalen und anormalen Entwickelung. By Johann Heinrich Meckel von Hemsbach. 8vo. Berlin, 1848.

Die rationelle Pferdezucht. Practische Leitfaden für die Zucht des Pferdes, insbesondere des grossen, starken, sogenannten englischen Halbblutpferdes. By Otto Albrecht Pauli. 8vo. Marienwerder, 1848.

Nouveau système d'amélioration des animaux domestiques. By $\mathbf{A}$. Pétéaux. 8vo. Paris, 1848.

Résumé de la nouvelle école d'équitation. By Charles Neuber Raabe. folio. Paris, 1848; and three other editions the same year.

Sur les moyens de perfectionner le cheval de guerre. Mémoire lu à l'académie des Sciences de Paris en 1848. By A. Richard. 8vo. Paris, 1848.

Horses, their Varieties, Breeding, and Management. By H. D. Richardson. 12 mo. Dublin, $1848 ; 1849 ; 12 \mathrm{mo}$. New York, 1852.

Die Wuthkrankheit bei den Haussäugethieren in ihrer pathologischtherapeutischen und polizeilich-socialen Beziehung. Ein meistens auf eigene Erfahrungen begrindeter, fur Aerzte und Nichtärzte bearbeiteter Beitrag zur Monographie dieses Uebels. By Wilhelm Rosenbaum. 8vo. Zerbst, 1848.

Bibliotheca medico-chirurgica pharmaceuto-chemica et veterinaria. Oder geordnete Uebersicht aller in Deutschland neu erschienen medicinisch-chirurgisch-geburtshiilflichen pharmaceutisch-chemischen und veterinär-wissen-schaftlichen Biicher. By Carl Johann Friedrich Wilhelm Ruprecht. 8vo. Gottingen, 1848.

Atlas bevattende de Anatomie des Paards in 34 steendrukplaten. By Toers Dierbergen Schubart. folio. Utrecht, 1848.

Praktischer Rathgeber für die Stalldienerschaft, Anweisungen zur Wartung und Ausbildung des Pferdes. By Louis Seeger 8vo. Berlin, 1848. 
1848. Указаніе средствъ къ пстпнномп и вьісшему познанію лошадеи. By Nicolai Telepnew. 12mo. St. Petersburg, 1848.

Loi du 20 Mai 1838 sur les vices et actions rédhibitoires, suivies d'un traité de clinique vétérinaire à l'usage des éléveurs et propriétaires de bestiaux. By Felix Thébaud. 32mo. Laval, 1848.

Analisi critica ragionata del rendiconto delle sperienze tentate sopra cavalli della regia truppa, per la cura del moccio del dottor Luigi Colomba. By Francesco Toggia. 8vo. Turin, 1848.

The Farmer's Directory, Agricultural Economy, Complete Farrier, Diseases of Horses, Cattle, Sheep, Swine, etc., also domestic management. By L. Towne. 4 to. London, [Circa 1848].

Renn-Kalender fiir Deutschland nebst mehreren Registern. By Carl Heinrich Vogler. 8vo. Berlin, 1848, etc.

Die Rotzkrankheit der Pferde heilbar. Veröffentlichung des neuesten Mittels zur Heilung des Rotzes und der Maassregeln, um gesunde Pferde vor Ansteckung zu sichern. By J. E. Wagenfeldt. 16mo. Bautzen, 1848.

Bemerkninger af F. W. Weinschenk ved Hr. Kammerherm, Landstutmester v. Bardenfleth's Erklaering til Hs. Maj. Kongen over de af Landmaendenes Forsamlinger Allerhöistsamme tilstillede Committee-belaekninger om Landets Heste-og Quaegavl. By F. W. Weinschenk. 8vo. Aalborg, 1848.

1849. Commission hippique de l'arrondissement de Douai. Questions à soumettre à la commission générale de la circonscription du depôt d'étalons d'Abbeville. Anon. 8vo. Paris, 1849.

Extract from the 'Moniteur' Agricole.'

Cours d'équitation militaire, à l'usage des corps de troupes à cheval, approuvée par son Excellence le ministre de la guerre. 8vo. Saumur, 1849.

Die Influenza der Pferde. Bericht iiber die Erfahrungen welche in Sïddeutschland hinsichtlich dieser Krankheit gemacht worden sind. Anon. 12mo. Carlsruhe, 1849.

An extract from the August number of the Thierarztlichen Zeitung.

Handbock för Ryttaren. Anon. 8vo. Malmö, 1849.

Industrial Library. No. 6, The Groom. Anon. 12mo. London, 1849. 
Instruction à l'usage des troupes à cheval. Ecole de voltige et du 1849. cavalier. Anon. 18mo. Brussels, 1849.

Ordonnance sur l'éxercise et les évolutions de la cavalerie, du 6 decembre 1829. Ecole du cavalier à cheval, Dragons. $32 \mathrm{mo}$. Paris, 1849.

Reglamento y ordenanza de S. M. para el ejercicio, evoluciones y maniobras de la caballeria, y dragones montados de sus ejercitos, y otros puntos relativos al servicio de estos cuerpos. Anon. 4to. Madrid, $1849 ; 1850$.

Рыспсты календаръ пा свьденія объ пспытаніяхъ лошадеп на рнспстыхь ппподрожахъ Въ С. Петербурів и Парскосе.льскпхъ рыспстыхъ въговъ. Санктпетероургъ Въ типорафін III отдАленпя собственнол его пмператорскаго велпчества канцеляріп. Anon. 8vo. St. Petersburg, $1849 ; 1850$.

Sympathiebüchlein oder die enthïlten naturlichen Zauberkräfte und Geheimnisse der Natur. Eine Sammlung von 338 erprobten sympathetischen Mitteln gegen viele Krankheiten bei den Menschen und Thieren. Von einem alter Schäfer. Anon. 8vo. Zurich, 1849.

Underretwing för (Dragon) Exerceerskolerne. Anon. Nestved, 1849.

The Australian Sportsman. Sydney, 1849, etc.

Ziekten en genezing der Paarden, Koeijen, Schapen, Zwynen, Honden, en andere Huisdieren. 'Ten gebruike der landslieden. By J. Bal. 8vo. Gent, 1849.

Die thierärztliche Geburtshülfe. Ein Handbuch für Thierärzte, Gestiitsbeamte und Landwirthe, sowie zum Gebrauche bei Vorlesungen, und zum Selbstunterricht. By Johann Wilhelm Baumeister. 8vo. Stuttgart, $1849 ; 1853$.

Haltung, Pflege und Behandlung der landwirthschaftlichen Haussäugethiere im gesunden und kranken Zustande. Zur Selbstbelehrung für Landwirthe, Viehbesitzer und zum Unterricht an landwirthschaftlichen Lehranstalten. By Carl Boehm. 8vo. Reutlingen and Leipzig, 1849.

The Stud for Practical Purposes and Practical Men. By Harry Hieover [Charles Brindley]. 8vo. London, 1849.

Traité des maladies des animaux domestiques avec des renseignements pour en connaitre l'âge et les vices rédhibitoires. By Brout. 8vo. Honfleur, 1849. 
1849. Cours élémentaire d'hippiatrique et de maréchalerie, a l'usage de la gendarmerie. By P. C. M. Cochet de Savigny. $18 \mathrm{mo}$. Paris, 1849.

The American Veterinarian: containing Diseases of Domestic Animals, their Causes, Symptoms, and Remedies; with Rules for Restoring and Preserving Health by good management; also for Training and Breeding. By S. W. Cole. 8vo. New York, [1849].

Nadere Mededeeling over de worming der Vagina en Urethra bij de vrauwelijke en die der Vesicula Seminales en Prostata bij de mannelijke Zoogdieren. By J. van Deen. 8vo. Gröningen, 1849.

Mémoire sur les maladies des cavités nasales des solipèdes que l'on confond souvent avec la morve, considérés principalement au point de vue pratique. By L. V. Delwart. 8vo. Brussels, 1849.

Traité de médecine vétérinaire pratique. By L. V. Delwart. 3 vols. 8 vo. Brussels, 1849-1853.

In Spanish:-Diccionario de Medicina veterinaria practica. Translated by Nicolas Casas de Mendoza. 4to. Madrid, 1850; 1854.

Programme d'un carrousel militaire servant de complément à l'instruction des recrues. [By Captain D'Elbee]. 4to. Versailles, 1849.

Remarques sur plusiers cas de pathologie observés chez les animaux. By E. Desmarest. 8vo. Paris, 1849.

Eine auf lange Erfahrung gegrundeter guter Rath an Kurhessens Pferdeziichter um grosse und gut ausgebildete Pferde zu erziehen. By Georg Ernst Adolph Wilhelm von Eschstruth. 8vo. Cassel, 1849.

Die Hippologie. By Johann Ernst Ludwig Falke. 8vo. Leipzig, 1849.

Propädentik oder encyclopädische Uebersicht der Thierheilkunde nebst ihrer neuern bessern Literatur. By Johann Ernst Ludwig Falke. 8vo. Leipzig, 1849.

Traité de l'élève du cheval dans le département de Lot-et-Garonne. By J. B. A. Goux. 8vo. Agen, 1849. 
Manuel de l'éleveur de chevaux, ou Exposition simple des principe, 1849. les plus rationnels d'élevage. By Goux. 8vo. Agen, 1849.

Bericht iiber eine Bereisung der vorziiglichsten ungarischen Gestiite Schäfereien und landwirthschaftlichen Anstalten, in der Monateu Juli und August, 1848. By Leopold Graf. 8vo. Vienna, 1849.

Nachträge zum ersten Theile des Lehrbuches der pathologischen Anatomie der Haus-Säugethiere. By Ernst Friedrich Gurlt. 8vo. Berlin, 1849.

Le bouvier modèle, traitant des soins à donner aux chevaux, à l'étables à la bergerie, à la porcherie, à la basse cour, etc. By Edouard Hocquard. 18mo. Paris, 1849.

Illustrative Farriery : A series of Lithographs of the different Varieties of Horses' Feet, the states Unshod and Shod by different Methods; with explanations of practical utility. By F. R. Hodgson. 4to. London, 1849.

Lettre d'un paysan à plusieurs autres, à propos de chevaux. [By E. Hubert]. 12mo. Paris, 1849.

Mittheilungen aus dem Veterinairfache. By Peter Jensen. 8vo. Dorpat, 1849.

Denkschrift iiber die Pferdezucht im Preussischen Staate. [By A. v. Saucken Jullienfelde]. 8vo. Berlin, 1849.

Le véritable et parfait bouvier moderne, contenant: 1. Part, de connaître, éléver, etc., tous les animaux domestiques; 2 . un traité sur les étangs et viviers; 3 . une instruction sur la manière de détruire les animaux nuisibles; 4. la legislation rurale; 5 . observations et découvertes nouvelles, etc. Ouvrage entièrement neuf, rédigé sur les manuscrits de Kerpelani. 12 mo. Paris, 1849.

Epizooties. Recueil des lois, réglements, mesures et moyens prescrits par l'autorité administrative, depuis 1713 , pour prévenir et arrêter les maladies contagieuses. Vices Redhibitoires. Extrait du code civil et loi du $20 \mathrm{Mai}, 1838$, suivis de modèles d'actes. By J. B. F. Larendu. 8vo. Paris, 1849.

Handledning i hästafveln. By Mathias Leyon. 8vo. Oerebro, 1849.

Handledning i Ridkonsten för Herrar och Damer, samt uti Konsten att köra två och fyra Hästar, förnämligast till selfundervisnig. By Mathias Leyon. 8vo. Malmö, 1849. 
1849. Zur Selbstlehrung fuir Reiter, sowohl von der Bürgerwehr-Cavalerie, als auch für angehende Reit-liebhaber. enthaltend die Commando's und Exercitien, Regeln und Vorschriften über den Sitz, die Zäumung. die Fuihrung, die Huilfen, das Satteln und die richtige Behandlung des Pferdes und seiner innern und aussern Krankheiten. By August Lotze. 12mo. Weimar, 1849.

Compendio de fisiologia umana $\mathrm{i}$ veterinaria. By Alessandro de Martini. 8vo. Naples, 1849.

The Horse's Mouth, showing the Age by the Teeth, containing a full description of the periods when the teeth are cut, the appearances they present, the tricks to which they are exposed, the eccentricities to which they are liable, and the diseases to which they are subject. By Edward Mayhew. 8vo. London, [1849; $1865]$.

Handleiding tot het gewennen van paarden aan de wapens, de schijf en het vuren. By B. van Merlen. 8vo. Amsterdam, 1849.

Er clen ondartende Lungesyge hos Quaeget, Snive og Springorm hos Hesten i Sjælland skjenket tilborlig Opmaerksamhed? By Jens Weibel Neergard. 8vo. Copenhagen, 1849.

Et Par Ord om den Kgl. Veterinairskole. By J. W. Neergard. 8vo. Copenhagen, 1849.

Einiges aus dem Gebiete der Reitkunst und Pferdekenntniss. Besonders berechnet für die Bediurfnisse und Vorkenntnisse der berittenen Infanterie-Offiziere. By Börries Christian von Oeynhausen. 8vo. Salzburg, 1849.

The Steeple Chase Calender and Hurdle Race Epitome for the season 1848-1849. By Joseph Osborne. 12mo. London, 1849.

De la race chevaline. By Eugène Perrault. 8vo. Rochefort, 1849.

Dictionnaire général de médicine et de chirurgie vétérinaire et des sciences qui s'y rattachent, etc. By Rey, Lecoq, Tisserant, and Tabourin. 8vo. Lyons, Paris, Labe, 1849 ; 1850.

Répertoire de médecine vétérinaire. 8vo. Brussels, 1849, etc.

Rapport détaillé, sur les moyens de régénérer les chevaux de guerre en France. Rapport adressée à l'Assemblée nationelle en 1849, au nom des comités de l'agriculture et de la guerre réunis. By $\mathrm{A}$. Richard. 8vo. [Paris, 1849]. 
Simple rectification d'une méprise de M. de Dampierre représentant 1849. des Landes, au sujet du rapport présenté au nom des comités de l'agriculture et de la guerre, à l'assemblée nationale, le 23 Mars, 1849, sur les haras et les remontes. By A. Richard (du Cantal). 8vo. Paris, 1849.

Notice sur un nouveau mode de collier, accompagnée de quelques considérations sur le trait et ses divers effets. By Jean Marie Antoine Marguerite Camille de Rochefort. 8vo. Paris, 1849.

From the "Spectuteur Militaire" (Cahier de Juillet, 1849).

Taschenbuch der Veterinair-Semiotik oder Anleitung zur Untersuchung und Bestimmung der Krankheiten der wichtigsten Hausthiere. By Johann Jacob Rychner. 16mo. Berne, 1849.

Observations sur la pousse de chevaux et l'emphysème de tous les animaux, et sur les moyens de les éviter. By Alexandre Sellier. 8vo. Rouen, 1849.

Observations on the Expansion of the Foot of the Horse. By Austin C. Shaw. 8vo. Dublin, 1849.

Méditations hippologiques. [By Ch. de Sourdeval]. 8vo. Paris, 1849.

An extract from Le Journal des Haras.

Manuel du Cavalier. By Orfaure de Tantaloup. 18mo. Paris, [Circa 1849].

Thierärztliches Wochen-Blatt. Organ des Vereins deutscher Thierärzte. Edited by Georg Nicklas. 4to. Ulm, 1849, etc.

De l'élevage du cheval, des courses et de l'amélioration des races chevalines en France. By Baron Charles Eugène de Cadier de Veauce. 8vo. Paris, 1849.

De Vrucht van den Mensch en van de Zoogdieren afgebeeld en beschreven en haren regelmattige en onregelmattige Ontwikkeling. By Willem Vrolik. folio. Amsterdam, 1849.

In Latin:- Tabulae ad illustrandam embryogenesin hominis et mammalium tam naturalem quam abnormem. Folio. Amsterdam, 1849.

Испытанія лошаден на Парскосельзкомь рнспстомь ппподрог 1850. въ 1850 году. Anon. 8vo. St. Petersburg, 1850, etc. 
1850. Anweisung zum Satteln und Packen bei der Preussischen Kavallerie. Von einem Preuss. Kavallerie Offizier. Anon. 12mo. Düsseldorf, 1850.

Courses de chevaux en France. Anon. folio. Paris, 1850.

Die äusseren Mängel und Gebrechen des Pferdes. Ein Taschenbuch fiir Pferdekäufer und Pferdeliebhaber mit einer Abbildung in Stahlstich nach einer Zeichnung des Professor Baumeister. Anon. 32mo. Stuttgart, 1850.

Directions for Treatment of Diseases of Horses, Cattle, Sheep, and Dogs. By a gentleman of many years experience and a master of Hounds. Anon. 12mo. Shrewsbury, 1850.

Guide de l'instructeur pour la méthode d'application de l'école du cavalicr et du peloton à pièd et à cheval, d'après la progression suivic à l'école de cavalerie à Saumur. Anon. 32mo. Paris, 1850 .

Manuel des Courses de Chevaux. Chevaux, Entraineurs, Jockeys, Parieurs, Hippodromes, Argot du Turf. Suivi de la Jurisprudence du Turf. Anon. [Circa 1850].

Loi du 28 Janvier 1850 sur les vices rédhibitoires. Expliquée et mise à la portée de tout le monde. By J. P. A. 8vo. Liege, 1850.

Historical Records of the Life Guards. 8vo. London, [Circa 1850].

Historical Records of the Royal Horse Guards. 8vo. London, [Cirea 1850].

Historical Records of the First (or King's) Dragoon Guards. 8vo. London, [Circa 1850].

Historical Records of the Second Dragoon Guards or Queen's Bays. 8vo. London, [Circa 1850].

Historical Records of the Third Dragoon Guards, Prince of Wales's. 8vo. London, [Circa 1850].

Historical Records of the Fourth (Royal Irish) Dragoon Guards. 8vo. London, [Circa 1850].

Historical Records of the Fifth (or Princess Charlotte of Wales') Dragoon Guards. 8vo. London, [Circa 1850].

Historical Records of the Sixth Dragoon Guards, Carabineers. 8vo. Lonilon, [Circa 1850]. 
Historical Records of the Seventh (or Princess Royal's) Dragoon 1850. Guards. 8vo. London, [Circa 1850].

Historical Records of the First (or Royal) Dragoons. 8vo. London, [Circa 1850].

Historical Records of the Second (or Scots Greys') Dragoons. 8vo. London, [Circa 1850].

Historical Records of the Fourth (Queen's Own) Dragoons. 8vo. London, [Circa 1850].

Historical Record of the Sixth (Inniskillings') Dragoons. Svo. London, [Circa 1850].

Historical Records of the Seventh (or Queen's Own Hussars). 8vo. London, [Circa 1850].

Historical Records of the Eighth (or King's Royal Irish) Hussars. 8vo. London, [Cirea 1850].

Historical Records of the Ninth (or Queen's Royal) Lancers. 8vo. London, [Circa 1850].

Historical Records of the Tenth (or Prince of Wales' Own) Hussars. 8vo. London, [Circa 1850].

Historical Records of the Eleventh (or Prince Albert's Own) Hussars. 8 vo. London, [Circa 1850].

Historical Records of the Twelfth (or Prince of Wales' Royal) Lancers. 8vo. London, [Circa 1850].

Historical Records of the Thirteenth Light Dragoons. 8vo. London, [Circa 1850].

Historical Records of the Fifteenth Hussars. 8vo. London, [Cirea $1850]$.

Historical Records of the Sixteenth (or Queen's) Lancers. 8vo. London, [Circa 1850].

Historical Record of the Seventeenth Lancers. 8vo. London, [Circa $1850]$.

Historical Records of the Cape Mounted Rifles. 8vo. London, [Circa $1850]$.

Historical Records of the Staffordshire Yeomanry Cavalry. [Circa 1850].

Ministère de l'agriculture et du commerce, Session de 1850. Question des Haras. 4to. Paris, 1850. 
1850. Traité de la réception des effets de harnachement pour les corps d'artillerie. Anon. 8vo. Paris, 1850.

The Sportsman's Pilot, Sporting Anecdotes, Record of Races, etc. - Edited by the 'Iron Mask.' [pseud.] 12mo. London, 1850.

Vierzig untriigliche und erprobte Mittel und Geheimnisse, die Krankheiten des Rotzes, der Druse, des Spates, der Verwundungen und viele andere wichtige Krankheiten an den Pferden leicht und sicher zu heilen. Ferner den Pferden das Köken abzugewöhnen, Unbändige leicht zı zähmen, das Durchgehen derselben zu verhindern, sie zum Ziehen zu bringen und ohne alle Kosten gesund, fett und die Haut glänzend zu machen Für Rossärzte, Kurschmeide, Oeconomen und jeden Pferdebesitzer. Anon. $12 \mathrm{mo}$. Ronneburg, [1850].

A Treatise on Brigade Movements of Cavalry. By Lieut.-Col. Angelo. Calcutta, 1850.

Icones anatomicae vulgarium Danicorum mammalium domesticorum Fasciculus osteologicus. By Henrik Bendz. folio. Hafniae, 1850.

Statistique de l'Etat Sanitaire et de la mortalité du cheval de Cavalerie. By J. Ch. M. Boudin. 8vo. Paris, 1850. Originallyappeared in the 'Spectateur Militaire,' October, 1849.

Treatise on the diseases of Horses, etc. By R. Bowers. 8vo. [Circa 1850 ].

Diseases of the Horse. By J. A. Briddon. 12mo. London, 1850.

Practical Horsemanship. By Harry Hieover [Charles Brindley]. 8vo. London, $1850 ; 1856$.

Manuel du maréchal ferrant. By A. J. Brogniez. 8vo. Brussels, 1850.

In Spanish:-Brogniez Cirujia veterinaria, compendidda, arreglada y adicionada con una Zoologia veterinaria aplicada a la Zootechnia, el Arte de herrar en frio y a fuego, y nociones sobre el forjado, etc. Translated by Nicolas Casas. 8vo. Madrid, 1855.

In German :-Der fertige Hufschmied oder Kunst des Huf. beschlags. Nach der Anleitung von M. Brogniez, Ritter des belg. Leop. Ordens und Professor au der Staatsveterinärschule. Ein Hand-und Hülfsbuch fïr Hüfschmeide, sowie für Landwirthe, Kavallerie Officiere und Pferdebesitzer im Allgemeinen. 8vo. Leipzig, 1856.

In Dutch :-Handboek voor den hoefsmid. 8vo. Brussels, 1851. 
Kleine Beiträge zur Anatomie der Haussäugethiere. By Carl 1850. Bernhard Bruehl. folio. Vienna, 1850.

Over de koopvernietigende gebreken en de waerborging in het verkoppen en verwisseln van huisdieren, volgens de grondregels van het burgerlyk wetbock, en de wyzigende wet van 28 January 1850. By Edward Campens. 8vo. Gent, 1850.

In French :-Des vices rédhibitoires et de la garantie dans les ventes et échanges d'animaux domestiques, d'après les principes du Code civil et la loi modificative du 28 Janvier, 1850. 8vo. Gent, 1850.

Ten gebruike van het landbouw-onderwys. Opbrenging en bestiering der nuttige huisdieren, volgens de wetten van de Levensleer, Werktuigskunst et Gezondheitsleer. By Edward Campens. 8vo. Gent, 1850.

In French :-Principes fondamentaux de la production chevaline, et du perfectionnement des races, professé par M. Richard, directeur de l'école des haras. 8vo. Gent, 1850.

Terapeutica, y materia medica. By Nicolas Casas de Mendoza. 8 vo. Madrid, 1850.

De la comparaison de l'estomac et des intestins dans nos espèces domestiques. By Gabriel Colin. 8vo. Paris, 1850.

Del buon governo dei cavalli. By Carlo Comparini. 8vo. Prato, 1850 .

The Advocate of Veterinary Reform, and outlines of Anatomy and Physiology of the Horse ... containing also a Veterinary Dictionary selected from the works of R. (F.) White of London, etc. By George H. Dadd. 8vo. Boston, 1850.

Pathologie spéciale, ou Descriptive des Principaux animaux domestiques, Parturition de principales Femelles domestiques. By L. V. Delwart. [1850].

De la domesticité chez les animaux et de la souche des animaux domestiques. By Dr. Deschamps. 8vo. Paris, 1850.

L'écurie et le cheval, fantaisie équestre par un palefrenier philosophe. By Jean Saint Rieul Dupouy. 32mo. Bordeaux, 1850.

Sur l'élève des chevaux en Belgique. By Pierre Douterluigne. 8vo. Brussels, 1850.

Compendium der Veterinär-Jurisprudenz. By Johann Ernst Ludwig Falke. 8vo. Brunswick, 1850. 
1850. Lehrbuch der Gesundheitspflege der landwirthschaftlichen Hausthiere. By Johann Ernst Ludwig Falke. 8vo. Leipzig, 1850.

Propädeutik und encyclopädische Uebersicht der Thierheilkunde nebst ihre neuern bessern Literatur. By Johann Ernst Ludwig Falke. 8vo. Leipzig, 1850.

Considérations sur l'amélioration des races d'animaux domestiques dans le grand-duché de Luxembourg. [By Eugène Fischer]. 8vo. Diekirch, [Circa 1850].

Wegweiser in die Thierheilkunst. By Christopher Joseph Fuchs. 8vo. Berlin, 1850.

Administration des haras. Atlas statistique de la production des chevaux en France. By Eugène Gayot. folio. Paris, 1850.

Cria Caballar en nuestras provincias del mediodia. By Jose Maria Giles. 4to. Ecijia. 1850.

Theorie und Praxis der Hufbeschlagskunst. Eine Anleitung zum Beschlag, zur Erkenntniss, Beurtheilung und Behandlung gesunde und kranke Hufe der Pferde, etc. By Johann Christoph. Gross. 8vo. Stuttgart, 1850.

Nosologie vétérinaire pratique. Onvrage utile à tontes personnes chargées du soin des chevaux, des bestiaux et des bêtes à laine. By Constant Guesdon. 12mo. Paris, [Circa 1850]; 6th edition, 1856.

Physiologie de toutes les races de chevaux du monde. By HavezMontlaville. 8vo. Paris, 1850.

The Gentleman's Stable Manual, or a Treatise on the construction of the Stable; also on the feeding and grooming of Horses, on the hygienic treatment of the sick horse, on shoeing, on the management of the hunter, and on Equine diseases and accidents, with the most scientific modes of treatment. By William Haycock. 8 vo. London, $1850 ; 1859$.

Amélioration de la race chevaline, Rapport fait au congrès des comices réunis du département de la Somme, Session de 1850. By E. Hecquet d'Orval. 8vo. Abbeville, 1850.

Denkschrift über die Beförderung der Landespferdezucht im preussischen Staate. By Friederich Rudolph Heine. 8vo. Zörbig, 1850. 
Das Zugpferd, dessell Eigenschaften, Behandlung und Gebrauch. 1850. Eine Sammlung vieljähriger Erfahrungssätze zum nuitzlichen Selbstunterrichte fiir Besitzer von Zugpferden und alle, die damit umgehen. By E. M. von Hermann. 8vo. Leipzig, 1850.

Reit-Instruction für die leichte Kavallerie. Von einem preussischen Kavallerie Officier [Major Conrad Herstatt]. 12mo. Diisseldorf, 1850.

Praktisches Handbuch der Chirurgie für Thierärzte. By C. H. Hertwig. 8 vo. Berlin, 1850.

Das Leben eines Kriegspferdes. Gezeichnet und radirt von Max Prätorius. By Wilhelm Hey. 4to. Gotha, 1850; 1853.

Militair-und Civil-Reiter-Schule neuerer Zeit. Zur griindlichen Anleitung in der einzig wahren Reiter-Praxis mit VII. OriginalSteindrücken. By Conrad von Hochstetter. Berlin, 1850.

Horse Guarls. By Lieut.-Col. Hort. 8vo. London, 1850.

Histoire des anciennes races chevalines du département de la Manche, adressée à M. Lavallette, commandant le dépôt des remontes de St.-Lô. By E. Houel. 8vo. St.-Lô, 1850.

Lettre d'un paysan à M. de La Rocque, à propos de chevaux. [By E. Hubert]. 12 mo. Paris, 1850.

Petit manuel de l'éleveur de chevaux, ou exposé simple des règles qui doivent guider clans les soins à donner aux poulinières et à leurs produits, suivi de considérations sur la production et l'amelioration du cheval. By Jacob. $12 \mathrm{mo}$. Nancy, 1850.

Petite Monographie des boiteries du cheval. By François Maxime Jacquemin. 8vo. Saumur, 1850.

Complete Farrier. By Knowlson. New York, 1850.

Unfehlbare Heilung des Milzbrandes der Pferde, Rinder, Schweine, Schafe und Ziegen mit homöopathischen Mitteln. By Christoph Ernst Julius Koch. 8vo. Gotha, 1850.

Ministère de l'agriculture et du commerce. Conseil supérieur des haras. Rapport sur les travaux de la session de 1850. By Christophe Léon Louis Juchault de La Moricière. 4to. Paris, 1850. 
1850. Oleczeniu chorób koni, bydla, swin, owiec i psow. Wyklad popularny dla uzytku gospodarza. By J. Lepkowski. 16mo. Wadowice, 1850 .

Projet d'une selle de troupe à lames mobiles; par un officier de cavalerie. [By Lieutenant Leurs]. 8vo. Brussels, 1850.

Poradnik weterynaryi gospodarczej dla uzytku ziemian i lubowników koni, z wykazem najlepszych owczarn w Szlazku i Krolestwie Polskiem. $Z$ jedenastoma tablicami rycin $w$ atlasie. By J. $\mathbf{H}$. Lewandowski. 8vo. Warsaw, 1850.

Handbuch der Anatomie der Hausthiere. Mit besonderer Beriicksichtigung des Pferdes. By Friedrich August Leyh. 8vo. Stuttgart, 1850.

Utkast till hästafvelssystem för Sverige, nied afseende på den enskilda landthästafveln. By Mathias Leyon. 8vo. Orebro, 1850.

Der Volkommene Pferdekenner, oder die Kunst, den Gesundheitszustand und die gewöhnlich verheimlichten Gebrechen des Pferdes sofort zu erkenmen, eine Kurze und durch genaue bildliche Bezeichnung veranschaulichte Darstellung aller Gebrechen und Krankheiten des Pferdes, etc. By Fürchtegott Ludwiger. $16 \mathrm{mo}$. Grimma and Leipzig, 1850.

Handleiding tot het gewennen van de paarden aan de wapens, de schijf en aan het vuren. By J. van Merlen. 8vo. Amsterdam, 1850.

Appréciation des chevaux, d'après les caractères du pouls et l'examen des formes et des habitudes extérieures. By Nicholas J. A. Minot. 8vo. Paris, 1850.

Manuel de l'éleveur, ou Méthode simplifiée de dressage des chevaux au montoir et au trait. By Count de Louis Edme Montigny. 8vo. Paris, 1850.

The Young Lady's Equestrian Assistant. By Captain A. F. Oaks. 8 vo. London, 1850.

Chirurgia weterinary jna praktyczna czyli wy klad sposobow wykonywania pospolitszych operacyi na zwierzctacs domowych do uzytku lekarzy weterynaryi i gospodarzy. By E. Ostrowski. 8vo. Warsaw, 1850.

Twelve Lectures on the Form and Action of the Horse, to which are appended some experimental enquiries into the Effects of Merlicine on Horses, comprising those of most of the mineral and vegetable poisons. By William Percivall. 8vo. London, 1850. 
These lectures were originally written for the 'Veterinarian,' 1850. where they appeared serially during $1842,3,4$.

Découverte des causes de la Morve et du Farcin. By E. L. Plasse. 8 vo. [Niort, 1850].

Réponse adressée à M. le ministre de la guerre au sujet de la découverte de la cause de la morve et du farcin. By L. E. Plasse. 8vo. [Poitiers, 1850].

Onu rätta tydningen af Side-utskotten pa rygg-och landt kotorna hos Mennisken och Däggdjuren. By Anders Adolph Retzius. 8 vo. Stockholm, 1850 .

Principes généraux sur l'amélioration des races de chevaux et autres animaux domestiques, à l'usage des écoles d'agriculture et de l'armée. By A. Richard. 8vo. Paris, 1850.

Rapport présenté à l'assemblée nationale au nom d'une commission spéciale nommée par cette assemblée pour etudier le haras de Saint Cloud fondé par le Roi Louis Phillippe. By A. Richard. 8vo. [Paris, 1850].

Laws and Practice of Horse Racing. By Captain C. Rous. $12 \mathrm{mo}$. London, $1850 ; 1852 ; 1866$.

Означенін кровног англископ лопади вт русскомт копнозаводств By J. S. 8vo. Moseow, 1850.

Ziichtung, Erziehung, Ausbildung des Pferdes im systematischen Zusammenhange. Vorschläge zu einer rationellen Organisation der Gestiite. Die Krankenpflege des Pferdes, nebst einer Abhandlung über den Maulriemen, ein nenes Zäumungsmittel zur besseren erhaltung und Verwendung des Pferdes. By Louis Seeger. 8vo. Berlin, 1850.

The Modern System of Farriery, comprehending the present entire improved mode of practice according to the rules laid down at the Royal Veterinary College, containing all the most valuable and improved remedies, accurately proportioned and properly adapted to every disease to which the Horse is incident, etc. By George Skeavington. 4to. London, [1850].

Société vétérinaire de la Marne, Compte-rendu des travaux de la Société. 8vo. Chalons sur Marne, 1850, etc.

Mélanges hippologiques, 1. L'étoffe et le sang; 2. production et éducation du cheval chez les anciens. By Charles de Sourdeval. 8vo. Tours, 1850 . 
1850. The Pictorial Gallery of English Race Horses, containing portraits of all the winners of the Derby, Oaks, and St. Leger Stakes during the last 20 years, and a history of the principal operations of the Turf. By George Tattersall. 8vo. London, 1850; 1851.

Bidrag till Generations-Orgarnernas Anatomi och Physiologie hos Menniskan och Däggdjuren. By Friedrich Wahlgren. 8vo. Lund, 1850.

The Equestrian's Manual ; or the Science of Equitation, with Advice to Purchasers of Horses, Saddlery, etc. By Samuel C. Wayte. 8vo. London, 1850.

Abridged and revised:-Graceful Riding; a Pocket Manual for Equestrians. 8vo. London, 1859.

1851 Abrichtungs-Reglement für die k. k. Kavalerie, 1851. 8vo. Vienna, 1851.

Bemaerkninger i Anledning af Stutterivœesenets Ordning ved Lov. Anon. 8vo. Copenhagen, 1851.

La Veterinaria tascabile, che insegna la maniere di curare le maladie dei cavalli specialmente in viaggio, colle regole per conoscere i loro difetti e loro eta. Anon. 12mo. Livorno, 1851.

Ministère de l'agriculture et du commerce. Compte rendu de l'administration des haras pour l'année 1850 , conformement à l'article 7 de l'arrêté organique du 11 decembre, 1848 (Paris, le 28 février 1851). 4to. Paris, 1851.

Notes sur l'hippiatrique à l'usage du peloton d'instruction des régiments d'artillerie. Anon. 32mo. Metz, 1851; 18mo. Metz, 1851.

Nuevo manual completo de veterinaria, que contiene el conocimiento general de los caballos, el modo de criarlos, de cuidarlos y de adiestrarlos; la descripcion de sus enfermedades y los mejores medios de curarlos, los preceptos sobre el herrage, etc. Anon. 8vo. Madrid, 1851.

Ordonnance sur l'exercise et les évolutions de la cavalerie, du 6 decembre, 1829. Ecole du cavalier à cheval. Chasseurs et Hussards. $32 \mathrm{mo}$. Paris, 1851.

Ordonnance sur l'exercise et les evolutions de la cavalerie, du 6 decembre, 1829, Ecole du cavalier à cheval. Lanciers. $32 \mathrm{mo}$. Paris, 1851. 
Regulations for Cavalry. Horse Guards. 8vo. London, 1851.

Sociedad de fomento de la criar caballar de España. Reglamento para las carreras y para los premios por construccion, bellezza y reproduccion. Anon. 8vo. Madrid, 1851.

Un mot sur 1 race arabe et sur le haras de St. Cloud. A non. 8vo. Paris, 1851.

Veterinary Homœopathy; comprising Rules for the General Treatment of Domestic Animals. Anon. 12mo. London, 1851.

Der homöopathische Haus-Thierarzt. Praktische Anweisung fur Landwirthe und Viehbesitzer iiberhaupt, alle Krankheiten und äusserlichen Verletzungen der Pferde, des Rindviehes, der Schafe, Ziegen, Schweine und Hunde auf homöopathischen Wege schnell und griindlich za heilen. By Christian Griem [pseud.] Quedlinberg and Leipzig, 1851.

Statistique raisonnée des animaux domestiques de l'arrondissement Fontenoy Vendee. By P. N. Ayraud. 8vo. Paris, 1851.

Rivista igienica del cavallo di truppa, ovvero nuove ricerche sulle cause della morva. By Danielli Bertacchi. 8vo. Turin, 1851.

Traité de l'organisation du pied du cheval, comprennant l'étude de la structure, des fonctions, et des maladies, de cet organe. By $\mathrm{H}$. Bouley. 8vo. Paris, 1851.

Manual de Veterinaria para uso de los oficiales de caballeria. By Pedro Briones, and Juan Abdon Nieto. 8vo. Madrid, 1851.

Eléments de chirurgie vétérinaire, a l'usage des études et de la pratique. By A. J. Brogniez. 8vo. Brussels, 1851.

Eenige lessen over praktische veerartseny. By Edward Campens. 8vo. Gent, 1851.

Opbrenging en bestiering der huisdieren in de verbetering der rassen By Edward Campens. 8vo. Gent, 1851.

Central-Zeitung für die gesammte Veterinär-medizin und ihre Hülfswissenschaften, mit vergleichender Bezugname auf die Menschenheilwissenschaft. Edited by Johann Martin Kreutzer. 4to. Erlangen, 1851, etc.

Historia organica de las armas de infanteria y caballeria. By the Count de Clonard. 7 vols. 4to. Madrid, 1851. 
1851. Les Chevaux du Sahara et les mours du desert, avec des commentaires par l'emir Abd-el-Kader. By General Melchior Joseph Eugène Daumas. 8vo. Paris, 1851 ; 1853; 12mo. Paris, 1854 ; 1858; 8vo. Paris, 1862.

In English :-The Horses of the Sahara, and the Manners of the Desert, ete. Translated by James Hutton. 8vo. London, 1863.

The Breeding and Economy of Live Stock, being the Results of Forty Years practical experience in the Management and Disposal of Cattle, Horses, Sheep, and Pigs. By James Dickson. 8vo. Edinburgh, 1851.

Sammlung von Abhandlungen betreffend alle Theile der praktischen Thierheilkunde und den Stand der Thierärzte. By J. F. C. Dieterichs. 8vo. Berlin, 1851.

In Dutch:-Hanlboek der Veeartsenykundige Heelkunde van J. F. C. Dieterichs. Uit het Hoogduitsch vertaald met eene Voorrede en eenige Aanteekeningen valn Dr. A. Numan. 8vo. Sneek, 1851.

In Swerlish:-Veterinair-Chirurgie, eller lära om Kreaturens yttre åkammor, dessas Behandling, samt chirurgiska Operationer. 3 vols. 8 vo. Hamburg, 1851

Die Hauptmängel sammtlicher Hausthiere fur Landwirthe, Viehschaner und Viehhändler. Nebst Anleitung zum Verhalten in vorkommenden Streitfällen. By Carl Gottfried Doll. 12mo. Markgröningen, 1851 ; 1857.

Le Maréchal expert français, contenant une connaissance exacte du cheval, etc. By Henri de Dombale. 12mo. Paris, 1851; $1853 ; 1855$.

Aphorismen iiber Reitwissenschaft und dazu gehörender Pferdebehandlung. By C. Gr. v. E. 8vo. Dresden, 1851.

El Eco Veterinario. Periodico cientifico y profesional. Mardrid, 1851, etc.

De betriegerijen, Kunstgrepen, roskammenen andere Streken welke in den Paardenhandel plaats grijpen. Eene Handleiding voor Eigenaars en Liefhebbers van Paarden, Paarden-artsen, Paarden-handelaren, Officieren der Kavalerie en artillerie. By Ignatz Ellerbrock. 8vo. Amsterdam, 1851 ; 1853.

Ricerche storico analitiche sugli scrittori di Veterinaria. By Giovanni Battista Ercolani. 2 vols. 8vo. Turin, 1851-54. 
Lehrbuch der allgemeinen Veterinär-Therapie. By Johann Ernst 1851.

Ludwig Falke. 8vo. Leipzig, 1851.

Praktische Anleitung zur allgemeinen und speciellen Viehzucht. Mit besonderer Riucksicht auf Selbst behandlung der Thiere in erkrankungs-und Ungliicksfällen. By Martin Fries. 8vo. Heilbronn, $1851 ; 8 \mathrm{vo}$. Mannheim, 1853.

Giornale di vétérinaria publicato per eura dei professori ed assistenti della regia scuola vétérinaria di Torino. Edited by G. B. Ercolani and D. Vallada; then by Giuseppe Lessona and Giuseppe Pellerini. 8vo. Turin, 1851, etc.

Ecole du cavalier au manége, basée sur les principes de l'ordonnance de cavalerie, à l'usage des instructeurs. By A. Guérin. 8vo. Saumur, 1851.

Veiledning i Lœren om Cavaleriets og Infanteriets Vaaben. By G. Hagerup. 1851.

On a remarkable effect of Cross-Breeding. By Alexander Harvey. 8 vo. Edinburgh, 1851.

Welche Einwirkungen können die neueren Prinzipien der Züchtung und Behandlung der Pferde auf die Haltung der Cavallerie Gewinnen? Eine Erörterung, welche Stände, Mitgliedern, Besitzern, Pferde-Händlern und Liebhabern auch zu empfehlen sein dürfte. By Wilhelm von Hassell. 8vo. Berlin, 1851.

Ручная конская кннгя, для ветеринаровъ, ремонтеровт, коннозаводчпковъ и вообше, любптелеп. Ву J. Hensler. $16 \mathrm{mo.}$ St. Petersburg, 1851.

Cavallerie-Katechismus. Fragen und Antworten uiber Reiterei. By Conrad Herstatt. 12mo. Duisseldorf, 1851.

Taschenbuch der gesammten Pferdekunde. Für jeden Besitzer und Liebhaber von Pferden. By Carl Heinrich Hertwig. 8vo. Perlin, $1851 ; 1857$.

De la Castration des Chevaux. By J. Lacoste. 8vo. Toulouse, 1851.

Sul perfezionamento del cavallo nella isola di Sardegna. By Giuseppe Lessona. 8vo. Vavallo, 1851.

Tankar i Sveriges hästafvelsfråge. By Mathias Leyon. 8vo. Orebro, 1851. 
1851. Wegweiser beim Pferdekauf oder Rath und Hülfe zur Beurtheilung des Pferdes, Enthïllung der Geheimnisse und Handelsvortheile der Pferdehändler, sowie auch der Kunstgriffe zum Vortheil des Käufer beim Mustern und beim Handel. By August Lotze. 8vo. Wiemar, 1851.

Betrachtungen ïber die Uebertragung der Rotzkrankheit vom Pferde auf den Menschen. By A. Louchard. 8vo. Teterow, 1851.

Carré imod en Cavalleri Attaque om Voebnings systemer. By X. A. [J. C. Lütken]. 8vo. Copenhagen, 1851.

Méthode abrégée de dressage des chevaux difficiles, et particulièrement des chevaux d'armes. By Count de Louis Edone Montigny. 8vo. Paris, 1851 .

Hesteavlens sörgelige Forfald i Danmark i det sidste halve Aarhundrede. Aarsagerne dertil og Midlerne til Sammes muelige Gjenophjelpning. En historisk-factisk Undersögelse, naermest anlediget ved de i Begyndelsen af dette Aar indkomme Commissionsforslag. By J. V. Neergard. 8vo. Copenhagen, 1851.

Leitfaden zur Abrichtung von Reiter und Pferd, nebst der Zäumungslehre und einem Anhange uiber Schulreiterei. Zum Behufe seiner theoretischen Vorträge am k. k. Militär-Central-EquitationsInstitute. By Borries Christian von Oeynhausen. 8vo Vienna, $1851 ; 1851 ; 1853$.

Cenni di storia naturale e igiena veterinaria. By Luigi Patellani. Milan, 1851.

Les Haras, ce qu'ils ont été, ce qu'ils sont, ce qu'ils devraient être. By Felix Person. 8vo. Paris, 1851.

E1 Regenerador de la Medicina Veterinaria. Edited by Florencio Paniagua. 4to. Madrid, 1851, etc.

Beurtheilung des Pferdes in Bezug seiner Brauchbarkeit für den Reit-oder Zug Dienst. By Heinrich Riege. 8vo. Berlin, 1851.

Cavalry Catechism. By Thomas Robbins. 16mo. London, 1851; $12 \mathrm{mo}$. London, 1864.

Betrachtungen iiber Pferdezucht im Allgemeinen und mit besonderer Rüchsicht auf die Provinz Westphalen. By General-Lieutenant Ludwig Johann Carl Gregor Eusebius Roth von Schreckenstein. 8vo. Carlsruhe, 1851. 
Руководство къ познанію лопаді Понаружному ея осмотру, 1851. составленное по лучим источншкамъ артіллеріп подполсовникомъ Рутенбергомъ. Изданіе второе съ прпложеніем' 167 пояснтельныхъ. By Ruten berg. 8vo. St. Petersburg, 1851.

Mémoires de John Spurr, l'hippomane. By N. de Saint-Albin. 8 vo. Paris, 1851.

Higiene veterinaria militas. By Fernando Sampedro. 8vo. Madrid, 1851.

Die Landwirthschaftschule. Eine systematisch geordnete Belehrung. uiber die gesammten Zweige des Acker-und Wiesenbaues, der Viehzucht, der Theirheilkunde, etc. Nebst einem Anhange uber die Anlage von Dörfern. By Anton Seyff. 8vo. Vienna, $1851 ; 1857$.

Immerwährender Trächligkeits-Kalender der nutzbarsten Hausthiere. By Karl Stein. 16mo. Rostock, $185 \mathrm{l}$.

Hints to the Yeomanry Cavalry of Great Britain. By Corporal-Major J. Stevenson. London, 1851.

Stud Book Français. Registre de chevaux de pur sang importes ou nés en France. 8vo. Paris, 1851, etc.

The Stud Farm, or Hints on Breeding for the Turf, the Chase, and the Road; addressed to Breeders of Race-horses and Hunters, to landed proprietors, and especially to Tenant Farmers. By Cecil [Cornelius Tongue]. 8vo. London, $1851 ; 1856$.

Studien und Erfahrungen im Bereiche der Pferdekunde. Eine Sammlung von Beobachtungen über das Wesen des Pferdes, die günstigen und ungünstigen Resultate der Ziichtung, Erziehung, Pflege, Training und Rennen, so wie auch uber das Wesen der Erbfehler, die Mechanik des Ganges und Belehrung uiber Geburtshïlfe und Jugendkrankheiten, wie überhaupt iber die Krankheiten des Pferdes und deren homöopathische Behandlung, etc., etc. By Theodor Traeger. 8vo. Sondershausen, 1851 .

Guide to the Breaking of Young Horses. By R. Turner. 8vo London, 1851.

Anleitung zur Pferdekenntniss, oder die Beurtheilung des Pferdes auf seine Vorziige und seine Mängel, nebst Rathgeber fuir Pferde kaiifer bei der Untersuchung von Pferden, und zur Sicherung gegen Betrug; Enthullung der Rosstäuscherkünste; Angabe der 
1851. auf den Pferdekauf bezüglichen Gesetze; Beschreibung der Kennzeichen der Gewährsmängel etc. Ein nützliches Handbuch für Jeden, welcher aus Neigung oder Beruf sich für das Pferd interessirt. By Johann Ludwig Wagenfeld. 8vo. and folio Atlas, Konigsberg, 1851 ; Leipzig, 1855.

Die Krankheiten der in der Landwirthschaft benutzten Haussäugethiere. Ein Handbuch beim Unterricht der Thierheilkunde auf Ackerbauschulen, sowie zum Selbstunterricht für Landwirthe. By Anton Ottomar Wernaer. 8vo. Halberstadt, 1851.

1852. The Book of Aids, or catechism in the system of equitation, practised at the Cavalry Riding Establishment. For the use of noncommissioned officers and assistants in Riding Schools. By authority of the Adjutant-General to the Forces. Anon. 8vo. London, 1852.

Cours complet de médicine vétérinaire pour M. M. les maréchaux de Paris et de la province. Anon. 16mo. Paris, 1852.

Instruction for Cavalleriets Exercis-og Remonteskole. Anon. 8vo. Christiana, 1852.

Jaarboekje van de Societeie tot Verbetering van het Paardenras in Nederland. 12mo. Haarlem, 1852, etc.

Mémoires de la Société impériale et centrale de médecine vétérinaire. 8 vo. Paris, 1852 , etc.

Principes d'équitation, de gymnastique et de natation, extraits de divers auteurs, Par un officier de cavalerie. Ánon. $32 \mathrm{mo}$. Maubeuge, 1852.

Règlement de la Société d'encouragement pour l'amélioration des races de chevaux en France. 12mo. Paris, 1852.

Yeomanry Regulations. 8vo. London, 1852.

Développement d'une question équestre relative au dressages des chevaux. By Count Alexis d'Abzac. 8vo. Paris, 1852.

Leichtfassliche Belehrung und Behandlung der verschiedenen Arten von Kolik, des Harnverhaltens, sowie der Trommelsucht und Verstopfung beim Pferde und Rinde. By Joseph Aigeldinger. 8 vo. Rottweil, 1852.

Annales de médecine vétérinaire. Edited by Delwart and Thienesse. 8 vo. Brussels, 1852, etc. 
Quarlrupeds of North America. By Audubon and Bachman. 8vo. 1852. New York, 1852-1854.

Cours d'équitation, adopté officiellement et enseigné à l'école de cavalerie et dans les corps de troupes à cheval. By Antonio Henri Phillippe Léon Comte d'Aure. Saumur, 1852 ; 5th edition, 18mo. Paris, 1859.

Ueber Pferdezucht, Reitkunst, Wettrennen, und Rennpferde. By Alexander von Bally. 8vo. Stuttgart, 1852.

Le Parfait Charron. By Louis Berthaux. 8vo. Dijon, 1852.

Allgemeines praktisches Vieharzneibuch der allopathischen und homœopathischen Thierheilkunde. Volständiger Unterricht zur Erkennung, Behandlung und Heilung der Krankheiten und Gebrechen der landwirthschaftlichen Hausthiere, Pferde, Rinder, Schafe, Schweine, Ziegen, Hunde, etc. By Moritz Beyer and Sig. v. Werneburg. 8vo. Leipzig, 1852.

In Polish:-Sposób i praktyezny, dla gospodarzy wiejskich nieobeznanych z weterynarya poznawania i leczenia chorób zwierzat domowych przez P. P. Werneburg i Beyer. Translated by $A$. $H$. 8vo. Worsaw, 1854 .

Blätter über Pferde und Jagd. Edited by C. H. Vogler. 24 vols. 8vo. Berlin, 1852-63.

Der thierarztliche Rathgeber in den innern und aussern Krankheiten der Pferde, Rinder, Schafe und Schweine. Ein Noth-und Huilfsbuch fiir Landwirthe und Viehbesitzer jeden Standes. By Carl L. Boehm. Svo. Vienna, $1852 ; 1853$.

Trattato di Zooiatria. By Siro Bonova. 8vo. Milan, 1852.

A Treatise on the Proper Condition for all Horses. By Harry Hieover [Charles Brindley]. 8vo. London, 1852.

L'Alimeutation par la viande de cheval. By E. F. Decroix. 8vo. l'aris, 1852 ; 1864.

Manuel de médecine vétérinaire. Extérieur, Anatomie, Physiologie, Ferrure et Parturition. By François Defays and Husson. 12 mo. Brussels, 1852.

Rapport présenté au nom de la section d'économie des animaux sur la concours pour des ouvrages et des mémoires sur l'amélioration, l'hygiéne et les maladies des animaux domestiques. By Onésime Delafond (Société nationale et centrale d'agriculture). Svo. l'aris, 1852. 
1852. Statistique générale et raisonnée des principaux animaux domestiques de l'arrondissements de Simoux-sur-Aude, suivie de la nomenclature, de la description et du traitement des maladies les plus communes qui attaquent nos espèces. By J. P. G. Deloupy. 8 vo. Sens à Toulouse, 1852.

Tratado de agricultura aplicada a la veterinaria. By José Echegaray. 8vo. Madrid, 1852.

Einige Worte zu William Miles Huf des Pferdes und dessen fehlerfreie Erhaltung. [By C. Graf von Einsiedel]. 8vo. Dresden, 1852.

Aigemeene paardenkenis. Eene handleiding voor eigenaars en liefhebbers van paarden, paarden-handelaren, paarden-artsen, officieren der Kavallerie en artillerie, etc., etc. Bevattende de naturlijke historie van het paard; de algemeene kennis der onderscheidene kleuran en rassen; kennis van den ouderdom; de kennis en beordling van de uitwendige deelen des paardes in den gezonden toestand, en naauwkeurige beschrijving en onderkenning der uitwendige gebreken; de onderkenning en beordeling der inwendige hoofdgebreken; de noodige voorzorgen en maatregeln, welke bij den koop en verkoop van paarden dienen in acht genomen te worden, om zich voor schade te hoeden. Benevens eene uitvoerige en naauwkeurige opgave der bedriegerijen en kunstgrepen, welke in den paardenhandel gepleega worden. By Ignatz Ellerbrock. 8 vo. Amsterdam, 1852 .

De droes van het paard. Eene op ondervinding steunende praktische handleiding; om den droes, zoo als hij in zijne verschillende vormen bij het paard voorkomt, op eene gemakkelijke en zekere wijze te onderkennen, en hem door eene doelmatige behandeling zeker en spoedig te genezen. Voor Landhuishoudkundigen, Veeartsen, Officieren der Kavallerie en Artillerie, Paardenhandelaars, Bezitters en Leifhebbers van Paarden. By Ignatz Ellerbrock 8vo. Oudewater, 1852.

Manuel des sous officiers d'infanterie et de cavalerie, à l'usage des écoles régimentaires du second degré. By Elie Fabre. 12mo. Paris, 1852.

Die allgemeine Veterinär-Pathologie und der Krankenexamen. By Johann Ernst Ludwig Falke. 8vo. Leipzig, 1852.

Die Anatomie und Physiologie der nutzbaren Hausthiere in ihrer praktischen Verbindung mit der Pathologie. By Johann Ernst Ludwig Falke. 8vo. Leipzig, 1852. 
Instruction de la cavalerie. Matériaux d'hippologie; $1^{\circ}$ revue retro- 1852. spective, Madame Isabelle; $2^{\circ}$ des aides suite à la position et à la tenue. By Antoine Flandrin. 8vo. Paris, 1852; 1855.

Instruction de la cavalerie. Quelques observations sur l'etât de la ques" tion. By Antoine Flandrin. 8vo. Saumur and Paris, 1852.

Etymologisches Wörterbuch der Veterinär-Medicin. Mit einem Anhang über Veterinär-Literatur. By J. Frey. 8vo. Stuttgart, 1852.

Grundziige der allgemeinen thierarztlichen Heilungslehre als Leitfaden beim Schul-und Selbst-Unterricht. By Christian Joseph Fuchs. 8vo. Erlangen, 1852.

Quadro zooetalogico, orvero guida per conoscere nel modo piu facile e pronto l'eta de gli aninali domestici dall'esame dei denti e della corna. By Luigi Ghilardi. folio. Milan, 1852.

Anleitung zur richtigen Zucht und Behandlung der Pferde. Inhaltend Aufzucht; Paarung der Pferde; Eigenschaften des Hengstes und der Stüte; Ernährung der Fohlen; Folengärten; Futterung, Wartung und Stallung der Pferde; Eigenschaften eines guten Arbeitspferdes; das Alter der Pferde zu erkennen; die Krankheiten der Pferde und ihre Heilung; Ertrag der Pferdezucht u. s. w. Nebst einem Anhang: Ueber die Behandlung des Hufs. Herausgeben unter Mitwirkung der Redaction der Agronomischen Zeitung. [By Dr. W. Hamm]. 8vo. Leipzig, 1852.

In Dutch:-Beknopte Handleiding tot de Kennis, het doelmatige Opfokken en Verzorgen van het Paard : de Behandeling van den Hoef, het Hoefbeschlag en het Aanbrengen van de eerste Hulp by sommige Ziektan van het Paard. Volgens eene Uitgare onder Medewerking van de Redactie der Agronomische Zeitung. Naar het Hoogduitsch bewerkt. Translated by A. J. de Bruijn. 8 vo. Amsterdam, 1852.

In Swedish:-Auviisning at uppföda. Ofwersättning. 8vo. Stockholm, 1852.

Elements of Veterinary Homœopathy, embracing Hints on the Application of Hydropathy. By William Haycock. 8vo. London, 1852.

Der rationelle Pferdezuchter. Zucht Wartung und Pflege der Pferde, ihre Krankheiten und deren homöopathische Heilung. Oder iiber die Natur des Pferdes, dessen Vollkommenheiten und Schönheiten : die Pferderacen und ihre Befähigung zur Dienstleistung, von der Aufzucht, Ernährung und Pflege der Pferde, wie auch die Beschreibung und homöopathische Heilung von 167 Pferde Krankheiten. By Joseph Heinrichs. 8vo. Leipzig, 1852. 
1852. Cours élémentaire d'equitation pour dames et pour hommes, apprenant l'art de monter á cheval sans le secours d'aucun maitre. By Gustave Henry. $12 \mathrm{mo}$. Rheims, 1852.

Frank Forrester's Field Sports of the United States and British Provinces of North America. [By Henry William Herbert]. 2 vols. 8 vo. New York, 1852.

The Quorndon Hounds, or a Virginian at Melton Mowbray. By Henry William Herbert. 12mo. Philadelphia, 1852.

Spezielle Naturgeschichte der Hausthiere. Ein praktisches Handbuch fiir Thierärzte, Landwirthe und alle Freunde der Hausthiere. By Dominik Hofer. 8vo. Regensburg, 1852.

Anleitung zur äussern Pferdekenntniss, zugleich als Rath beim Pferdekauf. Fiir Landwirthe, Cavalerie-Officiere, Pferdezuchter und Pferdeliebhaber. By Ferdinand Rudolph Jacoby. 8vo. Stolp, $1852 ; 1854$.

Eine Elementarlehre der Reitkunst. Ein Leitfaden zum Unterricht im Schul-und Campagnereiten, nebst einer kurzgefassten Reitlehre fur Damen und einem Anhange iiber das Verhalten auf Reitbahnen; allen Reitliebhaben vorzugsweise seinen Schulern und Schülerinnen. By Carl Anton Kappel. 8vo. Frankfort, 1852.

Elementos de Equitacion militar, para el uso de la Caballeria Española, escritos para los alumnos de la escuela militar de Equitacion. By Francisco de Laiglesia y Darrac. 8vo. Madrid, [Circa $1852] ; 1853$.

Un mot sur l'admistration des haras. By Count Savary de Lancosme Brèves. 8vo. Paris, 1852.

Della febbre in generale et della febbre degli animali domestici in particolari. By Carlo Lessona. 8vo. Turin, 1852.

Compendio de veterinaria, ou curso completo de zooiatrica domestica, approvada pelo Conselho superior de instrucçāo publica. By Jose Ferreira de Macedo Pinto. 8vo. Coimhra, 1852; 1854 .

Замұчанія о прптотовленіп лопадеп кт скачки гля г. офнцеровъ. Въ тппографіп III отдыленія собственноп его пмнераторскаго велпчества канцеляріп. [Ву P. Maesnow]. 12mo. St. Petersburg, 1852. 
Wahrnehmungen über die Rotzkrankheit der Pferde, ihre Ursachen 1852. und Verbanung. By Friedrich Marquart. 12mo. Olmuitz, 1852.

Die Zaumung mit der Kandare. From the posthumous paper's of Friedrich August Ludwig von der Marwitz. 8vo. Berlin, 1852.

О задачахъ пппологіп вг отнопеніп къ потребностям кавалеріп. - By A. Th. Middendorf. (Перепечатано пзъ 1 го тоня учекыхъ записокъ Императорскоп Академіп наукъ по лервому II третьему оудЊденіямъ.) 8vo. St. Petersburg, 1852 .

Des Animaux domestiques malades et des premiers soins à leur donner. By Miramont. 8vo. Paris, 1852.

Rapport de M. le Vicomte de Montigny. Ecuyer-professeur à l'Ecole des Haras, sur l'utilité et les avantages de la bride à mors régulateur combinée. (Extrait du Journal des Haras, Mai, 1852). 8 vo. Paris, 1852.

Lönnen for min 60 aarige, ved uomstödelige Kjendsgjerninger oplyste gavnlige Virken i Statens Interesse, eller et Par Ord til Finantsministeren, Grev Sponneck, i Anleding af haus Angreb paa mine suurt fortjente, ingenlunde rundelige Vartpenge. Tilligemed et flygtigt Blik paa vor Veterinairskole og vort Stutteri-Vaesens unvaerende Tilstand. By Jens Veibel Neergaard. 8vo. Copenhagen, 1852.

Aux éleveurs, aux cultivateurs, à tous les cavaliers. [By Casimir Noel]. 8vo. Paris, 1852.

Le cheval dompté et dressé par lui-même, ou théorie de la bride à mors régulateur combinée d'après une découverte physiologique, dedié aux éleveurs, aux cultivateurs, à tous les cavaliers, et à l'usage de tous conducteurs de chevaux d'attelage et de trait. - By Casimir Noel. 8vo. Meaux, 1852.

Pour bien poser la bride à mors regulateur, combinée par M. Casimir Noel. Avec la bride à mors regulateur, le cheval est dompté et dressé par lui-même. By Casimir Noel. 16mo. Meaux, 1852.

L'equitation naturelle enseignée en vingt-cing minutes avec la bride à mors régulateur sans gourmette et à rênes croisées. Dedié aux amazones et aux jeunes cavaliers. By Casimir Noel. 8vo. Paris, [1852]. 
1852. The Training of Cavalry Remounts. By Captain L. E. Nolan. 8 ro. London, $1852 ; 1861$.

In French:-Dressage des Chevaux de remonte par L. E. Nolan. Translated by Savin de Larclause. 8vo. Paris, 1872.

Belehrungen für den Oeconomen und Viehzuichter bei nachverzeichneten Krankheiten : Der Rotz, der Wurm und der Dummkoller der Pferde; Die Rinderpest, die Lungenseuche und die Perlsucht oder Franzosenkrankheit der Rinder; Die hitzige Maul-und Klauenseuche bei den Rindern, Schafen und Schweinen; Die ächten und unächten Pocken bei den Kiihen; der Milzbrand der Rinder, die Raude und die Pocken der Schafe, die Hundswuth. Sowie über die Eigenschaften des Schlachtviehes, welche das letztere zum Genusse untauglich und schädlich machen. Auf Anordnung des Königlich Sächsischen Ministeriums des Innern bekamnt gemacht. [By Professor Pieschel]. 8vo. Dresden, 1852.

Traité de maréchalerie vétérinaire. By A. A. Rey. 8vo. Paris, 1852 ; 1865.

The Horse: its varieties, breeding, and management. By H. D. Richardson. 8vo. London and Dublin, 1852.

Der Hufschmied, oder grundliche Anweisung, die Pferde auf die vollkommenste Art zu beschlagen. By Gotthold Rienecker. 8 vo. Suhl, 1852.

Die Beschälkrankheit und der Beschälausschlag der Pferde. Nach Beobachtungen pathologisch und therapeutisch bearbeitet. By C. Rodloff. 8vo. Birnbaum, 1852.

Grundzige zur gerichtlichen Veterinär-Medizin nach dem gegenwärtigen Standpunkte dieser Wissenschaft für Beamte, Juristen, Landwirthe, und Veterinär-Aerzte. By H. Rueckert. 8vo. Rostock, 1852.

Ueber den Bau und die Verrichtungen des Körpers unserer Hausthiere. Anatomisch-physiologische Einleitung in die Thierziichtungskunde, bearbeitet für den Landwirth, Gestiitsbeamten, Thierbesitzer, etc. By Adolph Rueff. 8vo. Stuttgart, 1852.

Ueber die Gewährleistung beim Handel mit Hausthieren. Eine critische Beleuchtung der Gesetze über Gewährmängel im Allgemeinen, nebst Mittheilung der betreffenden Rechtsnormen in Wuirtemburg. Ein Programm ausgegeben bey Gelegenheit der Jahresprïfung an der königl. wïrtemburgischen land-und forstwirthschaftlichen Akademie zu Hohenheim, an 28 August, 1852. By Adolph Rueff. 8vo. Stuttgart, [1852]. 
Cours d'hippologie contenant: $1^{\circ}$ la connaissance du cheval, $2^{\circ} 1852$. l'hygiène, $3^{\circ}$ l'industrie chevaline suivi d'un appendice sur la position du cavalier à cheval démonstrée par l'anatomie. Adopté officiellement et enseigné à l'école de Cavalerie et dans les Corps de Troupes à cheval, par Décision de M. le ministre de la guerre, en date 9 Avril, 1852. By Charles Casimir Beucher de Saint-Ange. 2 vols. 8vo. Saumur, 1852 ; Paris, 1854.

The same, abridged, 32mo. Saumur, 1852 ; Paris, 1853 ; 1854 ; 1855 .

Die Aufzucht, Wartung, Ernährung und Benutzung der Pferde, des Rindviehs, der Schafe. Ziegen und Schweine, nebst Angabe der bei denselben am haiifigsten vorkommenden Krankheiten, wie und mit welchen Mitteln dieselben gehoben werden können. Ein handbuch fuir kleinen Guts-und Bauerngutsbesitzer. By A. J. Schmid. 8vo. Berlin, 1852; 1853.

In Polish:-Chodowla, pielegnowania, zywienie i uzywanis koni, bydla, rogatego, owiec, koz i trzody chlewneji, oraz wysczegolnienie ich chorob zwyczajnych i sposobow leczenia. Ksiazka dorcezna dla posiedzicieli mniejszych dobr i wloscian. Translated by A. Zmudzinski. 8vo. Lezno, 1854.

Herr Baucher und seine Künste. Ein ernstes Wort an Deutschlands Reiter. By Louis Seeger. 8vo. Berlin, 1852.

Stable Practice; or hints on training for the Turf, the Chase, and the Road; with observations addressed to all who are concerned in Racing, Steeplechasing, and Foxhunting. By Cecil [Cornelius Tongue]. 8vo. London, $1852 ; 1857$.

Vierteljahresschrift fiir Wissenschaftliche Veterinärkunde, herausgegeben von den Mitgliedern des k. k. Wiener ThierarzneiInstituto. Edited by Dr. Franz Müller and Dr. Roell. 8vo. Vienna, 1852, etc.

Horse Training and Young Horsemanship. By N. Wiseman. London, 1852.

Rapport om det Kgl. Landstutteri og Hesteavlens Tilstand m. m. i Danmark afgiven til Indenrigsministeriet. By Georg Christian With. 8vo. Copenhagen, 1852 ; 1853 ; 1854 ; 1855; 1856; $1857 ; 1859$.

Anviisning til at Kjore, og til Hestes, Vognes og Seletoires Behandling 1853. For Kudske. Anon. 8vo. Copenhagen, 1853. 
1853. Das Alter eines Pferdes genau und sicher zu erkennen, dem Wechsel und den Veränderungen der Zähne, nebst Regeln, woraus Man schliessen kann, ein fehlerfreies Pferd zu kaufen. Für den Landmann und Pferdeliebhaber herausgegeben von einem vormaligen Schleswig-Holsteiner. Anon. 8vo. Hamburg, 1853.

Handbock för Ryttaren. Anon. 12mo. Malmö, 1853.

- On the deteriorated condition of our saddle-horses; the causes and the remedy. 'The State of our Cavalry, and the imperfect system under which this force and that of our army generally is administered. Anon. 8vo. London, 1853.

La Cavalerie considérée au point de vue de son harrachement et de son equipement. Anon. 8vo. [Circa 1853].

Ordonnance sur l'exercise et les evolutions de la cavalerie. Ecole du cavalier à cheval. Chasseurs et Hussards. 18mo. Paris, 1853.

Резултать испытаніп рыспстыхъ и другихъ лошаден въ Парскомъ Сель. Anon. 8vo. St. Petersburg, 1853.

Резултаты пспытаніи лошаді въ С. Петербургы зиою. Anon. 8vo. St. Petersburg, 1853.

Verzameling van Veeartsenijkundige Bijdragen, Uitgegeven door de Zuidhollandsche Maatschappij ter bevordering der Veeartsenijkunde. 8vo. Leyden, 1853.

Des tares molles et osseuses dans le cheval, considérations générales et très sommaires, nécessaires pour la complète intelligence des 31 pièces d'anatomie plastique à l'aide desquelles ont été réproduites les principales tares molles et osseuses qui affectent les membres du cheval. By Louis Auyoux. 8vo. Paris, 1853.

On the Breeding of Domestic Animals; being an Extract of the Practical part of a communication to the Royal Agricultural Society of England. By Valentine Barford. 8vo. London, 1853.

Le nouveau parfait bouvier, traité complet de l'élevage des bestiaux, contenant le Parfait Bouvier, le Parfait Herger, avec des instructions sur la manière de croiser, de propager les races; suivi de conseils sur l'administration d'une basse-cour, etc., et d'un précis sur les chevaux et les bétes de somme. By Berthaud. $12 \mathrm{mo}$. Paris and Limoges, 1853 ; Ebda, 1855 ; 1856. 
Traité du tic des chevaux et de la vieille courbature, ou procédés 1853. practique pour guerir ces deux vices. By Frédéric Bonneval. $12 \mathrm{mo}$. Paris, 1853.

Bipeds and Quadrupeds. By Harry Hieover [Charles Brindley]. 8vo. London, 1853.

Neuere Ansichten über die horizontale Ziigelfaust oder die rationelle Wirkung bei der Kanderenzugel in einer Hand. Dem Reiter-Publikum zur vorurtheilsfreien Prifung. By B. v. Cg. 8vo. Brandenburg, 1853.

Le Turf, ou les courses de chevaux en France et en Angleterre. By Eugène Chapus. $16 \mathrm{mo}$. Paris, 1853.

Bits of Turf. By Priam [Collins]. (Racing Times Office). $18 \mathrm{mo.}$ London, 1853.

Die Anwendung der Auskultation und Perkussion in den Krankheiten der Brusthöhle des Pferdes. By Dr. Jean Crocq. 12mo. Erlangen, 1853.

Nieuwe genees-en verloskundig handboek van het vee, bevattende een volledig onderrigt tot herkenning, behandeling en genezing van de ziekten en gebreken der paarden, koeijen, schapen, geiten, varkens, honden, kippen, ganzen, duiven, enz, of gids om zijne dieren zelve te kunnen bijstaan, ten dienste van den landman en veebezitter. By Jacob Adrian Dekker. 8vo. Leyden, [1853].

Précis hygiénique sur l'éducation des animaux en général suivi de conseils à M. M. les maitres de poste, entrepreneurs de diligences et autres, propriétaires, amateurs de chevaux, etc. By F. J. Dementhon. 8vo. Lyons, 1853.

Die Fehler und Gewahrsmängel und deren Kennzeichen bei den Pferden. Ein Leitfaden zum Gebrauche für Käufer und Verkäufer von Pferden, überhaupt für jeden Pferdebesitzer. By J. F. C. Dieterichs. 8vo. Leipzig, 1853.

De nederlandsche Paarden-Fokkerij, in haren geheelen Omvang. Bevaltende, algemeene Grondregelen der Paardenfokkerij; de Keus van Dek-hengst en Fok-Merrien; hunne Oppassing en Verpleging; Behandeling der Merrien in haren Draagtijd en Verpleging van den Spring-Hengst; Verlossing in natuurlijke en tegennatuurlijke Gevallen; Behandeling van de Ziekten der Veulen-Merrien et van het Veulen, bij en na de Verlossing. Behandeling der Merrien en van het veulen gedurende den 
1853. Zoogtijd en bij het Spenen van het Veulen; Verpleging der Veulens tot op hunnen vollen Wasdom als Paard; Hoef-Beslag bij jonge Paarden, en het Geshickt Maken tot den Arbeid en den Verkoop. Handleiding voor iederen Fokker van Paarden; voor alle Stoeterijen en Paarden-Fokkerijen; ten Dienste van Landbouwers, Paarden-Artsen, Officieren der Kavallerie en Artillerie. By Ignatz Ellerbrock. 8vo. Amsterdam, [1853].

Staatsthierarzneikunde. By Johann Ernst Ludwig Falke. 8vo. Leipzig, $1853 ; 1855$.

Matériaux d'hippigie (étude du cheval en santé). Leçons normales d'equitation militaire. De la position, et de la tenue. By $\mathbf{A}$. Flandrin. 8vo. Paris, 1853.

Hufbeschlags-Katechismus zum Unterricht und zur Selbstbelehrung für Hufschmiede, zunächst in den Grossherzogthïmern. Auf Veranlassung des Mecklenburgischen patriotischen Vereins abgefasst von Gustav Johann Georg Friedrich Flemming. 8vo. Laag, 1853.

I hvilken Wexelwirking staaer Brystkastens Form og Rumfang hos Huuspattedyrene med den ovrige Organisme, og hvad Hensyn bor man tage hertil ved Bedommelsen af Hesten? Opgave med Concurrencen til Lectorat ved den kongl, Veterinairskole I April 1853. By Christian Lauritz Früs. 8vo. Copenhagen, 1853.

Neuer Katechismus der deutschen Hufbeschlagkunst. Ein Handbuch für den Schulunterricht und die Selbstbelehrung. By Christian Joseph Fuchs. 8vo. Erlangen, 1853.

De l'hérédite de la morve et de la rage. By M. le commandeur de Gama Machado. 8vo. Paris, 1853. From the "Gazette des Hôpitaux," 29th Janiary, 1853.

Lehrbuch der allgemeinen Therapie fur Thierärzte. By August Carl Gerlach. 8vo. Berlin, 1853.

Opuscali d'ippologia ovvero ragionamenti sul cavallo. By Giuseppe Gherardi. 8vo. Firenze, 1853.

Mémoire sur les suites possibles de l'accouplement des animaux. domestiques. By Charles Girou de Buzareingues. 8vo. Paris, 1853. 
Beknopte handleiding tot verbetering van het paarden-ras in de 1853. Nederlanden. Benevens de voornaamste regelen bij het aanfokken van paarden in acht te nemen. Bv Christian Carel Goedhart. 8vo. Amsterdam, 1853.

Pedigree of the Thorough-bred Horse from three original sources. By W. J. Goodwin. folio. London, 1853.

Das Reitzeng und die Geschirre der Batterein und Kolonnen der Preussischen Artillerie nebst Anweisung zum Satteln, Schirren und Packen. By Carl Heinrich Eduard Graefe. 8vo. Berlin, 1853.

Die Zäumung des Pferdes mit dem Mors régulateur des Herr Cassimir Noël. By Carl Heinrich Eduard Graefe. 8vo. Berlin, 1853.

Einiege Worte über den Betrieb der bäuerlichen Pferdezucht. By Carl Graefe. 8vo. Wittenberg, 1853.

Beiträge zur vergleichenden pathologischen Anatomie der Gelenkkrankheiten. By Ernst Julius Gurlt. 8vo. Berlin, 1853.

Concurrence-Afhandling over Sporgsmaalet, Hvilken Indflydelse yttre Rogt og Pleie paa det unge Dyrs Udvikling og hvorledes indrettes de hensigtmaessigst for Follet og Folen. By Hans Jacob Hansen. Svo. Copenhagen, 1853.

Regler for Föllets og Folens Behandlung, samt de hensigtsmässigst. Midler til at fremme Udviklingen in den fordeelagtigste Retninge By Hans Jacob Hansen. 8vo. Copenhagen, 1853.

Bewegungslchre der geregelten Grund-gangarten des Pferdes, als Fundament der Reitkunde, zum Selbstunterricht für denkende Reiter und Pferdemaler. By T. H[einze]. 12mo. Leipzig, $1853 ; 1855$.

Kritische Peleuchtung des Miles'schen Hufbeschlags und Vergleichung desselben mit dem deustchen. By Friedrich Wilhelm Hoffmeister. 8vo. Berlin, 1853.

Cours de Science hippique professé à l'École des haras pendant les années $1848 ; 1849$; et 1850 . By Ephrem Hoüll. 8vo. Paris, 1853.

Katechismus der Pferdezucht, zugleich eine Beschreibung der Krankheiten der Zuchtthiere und Fullen enthaltend. By Ferdinand Rudolph Jacoby. 8vo. Stolp, 1853. 
1853. Die Nothwendigsten Huf-und Klaneneisen, und die Hauptregeln beim Hufbeschlage. Als Erlänterung zu der von dem stellvertretenden Lehrschmied Carl Friedrich Arndt angefertigten Sammlung von Muster-Hufeisen. By Peter Jessen. 8vo. Dorpat, 1853.

Практическя правила длі руководства прп покупюь лошадеп. By Jacob Johnson. 8vo. St. Petersburg, 1853.

Практиеске наставленіе къ учкежденію п содержанію хозапственныхъ конскихъ заводовъ. Ву А. Keikuatow. 8vo. St. Petersburg, 1853.

Zootechnie ou Science qui traite du choix des animaux domestiques, de leur conservation, de leur rendement et des principales maladies dont ils peuvent être affectées. By Charles Knoll. 2 vols. 8vo. Guebviller, 1853-54.

Grundriss der gesammten Veterinär-Medizin, mit ausfuihrlicher Darstellung aller in sanitäts-und vétérinär-polizeilicher, gerichtlicher, practischer und comparativ-wissenschaftlicher Hinsicht besonders wichtigen Krankheiten. Zum Gebrauche bei Vorlesungen fiir Studirende der Medizin, und zum Selbstgebrauche für Medizinalreferenten, Physicats-und practische Aerzte und Thierärzte. By Johann Martin Kreutzer. 8vo. Erlangen, 1853.

Elementos de Equitacion militar para el uso de la caballeria española ; escritos para los alumnos de la escuela militar de equitacion. By Francisco de Laiglesia y Darrae. 4to. Madrid, 1853.

Études basées sur l'anatomie et la physiologie pour servir à l'élève et au dressage des chevaux de selle. By Doctor P. E. Lamotte. 8vo. Paris, 1853.

Arsberättelse af Direktionen för hästafvels föreningen i Örebro Län. [By Mathias Leyon]. 8vo. Örebro, 1853.

Goda Råd i Hästafwel meddelade Örebro Läns Landtmän af Länets Hästafwels Förening. [By Mathias Leyon]. 8vo. Örebro, 1853.

De l'affection typhoide de l'espèce chevaline et der ses rapports avec la fièvre typhoide de l'homme. By Alexandre Benoit Loiset. 8vo. Lille, 1853.

Premier mémoire sur l'enzootie foudroyante (myélite dorso-lombaire) attaquant toutes les espèces herbivores dans le nord de la France. By Alexandre Benoit Loiset. 8vo. Lille, 1853. 
Gospodarz I., Rolnictwo II., Chodowanie i choroby koni, bydta i 1853. owiec III., Ogrodownictwo IV., Pszczelnictwo. By Ignatz Lyskowski. Svo. Brodnica, 1853.

Choix du Cheval, ou Appréciation de tous les caractères à l'aide desquels on peut reconnaitre l'aptitude des chevaux aux divers services. By J. H. Magne. 12mo. Paris, $1853 ; 1864 ; 4 t h$ eclition, 1875 .

Les Chevaux arabes de la Syrie. By J. Mazoiller. 8vo. Paris, 1853.

Appréciation du cheval, des qualités intrinsiques de cet animal pour le travail et la reproduction; guide-pratique indiquant les caractères à l'aide desquels on peut reconnaître avec précision; la force et le dégré de la force, lo fonds, l'ardeur, la paresse, la mollesse, le train, la vitesse ou la lenteur des mouvements, la nature du cheval, etc. A l'usage des cultivateurs, des éleveurs, des vétérinaires, des officiers de cavalerie, et de tous les propriétaires et amateurs des Chevaux. By Nicholas Jules Minot. 8vo. Paris, 1853.

Nouveau manuel complet de l'éducation et de l'hygiène du cheval. $\mathrm{By}$ Count Louis Edme de Montigny. 18mo. Paris, 1853.

Equitation des dames on guide de l'élève écuyer dédié à Mme. la Vicomtesse Drouyn de L'Huys. By Le Vicomte de Montigny. 8vo. Saumur, 1853 ; 8vo. Paris, 1858.

Lehrbuch der Anatomie des Pferdes mit vergleichender Berücksichtigen der übrigen Haussängethiere und physiologischen Bemerkungen. By Franz Müller. 8vo. Vienna, 1853.

Notice sur la bride à mors régulateur combinée d'apres une découverte physiologique. By Casimir Noel. 4to. Paris, 1853.

Petite théorie de la bride à mors régulateur sans gourmette et a rênes croisées, Extrait de l'équitation naturelle enseignée en vingt-cinq minutes. By Casimir Noel. 12mo. Paris, 1853.

Cavalry: its History and Tactics. By Capt. L. E. Nolan. 8vo. London, 1853 ; 3rd edition, 1860.

In French:-Histoire et tactique de la cavalerie, par L. E. Nolan, capitaine au 15 e de hussards de l'armée royale anglaise. Translated from the English with notes by Bonnean de Martray. 8vo. Paris, 1854. 
1853. De l'equitation qui convient aux Français et des races qui y sont propres. By Pinto. 8vo. Paris, 1853.

Hvilke Love gjaelde for Nedarvingen af Hunspattedyrenes Egenskaber paa deres Afkom, og hvorledes anvendes disse Love til Foraedling af Hesten? Concurrenceafhandling. By Ferdinand Victor Alphons Prosch. 8vo. Copenhagen, 1853.

- Horsemanship or the Art of Riding and Managing a horse, adapted for the guidance of Ladies and Gentlemen, on the Road and in the Field, with instructions for breaking in Colts and Young Horses. By Capt. M. Richardson. 8vo. London, 1853.

Méthode d'équitation sur de grandes lignes. By Colonel Aimé Theodore Marie Rigault de Rochefort. Svo. Paris, 1853.

Skizzen iiber Pferdezucht und Pferdewesen. Gesammelt auf einer Reise in England und Frankreich im Jahre 1852. [By Genl. Ritter]. 8vo. Vienna, 1853.

In Danish :-Skizzer, Hesteavl og Hestevaesen verlkommende. Samlede paa en Reise i England og Frankrig i Aaret 1852. Oversat fra det Tydske ifolge Foranstaltning af Kammerherre, forste Staldmester v Roepstorff. 8vo. Copenhagen, 1854.

Lehrbuch der Arzneimittellehre für Thierärzte. By M. F. Roell. 8vo. Vienna, $1853 ; 1866$.

Nowy lekarz, czyli sposoby leczenia koni, bydla, owiec i innych domowych zwierzat, tudziez karmienia i rozmnazania onych. Tlumaczenie uwienczonejo dziela pezez towarzystwo ekon. w Marchii, Edycya siodma, calkiem przerobiana i pomnozona wedlug 1790 wydanie orygin. By Johann Nicolaus Rohles. 12mo. Warsaw, 1853.

A Hindustani Work on the qualities of Horses. By Sa'ádatyár Khan. Lucknow, A.H. 1269, [A.D. 1853].

Guido allo studio dei contagi e simili morbi specifici. By Giulo Sandri. 16mo. Verona, 1853 ; Milan, 1857.

Homöopathischen Thierheilkunst. Ein ganz eigenthümlich eingerichtetes und dadurch sehr leicht fassliches und schnell Rathgebendes Hiulfsbuch fur jeden Viehbesitzcr, zunächts aber für den bei vorkommenden Viehkrankheiten meist ohne Rath und Hülfe dastehenden Landmänner, wonach er seine erkrankten Pferde, Rinder, Schafe, Schweine und Hunde auf die einfachste, 
schnellste, sicherste und wohlfeilste Art auf homöopathischen 1853. Wege selbst heilen und worin er vieles Wissenswerthe in Bezug auf diese Thiere erfahren kann. By Johann Christoph Schaefer. 8vo. Nordhausen, $1853 ; 1856$.

In English :-Veterinary IIomœopathy. Translated by W. H. Smith. 8vo. New York, 1855 .

Histoire critique et raisonnée de la production chevaline sur l'herbage de Saint Gervais (Vendée). By Charles de Sourdeval. 8vo. Paris, 1853.

The Structure and Diseases of the Horse, with their Remedies; also Practical Rules to Buyers, Breeders, Breakers, Smiths, etc. By W. C. Spooner. To which is prefixed an Account of the Breeds of the United States. Compiled by Henry S. Randall. 8vo. Auburn, 1853.

Nouveau traité de Matière Médicale de thérapeutique et de pharmacie vétérinaire. By François Tabourin. 8vo. Paris, 1853; 3rd edition, 2 vols. 8 vo. Paris, 1875 .

De la production chevaline en France et de l'intervention de l'Etât. By Eugène Tisserant. 8vo. Lyons, 1853.

Tydsskrift for veterinairer, redigeret og udgivet af $\mathrm{H}$. Bendz og H. Bagge, Lectorer ved den kongl. danske Veterinairskole. 8 vo. Copenhagen, 1853, etc.

Mittheilungen aus dem Innern von Russland zunächst für Pferdeliebhaber. Bericht des Professors der Dorpater Veterinärschule, Collegienrath's Fr. Unterberger, iiber die von ihm in den Sommerferien 1851, gemachte Reise. Gedruckt auf Verfügung der Oberbehörde. By Friedrich Unterberger. Svo. Dorpat, 1853.

In Russian :-Извчестія изъ внутренншхъ туъерніп, прену щественпо для люътелен лопалег. Отчегт профессоря дерптскаго встеринарнаго учнлища коллежкаго совьника Феодора Унтержергера опољзћћ. совершепноп пш во время.лॐтншхь вакаціп въ 1851 ощъ году. 8vo. Dorpat, [1853].

Du lait chez la fenme dlans l'état de santé et dans l'état de malarlie. Mémoire suivi de nouvelles recherches sur la composition du lait chez la vache la chèvre, la jument, la brebis et la chienne. By Dr. Max Vernois and Alfred Becquerel. 8vo. Paris, 1853.

Essai historique sur l'organisation de la cavalerie légère et principalment sur l'arme des chasseurs à cheval. By Baron Francois Simon Marie Jules Gay de Vernon. 8vo. Paris, 1853. 
1853. Manuale popolare di veterinaria a comodo d'ogni proprietario di Cavalli. By Alessandro Volpi. 8vo. Padua, 1853.

Ueber die Pferderace der Insel Oesel. Eine Abhandlung welche zur Erlangung der Magisterwiirde in den Veterinair-wissenschaften verfasst hat und mit Genehmigung des Hochverordneten Conseils der Dorpatschen Veterinairanstalt öffentlich vertheidigen wird Gottfried Weidemann aus Livland. By Gottfried Weidemann. 8vo. Dorpat, 1853.

Djurens sjukdomar och farsoter samt medlen till deras behandling och botande, jemte ett bihang. By Ludwig Westerberg. 8vo. Stockholm, 1853.

Die gewöhnlichsten innerlichen Krankheiten der Pferde und deren Heilung. By W. Winiker. 8vo. Tönning, 1853.

Rapport adressé à M. le Ministre de l'interieur sur une nouvelle épizootie qui a attaqué en 1851 et 1852 , des étalons et des juments poulinières des Hautes Pyrénées. By Yvart. 8vo. Paris, 1853.

1854. Ansichten uiber die auf dem Continente gemachten Versuche die Pferderace zu veredeln. Anon. 8vo. Comorı, 1854.

Compendium der praktischen Pferdekenntniss znm Unterricht für die Regiments-Schule des Königlich $3^{\mathrm{e}}$ Artillerie-Regiments. Als Manuscript gedruckt. Anon. 8vo. Magdeburg, 1854.

The Equestrian. A Handbook of Horsemanship containing plain practical rules for Riding, Driving, and the Management of the Horse: Anon. 12mo. London, [Circa 1854].

The Ladies' Equestrian Guide. Anon. 4to. London, 「Circa 18547.

Young Ladies' Equestrian Manual. Anon. 12mo. London, [Circa $1854]$.

Exerceer reglement for det Kgl. danske Cavalerie. Anon. 8vo. Copenhagen, 1854.

Handbock för Hussaren. Anon. $12 \mathrm{mo}$. Örebro, 1854.

Hesteavl og Hestevœsen vedk, overs. foranstaltet ved v. Roepstorff. Anon. 8vo. Copenhagen, 1854.

Instruction för Selning, Sadling och Packing. Anon. 8vo. Stockholm, 1854 . 
Instruction für den zur Abnahme der zu gestellenden Pferdewärter, 1854.

Handwerker und Mobilmachungspferde als Militair-Commissarius commandirten Offizier. Anon. 8vo. Berlin, 1854.

Извђстіе Московскаго обшества охотнпковт конскаго бъга о иснытаніяхъ лошадег въ 1854 году. Anon. 8vo. Moscow, 1854.

Le cheval, le bœuf, l'âne, le chameau, le dromadaire et autres quadrupèdes. Anon. $32 \mathrm{mo}$. Lille, $1854 ; 1857$.

On Cavalry Skirmishing. By a Cavalry Officer. Anon. London, 1854.

Ordonnance sur l'exercise et les evolutions de la cavalerie. 2e partie : Ecole du cavalier, du peloton et de l'escadron à cheval. Anon. 18 mo. Paris, 1854

A Proposal for the Re-Organization of the British Cavalry. Anon. 8 vo. London, 1854.

Systematisch-geordneter Bilder-Atlas für Landwirthe. Erste Section : Landwirthschaftliche Thierkunde. Erste Abtheilung: das Pferd und seine verschiedenen Racen. Anon. folio. Jena, 1854.

Underretning for Veterinaireleverne ved den kgl. Veterinairskole. Anon. 4to. Copenhagen, 1854.

Vollständiges Vieharzneibuch für kleine und grosse Landwirthe, sowie für Pferde-und-Viehbesitzer uiberhaupt. Mit besonderer Riucksicht auf Geburtshïlfe und Operationen, etc. Herausgegeben von drei praktischen Thierärzten. Anon. 8vo. Stuttgart, 1854.

Sketches in Stable, Road, Park, and Field. By Henry Alken. obl. 4to. London, 1854.

Die Fusskrankheiten der Pferde und des Rindviehes. By Matthias Anker. 2 vols. 8 vo. Bern and Zurich, $1854 ; 1857$.

On Outpost Duty. Cavalry. By Arentschildt. London, 1854.

Insuffisance en France, du cheval de guerre et de luxe; possibilité de l'obtenir en créant, dans les regiments de cavalerie, des écoles d'éleveurs au moyen du cheval plastique du Dr. Auzoux. By Louis Auzoux. 8vo. Paris, 1854.

Bibliografia militare italiana antica e moderna. By Mariano d'Ayala. 8vo. Turin, 1854. 
1854. Pferdezucht und Pferderennen Ein Wort zur Aufmunterung. By Georg Friedrich Bandow. 8vo. Berlin, 1854.

Zootechnie. Traité des maniements, des épreuves et des moyens de contention et de gouverne qu'on emploie sur les espèces domestiques chevaline, bovine, ovine et porcine, suivi de la coupe des animaux de boucherie en France et en Angleterre. By Antoine de Bardonnet des Martels. 8vo. Paris, 1854.

There is a German translation of this by Dr. C. H. Schmidt. 8vo. Weimar, 1856.

Oeurres complètes de F. Baucher. Méthode d'Equitation, basée sur de nouveaux principes, revue et augmentée ; suivi des Passetemps Equestres, Dialogues sur l'Equitation, nouveaux moyens équestres, derniers innovations, examen retrospectif, nouveau travail raisonné avec le caveçon. 8vo. Paris, $1854 ; 11$ th edition, 1859 ; 12th edition, 1864 ; 13 th edition, 1867.

Traité abrégé des connaissances extérienrs du cheval, ou moyens de reconnaitre l'aptitude des chevaux al1x differents services. By Louis Auguste Benjamin. 12mo. Paris, 1854.

Handbok i Djur-Medicinen. By Peter Magnus Billmann. 8vo. Ekesjo, 1854.

Veiledning i Rideundervijsningen ved Ritmester Carl Julius v. Bruhn. 8vo. Copenhagen, 1854.

Del capostorno e balordone dei cavalli. Utile ai proprietari e tenitori d'animali domestici. By Jacques Bujault. 18mo. Paris, 1854.

Cirujia Veterinaria By Nicolas Casas de Mendoza. 4to. Madrid, 1854.

Traité d'anatomie comparée des animaux domestiques. By Jean Baptiste Auguste Chaveau. 8vo. Paris, 1854.

There is a Translation of the above in English by George Fleming. 8vo. London, 1873.

Review of William Youatt's publication called 'The Horse.' By Bracy Clark. 4to. London, 1854.

Traité de physiologie comparée des animaux, considérée dans ses rapports avec les sciences naturelles, la médecine, la zootechnie et l'économie rurale. By Gabriel Colin. 2 vols. 8vo. Paris, $1854-1856$; $1871 ; 1872$.

Horse-shoeing as it is done, and as it ought to be. A letter addressed to the President and Members of the Saint John Agricultural Society. By A. Cuming. 8vo. London, 1854. 
The Modern Horse-Doctor, containing practical observations on the 1854. canses, nature, and treatment of disease and lameness in horses. By George H. Dadd. 12mo. Boston, 1854.

Traité de locomotion du cheval relatif à l'équitation. Nouvelles proportions. By Pierre Joseph Isidore Daudel. 8vo. Saumur, 1854 .

Principes généraux du cavalier arabe. By General Daumas. 32mo. Paris, 1854 ; $3 r d$ edition, 1855.

A German Translation of this by Lientenant Graeje. 8vo. Magdeburg, 1855.

Le Cheval de Guerre. By General Melchior Joseph Eugène Daumas. 32mo. Paris, 1854 ; 1855.

A German Translation by Lieutenant Graefe. 8vo. Nagdeburg, 1855 .

Verzeichniss von thierärztlichen Schriften. By Dieterrichs and Reckleben. 8vo. Berlin, [1854].

Пояснптельння замъччанія о Московскихъ конскихъ бъгахъ. Ву M. Dolomanow. 12mo. Moscow, 1854.

Veterinary Medicines, their Actions and Uses, and the diseases of the Domesticated Animals. By Finlay Dun. 12mo. Ediuburgh, $1854 ; 8$ vo. $1859 ; 1864 ; 1874 ; 1878 ; 1882$.

Die äussere Pferdekenntniss. By Arnold Dürler. folio. St. Gallen, 1854.

Praktisches Handbuch fur Oekonomen, Pferdeziichter, Pferdehalter und Pferdeliebhaber, über Pferdekeuntniss, Pferdehandel, die Entwickelung, Geburt und Ausbildung des Fohlens und die Heilmittel der gewöhnlichen Krankheiten des Pferdes. By Friedrich August Ehrhardt. 8vo. Insterburg, 1854.

Question importante négligée pendant la paix, rappelée par la guerre. l'élévage, le commerce, et la remonte. By Le Marquis d'Eurville de Grangues. 8vo. Paris and Caen, 1854.

Lehrbuch iiber die Diätetik oder Gesundheitspflege der landwirthschaftlichen Hausthiere. By Johann Ernst Ludwig Falke. 8vo. Leipzig, 1854.

Specielle Veterinär-Nosologie und Therapie. By Johann Ernst Ludwig Falke. 8vo, Leipzig, 1854. 26 
1854. Instruction de la Cavalerie. Nouvel état de la question. 1. Lettre à M. le comte d'Aure sur son ouvrage de $1853 ; 2$. Quelques observations sur la méthode de M. Baucher, dans son application à la cavalerie; 3. Notice et historique de l'Equitation Militaire jusqu'à ce moment. By Antoine Flandrin. 8vo. Saumur and Paris, 1854.

Das Seelenleben der Thiere insbesondere der Haussäugethiere im Vergleich mit dem Seelenleben des Menschen. Vortrage, gehalten zu Karlsruhe in der Gesellschaft 'Eintracht' im Winter 1853-54. By Christian Joseph Fuchs. 8vo. Erlangen, 1854.

Guide du Sportsman, ou traité de l'entrainement et des courses des chevaux. By Eugéne Gayot. 12 mo. Paris, $1854 ; 1865$.

Mittheilungen ans der thierärztlichen Praxis im Preussischen Staate. Mit Bewilligung Sr. Excellenz des Herrn Staatsministers v. Raumer aus den Veterinair-Sanitäts-Berichten der Koniglichen Regierungen. By August Carl Gerlach. 8vo. Berlin, 1854.

Vergleichende Odontographie. Darstellung des Zahnsystems der lebenden und fossilen Wirbelthiere. By Christopher Gottfried Giebel. 4to. Leipzig, 1854.

Рыспстые заводы въ россіп, составленпые изъ оппсеп владыльцевъ заводовъ комитета пмператорскпхъ ьыспстыхъ бћговъ раскоряднетялемъ. By N. Grajewski. 8vo. St. Petersburg, 1854.

L'Agriculture délivrée, ou Moyens faciles pour retirer de la terre quatre fois plus de revenue qu'elle en rapporte généralment. Cet ouvrage apprend aussi à préserver les chevanx et les mulets de la fluxion périodique. By Eugéne Grollier. 8vo. Louhans and Paris, 1854.

Caveleri-Emancipation. By C. Hoegh Guldberg. 8vo. Itter, 1854.

On Hysteria in the Mare, with illustrative cases. By William Haycock. 8vo. London, 1854.

Bemerkungen über Gegenstände der Militarischen Veterinärkunde. By F. Heusmann. 8vo. Hannover, 1854.

Société impériale et centrale d'agriculture. Du métissage général des chevaux français de selle et de carrosse par le pur sang anglais, comme moyen d'améliorer les premiers. Note lue à la Société imp. et centrale d'agriculture dans sa séance du 22 mars, 1854. By Huzard. 8vo. Paris, 1854. 
Fremstilling af Hesteavlens Tilstand i Danmark, ledsaget af en kort 1854.

Veiledning til den heusigtmaessigste Behandling af Hestetillaeget.

En af det Kongl-danske Landhunsholdningsselskab kronet Prisafhandling. By Knud Christian Keller. 8vo. Copenhagen, 1854.

Die Beurtheilung des Pferdes beim Ankauf. Vortrag in der Lesegesellschaft des Landwirthschaftlichen Hauptvereins zu Miunster, gehalten von dem Mltgliede. By Friedrich Wilhelm Carl Theodor Maria von Krane. 8vo. Münster, 1854.

Compendio de las generalidades de patologia y terapeutica veterinarias con nociones de policia sanitaria. By Ramon Llorente Lázaro. 8vo. Madrid, 1854.

De l'origine commune des chevaux arabes et des chevaux barbes. By A. Le Clercq. 8vo. Paris, 1854.

Notice détaillée sur la manière adoptée en Afrique pour établir les hommes et les chevaux de la cavalerie au bivouac. By Lecomte. $18 \mathrm{mo}$. Paris, 1854.

Saggio sui vizij redibitorij in veterinaria legale. By Alessandro Lemoigne. 8vo. Milan, 1854.

Tratrato della conformazione esterna del cavallo e dei principal animali domestici. By Carlo Lessona. 8vo. Turin, 1854.

Histoire naturelle vétérinaire. De l'œstre du cheval. By Guiseppe Lessona. 8vo. Paris, 1854.

Translated by L. Prangé. The Original appeared in the 'Giornale di Veterinaria' of 1852, then under the direction of the Brother, Carlo Lessona.

Guia de alveitar, ou vade-mecum de veterinario: memorial patologico e therapeutico, formulario pharmacologico. By Jose Ferreira de Macedo Pinto. 8vo. Coimbra, 1854.

The Life of a Race Horse. By John Mills. 12mo. London, 1854 ; 5th edition, 1856.

A French translation entitled:-Grandeur et décadence d'un cheval de course. 8vo. [Brussels], 1862.

Petit traité, ou Manuel dédié uniquement aux propriétaires, colons et fermiers des campagnes, concernant les maladies les plus contagieuses et dangereuses contre les animaux domestiques, manière de les connaitre et moyens pour les préserver et pour les traiter. By Israel Minvielle. 12mo. Bayonne, 1854. 
1854. Tracts on Cavalry Tactics. By Mortimer. 8vo. London, 1854.

Lehre vom Exterieur des Pferdes oder von der Beurtheilung des Pferdes nach seinen äusseren Formen. By Franz Müller 8vo. Vienna, $1854 ; 1868 ; 1875$.

Commentaires historiques et élémentaires sur l'équitation et la cavalerie. By Lieut.-Col. P. Mussot. 8vo. Paris, 1854.

Equitations-Studien. Mit besonderer Riicksichtsnahme auf den Unterricht in den Artillerie-Equitationen. By Alexander von Nadosy. 8vo. Vienna, 1854.

Afhandling om Danmarks Hesteavi. By M. L. Nathanson. 8vo. Randers, 1854.

Historisk-factisk Oplysning om vore Stutteriherrers soelsomme Commerce paa Hestenes Gebeet, fra Begyndelsen af dette Aarhundrede til Dato. Ned et Par Alvorsord til vor constituerede Landstutmester og hans Embedsvicarius, som skyldigt Svar paa uforskydlt Tiltale. Et Bidrag til Heste-og Menneskekundskab. By Jens Veibel Neergaard. 8vo. Copenhagen, 1854.

Die Kunst passende und fehlerfreie Pferde zu kaufen. By Carl Neubert. 8vo. Rosswien, 1854.

Dressage naturel et immédiat, du cheval; enseignement basé sur un effet physiologique, remarqué sur la bouche du Cheval. Dedié aux éleveurs. By Casimir Noel. 8vo. Paris, 1854; 1855.

An extract was published as:-Science naturelle science exacte. Extrait de la 2e partie du dressage naturel et immédiat du cheval. Enseignement basé sur un effet physiologique remarqué sur la bouche du cheval. Le moyen combiné sera appliqué avec succès à tout animal dont la bouche sera conformée à celle du cheval. 12 mo. Meaux, 1854 .

A M. M. les vétérinaires. Notice sur le feu français ou baume resolutif remplaçant le feu sur les animaux domestiques sans laisser aucune trace. By Jean Olivier. 18mo. Chalons, 1854.

Conseils aux cultivateurs sur l'hygiène pratique des animaux domestiques, ou Moyens de les entretenir en santé. By Mathurin Papin. 12mo. Renus, 1854 ; Paris, 1862.

Һесьды съ земледжцемъо лошадях и рогатомъ скоть йл о томъ что попадег и роготаго скота, должент дъ.тать прп воспи-

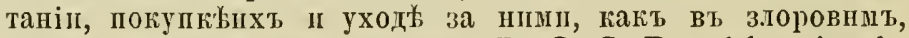
такт й въ больночт состояніп. Ву O. S. Paschkewitsch. 8vo. St. Petersburg, 1854. 
Abbozzo per un trattato di anatomia e fisiologia veterinaria. By 1854. Luigi Patellani. 8vo. Milan, 1854.

Guide dans le commerce des animaux domestiques. By B. Peyrow. 8vo. Nerac, 1854.

Recueil d'éléments d'hippologie, d'après M. M. Girard, le Baron Richerand, Milne Edwards, Lecoq, Gronier, Hurtrel d'Arboval, et Beucher de Saint Ange, destiné aux officiers et sous-officiers de cavalerie, dedié à Son Excellence M. le Maréchal Duc de Saldanha, etc., etc., commandant en chef de S. M. très-fidèle Le Roi de Portugal. By L. de Quillinan. 8vo. Paris, 1854.

Examen du cours d'équitation de M, d'Aure. By C. Raabe. 4to. Marseilles, 1854.

- Treatise on Horse taming. By John S. Rarey. 8vo. Ohio, 1854.

Republished in England as, "The Modern Art of Taming Wild Horses.' 8vo. London, 1858.

Also published with $J$. Mill's 'Directions,' 12 mo. 1858; with Scrutators's 'Horse and Hound,' Svo. 1858; and with J. $H$. Walsh's 'The Horse in the Stable and in the Field,' Chap. XII., Svo. 1866. Two other English editions appeared in 1858. The

- best is that by the Secretary to the first subscription of 5,000 guineas, entitled:-Horse Taming Horsemanship. Hunting. A new Illustrated edition of J. S. Rarey's 'Art of Taming Horses,' with the substance of the Lectures at the Round Honse, and Additional Chapters on Horsemanship for the Young and Timid. 8vo. London, 1859.

In French as:-L'Art de dompter les Chevaux. Traduit de l'anglais et précédé d'une Introduction. By F. de Guaita. 18mo. Paris, 1858. Also another translation:-Dressage du Cheval. La Méthode Rarey, mise à la portée de tous. By le vicomte de R. M. 8vo. Paris, 1858.

In Danish:-Konsten at dressere Heste. 8vo. Copenhagen, 1860.

Le fermier vétérinaire, ou Méthode anssi économique que facile de préserver et de guérir les animaux domestiques, et même les végétaux cultivés du plus grand nombre de leurs maladies. By François Vincent Raspail. 18mo. Paris and Brussels, 1854; $1855 ; 1856$.

In Spanish:-La veterinaria domestica o metodo tan econumico como facil de preservar y curar a los animales domesticos y a los vegetales cultivados de la mayor parte de sus enfermedades. 16mo. Madrid, 1855. 
1854. In Italian:-Il compagnuolo veterinario, ossia metodo economico e facile per preservar y guarire gli animali domestici ed anché $\mathrm{i}$ vegetali coltivati dal maggior numero delle loro malattie. $16 \mathrm{mo}$. Turin, 1856.

In Swedish :-Djur-och Wext-Läkebok-Praktiska anwisningar för landthushällare och djuregare att pä egan hand, efter författerens nya läkemethod, förebygga och behandla husdjurens och de odlade wexternas sjukdomar; samt att sjelf bereda dertill erforderliga, enkla och billiga läkemedel. 12mo. Stockholm, 1857.

Vices redhibitoires. Délai de la garantie. By Eugène Renault, 8vo. Paris, 1854.

L'Épigéonosie, ou la Peste universale qui règne depuis quelques années sur la globe terreste, spécialement dans l'air, l'eau, la terre, les mineraux etc.; mais où sont particulièrement traitées la maladie dite vénérienne des étalons et des juments, la maladie des pommes du terre, etc., et surtont la maladie des vignes. By Jean Baptiste Rhodes. 8vo. Auch, 1854.

Mémoire sur les maladies épizootiques, avec l'indication des remèdes propres à les guérir. By Ricourt. 12mo. Toulouse, 1854 .

Das Pferdefleisch als Nahrungsmittel aller Stände. Eine Anleitung wio dasselbe schmackhaft zubereitet und behandelt wird. Für Herrschafts-Bürger-und Soldatenkiichen. By Rudolph Rutta. $16 \mathrm{mo}$. Pesth, 1854.

Specielle Pathologie und Therapie der nutzbarsten Hausthiere für Thierärzte und gebildete Laien. By Johann Jacob Rychner. 8 vo. Berne, 1854.

Étude de l'appareil reproducteur dans les cinq classes d'animaux vertébrés, au point de vue anatomique, physiologique et zoologique. By G. J. Martin Saint-Ange. 4to. Paris, 1854.

Handbuch der Hausthierzucht. Als Leitfaden beim Uterricht in der Thierzucht auf Ackerbauschulen, nebst einem Anhang uiber englische Thierzucht. By Wilhelm Schönermark. 8vo. Brunswick, 1854.

Remarks on the Transport of Cavalry and Artillery. By Lt.-Col. Shirley. $12 \mathrm{mo}$. London, 1854.

Le Sport à Paris. Coutenant : le Turf la Chasse, le Tir au pistolet et à la carabine, les Salles d'Armes, la Boxe, le Baton et la Canne, etc., les Echecs, le Whist, etc. Edited by Eugène Chapus. 8vo. Paris, 1854, etc. 
Handbuch der Veterinair-Chirurgie. By Georg Strauss. 8vo. 1854. Vienna, 1854.

Landhesleavleni Danmark. Concurrenceskrift. By Franz Louis von Torp. 8vo. Copenhagen, 1854.

Il Veterinario o sia repertorio di zoojatria consecrato all'utile dell' agricoltura ed all progresso della medicina degli animali domestici e scienze affini. Edited by Lorenzo Corvini. 8vo. Milan, 1854, etc.

Delle prinicipali disposizione di polizia veterinaria, nel regno Lombardo-Veneto. By Alessandro Volpi. 8vo. Padua, 1854.

Het Leven der Dieren. By Willem Vrolik. 8vo. Amsterdam, 1854.

Neues Vieharzneibuch oder Unterricht wie der Landwirth seine Pferde, Rindvieh, Schafe, Schweine und Federvieh fiittern, warten und pflegen soll und deren Krankheiten leicht erkennen und wohlfeil, schnell und grindlich heilen kann. Nebst Anleitung zum Betriebe der Viehzucht. By J. E. Wagenfeldt. 8vo. [Circa 1854].

Auch ein Wort an Deutschlands Reiter. Entgegnung der Seegerschen Schrift. Anon. 8vo. Coblenz, 1855.

Corso completo di nosologia e terapia speciale veterinaria ad uso dei veterinari e dei medici che comprende la dottrina delle epizootie e la polizia zoojatrica. Anon. 8vo. Florence, 1855.

Darstellung des Pferddessin, Racen, Farben und Abzeichen. Als Erganzung fiir die Besitzer der ersten und zweiten Auflage von Anleitung zur Kenntniss des Aeussern des Pferdes von Professor Banmeister sowie fuir Pferdezuchter, Pferdeliebhaber, und Pferdebesitzer jeden Standes. Anon. 8vo. Stuttgart, [1855].

Das Dutzend Pferdeziichtungs-Glaubensartikel. Besprochen und verteutscht mit Riicksicht auf die Anordungen, welche für die 143 Hufenpächter der Grossherzoglich Oldenburgischen in Wagrien, Herzogthums Holstein. belegenen Giiter bestehen, durch Schnipp, Schnapp, Schnurr und Baselorum. Anon. 8vo. Hadersleben [1855].

Die Behandlung des Pferdes auf Ritten, nebst einer Anleitung zum Carrière-Reiten und Hecken-Springen; von einem k. k. Cavallerie-Offizier. Anon. 12mo. Vienna, 1855.

Extrait du réglement provisoire sur l'instruction à pied et à cheval dans les régiments d'artillerie. Instruction à cheval. Anon. $32 \mathrm{mo}$. Strasburg, 1855. 
1855. Höchst wichtige Geheimnisse für Pferdebesitzer. Anon. 8vo. Naumburg, 1855.

Homöopatiske Forsog, paa syge Huusdyr. 1 Deel Hesten. Anon. 8vo. Copenhagen, 1855.

Instruction for Exerceerskoleme ved det kgl. danske Cavalerie, Anon. 8vo. Odensee, 1855.

Instruction för Ryttaren. Anon. 12mo. Stockholm, 1855.

La nouvelle Maison rustique, encyclopedie manuel de toutes les sciences et de tous les arts dont un habitant de la campagne, proprietaire, fermier, cultivateur, peut tirer parti, etc., l'hygiène et la médicine domestiques, l'hippiatrique, l'art vétérinaire. Anon. 12 mo. Paris, [Circa 1855]; 3rd Edition, 1857.

Manuel du Cavalier. Ordonnance sur les exercises et les évolutions do la cavalerie, contenant les bases de l'instruction ministérielle du 21 Mars 1847, pour la charge et les feux des armes percutantes. Anon. 8vo. Saumur, 1855.

Regolamento per le corse di cavalli della Societa nazionale. Anon. 8vo. Turin, 1855.

Uddrag af Instructionen for Cavaleri-Exerceerskolers. Anon. 8vo. Randers, 1855.

British Cavalry at Balaklava. Vindication of Earl Lucan. 8vo. London, 1855.

Cavalry Tactics. School of the Trooper, of the Platoon, and of the Squadron. 2 vols. $18 \mathrm{mo}$. Philadelphia, 1855.

Ordonnance sur l'exercise et les évolutions de la cavalerie. Ecole du cavalier à cheval, Carabineers et cuirassiers. $32 \mathrm{mo}$. Paris, 1855 ; Strasburg, 1856.

Public Carriages of Great Britain. 12mo. London, 1855.

De la question Équestre et de Mme. Isabelle à l'école de cavalerie. By Le comte D'Aure. 8vo. Paris, 1855.

Anatomie clastique. Catalogue des préparations destinées à l'étude du cheval et clu bœuf. By Louis Auzoux. 8vo. Paris, 1855.

Anatomie clastique. Tablean synoptique du cheval. By Louis Auzoux. 8vo. Paris, 1855.

Cavalry, its uses in War. By N. L. Beamish. 8vo. 1855. 
Kort fremstilling af hestens bygning og liv, udarbeidet efter opfordring fra krigsministeriet af Prof. Dr. Henrik Bendz. lector i 1855. anatomie og physiologie ved den kongl. danske veterinairskole. 8vo. Copenhagen, 1855.

Le Parfait carrossier. By Louis Berthaux. 8vo. Dijon, 1855; 1862.

Ueber die Verbesserung der Hannoverschen Landes-Pferdezucht durch das Königliche Land-Gestiit zu Celle, nebst Vorschlägen zu dessen weiterer zeitgemässer Fortbildung, so wie zur gleichzeitigen Hebung unserer Pferdezucht durch die Privat-Beschäler. By Hermann Biedenweg. 8vo. Stade, 1855.

Der homöopathische Pferde-Arzt. Eine Darstellung der wesentlichen Erfordernisse fiir Gesunderhaltung des Pferdes, so wie der Grundsätze und Lehren der Homöopathie, mit ausfiihrlicher Angabe ihres Verfahrens zur Heilung der Krankheiten des Pferdes. By Carl Boehm. 8vo. Pesth, 1855.

Boletin de Veterinaria. Periódico oficial de la Sociedad Veterinaria de socorros mútuos. Anon. 8vo. Madrid, 1855.

Neues Thierarzneibuch oder die inneren Krankheiten der Pferde, Rinder, Schafe, Schweine und Hunde und deren Heilnng. By Friedrich Wilhelm Braungardt. 8vo. Leipzig, 1855.

Bulletin de la Société hippique de l'arrondissement d'Abbeville. 8vo. Abbeville, 1855, etc.

Inverse Elementary Tactics of Cavalry comprising simple, compound, and partial inversions, with the application of them to Regimental extensive and auxillary lines, also the conversion of the Squadron. By A. B. C. London, 1855.

Arte de Herrar. By Nicolas Casas de Mendoza. 4to. Madrid, 1855.

Cavalry Officers Journal. Edited by Humbley. 8vo. 1855, etc.

Leçons de science hippique générale ou traité complet de l'art de con. naître, de gouverner et d'élever le cheval. By Louis Baron de Curnieu. 8vo. Paris and Brussels, First part, 1855 ; Second part, 1857.

Haandbog for det Kgl. danske cavaleri. By Allan v. Dahl. 8vo. Kesvig, 1855.

Mémoire sur la contracture pelvienne comparée à la paraplégie sur le cheval. By Louis Demilly. 8vo. Chalons, 1855. 
1855. Nouvelles objections en faveur de la contracture pelvienne sur le cheval faites dans la séance de la société impériale et centrale de médecine vétérinaire du 12 juillet, 1855. By Louis Demilly. 8vo. Paris, 1855.

Praktischer Reitunterricht für Schule und Feld. By C. J. Diepenbrock. 12 mo. Basle, 1855.

Simple observations sur la ferrure. By Charles Dubourdieu. 8vo. Bordeaux, 1855.

Notice sur les races domestiques des chevaux, By Dureau de la Malle. 8vo. [One sheet] Paris, 1855.

Lehrbuch der gesammten Thierarzneiwissenschaft. By Johann Ernst Ludwig Falke. 8vo. Leipzig, 1855.

Der sicher und geschwind heilende Vieh-Arzt, oder Anweisung, wie man die Krankheiten der Pferde, des Rindviehes, der Schafe, Ziegen, Schweine und des Federviehes erkennen und bald und griindlich heilen kann. Nebst praktischer Anweisung zur erkenntniss des Pferdes für den Bauersmann. By F. C. Fischer. 8vo. Einbeck, 1855 .

Le Cavalier. Cours d'equitation pratique. By Victor Franconi. 12 mo. Paris, $1855 ; 1860$.

Veterinary Education....An Inaugural Lecture. By John Gamgee (Senr.). 8vo. London, 1855.

Die Haltung and der Sitz des Reiters. Ein Beitrag zur Geschichte der Reitkunst Mit Benutzung von "Mussot Commentaires sur l'equitation." By Carl Heinrich Eduard Graefe. 8vo. Weimar, 1855.

Deutschlands Sieger auf Englands Rennbahnen. Fiir alle guten Patrioten. By Pegasus [Graefe]. 8vo. Berlin, 1855.

Quelques observations sur l'amélioration des chevaux en Portugal. [By C. H. E. Graefe]. 8vo. Diiben, 1856.

Etat de crise de la production, élève, ses causes, son terme possible. La situation telle qu'elle est, écoles de dressage. By the Marquis d'Eurville de Grange. 8vo. Caen, 1855.

Guide de l'ami du Cheval revue scientifique et pratique. Edited by Count Savary de Lancosme-Brèves. 8 vo. Paris, 1855 , etc. 
Grrundzïge der Physiologie der Pflanzen und Thiere für den Landwirth. 1855. Zum selbst.Studium und für Schulen. Mit vielen in den Text gedruckten Abbildungen. By Wilhelm Hamm. 8vo. Leipzig, 1855 .

In Dutch:-Grundtrekken der natuurkunde van planten en dieren, ten dienste van landbouwers. Translated by C. E. Enklaar. 8vo. Zwolle, 1857.

Letter to Lord Panmure. Pamphlet on Cavalry. By Captain G. A. Hartmann. 15th Hussars. London, 1855.

La Plaine de Caen. Visit à l'établissement hippique de M. Basly. By Charles du Hays. 8vo. Mortagne, 1855.

Notices sur les courses d'Abbeville. Extrait du Bulletin de la Société hippique du mois d'Avril 1855. By E. Hecquet d'Orval. 8vo. Abbeville, 1855.

Light Horse. By Jacob Omnium [Matthew James Higgins]. 8 vo. London, 1855.

Horses and Hounds; a practical treatise on their management. By Scrutator [K. W. Horloch]. 8vo. London, 1855.

2nd edition, to which is added "Taming of Wild Horses." 8 vo. London, 1858.

Dressage par le surfaix cavalier des Chevaux de Cavalerie, d'attelage, et de course, en six et douze leçons. By Madame Marie Isabelle. 8vo. Paris, $1855 ; 1857$.

In English:-Surfaix Cavalier. A new system of breaking in and training horses. The breaking in and training of Cavalry and Race Horses and Hacks in twelve lessons, the breaking in of Carriage Horses in six lessons. 8vo. Londou, 1856.

Thierzeichnen. Erstes Heft. Das Pferd. Anfangsgruinde in Umrissen. By F. Kaiser. 8vo. Carlsruhe, 1855.

Instruktion über die marsch-und dienstfähige Einkleidung der Kavallerie besonders der Landwehr-Kavallerie, nebst einem Auhange iiber Satteldrückshaden und einer Anleitung zur Anfertigung und Anwendung der verschiedenen Strohmatten, Sattelpolster und anderer Hulfsmittel. By August Ferdinand Kirchhoewell. 8vo. Leignitz, 1855 .

Der Hausthier-Arzt. Ein zuverlassiger Rathgeber bei der Behandlung erkrankter Haussäugethiere mit besonderer Beriicksichtigung des Rindviehs. Nach den bewährtesten Heilmethoden der Wissenschaft und Praxis fur Landwirthe, Viehbesitzer und Thierärzte By F. X. Koerber. 8vo. Berlin, 1855. 
1855. A földmiveles es allattenyesztes. Utmutatas a mezei gazdasag sikeres es mineltöbb haszonnali üzesere. A hetedik kiadas utan fordította Galgoczy Karoly. By J. G. Koppe. 8vo. Pestl, 1855.

Lehrbuch der gerichtlichen Veterinär-Medizin. Zum Gebrauche bei Vorlesungen und zum Selbstunterrichte fuir Thierärzte, Gerichtsärzte, Richter, Advocaten, Landwirthe. By Johann Martin Kreutzer. 8vo. Erlangen, 1855.

Der Kavallerie-Unterofficier als Reiter, Reitlehrer und Zugfuihrer. Ein Leitfaden zur Selbstbelehrung für Kavallerie-Unterofficiere der Linie und Landıehr, sowie für 1 jährige Freiwillige der Kavallerie. Als Anhang: Der Felddienst der Kavallerie, und der Gar. nison-Wachdienst. By Herrmann Kruge. 8vo. Guhrau, 1855.

Elementos de patología especial veterinaria. By Ramon Llorente Lázaro. Svo. Madrid, 1855.

Aphorismen über Pferdezucht, den Sachsischen Landwirthen. By Gottfried Leberecht Lommatzsch. 8vo. Meissen [1855].

Memorabilien der Veterinär-Medizin in Russland. Herausgegeben von dem Verein der praktischen Thierärzte in St. Petersburg. Edited by Philipp Ludwig Theodor Busse. 8vo. St. Petersburg, 1855 , etc.

Traitement de la maledie de sang, ou Sang de rate chez les animaux domestiques. By N. J. A. Minot. 8vo. Meaux, 1855.

Hiilfsbuch beim theoretischen. Unterricht der Cavalleristen für jün gere Offiziere und Unter-offiziere. By von Mirus. 8vo. Berlin, $1855 ; 1868 ; 1874 ; 5$ th edition, 1877.

In English :-Cavalry Field Duty. London, 1873.

In French :-Aide-Mémoire du cavalier pour servir à l'instruction théorique des jeunes officiers et des sous officiers par le général Von Mirus. Translated by L. Le Maitre. 121no, Paris, 1874.

Progression du dressage des jeunes chevaux d'armes. By Count Louis Edme de Montigny. 8vo. Saumur, 1855.

Enteralgiologia Veterinaria. By Silvestre Blazquez Navarro. Svo. Madrid, 1855.

Un mot aux cultivateurs. Veritable hygiène du cheral. By Casimir Noel. 8vo. Paris, 1855.

Les Poulinières malgré elles. [By F. Person]. 8vo. Caen, 1855. 
Conseils du vétérinaire, ou moyens de conserver en santé les animaux 1855. de la ferme, de les secourer dans les maladies subites et dangereuses. By G. A. Pétry. Svo. Brussels, 1855.

Den lille praktische Stallmästereen, en oumbarlig handbok för Hästvänner och älskare af den moderna Ridkonsten. By B. H. Petterson. 8vo. Stockholm, 1855.

Lehrbuch der Hufbeschlagslehre mit Inbegriff der Lehre vom Klauenbeschlage. By Johann Pillwax. Svo. Vienna, 1855; 1871.

Hestens Ydrelaere og Pleie, et Udkast udarbeidet efter Opfordring fra krigsministeriet. By Ferdinand Victor Alphons Prosch. 8vo. Copenhagen, $1855 ; 2$ vols. 1860.

Improved Art of Farriery. By W. H. Prosser. [Circa 1855].

Horses, Hunting, and the Turf. By Major Rose. 8vo. Edinburgh and London, 1855.

Ueber Zäumung der Pferde, nebst kritischer Beschriebung der verschiedenen Zäumungs-Instrumente. Besonderer Abdruck aus Tennecker's Jahrbuch fiir Pferdezucht, Pferdekenntniss u. s. w. auf das Jahr, 1855. By Adolph Rueff. 12mo. Weimar, 1855.

Leitfaden in der Pferde-Kenntniss für Unteroffiziere der Kavallerie und Artillerie. By Johann Eduard Sabel. 8vo. Berlin, 1855.

Précis de la guérison de la morve chronique tuberculeuse du cheval, résultant de quarante années d'experiences opiniâtrément suivies, quoique coïteuses, et d'études approfondies sur cette matière. By J. F. Sage. 8vo. Aurillac, 1855.

Nuevo método de embocar bien todos los caballos y tratado sucinto de equitacion, para obtener buena mano de brida, adquirir firmeza en poco tiempo y saber ayudar al caballo, todo bajo principos originales, sencillos.y fáciles de comprender y ejecutar, por el Intendente D. Juan Segundo, comendador y caballero de varias reales y distinguidas órdenes, asi nacionales como estranjeros, sócio fundator de la Sociedad de Fomento de la cria caballar en Espana, e inventor de los bocados que llevan su nombre, y de un estribo de seguridad para las señoras a caballo. By 4to. Madrid, 1855 .

Remèdes et traitements pour les maladies des chevaux, rangés par ordre alphabétique et recueillis, d'apres les auteurs les plus distingués. By Thiriet-Stévenin. 8vo. Mézières, 1855. 
1855. O koniach, czyli krotki zbior zasad poznawanie, chodowania, leczenia i kucia koni. Drugie wydanie poprawione i pomnozone. By B. T[ykel]. 12mo. Warsaw, 1855 .

Törvényszéki állatorvostan. Közsegi orvosok, állatorvosok, közsegi döljárók számará. Kivonathan szerkeraté Posgay János Nyole fametszetiu ábrával. By Johann Elias Veith. 8vo. Pesth, 1855.

Catechism of Equitation, for the use of Cadets at Sandhurst College. By J. H. T. Warde. 12 mo. London, 1855.

1856. An Account of Tom Thumb, who trotted 100 miles in 10 hours and 7 minutes. Anon. 12mo. London, [Circa 1856].

De l'amélioration de la race chevaline en France. Anon. 8vo. Angoulême, 1856.

Den erfarne och pålitliga Hästläkaren, eller Bepröfwade och godkände botemedel för de mest gangbare hästsjukdomar. Samlede ur gamla och förfarne mäns och goda husballares skrifter. Till Landtmäns samt Dragoners, Ryttares och Kuskers tjenst. Anon. $12 \mathrm{mo}$. Stockholm, 1856.

Diccionario de Medicina, cirujía, farmacia, medicina legal, física, química, botánica, mineralogia, zoologia y veterinaria, sacado de las obras de Nysten, Bicheteau, O. Henry, J. Briard, Jourdan, etc., Aumentado con mas de 800 voces, e ilustrado con laminas intercaladas en el testo. Anon. 8vo. Madrid, 1856.

Manuel du maréchalerie rédigé par le conseil d'instruction de l'école de cavalerie, et approuvé par décision de M. le ministre de la guerre, en date du 31 octobre 1849 , pour l'usage des élèves maréchaux de l'école de cavalerie. Anon. 16mo. Saumur, 1856.

Reglement wegen Gestellung, Answahl und Abschätzung der Mobilmachungs-Pferde in der Provinz Brandenburg. Anon. 4to. Berlin, 1856.

Sichere Anleitung die Pferde zum (Yrabenspringen abzurichten. Durch langjährige Erfahrung erprobt von Schmidt Waldt. [pseud.] 8 vo. Eilenberg, [1856].

Théorie dans les chambres ou dans les écuries 9e régiment de cuirassiers. Anon. 8vo. Dijon, 1856.

Udtog af Instructionen for Caalleri-Exerceerskolerne. Anon. 8vo. Nestred, 1856.

Вйздка верховоп лошади. Соста впль гр. Ге. Санктпетербург'ь. Въ типографіп главнаго штаба его пмераторскаго величества по военноу чебпым заведеніямъ. Anon. 8vo. St. Petersburg, 1856. 
L'Amazone. Giomale della Societa nazionale delle corso. Edited 1856. by Puxeddla. 4 to. Turin, 1856, etc.

Annuaire officiel des steeple-chases. Edited by Mévelle, 1856.

Multiplication et amélioration des espèces chevaline, bovine, porcme et ovine dans le département d'Ille-et-vilaine. By Pierre Bellamy. 8vo. Rennes, 1856.

Manuel de médicine et de chirurgie vétérinaires. By Berthier. 8vo. Paris, 1856.

Maison rustique du XIX esiècle, contenant les meilleures méthodes de culture usitées en France et à l'étranger, etc. Avec 2,500 gravures représentant les instruments, machines, appareils, races d'animaux, arbres, plantes, légumes, serres, bâtiments ruraux etc. Terminé par des tables méthodique et alplabétique, etc., etc. 8vo. Paris, 1856.

The Articles on Hippology and the Veterinary Art are by Buegnot, Bouley, Maillard, Moll, Pressat, Renault, Rigot, and Yvart.

Honöopathischer Haus-und Schiffsarzt. Darstellung der wichtigsten Krankheiten und deren Behandlung nach homöopathischen Grundsätzen vom Doktor Ludwig Reichenbach. Nebst einem Anhang; Die wichtigsten Krankheiten der Hausthiere und deren homöopathische Behandlung vom Rathsthierarzt Carl Boehm. 8vo. Leipzig, 1856.

Tableaı synoptique d'embouchures comparées à l'usage des officiers, sous officiers de cavalerie et des amateurs de chevaux. By $\mathbf{R}$. de Bourg. folio. Nancy, [Circa 1856].

Die Augenkrankheiten der Pferde sowie anderer Hausthiere und deren Heilung. By Friedrich Wilhelm Braungardt. 8vo. Erfurt, 1856.

Hints to Horsemen, showing how to make money by Horses. By Harry Hieover [Charles Brindley]. 8vo. London, 1856.

I.e Cheval. By L. Marquis de Bruslard. [One sheet] 8vo. Paris, 1856.

Traité de menuiserie, a l'usage des carrossiers. By J. Chrys. Charpentier. 8vo. Paris, 1856.

Manuel de harnachement, à l'usage des troupes à cheval. By Capt. Laurent Cogent. 8vo. Paris, 1856. 
1856. Du moyen le plus propre d'utiliser la chair du cheval, de l'âne et du mulet. By A. Daunassans. 8vo. Toulouse, 1856.

Traité théorique et pratique de l'action rédhibitoire dans le commerce des animaux domestiques; contenant : la législation, la doctrine et la jurisprudence sur la matière ; la définition des vices rédhibitoires; l'explication détaillée des règles de la procédure, un Formulaire de tous les actes nécessaires et une table chronologique des jugements et arrêts. By Oscar Dejean. 8vo. Bordeanx, 1856 .

La gale des poules sur le cheval. Extrait du compte rendu de la Société Vétérinaire de la Marne (annee 1847). By Louis Demilly. 8vo. Rheims, 1856.

The Post and the Paddock : with Recollections of George IVth, Sam Chifney, and other Turf Celebrities. By the Druid [Henry Dixon]. 8vo. London, 1856.

Das neue herzogliche Marstallgebäude in Gotha. Auf höchsten Befehl Seiner Hoheit des regierenden Herzogs Ernst von SachsenCoburg-Gotha. By G. Eberhard. folio. Berlin and Potsdam, 1856.

O chowie i ulepszeniu rass koni z pogledem na chow koni w Anglii. By Philipp Eberhard. 8vo. Warsaw, 1856.

Leitfaden für den theoretischen Unterricht wie dieser in der Königlichen Militär-Reitschule betrieben wird, nebst einem aphoristischen, auf das nähere Verständniss der Reit-Instruktion zielenden Vorwort. By Carl Franz Michael von Elpons. 8vo. Berlin, 1856 ; 8vo. Hannover, 1877.

La système Wedlake pour le concassage des avoines, ou Moyen économique de nourrir un cheval pour à peu près un franc par jour. By Fenwick de Porquet. 8vo. Paris, 1856.

Catecismo elemental de agricultura prática, zootecnia, horticultura, jardinería, montes y plantios, con una breve reseña de economía $\mathrm{y}$ administracion rural, $\mathrm{y}$ un sencillo método de agricultura $\mathrm{y}$ aforo de líquidos y áridos. By Juan Zoilo Fernandez y Perez. $16 \mathrm{mo}$. Madrid, 1856.

Contains a special chapter on the Breeding and Management of the Horse.

La Morve et le farcin ne sont plus incurables. By Gautier-Mille. 8vo. Paris, 1856. 
Ressources en chevaux que la colonie d'Afrique offre à la consomma- 1856. tion de l'armée. By P. Eugène Géraud. 8vo. Paris, 1856.

Pedigree of the Thorough-Bred Horse, from the original sources. Comprising the most successful covering Stallions for the year. By W. J. Goodwin. folio. London, 1856.

Vollständiges Vieh-Arzneibuch. Oder gründlicher Unterricht alle innerlichen und äusserlichen Krankheiten der Pferde, der Schafe, Ziegen, Schweine und Hunde zu erkennen und zu heilen. By Johann Friedrich Gottlieb Grosskopf. 8vo. Prim, 1856.

Méthode de l'architecte en voitures. By Amable Guillon. 4to. Paris, 1856.

Traité complet ou trace général de tout l'équipage concernant le charron-carrossier, le serrurier et le sellier. By Amable Guillon. 4to. Paris, 1856.

Trattato popolare pel buen governo, per la moltiplicazione e pel miglioramento degli animali che servono alla economica campestre. By Giuseppe Haidvogl. 8vo. Mantua, 1856.

Das Pferd bei den Arabern. Aus dem VI. und VII. Bande der Druckschriften der philosophisch-historischen Classe der kaiserlichen Akademie der Wissenschaften besonders abgedruckt. By Dr. Freiherr Joseph v. Hammer-Purgstall. 4to. Vienna, 1856.

Turkish Cavalry Drill. By G. A. Hartmann. 12mo. London, 1856.

Thierärztliches Handbuch zur Belehrung für Landwirthe, Viehbesitzer, Thierärzte und Agenten von Viehversicherungs-Gesellschaften. Enthaltend das Wichtigste der Gesundheits-und Krankheitslehre der nutzbaren Hausthiere. By Julius Heinrich Heinrich. $12 \mathrm{mo}$. Magdeburg, 1856.

Lœren om de veterinaire Lœgemidler. By C. H. Hertwig. 8vo. Copenhagen, 1856.

Precetti elementari di ferratura teorico-pratica. By Georg Hitzinger. $16 \mathrm{mo}$. Milan, 1856.

Die Dressur des Reitpferdes (Campagne-und Gebrauchs-Pferdes) mit Ruicksichtnahme auf die Ausbildung von Soldatenpferden in Abtheilungen. By Friedrich Wilhelm Carl Theodor Maria Krane. 8vo. Münster, 1856.

Ueber das Sprunggelenk der Säugethiere und des Menschen (Aus den Druckschriften der k. Akademie der Wissenschaften). By Carl Langer. 4to. Vienna, 1856. 28 
1856. L'ami de l'éleveur. Reflexions pratiques sur l'espèce chevaline. By Phillippe Ursal Charles de Lastic Saint-Jal. 8vo. Paris, 1856.

Compendio de la Bibliografia de la Veterinaria Española, con algunas noticias historicas de esta ciencia en nuestra patria, y con las reglas de moral á que debe el Veterinario ajustar su conducta facultativa. By Ramon Llorente Lázaro. 8vo. Madrid and Santiago, 1856.

Zur geschichte der Pferdezucht in Mecklenburg. By Georg Chris. tian Friedrich Lisch. 8vo. Schwerin, 1856.

Kurzegefasstes Lexikon der gesammten Haus-und Landwirthschaft in einem Bande. Enthaltend in Artikeln in alphabetischer Reihenfolge den Acker-und Wiesenbau, die Viehzucht, die Thierheilkunde, den Garten-und Weinbau, etc. By William Loebe. 8vo. Leipzig, 1856.

Rapport sur le concours départemental d'animaux réproducteurs, tenu à Lille le 8 Septembre 1855 . By Alexandre Benoit Loiset. Svo. Lille, 1856.

English Cavalry in the Army of the East, 1854 and 1855. Divisional Orders and Correspondence whilst under the Command of Lieut.General the Earl of Lucan. K.C.B. London, 1856.

De la Péripneụmonie. By P. Macé. 12mo. Paimboeuf, 1856.

Leitfaclen für den Cavalleristen bei seinem Verhalten in und ausser dem Dienste. By von Mirus. 16mo. Berlin, $1856 ; 1863 ; 1867$; $1871 ; 1872 ; 1874 ; 1878$.

Another edition edited by G. v. Pelet-Narbonne, 16mo, Berlin, 1878.

Untersuchungen zur Naturlehre des Menschen und der Thiere. By Jacob Moleschott. 8vo. Frankfort, 1856.

Populäre Vorlesungen iiber Reiterei. Zur Unterhaltung und zum Selbstunterricht. By Ludwig Wilhelm Otto Digeon von Monteton. 8vo. Magdeburg and Leipzig, (1856).

Der praktische Hufbeschlag nach einer neuen und äusserst vortheilhaften Methode. Das Resultat vieljahriger und vielseitiger Erfahrungen und Erfindungen auf dem Gebiete der Hufbeschlagkunst. Mit einem Anlange: Die Rändekrankheit bei den Pferden und ihr schädlicher Einfluss auf den Cavallerie-Kriegsdienst inshesondere, nebst radikaler Heilung derselben. By Christoph Mussgnug. 8vo. Augsburg, 1856. 
Manuel d'hippiatrique, d'equitation et d'hygiène à l'usage de tous, ou 1856. étude de la connaissance intérieure et extérieure du cheval. By Lieut.-Col. P. Mussot. 2 vols. 8vo. Paris, 1856.

Nagra Anwisningar om sättet för uppfödande af en större och mera ulbildad Hästrace, Tillegnade Allmogen inom Elfsborg's Läu. By P. N-m. 12 mo. Wenersborg, 1856.

Historisk-factisk Notitser om vore angang fra berömte Stutterivæsen. By Jens Veibel Neergaard. 8vo. Copenhagen, 1856.

Erreurs et vérité. Un mot du système Baucher et sur les freins regulateurs. Offert aux amis du cheval. [By Casimir Noel]. 8vo. Paris, 1856.

Handledning för Ryttare och Hästwänner. By A. v. P. $16 \mathrm{mo}$. Göteburg, 1856.

Compendio de fisiologia veterinaria. By Luigi Patellani. 8vo. Milan, 1856.

Manuale di Anatomia e Fisiologia degli animali domestici. By Felice Perosino. 8vo. Turin, 1856.

Katechismus der Hufbeschlagskunst. Anleitung zum Selbstunterricht in Hufbeschlag. By Anton Heinrich Fritz Peters. 8vo. Schwerin, 1856.

Répertoire historique des chevaux de race pure en France. By Pontet. 8vo. Paris, 1856.

Grundtraek af Huusdyrens almindelige Sunhedspleie, udarbeidet naermest til Brug för Veterinairer og Landmaend. By Ferdinand Victor Alphons Prosch. 8vo. Copenhagen, 1856.

Uddrag af de Frederiksborgske Stutteriers Aarböger. By Ferdinand Victor Alphons Prosch. 8vo. Copenhagen, 1856.

Examen du "Traité de locomotion du cheval," relatif à l'équitation de M. J. Daudel. By C. Raabe. 4to. Marseilles, 1856.

La Locomotion; Histoire des chars, carrosses, omnibus, et voitures de tous genres. By D. Ramée. 12mo. Paris, 1856.

De la Cavalerie. By George de la Vallée de Rarecourt, Marquis de Pimodau. 8vo. Paris, 1856.

Anleitung zur Alterserkenntniss und Alterschatzung der landwirthschaftlichen Haussäugethiere, bearbeitet fïr Thierärzte, Landwirthe und Thierbesitzer. By Renggli. 8vo. Zurich, 1856. 
1856. Note sur les moyens d'augmenter la production animale de la France. By A. Richard. 8vo. Paris, 1856.

Rivista agronomica, Giornale di Agricoltura, Pastorizia, Veterinaria et science affini. Edited by Vincenzo Corsi. 8vo, Naples, 1856, etc.

The Post and the Paddock. By Druid [James Robinson]. 8vo. London, 1856.

The. 2nd edition appeared in the same year, with about 90 additional pages. Part of the above work was first printed in the "Sporting Review."

Turf Pencillings. By the Druid [James Robinson]. 8vo. London, [Circa 1856].

Die Kolik der Pfercle nebst zwei Anhängen über die Schlempmauke des Rindviehes und den Bandwurme der Sämmer. By Christian Rodloff. 8vo. Zirke, 1856.

Immerwährender Trächtigkeitskalender der nutzbarsten Hausthiere, nämlich der Pferde, Rinder, Schafe, Ziegen und Schweine. By August Roederer. $16 \mathrm{mo}$. Weimar, 1856.

Lehrbuch der Pathologie und Therapie der nutzbaren Hausthiere. By Moritz Friedrich Roell. 8vo. Vienna, 1856; 3rd edition, 1867.

In French:-Manuel de pathologie et thérapeutique des animaux domestiques. Translated by J. B. Derache and J. M. Wehenkel. 2 vols. 8 vo. Paris, 3rd edition, 1869.

Domace zivinozdrastvo $\mathrm{v}$ boleznich konj, govedja, ovac, presicev, kozin psov, ali nauk, kako mora kmetovavec svojo zivino rediti, ji streci jo kermiti in ozdravljati. Po nemski spisal. Poslovljena po devetnajstem natisu. S stirimi podobami. By Johann Nicolas Rohlwes. 8vo. Klagenfurt, 1856.

Della garanzia nel commercio degli animali domestici utili, secondo il vigente Codice universale austriaco, aggiuntevi formole di scritture, di contratti, di certificati peritali, etc. Operetta coronata di premio dalla Societa d'Incorraggiamento di Padova. By Giuseppe Nicola Rossi. 8vo. Vicenza, 1856.

Lettres sur les substances alimentaires et particulièrement sur la viande de cheval. By Is. Geoffroy Saint-Hilaire. $12 \mathrm{mo}$. Paris, 1856.

De l'usage alimentaire de la viande de cheval. Leçons faites an Muséum d'histoire naturelle par M. Isidore Geoffroy SaintHilaire. Recueillies et redigées par M. Camille Delvaille. 8vo. Paris, 1856. 
Elementos de Historia Natural Veterinaria. By Fernando Sam- 1856. pedro. 18 mo. Madrid, 1856.

La diathèse typhoïde du cheval et ses manifestations ordinaires dans l'armée. By A. Sanson. 8vo. Paris, 1856.

Ursprung der Rotzkrankheit und sichere Verhiitung derselben, fuir Thierärzte, Aerzte und Pferdebesitzer. By Friedrich Schmidt. 8vo. Attendorn, 1856.

Handbuch der Thierheilkunde, oder Anleitung, die Krankheiten der Haussäugethiere richtig $\mathrm{zu}$ erkennen, $\mathrm{zu}$ beurtheilen und $\mathrm{zu}$ heilen, mit Berucksichtigung der von der Homöopathie empfohlenen Arzneimittel und Angabe der in Deutschland vornehmlich aber in Preussen Geltung habenden polizeilichen und Gerichtlichen Maassnahmen. By Herrmann Seer. 8vo. Glogau, 1856.

Handbuch der Speciellen Pathologie und Therapie. By Werner Theodor Joseph Spinola. 8vo. 2 vols. Berlin, 1856-1858.

In Swcdish:-R Rådgifware för Landtmannen wid Behandling af Husdjurens Sjukdomar efter Dr. W. Th. Jos. Spinola. Translated by G. W. Sjöstedt. 8vo. Upsala, 1856.

Medicina Veterinaria, o sia igiene, fisiologia, patologia e terapia comparata. By Telesforo Tombari. 8vo. Rome, 1856.

Cours d'agriculture élémentaire et d'hygiène vétérinaire professés à la ferme-école des Plaines, canton de Neuvic, arrondissement d'Ussel (département de la Corrèze). By Philippe Comte d'Ussel. 8vo. Paris, 1856.

Die Naturgeschichte der nutzbaren Haussäugethiere. By Johann Elias Veith. 8vo. Vienna, 1856.

Manuel de l'éleveur de chevaux. By Felix Villeroy. 2 vols. 8vo. 1856

Trattato delle malattie epizootiche e contagiose degli animali domestici. By Alessandro Volpi. 8vo. Milan, 1856.

Manuel du roulage et des messageries, à l'usage des voituriers, rouliers, charretiers, entrepreneurs de transport par terre et par eaux, etc. contenant l'hygiène, les principales maladies et le choix des chevaux, classé et mis en ordre alphabétiquement. By Vuillermedunand. 18mo. Lons le Saulnier, 1856.

Calendrier des courses de Courtalain (1855). By le comte Alexandre de Walles. 8vo. Chateaudun, 1856. 
1856. The Horse in the Stable and in the Field; his varieties, management in health and disease, anatomy and physiology. By J. $\mathrm{H}$. Walsh. (Stonehenge), 8vo. London, 1856; London and New York, 1866. Another edition, 1873.

Manual of British Rural Sports, comprising shooting, hunting, fishing, coursing, hawking, racing, boating, pedestrianism, and the various rural games and amusements of Great Britain. By John Henry Walsh. 8vo. London, 1856.

In French:-Le Cheval Anglais; extrait du Manuel du Sport, publié à Londres en 1856 par Stonehenge. Translated by the Count J. de Lagondie. 8vo. Paris, 1860.

185\%. Calendrier officiel des courses de chevaux, publié sous les auspices de la Société d'encouragement pour l'amélioration des races des chevaux en France. By the Secretary of the Society of Encouragement (Jockey Club). Anon. 12mo. Paris, [Circa 1857].

De nieuwe Vee-arts. Practisch handboek voor elken veehouders. Aanwijzende middelen om de ziekten, welke zich dagelijks bij de paarden, het hornvee en de varkens openbaren te behandelen en te genezen. Bij een verzameld en nagelaten door iemand van eene grondige kennis en rijke ondervinding in dit vak. Anon. 8vo. Zierikzee, 1857.

Horse Owner's Guide and Complete Horse Doctor. Anon. $12 \mathrm{mo.}$ New York, [Circa 1857].

Instruction concernant le transport des chevaux ou mulets de remonte par les chemins de fer. Anon. 16mo. Paris, 1857. From the "Journal Militaive Officiel," 1855.

Les Courses de Chantilly, dédiê à Mme X. par un poëte inconnu. Anon. 12 mo. Tours, 1857 .

Magia naturalis innehållande mångfaldiga och säkra underrättelser om Hästars, Oxars, Kors, Qwigors, Tjurars, Kalfwars, Fars, Getters, Swinkreaturs m. m. ans och skötsel, samt botande wid påkommande sjukdomar, hwartill nästan alla läkemedlen hemtas inom Landtmannens eget distrikt. Dessutom åtskilliga tillförlitliga och försokta konster och hushallsgrepp, mett ett ord, det betydligaste af det som uti enskildta hushallningen forekommer, och som med allsingen eller ringa kostnad kan werkställas. Landtmannen till tjenst sammandragen utar de bästa kända hushållares dels tryeta dels otrzeta skrifter. Anon. 12mo. Stockholm, 1857. 
Mémoire adressé à la Société impériale et centrale de médicine vétér- $\mathbf{1 8 5 \%}$. inaire, le 15 décembre 1856 , de la cause réelle qui amène chez le cheval les deviations vicieuses et la plupart des vices du caractère. Du moyen à employer pour conserver sûrement dans les aplombs naturels la race chevaline. Anon. 8vo. [Meaux, 1857].

Petit Manuel du maréchal expert contenant: la manière de nourrir, pauser et gouverner les chevaux. Anon. 18mo. Epinal, 1857.

Praktische Anleitung zum Ankauf oder zur Abnahme von Pferden; zur Behandlung des Pferdehufs und dessen Beschlages, nebst Ansicht iiber Pferdeziichtung, für jiingere Kavalleristen Landwirthe und Pferdeliebhaber, von einem alten Kavallerie-Offizier. Anon. 8vo. Ratibor, 1857.

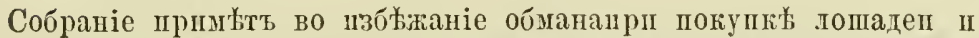
коровт. Сь прнсовокупленіемь правпль о ковке лоподеп, обязаниостен кучера но содерліаніп коровт. Anon. $24 \mathrm{mo.}$ St. Petersburg, i 1857.

Mémoire sur les tumeurs osseuses des jarrets du cheval (éparvins, courbes, et jardons). By Louis Alasonière. 8vo. NapoleonVendée, 1857.

Die Influenza der Pferde, mit besonderer Beriicksichtigung der Militar-Pferde. By Christian David Albrecht. 8vo. Potsdam, 1857.

Veterinär-Polizei und gerichtliche Thierheilkunde in Preussen für Thierärzte, angehende Departements-und Kreis-Thierärzte, Viehbesitzer, etc. By Johann Heinrich Herrmann Anacker. Svo. Prüm, 1857.

Opinion d'un médecin vétérinaire sur les effets réels des freins regulateurs perfectionnes, au point de vue de l'hygiène de la bouche. Notice addressée le ler Nov. 1856, au Recueil de médecine vétérinaire, Journal consacré à l'étnde et aux progrès de la médecine vétérinaire, etc. By Hippolyte Anginiard. Svo. Paris, 1857.

Petit Abrégé de médecine vétérinaire à l'usage des chasseurs. [By Arnault]. 32mo. Paris, 1857.

Tablean synoptique du pied du cheval. Anatomie Clastique. By Louis Auzoux. 8vo. Paris, 1857.

Nouvean système de harnachement pour la cavalerie. By Major L. Chr. Barth. 8vo. Wandsbeck, 1857. 
185\%. Precept and Practice. By Harry Hieover [Charles Brindley]. 8vo. Guildford, 1857.

Traité d'anatomie comparée des animaux domestiques. Appareils de la digestion, de la respiration de la dépuration urinaire, de la circulation, de l'innervation, des sens de la génération. By Jean Baptiste Auguste Chauveau. 8vo. Paris, 1857.

Petit Manuel vétérinaire pratique à l'usage des éleveurs, fermiers, et proprietaires ruraux. By Louis Chauvel. 12mo. Quentin, 1857.

The Habit and the Horse. A Treatise on Female Equitation. By Mrs. Stirling Clark. 4to. London, 1857 ; 1860.

In French:-Le Cheval et l'amazon, guide complet de l'equitation des dames. 8vo. Brussels, 1861.

Manual de veterinaria y equitacion. By Jose Ferrer de Conto. $18 \mathrm{mo}$. Paris, 1857.

Art hippiatrique, médicine théorique, et pratique vétérinaire reduite à sa plus simple expression. By Couesme. 8vo. Paris, 1857.

Anatomy and Physiology of the Horse with Anatomical and Questional Illustrations; containing also a Series of Examination on Equine Philosophy and Anatomy; with Instructions in reference to Dissection, and the mode of making Anatomical Preparations ; to which is added a Glossary of Veterinary Technicalities, Toxicological Chart and Dictionary of Veterinary Science. By G. H. Dadd. 8vo. New York, 1857.

Methode d'equitation et de dressage basée sur la mécanique animale contenant; I. Précis de l'equitation depuis Xenophon jusqu'à nos jours; 2. Étude méchanique du cheval; 3. Equitation proprement dite, ou école du cavalier, d'après une nouvelle méthode; 4. Equitation d'agrément, haute école, fariboles d'equitation, equitation de course, equitation des dames, suivie de dressage des chevaux de remonte, dédiée à la cavalerie. By Pierre Joseph Isidore Daudel. 8vo. Paris, 1857.

Les haras ce qu'ils n'ont pas fait ce qu'ils pourraient faire, par un ancien membre du jockey club à $\mathbf{M}$. le général Fleury aide de camp et premier écuyer de sa majesté l'empereur. [By the Marquis d'Eurville de Grangues]. 8vo. Paris, 1857.

Notice sur une nouvelle ferrure en glace. By François Defays. 8vo. Paris, 1857.

Notice additionelle sur la nouvelle ferrure à glace. By François Defays. 8vo. Brussels, 1857. 
De la ferrure des chevaux panards et cagneux. By François $185 \%$. Defays. 8vo. Brussels, 1857.

Description d'un appareil propre à remédier aux déviations du boulet chez le cheval (avec un dessin de M. Ed. Tschaggeny). By François Defays. 8vo. Brussels, 1857.

Kath und Hiilfe bei plotzlich eintretenden Krankheiten der grosseren Hausthiere bis zur Ankunft des Thierarztes. Für Landwirthe, Besitzer von Thieren, für Gemeinde-Vorsteher und Schutzen. By Joachim Friedrich Christian Dieterichs. 1 folio sheet, Berlin, 1857.

Erkenntniss des Alters der Pferde an den Zähnen. By Arnold Dürler. folio. St. Gallen and Berne, 1857.

Zootechnia, produccion animal. Segunda parte de la agricultura o sea la ciencia que enseña a multiplicar y mejorar los animales utiles al hombre. By Jose Echegaray. 8vo. Madrid, 1857.

Neue wichtige Erfindung wohlfeilster Pferdeernährung. Wie ernährt man ein Pferd für wenige Groschen täglich und zwei Pferde mit den Kosten eines Einzigen. By Fenwick de Porquet. $12 \mathrm{mo}$. Ulm, 1857.

An Essay on the Therapeutical Effect of Purgatives on the Horse. By J. Field. 8vo. London, 1857.

Ossevazioni critiche all'opuscolo de D. Giuseppe Lessona, sul perfezinnamento del cavallo nell'isola di Sardegna. By Paolo Gauglia. 8vo. Cagliari, 1857.

Chronique équestre, 1307-1848. By Eugène Gayot. 8vo. Chalons, 1857.

De la cryptorchidie chez l'homme et les principaux animaux domestiques. By Armand Goubaux. 8vo. Paris, 1857.

Éléments de chirurgie vétérinaire. By Dr. Jean Gourdon. 2 vols. 8vo. Paris, 1854-1857.

Étude sur le Cheval de guerre. By Louis Pierre Goyau. 8vo. Paris, 1857.

Pedigree der Vollblut-Hengste, welche in Deutschland bis einschliesslich 1857 zur Zucht benutzt worden sind. [By Capt. Graefe]. 8vo. Berlin, 1857. 
1857. Bericht iiber das Veterinärwesen im Konigreich Sachsen fiir das Winter-Semester 1856-57. Herausgegeben von der Koniglichen Commission fïr Veterinärwesen durch Professor Kaubner. Zugleich als Programm der Koniglichen Thierarzneischule für das Schuljahr, 1856-57. 8vo. Dresden, 1857.

Frank Forrester's [pseud.] Horse and Horsemanship of the United States and British Provinces of North America. [By Henry William Herbert]. 2 vols. 8vo. New York, 1857.

Famous Race and Trotting Horses of America from 1818 to 1856. By Henry William Herbert. 8vo. New York and London, 1857.

Die Pferdezucht Würtembergs. Abbildungen ausgezeichneter ZuchtPferde aus den Privat-Gestuiten S. Maj. des Konigs und dem Koniglichen Landgestiute. Herausgegeben unter Mitwirkung der Königlichen Land-Gestüts-Commission. 15 Tafeln in Farbendruck, nach dem Leben gezeichnet und lithographirt von L. Voltz, und E. Völckers. The text by Edward Hering. 4to. Stuttgart, 1857.

Lehrbuch der Thierheilkunde in alphabetischer Ordnung herausgegeben. By A. Herzog. 8vo. Philadelphia, 1857.

Neu zusammengestellter Hufbeschlagkasten. By Peter Jessen. 8vo. St. Petersburg, 1857.

Le Jockey Club, journal illustré de la fashion, du turf, du sport, et des compagnies équestres. 4to. Paris, 1857, etc.

Physiognomie du dépôt de remonte de Saint-Maixent. Elève du cheval et industrie mulassière dans le département des Deux-Sèvres. La Remonte actuelle. By Auguste Gabriel Olivier Lemaitre. 8 vo. Saint-Maixent, 1857.

Della conformazione esterna delle differenti specie de animali domestici. By Carlo Lessona. 8vo. Turin, 1857.

Considerazioni igieniche sull'alimentazione dei cavalli. By Giuseppe Levi. 8vo. Florence, 1857.

Théorie pratique, progressive et méthodique de l'ordonnance de 1829, sur la exercises et les evolutions de la cavalerie, rédigée d'après les progressions actuellement suivies à l'école de Saumur. By Albert Liasse. 18mo. St. Germain-en-Laye, 1857.

Observations sur la théorie pratique, progressive et méthodique. By Albert Liasse. 8vo. Avignon, 1857. 
Morgan Horses: A Premium Essay of the Origin, History, and 1857. Characteristics of this Remarkable American Breed of Horses; tracing the Pedigree from the Original Justin Norgan, through the most noted of his progeny, down to the present time; to which are added, Hints for Breeding, etc., etc. By D. C. Linsley. $12 \mathrm{mo}$. New York, 1857.

Landwirthschaftliche Thierheilkunde. Ein Leitfaden des thierärztlichen Unterrichtes an ökonomischen Lehranstalten. By A. Masch. 8vo. Vienna, 1857.

History of the Turf in South Carolina. By Miliken. [Circa 1857].

Outlines of veterinary homcopathy; comprising horse, cow, dog, sheep, and hog diseases, and their homoopathic treatment. By James Moore. 8vo. Manchester, 1857 ; 1859; 5th edition, London, $1867 ; 1871$.

Taschenbuch für Pferdekenner und Pferdeliebhaber. Ergebnisse einer mehr als siebzigjährigen Ausubung des Pferdehandels. Nebst einem Anhange, selbst erlebte Anekdoten im Pferdehandel enthaltend. By Abraham Mortier and Dr. C. F. Lentin. 8vo. Dessau, 1857.

Un mot de la dynamique animale. Étude du Cheval. [By Casimir Noel]. 8vo. Meaux, 1857.

Rapport à M. le général Fleury, premier écuyer de S. M. l'empereur. By Eugène Perrault. 4to. Paris, 1857.

Réponse à un Rapport de M. Reynal, chef de service à l'École impériale vétérinaire d'Alfort, discnté à la Société Impériale et centrale de médecine vétérinaire, dans la séance extraordinaire du 22 Janvier, 1857. By L. E. Plasse. 8vo. [Poitier, 1857].

Udstillingen i Wien 1857. Stutterierne Babolna, Kisber, Mezohegyes og Trakehnen. By Ferdinand Victor Alphons Prosch. 8vo. Copenhagen, 1857.

Locomotion du Cheval, Examen des traités de de l'extérieur du cheval et des principaux animaux domestiques, de F. Lecoq, et de physiologie comparée des animaux domestiques, de G. Colin. By C. Raabe. 4to. Paris, 1857.

Locomotion du cheval. Examen des allures, selon M. H. Bouley. By C. Raabe. 8vo. Paris, 1857. 
1857. Étude du Cheval de service et de guerre, suivant les principes élémentaires des sciences naturelles appliqués à l'agriculture. By Antoine Richard (du Cantal). 12mo. Paris, 1857.

3rd edition-précédé de lettres du General Daumas et de l'Emir Abd-el-kadr sur le cheval arabe, et de reflexions à ce sujet. $12 \mathrm{mo}$. Paris, 1859. 5th edition, 12mo. Paris, 1874.

Rapports sur les races animales domestiques de l'Algerie. By A. Richard. 8vo. Paris, 1857.

Traité complet du chirurgie et de médecine vétérinaires. By Henri de Rozières. 2 vols. 8 vo. Paris, 1857.

Ueber Bau und Einrichtungen der Pferdestallangen. Besonderer Abdruck aus S. v. Tennecker's Jahrbuch für Pferdezucht, etc. auf das Jahr 1857. By Adolph Rueff. 12mo. Weimar, 1857.

Le Bauchérisme réduit à sa plus simple expression, on l'art de dresser les chevaux d'attelage, de dame, de promenade, de chasse, de course, d'escadron, de cirque, de tournoi, de carrousel. Programme des Cours d'Equitation Civile et Militaire professés à Bruxelles, Malines, Coblentz, Prague, Vienne, Breslau, Naples, etc. Suivi de notes militaires (organisation, instruction de l'armée, academie militaire), avec planches représentant le travail de Buridau, Capitaine. By Louis Joseph Rul. 8vo. Paris, 1857.

Die nuitzlichen Hausthiere, das Pferd, das Rind, das Schaf, in Bezug auf Altersbestimmung Zucht, Fïtterung, Mastung, Ankauf und Verkauf. Mit 8 naturgetreuen Tafeln in Farbendruck. By Gustav Swoboda. 8vo. Vienna, 1857.

Bildnisse vorzüglicher Hengste aus dem königlich Hannoverschen Landgestiit Celle. By Emil Volkers. folio. Hamburg, 1857.

Die regelrechte Fahrkunst, oder: Grundliche Anleitung zum praktischen Fahren und Einfahren junger Pferde sowohl für Herrschaften und Equipagen-Besitzer, die sich selbst dafür interessiren, wie auch für Kutscher, die es griundlich erlernen und sich darin vervollkommnen wollen. Nach englischen Grundsätzen und Englischer Methode, so wie nach 21 jähriger Erfahrung. By Johann Friedrich Witte. 8vo. Berlin, 1857

Wochenschrift für Thierheilkunde und Viehzucht. Edited by Th. Adam, Dr. May, and Georg Nicklas. 8vo. Augsburg 1857 ; etc. 
Ksiega rodowa koni, czystej krwi angielskiej i arabskiej, wraz hzic 1857. pochodzeniem, znajdujacych sie w Galicyi. By Erazmus v. Wolanski. 8vo. Lemberg, 1857.

Fortegnelse over den Kgl. Veterinair.og Landboh iskoles Bibliothek. 1858. Anon. 8vo. Copenhagen, 1858.

Ledetraad ved Underviisn i skolen for Militaire Beslagsmede. Anon. 8vo. Copenhagen, 1858.

Rules for Keeping a Horse. Anon. 18mo. 1858.

Le Cultivateur vétérinaire. By Henri Arrault. 12mo. Paris, 1858.

Remarks on the Organization of the British Cavalry. By Valentine Baker. 8vo. London, 1858.

American Horse Tamer. By J. Bentwright. 8vo. New York, 1858.

The Sporting World. By Harry Hieover [Charles Brindley]. 8 vo. London, 1858.

Dictionnaire usuel de chirurgie et de médecine vétérinaires, rédigé d'après les travaux de Bourgelat, Vitet, Huzard, etc. By Beugnot. 2 vols. 8 vo. Paris, $1858 ; 1859$.

Circus and Circeusian Games. By Richard Burgess. 8vo. [Circa $1858]$.

Annuaire du Sport en France. Guide complet du Sportsman. Dates des Courses. Classement des hippodromes, etc. By Eugène Chapus. 18 mo. Paris, 1858.

Le Vétérinaire. Ouvrage pratique. By Jules Clément. $12 \mathrm{mo.}$ Paris, 1858.

The Edinburgh Veterinary Review and Annals of Comparative Pathology. 8vo. Edinburgh, 1858-1865.

The Veterinarians Vade Mecum. By John Gamgee. (Senr.) 8vo. Edinburgh, 1858.

Observations sur la contagion chez les animaux domestiques. By L. Gillet. 8vo. Paris, 1858.

The Horse not originally imported into America from the East. By Francis S. Holmes. 8vo. Charleston, [1858].

Das Preussische Veterinärmedicinalwesen. Aus Amtlichen Quellen dargestelt. By W. Horn. 8vo. Berlin, 1858. 
1858. The Horse Book, being simple rules for Managing and Keeping a Horse. To which are added a few words on the Horse's Eye, Foot, and Stomach, with hints on Draught. By Edward King. 12 mo. London, 1858 ; 16 mo. New York, 1866.

Croisement de la race chevaline en France. By J. Klein. 8vo. Paris, 1858.

Traité de Pathologie Vétérinaire. By L. Lafosse. 2 vols. 8vo. Toulouse and Paris, 1858-1862.

Nouveau manuel complet du vétérinaire. By Lebeaud. $18 \mathrm{mo}$. Paris, 1858.

Annuaire des vétérinaires. By V. Mazurkiewicz. 16mo. Paris, 1858 , etc.

Operette bibliografiche del Cavallo. By Guiseppe Molini. 8vo. Firenze, 1858.

Mine Anskuelser om Danmarks Hesteavi. By M. L. Nathanson. 8vo. Randers, 1858.

Foredrag i Anledning af en Comitee betcnkning om mit Forslag till Danmarks Hesteavis Forbedring. By M. L. Nathanson. 8vo. Randers, 1858.

Silk and Scarlet. By the Druid [James Robinson]. 8vo. London, 1858.

Telier's System of Horse Taming. By Frederick Taylor. $12 \mathrm{mo}$. London, 1858.

Guide élémentaire et progressive de la methode Baucher, à la portée de tous, ou l'art de dresser les chevaux de promenade, de chasse, d'escadron et de carrousel. By P. Teulierès. 8vo. Rouen, 1858.

Nogle Bemaerkninger vort Hestevoesen Vetraeffende. By F. L. Torp. 8vo. Copenhagen, 1858.

Horse Taming made easy. By C. Rous. 16mo. London, 1858.

Метода выъзаки лошадп на повыхъ основаніахъ перевель сънъмецкаго пा дополншль драгунскаго его пмператорскаго высочества наслъдлника Цесаревича полка Вегенеръ. Ву Wegener. 4to. Woronetz, [Circa 1858].

1859. Die Zähmung und Dressur der Pferde. Beleuchtung der Rarey'schen Zähmungsmethode. Anon. 8ro. Leipzig, 1859. 
The Horse-shoer's Manual. 12mo. New York, [Circa 1859].

Things worth knowing about Horses. By Harry Hieover [Charles Brindley]. 8vo. Guildford, 1859.

Carriage Builder's and Harness Maker's Art Journal. 4to London, 1859-61.

Form of the Horse as it is open to the inspection of the ordinary observer. By James Crawford Ledbie Carson. 8vo. Dublin, 1859; 8vo. London, 1862.

Projet de règlement sur le service strategique de la cavalerie avant le combat. By Dérigny. [Circa 1859].

Park Riding, with some remarks on the Art of Horsemanship. By J. R. Dunbar. 8vo. London, 1859.

Horse Railways. A Practical Treatise on Street or Horse Power Railways. By A. Easton. 1859.

Des meilleures dispositions à donner aux écuries. By Eugène Gayot. 8vo. Paris, 1859.

Manuel d'Équitation, ou essai d'un progression pour servir au dressage prompt et complet des chevaux de selle, et particuliérement des chevaux d'armes, précédé d'une analyse raisonnée du Bauchérisme. By G. A. Gerhardt. 8vo. Paris, 1859 and 1869.

Rapport sur l'état sanitaire des Chevaux au camps de Châlons (année 1858). By J. B. A. Goux. 8vo. Paris, 1859.

Das Pistol, dessen Theile, Behandlung und Gebrauch. Für Avancirte der Kavallerie und reitenden Artillerie. By Prem. lieut. Gräfe. 1859.

Hints to Horsekeepers; a Complete Manual for Horsemen. By Henry William Herbert. 8vo. New York, 1859.

The Horse and his Master. Hints on Breeding, Breaking, etc. By Vere D. de Vere Hunt. 8vo. London, 1859.

Haandbog i Husdgravlen. By H. Jensenius. 8vo. Christiana, 1859.

Der Kavallerie-Unteroffizier als Reiter, Reitlehrer und Zugführer. Ein Taschenbuch für jüngere Kavallerie-Offiziere. By Major H Kruge. 8vo. Guhran, 1859. 
1859. Hippo-Lasso, appareil compressif servant à maitriser le cheval, le mulet, et généralement les grands quadrupèdes domestiques, difficile a manier par suite de leur caractère méchant, rétif ou sauvage. By C. Raabe and Lunel. 8vo. Paris, 1859.

Hunting Sketches. By Druid [James Robinson]. 12mo. London, 1859.

Traité sur l'art de dompter et de dresser les chevaux et les taureaux vicieux et méchants. By Alexandre Rufener. 8vo. Paris, 1859.

Arte de herrar y forjar. By Sainz. 8vo. Zaragoza, 1859.

Organization of Irregular Cavalry. By Frederick Taylor. 8vo. Calcutta, 1859.

1860. Obituary notice of Lord Londesborough. With an account of his Stud, etc. Printed for private circulation. Tadcaster, 1860.

Horses and Roads ; or How to Keep a Horse sound on his Legs. By Free-Lance. [Pseud.] 8vo. London, [Circa 1860].

Extrait de l'Ordonnance de Cavalerie pour Sous-officiers et Brigadiers, Par un Capitaine Instructeur de l'Ecole de Cavalerie de Saumur. Anon. $32 \mathrm{mo}$. Paris, 1860.

Réglement sur l'exercise et les manœuvres de la cavalerie belge. École du cavalier à cheval. École du peloton à cheval, École de l'escadron à cheval. Evolutions de Brigade Anon. [Circa 1860].

La Question Chevaline dans ses rapports avec la production du Cheval de l'armée et de luxe. By Bernard Abadie. 8vo. Paris, 1860.

Mounted Rifles. By Lieut.-Col. Acland. London, 1860.

Races Chevaline de la Belgique. By Douterluigne Airie. [Circa 1860 ].

Guide complet du peintre en voitures. By Arlot. 8vo. Paris, 1860.

Essai sur la cavalerie irregulière. By E. Arnous-Rivière. 8vo. Paris, 1860.

Question Chevaline. By Antonio Henri Phillippe Léon Comte d'Aure. 8vo. Paris, 1860.

Encore la question Chevaline. By Antonio Henri Phillippe Léon Comte d'Aure. 8vo. Paris, 1860.

Insuffisance des Chevaux forts et légers, du cheval de guerre et de luxe. By Dr. Louis Auzoux. 8vo. Paris, 1860. 
Avenir de la Cavalerie. Examen technique des ouvrages publiés sur 1860.

l'ordonnance du 6 Decembre 1829, tactique des trois armes dans

l'esprit de la nonvelle guerre. By le général baron Leopold Michel Martial Azémar. 3 vols. 8vo. Paris, 1860.

Avenir de la Cavalerie. Son rôle dans les batailles, suivi d'une projet du création des zouaves montés. By le général baron Léopold Michel Martial Azémar. 8vo. Paris, 1860.

Nécessité pour la France d'une puissante armée, et considérations nouvelles sur les troupes à cheval. By Th. Rob. Bugeaud. Paris, 1860.

In Vol. IV. of the Nouvelle Bibliotheque Mititaire d'Elite; and followed by Baron Azémar's "Avenir de la Cavalerie."

De la Morve et du Farcin, communiquées par infection médiate ou immédiate du cheval à l'homme de guerre et des moyens pratiques propres à en diminuer la fréquence dans l'armée. By Bernier. 8vo. Paris, 1860.

Construction of Horse Railways. By C. Burn. 1860.

Note sur la nature et le traitement chirurgical de l'ophthalmie ou fluxion périodique du cheval. By Dr. Didot. 8vo. Paris, 1860.

Treatise upon Horsemanship. By Lieut. Eicke. 8vo. London, 1860.

Diseases of Horses, their Pathology, Diagnoses, and Treatment; to which is added a complete Dictionary of Equine Materia Medica. For the Use of Amateurs. By Hugh Dalziel. London, [Circa $1860]$.

Kunsten ei at bedrages af Hesteprangere. By Chr. P. J. Fogh. [Cirea 1860].

Synopsis of a Course of Lectures on Veterinary Medicine and Surgery. By John Gamgee (Senr.). 12mo. Edinburgh, 1860.

Traité de la castration des animaux domestiques. By Dr. Jean Gourdon. 8vo. Paris, 1860.

Memorandum for the Guidance of Cavalry employed in Ontpost duty at Aldershot. By Sir Hope Grant. 12mo. London, [Circa 1860].

Dressage de Cheval du Guerre suivi du dressage des chevaux rétifs, des sauteurs aux piliers et en liberté. By A. Guérin. 8vo Paris, $1860 ; 1861$. 
1860. Dictionnaire généalogique de la race pure, pour remonter à l'origine des chevaux et juments de pur sang anglais qui ont été introduits en France. By Charles Du Hays. 8vo. Paris, 1860 ; Brussels, 1863.

The Horse and his Rider. By Sir Francis Bond Head. 8vo. London, 1860.

Les Chevaux de pur sang en France et en Angleterre. By Ephrem Houë1. 2 vols. 8vo. Paris, 1860-1866.

L'Industrie privée et l'administration des haras. Réponse à M. le baron de Pierres. By E. Houël. 8vo. Paris, 1860.

Reiterwesen. Ein Handbuch für berittene Offiziere der Infanterie. Enthaltend: Pferdekenntniss, Reitinstruction, Ajustemente des Pferdes mit Cattel und Zaum, die Wartung und Pflege, der Hufbeschlag, die wichtigsten Krankheiten des Pferdes, die gebräuchlichsten Operationen am Pferde und dic Fourage. By Major H. Kruge. 1860.

Nouveau système d'Enfrenèment B. S. G. D. G. servant addresser, diriger, dominer les chevaux les plus difficile. By J. B. Lachaume. Paris, 1860.

Réponse a M. le baron d'Azemar, Colonel de lanciers auteur de l'ouvrage Avenir de la cavalerie (partie purement equestre du 3re volume). By Count Savary de Lancosme Breves. 8vo. Paris, $1860 ; 1861$.

Nouveau Manuel complet du bourrelier et du sellier. By Lebrun. 18 mo. Paris. 1860.

Leçons d'hippologie. By Eugène Lemichel. 8vo. Paris, 1860.

Cours élémentaire d'hygiène hippique. By N. C. Malfrain. 8vo. Paris, 1860.

The Illustrated Horse Doctor, being an accurate and detailed account, accompanied by more than 400 pictorial representations characteristic of the various diseases to which the Equine Race are subjected, together with the latest mode of treatment and all the requisite prescriptions written in plain English. By Edward Mayhew. 8vo. London, 1860; New York, 1861; 9th edition, 8vo. London, 1876.

Essai sur les proportions du cheval et son anatomie externe comparée à celle de l'homme, à l'usage des ecuyers militaires ou civils, et des Artistes. By J. P. Mégnin. 4to. Paris, 1860. 
E1 Memorial de Caballeria. Madrid, 1860 ; etc.

Du Passé et de l'avenir des haras. Recherches sur le commerce, les dénominations et la production des chevaux, principalement en France avant 1789. By Francisque Michel. 8vo. Paris, 1860.

The Book of Field Sports and Library of Veterinary Knowledge. By H. D. and William J. Miles. 4to. London, 1860-1867.

General remarks on Stables and examples of Stable Fittings. By William J. Miles. 8vo. London, $1860 ; 1873$.

L'Administration des haras et l'industrie privée. By le barou de Pierres. 8vo. Paris, 1860.

Light Cavalry for India. By Neale Porter. [1860].

Examen de la théorie de la similitude des angles de M. Le General Morris (Jan. 1859) comprenant $1^{\circ}$ Aperçu de cette théorie extrait de l'essai sur l'exterieur du cheval; $2^{\circ}$ Aperçu des modifications apportés au tracé de M. le General Morris; $3^{\circ}$ Tracés de cette méthode selon les auteurs ci-après désignés: Morris, St. Auge, Raabe; $4^{\circ}$ Aperçu explicatif de la théorie du tracé de M. le capitaine Raabe. By C. Raabe. Paris, 1860.

L'Age du Cheval, suivi d'un exposé des ruses employées par les maquignons et des moyens de les déjouer. By H. Robinson. $12 \mathrm{mo}$. Paris, 1860.

Nouvelle bride dite à voile, pour arrêter à volonté les chevaux emportés, ou qui ont pris le mors aux dents. By Alexandre Rufener. In French and German, 8vo. Paris, 1860.

The Wild Sports of India, with remarks on the Breeding and Rearing of Horses and the Formation of Light Irregular Cavalry. By Capt. H. Shakespear. 8vo. London, 1860.

Treatise on the Horse's Foot. By James Turner. 8vo. [Circa 1860].

Twenty Four Portraits of Brood Mares belonging to the Royal Stud at Hampton Court. By C. W. With descriptions. folio. 1838.

Libro de los hierros o marcas que usan los criadores para sus ganados caballares, rectificados por fin del año 1859 , reunidos por los establecimientos de remonta, recopilados por la subdirecciou de los mismos, y mandado imprimir. By Sr. D. D. Juan Zabala, Director General de Caballeria. folio. Cordoba, 1860. 
1861. Album de la Caballeria Española des de sus primiteros tiempos hasta el dia; publicada por la direccion general del arma. folio. Madrid, 1861. Contains 70 coloured lithographic plates, with explanations.

Equitation et dressage. Methode Joseph. [Anon]. 16mo. Paris, 1861.

Confessions of a Horse Coper. Anon. 1861.

The Horse; How to Ride him. By Butler. 1861 ; 1865.

Reitsystem für Lehrer der Kavallerie und Artillerie zur Ausbildung von Recruten zu Pferde und zur Dressur von Remonten. By D. F. Bötticher. 8vo. Berlin, $1861 ; 1877$.

Memorial thérapeutique du vétérinaire praticien pour 1861. By Henri Bouley and A. Sanson. 24mo. Paris, 1861.

Nachrichten und Betrachtungen iiber die Thaten und Schicksale der Reiterei in den Feldzigen Friedrich II. und in denen neuerer Zeit. By Von Canitz. 1861.

Cavalry Brigade Movements. By Lieutenant-General the Earl of Cardigan. 8vo. London, 1861.

De la Reconstruction du cheval sauvage primitif par la réunion, chez un type idéal, de ses caractères spécianx et spécifiques. By Dr. Cornay. 12mo. Paris, 1861.

La Cria Caballar en España. By Coronel D. Juan Cotarelo y Garastazu. folio. Madrid, 1861.

Nouveau manuel pratique indispensable aux propriétaires d'animaux. Instructions pratiques pour traiter soi-même les affections et les maladies les plus fréquentes et les plus subtiles. By J. B. S. Elluin. 18mo. Paris, 1861.

Notes on Shoeing Horses. By Frederick Wellington John Fitzwygram. 4to. London, 1861 ; 8vo. London, 1863.

General and Descriptive Anatomy of the Domestic Animals. By John Gamgee (Senr.), and James Law. 2 vols. 8vo. Edinburgh, 1861.

Our Domestic Animals in Health and Disease. By John Gamgee (Senr.). 8vo. Edinburgh, 1861-64.

Unsere Pferde; ein Beitrag zur deutschen National Oeconomie. By R. Jannasch. 8vo. Dresden, 1861. 
Des Causes de la dégénération des races chevalines en France et des 1861. mesures à prendre pour obtenir leur complète régénération. By Paul de Lalaubie. 8vo. Paris, 1861.

Seconde Réponse a M. le baron d'Azémar, colonel de lanciers, auteur de l'ouvrage Anenir de la cavalerie (partie purement equestre du 3e volume). By Count Savary de Lancosme-Brèves. 8vo. Paris, 1861 .

Der Fuss des Pferdes in Riicksicht auf Bau, Verrichtungen und Hufbeschlage. Gemeinfasslich in Wort und Bild dargestellt. By A. G. T. Leisering and H. M. Hartmann. 8vo. Dresden, $1861 ; 1866 ; 1870 ; 1876$.

Anatomy of the external form of the Horse; with explanations. By James Lupton. folio. London, 1861.

The Book of Aids, whole system of Equitation. By Thomas Martin. $12 \mathrm{mo}$. London, 1861.

Aide Mémoire d'administration à l'usage des Sous-officiers de Cavalerie. By Victor Milet. 8vo. Paris, 1861.

La Connaissance générale du cheval; étude de zootechnie pratique, par les auteurs de l'Encyclopédie pratique de l'agriculture, sous la direction de L. Moll et Eugène Gayot. 8vo. Paris, 1861.

Les Haras et l'industrie chevalıne. By Count de Morgan-Frucourt. 8vo. Paris, 1861.

L'Equitation pratique. By Jules Pellier. Junr. 12 mo. Paris, 1861 ; $1863 ; 1875$.

De la Cavalerie. Reflexions sur les idées émises au sujet de la diminution et de la transformation de cette arme. By Bruno Renard. 8vo. Paris, 1861.

Mémoire sur l'application du système Rarey. By A. Ricquet. 8vo. Paris, 1861.

Chevaux de selle, de chasse, de course et d'attelage. Manuel complet de l'éleveur et du propriétaire de chevaux. By H. Robinson. 8vo. Paris, 1861.

Guide hygiénique et chirurgical pour la castration et le bistournage du cheval, du taureau, de la vache, du bélier, du verrat, etc. By E. Serres. $12 \mathrm{mo}$. Paris, 1861.

Recollections of a Horse Dealer. By Frederick Taylor. 8vo. London, 1861. 
1861. Kurze Anleitung zur Ertheilung des Reitunterrichts, sowie zum Selbstunterricht für Kavallerie-und Infanterie-Offiziere. Mit erläuternden Abbildungen. By General Treyden v. Trotta. 1861.

1862 Handbuch der gerichtlichen Thierheilkunde. Anon. 8vo. Berlin, 1862.

Instructions sur le travail individuel dans la Cavalerie, le tir du fusil et du pistolet. Traité sur la ferrure. Anon. 3 parts. 4 to. 8 vo. and $18 \mathrm{mo}$. Paris, 1862.

Etudes sur les cavaleries étrangères. Cavalerie anglaise. 1re partie: Equitation militaire, Ecole du cavalier à pied. Ecole de division et d'escadron. 2re partie: De l'ordre en colonne avec distance; de l'ordre de colonne serrée, de l'ordre en bataille. Par un Officier du Cavalerie. Anon. 8vo. Paris, 1862; 1863.

Regulations for the Instruction, Formation, and Movements of the Cavalry. Horse Ginards. 8vo. London, 1862.

Un Mot sur l'étiologie de la Morve et du farcin. By R. F. Baillif. 8vo. Paris, 1862.

Le Cheval de la Plata comme cheval de Guerre, son importation pour la remonte de l'armée, état de la question. By Charles Barbier. 8vo. [Circa 1862].

Formulaire vétérinaire, suivi d'un mémorial thérapeutique. By $\mathbf{A}$. Bouchardat. $18 \mathrm{mo}$. Paris, 1862.

Country Gentleman, Sporting Gazette and Agricultural Journal, London, 1862, etc.

Notice et documents historiques sur les Chevaux Orientaux. By E. Duhousset. 8vo. Paris, 1862.

Auch ein Wort ïber die Ausbildung der Cavallerie. By S. von E—_. 8vo. Berlin, 1862.

Horses and Stables. By Frederick Wellington John Fitzwygram. 8 vo. London, $1862 ; 1886$.

Achat du Cheval, ou Choix raisonné des Chevaux d'apres leur conformation et leurs aptitudes. By Eugène Gayot. 12mo. Paris, 1862.

Equitation Militaire. Mémoire analytique, critique, et pratique sur le dressage, et la conduite du cheval de guerre. By G. A. Gerharcit. 8vo. Paris, 1862. 
Der Oberstallmeister, ein Lebensbild aus dem achtzehnten und neunzehnten Jahrhundert. By Oberst lieut. v. Gilsa. 1862.

Elémens d'hygiène Vétérinaire. By Godin. [Circa 1862].

Le Cheval Méchanique. By Colonel v. Hamel. 8vo. Stuttgart, 1862.

Hippologische Blätter, Centralorgan fuir die Interessen der Pferlezucht in Oesterreich. Edited by L. Bergmann. folio. Vienna, 1862, etc.

Le Vetérinaire pratique. By Edouard Hocquard. 8vo. Paris, [Circa 1862]; 6th edition, 1873.

Horse Warranty. A plain and comprehensive guide to the various points to be noted, shewing which are essential and which are unimportant. By Peter Howden. 16mo. London, [1862].

Stable Architecture. By Thomas Edward Knightley. folio. London, 1862.

Un mot sur le cheval français. By $T$. de Bourrousse de Laffore. 8vo. Paris, 1862.

Notes anatomiques sur l'opération de l'hyovertébrotomie ou ponction des poches gutturales des solipèdes. By Felix Lecoq. 8vo. Paris, 1862.

Essai sur l'enseignement de la médecine vétérinaire en Belgique. By J. B. Legrain. 8vo. Paris, 1862.

Cours d'hippologie, ou Abrégé de l'anatomie de la physiologie, de l'extérieur, de la maréchalerie, des maladies et des accidents dont le cheval est le plus souvent attient. By E. Lescot. $18 \mathrm{mo}$. Paris, 1862.

Instructions for the Field Service of the United States Cavalry. By G. B. McClellan. 12mo. Philadelphia, 1862.

- European Cavalry. By G. B. McClellan. 12mo. Philadelphia, 1862.

Mémoire sur les principaux systémes de ferrure. By Merche. 8vo. Paris, 1862.

La Tactique française-Cavalerie. By Captain Le Luyer Morvan. 8vo. Paris, 1862.

Lehrbuch der Physiologie der Haussäugethiere fur Thierärzte und Landwirthe. By Franz Müller. 8vo. Vienna, 1862; 1871. 
1862. Les Régiments de fer. Origine de la grosse cavalerie en France, cavaliers et piétons, le casque et la cuirasse. L'emploi des armes à feu dans la cavalerie. Les cuirassiers, la journée du cavalier. By Baron F. de Reiffenberg. 8vo. Paris, 1862.

Der Sporn. Zentralblatt für die Gesammt-Interessen des deutschen Sports. Organ der Landes-Vollblut-Zucht. 4to. Berlin, 1862, etc.

Life of a Nag Horse with practical directions. By Frederick Taylor. 12mo. London, 1862.

Coup l'œil sur les races chevalines françaises. By E. T[estarode]. 8 vo. Paris, 1862.

Aperçus équestres au point de vue de la méthode Baucher. By I. Wachter. 12mo. Paris, 1862.

1863. Guide du parieur aux courses, contenant les combinaisons les plus favorables, les règlements du Jockey Club et du salon, des courses ainsi que les tables de proportion nécessaires à tous les paris, par un vieux sportsman. 8vo. Paris, 1863.

Horse Racing, its History and Early records of the Principal and other Race Meetings, with Anecdotes. 8vo. 1863.

La Cavalerie. Sa situation actuelle, et son amélioration prochaine. 8vo. Paris, 1863.

Manual of Drill for Mounted Rifle Volunteers. Horse Guards. $12 \mathrm{mo}$. London, 1863.

Regulations for the Movements and Formations of a Division or Brigade of Cavalry. $12 \mathrm{mo}$. London, 1863.

Réponse aux attaques dirigées contre l'arme de la Cavalerie. By Général Ambert. 8vo. Paris, 1863.

Blätter für Pferdezucht und Central Organ für hippologische Vereine, Gestiitswesen, Pferde-Dressur, Händler, etc. 4to. Leipsic, 1863, etc.

Ordonnance sur l'exercise et les évolutions de la Cavalerie du 6 decembre 1829, appropriée à chaque arme, modifiée d'apres les decisions ministerielles, qui ont paru jusqu'a ce jour, annotée et augmentée d'une instruction pratique pour donner la leçon sur le terrain. By A. Bué. 8 vols. 18mo. Paris, 1863.

Impôt sur les voitures et chevaux. By Jean Baptiste Auguste Chouveau. 8 vo. Toulouse, 1863. 
La Cavalerie considérée au point de vue de son harnachement et de 1863. son équipement. By Capt. L. Cogent. 8vo. Paris, 1863.

Cavalry Tactics or Pegulations for United States Cavalry. By P. St. George Cooke. 16mo. New York, 1863, 1872.

Conduite et gestion du Cheval, du bœuf, et du mouton en Champagne. By L. N. Demilly. senr. 12mo. Paris, 1863.

Les Chevaux et les courses en France: Manuel de l'amateur. By Urbin Desvaux. 16mo. Paris, 1863.

Traité de maréchalerie par le nouveau système de ferrure en caout chouc durci. By J. B. S. Elluin. 18mo, Paris, 1863.

Die Hippologische Literatur von 1848 bis einschliesslich 1857. Verzeichniss der in diesem Zeitraum uiber Alles was das Pferd betrifft erschienenen Bücher, mit biographischen Notizen iber die Verfasser. By Carl Graefe. 8vo. Leipzig, 1863.

Traité de la ferrure des chevaux. By Captain Guendeville. 12mo. Paris, 1863.

Les Courses en France, en Belgique et à Bade. Origines, performances, et produits dés vainqueurs des principaux prix dans ces diverses contrées. Tableaux de tous les prix groupés par reunions de courses. By Charles Du Hays. 8vo. Brussels, 1863.

Pferd und Reiter, oder die Reitkunst in ihren ganzen Umfange. Nach rationeller, allein auf die Natur der Menschen, sowie des Pferdes gegründet, rasch und sicher zum Ziele führend. By T. Heinze. 8vo. Leipsic, $1863 ; 1873 ; 1877$.

Le Cheval de selle à ses differents usages, ou Méthode pour l'education et le dressage du Cheval. By Louis d'Illiers. Svo. Paris. 1863.

Manuel de l'étalonnier et de l'éleveur. By F. C. J. Liégeard. $12 \mathrm{mo}$. Paris, 1863.

Modern Practical Farrier, containing a complete system of the Veterinary Art. By Henry Downes Miles. 4to. London $1863 ; 1864$.

Stable Secrets; or Puffy Doddles, his Sayings and Sympathies. By John Mills. 8vo. London, 1863; 1865.

Cavalry Drill and Sabre Exercise. By George W. Patten. 16mo. New York, 1863. 
1863. Des Mraladies virulentes comparées chez l'homme et chez les animaux. By Dr. M. Peter. 8vo. Paris, 1863.

Méthode de haute école d’équitation. By C. Raabe. 8vo. Marseilles, 1863.

With Atlas in 4 to.

Cavalry, its History and Management in War, By J. Roemer. 8vo. New York, 1863.

Beiträge zur Kenntniss der fossilen Pferden und zu einer vergleichenden Odontographie der Hausthiere im Allgemein. By Ludwig Rüttimeyer. 8vo. Basle, 1863.

Reprint from the Verhandlungen der Naturforsch. Gesellschaft in Basle.

Notions usuelles de médecine vétérinaire. By André Sanson. 12 mo. Paris, 1863 ; fth edition, Paris, 1873.

Biographisch-literarisches Lexicon der Thierärzte aller Zeiten und Länder, sowie der Naturforscher, Aerzte, Landwirthe, Stallmeister u. s. w., welche sich um die Thierheilkunde verdient gemacht haben. By G. W. Schrader. 8vo. Stuttgart, 1863.

Einiges iiber die Ausbildung der Reiter und Pferde bei der Königl. preussichen Militar-Reitschule mit Bezugnahme auf die Broschüre "Auch ein Wort iiber die Ausbildung der Kavallerie von S. v. E." By E. F. Seidler. 8vo. Berlin, 1863.

Riding and Driving. By J. H. Walsh [Stonehenge]. London, 1863.

1864. De l'equitation et de la haute école, ayant pour base la position de jambette, par un amateur d'equitation. Anon. 8vo. Paris, 1864.

Five Hundred Questions and Answers offered to the Subalterns of the British Cavalry. By a Brother Subaltern. Anon. 8vo. London, 1864.

Our Native Cavalry. By Ghorchurra. [psend.] 8vo. Meerut, 1864.

Instruction sur le dressage du cheval de troupe. Anon. Paris, 1864.

Méthode du dressage du cheval de troupe. Anon.12mo. Paris, 1864.

Manuel du Cocher. By Jean André. 18mo. Paris, 1864.

Considerations générales sur l'emploi du cheval Hongre affecté aux transports rapides et aux services publiques des grandes villes. By Louis Auguste Benjamin. 8vo. Paris, 1864. 
The Horses of Antiquity, Middle Ages, and Renaissance. From the 1864. earliest monuments down to the XVIth Century. By P. Charles Berjeau. 4to. London, 1864; 1875.

Handy book for United States Cavalry. By Brigadier General Cooke. Philadelphia, 1864.

Pure Saddle Horses and how to breed them in Australia. By E. M. Curr. 8vo. Melbourne, 1864.

A Mamual for Cavalry. By Brigadier General W. L. Elliott. 16mo. Philadelphia, 1864.

La Liberté des Haras et la Crise Chevaline en 1864. By Count Louis Alexander de Careil Foucher. Svo. Paris, 1864.

De Cheval Oriental, et de son emploi dans l'amélioration des races françaises. By Dr. Jean Gourdon. 8vo. Paris, 1864.

Les Trotteurs ; Origines, performances, et produits des individualitiés qui ont le plns marqué les courses au trot. Tables des meilleures vitesses pour quatre kilomètres et au dessus. Nombre des courses dont le temps a été constaté chaque année. By Charles du Hays. 8vo. Brussels, 1864.

Comment les races chevalines se forment et se conservent. Alliances consanguines. By J. B. Huzard (fils). 8vo. Paris, 1864.

Notice sur une selle de troupe à lames mobiles divisées. By A. Leurs. 8 vo. Paris, 1864.

Race chevaline. Espèces bovine, asine, et ovine. By N. C. Malfrain. 8vo. Paris, 1864.

The Illustrated Horse Management, containing descriptive remarks upon Anatomy, Medicine, Shoeing, Teeth, Food, Vices, Stables; likewise a plain acconnt of the situation, nature, and value of the different points; together with comments on Grooms, Dealers, Breeders, Breakers, and Trainers. By Edward Mayhew. 8vo, London, $1864 ; 1867 ; 1876$.

Memoire sur le crapand du cheval, sa nature, et son traitement. By J. P. Mégnin. 8vo. Paris, 1864.

Instruction pratique pour faciliter l'élevage et le dressage des chevaux de selle et d'attelage. By Alphonse Morin. Svo. Paris, 1864.

Annuaire du turf continental By Ernest Parent. 12mo. Paris, 1864. 
1864. Cavalerie, Evolutions de Brigade. By le Baron de Richepance. Paris, 1864.

Lettres sur l'hippophagie. By Dr. E. Robinet. 12mo. Paris, 1864.

Nouvelle études historiques, critiques et experimentales sur la contagion de la morve et espécialement de la morve chronique. By F. Saint Cyr. 8vo. Paris, 1864.

Sporting Opinion. Folio. London, 1864, etc.

Cours d'hippologie, à l'usage de M. M. les officiers de l'armée, de M. M. les officiers des Haras, les vétérinaires, etc. By A. Vallow. 8vo. Saumur, 1864; 4th edition enlarged, 2 vols, 1873.

Abrégé d'hippologie à l'usage des sous officiers de l'armée. By $\mathbf{A}$. Vallow. 12mo. Paris, $1864 ; 1867 ; 1871 ; 1873$.

De l'infanterie et de la cavalerie dans l'art militaire, histoire et réformes. By J. F. de Métivier de Vals. 8vo. Paris, 1864.

1865. Cavalry Sword Exercise. Horse Guards. Svo. London, 1865.

Entweder-Oder. Was muss für die vaterländische Pferdezucht geschehen? 8vo. Wittenberg, 1865 .

Règlements sur les théories à pied et à cheval, et sur la conduite des voitures et des mulets de but pour les troupes du train des équipages militaires. Ministre de la guerre. 12mo. Paris, 1865.

Regulations for the Instruction, Formations, and Movements of the Cavalry. Horse Guards. 8vo. London, 1865.

Taschenbuch fiir Kavallerie-Mannschaft-Schuelen. In Fragen und Antworten. Deutsch und böhmisch. 8vo. Vienna, 1865.

General Principles of the Non-Pivot System of Cavalry Drill. By Valentine Baker. 8vo. Dublin, 1865.

Die literarischen Erscheinungen der letzen 20 Jahre 1845-1864 auf dem Gebiete der Kriegswissenschaft. By Eduard Baldamus. 8vo, Leipzig, 1865, etc.

Notes et documents cnncernant l'administration des Haras en Normandie. By J. de Robillard de Beaurepaire. 8vo. Paris, 1865 .

Mounted Rifle or Volunteer Irregular Cavaliy. Manual of Drill, with Diagrams. By Lieut.-Col. Bower. $12 m$. [Circa 1865]. 
History of the United States Cavalry. By A. G. Brackett. New 1865. York, 1865.

Landwirthschaftliche, iiber Pferdezucht und gestutswesen. By Briefe. 8vo. Dillingen, 1865.

Les Courses de Marsielle et l'élevage du cheval dans le sud-est de la France. By Hippolyte de Broye. 8vo. Marsielles, 1865.

Dictionnaire de l'art vétérinaire à l'usage des cultivateurs et des gens du monde. By Charles de Bussy. 12mo. Paris, 1865.

Formulario Universal de Veterinaria, o guida pràtico del veterinario y del farmacéntico. By Nicolás Casas de Mendoza. Svo. Madrid, 1868.

Epsom, Chantilly, Bade. By Hiéron [Eugène Chapus]. $12 m$ m. Paris, 1865.

The Horse trainer's and Sportsman's Guide. By Digby Collins. 8vo. London, 1865.

A German translation by Otto Mayr. 8vo. Vienna, 1877.

The Turf and the Race Horse, describing trainers and training, the stud farm, the sires and brool mares of the past and present, and how to breed and rear the racehorse. By R. H. Copperthwaite. 8 vo. London, $1865 ; 1866$.

Gladiateur et le haras de Dangu, à MI. le comte Fréderic de Lagrange. By Louis Demazy. 18mo. Paris, 1865.

Plain Rules for the Stable. By John Gamgee, senr. and John Gamgee, junr. 8vo. London, 1866.

Causeries chevalines. By Alexandre Gaume. 12mo. Paris, 1865.

Traité des resistances du cheval, ou Méthode raisonnée de dressage des chevaux difficiles, domnant la solution de tous les problèmes embarrassants qui peuvent se présenter dans le dressage du cheval de selle, et en général dans la pratique de l'equitation, et philosophie hippique déduite de la physiologie et de la méchanique animales. By G. A. Gerhardt. 8vo. Paris, 1865.

De la Pyétite chronique chez le cheval. By Désiré Guilmot. 8vo. Paris, 1865.

Our Saddle Horses. By Ker B. Hamilton. 8vo. London, 1865. 
1865. Guide du Marchand de Chevaux et du consommateur. Recueil sommaire des meilleures foires de France, leur composition, leur importance. By Charles Du Hays. 16mo. Paris, 1865.

Practical Lessons on Hunting and Sporting. By Scrutator [K. W. Horloch]. 8vo. London, 1865.

Les Chevaux Français en Angleterre. By Ephrem Houël. 8vo. Paris, 1865.

Army Equipment, Part I., Cavalry. Horse Guards. By Henry Montague Hozier. 8vo. London, 1865.

Programme élémentaire d'un cours d'art et d'histoire militaires appliqué à la cavalerie. By Emile Humbert. 8vo. Paris, 1865.

Unsere Pferde als Folgen der Anglomanie. By R. Jannasch. 8vo. Coburg, 1865.

Guide pratique vétérinaire, on Memento thérapeutique. By J. Lacassin. 32mo. Paris, 1865.

Le Cheval, l'âne et le mulet; extérieur, race élevage, entretien, utilisation, etc. By Pierre Aristide Adolph Lefour. 12mo. 1868.

Handlening i ridkonsten samt uti Konsten att Kora. By M. Leyon. 8vo. Stockholm, 1866.

Des Chevaux, Observations sur la manière de les dresser, de les employer, de les nourrir, etc. By E. Loiselet. $12 \mathrm{mo}$. Paris, 1865.

The Handy Horse Book, or Practical Instructions in Driving, Riding, and the general care and management of Horses. By A Cavalry Officer [Magenta]. 8vo. London, [1865]. 3rd edition 1867; $1871 ; 1881$

L'Ami du Cheval; simples conseils sur l'élève, l'hygiène, la médicine et l'achat des chevaux. By J. P. Mégnin. 18mo. Paris, 1865.

De l'Origin de la ferrure du cheval. By J. P. Mégnin. 8vo. Paris, 1865 .

Manuel des piquers, cochers, grooms et palefreniers, à l'usage des Écoles de dressage et d'Equitation de France. By Count Louis Edme de Montigny. 12mo. Paris, $1865 ; 186 \tau ; 1873$.

Fragen und Antworten zur Abrichtung des Reiters und Pferdes für der Pferdeliebhaber. Ein Handbuch iiber Pferdekenntniss im Weiteren Sinne. Fiir Besitzer, Ziichter und Liebhaber der Pferde in allen Ständen und Ländern. By B. C. v. Oeynhausen. 8vo. Vienna, 1865. 
Dictionnaire Vétérinaire Homœopathique pour traiter soi même les maladies des animaux domestiques. By Prost-Lacuzon and H. Berger. 12mo. Paris, 1865.

Leitfaden fuir Kavalleristen und Pferdebesitzer. Ein Rathgeber in der prakt. Pferdekenntniss. By A. v. Rabenen. 16mo. Berlin, 1865.

Idées pratiques sur la cavalerie. By Count de Rochefort. 8vo. Paris, 1865.

Treatise on Drill and Manœuvres of Cavalry combined with Horse Artillery. By Major-General M. W. Smith, C.B. 8vo. London, 1865.

The Robinson Horse Shoe. By C. W. Tayleure. New York, N.D. [Circa 1865].

Historique du 2e régiment de chasseurs à cheval depuis sa création jusqu'en 1864. By François Simon Marie Jules Gay de Vernon. Svo. Paris, 1865.

Vorschläge zur Förderung der Pferdezucht mit besonderer Beriicksichtigung der Rheinprovinz. By J. Waldschmidt. 8vo. Bonn, 1865 .

Rollwagenbuchlein. By J. Wickram. Leipzic, 1865.

Ueber cavalleristiches Reiten. By W. von Willisen. 8vo. Dessau, $1865 ; 1873$.

Die Pferdezucht in der Schweiz. Ein Wegleiter für die Diskussion dieser Frage durch die Gesellschaft schweizer. Thierärzte. By R. Zangger. 8vo. Bern, 1865.

Dienst-Reglement für das k. k. Heer. 3. Theil, Kavallerie. 8vo. 1866. Vienna, 1866.

Kritische Gedanken tiber die bayerische Cavallerie nach dem Feldzuge 1866. 8vo. Munich, 1867.

Gedanken iiber die Vergangenheit, Gegenwart und Zukunft der preuss. Cavallerie. 8vo. Stendal, 1866.

Gesetz für den Münchener Rennverein. 8vo. Munich, 1866.

Allgemeine Lehrsätze der Pferdezucht in neuem Gewande. By Justinus Redivivus. [pseud.] 8vo. Wittenberg, 1866.

Tibskrift Skanska häst-föreningens 1866. Anon. 4to. Malmö, 1866. 
1866. Nouvel essai sur l'équitation et seconde expression du baucherisme; par un amateur. (docteur en médecine). Anon. 8vo. Paris, 1866.

Katechetischer Veterinär-Unterricht . . . fir Cadeten, etc. By C. Ableitner. 12mo. Dillingen, 1866.

Bibliotheca militaris et hippologica; ocler systematisch geordnete Uebersicht aller im deutschen Buchhandel auf dem Gebiete der Kriegswissenschaft und der Pferdekunde neu erschienenen Bïcher, Zeitschriften, und Landkarten. By A. Buchting. 8vo. Nordhausen, 1866.

Rathgeber uiber Pflege und Fütterung der Pferde im Kriege. Fïr Kavaleristen. By Fr. Dominick. 8vo. Berlin, 1866.

Handbuch für Pferdebesitzer. By C. Fortwängler. 8vo. Vienna, 1866.

A System of Medecine. Edited by J. Russell Reynolds. Vol. I. Art. Glanders-Equinia. By Arthur and John Gamgee. 8vo. London, $1866 ; 1870$.

Four Years in the Saddle. By Colonel Harry Gilmore. 12 mo. London, 1866.

Die topographische Myologie des Pferdes mit besonderer Beriicksichtigung der locomotorischer Wirkung der Muskeln. By $\mathbf{K}$. Günther. 8vo. Hannover, 1866.

Some Account of the Popular Belief in Animated Horse-Hairs in the Play of Antony and Cleopatra. By J. O. HalliwellPhillips. sm. 8vo. London, 1866. Privately Printed.

Le Cheval percheron. Production, élevage, dégénérescence de la race, etc. By Charles du Hays. 12mo. Paris, 1866.

Words of Command employed in manœuvring a Regiment of Cavalry, a Battalion of Infantry, and a Battery of Artillery. By Henry W. L. Hime. 8vo. London, 1866.

Stallhaltung und Stallpflege. By U.v. Hochwächter. 8vo. Berlin, 1866.

The Illustrated Sporting Life and Pastoral Register. Sydney, 1866.

Afterwards the Sydney Sporting Life and publisherl without illustrations. 
Horse training made easy, being a new and practical system of teach- 1866. ing and educating the Horse ... to which is appended an Essay on Shoeing. By Robert Jennings. 8ro. Philadelphia, 1866.

Husdjursskotselns hufoudgrunder. By C. A. Lindqvist and J. Arrhenius. Svo. Stockholm, 1866.

Encyclopädie fiir Pferdefreunde, Pferdebesitzer und Pferdezuichter. By K. Löffer. 8vo. Berlin, 1866.

Système d'attelags permettant d'employer immédiatement au trait tout cheval de cavalerie, pris dans le rang, sellé, paqueté et monté par son cavalier. By Colonel Charles Martin. 8vo. Paris, 1866.

Die k. k. Militär Gestiite in Oesterreich. Kisbér, Babolna, Mézohegyes, etc. By Otto Mayr. Svo. Vienna, 1866.

Comnaissances complètes du cavalier, de l'ecuyer et de l'homme de cheval, divisées en quatre parties. By Felix Van der Meer. Svo. Brussells, 1866.

Le Fer Elastique. Nouveau système de ferrure. By Charles Peillard. 8vo. Paris, 1866.

Afterwards published in 12mo. Paris, $1868 ; 1871$, under the title:- "Le Fer Elastique. Ferrure physiologique."

Recherches historiques sur le cheval antique et les anciennes cavaleries. By G. Recordon. 8vo. Paris, 1866.

Les Lanciers. ByBaron Frédéric de Reiffenberg.18mo.Paris, 1866.

Les Dragons. By Baron Frédéric de Reiffenberg. 8vo. Paris, 1866.

Reitkunst und Dressur nach dem Naturgesetze. By K. L. Schilling v. Kannstatt. 8 vo. Stuttgart, 1866.

Der hufbeschlags des Pferdes. Fur Landwirthe und Hufschmiede bearbeitet. By P. Schmelz. Svo. Marburg, 1866.

Mittheillungen aus der thierarztlichen Praxis in Kurhessen. By C. Schmidt. Gottingen, 1866.

Die Fortscluritte in der Pferde-Dressur und Behandlung nebst einigen praktische Lehren für den Reiter und Besitzer. By H. Fr. J. v. Schönberg. Svo. Naumburg, 1866.

Berichtigungen und Ergänzungen für die Beiträge zu einem Gestütbuch von Trakehnen. By H. Stillfried. 8vo. Berlin, 1866.

Tidskrift för veterinär medicin o. husdjurs-skötsel. Edited by F. Lundberg. 8vo. Stockholm, 1866-74. 
1866. Questions and Answers for Cavalry Subalterns. By Twyford. 8vo. London, 1866.

Connaissances complètes du cavalier, de l'ecuyer, et de l'homme de cheval. By Felix Vandermeer. 8vo. Brussels, 1866.

Abrégé d'hippologie. Connaissance pratique du Cheval. By A. A. Vial. Svo. Paris, 1866.

1867. Anleitung zur praktischen Einuibung des Felddienstes für die Kavallerie. $16 \mathrm{mo}$. Vienna, 1867.

A propos de la Reorganisation de l'armée. Cavalerie. 8vo. Paris, 1867.

Our Great American Horses. By Hambletonian. New York, 1867.

Regulativ der Militär-Reit Anstalt. Anon. 8vo. Dresden, 1867.

Reiter Gedanken. Anon. 8vo. Munich, 1867.

Zusammenstellung cler im Ressort der Abtheilung für das RemonteWesen im Kriegs-Ministerium bestehenden hauptsächlichsten Bestimmungen. Anon. Svo. Berlin, 1867.

Kurz-und leichtfasslicher Unterricht iiber den Hufbeschlag der Pferdes. By K. Ableitner. 12mo. Dillingen, 1867.

Almanach du Sport, courses de chevaux, paris, poules, régates, chasses, villégiatures, ete. $16 \mathrm{mo}$. Paris, 1867, etc.

Allgemeines Gestiit-Buch. Ein Verzeichniss der Vollblut Pferde, etc. By F. André. 8vo. Berlin, 1867.

Hunting and Steeple Chasing. By C. Clarke. 1867.

Der militärische Reitunterricht. By A. Dachenhausen. 8vo. Hanover, 1867.

Treatise on Harness, Saddles, and Bridles; their history and manufacture from the earliest times down to the present period. By A. Davis. 8 vo. London, 1867.

Les Ferrures pathologiques, ou application raisonnée de la ferrure au traitement des affections du pied et des membres. By François Defays. 16mo. Paris, 1867; 1868.

The Law relative to the sale and purchase of Horses, comprising notices of the statutes and decisions thereon and the leading cases on warranties, with a copious index and a Table of the authorities cited and observations on letting, hiring, and driving horses. By Charles Egan, 8vo. London, 1867. 
Manuel de l'entraîneur. By J. de Gemeiner. 18mo. Paris, 1867. $186 \%$.

Ueber die Wartung und den Gebrauch des Dienst-Luxus-und JagdPferdes. By G. Glasenapp. 8vo. Berlin, 1867.

Über Verwendung und Ausbildung der Kavallerie mit besonderer Berücksichtigung des Feldzuges von 1866. By Görgey von Görge. 8vo. Berlin, 1867.

Conseils aux éleveurs de chevaux. By Charles du Hays. 12 mo. Paris, 1867.

Handledning i ridkonsten. By E. Henriques. 16mo. Stockholm, 1867.

Vorbereitung zur Arbeit oder die Kunst seine Pferde leistungsfähiger zu machen und langer bei vollkommener Kraft zu erhalten. By U. v. Hochwachter. 8vo. Neuwied, 1867.

Die Pferdekrankheiten und deren homöopathische Heilung. By G. Hubner. 8vo. Berlin, 1857.

Cavalry Swordsman. By Alfred Hutton, 8vo. London, 1867.

Die französischen Pferde-Racen, ihre Charakteristik und Bezugsorte. By F. R. Jacoby. 8vo. Enfurt, 1867.

Die erste Ausstellung von Pferden aus Gesammtrussland. In Moscau, im September, 1866. Nach authent. Quellen. By Peter Jessen. 8vo. Dorpat, 1867.

Anleitung zum Ertheilen eines systematischen Unterrichts in der Soldatenreiterei, auf Grundlage der fuir die preussische Armee gegebenen Bestimmungen. By Fr. H. Krane. 8vo. Berlin, 1867 ; 3rd edition, $1868 ; 1875$.

Recherches sur la vitesse du cours du sang dans les artères du cheval au moyen d'un nouvel hémadrographe. By Dr. Louis Lortet. 4to. Paris, 1867.

Kurze Uebersicht der Dressur des Kampagnepferdes. By Major Freiherr v. Lutzow. 8vo. Berlin, 1867.

Anatomical Outlines of the Horse. By J. A. Macbride. 8vo. London, [1867].

2nd edition edited by T. W. Mayer. 8vo. Cirencester, 1878.

Rapport sur les progrès de la médicine vétcrinaire depuis 25 ans. By J. H. Magne. 8vo. Paris, 1867. 
1867. Allgemeines österreichisches und ungarisches Gestütbuch. Verzeichniss der in Oesterrich und Ungarn befindliche englische VollblutPferde, nebst deren Abstammung. By Otto Mayr. 8vo. Vienna, $1867 ; 1875$.

La Maréchalerie Française, son histoire depuis son origine, jusqu'à nos jours. By J. P. Mégnin. 8vo. Paris, 1867.

Manuel des courses, France, Angleterre, Belgique, Allemagne, Dictionnaire du turf. By vicomte H. de Mirabal. 12mo. Paris, 1867.

De la Ferrure du cheval. Examen pratique du mode de ferrure inauguré par M. Charlier. By Alphonse Morin. Svo. Paris, 1867.

Die Behandlung des Pferdes auf Reitten nebst einer Anleitung zun Ueben desselben im Galoppiren und Springen. By K. Newte. 8vo. Olmuitz, 1867.

Dictionnaire des courses. By Eugène Paz. 32mo. Paris, 1867.

Le Handicap. Manuel populaire des Courses. Explications élémentaires sur les courses plates et steeple-chases. Vocabulaire des termes de courses. By Olivier Pichat. 12mo. Paris, 1867.

Das diatetische Heilverfahren bei Pferden und der engl. Hufbeschlag. By E. Renner. 8vo. Breslau, 1867.

The Mule, a Treatise on the Breeding, Training, and uses to which he may be put. By Harvey Riley. 12mo. New York, 1867.

Die Pferde des Alterthums. By A. Schlieden. 8vo. Neuwied, 1867.

Leitfaden für Reitunterricht. By J. Seinsheim. 8vo. Munich, 1867.

Haudbok i husdjursskötseln I. Hastens ytterlära. By Gutaf Wilhelm Sjostedt. 8vo. Stockholm, [1867]; 1868.

Cavalry Outpost Drill and Skirmishing. By Major-General M. W. Smith. C.B. London, 1867.

Auszug aus der Reit Instruktion für Unteroffiziere und die 2. Reitklasse. Nebst einer Anhang iber Stallpflege und Behandlung des Hufes. By Frhr. v. Strombeck. 8vo. Berlin, 1867.

The Veterinary Gazette. 8vo. London, 1867, etc.

Traité d'Hippologie, connaissance pratique du cheval. By A. A. Vial. 8vo. Paris, 1867. 
Der Pferdezuichter. Anleitung zur Kenntniss der gesammten Pferde- 1867. wissenschaft. Ein Handbuch fuir Pferdebesitzer und Pferdeliebhaber. By F. Villeroy and A. Müller. 8vo. Mainz, 1867.

An Address on Veterinary Medicine. By F. D. Weisse. 1867.

P. M. om hästens yttre delar, ålder, färg, sjukdomar, etc. By A. L. Wemberg. $12 \mathrm{mo}$. Gotenburg, 1867.

Drei Fragen zı unserer Gestüts-und Landespferdezucht und deren versuchsweise Beantwortung. By G. Zoppretz. 8vo. Ravensburg, 1867.

Abhandlung über das Bändigen und Einfahren stütziger Pferde, über 1868. Auspannungs-Zugs-und Fuhrwerkstheorie. Anon. 8vo. Laibach, 1868.

Einige Worte über die Fechtiibungen der deutschen Kavallerien. Von einem preussischen offizier. Anon. 1868.

Étude sur la cavalerie légère. Formation d'escadrons d'éclaireurs à cheval. Anon. 8vo. Paris, 1868.

Gedanken-Reiter und Reiter-Gedanken. Anon. 8vo. Munich, 1868.

Grundzüge der Organisirung von Anstalten und Verkehrungen zur Hebung der Pferdezucht in Oesterreich Anon. 4to. Vienna, 1868.

Idées pratiques sur les manœvres de la cavalerie par un officier de cavalerie. Anon. 8vo. Paris, 1868.

Kurzegefasste, Theorie, des Reitunterrichts im Anschlusse an die Vorschriften fiir den Reitunterricht der k. b. Cavallerie. Anon. 8vo. Munich, 1868.

Vorschriften über Anlegung der Pferde-Equipage und des Gepäcks für die königl. sächs. Cavallerie 1867. Anon. 8vo. Dresden, 1868.

Das Kriegspferd im gesunden und kranken Zustande. By $\mathrm{K}$. Ableitner. 8vo. Stuttgart, 1868.

Conseils en action dounés aux cochers et aux charretiers, et suivis d'une conférence sur le cheval, son histoire naturelle, ses travaux, etc. By B. de Beaupré. 12mo. Paris, 1868.

Die preuss. Kavallerie in der Kampagne 1866. By L. v. Besser. 8vo. Berlin, 1868.

Handbok i farmakologien för veterinärer. By F. G. Bjorklund. 8 vo. Stockhulm, 1868. 
1868. A. B. C. des Courses. By Canter. 18mo. Paris, 1868.

Projet d'une nouvelle organisation pour la cavalerie française. By Captain L. Cogent. 8vo. Paris, 1868.

Modern Cavalry, its organization, armament, and employment in War. With an appendix containing letters from General Fitzhugh Lee, Stephen D. Lee, and F. L. Rosser of the Confederate States Cavalry, and Colonel Jenyn's system of non-pivot drill in use in the 13th Hussars. By George T. Denison. 8vo. London, 1868.

In German:-Die Cavallerie nach dem Geiste der jetzigen Kriegsführung: ihre Organisation Bewaffnung und Verwendung im Kriege. Translated by E. v. Xylander. 8vo. Munich, 1870.

In Swedish:-Nutidens Kavalleri, dess organisation, etc. Translated by G. M. Björnstjerna. 8vo. Stockholm, 1873.

Une Question historique. L'Hippophagie, 1720-1868. By Abbé V. Dufour. 18mo. Paris, 1868.

On Seats and Saddles, Bits and Bitting, and the prevention and cure of restiveness in Horses. By Francis Dwyer. 8vo. London, $1868 ; 1869 ; 1879$.

Die rationelle Hufbeschlagslehre nach den Grundsätzen der Wissenschaft und Kunst. By W. E. A. Erdt. 8vo. Breslau, 1868.

Einige worte zur sächsischen Pferdezuchtfrage. By H. Erler. 8vo. Dresden, 1868.

Manuel de l'éleveur du cheval croisé en Belgique. By Modeste Fœlen. 18 mo. Paris, 1868.

Manuel populaire sur les soins à donner aux chevaux, ânes, et mulets employés au travail dans les champs, ou dans l'industrie. By Modeste Fœlen. 12mo. Paris, 1868.

La Vallée d'Auge et la côte Deauvillaise. By Charles Du Hays. 8vo. Paris, 1868.

De la cavalerie française. By Captain A. Hocquet. 8vo. Paris, 1868.

Abrégé d'équitation à l'usage de M. M. les officiers d'infanterie, des élèves des écoles préparatoires et de toutes les personnes qui désirent apprendre à monter à cheval, suivi de quelques notions indispensables concernant le harnachement, l'hygiène et les allures du cheval By Lieut. Hoecker. 8vo. Luneville, 1868. 
Die Krankheiten der Pferde und Fullen und deren homöopathische 1868. Behandlung. By G. Lackner. 8vo. Lübeck, 1868.

Försök till praktisk framställn af grunder for fältridning. By P. Lindblad. $12 \mathrm{mo}$. Stockholm, 1868.

Beskrifn öfver husdjurens sjukdomar. By F. Lundberg. 8vo. Stockholm, 1868.

Nouveau traité des formes extérieures du cheval. By Merche. 8vo. Paris, 1868.

A Handy Book of Horse Law, and of the Laws Relating to Field Sports. By C. G. Merewether. 8vo. London, 1868.

Modern Practical Farriery, a complete guide to all that relates to the Horse. By William J. Miles. 2 vols. 4to. London, [1868-69]; [1873-74].

Illustrations and anatomical plates by $B$. Hering.

Apperçu historique sur les institutions hippiques et les Races chevalines de la Russie. By J. Moerder. 8vo. St. Petersburg, 1868. In German :-Die Pferdezucht in Russland. Ein kurzer Ueberblick. 8vo. Vienna, 1873.

Deutsches Gestiit-Album. Photographien vorziiglicher Pferde in den Gestiuten Deutschlands. By $H$. Athaldensl. Nathusius and A. Krocker. folio. Berlin, 1868-71.

Oriental Sporting Magazine. 8vo. Calcutta, 1868, etc.

La Maréchalerie, ou la Ferrure des animaux domestiques. By $\mathbf{A}$. Sanson. 12mo. Paris, 1868.

Gedanken iber einige kavalleristische Angelegenheiten. By W. Siegmann. 8vo. Leipzig, 1868.

Der Hufbeschlage und die Pflege des Hufes. By Starke. 8vo. Minden, 1868.

Tidning for hastvanner. Edited by C. G. Wrangel. 4to. Jönk, 1868.

Studio sullo stato attuale della produzione equino in Italia. By Giudetti Vercelli. [Circa 1868].

Notizen über die Pferdezucht, Gestuteinrichtungen, Veterinärschulen, Marstalle und Trainiranstalten in Deutschland und Frankreich. By A. Vogelmann. 8vo. Karlsruhe, 1868. 
1869. Avenir de la Cavalerie en campagne. Anon. Svo. Paris, 1869.

Brigade and Divisional Movements of Cavalry. Horse Guards. 8vo. London, 1869.

Das Exerziren eines Eskadron zu Fuss. Ein Auszug aus d. ExerzierReglement fiir die Kavallerie der Königl. preuss. Armee v. 5 Mai 1855. Anon. 8vo. Potsdam, 1869.

Exerziren einer Escadron zu Pferde. Ein Auszug aus d. ExerzierReglement fiir die Kavallerie der königl. preuss. Armee vom 5 Mai 1855. Anon. 8vo. Potsdam, 1869.

Instruction iiber das Scheibenschiessen der mit Hinterladungs-Carabinern bewaffneten K. S. Reiter-Regimenter. Anon. 8vo. Dresden, 1869.

Instruction iiber das Scheibenschiessen der mit Zündnadel-Karabinern bewaffneten Kavallerie-Regimenter. Anon. 8vo. Berlin, 1869.

Normen fuir die Behandlung in der k. k. Armee. Anon. 8vo. Vienna, 1869.

Notiz-Buch für den Unteroffizier der Kavallerie, Feld Artillerie u. d. Train. Anon. 16mo. Berlin, 1869 ; 6th edition, 1874.

Pferderennen, Pferdezucht und der Badische Rennverein in Mannheim. Anon. 8ro. Mannheim, 1869.

Reglement fuir die Rennbahnen im preussischen Staate. Anon. 8vo Berlin, 1869.

The Book of Aids, or Catechism of the System of Equitation. Anon. Canterbury, 1869.

Traineringen, el. Konsten att bibringa sui häst högsta möjliga drag af capacitet o. fortfaraude bibehalla honom deri. Anon. 8vo. Lund, 1869.

Den pålitlige hast-o voskaps-läkaren. By Peter Christian Abildgaard. 12mo. Stockholm, [1869]; 7th edition, 1874.

De la Cavalerie dans le passé et dans l'avenir. By Colonel d'Andlau. 8vo. Paris, 1869.

The Horseowner's and Stableman's Companion, or Hints on the Selection, Purchase, and General Management of the Horse. By George Armatage. London, 1869. 
Notions d'Equitation à l'usage de M. M. les Officiers d'Infanterie. 1869. By H. d'Assézat. 18mo. Paris, 1869.

Der preussischer Militär-Rossarzt. Eine Sammlung von Verordnungen welche über die Rossärzte der preussischer Armee entweder direct erlassen worden sind, oder auf dieselben Anwendung finden. By E. Bierlich. 8vo. Berlin, 1869.

Den erfarne djurläkaren el. hushållsdjurens sjuksdomar kannetecken, etc. By Peter Magnus Billman. 12mo. Stockholm, [1869]; 5th edition, 1873.

De l'entrainement des Chevaux de Troupe. By T. Bonie. 8vo. Paris, 1869.

Les Races chevalines en France. By Count Guy de Charnacé. 12 mo. Paris, 1869.

Occasional Papers on Veterinary Subjects, with a Memoir by R. O. Pringle. By William Dick. Svo. London, 1869.

Das Gestiit zu Torgel oder die Pferdezucht in Livland. By J. Dimse. 8vo. Riga, 1869.

Nature et pathogenie de la morve. Études de pathologie comparée. By H. P. Filet. 8vo. Paris, 1869.

Horse Shoes and Horse Shoeing. Their Origin, History, Uses, and Abuses. By George Fleming. 8vo. London, 1869.

Das Offizierspferd. Praktischer Rathgeber insbesonders für berittene Infanterie-officiere und Pferdebesitzer iiberhaupt. By L. Frey. 16mo. Darmstadt, 1869.

La vérité sur la méthode Baucher (ancienne et nouvelle). Observations critiques, précédés d'une petite étude sur l'intelligence du cheval dans ses rapports avec l'equitation. By G. A. Gerhardt. 8vo. Paris, 1869.

Gun, Rod, and Saddle. By Parker Gillmore. 8vo. London, 1869.

Ferrure du cheval. Organisation, maladies, hygiène du pied. By Louis Pierre Goyau. 12mo. Paris, 1869.

Gedanken iiber berittene Infanterie (Dragoner). By M. Gr[adinger?] 8vo. Munich 1869.

Remarks on the Organization of the Bengal Cavalry. By H. 1869.

Le Cheval en France depuis l'époque gauloise jusqu'à nos jours. Géographie et institutions hippiques. By Ephrem Houël. 8vo. Paris, 1869. 
1869. Manuel de petit éleveur de poulains dans le Perche, et spécialement dans le Perche d'Eure-et-Loir. By J. B. Huzard (fils). $12 \mathrm{mo}$. Paris, 1869.

Equitation, haute école et courses de chevaux. By B. J. Jullien. 12 mo. Paris, 1869.

Traité de pharmacie vétérinaire chimique théorique et pratique. By J. Ph. Lebas. 8vo. Paris, [1869]. 7 th edition edited by $E$. Dautreville and $E$. Clement, 1875.

Dermatologie hippique, ou Traité de l'organisation et des maladies de la peau du cheval. By J. P. Mègnin. 8vo. Paris, 1869.

Gang des Pferdes und Sitz des Reiters. By B. C. v. Oeynhausen. 4to. Vienna, 1869.

Horse and Horseman. By C. S. March Phillipps. 18mo. London, 1869.

A. B. C. du Sportsman. Connaissance pratique de l'extérieur du cheval. By Honoré Pinel. 8vo. Paris, 1869.

Modern Tactics of the Three Arms, with Notes on Cavalry Equipment. By Major-General M. W. Smith. C.B. London, 1869.

Descriptive Anatomy of the Horse and Domestic Animals, chiefly compiled from the MSS. of Thomas Strangeways and F. Goodsir. By J. Wilson Johnstone and T. J. Call. 8vo. London, 1869.

Le Carnet du peintre en voitures. By Brice Thomas and Gastellier. 4 to. Paris, 1869.

Der Gang der Dressur des Remontepferdes. By E. v. Troschke. 8vo. Münster, 1869.

Pharmacopoia for the Use of Practitioners and Students of Veterinary Medicine. By R. V. Tuson. 8vo. London, 1869.

Zur Taktik der Cavallerie. Betrachtungen v. L. W. 8ro. Vienna, 1869.

Vorschläge zur Hebung der Landes-Pferdezucht. By WedemeyerSchönrade. 8vo. Berlin, 1869.

Die Anatomie des Pferdekorpers in ihrer praktischen Anwendung auf die äussere Form den lebenden Thieres bildlich dargestellt und beschrieben. By L. Wentz. folio. Carlsruhe, 1869. 
Agra tankar i stuteri-reorganisationsfrågan. By C. G. Wrangel. 1869. 8 vo. Stockholm, 1869.

Betankade ang. stuteriväsendet afgifvet. d. 17 dec. 1869 af Komi- 1870. terade. Anon. 4to. Stockholm, 1870.

Die Kavalleriefrage in Suddeutschland. Material zur Bildung eines selbst-ständliches Urtheils iiber diese Frage. Herausgegeben von einen siiddeutschen Fachmann. Anon. 8vo. Stuttgart, 1870.

Flïchtige, Betrachtungen, iiber Umwandlung des Königl, preuss. Militär-Reit-Instituts in einer Central-Reitschule für das gesammte deutsche Heer. 8vo. Berlin, 1870.

Handbuch für Reserve und Landwehr-Kavallerie Offiziere, sowie für Einjahrig-Freiwillige der Kavallerie. Nach amtlichen Quellen bearbeitet. 8vo. Berlin, 1870.

Kommando-Worte-Aviso und Signale aus dem Abrichtungs-Reglement für die k. k. Kavallerie. 8vo. Vienna, 1870.

Manual and Firing Exercises for the Cavalry Carbine (Westley Richards). 8vo. London, 1870.

Standing Orders of the 19th Hussars. 8vo. Canterbury, 1870.

Taschenbuch für Pferdeliebhaber, oder gründliche Anweisung, in kurzer Zeit ein praktischer Pferdekenner und Reiter zu werden, wie auch junge Pferde schulgerecht zuzureilell. Nebst einer Anhang enthaltend: Hausmittel bei Krankheiten der Pferde, etc. 8vo. Brunswick, 1870.

Three letters on the Horse, Master, and Donkey. By Blunt Spurs [Pseud.] 8vo. London, 1870.

Trotting Record for 1869. New York, 1870.

Ueber den Congress deutscher Pferdezüchter, wie solcher am 21 Febr. 1870 in Berlin zusammentrat. 8vo. Grimma, 1870.

Ueber die Thätigkeit und Verwendung der Kavallerie in Feldzuge 1866 in Böhmen und am Main. Svo. Berlin, 1870.

Vorschriften iiber das Pferdewesen des k. k. Heeres. 4to. Vienna, 1870.

Zur Taktik der Reiterei. 8vo. Stuttgart, 1870.

Notes on Horses for Cavalry Service. By Major Arnold. New York, [Circa 1870]. 
1870. Cavalleristische Mosaiken. By L. v. Besser. 8vo. Berlin, 1870.

Ueber die Aufgaben und die Verwendung der Reiterei im Kriege und iiber ihre Vorbereitung dazu im Frieden. Vortrag. By Bismarck-Bohlen. 8vo. Berlin, 1870.

Etude sur la Combat à pied de la cavalerie. By T. Bonie. Svo. Paris, [1870].

De l'alimentation des enfants et des adultes dans une ville assiégée et en particulier de la viande de cheval. By Dr. Ed. Bourggoin. 8vo. Paris, 1870.

Anteck : 2 om Preussens häst-avel o. Stuterier. By C. F. Boy. 8 vo. Stockholm, 1870.

Centrall-Blatt für die Gesammt-Interessen des deutschen Sport. Officieller Organ des oesterreich. Jockey Club und der Gesellschaft zur Præmiirung gut dressirter Campagne Pferde. Edited by C. Suchomel. folio. Vienna, 1870 , etc.

De l'Équitation consiclérée au point de vue physiologique, hygiénique et thérapeutique. By Dr. R. Chassaigne. 8vo. Paris, 1870.

Trois Journées d'exploration, par une division de Cavalerie en avant d'une armée sur la ligne d'operations de Chalons à Metz. Essai d'après la carte avec un croquis des marches en trois Couleurs. By Maxime Cherfils. 8vo. Paris, 1870.

Campagne-Reiterei und Remonte Dressur. By E. v. Colomb. 8 vo. Berlin, 1870.

American Reformed Horse Book ; Breeding, Rearing, and Management on reformed system of practice. By G. H. Dadd. 8vo. New York, $[1870] ; 1875$.

Theoretisch-practische Anleitung zur Ausuibung den rationellen Hufbeschlags. By Fr. Dominick. 8vo. Berlin, 1870.

Dictionnaire Vétérinaire a l'usage des cultivateurs et des gens du monde. By L. Félizet. 16mo. Paris, 1870.

Znd edition with an introduction by J.A.Barral. 8vo. Paris, 1883.

Introduction to the Osteology of the Mammalia. By Prof. W. H. Flower. 8vo. London, 1870.

Kulturpflanzen und Hausthiere in ihrem Übergang aus Asien nach Griechenland und Italien, etc. By Victor Hehn. 8vo. Berlin, $1870 ; 1874 ; 1877$.

In English as:--The Wanderings of Plants and Animals from their First Home. 8vo. London, 1885. 
Kort afh. om hästens fot, hofbeslaget för hästar o. oxar samt sjuks- 1870 . domarue hos husdjuren. By G. V. Hofling. 12mo. Skara, 1870.

Ein Reit-Institut für die Kavallerie. Neujahrstraum einen alten Kavalleristen. By F. v. K. 8vo. Berlin, 1870.

Linné o. veterinär-vetenskapeu. By J. G. H. Kinberg. 12 mo. Stockholm, [1870].

Anleitung zur Ausbildung der Kavallerie-Remonten. By Fr. v. Krane. 8vo. Berlin, $1870 ; 1879$.

Comparative Longevity in Man and the Lower Animals. By E. R. Lankester. 8vo. London, 1870.

Étude sur les races de Chevaux de la Russie. By Jules Chevalier de La Teillais. 8vo. Renmes, 1870.

Minnesbok for veterinär i tjenst. By C. A. Lindqvist. 8 vo. Stockholm, 1870.

The External Economy of the Horse. By James Irvine Lupton. 8vo. London, [Cirea 1870].

Traité de médecine vétérinaire pratique et d'hygiène. By P. Luton. 8vo. Paris, 1870.

The Law of Horses. By Charles Francis Morrell. 8vo. London, [Cirea 1870].

Les Origines du cheval domestiques, d'après la paléontologie, la zoologie, l'histoire et la philologie. By C. A. Piétrement. 8vo. Paris, 1870.

Der grosse Kavallerie-Kampf bei Stresetitz in der Schlacht von Königgrätz am 3 Juli 1866. By B. Quistorp. 8vo. Neisse, 1870.

Théorie raisonnée de l'école du cavalier à cheval, a l'usage de M. MI. les instructeurs, iudiquant les modifications à faire à l'ordonnance du 6 Dec. 1829, sur les exercises et les evolutions de la Cavalerie, etc. By C. Raabe. Paris, 1870.

Études sur la cavalerie française. By Baron Frederic de Reiffenberg. 18mo. Paris, 1870.

Progression méthodique de dressage avec un simple filet de tous les chevaux de Cavalerie. By L. Rul. Paris, 1870. 
1870 Veterinary Aide Memoire, and Receipt Book for the Use of Non-professional Horse Owners in India. By William Henry Ryves. 8vo. Calcutta, 1870.

Another edition, 1873 ; 6 th edition revised by C. L. Brown and W. H. Kemp. 8vo. Calcutta, 1884.

Deutches Gestuit Album. Photographien vorzuigliche Pferde. Вy $\mathbf{H}$. Schnabeli. folio. Berlin, 1870-74.

Deutsches Gestuit-Buch. Geschichte und Beschreibung deutscher Gestite. By J. Schwartz and A. Krocker. 8vo. Berlin, 1870.

Das königl. preussische Hauptgestiit Gradetz. Mit Stammtafeln und Situationsplänen. By J. Schwartz. 8vo. Berlin, 1870.

Cavalry Equipment. By Major General M. W. Smith, C.B. London, 1870.

Le nouvel armement de la cavalerie depuis l'adoption de l'arme se chargeant par la culasse pour l'infanterie. By C. J. Tackels. 8vo. Cassel, 1870.

Traité de menuiserie en voitures. By Brice Thomas. 4to. Paris, 1870.

Un Voyage Manœuvre de Cavalerie. By Col. J. V. Verdy du Vernois. 18mo. Berlin, [1870].

Records of the Staffordshire Yeomanry. By P. Webster. London, 1870.

1871 Andeutungen für die praktischen Uebungen der Kavallerie. Anon. 8 vo. Szegedin, 1871.

Cavalry sword, carbine, pistol, and lance exercise. Horse Guards. 8 vo. London, 1871.

Undervisning i fälttjensten för Kavalleriet. Anon. 12mo. Stockholm, 1871.

Dienst-Unterricht für den sächs. Cavalleristen. Anon. 8vo. Pirna, 1871.

Exerciereglemente för Kavalleriet. Anon. 12mo. Stockholm, 1871.

Horses : their Rational Treatment and the Causes of their Deterioration. Anon. 8vo. Londor, 1871.

Remonte und Augmentation. Alphabetisch geordnete Sammlung von Pferde-Namen. Eine Kameradschaftliche Gabe für Escadronund Batterie-Chefs, Rittmeister beim Train, sowie für alle Pferdefreunde. $32 \mathrm{mo}$. Celle, 1871. 
Die zukinftige deutsche Militär-Reitakademie. Eine Erwiderung 1871. auf die jiingst bei Mittler and John in Berlin gedruckte Scrift, "Flïchtige Betrachtungen uiber Umwandlung des königl. preuss. Militär-Reit-Instituts in einer Central-Reit-Schule für das gesammte deutsche Herr." 8vo. Leipzig, 1871.

Sicherheits-und Nachrichtendienst für den Gebrauch der EscadronsSchulen. 8vo. Szegedin, 1871.

Positıve Vorschlage zur Hebung der Pferdezucht mit Privat-Staatsund Landgestüten. By K. Ableitner. 8vo. Vienna, 1871.

Campagne de 1870. La Cavalerie française. By T. Bonie. 12 mo. Paris, 1871.

In English:-The French Cavalry in 1870 with its tactical results. Translated by Lieutenant C. F. Thomson. 8vo. London, 1873.

In German:-Die französische Kavallerie. Translated by K. von S. 8vo. Berlin, 1872.

In Swedish:-Kriget 1870. Franska Kavalleriet. Translated by K. Bohnstedt. 8vo. Stockholm, 1873.

Les Cliasseurs de France à la réorganisation de l'armée en 1871. By Count Frédéric de $C^{* * *}$. 8vo. Aachen, 1871.

Manuel du Cavalier; l'equitation sans maitre, suivi d'un traité d'hippologie. By Philippe Desclée. 32mo. Paris, 1871.

Die landwirthschaftliche Fohlenzucht. By W. Dilg. 8vo. Vienna, 1871.

Einige Worte über die Schur des Pferdes von Standpunkte der Gesundheitspflege. By R. A. Duliège. 8vo. Vienna, 1871.

Yeomanry Cavalry. By C. J. E. London, 1871.

Slow Horses made Fast, and Fast Horses made Faster. By J. Elderkin. New York, 1871.

Vétérinaire campagnard; manuel génevois de médicine vétérinaire pratique et usuelle. By J. C. Favre. 12mo. Paris, 1871; 1872.

Animal Plagues, their History, Nature, and Prevention. By George Fleming. 8vo. London, 1871 ; 16mo. London, 1876.

Notes on Cavalry. By Col. Charles Craufurd Fraser, V.C C.B. Agra, 1871. 
1871. Hippologisk teckenlara. By A. Frison v. Zweigberg. $12 \mathrm{mo}$. Lund, 1871.

A Treatise on Horse Shoeing and Lameness. By Joseph Samson Gamgee. 8vo. London, 1871.

Tankar om kavalleriets användn. o. utbildn. By Görgey von Görge. 8vo. Stockholm, 1871.

Notes sur la Cavalerie. By A. Guérin. Svo. Paris, 1871.

The Hub, a monthly Magazine for Carriage Builders. New York, 1871-1876.

Conference d'Etat Major. La Cavalerie et son armement depuis la guerre de 1870. By Baron Auguste Lahure. 8vo. Brussels, 1871 ; 8vo. Paris, $1872 ; 16 \mathrm{mo}$. Brussels, 1873.

Aux cultivateurs. Du Cheval, sa reproduction, son élevage et son commerce. By Gabriel Lapotre. 8vo. Paris, 1871.

The Loriner. Opinions and Observations on Bridle-Bits and the suitable bitting of Horses. By Benjamin Latch ford 4 to. London, 1871.

Containing A Treatise on the suitable bitting of Horses, with a description of a new system of Bridle-Bits. Invented by Don Juan Segundo. Translated from the Original Spanish Manuscript which was dedicated to H.M. George IV. in 1832, and first printed with the "Loriner," by Benjamin Latchford.

Du Rôle et de l'emploi de la cavalerie, aux differentes époques. By A. Lefebvre. $16 \mathrm{mo}$. Namur, 1871 ; Paris, 1872.

Le Jeu sur les courses de chevaux. By $H$. Lefèvre. 12mo. Paris, 1871.

Die Remontirung der Preussische Armee in ihrer historischen Entwickelung und jetzigen Gestaltung. By E. O. Mentzel. 8vo. Berlin, 1871.

Handbook of the Veterinary Art. By Pardon. 1871.

Memoire sur les Chevaux à trentequatre côtés. By C. A. Piétrement. 8vo. Paris, 1871.

Der Percheron, seine Zucht in Frankreich, in welcher Weise bei uns möglich und wie einzufuhren. By A. Plotz. 8vo. Berlin, 1871.

Aphorismen iuber Reitunterricht, Distanzreiten und Stallwart. By Frhr. v. Riedheim. 16mo. Munich, 1871.

Beiträge zur Hebung unserer Landes-Pferdezucht mit specieller Berücksichtigung der Provinzen des südlichen Oesterreichs. By E. Schwarzl. 8vo. Vienna, 1871. 
Anleitung zur Behandlung der Remonten. Als Anhang zum Reitun- 1872. terricht für die Kavallerie. Anon. 8vo. Berlin, 1872.

Zeitgemásse Ansichten über die Kavallerie. Anon. 8vo. Berlin, 1872.

A Proposed New Drill for the Manœuvring of Cavalry on a system without Pivots. Compiled by "Light Cavalry." Anon. Madras, 1872.

Historiques des remontes depuis les Romains, suivi d'un projet d'organisation d'une landwehr hippique. By L. L. Anon. 12 mo. Paris, 1872.

Horse-owner's Companion : hints on selection, purchase, and management of the Horse. By the Happy Hours' Company. Anon. 16mo. New York, 1872.

Betrachtungen uiber die Formation, Verwendung und Leistungen der Reiterei, angeregt durch die Schrift : La Cavalerie française, par Bonie. 8vo. Berlin, 1872.

Anleitung zum Ertheilen einen systematischen Unterrichts in der Soldatenreiterei. auf Grundlage der fïr die preuss. Armee gegebenen Bestimmungen. Mit Nachträgen über das "Nehmen von Hindernissen und das Englisch-Traben." 8vo. [Circa 1872]. Brd edition, 1875.

Instructions for Cavalry Outposts, provisionally approved for Autumn Manœuvres. London, 1872.

Reglement fïr die Flachrennen und Rennen mit Hindernissen im pressischen Staate. 8vo. Berlin, 1872.

Standing Orders for India of the 5th R. I. Lancers. Sealcote, 1872.

Unasked Advice. A Series of Articles on Horses and Hunting. By Impecuniosus. [pseud.] 8vo. London, 1872. Reprinted from the "Field."

Vorschriften für den Unterricht der Kgl. bayer. Cavalerie. Unterricht des Reiters zu Fuss, zu Pferd, des Gliedes, des Zuges, dann der Escadron und des Regimentes zu Pferd. 16mo. Bamberg, 1872.

Lüge und Wissenschaft. Neues zu Altem. Für Offiziere aller Waffen, insbesondere fuir jene der Infanterie und der Reiterei. By Arkelay. 8vo. Frankfurt, 1872. 
1872. Die Erscheinungen der deutschen Literatur auf dem Gebiete der Kriegswissenschaft und Pferdekunde 1865-1871. By Edward Baldamus. 8vo. Leipzig, 1872.

Notes on the Cavalry Drill, or Instructions in the Cavalry Regulations. By Lieutenant Bowdler Bell. Sealcote, 1872.

Fond et Vitesse d'une troupe de Cavalerie en Campagne. Influence du poids et de la nouriture sur le fond et la vitesse. Équitation militaire. Entrainement. By F. Bonie. 12mo. Paris. 1872.

In German:-Leistung und Schnelligkeit einer Reitertruppe im Felde, Einfluss des Gewichtes und der Emährung auf Leistung und Schnelligkeit, Soldatenreiterei, Trainirung, 1873.

In Sicedish:-Om Kavalleriets snabbhet o uthållighet i fällt. Translated by A. Ridderstolpe. 8vo. Stockholm, 1873.

De la Condition des chevaux de chasse en France. By Count Le Couteulx de Canteleu. 12mo. Paris, 1873.

Agenda-formulaire du vétérinaire practicien pour 1872. By $\mathbf{E}$. Clément. 24mo. Paris, 1872.

Réorganisation de la cavalerie. Question du harnachement. By Captain L. Cogent. 8vo. Paris, 1872.

Entwurf zu allgemeinen Regeln für die Aufstellung und den Gebrauch grösserer Cavallerie-Abtheilungen. By E. v. Colomb. 8vo. Neisse, 1872.

La Cavalerie légère. By Colonel baron Daru. 8vo. Paris, 1872.

Universal Bit. Riding or Driving, with six assorted Mouths and Spanner. By A. Davis. 8vo. London, 1872.

Noch ein wort weil es Noth thut, zum Hufbeschlage. By Graf Einsiedel. 8vo. Bautzen, 1872.

Dictionnaire du sport français. Courses, Chevaux, Entraînement, langue dı turf, célébrités du turf, paris et parieurs, règlements, hippodromes. By Ned Pearson [Baron d'Etreillis]. $12 \mathrm{mo}$. Paris, 1872.

De l'equitation dans les regiments de cavalerie en Prusse. By $\mathrm{H}$. de la $F^{* * *}$. 12 mo. Paris, 1872.

Étude sur l'emploi des corps de cavalerie au service de sûreté des armées. By A. Fischer. 12mo. Paris, 1872. 
Étude sur la tactique de la Cavalerie. By A. de Formanoir. 1872. 16 mo. Paris, 1872.

Practical Horse Shoeing. By George Fleming. 8vo. London, $1872 ; 1873$.

Das Reitpferd. Erkennen des Alters, Ankauf, Pflege-und Wartung. Krankheiten, etc. Trainiren. Sport. By L. Frey. 8vo. Darmstadt, 1872.

Ordonnance sur le service intérieur des troupes à cheval, du 2 Nov. 1833. Annotée de toutes les dispositions qui l'ont modifiée jusqu'au 1 Avril 1859, et augmentée du decret du 10 Août, 1872. By Al. Garrel. 8vo. Paris, 1872.

Horse Training madle Easy. By R. Jennings. New York, 1872.

Die Reitkunst in ihrer Anwendung auf Campagne-Militär und Schulreiterei. By A. Kästner. 8vo. Leipzig, 1872; 3rd edition, 1876.

Essai sur une organisation nouvelle de la cavalerie. By A. Laferrière. 8vo. Paris, 1872.

Charcutier, Boncher et Equarrisseur, contenant l'Art de préparer et de conserver les differentes parties du Porc, les maniements et le dépeçage du Bouf, de la Vache, du Taureau, du Vean, du Mouton, du Porc et du Cheval, et traitant de l'utilisation des débris. By Le Brun and W. Maigne. 8vo. Paris, [Circa 1872].

Le Cheval et le mulet; caractères, organization, races, hygiène. Cours d'hippologie, professé à l'ecole spéciale militaire de Saint-Cyr. By Eugène Lemichel. 12 mo. Versailles, 1872.

De la Gale du cheval etudiée dans ses trois variétés, sarcoptique, psoroptique, et symbiotique, et des animalcules qui la produisent. By J. P. Mégnin. 8vo. Paris, 1872.

Instruction sur la manière de harnacher et de charger les chevaux d'artillerie. By C. Muguardt. Brussels, 1872.

Outpost Duty and Reconnaissance. By Major General Napier. London, 1872.

Ueber die Lage der Landespferdezucht in Preussen. By H. Althaldensleben Nathusius. 8vo. Berlin, 1872.

Das system der Frühreife und seine Anwendung auf unsere RemonteZucht und Dressur. By A. Plötz. 8vo. Berlin, 1872. 
1872. Die Entstehungsgeschichte des englischen Vollblutpferdes, sein Einfluss auf die Pferdezucht und Cultur Englands. Eine hippolgische Skizze aus sachverständige Autoren zusammengestelt und erläutet. By A. Plötz. 8vo. Halberstadt, 1872.

Horse Taming. By W. J. Powell. Philadelphia, 1872.

Beitrag zur Pferdezucht in der Provinz Posen. By F. Rahm. 8vo. Bromberg, 1872.

Cavalerie. By Charles Joseph Aloyse Riva. 16mo. Paris, 1872.

Gedanken iiber eine Verbesserung des Reitwesens und der Stallpflege bei den Infanterie. By Richard Schoenbeck. 8vo. Berlin, 1872.

Einige Beiträge zur theoretischer und practischer Veterinär-Wissenschaft, etc. By Konrad Ludwig Schwab. 8vo. Munich, 1872 .

Anleitung zum Voltigiren, sowohl auf dem hölzernen Voltigirbock als auf dem lebendigen Pferde, nebst kurzer Unterweisung, die Pferde an das ruhige Stehen hierzu zu gewöhnen; zuvörderst dem Unterrichte in Kavallerie-Abtheilungen angeeignet, jedoch auch für Privat-Reitbahnen anwendbar. By E. F. Seidler. 8vo. Berlin, 1872.

Die Dressur des Pferdes fiir Kavallerie-Offiziere, angehende Bereiter und Freunde der Reitkunst. Die systematische Dressur des Campagne-und Gebrauchs-Pferdes mit Berucksichtigung junger Pferde, deren Körper noch nicht Kräftig ausgebildet ist. Die Dressur diffiziler Pferde, die Korrektion verdorbener und böser Pferde, erläntert durch Hinweisungen auf den geregelten und ungeregelten Mechanismus und die in Disharmonie gestellten Muskelkräfte des Pferdes, nebst Anleitung zur theilweisen und auch zur speciellen Bearbeitung des Pferdes an der Hand, ohne und mit dem spanischen Reiter. By E. F. Seidler. 8vo. Berlin, 1872; 1878.

Beitrag zum Reitunterrichte. By Graf D. Széchényi. 4to. Pesth, 1872.

Rationelle Ausbildung des Reiters und Pferdes in der Kavallerie oder wie man schnell und gut reiten lernt, und sich jedes zum Reitdienste geeignete Pferd auf rationelle Weisse am schnellsten und sichersten thätig macht. By J. Waldschmidt. 8vo. Berlin, 1872. 
Betrachtungen iiber die Thätigkeit und Leistungen der Cavallerie in 1872. Kriege 1871. By D. H. Walter. 8vo. Cassel, 1872.

In Swedish:-Betraktelser öfver Kavalleriets verksamlet o. bedrifter under 1870-71 ärs krig. Translated by E. Grönvall. 8vo. Stockholm, 1873.

Vorschlage zur Hebung der Pferdezucht. By von WedemeyerSchönrade. 8vo. Berlin, 1872.

Veterinary Surgery. By W. Williams. 8vo. London, 1872; 1876.

Minnesblad för Lakare o. apotekhara sant veterinärer, etc. By $\mathbf{A}$. H. Wistrand. $12 \mathrm{mo}$. Stockholm, 1872.

Nordiska stuteriboken. By C. G. Wrangel. folio. Stockholm, 1872.

Conferences Militaires Françaises, sur la Cavalerie dans le passé et 1873. dans l'avenir. Anon. Paris, 1873.

Das Exerzieren zu Pferd nach dem neuesten Reglement. Anon. 8vo. Augsburg, 1873.

Der Kavallerie-Unteroffizier, als Zugführer, Flïgel und schliessender Unteroffizier in der Eskadron und das Exerzieren des Zuges (die Zugschule) Nach dem königl. preuss. Exerzier-Reglement und anderen Allerhöchsten Bestimmungen. Anon. Svo. Berlin, 1873.

Equipment Table for British Cavalry in India. Anon. 1873.

Handbok i fälttjenstens inöfvande vid kavalleriet. Anon. 8vo. Stockholm, 1873.

Ideen über Kavallerie Verwendung und Bewaffinung im Sinne der neuen Infanterie-Taktik. Von einem össterr. Kavallerie Officier. Anon. 8vo. Vienna, 1873.

Non-Commissioned Officer's Handy Book for Cavalry and Engineers. Anon. Calcutta, 1873.

Outpost Duties of the 10th Royal Hussars. Anon. London, 1873.

Règlement sur le service Interieur la police, et la discipline de Cavalerie. Anon. Brussels, 1873.

Report on East India Government Studs. Anon. 1873.

Standing Orders of the 10th Royal Hussars. ' London, 1873. 
1873. Instruction für die Waffenübungen der Kavallerie. 8vo. Berlin, 1873.

Instructions for Cavalry Outpost duties. Horse Guards. 8vo. London, 1873.

Exercir-Reglement für die Kavallerie der königl. preussischen Armee vom 5 Mai 1855, Neu abdruck unter Bericksichtigung der durch Allerhöchste Cabinets-Ordre vom 9 Jan. 1873, zur versuchsweise Einfiihrung. Folio. Berlin, 1873.

Die bedeutendsten Rennpferde in anerkannt vorzïglichen Photographien. Eine Collection von circa 75 Blätter. Folio. Berlin, 1873.

Organization and Equipment of Cavalry. By Valentine Baker. U. S. Institution, London, 1873.

Cavalry at the Camp. By O. Barnes. Allahabad, 1873.

Catechism of Cavalry Reconnaissance, Marches, and Outposts. By Bowdler Bell. 8vo. London, [Circa 1873].

Hofbeslagslära. By O. Pehrsson Bendz. 8vo. Lund, $1873 ; 4 t h$ edition, 1876.

Om hästkulturen i Normandiet. By O. Pehrsson Bendz. 8vo. Lund, 1873.

Aus der Campagne 1870-71. Der Ehrentag der deutschen Cavallerie am 16 Augt, 1870 bei Vionville und Mars la Tour. By L. v. Besser. 8vo. Berlin, 1873.

In English :-French and Prussian Caralry in the Battle near Vionville and Mars le Tour. Translated by T. H. Smith. Svo. Umballa, 1873.

Notes from a Hunting Box not in the Shires. By Georgina Bowers, obl. folio. 1873.

Anleitung zur Ertheilung des Rekruten-Reit-Unterrichts bestimmt zum Gebrauch der Offiziere und Unteroffiziere der Feld-Speciel der Reitenden Artillerie. By v. Corvisart-Montmarin. 8vo. Berlin, 1873.

Cinésie équestre. Nouvelle étude de cheval et principes inedits d'équitation rationnelle et de haute école. By Emile Debost. 8vo. Paris, 1873. 
Horse shoeing as it is and as it should be. By William Douglas. 1873. 8 vo. Edinburgh, 1873.

Correspondence on Mounted Riflemen. By Lord Elcho. London, 1873.

Les Chevaux de pur sang; physionomie des écuries de courses françaises, propriétaires, entraineurs, jockeys. By Baron d'Etreillis [Ned Pearson]. 18mo. Paris, 1873.

Ein Cavallerie-Regiment im Aufklärungsdienste vor einem grösseren Heereskörper. By Forster and Pidoll. 8vo. Vienna, 1873.

In Swedish:-Ett Kavalleriregementes rekognoseringstjenst. Translated by H. Biel. 8vo. Stockholm, 1874.

Leitfaden des Pferdewesens für k. k. Cadeten und Officiers-Aspiranten. By Görgey von Görge, Toporez, and E. Bauer. 8vo. Vienna, 1873.

Une Conférence sur le commerce des chevaux. By Louis Pierre Goyau. 8vo. Paris, $1873 ; 1874$.

Wochen in Mezöhegyes. Mittheilungen über das königl. ungar. Staatsgestut daselbst. By C. Hahn. 8vo. Stuttgart, 1873.

What to observe and how to report it; being Instructions in Reconnaissance for Non-Commissioned Officers of Cavalry. By Major Lonsdale Augustus Hale. Chatham, 1873. Chapter 4, Reprinted, 16mo. 1876.

Accidents to Horses on Carriage-way pavements. By William Haywood. 8vo. London, 1873.

Privately printed.

Questions d'organisation sur la cavalerie. By A. Hocquet. $12 \mathrm{mo}$. Paris, 1873.

Du cheval de Service; production, élevage et dressage. By Ephrem Houël. 12mo. Paris, 1873.

The Employment of Cavalry in War. By Captain Henry Montague Hozier. 8vo. London, 1873.

Races chevalines de France et de Belgique, leurs caractères, leur production et leur élevage. By F. R. Jacoby. 8vo. Paris, 1873.

Reiten und Dressiren. By F. Joseph. 8vo. Berlin, 1873.

Kritische Blicke auf die Deutsche Kavallerie nach dem Kriege 1870-71. By Major F. Le Fort. 8vo. Berlin, 1873. 
1873. Etude sur la locomotion du Cheval et des quadrupèdes en général, considerée dans ses rapports avec l'equitation et la représentation des quadrupèdes à toutes les allures et à toutes les variétés de ces allures. By Jules Lenoble du Teil. 4to. Paris, 1873.

Die Pferdezucht im Grossherzogthum Baden; ihre Vergangenheit, Gegenwart und anzustrebende Zukunft. By A. Lydtin. 8vo. Karlsruhe, 1873.

Medicine vétérinaire rurale, par un vétérinaire agronome $[\mathrm{J}, \mathrm{H}$. Magne]. 12mo. Paris, 1873.

Hints on the Working and Duties of Cavalry, when in Brigade and Division. By John Henry Porter Malcolmson. Kurrachi, 1873.

Der Remonte Transportfuihrer. Uebersichtliche Zusammenstellung der darauf beziiglichen publizirten Bestimmungen uiber RemonteTransport; deren Instradirung, Verpflegung und Kostenliquidation. By Merres. 1873.

Die Pferdezucht in Russland. Ein kurzer Ueberblick. By J. Moerder. 8vo. Vienna, 1873.

Manuel de l'éleveur de poulains dans le Perche, et particulièrement dans le département d'Eure-et-Loir. By Alphonse Morin. 12 mo. Paris, 1873.

The Perfect Horse: How to know him, how to breed him, how to train him, how to shoe him, how to drive him. By William Henry Murray. 8vo. Boston, 1873.

Abrichtung von Reiter und Pferd, nebst der Zäumungslehre. By Oeynhausen. 8vo. Vienna, 1873.

Le Cheval usuel ou de demi-sang. By Honoré Pinel. 8vo. Paris, 1873.

Gedanken eines Truppen Offiziers über Werth, Verwendung und Kräfte-verhaltniss der Cavallerie-Waffe. Vortrag, gehalten am 7 Februar 1873 im Wiener Militär Casino. By Freiherr v. Pach zu Bernegg. 8vo. Vienna, 1873.

Sicherheitsdienst für die Kavallerie. Nach den Allerhöchsten Verordnungen vom 29 Juni 1861 und nach den sonst hergebrachten Formen zusammengestellt durch O.W.V.R. 16mo. Potsdam, 1873.

Traité de jurisprudence vétérinaire. By A. A. Rey. 8vo. Paris, $1873 ; 1874$. 
Das Pferd in seinen Racen, Farben und Gangarten. Eine hippolog- 1873. ische Monographie. Zugleich Text für die Wandtafeln zur Darstellung der Racen, Farben und Gangarten des Pferdes. By A. Rueff. 8vo. Stuttgart, $1873 ; 1874$.

Notes on Cavalry Service. By Major John Cecil Russell. 12 mo. London, 1873.

Recherches sur la nature des affections typhoïdes du cheval. By J. B. V. Salle. $12 \mathrm{mo}$. Paris, 1873.

Das Soldatenfreundes. Instructionsbuch fiir den Cavalleristen. By L. Schneider. 8vo. Berlin, $1873 ; 1874$.

Re-organisation de la Cavalerie. By Tripard. 8vo. Paris, 1873.

Newmarket and Arabia, an examination of the descent of Racers and Coursers. By Roger D. Upton. 8vo. London, 1873.

Die Stallhaltung der Pferde. Für militär und civil Oeconomen, Thierärzte, Architecten Beamte und Alle, die sich um des Pferdes und unserer Hausthiere Wohl und Wehe bekiimmern wollen oder sollen. By C. Weber. 8vo. Wurzberg, 1873.

Ueber Cavalleristisches Reiten. By Willisen. 8vo. Dessan, 1873.

Lecture on Mounted Riflemen. By Lieutenant-Colonel Wood. London, 1873.

Die Landespferdezucht in Wiirtemberg. Ein Programm ausgegeben am 20 Novr. 1872 bei der 54, Stiftungsfeier der k. Würtemb. Land-und forstwirthschaftl. Akademie Hohenheim. By W. Zipperlen. 8vo. Ulm, 1873.

Cavalry Outpost Duties. By a Cavalry Officer. Anon. Secun- 1874. derabad, 1874.

Instruktion fiir den Cavalleristen und sein Verhalten in und ausser dem Dienste. Von einem Stabs.Offizier. Anon. 16mo. Braudenburg, $1874 ; 1878$.

Mittheilungen iiber das Oldenburgische schwere Wagenpferd. Anon. 8vo. Oldenburg, 1874.

Peeps into the Human Hive. Anon. 2 vols. 8vo. London, 1874. Contains an essay on Buying Horses, and the Tricks of Dealers.

Praktisk underoisningskurs i ritkonoten. Anon. 4to. Stockholm, 1874. 
1874. Andeutungen iiber den Gebranch des Säbels zu Pferde bei den Fechtibungen und in Ernstkampfe. 8vo. Vienna, 1874.

Cavalry Movements, provisionally approved for Cavalry at Aldershot. Svo. London, 1874.

Description détaillée du harnachement de cavalerie modele 1874 adopté par decision ministerielle du 29th Nov. 1874. Paris, 1875 .

Die grossen Kavallerie-Manöver in der Preussischen Armee. 8vo. Berlin, 1874.

Die Preisträger, bei der internationalen Pferde Ausstellung vom 18-27 Septr. 1873 der Weltausstellung in Wien. Folio. Vienna, 1874.

Instructionsbuch für den Cavalleristen. 8vo. Hannover, 1874.

Instruktion iiber das beim Auftreten des Rotzes unter den Pferden der Truppen zu beobachtende Verfahren. 8vo. Berlin, 1874.

Hippologische Mittheilungen und Notizen, uiber die Natur, Eigenschaften, Pflege und Verwendung des Pferdes. 8vo. Vienna, $1874 ; 1878$.

The Anuals of the Irregular Horse. Anon. Allahabad, 1874.

United States Army Cavalry Tactics. New York, 1874.

The Barb and the Bridle, a handbook of equitation for Ladies and manual of Instruction in the science of Riding, from the preparatory suppling exercises on foot to the form in which a lady should ride to Hounds. By Vielle Moustache [pseud.] Anon. 8vo. London, 1874.

Geschichte des Zieten'schen Husaren Regiments. By Freiherr v. Ardenne. 1874.

Le Meilleur de nos Serviteurs, le cheval. Comment il doit être traité et gouverné. Son histoire naturelle. Ses travaux et ses souffrances. By B. de Beaupré. 12mo. Paris, 1874.

Om militär-veterinärväsendet i Preussen o. Frankr. By O. Pehrsson Bendz. 8vo. Lund, 1874.

Tabla öfver hästens alder. By O. Pehrsson Bendz. folio. Lund, 1874.

Cavalry Memoranda. By Lance Corporal Cavandini. Bangalor 1874. 
Memoire sur le service de sureté stratégique de la cavalerie. By 1874. Maxime Cherfils. 12 mo. 1874.

Cavalry Compendium. By J. Congdon. Philadelphia, 1874.

Anleitung zur Ertheilung der Rekruten-Reit-Unterrichts. Nach Remonte. By v. Corvisart-Montmarin. 8vo. Berlin, 1874.

The Horseman's Stable Companion, with valuable receipts; also a list of all works published since 1860 of interest to the Sportsman or Country Gentleman. By A. Davis. 8vo. London, 1874.

Des moyens d'augmenter la production et de prolonger la conservation du cheval de guerre. By Emile Decroi. 12mo. Paris, 1874.

Le Cheval; Etudes sur les allures, l'extérieur et les proportions du cheval, Analyse de tableaux représentent des animaux. By E. Duhousset. 8vo. Paris, $1874 ; 1881$.

How to keep a Horse at a cost of $£ 10$ to $£ 12$ a-year, in fine condition to ride and drive. By Kinard B. Edwards. 8vo. London, 1874.

Die Kolik der Pferde. By F. Friedberger. 8vo. Berlin, 1874.

Le Vétérinaire actif. By Guilbert. 8vo. Paris, 1874.

Der Halbblutzuchter. Wochenschrift fur Pferdezucht und Volkswirthschaft. Officielles Organ der Section 6 (für Pferdezucht) der k. k. Landwirthschafts-Gesellschaft. Edited by C. Suchomel. folio. Vienna, 1874, etc.

Mounted Infantry. By Captain Hogg. 8vo. Bombay, 1874.

England's Horses for Peace or War, their origin, improvement, and Scarcity. By Vere D. de Vere Hunt. 8vo. London, 1874.

Die Reiterei in der Schlacht bei Vionville und Mars la Tour am 16 August 1870. By Kaehler. 8vo. Berlin, 1874.

Seydlitz in seiner Bedeutung fiir die Reiterei von damals und jetzt. Vortrag in der Militär-Gesellschaft zu Berlin am 100 jahr. Gedachtnisstage seines Todes am 7 Novbr. u. 10 Decbr. 1873 geh. By Kaehler. 8vo. Berlin, 1874.

Hippologische Karte von Oesterreich. Auf Veranlassung d. hohen k. k. Ackerbau-Ministeriums bearbeitet. By Kossen v. Sterneck. 4to. Vienna, 1874. 
1874. Le cheval et son chevalier. By Count J. de Lagondie. 2 vols. 12 mo. Paris, 1874.

Country House Essays, containing Horses and Riders, etc. By John Latouche. London, 1874.

Hjelpreda vid den forsta behandlingen af husdjurens allmännaste sjukdomar. By C. A. Lindqvist. 12mo. Stockholm, [18747; 1875.

Jahresberichte iiber die Veränderungen und Fortschritte im Militärwesen. By Von Loebell. 1874, etc.

Die Zucht, Pflege und Veredelung des Pferdes. Encyclopädie fiir Pferdefreunde, Pferdebesitzer und Pferdeziichter. By K. Löffler. 8 vo. Berlin, 1874.

En Avant. Essays on Native Cavalry. By Major MacKenzie. 8vo. Allahabad, 1874.

Historical Record of the 18th Hussars. By Harold Esdaile Malet. 8vo. London, 1874.

Le Cheval. Manuel du cavalier suisse. By B. J. Mercier. $12 \mathrm{mo.}$ Paris, 1874.

Die Pferdezucht. Mit zahlreichen Racebildern und anderen in den Text gedruckte Holzschnitten. By C. F. Müller and G. Schwarznecker. 8vo. Berlin, 1874-79.

Die Zäumung des Pferdes mit ihren jetzigen Mängeln und die Universal Kandare, als verbesserte Zäumung. By Graf von Münster. 8vo. Breslau, 1874.

Beitrag zur Lösung der Frage iiber Landespferdezucht. By B. C. v. Oeynhausen. 8vo. Vienna, 1874.

De la Cavalerie dans le service de sureté stratégique. By N. Pajol. 12 mo. Paris, 1874.

Manuel d'Equitation. By Louis Charles Pellier, Senr. 8vo Paris, 1874.

Die Stallkunde. Ein niitzliches Handbuch fïr alle Pferdebesitzer und Jene, welchen Pferde zur Aufsicht oder Behandlung anvertraut sind und insbesondere für jeden berittenen Militär. By $\mathbf{A}$. Petricic. 8vo. Vienna, 1874. 
Das Halblutpferd als Cavalleriepferd, seine Leistungsfähigkeit und die 1874.

Erhohung derselben durch Erziehung, Dressur und Trainirung. Eine hippologische Cavalleristische Studie. By A. Plötz. 8vo. Halberstadt, 1874.

Down the Road, or Reminiscences of a Gentleman Coachman. By C. T. S. Birch Reynardson. Svo. London, $1874 ; 1875$.

The Book of the Horse: (Thorough-Bred, Half-Bred, Cart-Bred), Saddle and Harness, British and Foreign, Hints on Horsemanship, the Management of the Stable; Breeding, Breaking, and Training for the Road, the Park, and the Field. By S. Sidney. 4to. London, 1874 ; a new edition, 4to. London, 1881.

Story of the Godolphin Arabian. By J. B. Stephens. 8vo. London, 1874.

Histoire de la cavalerie française. By General Susane. 3 vols. $12 \mathrm{mo}$. Paris, 1874.

Rapport au général Morin, directeur du Conservatoire des arts et métiers, sur l'enseignement technique de la construction des voitures. By Brice Thomas. 8vo. Paris, 1874.

Cavalry Tactics assimilated to the Tactics of Infantry and Artillery. By Major-General Emory Upton. New York, 1874.

Studien über Truppenführung. I)ie Kavallerie-Division im Armeeverhande. By General J. Verdy du Vernois. 8vo. Berlin, 1874.

Die Kriegsführung der neuesten Zeit und deren Einfluss auf die Verwendung Organisation, Ausriistung und Taktik der Cavallerie. By D. H. Walter. 8vo. Cassel, 1874.

In Swedish :-Nyare tidens krigföringssätt o. dess inflytande på kavalleriets anvandn, etc. Translated by E. Grönvall. Svo. Stockholm, 1875.

Der Hufschmidt. Anleitung zum Selbstunterricht im Hufbeschlag. By E. Walther. 8vo. Bautzen, 1874.

Ueber Erkelunung des Alters beim Pferd und eine Anhang Gewahrkrankheiten und Gewahrfristen beim Viehhandel. By E. Walther. 8vo. Bautzen, 1874.

Geschichte des Rheinischen Kürassier Regiments Nr. 8. By Lieut. A. von Wellmann. 4to. Hannover, 1874. 
1874. Die theoretische Ausbildung des Cavallerie Unteroffiziers in der Kenntniss des Terrains und dem Felddienste. Leitfaden für den Unterricht und zur Selbstunterweisung. By H. Weyhern. 8vo. Cassel, 1874.

Principles and Practice of the Veterinary Art. By W. Williams. 8vo. London, 1874.

The Trotting Horse of America. By Hiram Woodruff. Pliladelphia, 1874.

Das Pferd im gesunden und kranken Zustande. Ein unentbehrliche Handbuch für jeden Pferde-Besitzer. By L. Zech. 8vo. Vienna, 1874 .

Die Gesundheitspflege der Pferde in Bezug auf Benutzung. By A. $Z$ ünde1. 8vo. Stuttgart, $1874 ; 1882$.

1875. Agenda du vétérinaire praticien pour 1875. Anon. 12mo. Paris, 1875.

Le Cirque Franconi, détails historiques sur cet établissement hippique et sur ces principaux écuyers, recueillis par une chambrière en retraite. Anon. 8vo. Paris, 1875.

Carbine Exercises and Musketry Instruction for the Cavalry of the Army of India. Calcutta, 1875.

Cavalry Regulations, for Instruction, Formations and Movements. 8 vo. London, 1875.

Cours abrégé d'hippologie à l'usage des sous officiers, des brigadiers et éleves brigadiers des corps de troupe à cheval, rédigé par les soins de la commission d'hygiene hippique; approuvée par la ministere de la guerre, 2 Avril, 1875. 32mo. Paris, 1875.

École de voltige militaire, et l'école du chevalier à cheval. Brussels, 1875 .

Exercir Reglement für die k. k. Kavallerie. 8vo. Vienıa, 1875.

Handbook for Military Artificers, containing Instructions for collar makers, etc. Horse Guards. $12 \mathrm{mo}$. London, 1875.

Instruction du 16 Aout 1875 sur l'emploi en campagne du Sifflet dans la cavalerie. Paris, 1875.

Instruction pratique sur le service de la cavalerie en campagne, approuvée par le ministere de la guerre, 17 fevrier. Paris, 1875.

Movements of Cavalry provisionally adopted. Horse Guards. 8vo. London, 1875. 
Instructions in the Duties of Cavalry, Reconnoitring an Enemy, 1875. Marches, Outposts, etc For the nse of Auxilliary Cavalry. Horse Guards. 8vo. London, 1875.

Règlement sur les exercises de la cavalerie. École du peloton à cheval. École de l'escarlron à cheval. École du Régiment. Approuvé par le ministere le la guerre, le 12 juillet 1875 . 18 mo. Paris, 1875.

Reglemente för skjutofningarna vid Kavalleriet. 12mo. 1875.

Standing Orders for the Bengal Cavalry. Simla, 1875 .

Verhandlung der Commission ziir Forderung der Pferdezucht in Preussen. By F. Alpert. 8vo. Leipzig, 1875.

I. Constitution de la Cavalerie. II. Service de la Cavalerie en campagne. By Augey-Dufresse. 8vo. Paris, 1875.

Der Cavallerist im Gefecht zu Fuss. Eine Anleitung zur Instruction mit Berucksichtigung der allerhöchsten Bestimmung. By v. B. 8vo. Berlin, 1875.

Die Erscheinungen der deutschen Iiteratur auf dem Gebiete der Kriegswissenschaft und Pferdekunde 1870-1874. Mit einem Anhange: Die wichtigsten Karten und Pläne Europa's. Systematisch und mit alphabetischen Register. By Eduard Baldamus. 8vo. Leipzig, 1875.

Studie iiber Taktik der Cavallerie. By Ernst Beck. Svo. Vienna, 1875.

Les Aides du Cavalier, ou simples observations sur l'art de conduire et de dresser les Chevanx; suivi de Dialogues : simplification du dressage, etc. Dediée aux jeunes gens appelés à faire partie de l'armée dans la cavalerie. By F. Burdelot. 8vo. Paris, 1875.

Aus der Säbeltasche einen alten Kavalleristen Erzählungen. By Fr. v. Crane. 8 vo. Berlin, 1875 .

Zusammenstellung der Rang-und Quartierlisten der konigl. preuss. Cavallerie von der Reorganisation der Armee nach den Freiheitskriegen und dem Erscheinen der erste Rangliste von 1817 an, bis auf die neueste zeit. By Baron von $\mathrm{D}^{* * *}$. 8vo. Stendal, 1875 .

Méthode progressive applicable an dressage du cheval de troupe, d'officier et d'amateur. By M. F. Dutilh. 8vo. Paris, 1875. 
1875. The Armament of Cavalry. By Lieut. G. H. Elliott. Allahabad, 1875.

A Manual of Veterinary Sanitary Science and Police. By George Fleming. 2 vols. 8 vo. London, 1875.

Cheval de chasse et de service; l'hygiène, la condition, le traitement, suivi d'un manuel veterinaire. By Baron de Fleury. $12 \mathrm{mo}$. Paris, 1875.

Bits and Bearing Reins. By Edward Fordham Flower. 8vo. London, 1875.

Ein Beitrag zur Rassekunde unserer Pferde. By L. Franck. 8vo. Berlin, 1875.

Die Hausthierraçen. Mit Zeichnıngen von H. Schenck. By C. Freytag. 8vo. Halle, 1875-1877.

Circus Life and Circus Celebrities. By Thomas Frost. 8vo. London, 1875.

Élève du cheval de gnerre et du cheval de luxe en Belgique. By Jules Gerard. 8ro. Brussels, 1875.

Leitfaden beim theoret. Unterricht der Kavalleristen. II. Der ältere Kavallerist. By Görgey von Görge. 16mo. Berlin, 1875.

A Chapter on Outposts. By Edward Bruce Hamley. 8vo. London, 1875.

Horses. Law of Bargain. By Hanover. Cincinati, 1875.

Die Hannoversche Cavallerie und ihr Ende. By W. V. Hassell. 8vo. Hanover, 1875.

Training and Horse Management in India. By Captain Matthew Horace Hayes. 8vo. Calcutta, $1875 ; 1878$; fth edition, 1885 .

Projet de création d'une jumenterie arabe par province en Algérie. By G. de Landemont. $12 \mathrm{mo}$. Paris, 1875.

Traité complet d'équitation. Cours élémentaire. By C. Mansuy. 8 vo. Paris, 1875 .

Oesterreichisches Pferde-Stammbuch. Verzeichniss der in den österreich. Kronländern zur Zucht verwendeten Hengste, Stiiten und ihrer Nachkommen. By Otto Mayr. 8vo. Vienna, 1875. 
Die Kraftproduction und der Kraftverbrauch im Pferde vom kavaller- $18 \% 5$. istischen Standpunkte. By F. Frhr.v. Muhlwerth-Gartner. 8 vo. Teschen, 1875 .

Esquisses historiques sur la cavalerie chez les peuples anciens et modernes. By Th. Pasquier. 12mo. Paris, 1875.

Handbuch zur Ertheilnng des theoretischen Unterrichts iber Reiten an Unteroffiziere der Kavallerie und zum Selbstunterricht fiir angehende Reitlehrer. By G. V. Pelet-Narbonne. Svo. Vienna, $1875 ; 1879$.

Nouveaux documents sur quelques points de l'histoire du cheval, depuis les temps paléontologiques jusqu'a nos jours. By C. A. Piétrement. 8vo. Paris, 1875.

Die Pferdezucht-Frage in den im Reichsrathe vertretenen Konigreichen und Ländern. By Ladisl. Graf v. Rozwadowski. 8vo. Vienna, 1875.

Traité d'obstétrique vétérinaire. By Fr. Saint Cyr. 8vo. Paris, 1875 .

Handbok i förlossningskonsten för veterinärer o. uppfodare af husdjur. By Gustaf Wilhelm Sjostedt. 8vo. Stockholm, 1875.

Handbok för veterinärer vid uppsättande af utlåtanden. By W. T. J. Spinola. 8vo. Crönstad, 1875.

Sveriges Veterinär-författninger. Edited by C. R. Walter. Svo. Stockholm, 1875, etc.

Tidskrift fir veterinärer o. landthushallare. Edited by J. G. H. Kinberg. 8vo. Stockholm, 1875, etc.

Causeries d'un homme du cheval. By Bergeaud de Verneuil. 8vo. Paris, 1875 .

The Veterinary Journal and annals of comparative pathology. Edited by G. Fleming. 8vo. London, 1875, etc.

The Veterinary Vade Mecum, a Manual on the Horse, Cow, Dog, and Sheep. Edited by Lord and Rush. 8vo. 1875.

Die Cavallerie Manöver bei Totis. By Joh. Frhr. v. der Waldstatten. Svo. Teschen, 1875 .

Die Gesundheitslehre des Pferdes ; oder die Lehre von der Ernährung Fütterung, Stallung, Pflege, Wartung und sonstigen Behandlung desselben. By J. J. Worz. 8vo. Ulm, 1875. 36 
1876. Ansichten eines Infanteristen iiber clas Feuergefecht bei den Kavallerie-Divisionen. [Anon]. 8vo. Hanover, 1876.

Der Turf. Wörterbuch fïr Pferde-Rennen, enthaltend in alphabetisch geordneter Reihenfolge die gewohnlichsten auf die Pferderennen und den Rennsport Bezug habende Worte und Ausdriicke, nebst deren Erklärung. 32mo. Vienna, 1876.

Exercir Reglement für die Kavallerie. vom 5 Juli 1876. 8vo. Berlin, 1876.

Instruction sur le service de la cavalerie eclairant une armée, approuvée par le ministre de la guerre le 27 juin 1876 . Paris, 1876.

Instructions for Cavalry Adranced and Rear Guards. Horse Guards. 8vo. London, 1876.

Instructions for Cavılry Outpost Duties. 12mo. London, 1876.

Notizen für Unteroffiziere der k. k. Kavallerie. 16mo. Teschen, 1876.

Reglement iiber die Remontirung der Armee. 8vo. Berlin, 1876.

Règlement sur l'instruction à cheval dans les corps de troupes de l'artillerie approuvé par le ministere de la guerre le 28 Oct. 1876. Paris, 1876.

Regulations for the Movements and Instruction of Cavalry. Horse Guards. 8vo. London, 1876.

Tenth Royal Hussars. Rules for Skirmishing. Agra, 1876.

Verhandlungen der auf den 25 Mai 1876 in das k. k. AckerbauMinisterium einberufenen Pferdezucht-Enquete. 8vo. Vienna, 1876.

Practische Anleitung zur Bearbeitung des Pferdes an der Longe. By v. Arnim. 16mo. Hanover, 1876.

Deutsche Reiterei in den Kriegen der Urzeit und den frühen Mittelalters, etc. By Becker. 8vo. Karlsruhe, 1876.

Grundzuige der Mechanik des Hufes und ein darauf gestiitzen naturgemässen Diätetik desselben. By P. R. Brucher. 8vo. Hanover, 1876.

Detached duties of Yeomanry Cavalry under the Mobilization Scheme. By C. J. E***. London, 1876. 
Text Book of Veterinary Obstetrics, including the Diseases and 1876 Accidents incidental to pregnancy and parturition in the Domesticated Animals. By George Fleming. 8vo. London, 1876.

Beitrag zur vergleichen den Beurtheilung der Pferdezucht-Verhältnisse aller deutschen Länder. Die Pferdezucht in Mecklenburg. By H. Hävernick. 8vo. Leipzig, 1876.

Promemoria und 99 Thesen zur deutschen Pferdezuchtfrage. By $\mathrm{H}$. Hävernick. 8vo. Leipzig, 1876.

Pferd und Fahrer; oder die Fahrkunde in ihrem ganzen Umfange, mit besonderer Berüchsichtigung von Geschirren, Wagen, und Schlitten. By Thdr. Heinze. 8vo. Leipzig, 1876.

Redressur oder Behandlung der Mängel und Untugenden bei Reitpferden. By Hermann Hilcke. 8vo. Brünn, 1876.

Notes and reports on the Carriages of the International Exhibitions. By G. N. Hooper. London, 1862 ; Dublin, 1865 ; Paris, 1867 ; Reporter for the Society of Arts, Paris, 1855 ; London, 1876.

Die Kavalleristischen Reglements und Instructionen Friedrich des Grossen. Ein Gedenkblatt seiner Thätigkeit auf dem Gebiete der Bildung, Erziehung und Verwendung des Heeres. By Oberst lieut. Kaehler. 1876.

Hippodromos. Einiges uiber Pferde und Rennen im griech. Alterthum. By Geo. Graf. Lehndorff. 8vo. Berlin, 1876.

Coaching; with Anecdotes of the Road. By Lord William Pitt Lennox. 8vo. London, 1876.

Der Felddienst der Cavallerie. Zum Gebrauche für Offiziere der Waffe. By Frhr. v. Loe. 8vo. Bonn, 1876.

Annals of the Road, or notes on Mail and Stage coaching in Great Britain. By Harold Esdaile Malet. To which are added Essays on the Road by Nimrod. 8vo. London, 1876.

Oesterreichishe Zeitschrift für Hippologie und Pferdezucht. 4to. Vienna, 1876, etc.

From Everglade to Canon with the Second Dragoons (2nd W. S. Cavalry). By Rodenbough. 8vo. New York, 1876. 
1876. Instruktionen betreffend die Erziehung, Ausbildung, Verwendung und Fiihrung der Reiterei von dem einzelnen Manne und Pferde bis zur Cavallerie-Division. Zusammengestellt durch von VollardBockelberg, eingeleitet durch Kaehler. Mit dem Bildniss des Generals von Schmidt. By Carl v. Schmidt. 8vo. Berlin, 1876.

In French :-Instructions relatives à l'instruction, l'education, l'emploi et la conduite de la cavalerie, depuis le cavalier isolé, jusqu'a la division de Cavalerie. Translated by le Capitaine Weil. [Paris, 1877].

Das girettene Pferd, seine Anwendung, Wartung und Pflege. Ein Hiilfsbuch fïr den berittenen Infanterie-Offizier, sowie jeden Besitzer von Dienst-und Luxus-Reitpferden. By Rich. Schönbeck. 8vo. Magdeburg, 1876.

Ueber das Pferdewesen bei der Infanterie. By Rich. Schönbeck. 8vo. Berlin, 1876.

Die theoretische Ausbildung der Rekruten der Kavallerie in Feld. dienst. By Ludwig Frhr. v. Siefried. 8vo. Berlin, 1876.

Outlines of Equine Anatomy. A Manual for Veterinary Students. By J. H. Steel. Svo. London, 1876.

Beitrag zu den Kavallerie-Uebungsreisen. By General J. Verdy du Vernois. 8vo. Berlin, 1876.

Die Anatomie des Pferdes. By C. F. Voigtlaender. folio. Dresden, 1876.

Die Cavalerie-Manover zu Bruck a. d. Leitha im Herbste 1875. By Waldheim. 8vo. Vienna, 1876.

Ansichten iiber Ausbildung eines Eskadron nach den Anforderungen der Jetztzeit. By Hann. v. Weyhern. 8vo. Berlin, 1876.

Die Staats-und Landespferdezucht-Anstalten Wiirtembergs, mit einer Einleitung iber ihre geschichtliche Entwickelung aus den vormaligen fürstlichen Hofgestiiten, nebst einer Darlegung der fruheren und jetzt Betriebsweise der würtembergische Landespferdezucht. By J. J. Worz. 8vo. Ulm, 1876.

Draft Book of Centennial Carriages displayed in Philadelphia, 1870. Anon. folio. New York, 1877.

Karabiner-Schiess-Instruktion für die Kavallerie. Anon. $16 \mathrm{mo.}$ Berlin, 1877. 
Leitfaden zum Unterricht der im Traindienst auszubildenden Kaval- 1877. lerie-Unteroffiziere und Gefreiten. Anon. 16mo. Berlin, 1877.

Exercir-Reglement (im Auszuge) fuir die Cavalerie der k. b. Armee. $16 \mathrm{mo}$. Bamberg, 1877.

Instruktion iber den Kavalleriefelddienst. Auszug fiir Ulaneregimenter. 8vo. Hannover, 1877.

Der rechte Vorderfuss im Naturzustande vor dem Beschlage und dessen stufenweise Veränderung durch fehlerhaften Beschlag. Folio. Bautzen, 1877.

Règlement sur l'instruction à cheval dans les escadrons du train des equipages militaires, approuvé par la ministere de la guerre, le 31 janvier 1877. Paris, 1877.

Vergische Lanziers-Westfälische Husaren Nr. 11. By Frhr. v. Ardenne. 1877.

Every Man his own Horse Doctor. By G. Armatage. 8vo. London, 1877.

2nd edition under the title:-The Veterinarian's Pocket Remembrancer, containing Concise Virections for the treatment of Urgent or Rare cases, embracing Semeiology, Diagnosis, Prognosis, Surgery, Therapentics, Detection of Poisons, Hygiene, ete. $18 \mathrm{mo}$. London, 1884 .

Sammlung von Gestiits-Brandzeichen der Staats-und Privat-Gestiite Europas u. d. Orient. By Carl Brauer. 8vo. Dresden, 1877.

Das Pferd. Belehrmengen uiber Wartung und Pflege, Fehler und Mängel, Krankheiten und Alter des Pferdes. Nebst Regeln zum Schutz gegen Uebervortheilungen beim Ankaufe und einer Anhang: Ein Pferd um die Hälfte billiger, als nach bisher Weise, zu ernähren. By Ferdinand Dallmann. 8vo. Vienna, 1877.

History of Cavalry from the earliest times, with lessons for the future. By George T. Denison. 8vo. London, 1877.

Horses and Harness. A Sequel to Bits and Bearing Reins. By Edward Fordham Flower. 8vo. London, 1877.

Regulations for the Instructions and Movements of Cavalry. By Colonel George Crommelin Hankin. Meerut, 1877.

Einiges über die derzeitigen Verhaltnisse der Landespferdezucht in Nieder-Oesterreich. By Dominik Graf Hardegg. 8vo. Vienna, $187 \pi$. 
1877. Veterinary Notes for Horse Owners; an Every-day Horse Book. By Matthew Horace Hayes. 8vo. London, 1877; 3rd endition, 1885 .

Erfahrungen eines alten Reiters. Rathschläge für Pferdebesitzer und angehende Reiter. Reitenlernen ohne Lehrer, Behandlung junger, bösartiger verrittener Pferde, sowie das Zureiten und Einfahren derselben und Fohlenzucht. By Alexander Graf v. Keller. 8vo. Leipzig, 1877.

Horse Warranty on the Purchase and Sale of Horses, with hints as to methods of procedure in cases of Dispute. By Francis Henry Lascelles, L.L.B. 8vo. London, 1877.

The Farmer's Veterinary Adviser. By J. Law. 8vo. Ithaca, U.S., 1877 ; 8vo. London, 1884.

De l'instruction théorique de la recrute de cavalerie dans le service de campagne. By Lufried. Brussels, 1877.

Leib-Husaren-Regiment Nr 2 im Kriege gegen Frankreich, 1870-71. Ein Beitrage zur Geschichte des Regiments. By Mackenzen. 1877.

Ueber die Reitkunst. By L. W. Otto Digeon v. Monteton. 8vo. Stendal, 1877.

On Horse Breaking. Founded on Experience obtained in England, Australia, and America. By Robert Moreton. 8vo. London, 1877 ; Edinburgh, 1883.

Horses and Riding. By George Neville. 8vo. London, 1877.

Sicherheitsdienst fuir die Kavallerie. Nach den Allerhochsten Verordnungen vom 17 Juni 1870 und nach den sonst hergebrachten Formen zusammergestellt von Otto Wilh. v. R. Verbessert und vermehrt durch H. v. W. 16 mo. Potsdam, 1877.

Die Leitung und Ertheilung des Reitunterrichts bei einem FeldArtillerie-Regiment, auf Grund der Reitinstruktion fuir die konigl. preuss. Kavallerie und der gegebenen hoheren Bestimmungen. By V. Ramm. 8vo. Berlin, 1877.

Die Literatur der Feuerwehr-und Turnwesens, der Heilgymnastik, Fahr-, Fecht-, Reit-, Ring-, Schwimm- und Tanzkunst, sowie des Boxens, Ruderns, Schlitt-schuhlaufens und der Fingergymnastik von 1860-1877. By Hermann Schmidt. 8vo. Prague, 1877.

Black Beauty; The Autobiography of a Horse. Translated by A. Sewell. 12 mo. London, 1877. 
Das Pferd. Erfahrungen aus meinem Leben iiber den Einkauf, die 1877. Pflege, den Hufbeschlag, das Reiten des Pferdes und die Fahrkunst. Für alle Pferdefreunde in gereimten Versen. Als nachlass d. Verstorbenem nebst Einfiilırungswort. Herauscegeben von Rich. v. Meerheim. By J. S. Trautvetter. 8vo. Dresden, 1877.

Officielles Gestiits-Buch für Oesterreich-Ungarn enthaltend die in Oesterreich-Ungarn befindlichen Vollblutpferde englische Abstammung Herausgegeben von der Gestutsbuch Commission des Jockey Club für Oesterreich. By C. Wackerow. 8vo. Vienna, 1877 , etc.

Die Reiter-Regimenter der konigl. preussischen Armee 1571-1876 graphisch dargestellt. By A. v. Wellmann. 4to. Hamnover, 1877.

Strategische Kavallerie-Manover. Studien und Vorschläge, angeregt durch die grossen strategischen Manover der russ. Kavallerie an der Weichsel in Herbste, 1876. By Geo. Cardinal v. Widdern. 8vo. Gera, 1877.

Notes on Cavalry Tactics, Organisation, etc. By a Cavalry Officer. 1878. Anon. 8vo. 1878 .

Nozioni elementari pel soldato di cavalleria, raccolte pel uso del reggimento cavalleria Monferrato. Anon. 32mo. Padua, 1878.

In zwolfter Stunde. Ein Wort an den Reichsrath in Sachen unserer Landespferdezucht von einem Nicht-Steuerer. Anon. 8vo. Vienna, 1878.

Anleitung zur Kenutniss des Pferdes. 16 mo. Frauenfeld, 1878.

Das Feuergefecht der Kavallerie. Ansichten eines alten Kavalleristen mit Riicksicht auf die nenere Kriegfiihrung. 8vo. Munich, 1878.

Kommando-Tabelle für die Kavallerie. Auf Grund des ExerzirReglements vom 5 Juli, 1876. 8vo. Potsdam, 1878.

Manual and Firing Exercise for the Martini-Henry Carbine. Horse Guards. 12 mo. London, 1878.

Manual for Regimental Transport Cavalry. London, 1878.

Wegweiser auf dem Felde der Campagne-Reiterei. Lehrbuch zum Gebrauch beim Theoretischen Reit-Unterricht; sowie zur Selbstbelehrung und Unterhaltung. 8vo. Berlin, 1878. 
1878. Zum Unterrichts fiir die k. b. Cavalerie. Gesammelt aus den Reglements und anderen militärischen Werken. 400 Fragen und Antworten. 16mo. Bamberg, 1878.

Die mecklenburgische Pferdezucht. By Graf v. A. Bernstorff. 8 vo. Stavenhagen, 1878.

The Bedouins of the Euphrates Valley. By Lady Anne Blunt. Edited with a Preface and some account of the Arabs and their Horses, by Wilfred S. Blunt. 2 vols. 8vo. London, 1878.

Nozioni elementari per la cavalleria. By Col. Cagni. 32mo. Verona, $1878 ; 1879$.

Studien iiber die Ausbildung der Artillerie-Remonten. By v. Corvisart-Montmartin. 8vo. Berlin, 1878.

The Clydesdale Stud Book; Retrospective volume containing Pedigrees of Stallions foaled previously to 1st January, 1875. Collected and Compiled by the Right Honorable the Earl of Dunmore 8vo. London, 1878. Privately printed.

Horseback Riding from a Medical point of view. By G. Durant. $12 \mathrm{mo}$. New York, 1878.

The examination of Horses as to soundness; a text book for pro fessional men and amateurs. A course of lectures remodelled and enlarged, delivered at the Veterinary College, with an Appendix on the law of Horses and Warranty. By William Fearnley. 8ro. London, 1878.

American Farrier and Horse Doctor. Notes from the Best Authors. By C. Forrest. 12mo. London and New York, 1878.

Stnt-Buch des konigl. preuss. Haupt-Gestits Trakehnen. By J. P. Frentzel. 8vo. Berlin, 1878.

Our Horses; Being anecdotes from Personal Experience of Individual Horses, with brief practical hints on Breeding. Buying and Selling, Breaking, Shoeing, Doctoring, etc. By Colonel Edmund Armitage Hardy. 8vo. London, 1878.

American Roadsters and Trotting Horses. By H. T. Helm. 4to Chicago, 1878.

Instruction für den Offizier-Pferde-Burschen. By L. v. Heydebrand und der Lasa. 8vo. Leipzig, 1878. 
Das Pferd des Infanterie-Offiziers. Unterweisung über das Pferd im 1878. Allgemeinen, seine Fehler, Krankheiten und Untugenden, sowie iiber Ankauf, Stallpflege und iiber seinen Gebrauch. By L. v. Heydebrand und der Lasa. 8vo. Leipzig, 1878.

Die Pferdezucht in Oesterreich-Ungarn nach ihren Haupt-Typen dargestellt. By Chrf. Josch. 8vo. Klagenfurt, 1878.

Hülfsbuch zur Ertheilung praktischen Instruktion über den CavallerieKarabiner für Unteroffiziere. Mit Zugrundelegung dervom Konigl. Kriegs-Ministerium gegebenen Instruktion bearbeitet und durch 16 Zeichnungen der Schlusstheile erläutert. By Eph. Klatte. 8vo. Berlin, 1878.

Das konigl. preussische Garde-Husaren Regiment im Feldzuge gegen Frankreich 1870-71. Ein Beitrag zur Geschichte des Regiments. By Baron von Kottwitz. 1878.

Die Uebungen der in Herbst 1877, bei Darmstadt zusammengezogenen Cavallerie-Division. Nach den Acten des Commandos dieser Division zusammengestellt. By C. v. L. 8vo. Darmstadt, 1878.

Über die Pferdezucht im Grossherzogthum Oldenburg, nach von 1824-77, angestellten Beobachtungen. Ueber die Pferdezucht der Marschgegenden unseres Landes, besonders des Stedingerlandes. Ueber den Vorfall der Harnblase bei Pferden. Ueber den Bau des Hufes und den Beschlag desselben. Ueber die Schweineseuche (Milzbrand) Beobachtungen von 1843-77. By F. L. W. Loennecker. 8vo. Oldenburg, 1878.

Ueber den Werth und die Anwendung der Freiubungen im Reitunterricht. By von C. M. 8vo. Berlin, 1878.

Riding Recollections. By G. J. Whyte Melville. 8vo. London, 1878.

Gedanken über Reorganisation der Cavallerie. By Arndt. v. Plotz. 8vo. Berlin, 1878.

Skizzen zur Geschichte des Pferdes des Reit-1nnd Fahrwesens. Zusammengestellt nach den neuesten und besten Quellen. By Gust. Pokorny. 8vo. Prague, 1878.

Militärischer Dienstunterricht für die Kavallerie des deutschen Reichsheers. Zunächst für Einjährig-Friewillige, Offizier-Aspiranten und jiingere offiziere des Beurlaubtenstandes. By B. Poten. 8vo. Berlin, 1878. 
1878. Geschichte des ersten Badischen Leib-Dragoner-Regiments $\mathrm{Nr} 20$ und dessen Stamm-Regiments, des Badischen Dragoner Regiments von Freystadt von 1803 bis zur Gegenwart. By Prem. lieut. Ran. 1878.

Das k. k. Militar-Thierarznei-Institut in Wien wahrend den ersten Jahres seines Bestehens. Eine historische Skizze. By M. F. Roll. 8vo. Vienna, 1878.

Commandoschale oder sämmtliche Commando der für die $\mathrm{k}$. bayer. Cavalerie vorgeschriebenem Reglements. By Freiherr v. Rotenham. $16 \mathrm{mo}$. Bamberg, 1878.

Pocket Manual of Homœopathic Veterinary Medicine. By E. H. Ruddock. 12mo. London, 1878.

Handbüchlein zum Gebrauch bei Abrichtung des Remontepferdes. By W. Rudorff. 16mo. Hannover, 1878.

Role of Horse Artillery in a Campaign. By Lieut. Trotter, R.H.A. Prize Essay. Royal Artillery Institution Gold Medal, 1878.

Der Strategische Dienst der Kavallerie. Historische-didaktische Studie. By D. H. Walter. 8vo. Berlin, 1878.

Die russischen kavallerie-Divisionen und die Armee Operationen im Balkan-Feldzuge 1877-78. Nach den Veroffentlichungen aus den russischen Operationsakten bearbeitet. By Capt. Cardinal v. Widdern. 1878 .

1879. Grundsätze der Reitkunst. Für den theoretischen Reitunterricht auf Kavallerie Schulen dargestellt. Anon. 8vo. Berlin, 1879.

Instruktionsbuch für den Cavalleristen. Von einem konigl. preuss. Cavallerie-Offizier. Anon. 16mo. Hannover, 1879.

Karabiner-Schiess-Instruktion für die Kavallerie, abgeändert für den Train. Anon. 16mo. Berlin, 1879.

Leitfaden für die Ertheilung sowolnl des theoretischen wie des praktischen Reitunterrichts von einem Kavallerie-Offizier. Anon. $16 \mathrm{mo}$. Verden, 1879.

Neuer Schauplatz der Künste und Handwerke. Mit Berïcksichtigung der neuesten Erfindungen. Herausgegeben von einer Gesellschaft von Kunstlern, technischen Schriftstellern und Fachgennossen. 8vo. Weimar, 1879. 
Oesterreichisch-ungarischer Renn-Kalender. Herausgegeben von der 1879. Redaktion des "Sport." Anon. 8vo. Vienna, 1879.

Caricamento della fucina da cavalleria. Anon. 18mo. Rome, 1879.

Stalldienst. Bilder aus dem Reiterleben. By Th. Alten. 8vo. Luneburg, 1879.

Der Kavallerie-Unteroffizier als Rekruten-und Reitlehrer sowie als Zugführer, Fliugel-und schliessender Unteroffizier. By Balthasar. 8vo. Berlin, 1879.

Handbuch der Pferdekunde. Für Offiziere und Landwirthe. By L. Born and H. Möller. 8vo. Berlin, 1879.

The Centaur. A weekly Record of the Road. Folio. London, 1879 , etc.

The Horse's Foot and how to Shoe it. Anatomy and Diseases. By J. R. Cole. 8vo. Cincinnati, 1879.

Cenni sull'industria del piede equino, ossia ferri da cavallo in rapporto ai principii della scienza, della pratica e dell'economia, con testie note del prof. Brambilla. By Eligio Collella. 8vo. Milan, 1879.

Lessons in Horse Judging, with instructions for the Summering of hunters. By William Fearnley. 8vo. London, 1879.

Come avviene lo spostamento dell'asse triangolose nella podoflemmatite cronica del cavallo e relativa proposta di cura : lettera al prof. L. Lombardini. By G. Fogliata. 8vo. Pisa, 1889.

Gestütsbuch. Herausgegeben von der Gestiitsbuch-Commission des Jockey Club für Oesterreich. Edited by C. Wackerow. 8vo. Vienna, 1879.

Leitfaden beim theoretischen Unterricht der Kavalleristen. Von G[örgey] v. G[örge] Mit Instruction über den Kavallerie-Karabiner M. 1871 Bearbeitet voul Balthasar. 16mo. Berlin, 1879.

Die deutsche Literatur auf dem Gebiete der Pferdekunde von 1850 1879. In 30 Rubriken systematisch zusammengestellt. By O. Gracklauer. 8vo. Leipzig, 1879.

Die Cavallerie des Deutschen Reichs. Derselben Entstehung, Entwickelung und Geschichte, nebst Rang-, Quartier-, AnciennetätsListe und Uniformisung. By R. v. Haber. 8vo. Hannover, 1879 . 
1879. Études hippiques. By L. Herbin. 4to. Paris, 1879.

Behandlung und Dressur junger Pferde nach neueren Grundsätzen. By C. Herstatt. 16 mo. Neuwied, 1879.

Illustrirte Blatter für Reiter und Pferdefreunde. Mit OriginalZeichnungen von Emil Adam, O. Fickenscher, und H. Lang. 4to. Stuttgart, 1879, etc.

Illustrirte Sport-Zeitung. Edited by MI. v. Laaba. folio. Berlnn, 1879, etc.

Die preussische Reiterei von 1806 bis 1876 in ihrer inneren Entwickelung. A us authentischen Aktenstücken dargestellt. By Kaehler. 8vc. Berlin, 1879.

Officielles organ des Jockey Club für Oesterreich, des TrabennVereines, des Reiter-Club in Wien, des Vollblutzucht-Vereines für Oesterreich und der Gesellschaft zur Prämirung gut dressirter Campagne-Pferd. By Freiherr v. Karst-Karstenwerth. folio. Vienna, 1879.

A Treatise on the Horse and his Diseases. By B. J. Kendall. 8vo. Vermont, 1879.

Dizionario dei termini antichi e moderni delle scienze mediche e veterinarie. By Antonio Longhi and Ernesto Tirinanzi. 8vo. Milan, 1879.

Die Rosshaarspinnerei Deutschlands. By F. Maurer. 8vo. Berlin, 1879.

Die Mechanik und das Pferd. Studien Fragmente iiber die Bewegung des Thierkörpers, mit Bezug auf die daraus resultirende mecanisch begründeten Hilfen. By E. F. Migotti. 8vo. Vienna, 1879.

Leitfaden des practischen Hufbeschlags. By Wilhelm Mohr. 8vo. Wurzburg, 1879.

Der Sicherungs und Nachrichtendienst eines Eskadron. Systematischer Abrichtungs-Vorgang. By Carl Morawetz. 8vo. Vienna, 1879.

Die Ausbildung des Kavalleristen und dem Kavallerie-Karabiner 1871. By O. Natzmer. 8vo. Potsdam, 1879.

Le Cavalier en temps de paix et en temps de guerre, ses devoirs et ses droits. By Th. Pasquier. 18mo. Paris, 1879.

Hippologische Studien, als Lehr-und Lernbehelf für Artillerie-Regiments-Equitationen. By Geo. Petzer. 8vo. Vienna, 1879. 
The Horse : A book for the People. By B. Pitcher. 8vo. Chicago, 1879. 1879.

Kunstliche Mittel zu nützlichen Zwecken. Ein Beitrag zur praktischen Pferde-Dressur (mit Benutzigung von Guérinier's "École de cavalerie.") By Paul Plinzner. 8vo. Stendal, 1879.

El Caballo, su historia, origen de ciertas razas, y nociones sobre los cruzamientos y mestizages de estas. By Don Andres Parlade y Sanchez de Quiros. 4to. Madrid, 1879.

Osterreichische Monatsschrift für Hippologie und Pferdezucht. Offizielles organ der VI. Section (fiir Pferdezucht) der k. k. Landwirthschafts-Gesellschaft in Wien. Edited by Ludwig Ramshak. 4to. Vienua, 1879, etc.

The History of the British Turf, from the Earliest Times to the Present Day. By James Rice. 2 vols. 8vo. London, 1879.

Theoretische Instruktion über Reiterei für die Unteroffiziere der Kavallerie. By Richter. 8vo. Berlin, 1879.

Rathschläge zum vortheilhaften Betriebe der Pferdezucht an kleine Ziichter. By W. v. Sporcken-Ludersburg. 8vo. Celle, 1879.

Leitfaden für den Unterricht der zweiten Reitklasse. Auf Grundlage der preuss. Reit-Instruktion. By Freiherr v. Strombeck. 8vo. Darmstadt, 1879.

The English Stud Book of Cart Horses, the first volume containing Pedigrees of Stallions foaled previous to January 1st, 1877, also two Essays on the Breeding, Rearing, Feeding, and Management of Cart Horses. 8vo. 1880.

Istruzioni sulla divisa della truppa delle armi di fanteria, cavalleria, artiglieria e genio. Svo. Rome, 1880.

Published by the Ministero della Guerra.

Libro genealogico (Stud book) dei cavalli di puro sangue importati o nati in Italia, e registro dei prodotti incrociati. 8vo. Rome, 1880 , etc.

Published by the Ministero di Agricoltura.

Regolamento d'istruzione e di servizio interno per la cavalleria, sevizio in marcia. $32 \mathrm{mo}$. Rome, 1880.

Published by the Ministero della Guerra. 
1880. Die Erscheinungen der deutschen Literatur auf dem Gebiete der Kriegswissenschaft und Pferdekunde, 1875-1879, mit einem Anhange, etc. By Edouard Baldamus. 8vo. Leipzig, 1880.

Englischer Hufbeschlag. Praktische Anleitung für Hufschmiede und Pferdebesitzer. By Heinrich Behrens. 8vo. Berlin, 1880.

Questions and Answers in Part III. of the Cavalry Regulations. By C. W. B. Bell. 8vo. London, 1880.

Rapport sur la Carrosserie. By N. Belvallette. 8vo. Paris, 1880.

Reminiscenza d'igiene ippico-militare pratica. By D. Bertachni. 8vo. Turin, 1880.

The Suffolk Stud Book. By H. Biddell. 8vo. Diss, 1880 .

Leaves from a Hunting Journal. By Georgina Bowers. 4to. London, 1880.

The Hunting Counties of Great Britain, their facilities, character and requirements. By Brooksby. 8vo. London, 1880.

Due parole sul sangue degli animali e sull' abuso del salasso negli equini e nei borini. By Enrico Bucalossi. 8vo. San Miniato, 1880.

Sulla origine del ferro da cavallo. By G. B. Caviglia. $12 \mathrm{mo}$. Rome, 1880.

Reprinted from the Rivista Militare Italiana.

Sulla alimentazione dei cavalli di truppa. By G. B. Caviglia. 8vo. Rome, 1880.

Reprinted from the Rivista Militare Italiana, 1880.

Horse's Teeth; a treatise on their developement, pathology and dentistry. By W. H. Clarke. 8vo. New York, 1880.

Coach Builders' Art Journal. 4to. London, 1880, etc.

Guide for the proprietors of Horses. By Frederick Daniel. 8vo. Calcutta, 1880.

The Race Horse in Training, with some Hints on Racing and Racing Reform. By William Day. 8vo. London, 1880; 1886.

Der Kavallerie-Karabiner M. 71. Beschreibung und Gebrauch. Nach den offiziellen Instruktionen bearbeitet. By Dewall. 8vo. Berlin, 1880 . 
El Indispensable a los Veterinarios y Albeitares. By Rafael 1880. Espejo. $12 \mathrm{mo}$. Madrid, 1880.

Il cavallo commerciale ed il cavallo militare: lettera al prof. Lanzilotti Bondanti By Giacinto Fogliata. 8vo. Milan, 1880. Reprinted from the Clinica Veterinaria.

La Cavalerie pendant la campagne de Prusse (1806) d'après les archives de la Guerre. By P. Foucart. 12mo. Paris, 1880.

Carriage Painter's Manual. By F. B. Gardner. 8vo. New York, 1880.

Recherches sur l'équitation militaire. By Alexandre Gaume. $12 \mathrm{mo}$. Paris, 1880.

Leitfaden bei der Instruktion der Rekruten und der älteren Mannschaften der Kavallerie von G[örgey] von G[örge]. Nach den neuesten Verordnungen bearbeitet von Balthasar. $16 \mathrm{mo}$. Berlin, 1880.

The Horsekeeper. A Journal for Livery Stable Keepers, Omnibus Proprietors, etc., London, 1880.

Principes d'equitation. By E. Lalanne. 12mo. Paris, [1880].

Die Kavallerie-iibungs-reise in Mähren. By E. Merta. 8vo. Vienna, 1880.

Dell' igiene e della medicina veterinaria nel comune di Vigevano. By Epaminondo Moroni. 8vo. Vigevano, 1880.

Manual of pharmacy for the Student of Veterinary Medicine. By W. J. T. Morton. 8vo. London, 1880.

Conseils pour dressage des chevaux difficiles, précédés d'une lettre de M. Pelier père. By F. Musani. 8vo. Paris, 1880.

Horses and Horsemen. By Mrs. Power O'Donoghue. London, 8 vo. 1880.

Intorno alla degenerazione amiloide negli animali e particolarmente nel cavallo. By Giampietro Piana. 8vo. Milan, 1880. Reprinted from the Clinica Veterinaria, 1880.

The International Dictionary for Naturalists and Sportsmen in English, French and German, containing the terms used in Hunting, Shooting, Fishing, etc., Natural History, and the Sciences. By Edwin Simpson-Baikie. 8vo. London, 1880. 
1880. An Essay on Cart Horses. By W. F. Smith. 8vo. Bromley, 1880.

Le cheval à côte de l'Homme. By C. De Sourdeval. 12mo. Paris, [1880].

Cavalerie Francaise, 1870-1879. By C. de Vittré. 12mo. Paris, 1880 ,

Sketches in the Hunting Field. By Alfred E. T. Watson. 8vo. London, 1880.

1881. Annuaire de la Carrosserie. 8vo. Paris, 1881.

The Horse Breeder's Register. Folio. Wisbech, 1881.

Beiträge zur Geschichte de Oesterreichischen Kavallerie . . . in Feldzugen d. xviii. Jahr. hundert und in jenen der neuesten Zeit. Anon. Vienna, 1881.

Le corse dei cavalli in Italia, 1881. Anon, 16mo. Treviso, 1881.

Regolamento per l'amministrazione, la contabilità ed il servizio interno dei depositi d'allevanento cavalli. Anon. 8vo. Rome, 1881. Published by the Ministero del Guerra.

Il libro del soldato di cavalleria. Anon. 16mo. Piacenza Florence, 1881.

Sulle razze equine di Campitello. Anon. 8vo. Mantova, 1881.

The Horse and how to manage him. Anon. 8vo. London, [1881].

- On Horseback, in the School and on the Road. By E. L. Anderson. 12mo. London, 1881.

How to Ride and School a Horse, with a System of Horse Gymnastics. By Edward L. Anderson. 8vo. London, 1881.

Corso magistrale di equitazione saggio d'un maestro per l'istruzione degli allievi e delle maestre nelle ippiche discipline. By Achille Angelini. 8vo. Florence, 1881.

Die Bearbeitung des Remonte-Pferdes. By C. von Arnim. 8vo. Berlin, 1881.

Vescicatorio liquido per le zoppicature dei cavalli e bovini : istruzioni e documenti. By Pietro Azimonti. 8vo. Milan, 1881.

Album de la cavalerie française, 1635-1881. By Count L. de Bouillé. obl. folio. Paris, 1881. 
Uber die Organisation der Cavallerie. By Brix. 8vo. Berlin, 1881. 1881.

American turf register and racing calendar (annual). By S. D. and

L. C. Bruce. New York, 1881.

L'uso della legatura elastica in chirurgia veterinaria : rivista sintetica.

By Alessandro Lanzilotti Buonsanti. 8vo. Milan, 1881.

Practical Treatise on Coach Building. By J. W. Burgess. London, 1881.

A History of the Ayrshire Yeomanry. By W. S. Cooper. 8vo. Edinburgh, 1881.

Nouveaux cas de didactylie chez le cheval et interprétation de la polydactylie des équides en général. By Ch. Cornevin. 8vo. Lyons, 1881.

The Horseman's Guide. By G. P. Delisser. New York, 1881.

Cavalleria in guerra. By G. Eccheli Del Dosso. 16mo. Turin, 1881.

I depositi d'allevamento cavalli in Italia. Memoria. By Clemente Doux. 8vo. Roma, 1881.

Le Cheval: allures, exterieur, proportions. By E. Duhousset. 8 vo. Paris, 1881.

Tratado de la Cria Caballar Mular y Asnal y Nociones de Equitacion. By Rafael Espejo y del Rosal. 8vo. Madrid, 1881.

- Riding on the Flat and Across Country. A Guide to Practical Horsemanship. By Capt. Matthew Horace Hayes. 16mo. London, 1881 ; 1884.

Clever Dogs, Horses, etc. By S. Hibberd. 8vo. New York, 1881.

The Percheron Horse. By C. Du Huys. 8vo, New York, 1881.

Jagttagelser vedrorende Hesteavlen i England og Nordfrankrig. By J. Jensen. 8vo. Copenhagen, 1881.

Della nevralgia brachiale del cavallo : lezione. By Giuseppe Levi. 8vo. Milan, 1881.

The Horse as he Was, as he Is, and as he Ought to Be. By James Irvine Lupton. 8vo. London, 1881.

Beiträge zur Geschichte der fossilen Pferde insbesondere Italiens. By C. J. F. Major. Berlin, 1881. 38 
1881. The Perfect Horse. By W. H. H. Murray. New York, 1881.

- Ladies on Horseback, Park Riding and Hunting, with Hints on Costume and Numerous Anecdotes. By Mrs. Power O'Donoghue. 8vo. London, $1881 ; 1886$.

The Light Cavalry Brigade in the Crimea. Extracts from the Letters and Journal of General Lord George Paget during the Crimean War. 8vo. London, 1881-85.

The Dress, Horses and Equipment of Infantry and Staff Officers. By Captain Henry Hallam Parr. 8vo. London, 1881.

De la variole du cheval, horse-pox, etc. By J. Philippe. 8vo. Rouen, 1881.

Vulgarisation de l'equitation. By J. A. F. J. Pigouche. $12 \mathrm{mo}$. Paris, 1881.

On Equus Prjevalski, a new species of Wild Horse discovered in Central Asia. By M. Poliakoff. St. Petersburg, 1881.

Sopra lo Strato intergranulare della retina" del cavallo. By $\mathbf{R}$. Rampoldi. 8vo. Pavia, 1881.

Le trotteur aux Etats-Unis. By J. Roussel. 12mo. Paris, 1881.

La Russie Chevaline et les courses de résistance. By Paul Salvi. 8vo. Milan, 1881.

Instructions for training Cavalry. By C. von Schmidt. 8vo. London, [1881].

Horse Portraiture. By J. C. Simpson. New York, 1881.

The Horse in Motion, as shown by Instantaneous Photography, with a Study in Animal Mechanics, founded on Anatomy, and the Revelations of the Camera, in which is demonstrated the theory of Quadrupedal Motion. By J. O. B. Stillman. 4to. Boston, 1881 ; 4to. London, 1882.

Elementi di giurisprudenza medico-veterinaria. By C. Vallada. 8vo. Turin, 1881.

Exterior de los principales animales domésticos, y mas particularmente del caballo, o sea estudio de sus formas externas, bellezas y defectos, buenas y malas cualidades con arreglo al servicio ó género de produccion à que se les dedique. By Santiago de la Villa y Martin. 8vo. Madrid, 1881. 
Hygiène du Cheval de troupe. By H. Wolff. 8vo. Paris, 1881. 1881.

Regulations for the Veterinary Department of the Horse Guards. 8vo. 1882. London, 1882.

Famous Racing Men, and Tales of the Turf. By Thormanby [pseud]. 8vo. London, 1882.

Istruzione per la requizione dei quadrupedi per il servizio del $R$. esercito. Anon. $32 \mathrm{mo}$. Rome, 1882.

The Horse and his ways. Anon. 16mo. London, [1882].

Stud Groom. Management of the Horse. Anon. 8vo. Norwich, 1882.

Racing Analysis. Anon. 8vo. Birmingham, [1882], etc.

Brevi cenni sull'oggetto della medicina veterinaria legato coll'esistenza dell' uomo. By Antonio Adriano. 16mo. Milan, 1882.

A System of School Training for Horses. By Edward $L$. Anderson. 8vo. London, 1882.

Leitfaden der Veterinär-Polizei für Stadt-und Bezirksärzte, Thierärzte, Sanitäts-Beamte, sowie für Physikats-Candidaten. By Ant. Barauski. 8vo. Vienna, 1882.

La polizia sanitaria veterinaria considerata nei reguardi dell'igiene e dell'economia pubblica: : memoria letta nell'adunanza generale della Societa veterinaria toscana del 21 febbraio, 1882. By Calmiero Bianchi. 16mo. Turin, 1882.

Die Krankheiten des Herzens des Pferdes. By F. Blazecovic. 8 vo. 1882.

Cenni sulle condizioni medic̀o-veterinarie della provincia di Verona nell' anno, 1880. By Felice Bruni. 8vo. Verona, 1882.

La caballeria en los ejércitos modernos. By Vicente de Cortijo. 8 vo. Madrid, 1882.

Il cavallo di truppa italiano, e la sua razione alimentare. By Alessandro Costa. 8vo. Milan, 1882.

Reprinted from the Clinica Veterinaria.

Die Pferdestaupe. Eine Monographie nach eigenen Beobachtungen. By W. Dieckerhoff. 8vo. Berlin, 1882.

Tratado de higiene veterinaria y de policia sanitaria de los animales domesticos. By R. Espejo y del Rosal. 4to. Madrid, 1882. 
1882. The Simple Ailments of Horses; their Nature and Treatment By W[illiam] F[earnley]. 8vo. Edinburgh, 1882.

Manuale di ippo-podologia. By Giacinto Fogliata. 8vo. Pisa, 1882.

Le Dressage des Chevaux. By G_. 12mo. Paris, 1882.

Doppio compito della cavalleria. By G. Gnecco. 8vo. Rome, 1882. Reprinted from the Rivista Militare Italiana.

Manuale di veterinaria popolare, ossia Istruzioni per l'allevamento, il governo, le compere, etc. degli animali domestici. By G. Gorini. $16 \mathrm{mo}$. Milan, 1882.

De l'Extérieur du Cheval. By A. Goubaux and G. Barrier. 8vo. Paris, 1882.

The Horse Owner's Safeguard. A Handy Medical Guide for every man who owns a Horse. By G. S. Heatley. 8vo. Edinburgh, 1882.

Provvedimenti per una maggiore produzione equina nella provincia di Mantova. By Cesare Meneghini. 8vo. Mantova, 1882.

Stable Management, and the Prevention of Diseases among Horses in India. By J. J. Meyrick. 8vo. London, 1882.

Nouveau manuel de l'éducation du cheval. By Count F. de Montigny. 18mo. [Paris], 1882.

On Horse Breaking. By R. Moreton. 8vo. London, 1882.

Das Vollblutpferd als Regenerator. Gedanken uiber Deutschlands Pferdezucht. By G. Graf. zu Münster. 8vo. Berlin, 1882.

Das schwere Arbeitspferd mit besonderer Rücksicht auf den Clydesdale. Reiseeindricke, Erfahrungen und Betrachtungen. By H. v. Nathusius. 8vo Berlin, 1882 .

Les chevaux dès les temps préhistoriques et historiques. By C. A. Piétrement. Paris, 1882.

Was können wir zur Hebung der Reitkunst thun? By P. Plinzner. 8 vo. Stendal, 1882.

The Management and Treatment of the Horse in the Stable, Field, and on the Road. By a Stud Groom [William Proctor]. 8vo. Norwich, 1882 ; Hertford, 1883. 
Die Seuchen und Herdekrankheiten unserer Hausthiere, mit Rück- 1882. sicht auf die Zoonosen des Menschen. By H. Pütz. Stuttgart, 1882.

Tutti veterinarii : ossia, l'agricoltore veterinario, etc. Versione di F. Meris. By H. Reuner and M. Rothernel. 4to. 1882.

An Essay on the Breeding and Management of Draught Horses. By R. S. Reynolds. 8vo. London, 188 \%

Les Martyrs du Travail: Le Cheval. By A. E. Roche. $18 \mathrm{mo.}$ Paris, 1882.

L'Ane. Medicine vétérinaire. By A. E. Roche. 18 mo. Paris, 1882.

Deposito cavalli stalloni di Crema. By Rossi. 8vo. Crema, 1882.

Die Bein-und Hufleiden der Pferde, ihre Entstehung, Verhïtung und arzneilose Heilung. By Spohr. 8vo. Berlin, 1882; 1883.

Scraps from my Sabretasche. Adventures while in the 14 th Dragoons. By G. C. Stent. 8vo. London, 1882.

How to outwit the Horse. Anon. 8vo. London, [1883].

1883.

Paris à Cheval. By Crafty [pseud.] 4to. Paris, 1883.

The Saddle Horse: a New Method of Teaching Riding and Training by means of Pictures from the Life. By E. L. Anderson. 8vo. Edinburgh, 1883.

2nd edition, containing some observations upon the mode of changing lead in the galop. 8vo. Edinburgh, 1886.

Carriages, Roads and Coaches. By S. Berdmore. 8vo. London, 1883.

The Architecture of the Stables and Country Mansions. By John Birch. 4to. Edinburgh, 1883.

Half-bred Horses for Field or Road. By Earl Cathcart. From the Journal of R. A. S. E., 1883.

Aide Memoire de l'officier de cavalerie en campagne. By Jacques de Chabot. 18 mo. Paris, 1883.

The Smithy and the Forge: a Rudimentary Treatise, including Instruction in the Farrier's Art, with a chapter on Coach Smithing. By W. J. E. Crane. London, 1883.

Les Allures du cheval, démontrées à l'aide d'une planche coloriée, découpée et articulée. By Ed. Cuyer. 4to. Paris, 1883. 
1883. Das strategische Kavallerie Manöver unter General Gurko im südlichen Russland Herbst 1882, und die Reformbestrebungen in der russischen Kavallerie. By A. v. Drygalski. 8vo. Berlin, 1883.

L'Asino nella leggenda e nella letteratura, etc. By Giuseppe Finzi. 8vo. Turin, 1883.

Écuyers et Cavaliers, autrefois et aujour d'hui By le Baron d'Etreillis. 8vo. Paris, 1883.

Compagnie General des Voitures. Études experimentales sur l'alimentation du cheval de trait. By L. N. Grandeau and A. Leclerc. 2 parts, 4to. Paris, 1882-83.

The Sportsman's Gazetteer and General Guide . . . Revised, enlarged and brought down to date by the Author. By Charles Hallock. 8vo. New York, 1883.

Indian Racing Reminiscences, being entertaining narratives and anecdotes of Men, Horses and Sport. By M. Horace Hayes. 16 mo London, 1883.

Horse Breeding Recollections. By Count G. Lehndorff. 8vo. London, 1883.

Kritische Beleuchtung des Reit-und Pferdedressur-Systems d. E. Frhrn. v. Troschke . . . in der Anwendung auf die Kampagne-

.. Reiterei. By Lehnert. 8vo. Rastatt, 1883, etc.

Zur Zahmung des Pferdes und die Kandaren-Einsatz-Garnitur. By G. Graf zu Münster. 4to. Dresden, 1883 .

Carriage Builder's Tour in America. By H. Mulliner. 8vo. Leamington, [1883].

The Cream of Leicestershire. By Captain Pennell-Elmhurst 8 vo. London, 1883.

Harness as it Has Been, as it Is, and as' it Should Bee. 'With Remarks on Traction and the use of the Cape Cart by Nimshivich. [John Philipson.] 8vo. Newcastle-upon.Tyne, 1883.

Hippognosie ou Connaissance complète du cheval. By Honoré Pinel. 12mo. Paris, 1883.

Breeding of Horses for Use. By F. Rane. 8vo. London, 1883.

Text Book of Equine Medicine. By W. Robertson. 8vo. London, [1883]. 
The Horse and Dog, not as they are, but as they should be. Old and 1883. Erroneous Theories relative to the management of the Horse, brought face to face with the Facts of the Nineteenth Century. Together with an elaborate and scientific essay on Horse-shoeing; also the ordinary Diseases of Horses and Dogs and their Treatment, with many valuable recipes; how to tell a Horse's Age up to 21 years, and a full explanation as to how Horses and Dogs are taught numerous tricks. By $\mathrm{H}$ [amilton] Sample, 8vo. [San Francisco, 1883].

Privately printed

Die Zäumung des Pferdes in Theorie und Praxis, ete. By Richard Schoenbeck. 8 vo. Berlin, 1883.

Turfbuch für 1883. By V. Silberer. Vienna, 1883.

Das Training des Rennpferdes. By V. Silberer and G. Ernst. Vienna, 1883.

The History of the Shire Horse, and the Origin of the English Cart Horse. By Frederick Street. 8vo. London, 1883.

Der Lungenbrand der Pferde. By F. A. W. Tappe. 8vo. 1883.

Zeitschrift für Pferdekunde und Pferdezucht. 4to. Stuttgart, 1883 , ete...

The Hackney Horse, as it is, and as it was. A look into some Old 1884. Records. Live Stock Journal Almanae for 1884 .

Illustrated Sports: an illustrated Monthly Journal, with which is incorporated Goy's Calendar of Sports.' Folio. London, 1884, ete

How did it run! answered at sight. Racing form to date... alphabetically arranged. 8vo. London, 1884 , ete.

The vorapper beurs the additional title: "The A B C Racing Guide."

Confessions of a Horse Dealer. Anon. 8vo. London, 1884.

Fanous Horses of America. 59 Portraits of Ameriean Turf Celebrities past and present, with short biographies. Anon. 4to. London, 1884.

Die Thätigkeit der Kavallerie-Divisionen in Kriege. Anon. 8vo. Berlin, 1884.

Sportsman's Friend, or Turf Ready Reckoner, ete. Anon. 8vo. Birmingham, 1884. 
1884. Über die Treffen-Taktik der Kavallerie. Anon. 8vo. Berlin, 1884.

Was haben wir von der russischen Kavallerie zu erwarten? Anon. 8vo. Hannover, 1884.

The Tale of a Horse. By Blinkhoolie [pseud.] 8vo. London, 1884.

$\checkmark$ Across Country. By Wanderer [pseud.] 8vo. London, 1883.

Types of the Turf: Anecdotes and Incidents. By Rapier [pseud.] 8vo. London, 1883.

Horses, Horsemen and Horsemanship, an Anecdotic Medley. By Thormanby [pseud.] 8vo. London, 1885 .

Amelioration de l'espèce chevaline par des accouplements raisonnés. By L. Alasonière. 8vo. Paris, 1884.

Tratado completo de las enfermedades de las animales domésticos, y aves de corral, descritas segun los ultimos adelantos de la ciencia. By B. Arago. 8vo. Madrid, 1884.

Zähmung und Abstammung des Pferdes. By A. Barauski. 8vo. Vienna, 1884.

The Cleveland Bay Stud Book, 8vo. Marton, 1884.

Hippologisches Worterbuch enthaltend eine kurze Erklärung der im Gebiet der Pferdekunde und Reitkunst am häufigsten gebrauchten Wörter und Ausdriicke, etc. By P. Deseler. 8vo. Stuttgart, 1884.

Grundriss der Geschichte der Thierheilkunde. Für Thierärzte und Studirende bearbeitet. By Dr. Friedrich Eichbaum. 8vo. Berlin, 1881.

Handbuch der vergleichenden Histologie und Physiologie der Haussaïgethiere. By W. Ellenberger. Berlin, 1884.

The Season 1883-4. A Saturday with Sir W. W. Wynn's Hounds at Whitechurch Station (in verse). By J. M. Etches. 8 ro. London, 1884.

The Harness Maker's Illustrated Manual. A Practical Guide for Manufacturers of Harness, Pads, Gig Saddles, etc. By W. N. Fitzgerald. 8vo. London, 1884.

Carriage Trimmer's Manual, Guide Book and Illustrated Technical Dictionary. A Practical Treatise for the Carriage Trimmer. By W. N. Fitzgerald. 8vo. London, 1884. 
Fore's Sporting Notes and Sketches, a Quarterly Magazine. 8vo. 1884. London, 1884, etc.

Racing Life, Racing Tales, etc. By Joseph Glover. 16mo. Manchester, 1884.

The Hackney Stud Book. Prepared under the direction of the Editing Committee of the Council of the Hackney Stud Book Society, with an Historical Introduction by Henry F. Euren, Secretary, Norwich, 1884, etc.

Hackney Carriage Guardian. 4to. London, 1884, etc.

La Femme à cheval. Théorie. Pratique, Anecdotes. By le vicomte de Hédonville. $16 \mathrm{mo}$. Paris, 1884.

Das Wachstum der Menschlichen Nagels und des Pferdehufs. By J. Henle. 4to. Güttingen, 1884.

Die Amazone. Einfiihrung in das Gebiet der edlen Reitkunst für Damen. By Leopold von Heydebrand und der Lasa. 8vo. Leipzig, 1884.

Handbuch fur Halbblutzuichter. By Leopold von Heydebrand und der Lasa. 8vo. Vienna, 1884.

Einfache Einleitung zur Pferde-Wartung für Offizierbursche und angehende Pferdewärter. By J. Höfer. 8vo. Cohurg, 1884.

The American Horsewoman. By E. Karr. 8vo. Boston, 1884.

Encyklopädie der gesammten Thierzucht mit Inbegriff aller einschlägigen Disciplinen und der speciellen Etymologie. Handwörterbuch für praktische Thierärzte, Thierziichter, Landwirthe und Thierbesitzer iiberhaupt. Unter Mitwirkung von Ableitner, Anacker, Azary, etc. By A. Koch. 8vo. Vienna, 1884.

Cavalerie. By Baron A. Lahure. 8vo. Paris, 1884.

Dictionnaire de médecine, de chirurgie de pharmacie, de l'art vétérinaire et des sciences qui s'y rapportent. By E. Littré. 8vo. Paris, 1884.

Tratado de patologia general veterinaria. By Martine $z$ y Miranda. 8vo. Madrid, 1884.

Fossile Pferde aus deutschen Diluvial-Ablagerungen und ihre Beziehungen zu den lebenden Pferden. By A. Nehring. 8vo. Berlin, 1884 
1884. The Charlier Horse Shoe: Its Advantages, Disadvantages and Use. By William Pallin. 8vo. Dublin, 1884.

Der Kavallerie-Unteroffizier im innern Iienst der Eskadron. By v. Pelet-Narbonne. 8vo. Berlin, 1884.

The Best Season on Record. By Captain Pennell-Elmhurst 8vo. London, 1884.

Précis de police sanitaire vétérinaire, on Exposé des mesures sanitaires applicables aux animaux en France et en Algérie. By F. Peuch. 12mo. Paris, 1884.

Das Pferd. Organ für die gesammten auf das Pferd beziigliche Interessen. 4to. Dresden, 1885, etc.

Traité d'équitation pratique au point de vue de la promenade et de la chasse. By Charles de Poly. 8vo. Paris, 1884.

Die Dame als Reiterin. Informirung iiber die Reitkunst der Damen. By Ads. Schlaberg. 8vo. Berlin, 1884.

Aide-Mémoire du vétérinaire médicine, chirurgie, obstétrique, formules, police sanitaire, et jurisprudence commerciale. By Jules Signol. 18mo. Paris, 1884.

Turf-Lexicon. By V. Silberer. 8vo. Vienna, 1884.

Cavalry in Modern War. By F. C. Trench. 8vo. London, 1884. Brackenburg (C. B.) Military Handboolis, Vol. 6.

La Cavalerie française en 1884. By Ubiez. 12mo. Paris, 1884.

Observations on the Preservation of Hoofs, and the Designing of Hoof Trophies. By Rowland Ward. 8vo. London, 1884.

Die 10 Gebote des Pferdebesitzers. Die Ursachen der friihzeitigen Gliedmassen-Abnuitzung der Pferde und die Mittel, diesem Ueberstande erfolgreich entgegenzuwirken. By $\mathrm{H}$. Weiss. kopf. 8vo. Augsburg, 1884. 2nd edition, ibid.

An episode in the career of a Charger. By Thomas Willis, folio. London, 1884.

1885. The Lunar Month Summary of Past Racing. Anon. 8vo. London, 1885 , etc.

Notes sur les cavaleries étrangères. Paris, 1885. 
A New Book of Sports. Reprinted from the Saturday Review. 1885. Anon. 8vo. London, 1885.

Contains, Coaching, p. 165. Riding Schools, p. 173.

Illustrated Stable Maxims. To be hung in Stables for the use of Grooms and Stablemen, on sheet. London, 1885.

The Badminton Library-Hunting. By the Duke of Beaufort and Mowbray Morris. 8vo. London, 1885.

Lehrbuch der Veterinär-Augenheilkunde für den Unterricht und praktischen Gebrauch. By F. Blazekovic. 8vo. Vienna, 1885.

Selbstunterricht in der Pferde-Kenntniss. By P. Brand. 8vo. Frankfurt, 1885.

El caballo, su constitucion, resistencia y conservacion. By C. Fernández de Castroverde. 4to. Barcelona, 1885.

Sport. By Bromley Davenport, M.P. Illustrated by LieutenantGeneral Crealocke, C.B., C.M.G., 4to. London, 1885.

Recherches expérimentales sur la viande de cheval et sur les viandes insalubres au point de vue de l'alimentation publique. By E. Decroix. 8vo. Paris, 1885.

Nouvelle Méthode d'escrime à cheval. By Captain Dérué. $12 \mathrm{mo}$. Paris, 1885.

Patroclus and Penelope: a Chat in the Saddle. By Theo. A. Dodge. Boston and Edinburgh, 1885.

Hound and Horn: or the Life and Recollections of George Carter, the Great Huntsman. By I. H. G. London, 1885.

De l'Instruction à cheval dans les régiments d'artillerie de campagne. By A. Girette. 8vo. Paris, 1885. Reprinted from the Revue d'Artillerie.

Veterinary Pharmacology and Therapeutics. By J. B. Gresswell. 12 mo. London, 1885.

Equine Medicine: A Manual of its Theory and Practice, for the use of Veterinary Surgeons, Students, and Owners of Horses. By J. Brodie Gresswell and Albert Gresswell. London, 1885.

Betrachtungen über den Felddienst der Kavallerie. By Lieutenant v. Haugwitz. 8vo. Berlin, 1885. 
1885. Soundness in Horses. By M. Horace Hayes. 8vo. London, 1885.

The Points of the Horse. A familiar Treatise on Equine Conformation. By M. H. Hayes. obl. 4to. London, 1885.

Practical Veterinary Remedies. A useful Handbook on Medicine, describing its Properties, Action, Uses, and Doses. By G. S. Heatley. 8ro. Edinburgh, 1885.

'The History of Newmarket and the Annals of the Turf, from the Earliest Times to the End of the Seventeenth Century. By J. P. Hore. Vol. 1, London, 1885.

Die einheitliche Rert-und Fahr-Ausbildung der Feld-Artillerie. By . Captain Hube. 8vo. Berlin, 188 s.

The Steeplechase Horse : How to Select, Train and Ride Him. With notes on Accidents and Diseases, and their Treatment. By Captain J. Humfrey. 8vo. Calcutta, 1885.

Lettres sur la cavalarie. By E. Jaeglé. Paris, 1885.

Gemeinverständlicher Leitfaden der Anatomie und Physiologie der Haussäugethiere. By Dr. H. Kaiser. 8vo. Berlin, 1885.

Précis de thérapeutique vétérinaire avec données scientifiques spéciales sur les effets des alcaloides. By M.Kaufmann.12mo.Paris, 1885.

Atlas der Anatomie des Pferdes und der iibrigen Hausthiere fiir Thierärzte und Studirende der Veterinärkunde, landwirthschaftliche Lehranstalten und Pferdeliebhaber iiberbaupt. By A. G. S. Leisering. folio. Leipzig, 1885, etc.

British Museum Mammals. Catalogue of the Fossil Mammalia, Part II., Ungulata. By R. Lydekker. 8vo. London, 1885.

Comment il faut choisir un cheval, comnaissances pratiques sur l'anatomie, l'extérieur, les races. Principes pour essayer les chevaux de selle et d'attelage. By the Comte de Montigny. $16 \mathrm{mo}$. Paris, 1885 .

In Saddle and Stable. The Common Sense of Riding. By Mrs. Power O'Donoghue. 16mo. London, 1885.

On Mammalian Descent: The Hunterian Lectures for 1884. Being nine lectures delivered in the theatre of the Royal College of Surgeons during February, 1884. By W. Kitchen Parker. 8 vo. London, 1885.

Contains:-Fossil Horses, p. 144; Evolution of the Horso Tyре, р. 202. 
Der schwarze Staar der Pferde. Eine diagnostische und forensische 1885. Studie. By J. Peters. 8vo. Berlin, 1885.

Draught. The Worshipful Company of Coach Harness Makers' First Prize Essay. By William Philipson. 8vo. Newcastle-onTyne and London, 1885.

The Technicalities of the Art of Coach-body-making. By John Philipson. London, 1885.

Our Horses; or the Best Muscles, controlled by the Best Brains. By Alfred Saunders. 8vo. London, 1885.

Instruktionen betr. die Erziehung, Ausbildung Verwendung und Führung der Reiterei von dem einzelnen Manne und Pferde bis zur Kavallerie-Division. By Carl von Schmidt. 8vo. Berlin, 1885 .

The Mammalia in their Relation to Primæval Times. By Oscar Schmidt. 8vo. London, 1885.

Das Gymnasium des Pferdes. By Gustav Steinbrecht, 8vo. Potsdam, 1885.

Neues, illustrirtes Haus-Thierarzneibuch. Eine ausfiihrliche Beschriebung der Zucht und Haltung sämmtlicher Hausthiere, der inneren und ausseren Krankheiten und deren Behandlung, der Thiersenchen, der Haupträngel einschliesslich der beziiglichen Gesetze, sowie der Geburtshilfe und des Hufbeschlags. By V. Strebel and E. Reicherter. 8vo. Reutlingen, 1885.

Tabellen für das Turnen der Truppen zu Pferde. By von Thumen. $16 \mathrm{mo}$. Nordhausen, 1885.

La Cavalerie aux manœuvres des 4 e et 17 e corps. By Ubiez. 8 vo. Paris, 1885.

Reprinted from the "Revue de Cavalevie."

Die Verwendung der Cavallerie im Gefechte abgeleitet aus dem Wesen und den Eigenshaften der Waffe. By Wilhelm Edler von Vivremont Lahousen. 8vo. Vienna, 1885.

Cavalleristische Versuche. By Hann von Weyhern. 8vo. Berlin, 1885 .

Horse and Man : their Mutual Dependence and Duties. By J. G. Wood, M.A. Svo. London, 1885. 
1885. Anleitung zur Kenntniss und Gesundheitspflege des Pferdes. By E. Zschokke. $12 \mathrm{mo}$. Zurich, 1885.

1886. Portraits of celebrated Race Horses of the Past and Present Centuries, in chronological order, commencing in 1702 and ending in 1870 , together with their respective pedigrees and performances recorded in full. Anon. London, 1886.

Organisation et rôle de la cavalerie française pendant les guerres de 1800 à 1815 . Anon. 8vo. Paris, 1886.

A travers la Cavalerie: Organisation, Mobilisation, Instruction, Administration, Remontes, Tactique. Anon. 8vo. Paris, 1886.

Dressage du cheval de chasse, suivant la méthode de teu M. le commandant Dutilh, par un de ses élèves. Anon. 8vo. Paris, 1886.

Le Cheval, Extérieur, Structure et fonctions, Races. By E. Alix and E. Cuyer. 4 to. Paris, 1886.

Vice in the Horse, and other Papers on Horses and Riding. By E. L. Anderson. 8vo. Edinburgh, 1886.

Horse Racing in France, a History. By Robert Black. London, $1886 ; 1887$.

La Cavalerie de seconde ligne en France et à l'étranger. Appels et période d'instruction. By R. Brunet. 18 mo. Paris, 1886.

Les Haras de France. By Edouard Cavailhow. 12mo. Paris, 1886.

Nouveau Manuel du Cocher, contenant une étude sur les principales races de chevaux, des notions d'hygiène, de dressage, etc. By E. Court. 12 mo. Paris, 1886.

Practice of Veterinary Medicine and Surgery. By E. Courtenay. 8 vo. London, 1886.

The Practical Horse Keeper. By George Fleming, LL.D., F.R.C.V.S. 8vo. London, 1886.

Die Reiter und die Rittercenturien zur Zeit der römischen Republik. By Bh. Gerathewohl. 8vo. Munich, 1886.

The Equine Hospital Prescriber, drawn up for the use of Veterinary Practitioners and Students. By J. B. and A. Gresswell. $12 \mathrm{mo} .1886$.

Diseases and Disorders of the Horse. A Treatise of equine Medicine and Surgery. By.J. B. and A. Gresswell. 8vo. Leeds, 1886. 
Zur Gesundheitslehre des Pferdes nach practischen Erfahrungen. 1886. I. Thl. Die rationelle Fütterung und die wichtigsten Futtermittel. II. Thl. Der Pferdestall und seine sanitären Erfordernisse. By W. Haase. 8vo. Berlin, 1886.

Every man his own Veterinarian. A Practical Manual of the Diseases of Domestic Animals. By G S. Heatley. 8vo. Edinburgh, 1886.

Hippologie. Etude du cheval. By J. Hughes. 8vo. Paris, 1886.

Manual pratique d'equitation. By Le Brun-Renaud. 12mo. Paris, 1886 .

Études sur la Cavalerie. By H. Martin. 8vo. Paris, 1886.

Comment il faut dresser un cheval. Connaissances pratiques d'hippologie, principes d'attelage, etc. Avec 81 vignettes. By le Comte de Montigny.

Das Hauptgestiit Beberbeck unter preussischer Verwaltung. Mit einem Anhang: Ueber den gegenwärtigen Zustand des preussischen Gestuitswesens. By Paalzow. 8vo. Berlin, 1886.

Le Cheval. Traité complet d'hippologie, suivi d'un cours d'équitation pour le cavalier et la dame. Avec 182 vignettes et figures. By E. Santini.

Bibliographie générale des ouvrages sur la chasse, la vénerie et la fauconnerie, publiés ou composés depuis le $\mathrm{xv}^{\mathrm{e}}$ siècle jusqu'à ce jour en français, latin, allemand, anglais, espagnol, italien, etc. Avec des notes critiques et l'indication de leur prix et de leur valeur dans les différentes ventes. By $R$. Souhart. 8vo. Paris, 1886.

The Badminton Library-Racing. By the Earl of Suffolk and Berkshire, and Mr. W. G. Craven, with a contribution by the Hon. F. Lawley.- Steeplechasing. By Arthur Coventry and Alfred E. T. Watson. 8vo. London, 1886.

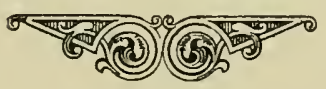





\section{A P PENDIX.}

Wahrhafftige vnd eygentliche Contrafactur, vnd Formen, der Zäu- 1562. mung vnd gebiss, zu allerley mängeln vnnd vndterrichtung der Pferdt, sampt jren zugehörenden Nassbändern, Cauczonj, Stegreyf, Sporn, vnnd anderem, so zur Reytterey erfordert wird. By Hans Kreutzberger. folio. Augsburg, 15.62.

Gemaltes Bissbuch. (Kinnketten). By Pietro Ant. Ferrari. folio. 1629. 1629

A German translation of the original Italian work, 1602.

Diverses Figures et Manieges de Cheraux. Jy Le Marquis De 1660. Sourches. small 4to. [Circa 1660].

The School of Recreation; or a Guide to the most ingenious exercises of Hunting, Riding, Racing, Hawking, Temnis, Cock-fighting,

1684. Angling, etc. By R. H. 12mo. London, 1684; 1720.

Cavalcade ende Triumph-Waghens de welcke toeghernst door de Jonckheydt de Scholen . . . der Societeyt Jesu in de Stadt van 1790. Brussel sullen vertoont worden den 21 Julii, 1698. Anon. 4to. Brussels, 1770.

Trattati completi sul cavallo, sul bue, sugli asini, sui muli, etc. By 1793 Sav. Scrofani. 8vo. Venice, 1793 .

Loyal Volunteers of London and Environs, Infantry and Cavalry in their respective Uniforms, representing the whole of the Manual, Platoon, and Funeral Exercises. By T. Rowlandson. 4to. London, 1799.

Die Geschichte des Pferdes, sein Ursprung, seine körperlichc und psychische Charakteristik, seine Varietäten und Stammverwandten. Nebst einem Anbange W. Youatt's: Ueber die Krankheiten des Pferdes, Nach dem Englischen bearbitet von Dr. F. M. Duttenhofer, By William C. L. Martin. 8vo. Stuttgart, 1851.

A translation of Martin's History of the Hor'se, vide 1845. 40

1851. 
1876. Analysis of the Derby, Oaks, and Grand Prix de Paris. By Snap. [pseud.] 8vo. London, 1876.

1877. Der Fenergefecht der Kavallerie. Anon. 8vo. Munich, 1877.

Cavalerie et forteresses. By A. L. Cambrelin. 8vo. Gand, 1877.

1878. Méthode d'Equitation. By L. Emery de Collomb. 8vo. Brussels, 1878.

Instructions on the Employment of the Whistle for Cavalry Outposts.

By W. L. Twentyman. 16mo. [Dublin? 1878].

The World on Wheels. By E. M. Stratton. 8vo. New York, 1878

Manual for Transport (Cavalry). Horse Guards. 16mo. London, 1878.

Du Cheval de Guerre et des soins qu'il réclame. By H. J. T. Aerts 8vo. Brussels, [1878].

Landwirthschaftliches-Gesellschaft. Monatsschrift fiir Hippologie und Pferdezucht. folio. Vienna, 1878, etc.

1879 Dentition of the Horse. By C. E. Curtis. 8vo. Alton, 1879.

Die Brusthöhle des Pferdes. By F. Eichbaum. 8vo. 1879.

Directions for Preserving Carriages. By Hooper \& Co Single sheet folio. London, [1879].

Traité de médicine légale vétérinaire. By J. B. Dessart. 8vo. Brussels, 1879.

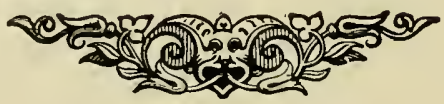


INDEX TO

NAMES OF AUTHORS. 



\section{INDEX TO \\ NAMES OF AUTHORS.}

A. J. P. 1850

Abadie. Bernard 1860

Abd Ullah Ben Safi. 1407

Abd Ullah Khan Behadoor Firuz Jang. 1650

Abildgaard. Peter Christian 1770

Ableitner. C. 1866. 1867. 1868. 1871

Abzac. Alexis d' 1852

Acland. Lt. Col. 1860

Adams. John 1799

Adams. Williams Bridges 1837

Adriano. Antonio 1882

Aerts. H. J. T. 1878

Aguilar. Pedro de 1572

Ahmad. Nizam Ud-Din 1660

Aigeldinger. Joseph 1852

Ainslie. Major 1843

Airie. Douterluigne 1860

Alasonière. Louis 1857. 1884

Albrecht. Christian David 1857

Aldéquier. Flavin D' 1843

Aldrovandus. Ulysses 1616

Alexandre. 1848

Alix. E. and E. Cuyer 1886

Alken. Henry 1816. 1824. 1854

Allen. 1737

Allen. John 1825

Allen. R. L. 1848

Allen. William 1798

Allesandro. Giuseppe 1711

Almond. Robert 1673 
Alpert. F. 1875

Alpin. J. F. 1831

Alten. Th. 1879

Alton. E. D' 1810

Ambert. Genl. 1863

Ambros Miguel Nicolas 1686

Amman. Josz. 1569. 1584

Ammianus Marcellinus. 360

Ammon. Carl Wilhelm 1802. 1804. 1805. 1807.1808.

Ammon. Georg Gottlieb 1829. 1833

$[1809$

Ammon. K. H. 1834

Amoreux. P. J. 1810

Anacker. Johann Heinrich Hermann 1857

Andlau. Col. d' 1869

Andrada. Pedro Fernande $z$ de 1580

Andrade. Antonio Galvao de 1678

Andrade. Manoel Carlos de 1790

André. J. Ch. H. 1805

André. F. 1867

André. Jean 1864

Angelini. Achille 1881

Angelo. Henry 1840. 1850

Anginiard. Hippolyte 1857

Anker. Matthias 1854

Apperley. Charles James 1831. 1835. 1837. 1838.1842

Apsyrtus. 322

Aquino. Gio. Paolo d' 1636

Arago. B. 1884

Arboval. Hurtrel D' 1838

Arcellazzi. Stefāno 1813

Ardenne. Freiherr v. 1874. 1877

Arentschildt. 1854

Arias de Avila. Juan 1590

Arkelay. 1872 
Arlot. 1860

Armatage. George 1869.1877

Arnault. Henri 1857. 1858

Arnim. 1876. 1881

Arnold. Maj. 1870

Arnous-Rivière. 1860

Arredondo. Martin 1661

Ascheberg. J. H. 1836

Assézat. H. d' 1869

Astley. John 1584

Astley. Philip 1774. 1800

Aubert. P. A. 1836. 1842. 1845

Auboyer. 1845

Aubry. Charles 1833

Augey-Dufresse. 1875

Aure. Antonio Henri Phillippe Comte d' 1842. 1844.

Autenrieth. Fr. 1832

[1845. 1855. 1860

Authenrieth. Chr. Fr. 1823

Autheville des Amourettes. Louis d' 1756

Auzoux. Louis 1848, 1853. 1854, 1855. 1857. 1860

Ayala. Mariano d' 1854

Aygaleng. 1809

Aymon. Roche-1817. 1828

Ayraud. P. N. 1851

Azémar. Michel Martial 1860

Azimonti. Pietro 1881

Bach. Chr. de. 1829

Badcock. John 1829

Baikie. Edwin Simpson- 1880

Baillif. R. F. 1862

Baily. $\quad 1825$

Baily. Edward 1746

Baker. Henry 1760 
Baker. Valentine 1865. 1873

Bal. J. 1849

Balassa. Constantine 1828. 1835

Baldamus. Edouard 1865. 1875. 1880

Balle. Peter Wilhelm v. 1830. 1834. 1845

Bally. Alexander von 1852

Balme. Mottin de 1a 1773. 1776

Balthasar. 1879. 1880

Bamford. 1824

Bandow. Georg Friedrich 1854

Barauski. Ant. 1882. 1884

Barbier. Charles 1862

Bardonnet des Martels. Antoine de 1854

Baret. Michael 1618

Barford. Valentine 1853

Barker. Christopher 1575

Barnes. Bernes or Berners. Juliana 1486

Barnes. O. 1873

Barth. K. M1dr. 1812

Barth. Major L. Chr. 1857

Barthelemy. 1830

Bartlett. J. 1754. 1758. 1764

Basta. George 1612

Basserie. Col. Paul 1847

Bates. 1835

Baucher. F. 1833. 1834. 1840. 1842. 1844. 1854

Baumeister. Johann Wilhelm 1845. 1848

Beamish. N. L. 1855

Beaufort. Duke of, and Mowbray Morris. 1885

Beaumgarten-Crusius. Gottlob August 1796

Beaumont. Imbotti de 1679

Beaupré. B. de 1868. 1874

Beaurepaire. J. de Robillard de 1865

Beck. C. Ch. 1826 
Beck. Ernst 1875

Becker. 1876

Beckmann. Johann 1780

Begrières. Carbon de 1725

Beham. Hans Sebald 1528

Behrens. Heinrich 1880

Bel. Charles Vial de Saint-1791. 1793

Bell. Bowdler 1873

Bell. Charles 1806

Bell. C.W.B. 1880

Bell. Thomas 1837

Bellamy. Pierre 1856

Belvallette. N. 1880

Benavides. Francisco 1732. 1734

Bendz. Henrik 1850. 1855

Bendz. O. Pehrsson 1873. 1874

Benfert. J. R. 1832

Benjamin. Louis Auguste 1854. 1864

Benkendorff. Genl. 1831

Bentwright. J. 1858

Benvenuti. G. 1760

Berdmore. S. 1883

Berenger. Richard 1771

Berghofer. Ludwig 1818

Berjeau. Charles 1864

Bernad. Francisco Pasqual 1757

Bernier. 1860

Bernstorff. Graf von A. 1878

Bertacchi. Danielli 1851. 1880

Berthaud. 1853

Berthaux. Louis 1852. 1855

Berthelot. 1846

Berthier. 1856

Besser. L. v. 1868. 1870. 1873 
Bewick. Thomas 1790

Bianchi. Calmiero 1882

Biddell. H. 1880

Biedenweg. Hermann 1855

Biel. Baron Gli. Wlh. Ldw. 1830

Bierlich. E. 1869

Billing. Thomas Samuel 1837

Bingley. Thomas 1838. 1839

Bingley. W. 1809

Binz. Gervas 1807

Binz. Pantal. 1824

Birac. Le Sieur de 1688

Birch. John 1883

Bishop. William 1846

Bismark. Graf von 1820. 1822. 1827

Bismark-Bohlen 1870

Bjorklund. F. G. 1868

Black. Robert 1886

Blaine. Delabere Pritchett 1799. 1802. 1803. 1840

Blaine. Ephraim 1831

Blazecovic. F. 1882. 1885

Bleiweis. Johann 1838

Blome. Richard 1686

Blumenschein. Mar. Geo. 1787

Blumhoser. M. 1811

Blunt. John 1773

Blunt. W. Scawen 1880

Blunt. Lady Anne 1878

Boardman. Thomas 1802

Bobadilla. Antonio 1823

Bocksperger. Hansen 1569

Böhmen. M. 1802

Boehm. Carl L. 1852. 1855. 1856

Bötticher. D. F. 1861 
Bohlmann. Carl. Fried. 1821

Boigne. Charles de 1843

Boisdeffre. Chevalier de 1790

Bojanus. Ldw. H. 1811. 1815

Bonie. T. 1869. 1870.1871. 1873

Bonifaz. Gaspar 1635

Bonino. G. 1842

Bonneval. Frédéric 1853

Bonova. Siro 1852

Bonsi. Francisco 1757. 1786. 1795. 1802. 1825

Borges. Alvarez 1680

Boswell. Edward 1798

Bötticher. D. F. 1861

Bouchardat. A. 1862

Boudin. J. Ch. M. 1850

Bouillé. Count L. de 1881

Bouley. 1856

Bouley and Dupuy. 1838

Bouley and Sanson. 1861

Bourg. R. de 1856

Bourgelat. Claude 1744. 1750. 1765. 1767. 1768. 1770.

Bourgoin. Ed. 1870

Bower. Lt.-Col. 1865

Bowers. Georgina 1873. 1874

Bowers. R. 1850

Brack. F. de 1831

Bracken. Henry 1742

Brand. P. 1885

Brauell. J. Fr. 1825

Brauer. Carl 1877

Braungardt. Friedrich Wilhelm 1855. 1856

Braunschweig. Carl von 1823

Brézé. Comte de 1769. 1772

Briddon. J. A. 1850 
Bridges. Jeremiah 1752

Briefe 1865

[1857. 1859

Brindley. Charles 1845. 1848. 1850. 1852. 1853. 1856.

Briones. Pedro and Juan Abdon Nieto 1851

Brivet. C. 1844

Brix. 1881

Brogniez. A. J. 1839. 1850. 1851

Brooksby [pseud] 1880

Brosche. Joh. R. Jos. 1812

Brout. 1849

Brove. Solomon de la 1593

Brown. C. F. 1829

Brown. Capt. Thomas 1830

Brown. Thomas 1846

Browne. T. 1624

Broye. Hippolyte de 1865

Brucher. P. R. 1876

Brück. J. A. F. 1787

Brückner. L. $18: 9$

Brudermann. Rudolph v. 1843

Brueh1. Bernhard 1850

Bruggemann. J. G. 1817

Brugnone. Gio. Antonio 1774. 1781

Bruhn. Carl Julius v. 1854

Brun-Renard. Le 1886

Brunet. R. 1886

Bruni. Felix 1882

Brunn. A. Fr. 1825

Brunot. 1826. 1831

Brunswig. Fr. Wilh. 1831

Bruslard. L. Marquis de 1856

Bruyn. Abraham de 1577

Bryce. John 1678

Bryon. Thomas 1841 
Bucalossi. Enrico 1880

Bucellati. Alessandro 1845

Buchoz. Pierre Joseph 1772

Buchting. A. 1886

Budd. Richard Hayward 1816

Bué. A. 1863

Buegnot. 1856

Buerger. H. 1830

Buffon. George Louis Leclerc Comte de 1849

Bugeaud. Th. Rob. 1841. 1860

Bujault. Jacques 1854

Buksh. Mohammed 1839

Bultzingstoven. Ritter von 1840

Bunbury. H. 1787

Buonsanti. Alessandro Lanzilotti $188 \mathrm{I}$

Burdelot. F. 1875

Burdon. William 1730

Burger. H. 1823

Burgess. J. W. 1881

Burgess. Richard 1858

Burgsdorf. C. F. W. 1820. 1827

Burke. B. W. 1806

Burn, C. 1860

Busch. J. D. 1797. 1806

Bussy. Charles de 1865

Butler. 1861

C. L. W. 1610

C... Frédéric de 1871

Cagni. Col. 1878

Calloet. Querbrat 1666

Calvo. Fernando 1580

Calvo y Cavero. Juan Francisco 1789

Cambrelin. A. L. 1877 
Camerarius. Joachim 1539

Campagnole. Genl. 1814

Campanella. Tommaso 1570

Campens. Edward 1850. 1851

Canstatt. Dr. and Dr. Eisenmann 1848

Canter. 1868

Cappello. G. B. 1588

Capuron. Joseph 1806

Caputo. Francesco 1841

Cardigan. Earl of 1861

Cardıni. François 1845

Carraciolo. Pasquali 1566

Carruthers. 1825

Carson. James Crawford Ledbie 1859

Casas de Mendoza. Nicolas 1832. 1833. 1834. 1843. 1844.

[1846. 1847. 1850.1854. 1868

Castroverde. C. Fernandez de 1885

Cathcart. Earl 1883

Cato. Marcus Porcius 1472

Cavailhon. Edouard 1886

Cavandini. 1874

Cavendish. William. Duke of Newcastle. 1667

Cavero. Francisco Garcia 1727. 1728. 1729. 1731.

Caviglia. G. B. 1880

[1732. 1740. 1756

Cespedes y Velasco. Franciscus de 1609

Chabannes. 1827

Chabert, Flandrin and Huzard 1789. 1796

Chabot. Jacques de 1883

Chacon. Ferdinando 1551

Chambre. Le 1657

Chapus. Eugène 1853. 1858. 1865

Charlier. Pierre 1845

Charnacé. Guy de 1869

Charpentier. J. Chrys. 1856 
INDEX TO NAMES OF AUTHORS.

Chassaigne. Dr. R. 1870

Chauvel. Louis 1857

Cheny. 1729

Cherry. F. C. 1820. 1842

Chervils. Maxime 1870. 1874

Chifney. Samuel 1800. 1804

Chuteau. Olivier 1834

Cito. Giovanni Antonio 1590

Clam. Dupaty de 1769. 1771

Clarendon. Thomas 1843. 1847

Claridge. John 1670

Clark. Bracy 1796. 1805. 1810. 1813. 1814. 1815. 1816.

1818. 1822. 1824. 1825. 1826. 1829. 1830. 1831. 1832.

1833. 1835. 1838. 1839. 1840. 1854. 1855

Clark. James 1770. 1776. 1788

Clark, Mrs. Stirling 1857

Clarke. C. 1867

Clarke. W. H. 1880

Clater. Francis 1783

Clément. E. 1872

Clément. Jules 1858

Clerk. Chevalier Le 1700

Clifford. Christopher 1585

Cline. Henry 1804

Clonard. Count de 1851

Cochet de Sávigny. P. C. M. 1849

Coesme. 1857

Cogent, Laurent 1856. 1863. 1868. 1872

Cole. J. R. 1879

Cole. S. W. 1849

Coleman. Edward 1798. 1800. 1801

Colin. Gabriel 1850. 1854

Collella. Eligio 1879

Collins. 1853 
Collins. Digby 1865

Collomb. L. E. de 1878

Colomb. E. von 1870. 1872

Colùmella. A.D. 20

Comparini. Carlo 1850

Conde. Pedro Garcia 1685

Congdon. J. 1874

Conti. E. 1823

Conto. José Ferrer de 1857

Cooke. P. St. George 1863. 1864

Cooper. W. S. 1881

Copperthwaite. R. H. 1865

Corbet. Henry 1845

Cordier. 1824

Cornay. Dr. 1861

Cornevin. Ch. 1881

Corte. Claudio 1573

Cortijo. Vicente de 1882

Corvisart-Montmartin. Von 1873. 1874. 1878

Costa. Alessandro 1882

Cotarello y Garastazu. Juan 1861

Couteleux de Canteleu. Count de 1872

Court. E. 1886

Courtenay. E. (Appendix) 1886.

Cox. Nicholas 1674

Crafty. [pseud.] 1883

Crane. Fr. v. 1875

Crane. W. J. E. 1883

Crescenzi. Pier de 1300

Cresset. John 1672

Creutsberger. John 1591

Cromwell. Oliver 1654

Cros. Giovanni 1824

Crusius. Gottlob August Beaumgarten 1796 
Culombre. Augustino 1518

Cuming. A 1854

Cumming. 1809

Curnieu. Baron Louis de 1841. 1848

Curr. E. M. 1864

Curtis. C. E. 1879

Cusac. 1822

Cuvier. Baron George Leopold Chrétien Frederic Cuyer. Ed. 1883

[Dagobert 1816

Cyr. Fr. Saint 1875

D. . . Baron von 1875

Dachenhausen. A. 1867

Dadd. George H. 1850. 1854. 1857. 1870

Dalbiac. James Charles 1806

D'Aldéquier. Flavin 1843

Dallmann. Ferdinand 1877

D'Alton. E. 1810

Dalziel. Hugh 1860

Damoiseau. Louis 1839

Dandolo. Aluise 1722

Daniel. Frederick 1880

Daniel. William 1801

D'Arboval. Hurtrel 1838

Daru. Colonel Baron 1872

Daudel. Pierre Joseph Isidore 1854. 1857

Daum. Heinrich 1788

Daum. Ludwig 1813. 1817. 1819. 1820. 1821. 1822

Daumas. Melchior Joseph Eugène 1851. 1854

Daunay and Lignée. P. 1841

Daunassans. A. 1856

D'Aure. Antonio Henri Phillippe Léon Comte 1844

Davenport. Bromley 1885

[1845. 1855, 1860

Davidis. Henriette 1848 
INDEX TO NAMES OF AUTHORS.

Davidson. William 1880

Davila y Heredia. Andres 1674

Davis. A. 1867. 1872. 1874

Day. William 1795

Debost. Emile 1873

Decker. Carl v. 1818

Decroix Emile F. 1852. 1874. 1885

Deen. J. van 1849

Defays. François 1857.1867

Defays and Husson. François 1852

Deigendesch. Johann 1744

Dejean. Oscar 1839. 1856

Dekker. Jacob Adrian 1853

Delafond. Onésime 1838. 1841. 1842. 1843. 1852

D'Elbee. Capt. 1849

Delcampe. Le Sieur 1664. 1712

Delisser. G. P. 1881

Deloupy. J. P. G. 1852

Delwart. L. V. 1849. 1850

Delmazy. Louis 1865

Dementhon. F. J. 1853

Demilly. Louis 1855. 1856. 186.3

Denis. Le Vaillant de St. 1789

Denison. George T. 1877

Dérigne. 1859

Dérué. 1885

Desclée. Phillippe 1871

Deseles. P. 1884

Desmarè. E. 18201849

Dessart. J. B. 1879

Desvaux. Urbin 1863

Dewall. 1880

Dick. William 1869

Didot. Dr. 1860 
INDEX TO NAMES OF AUTHORS.

Didrichsen. D. 1801

Dieckerhoff. W. 1882

Diepenbrock. C. J. 1855

Dieterichs. J. F. C. 1820. 1822. 1823. 1824. 1825. 1828.

[1831. 1834. 1835. 1842. 1851. 1853. 1857

Dietrichs and Reckleben. 1854

Dilg. W. 1871

Dimse. J. 1869

Disney. John 1806

Dittmer. Adolphe 1842

Dixon. Henry [Druid] 1856

Dobrizhoffer. 1784

Dodge. Theo. A. 1885

Dolffs. Theod. v. 1842

Doll. Carl Gottfried 1851

Dolomanow. M. 1854

Dombale. Henri de 1851

Dominick. Fr. 1866. 1870

Dompierre. De Preseau de 1788

Donaghue. Mrs. Power O' 1881

Donovan. E. 1820

Dosso. G. Eccheli del 1881

Douglas. William 1873

Douterluigne. Pierre 1850

Doux. Clementi 1881

Dow. G. J. 1846

Downing. Joseph 1797

Drouville. J. B. 1811

Drygalski. A. v. 1883

Duarte. Rei de Portugal 1434

Dubourdier. Charles 1855

Dubroca. 1844. 1845. 1846

Dufour. Abbé v. 1868

Dufresse. Augey 1875 
Du Hays. Charles 1860. 1868

Duhousset. E. 1862.1874. 1881

Duliège, R. A. 1871

Dun. Finlay 1854

Dunbar. J. R. 1859

Dunmore. Earl of 1878

Dupouy. Jean Saint Rieul 1850

Dupuy. 1817

Durant. G. 1878

Dureau de la Malle. 1855

Dürler. Arnold 1854. 1857

Dutilh. M. F. 1875

Duttenhofer. F. M. 1848

Dwyer. Francis 1868

E. C. Gr. v. 1851

E. C. J. 1871.1876

Easton. A. 1859

Eberhard. G. 1856

Eberhard. Philipp 1856

Echegaray. Jose 1852. 1857

Eckert. P. 1832

Edwards. Kinard B. 1874

Egan. Charles 1867

Egan. Pierce 1825

Ehrhardt. Friedrich August 1854

Ehrmann. Joh. Chr. 1778. 1779. 1780

Eichbaum. Dr. Friedrich 1879. 1884

Eicke. Lieut. 1860

Einsiedel. C. Graf von 1852

Eisenberg. Baron d' 1727

Eisenmann. Dr. 1848

Elbee. Capt. D' 1849

Elcho. Lord 1873 
Elderkin. J. 1871

Eléoute. 1835

Eliot. Francis Percival 1797

Ellenberger. W. 1884

Ellerbrock. Ignatz 1851. 1852. 1853

Elliott. G. H. 1875

Elliott. W. L. 1864

Ellis. William 1759

Elluin. J. B. S. 1861. 1862

Elmhurst. Pennell [Brooksby] 1880. 1883. 1884

Elpons. Carl Franz Michael von 1856

Engelhardt. Jh. 1803

Engelhart. Ant. 1834

Enslin. Theod. Chr. Fr. 1825

Ercolani. Giovanni Battista 1851

Erdelyi. Michel von 1814. 1819. 1820. 1827. 1829

Erdt. W. E. A. 1868

Erkens. H. J. 1835

Erler. H. 1868

Esbach. Peter 1727. 1747, 1753. 1764

Espejo y de Rosal. Rafael 1880. 1881. 1882

Esteves. Affonso 1425

Etches. J. M. [Ashwood] 1884

Etreillis. Baron d' 1872. 1873. 1883

Eurville de Grangues. Le Marquis d' 1854

Eyquem. Michel 1580

F... H. de la 1872

F ... L. A. 1814

Faber. P. D. and C. F. 1830

Faber. Dr. Wilhelm Everhard 1846

Fabre. Elie 1852

Fabricy. Gabriel 1764

Fairfax. J. 1795 
Falke. Johann Ernst Ludwig 1848

Fawconer. Thomas 1770

Fearnley. William 1878. 1879. 1882

Félizet. L. 1870

Fellenberg. L. R. de 1841

Felton. William 1794

Fenwick de Porquet. 1856. 1857

Ferguson. H. 1843

Fernandez y Perez. Juan Zoilo 1856

Feron. John 1803

Ferrer de Conto. Jose 1857

Ferraro. Pietro Ant. 1602. 1629

Ferrero. Ponsiglione 1837

Feuring. G. L. 1821

Field. J. 1857

Filet. H. P. 1869

Finzi. Giuseppe 1883

Firuz Jang. 1650

Fischer. A. 1872

Fischer. E. 1850

Fischer. G. M. S 1832

Fitzgerald Baron de Ros. Lord William Lennox Lascelles 1844

Fitzgerald. W. N. 1884

Fitzwygram. Frederick Wellington John 1861.1862

Flandrin Antoine 1852. 1853. 1854

Fleming. George 1869. 1871. 1872. 1875. 1876. 1886

Flemming. Gustav Johann Georg Friedrich 1853

Fletchier. E. 1670

Flint. William 1815

Florke. Heinr. Gust. 1818

Flower. Edward Fordham 1875. 1877

Flower. Prof. W. H. 1870

Fœlan. Modeste 1868 
Fogg. Jos. 1837

Fogh. Chr. F. J. 1860

Fogliata. Giacinti 1879. 1880. 1882

Folard. Jeau Charles de 1733

Ford. Richard 1791

Fore. 1884

Formanoir. A. de 1872

Forrest. C. 1878

Forrester. William 1788

Forstor and Pidoll 1873

Fortwäugler. C. 1866

Fouan. Charles de 1845

Foucart. P. 1880

Foucher. Louis Alexander de Cariel 1864

Foquet. Jeau 1671

Francisci. E. 1670

Franck. L. 1875

Franconi. Victor 1855

Fraser. Charles Craufurd 1871

Freeman. Strickland 1796. 1806

Freis. Christian Lauritz 1853

Freis. Martin 1851

Frentzel. J. P. 1878

Frey. J. 1852

Frey. L. 1869. 1872

Freytag. C. 1875

Friedberger. F. 1874

Fritsch. C. A. 1848. 1849. 1850. 1851. 1852. 1853

Froriep. Robert 1845

Frost. Thomas 1875

Frucourt. Morgan-1861

Fuchs. Christian Joseph 1852. 18531854

Fuhrmeister. Friedrich 1842

Funke. Carl Friedrich Wilhelm 1836 
INDEX TO NAMES OF AUTHORS.

Fusco. Raffaele 1822

Fuss. Frz. 1797

G. J. H. 1885

G. L. 1708

Gaab. J. 1770

Gajani. G. Batista 1619

Gali. Galdericus 1619

Galiberto. G. B. di 1650

Galisset. Ch. and J. Mignon 1812

Gallego. Fr. Pedro 1629

Gama Machado. 1853

Gamgee. John 1855. 1858. 1860. 1861. 1865. 1866

Gamgee. Joseph Samson 1871

Gard. 1809

Gardner. F. B. 1880

Garrel. Al. 1872

Garsault. F. A. de 1741. 1756. 1769

Garzoni. Marino 1692

Gauglia. Paolo 1857

Gaume. Alexandre 1865. 1880

Gautier. Mille 1856

Gayot. Eugène 1848. 1850. 1857. 1862

Gemeiner. J. de 1867

Genoillac. Jacques de 1547

Georgio. S. R. J. 1840

Gerathewohl. Bh. 1886

Géraud. Eugène 1856

Gerber. Jos. Volmar 1832

Gerhardt. G. A. 1859. 1862. 1865

Gerlach. Carl 1853. 1854

Gervais. 1847

Gesner. Conrad 1551

Gherardi. 1853 
Ghilardi. Luigi 1852

Gibbons. $\Upsilon 1825$

Gibson. Hope 1848

Gibson William 1720.1721.1750.1755

Giebel. Christopher Gottfried 1854

Giles. Jose Maria 1836. 1848

Gillmore. Parker 1869

Gilmore. Harry 1866

Gilpin. William 1791

Gilsa. Oberst-Lieut. 1862

Girard. J. 1807. 1827.1832.1841

Girette. A. 1885

Girou de Buzareingues. 1853

Giustiniani. Henri de 1841

Gjersing. Niels 1826

Glasenap. G. 1867

Glover. Joseph 1884

Gmeiner. Fr. v. 1803

Gnecco. G. 1882

Godin. 1862

Goedhart. Christian Carel 1853

Goodwin. J. 1820. 1821

Goodwin. W. J. 1853. 1856

Görgey von Görge. 1867. 1871. 1873. 1875

Gorini. G. 1882

Gosden. T. 1791

Gotthard. Joh. Chr. 1800

Götz. F. J. 1830

Goubaux. A. and G. Barrier 188?

Gourdon. Dr. Jean 1857. 1860. 1873

Goyau. Louis Pierre 1857. 1869. 1864

Goux. J. B. A. and Merche. 1844. 1849. 1859

Gracklauer. O. 1879

Gradinger. M. 1869 . 
Graefe. Carl Heinrich Edouard 1853. 1855. 1857. Graf. Leopold 1846. 1847, 1848. 1849

Grajewski. 1836. 1854

Grangues. Dr. Eurville de 1854. 1855. 1857

Grant. Sir Hope 1860

Greenwood. G. 1839. 1840. 1843

Grellier. Louis 1802

Gresswell. J. B. 1885

Gresswell. J. B. and Albert 1885. 1886

Greve. Bern. Ant. 1814

Grey. Thomas de 1639

Gribble. Samuel 1829

Griffiths. William 1784

Grison1. Federico 1552

Grogniez. 1883

Grollier. Eugène 1854

Gross. Johann Christopher 1850

Grosskopf. Johann Friedrich Gottlieb 1856

Gueldberg. C. Hoegh 1854

Guendeville. Capt. 1863

Guérini. A. 1851. 1860. 1871

Guérini. A. 1724

Guerinière. Fr. Robichon de la 1733. 1740

Guevary. Antonio de 1529

Guilbert. 1874

Guillet. Le Sieur de 1680

Guillon. Amable 1856

Guilmot. Désiré 1865

Günther. Friedrich August 1836

Gunther. J. H. F. 1830. 1837

Günther. K. 1866

Gurlt. Ernst Frederick 1824. 1831. 1832. 18371849

Gurlt. Ernst Julius 1853 
H. 1869

Haase. W. 1886

H. R. 1684

Haber. R. von 1879

Hackett. William 1811

Hadrian. The Emperor A. D. 120

Hafftendorn. Jh. Sam. 1832

Hagemann. Berthold H. 1845

Hagerup. G. 1851

Hahm. C. 1873

Haidvog1. Giuseppe 1856

Halberbach. Ch. 1822

Hale. Lonsdale Augustus 1873

Halliwell-Phillips. J. O. 1886

Hallock. Charles 1883

Hamel. Col. v. 1862

Hamilton. Ker B. 1865

Hamley. Edward Bruce 1875

Hamm. Dr. W. 1852. 1855

Hammer-Purgstall. Joseph von 1856

Hamont. P. N. 1842. 1843

Hanger. George 1814

Hankin. George Crommelin 1877

Hanover. 1875

Hansen. Hans Jacob 1853

Hardegg. Dominik graf 1877

Hardy. Edmund Armitage 1878

Harewood. H. 1835

Harguinez. 1750

Hartmann. Capt. G. A. 1855. 1856

Hartmann. Johann Georg 1786

Hartmann. Dr. 1848

Hartmann. Leopold von 1777

Hartung. W. Fried. 1834 
INDEX TO NAMES OF AUTHORS.

Harvey. Alexander 1851

Hassel. Wilhelm von 1851. 1875

Haubner. Gottlieb Car1 1838

Haupt. Wilhelm 1845

Hauzwitz. Lieut. von 1885

Havemann, August Conrad 1792. 1820

Hävernick. H. 1876

Havez-Montlaville. 1850

Haxthausen. F. L. 1839

Haycock. William 1850. 1852. 1854

Hayes. Matthew Horace 1875. 1877. 1881. 1883. 1885

Hayne. Anton. 1830. 1814

Hays. Charles Du 1855. 1860. 1853. 1865. 1867. 1868

Haywood. William 1873

Head. Sir Francis Bond 1860

Heatley. G. S. 1882. 1883. 1885. 1886.

Heber. Reginald 1752

Hecquet d'Orval. E. 1855

Hédouville. Le Vicomte de 1884

Heine. Fried. Rudolph 1850

Heinrich. Julius Heinrich 1856

Heinrichs. Joseph 1852

Heinze. Theodo. 1853, 1863. 1876

Hellmund. J. M. 1848

Helm. H. T. 1878

Helmsbrecht. F. M. 1795

Hemsbach. Johann Heinrich Meckel von 1848

Henle. J. 1884

Henriques. E. 1867

Henry. Gustave 1852

Hensler. J. 1851

Hentze. H. W. 1848

Herbert. Henry William 1852

Herbin. L. 1879 
Herbst. Johann August 1836

Hering. Ed. 1832. 1834. 1857

Herklotz. J. Geo. 1802

Hermann. J. 1840

Hermann. E. M. von 1850

Herrstatt. C. 1879

Hersenberger. 1844

Hershberger. H. R. 1845

Herstatt. Conrad 1850. 1851

Hertwig. C. H. 1850. 1851. 1856

Hervard. Jean 1599

Herzog. A. 1857

Hess. Aldolph 1839

Heusmann. F. 1854

Hey. Wilhelm 1850

Heyde. W. G. von der 1847

Heydebrand und der Lasa. Leopold von 1878. 1884

Heydebrand und der Lasa. Thassile von 1828

Hibberd. S. 1881

Higgins. Matthew James 1855

Hilcke. Hermann 1876

Hime. Henry W. L. 1866

Hinde. Capt. 1778

Hitzinger. George 1856

Hiver. Joseph 1783. 1787

Hochstatter. Conrad v. 1821. 1850

Hochwachter. U. v. 1866

Hocquard. Edouard 1849. 1862

Hocquet. Capt. A. 1868. 1873

Hodgson. F. R. 1849

Hodgson. Thomas 1824

Hoecker. Lieut. 1868

Hoehning. C. F. J. 1848

Hoerdt. Sigm. 1822. 1830 
Hofacker. Dan. 1822. 1823. 1825. 1828

Hofer. Dominik 1852

Hofer. J. 1884

Hoffman. J. B. 1828

Hoffmann. Carl 1803. 1805

Hofling. G. v. 1870

Hofmeister. G. C. M. 1828

Hogelmuller. Geo. 1811

Hogg. Capt. 1874

Holmes. Francis S. 1858

Hookham. Thomas 1836

Hooper. G. N. 1876

Hooper \& Co. 1879

Hore. J. B. 1885.1886

Horlock. K. W. 1855. 1865

Hormann. J. 1834

Horn. W. 1834

Hort. Lt.-Col. 1850

Hostun. L. H. Pons D' 1806

Houël. Ephrem 1841. 1842. 1843. 1848. 1850. 1853.

Howden. Peter 1862

Hozier. Henry Montague 1865. 1873

Hube. Capt. 1885

Hubert. E. 1849. 1850

Hubner. G. 1867

Hugues. 1800

Hugues. J. 1886

Hulfreich. Erdmann 1787

Humbert. Emile 1865

Humfrey. J. 1885

Humphreys. R. 1824

Hunersdorf. Ludwig 1791. 1846

Hunt. Vere D. de Vere 1859. 1874

Hunter. James 1796 
Husson. 1852

Hutton. Alfred 1867

Huys. Charles Du

Huzard. J. B. 1789. 1797. 1805. 1829. Fils. 1854. 1864.

Illiers. Louis d' 1863

Im-Thurn. Ed. 1836. 1841

Isabelle. Madame Marie 1855

Ithen. Joseph Ant. 1829

Itier. Alex. 1842

Jacob, Giles 1718

Jacob. 1850

Jacobi. C. W. 1809

Jacoby. Ferdinand Rudolph 1852. 1853. 1857. 1873

Jacquemin. François Maxime 1826. 1850

Jaeglé. E. 1885

Jal. Phillippe Ursal Charles de Lastic Saint 1856

Jannasch R. 1861. 1865

Janné. Auguste Jacques 1848

Jardine. Sir W. 1833

Jause. Fr. 1817

Jennings. Robert 1866. 1872

Jensen. J. 1881

Jensenius. H. 1859

Jenyns. Leonard 1838

Jervis. John 1827

Jessen. Peter 1849. 1853. 1857. 1867

Jewett. Paul 1795

Joce. J. 1844

John. Christian Friedrich 1848

John. Jacob 1853

Johnson. J. B. 1831

Josch. Chrf. 1878 
Joseph. F. 1873

Julius Africanus. Sextus A.D. 225

Jullien B. J. 1869

Jullienfelde. A. v. Saucken 1819

Jush. Ant. Fr. 1819

K. F. v. 1870

Kaehier. 1874. 1876. 1879

Kaestner. Christian Nicolaus 1842

Kahn. Fr. 1789

Kaiser. F. 1855

Kaiser. D. H. 1885

Kappel. Carl Anton. 1852

Karacsay de Wallje-Szaka. Fedr. v. 1832

Karr. E. 1881

Karst-Karstenwerth. Freiherr von 1879

Kayser. C. F. 1831

Kegel. Karl 1819. 1820

Keller. Alexander Graf von 1877

Keller. Knud Christian 1854

Kendall. B. J. 1879

Kerpelani. 1849

Kersting. Joh. Adam 1788. 1789

Kertitschka. J. 1827

Kielmeyer. Carl. Fr. v. 1814

Kinberg. J. G. H. 1870

King. Edward 1858

Kirchhœwell. August Ferdinand 1855

Kirke. John 1805

Kirtland. G. 1882

Kitchener. Dr. William 1848

Klatte. Ephr. 1804. 1805. 1807. 1814. 1815. 1817. 1818.

Klein. J. 1858 
INDEX TO NAMES OF AUTHORS.

Klemm. Heinrich 1847

Knight. Charles 1825

Knight. 1840

Knightley. Thomas Edward 1862

Knobelsdorff. 1820

Knowlson. 1850

Koch. A. 1884

Koch. Christopher Ernst Julius 1850

Köllner. Heinr. August 1789. 1790. 1791

König. Jos. 1820

Konigstadter. Frz. Ldw. 1804

Koppe. J. G. 1855

Körber. F. X. 1839. 1848. 1855

Kossen v. Sterneck. 1874

Kottwitz. Baron v. 1878

Krane. F. W. C. T. M. von 1854. 1856. 1870

Krane. H. 1867

Kruetzberger. Hans 1562

Kruetzer. Jh. Mart. 1818. 1833. 1840. 1855

Krocker. A. 1870

Kruge. Hermann 1855. 1859. 1860

Kruger. Aldolph. 1831

Kuers. F. A. 1839. 1841

Laborde. E. 1835

Lachaume. J. B. 1860

Laffore. T. de Bourrouse de 1862

Lafosse. Etienne Gillaume 1749. 1754. 1766. 1772.

Lafosse. L. 1858

$[1776$

Lagondie. J. de 1874

Lahure. A. 1884

Laiglesia y Darrae. Francisco de 1852

Lalanne. E. 1880

La Moriciere. C. L. L. J. de 1850

$[1860$

Lancosme-Breves. Count Savary de 1842. 1843. 1852. 
INDEX TO NAMES OF AUTHORS.

Landemont G. De 1875

Langenbacher. J. 1811

Lappe. Fr. Karl 1816

Lapuerta y Chaquet. Miguel Pedro 1781

Lavendu. J. B. F. 1849

Lascelles. Francis Henry 1877. 1881

Lassaigne. J. L. 1841

Latouche. John 1874

La Tour-Du-Pin-Chambly. 1841

Laubender. Bernard 1803. 1805. 1811. 1812. 1816

Laulanhier. A. de 1843

Lavocat. A. 1840

Law. J. 1877

Lawrence. John 1796. 1829 , 509

Lawrence. Richard 1800. 1801. 1809. 1816

Lawson. A. 1820

Lawson. J. 1797

Lazaro. Ramon Llorente 1854. 1855. 1856

Laziroules. G. Bergasse de 1843

Lebas. J. Ph. 1841. 1869

Lebeaud. 1858

Leblanc. Urbain 1840. 1841. 1848

Lebrun. 1860

Lebrun. and W. Maigne 1872

Le Chambre. 1657

Leclerc. A. 1883

Lecomte. 1854

Lecoq. Felix 1843. 1862

Lecornue. L. 1843

Lefebvre. A. 1871

Lefèvre. H. 1871

Legrain. J. B. 1862

Legros. 1840. 1843

I,ehmann. 1801 
Lehndorf. G. 1883

Leisering. A. G. T. 1885

Le Maire. 1843

Lemaitre. Auguste G. O. 1857

Lemichel. Eugène 1860. 1872

Lemoigne. Alessandro 1854

Lengerke. Alex. v. 1827

Lentin. Dr. C. F. 1857

Lescot. E. 1862

Lespinats. A. de 1847

Lesley. Genl. 1642

Lessona. Carlo 1827. 1833. 1834. 1852. 1854. 1857

Lessona. Giuseppe 1854

Letang. Antoine de 1832

Letang. George Nicholas Marc Baron de 1812

Leuckart. Fried. Siegm. 1832

Levi. Giuseppe 1857. 1881

Lewaneu. Jos. Arn. von 1817

Leyh. Fried. August 1850

Leyon. Mathias 1818. 1849. 1850. 1851. 1853. 1865

Liasse. Albert 1857

Liberati. F. 1639

Liégeard. F. C. J. 1863

Ligne. Charles Joseph Prince de 1780

Lignée. 1841

Lindenau. A. Fr. Aug. 1804. 1806. 1831

Lindqvist. C. A. and J. Arrhenius 1866. 1870. 1874

Linsley. D. C. 1857

Lisch. George Christian Friedrich $18 \pi 6$

Littré. E. 1884

Lloyd. G. 1815

Locatelli. Ant. 1825

Loebe. William 1856

Loebell. Von 1874 
INDEX TO NAMES OF AUTHORS.

Loennecker. F. L. W. 1878

Löffler K. 1866. 1874

Loiset. Baptist 1826. 1828. 1853. 1856

Loiset. E. 1865

Lombardi. Vincenzo 1773

Lommatszch. Gottfried Leberecht 1855

Lortet. Dr. Louis 1867

Lotze. August 1849. 1851

Loubat de Bohun. 1781

Louchard. A. 1847. 1851

Low. David 1840

Loweneck. Fr. von 1824

Lower. Richard 1668

Lucan. Earl of 1856

Lucas. William 1786

Ludwiger. Furchtegott 1850

Lufried. 1877

Lundberg. F. 1868

Lupke. Joh. Chr. Gottfr. 1828. 1834

Lupton. James Irvine 1870. 1881

Lütken. J. C. 1851

Luton. P. 1870

Lutzow. Von 1867

Lydekker. R. 1885

Lyskowski. Ignatz. 1853

M. C. 1878

M. T. T. D. E. 1647

Macbride. J. A. 1867

Macé. P. 1856

Macedo-Pinto. Jose Ferreirer de 1852. 1854

Machado. Gama-1853

Machay. C. B. von 1819

Machuca Bernardo de Várgas 1600. 1621 
INDEX TO NAMES OF AUTHORS.

Mac Kenzie. Maj. 1874

Mackenzen. 1877

Maesnow. P. 1852

Magne. J. H. 1842. 1845. 1853. 1867. 1873

Maher. Capt. 1833

Mahlbeck. Wilh. Fr. 1815

Maigne. W. 1872

Maillard. 1856

Major. C. J. F. 1881

Malatesta. Alex. Massarius 1600. 1605. 1660

Malbie. Nicholas 1576

Malcolmson. John Henry Porter 1873

Maleden. L. de 1803

Malet. Harold Esdaile 1874

Malfrain. N. C. 1860. $186 t$

Mansuy. C. 1875

Manzanas. Eugeno 1570

Maples. John 1776

Marcellinus. Ammianus A. D. 360

Markham. Gervase 1593. 1596. 1599. 1605. 1607. 1610.

1611. 1615. 1616. 1620.1625. 1656. 1681

Markland. James Heywood 1828

Marquard. George 1848

Marquart. Friedrich 1852

Marschalk. Georg 1642

Marshall. John 1787

Martin. 1843

Martin. William Charles Linnœus 1840. 1845

Martinez y Miranda 1884

Martini. Alessandro de 1849

Marwitz. F. A. L. von der 1852

Mascall Leonard 1587

Masch. A. 1857

Massa. Vincenzo 1829. 1830 
Massé. Jean 1568

Maurer. F. 1879. 1881

Maxwell. 1833

Mayer. John 1819

Mayhew. Edward 1849. 1860. 1864

Mayr. Fr. Ant. 1797

Mayr. Otto 1866. 1867. 1875

Mazoiller. J. 1853

Mazurkewitz. v. 1858

Mazzucchelli. Federigo 1802. 1805

Mc. Clellan. G. B. 1862

Meer. Felix Van der 1866

Megnin. J. P. 1860. 1864. 1865. 1867. 1869. 1872

Melfort. Drummond de 1776

Melgarejo. Jose de Morla 1738

Melville. Whyte- G. J. 1878

Melzo. Fr. Lodovico 1611

Meneghini. Cesare 1881

Menezes. Fernando Telles de 1600

Menou. René de 1612

Mentzel. E. O. 1371

Merche. 1862.1868

Mercier de l'Eure. 1841

Mercier. B. J. 1874

Merewether. C. G. 1868

Merk. Theodore 1815. 1819. 1820. 1833. 1840

Merlen. B.Van 1837. 1840. 1842. 1846. 1849. 1850

Merres. 1873

Merrick. William 1788

Merta. E. 1880

Meyer. C. D. 1793

Meyer. F. 1841

Meyer. G. 1803

Meyrick. J.J. 1882 
INDEX TO NAMES OF AUTHORS.

Michaelis. Johann David 1771

Michel. Francisque 1860

Micheli y Marques. Jos. 1662

Middendorf. A. Th. 1852

Miglio. Antonio 1824

Mignon. D. J. 1841. 1842

Migotti. E. F. 1879

Miles. Henry Downes 1863

Miles. W. J. 1845. 1868

Miles. H. D. and William 1860

Miliken. 1857

Mills. James 1843

Mills. John 1776

Mills. John 1845. 1854

Mills. John 1863

Milton. William 1810

Moerder. J. 1868

Moiroud. L. 1842

Mohammed Ali Hazin. 1716

Mohammed Buksh. 1839

Mohr. Wilhelm 1879

Moleschott. Jacob. 1856

Molini. Giuseppe 1858

Moll. 1856

Möller. H. 1879

Montaigne. Michel-Eyquem de 1580

Montendre. Achille de 1840. 1845

Montes. Juan Antonio 1787

Monteton. L. W. O. D. von 1856. 1877

Montfaucon de Rogles. 1778

Montigny. James Edone Count de 1851. 1852. 1853.

Montlaville. Havez 1850

[1855. 1865. 1885. 1886

Montmarin. Corvisart 1873. 1874. 1878

Montmorency. Lt.-Col. 1820 
Monray. Chevalier de 1796

Monto y Roca. 1742

Moorcroft. William 1795. 1800

Moore. James 1857

Morabda. Jose Andres de 1732

Morawetz. Carl 1879

Morel. F. L. 1823

Moreton. Robert 1877. 1882

Moricière. C. L. L. J. de La 1850

Morin. Alphonse 1864. 1867. 1873

Morland. George Hanby 1811

Morland. Thos. Henry 1792

Moroni. Epaminonda 1880

Morrell. Charles Francis 1870. 1871

Morris. Mowbray 1886

Mortgens. Abraham 1824

Mortier. Abraham 1857

Mortimer. 1854

Morton. W. J. T. 1837. 1840. 1844. 1880

Morvan. Le Luyer 1862

Mottin de la Balme. 1773. 1776

Mousis. B. 1841

Moussy. De 1834

Muguardt. C. 1872

Muhlwerth-Gartner. 1875

Muller. Alexandre 1816

Muller. C. F. 1874

Müller. Franz 1853. 1854. 1862

Muller. Joh. Gottlieb 1832

Muñoz de Peral. 1640

Münster. Graf von 1874. 1882

Münter. Joh. Carl C. 1791

Murray. William Henry 1873. 1881

Murrell. 1823 
Musani. F. 1880

Mussgnug. Christoph. 1856

Mussot. P, 1854. 1856

N ...m. P 1856

N. P. J. B. 1810

Naaldwyck. Petrus A. 1531

Nadosy. Alexander von 1854

Nalhorst. J. Th. 1835

Napier. Maj.-Genl. 1872

Narbonne. Pelet- 1875. 1884

Nathanson. M. L. 1842. 1854. 1858

Nathusius. Athaldens1 1868. 1872. 1882

Natzmer. O. 1879

Naumann. Bernhard 1780

Naumann. Johann Georg 1795. 1800. 1811

Navarette. Francisco Perez de 1626

Navarro. Silvestre Blazquez 1855

Neergard. Jens Weibel 1800. 1814. 1828, 1841. 1842.

Nehring. A. 1884

[1849. 1851. 1852.1854.1856

Neubert. Carl 1854

Neville. George 1877

Neville. L. 1796

Newcastle. Guillaume Marquis et Comte de 1657. 1667

Newte. K. 1867

Noacke. C. A. 1826

Noel. Casimir 1852. 1853. 1854. 1855. 1856. 1857

Nolan. L. E. 1852. 1853

Noue. Pierre de La 1620

Noveli. Nicolas Rodrigo 1726

Numan. A. 1834

Nüsken. Friedr. 18281838 
INDEX TO NAMES OF AUTHORS.

Oaks. A. F. 1850

O'Donoghue. Mrs. Power 1880. 1881. 1885

Oebschelwitz. L. W. F. van 1761. 1766

Oehlmann. Karl Aug. 1789. 1800. 1802

Oeynhausen. B. C. v. 1849. 1851. 1865. 1869. 1873.1874

Oliphant. George Henry Hewitt 1847. 1882

Olivier. Jean 1854

Orr. G. 1803

Orval. Hacquet D' 1850

Osbaldison. William A. 1792

Osbourne. Joseph 1849

Osmer. William 1756

Osorio y Vega. Manuel Alvarez 1733

Ostrowski. E. 1850

Oudinot. Nicholas Charles Victor d' 1840. 1842

P. A. von 1856

Paalzow. 1886

Pach zu Bernegg. Freiherr v. 1873

Pacheco. Francisco Pinto 1670

Packe. Edmund 1834

Paget. Lord George 1881

Pajol. N. 1874

Pallin. William 1884

Palmieri. L. 1625

Panciroli. Guido 1599

Papa. Fran. 1845

Papin. Mathurin 1854

Pardon. 1871

Parent. Ernest 1864

Parr. Henry Hallam 1881

Paschkewitsch. O. S. 1854

Pasquier. Th. 1875. 1879

Patellani. Luigi 1851. 1854. 1856 
Patten. George W. 1863

Paul. Saint-Ange Saint- 1842

Pauli. Otto Albrecht 1848

Paulina. Nicolo and Luigi Santa 1696

Paz. Eugène 1867

Peacham. Henry 1639

Peall. Thomas 1814

Pearson. Robert 1811

Peck. William 1814

Peillard. Charles 1866

Pelet-Narbonne. G. v. 1875. 1884

Pellier. Jules 1861

Pellier. Louis Charles 1823. 1874

Pembroke. Henry Earl of 1761

Pennant. Thomas 1768. 1771

Percival. William 1823. 1829. 1834, 1850

Perez. Luyz 1568

Perez de Navarette. Francisco 1626

Perrault. C. and E. Fletchier 1670

Perrault. Eugène 1849. 1857

Perosino. Felice 1856

Persch. Joh. Fried. 1840

Person. Felix 1841. 1851. 1855

Pessina. Ign. Jos. 1822

Pétéaux. A. 1848

Peter. Dr. M. 1863

Peterka. Joh. 1827

Peters. Anton H. F. 1856

Peters. J. 1886

Petersen. Geo. Fr. 1796

Petricio. A. 1874

Pétry. G. A. 1855

Pettersen. B. H. 1855

Petzer. Geo. 1879 
Peuch. F. 1884

Peyrow. B. 1854

Philippe. J. 1881

Philipson. John 1883. 1885

Phillipps. C. S. March 1869

Piana. Giampietro 1880

Pichat. Olivier 1867

Pick. William 1785. 1803

Pierres. Le Baron de 1860

Pieschel. Professor 1852

Piétrement. C. A. 1870. 1871. 1875. 1882

Piggott. Lieut. 1794

Pigouche. J. A. F. J. 1881

Pilger. Friedrich 1803

Pillwax. Johann 1855

Pinel. Honoré 1869. 1873. 1883

Pinto. Jose Ferreira de Macedo- 1852. 1854

Plasse. E. L. 1850. 1857

Plinius Secundus. C. D. 50

Plinzner. P. 1882

Plon. Jh. Chr. 1790

Plotz. A. v. 1871. 1872.'1874. 1878

Ploucquet. Wilhelm G. 1780. 1790

Pluvinel. Antoine 1623

Pokorny. Gust. 1878

Poliakoff. M. 1881

Politz. Andr. 1803

Pollnitz. G. L. v. 1810. 1811. 1815. 1818. 1819. 1820

Poly. Charles de 1884

Pomar. Pedro Pablo 1789. 1793

Pompee. Du Breuil 1669

Pond. John 1753

Pons D'Hostun. L. H. 1806

Pontet. 1856 
INDEX TO NAMES OF AUTHORS.

Porquet. Fenwick de 1857

Porter. Neale 1860

Porterie. Major de La 1754

Poten. B. 1878

Pouloti. Esprit Paul de La Font 1787. 1*

Powell. W. J. 1872

Powis. Richard 1814

Pownall. T. 1771

Préval. Claude Antoine Hippolyte de 18*

Prinz. Carl Glob. 1841

Prizelius. Joh. Gfr. 1771. 1777

Procter. William 1882

Prosch. Ferdinand Victor Alphons 1853. 1855. 185

Prosser. Thomas 1786

Prosser. W. H. 1855

Pütz. H. 1882

Pye. H. J. 1735

Quabeck. Charles Goupy de 1841

Quillinan. L. de 1854

Quistorp. B. 1870

R. Otto Wilhelm v. 1873.1877

Raabe. C. W. 1845 1848. 1854. 1856. 1857. 1860. 1863.

Radcliffe. F. P. D. 1838

Rahm. F. 1872

Raimund. Carl 1845

Rainard. J. 1845

Ramée. D. 1856

Ramirez. Baltasar Francisco 1629

Ramm. v. 1877

Rampoldi. R. 1881

Rane. F. 1883

Rapier. [pseud.] 1883 
Rarecourt. George de la Vallée 1856

Rarey. John S. 1854

Raseman. 1820

Raspail. François Vincente 1854

Rath. C. F. 1841

Recleben. 1854

Recordon. G. 1866

Reeves. George 1838

Reeves. John 1757

Rego. Antonio Peregra 1679

Reicherter. E. 1885

Reiffenberg. F. de 1862.1866. 1870

Reinecker. Gottl. 1827. 1828. 1852

Reitzenstein. Wolf. Chrfr. 1764

Renard. Bruno 1861

Renatus. Vegetius 1524

Renault. Eugène 1840. 1854

Renggli. 1856

Renner. H. and Rothernell 1882

Reska. Ignaz. 1837

Retzius. Anders Adolph 1850

Revarchon. Hector 1825

Rex. Joh. Carl Frae 1790

Rey. A. A. 1852.1873

Reynardson. C. T. S. Birch 1874

Reynolds. R. S. 1882

Rhodes. Jean Baptiste 1854

Ribbe. Joh. Chr. 1821

Ribero. Antonius Ludovicus 1671

Richard. A. 1847. 1848. 1849. 1850. 1856. 1857.

Richardson. H. D. 1848. 1852

Richardson. Capt. M. 1853

Richepance. Baron de 1864

Ricourt. 1854 
Ricquet. A. 1861

Ridinger. Joh. Elias 1722. 1734. 1752. 1754.1760. 1761.

Riedheim. Frhr. v. 1871

Riege. Heinrich 1851

Riem. Joh. 1786. 1805

Rigau. Ed. 1845

Rigault. 1810

Rigot. F. J. J. 1824. 1840

Risueno. Carlos 1829. 1834

Ritter. Genl. 1853

Riva. C. J. A. 1872

Rivet. Capt. 1847

Robbins. Thomas 1851

Rober. Fr. Aug. 1794

Roberts J. and H. 1760

Roberts. Mary 1841

Robertson. D. 1771. 1790

Robertson. W. 1883

Robinet. Dr. E. 1864

Robinet. Jos. 1777

Robinson. H. 1861

Robinson. James 1856. 1858. 1859. 1860

Robredo y Villaroya. S. 1744

Rochau. A. de. 1828

Roche. A. E. 1882

Roche-Aymon. Comte de la 1817. 1828

Rochefort. J. M. A. M. C. de 1849. 1853. 1865

Rodenbough. 1876

Rodet. J. B. C. 1840

Rodloff. Christian 1852. 1856

Rodriguez. Bernardo 1790

Roederer. August 1856

Roell. M. F. 1853. 1856

Roemer. J. 1863 
Rogles. Monfaucon de 1778

Rohles. Joh. Nic. 1780, 1783. 1804. 1806. 1816. 1819

Roimonda. Eugenio 1626

[1822, 1842, 1853, 1856 .

Rowlandson. T. 1799

Rose. Maj. 1855

Roserus. P. F. 1780

Rosselmini. Niccolo 1764

Rosenbaum. Wilhelm 1848

Rossi. 1882

Rossi. Giuseppe Nicola 1856

Rossier. J. B. 1832

Rotenham. Freiherr v. 1878

Roth von Shreckenstein. Ludw. Joh. Carl Gregor Eusebius 1851

Rous. C. 1850.1858

Roussel. J. 1881

Royo. Domingo 1734

Rozières. Henri de 1857

Rozwadowski. Ladislau Graf v. 1875

Rückert. Ernst. Ferd. 1839

Ruddock. E. H. 1878

Rudorff. W. 1878

Rueckert. H. 1852

Rueff. Adolph. 1852. 1855. 1857. 1873

Ruel. Jean 1530

Rufener. Alexandre 1859. 1860

Ruff. William 1842

Ruffus. Laurentius 1462

Ruini. Carlo 1598

Ru1. Louis Joseph 1857. 1870

Rumpelt. Geo. Ludw. 1786. 1801

Ruprecht. Carl Johann Friedrich Wilhelm 1848

Russell. John Cecil 1873

Rutenberg. 1851 
Rutta. Rudolph 1854

Rüttimeyer. Ludwig 1863

Rychner. J. J. 1828. 1839. 1842. 1849. 1854

Rydge. John 1832

Ryding. William 1801

Ryves. William Henry 1870

S. J. 1850

Sa'ádatyár Khan. 1853

Sabel. Johann Eduard 1855

Sablon. J. B. 1844

Sadler. Richard 1587

Sage. J. F. 1855

Saint Albin. N. de 1851

Saint Ange. C. J. Martin 1854

Saint Ange. Charles Casimir Beucher de 1852

Saint Bel. Charles Vial de 1791. 1793

Saint Cyr. F. 1864. 1875

Saint Hilaire. Isidore Geoffry. 1856

Saint Jal. P. U. C. de Lastic 1856

Saint-Paul. Saint-Ange 1842

Saint Paul. Fr. Wilh. Leop. v. 1804

St. Denis. Le Vaillant de 1789

Sainz. 1859

Saive. Maximilien de 1844

Salle. J. B. V. 1873

Salvi. P. 1881

Sallwurk. Fr. H. v. 1817

Salzedo. Gregorio de Tapia y 10643

Salzmann. J. G. 1829

Sampayo. Joao Teyxeira de 1710

Sampedro. Fernando 1851. 1856

Sampedro. Guillermo 1830. 1833. 1840

Sample. Hamilton 1883 
Sander. Jh. K. H. 1789. 1810. 1812

Sandri. Giulio 1824. 1853

Sanson. André 1856. 1861. 1863. 1868

Santa Maria de Carmel. Onorato 1761

Santini. E. 1886

Santos. Antonio 1835

Sarchiano. 1826

Saucerotte. Ed. 1837

Saunders. Alfred 1885

Saunier. Gaspard de 1734. 1749. 1756

Savary de Lancosme Brèves. 1852. 1860. 1861

Savigny. P. C. M. Cochet de 1849

Scacco. Filippo 1553. 1591. 1602

Schaefer. Johann Christoph. 1853

Schäfer. Joh. Geo. 1795

Schanzel. Ignatius 1800

Schauenberg. Baron de 1838

Schilling von Kannstatt. 1866

Schlaberg. A. 1884

Schlichting. Aloys 1820. 1822

Schlieden. A. 1867

Schmelz. P. 1866

Schnabeli. H. 1870

Schneider. L. 1873

Schoenbeck. Richard 1872. 1876. 1883

Schönberg. H. Fr. J. v. 1866

Schönermark. Wilhelm 1854

Schrader. F. A. 1833

Schrader. G. W. 1862

Schreckenstein. L. J. C. G. E. Roth von 1851

Schreiner. Frz. Xav. Jos. 1829

Schubert. Toers Dierbergen 1848

Schultes. Joseph 1840 [1820. 1826. 1831. 1840. 1872

Schwab. Konrad Ludwig 1803. 1814. 1815. 1817. 1819 
INDEX TO NAMES OF AUTHORS.

Schwabe. Ernst 1803

Schwartz. J. 1870

Schwartz. J. and A. Krocker. 1870

Schwarzl. E. 1871

Scott. John 1820

Scott. William Henry 1818

Scrofani. Sav. 1793

Sebald. Geo. Fr. 1806. 1812. 1815

Seeger. Louis 1848. 1850. 1852

Seer. Hermann 1856

Segundo. Juan 1855

Segundo. J. and Col. von Schepeler. 1829

Seidler. E. F. 1842. 1843. 1844. 1863. 1872

Seinsheim. J. 1867

Selincourt. 1683

Sellier. Alexandre 1849

Serres. E. 1861

Seyff. Anton. 1851

Shakespear. Capt. H. 1860

Sharkey Pat. 1790

Shaw. Austin C. 1849

Shipp. John 1806

Shirley. Lieut.-Col. 1842. 1854

Sidney. S. 1874

Siefried. Ludwig Frhr. v. 1876

Siegmann. W. 1868

Signol. Jules 1884

Silberer. V. 1883. 1884

Silberer. V. and G. Ernst 1883

Silberschmidt. Fr. 1829

Simon. Heinr. 1811

Simpson. J. C. 1881

Sind. J. B. de 1764. 1766. 1768. 17691770

Sjostedt. Gustav Wilhelm 1867. 1875 
Skeavington. George 1850

Skeene. Capt. 1808

Small. Matthew 1833

Snape. Andrew 1683

Snape. Edward 1805

Sohr. Wilh. 1829

Solleysel. Jacques de 1664

Soria. Manoel Barradas 1700

Souhart. R. 1886

Sourches. Le Marquis de 1660

Sourdeval. Ch. de 1849. 1850. 1853. 1880

Spilsbury. Francis 1785

Spinola. W. T. J. 1839. 1814. 1856. 1875

Sphor. 1882

Spooner. W. C. 1840.1853

Stanley. Edward 1827

Starke. 1868

Steel. J. H. 1876

Stein. Karl 185]

Steinbrecht. Gustav 1885

Steinhoff. Fr. L. C. 1824. 1840

Steinhoff. Th. Eph. 1795

Stent. G. C. 1882

Stephan. Hein. Wilh. 1843

Stephen. Sir George 1835

Stephens. 1843

Stephens. J. B. 1874

Stevenin. Thiriet- 1855

Stevenson. J. 1851

Steward. Charles 1821

Stewart. John 1830. 1831. 1833

Stillfried. H. 1866

Stillmann. J. O. B. 1881

Stovin. Aistroppe 1794 
Stradan. Johan 1578

Strangeways. Thomas 1869

Stratton. E. M. 1878

Strauss. G. 1832. 1854

Strebel. V. 1885

Street. Frederick 1883

Stroh. Wilhelm 1845

Strombeck Frhr. von 1867

Strubenberg. Johan Wilhelm von 1662

Suarez. Alonso 1564

Surtees. R. S. 1831

Susane. Genl. 1874

Swoboda. Gustav 1857

Swoboda. Max. 1820

Sylva. Pedro da 1751

Széchényi. D. 1872

Szelinski. Georg 1883

Tabourin. Fr. 1849. 1853

Tackels. C. J. 1870

Tacquet. Jean 1614

Tagliacozzo. M. Filippo Sacchoda 15531591

Tamm. F.J v. 1765.1785

Tantaloup. Orfaure de 1849

Tapia y Salzédo. Gregorio de 1643

Taplin. William 1788. 1803

Tappe. F. A. W. 1883

Tardieu. Ambroise 1843

Tattersall. George 1842. 1843. 1844. 1850

Taubert. 1845

Tayleure. C. W. 1865

Taylor. Frederick 1859. 1861. 1862

Taylor. Joseph 1808

Teast. 1821 
Telepnew. Nicolai 1849

Teller. Fried. Aug, 1830

Tennecker. S. von 1794. 1797. 1798. 1799. 1801. 1802.

1803.1805. 1815.1816.1819.1820.1821.1822.1823.1825.

Testarode. E. 1862

$[1827.1828$

Thébaud. Felix 1848

Thielman. Chr. 1818

Thilenius. M. G. 1796

Thiroux. Charles 1799

Thomann. Edm. 1884

Thomann. C. Fd. 1831

Thomas. 1829

Thomas. Brice 1869. 1870. 1874

Thomas. Clément 1846

Thon. Chr. Fr. G. 1824

Thümen, Von 1885

Thurn. Ed. Im- 1836. 1840

Tiberius. 400

Tiden. Lars 1841

Timlich. C. 1796

Tindall. John 1814

Tisserant. E. 1849. 1853

Tocqueville. Le Vicomte de 1842

Toggia. Francesco 1846. 1848

Toggia. Francis 1786. 1807. 1812. 1819

Tombari. Telesforo 1856

Tongue. Cornelius 1851. 1852

Torcy. Marquis de 1843

Torp. Franz Louis von 1854

Touchemoulin. Aegidius 1830

Towne L. 1848

Traeger. Theodor 1846. 1851

Trautenberg. E. A. 1796

Trautvetter. J. S. 1877 
Trench. F. C. 1884

Treyden von Trotta. 1861

Tripard. 1873

Troschke. E. von 1869

Trotter. Lieut. 1878

Trutta. Gio. Battista 1699. 1785

Tscherning. H. C. 1843

Tscheulin. Geo. Fr. 1804.1810. 1811. 1815. 1817. 1819.

Tuczek. F. W. 1846

Tuffnell. F. 1832

Turenne. Joseph de 1844. 1845

Turner. James 1860

Turner. R. 1851

Tuson. R. V. 1869

Tuting. William 1770

Twentyman. W. L. 1878

Twici. Guyllame 1307

Twyford. 1866

Tykel. B. 1855

Tyndale. W. 1801

Ubiez. 1884. 1885

Uden. Fr. 1800

Ueberacker. J. 1821

Unterberger. Friedrich 1853

Upton Roger D. 1873. 1874

Ussel Phillippe Comte d' 1856

Vaillant St. Denis. Le 1789

Vallada. C. 1881

Valle. Pietro della 1650

Vallow. A. 1864

Vals. J. F. de Metivier de 1864

Vandermeer, Felix 1866

Vandermulin. 17C0 
Vargas Machuco. Bernardo de 1600.1621

Varro. Marcus Terentius 1472

Vatel. P. 1828

Veauce. Charles Eugène de Cadier de 1849

Vegetius Renatus. 1524

Veiss. Giuseppe $18: 9$

Veith. Johann Elias 1817. 1826. 1855. 1856

Velasco. Franciscus de Cespedes y 1609

Velten. Hanns-Tob 1835

Veltheim. R. von 1820

Vercelli. Giudetti 1868

Verdy du Vernois. A. M. F. de 1784

Verdy du Vernois. Col. J. V. 1870. 1874. 1876

Vergnaud. 1884

Vergnaud. Amand Denis 1834

Verheyen. S. 1845

Verneuil. Bergeaud de 1875

Vernois. Dr. Max 1853

Vernon. F. S. M. J. Gay de 1810. 1853. 1865

Veyssière. J. B. 1840

Vial. A. A. 1866. 1867

Viborg. Eric Nissen 1800.1801. 1809.1821.1824. 1826

Vierordt. Er. Jac. 1800

Villa y Martin. Santiago de la 1881

Villalobos. Simon de 1605

Villaroya. Sebastian Robredo y 1744

Villery. Felix 1856

Vines. Richard 1830. 1833

Vinci. Leonardo di 1499

Vitet. Louis 1771

Vittré. C. de 1880

Vix. Dr. Karl W. 1834

Voetsch. Louis 1842

Vogeli. Felix 1833. 1835. 1836 
INDEX TO NAMES OF AUTHORS.

Vogelmann. A. 1868

Vogler. Carl Heinrich 1847. 1848

Voigtlander. C. F. 1876

Volkers. Emil 1857

Volpi. Alessandro 1853. 1854. 1856

Vrolik. Willem 1849. 1854

Vuillermedunand. 1856

Vyner. R. T. 1841

W. C. 1860

W. L. 1869

Wachter. L. 1862

Wackerow. C. 1877

Wagenfeld. Ludwig 1832. 1840. 1851

Wagner. Chr. Gfr. 1816

Wagenfeldt. J. E. 1848. 1854

Wahlgren. Friedrich 1850

Walch. C. 1831

Waldheim. 1876

Waldinger. Hieron 1805. 1808. 1809. 1812.1814. 1815.

Waldschmidt. J. 18651872

Waldstatten. Joh. Frhr. v. der 1875

[1820. 1830

Walker. B. 1770

Walker. Donald 1834

Wall. Richard 1768

Walles. Alexandre de 1856

Wallhausen. Jean Jacques de 1616

Wallis. Thomas 1764

Wallje-Szaka. Fedor von Karacsay de 1832

Wallmerode. Fr. M. Fr. v. Bouwinghausen. 1777. Walsh. John Henry 1856. 1863

Walter. 1795

[1779, 1790. 1791

Walter. D. H. 1872. 1874. 1878

Walther. E. 1874

47 
Ward. 1650

Ward. James 1823

Ward. Rowland 1884

Ward. William 1776

Warde. J. H. T. 1855

Watson. E. T. 1880.1886

Wayte. Samuel C. 1850

Weal. Jer. 1830

Weatherby. E. and J. 1837

Weber. C. 1873

Weber. M. J. 1824

Webster. P. 1870

Weckerlin. August von 1846

Wedermeyer.Schönrade. 1869. 1872

Wefeld. J. A. v. 1779

Weidemann. Gottfried 1853

Weidenkeller. Joh. Jac. 1818. 1822. 1825. 1828. 1831.

Weinshenk. F. W. 1848

Weisskopf. H. 1884

Wellmann. A. v. 1874. 1877

Wentz. L. 1869

Wernaer. Anton Ottomar 1851

Werneburg. Von 1852

Werner. Jh. Gli. 1793. 1821

Westerberg. Ludwig 1853

Weyhern. H. 1874. 1876

Weyrother. A. de 1804

Weyrother. Max von 1814

White. James 1800. 1801. 1804. 1815

Whyte. James Christie 1840

Wickram. J. 1865

Widdern. Geo. Cardinal von 1877. 1878

Wiehe. Jh. Geo. W. 1771

Wilhelmi. A. P. 1832. 1837 
INDEX TO NAMES OF AUTHORS.

Wilkinson. William 1818

Will. Ant. 1817

Williams. W. 1872.1874

Willis. Thomas 1884

Willisen, W, von 1865. 1873

Wilson. Yorick 1801

Winiker. W. 1853

Winter. J. W. 1846

Winter. Georg Simon de 1672. 1678

Wiseman. N. 1852

Wistrand. A. H. 1872

With. Geo. Chr. 1840. 1841. 1852

Witte. Johann Friedrich 1857

Wohlen. Chr. 1830

Wolanski. Erazmus von 1857

Wolff. H. 1881

Wolstein. Jh. Gli. 1779. 1787. 1792. 1805. 1807

Wood. J. G. 1885

Wood. Lieut.-Col. 1873

Woodruff. Hiram 1874

Wopperer. Th. 1799. 1800

Worz. J. J. 1875. 1876

Wrangel. C. G. 1869. 1872

Wüppermann. Ludwig Phil. 1832

Xenophon. B.c. 380

Youatt. William 1831. 1839

Young. Sir W. 1801

Yvart. 1853

Zabala. Juan 1860

Zamora. Jose Perez 1735

Zamora. Pedro Lopez 1588 
Zangger. R. 1865

Zech. L. 1874

Zehentner. Jos. Eph. 1753. 1754

Zeiher. Joh. Cr. 1771

Zen. Anania 1658

Zerrener. Theodor Ferdinand 1841

Zipf. Stephen 1807

Zipperlen. W. 1873

Zorn. Chr. 1768

Zschokke. E. 1885

Zündel. A. 1874

Zuniga y Arista. Gregorio de 1705

Zyllnhardt. Karl v. 1782

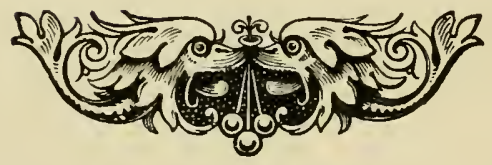




\section{INDEX}

TO VARIOUS SUBJECTS INCLUDED UNDER THE HEADING OF

\section{WORKS ON}

\section{HORSES AND EQUITATION}

NAMELY :-

BREEDING

Equitation and Stable

Management

RACING

Horse's Foot, Shoes

\section{AND Shoeing}

Harness, Bits and Bitting

Driving \& CoAchbuilding

Uses after Death

Laws Relating to

Warrantry, Acts etc.

Miscellaneous
Natural History, Anatomy, Physiology and External Cavalry FORM

Veterinary

The Chase

Age of Horse, Dentition And Dentistry

Stable Architecture

Artistic

Mules and Asses

BRANDS

Pageantry, Chariots, etc. 



\section{INDEX TO SUBJECTS.}

\section{BREEDING.}

Kurrat ul-Mulk $\quad \ldots \quad \ldots \quad 1478$

Blundevile $\ldots \quad \ldots \quad \ldots \quad \ldots \quad 1565$

$\begin{array}{lllll}\text { Carraciolo } & \ldots & \ldots & \ldots & 1566\end{array}$

$\begin{array}{lllll}\text { Fugger } \quad \ldots & \ldots & \ldots & 1578\end{array}$

$\begin{array}{lllll}\text { Sadler } & \ldots & \ldots & \ldots & 1587\end{array}$

$\begin{array}{lllll}\text { Zamora } & \ldots & \ldots & \ldots & 1588\end{array}$

$\begin{array}{lllll}\text { Tacquet } & \ldots & \ldots & \ldots & 1614\end{array}$

$\begin{array}{lllll}\text { Baret } \quad \ldots & \ldots & \ldots & 1618\end{array}$

De Grey $\quad \ldots \quad \ldots \quad \ldots \quad 1639$

$\begin{array}{lllll}\text { Markham } & \ldots & \ldots & \ldots & 1656\end{array}$

$\begin{array}{lllll}\text { Calloet } & \ldots & \ldots & \ldots & 1666\end{array}$

$\begin{array}{lllll}\text { Francisci } & \ldots & \ldots & \ldots & 1670\end{array}$

De Winter ... $\quad \ldots \quad \ldots \quad 1672$

Garzoni $\quad \ldots \quad \ldots \quad \ldots \quad 1692$

$\begin{array}{lllll}\text { Guerini } & \ldots & \ldots & \ldots & 1724\end{array}$

Osmer

Wallis

Wall

Brézé

Sind

Michaelis

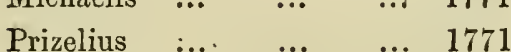

Mills

Ward

Brugnone

Anon

Pouloti

Dompierre

Gosden
Pomar

$\begin{array}{llll}\ldots & \ldots & \ldots & 1793\end{array}$

Helmbrecht and Naumann... 1795

Petersen

1796

Wopperer $\quad \ldots \quad \ldots \quad \ldots \quad \ldots \quad 1800$

Rumpelt

... 1801

Maleden

... 1803

$\begin{array}{lllll}\text { Orr } \ldots & \ldots & \ldots & \ldots & 1803\end{array}$

$\begin{array}{lllll}\text { Pick ... } & \ldots & \ldots & \ldots & 1803\end{array}$

$\begin{array}{llllll}\text { Stud-book } & \ldots & \ldots & \ldots & 1805\end{array}$

Huzard $\quad \ldots \quad \ldots \quad \ldots \quad \ldots \quad 1803$

$\begin{array}{lllll}\text { Klatte } & \ldots & \ldots & \ldots & 1805\end{array}$

$\begin{array}{lllll}\text { Wolstein } & \ldots & \ldots & \ldots & 1805\end{array}$

Rohlwes $\quad \ldots \quad \ldots \quad \ldots \quad 1806$

$\begin{array}{lllll}\text { Kail ... } & \ldots & \ldots & \ldots & 1811\end{array}$

Morland $\quad \ldots \quad$... $\quad \ldots \quad 1811$

Campagnola... $\quad \ldots \quad \ldots \quad 1814$

$\begin{array}{lllll}\text { Hanger } \quad \ldots & \ldots & \ldots & 1814\end{array}$

$\begin{array}{lllll}\text { Klatte } & \text {... } & \text {... } & \text {.. } & 1814\end{array}$

$\begin{array}{lllll}\text { Waldinger } & \ldots & \ldots & \ldots & 1814\end{array}$

Bojanus $\quad \ldots \quad \ldots \quad \ldots \quad 1815$

Flint $\quad \ldots \quad \ldots \quad \ldots \quad 1815$

$\begin{array}{llllll}\text { Berghofer } & \ldots & \ldots & \ldots & 1818\end{array}$

$\begin{array}{llllll}\text { Florke } \quad \ldots & \ldots & \ldots & 1818\end{array}$

$\begin{array}{llllll}\text { Mayer } & \ldots & \ldots & \ldots & 1819\end{array}$

$\begin{array}{lllll}\text { Burgsdorf } & \ldots & \ldots & \ldots & 1820\end{array}$

$\begin{array}{llllll}\text { Erdelyi } \quad \ldots & \ldots & \ldots & 1820\end{array}$

$\begin{array}{lllll}\text { Kegel } \quad \ldots & \ldots & \ldots & 18 \% 0\end{array}$

$\begin{array}{lllll}\text { Tennecker } & \ldots & \ldots & \ldots & 1820\end{array}$

Walther $\quad \ldots \quad \ldots \quad \ldots \quad 1820$ 


\section{BREEDING-Continued.}

\begin{tabular}{|c|c|c|c|c|c|c|c|c|c|}
\hline \multicolumn{2}{|c|}{ Weidenkeller } & $\cdots$ & $\ldots$ & 1822 & Lindenau & 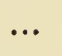 & $\cdots$ & $\cdots$ & 1831 \\
\hline Archiv. & $\ldots$ & $\ldots$ & $\ldots$ & 1823 & Weidenkell & & $\ldots$ & $\ldots$ & 1831 \\
\hline Dutoict & ... & ... & $\ldots$ & 1823 & Benfert & $\ldots$ & $\ldots$ & $\ldots$ & 1832 \\
\hline Tennecker & $\ldots$ & $\ldots$ & $\ldots$ & 1823 & Hippologisc & he $B$ & ter & $\ldots$ & 1832 \\
\hline Dieterrichs & ... & $\ldots$ & ... & 1824 & Letang & $\ldots$ & $\ldots$ & ... & 832 \\
\hline Viborg & ... & $\ldots$ & ... & 1824 & Minutoli & $\ldots$ & $\ldots$ & ... & 832 \\
\hline Dieterrichs & ... & ... & ... & 1825 & Rossier & ... & ... & $\ldots$ & 832 \\
\hline Hofacker & ... & ... & ... & 1825 & Anon & $\ldots$ & $\ldots$ & $\ldots$ & 833 \\
\hline Smith & ... & $\ldots$ & $\ldots$ & 1825 & Ammon & $\ldots$ & $\ldots$ & ... & 833 \\
\hline Gjersing & $\ldots$ & $\ldots$ & $\ldots$ & 1826 & Ammon & $\ldots$ & $\ldots$ & ... & 834 \\
\hline Burgsdorf & ... & $\ldots$ & ... & 1827 & Chuteau & $\ldots$ & ... & $\ldots$ & 834 \\
\hline Chabannes & ... & ... & ... & 1827 & Journal & $\ldots$ & $\ldots$ & $\ldots$ & 834 \\
\hline Erdelyi & ... & $\ldots$ & ... & 1827 & De Moussy & .. & ... & ... & 1834 \\
\hline Kertitshka & ... & $\ldots$ & ... & 1827 & Herbst & $\ldots$ & ... & $\ldots$ & 1836 \\
\hline Lengerke & ... & ... & $\ldots$ & 1827 & Schmid & $\cdots$ & $\ldots$ & ... & 1837 \\
\hline Ammon & ... & $\ldots$ & $\ldots$ & 1828 & Thacker & ... & $\ldots$ & $\ldots$ & 1837 \\
\hline Hofacker & ... & $\ldots$ & $\ldots$ & 1828 & Weatherby & ... & $\ldots$ & $\ldots$ & 1837 \\
\hline Hofmeister & ... & $\ldots$ & $\ldots$ & 1828 & Montendre & $\ldots$ & $\ldots$ & ... & 840 \\
\hline Klatte & ... & $\ldots$ & ... & 1828 & D'Oudinot & $\ldots$ & ... & $\ldots$ & 1840 \\
\hline Neergard & ... & ... & ... & 1828 & Vernon & $\ldots$ & ... & ... & 1840 \\
\hline Pogge & ... & $\ldots$ & ... & 1828 & Anon & ... & $\ldots$ & $\cdots$ & 1841 \\
\hline Rochau & ... & $\ldots$ & ... & 1828 & L'Argus des & $\mathrm{Ha}$ & $\ldots$ & $\ldots$ & 1841 \\
\hline Tennecker & ... & $\ldots$ & ... & 1828 & Bugeaud & ... & ... & ... & 1841 \\
\hline Weidenkelle & & $\ldots$ & ... & 1828 & Curnieu & $\ldots$ & $\ldots$ & $\cdots$ & 1811 \\
\hline Ammon & ... & $\ldots$ & ... & 1829 & Daunay and & $\mathrm{Lig}$ & e... & ... & 1841 \\
\hline Brown & ... & $\ldots$ & ... & 1829 & Chambly & $\ldots$ & ... & ... & 1841 \\
\hline Huzard & ... & $\ldots$ & ... & 1829 & A. de C. & $\ldots$ & ... & $\ldots$ & 1842 \\
\hline Ithen & ... & $\cdots$ & ... & 1829 & Dieterrichs & $\ldots$ & .. & ... & 1842 \\
\hline Lawrence & ... & $\ldots$ & ... & 1829 & Dittmer & . & ... & $\ldots$ & 1842 \\
\hline Schreiner & ... & $\ldots$ & $\ldots$ & 1829 & Houël & & $\ldots$ & $\cdots$ & 1842 \\
\hline Sohr & ... & $\ldots$ & ... & 1829 & Itier ... & $\ldots$ & $\ldots$ & $\ldots$ & 1842 \\
\hline Gunther & $\ldots$ & .. & $\ldots$ & 1830 & Bréves & $\ldots$ & . & $\ldots$ & 1842 \\
\hline
\end{tabular}


BREEDING-Continued.

$\begin{array}{lllll}\text { Nathanson } & \ldots & \ldots & \ldots & 1842\end{array}$

$\begin{array}{lllll}\text { Neergard } \quad \ldots & \ldots & \ldots & 1842\end{array}$

$\begin{array}{lllll}\text { D'Oudinot } & \ldots & \ldots & \ldots & 1842\end{array}$

$\begin{array}{llllll}\text { Seidler } \quad \ldots & \ldots & \ldots & 1842\end{array}$

$\begin{array}{lllll}\text { Tocqueville ... } & \ldots & \ldots & 1842\end{array}$

$\begin{array}{llllll}\text { Boigne } & \ldots & \ldots & \ldots & 1843\end{array}$

Girardin and Torcy... $\quad \ldots \quad 1843$

Grammont ... $\quad \ldots \quad \ldots \quad \ldots 1843$

Hamont $\quad \ldots \quad \ldots . \quad \ldots \quad 1843$

Laborde $\quad \ldots \quad \ldots \quad \ldots \quad \ldots 1843$

Bréves $\quad \ldots \quad \ldots . \quad \ldots \quad 1843$

$\begin{array}{lllll}\text { Laulanhier } & \ldots & \ldots & \ldots & 1843\end{array}$

$\begin{array}{lllll}\text { Girardin } \quad \ldots & \ldots & \ldots & 1844\end{array}$

Sablon $\quad \ldots \quad \ldots \quad \ldots \quad 1844$

Saive $\quad \ldots \quad \ldots \quad \ldots \quad 1844$

$\begin{array}{lllll}\text { Turemne } & \ldots & \ldots & \ldots & 1844\end{array}$

Auboyer $\quad \ldots \quad \ldots \quad \ldots \quad 1845$

D'Aure $\quad \ldots \quad \ldots \quad \ldots \quad 1845$

Baumeister ... $\quad \ldots \quad \ldots \quad 1845$

Jäger $\quad \ldots \quad \ldots \quad \ldots{ }^{\prime} 1845$

Montendre ... $\quad \ldots \quad \ldots \quad 1845$

Raimund $\ldots \quad \ldots \quad \ldots \quad 1845$

Rainard $\quad \ldots \quad \ldots \quad \ldots \quad 1845$

$\begin{array}{lllll}\text { Turenne } & \ldots & \ldots & \ldots & 1845\end{array}$

$\begin{array}{lllll}\text { Anon } & \ldots & \ldots & \ldots & 1846\end{array}$

$\begin{array}{lllll}\text { Dubroca } & \ldots & \ldots & \ldots & 1846\end{array}$

Weckherlin... $\quad \ldots \quad \ldots \quad 1846$

$\begin{array}{lllll}\text { Basserie } \quad \ldots & \ldots & \ldots & 1847\end{array}$

$\begin{array}{llllll}\text { Lespinats } & \ldots & \ldots & \ldots & 1847\end{array}$

$\begin{array}{llllll}\text { Volger } \quad \ldots & \ldots & \ldots & 1847\end{array}$

Kurze Auleitung $\quad \ldots \quad \ldots \quad 1848$

Quelques Mots $\quad \ldots \quad \ldots \quad 1848$
Socièsé d'Agriculture $\quad \ldots \quad 1848$

Delafond $\quad \ldots \quad \ldots \quad \ldots \quad 1848$

$\begin{array}{llll}\text { Dieterrichs ... } & \ldots & \ldots & 1848\end{array}$

$\begin{array}{lllll}\text { Fritsch } & \ldots & \ldots & \ldots & 1848\end{array}$

$\begin{array}{lllll}\text { Gayot } & \ldots & \ldots & \ldots & 1848\end{array}$

$\begin{array}{lllll}\text { Pauli } & \ldots & \ldots & \ldots & 1848\end{array}$

$\begin{array}{lllll}\text { Pétéaux } & \ldots & \ldots & \ldots & 1848\end{array}$

$\begin{array}{lllll}\text { Richard } \quad \ldots & \ldots & \ldots & 1848\end{array}$

Richardson ... $\quad \ldots \quad \ldots \quad 1848$

Weinschenk $\quad \ldots \quad \ldots \quad 1848$

$\begin{array}{llllll}\text { Anon } & \ldots & \ldots & \ldots & 1849\end{array}$

$\begin{array}{lllll}\text { Brindley } \quad \ldots & \ldots & \ldots & 1849\end{array}$

Eschstruth ... $\quad$.. $\quad$.. 1849

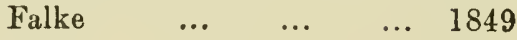

$\begin{array}{lllll}\text { Goux } & \ldots & \ldots & \ldots & 1849\end{array}$

$\begin{array}{lllll}\text { Graf } & \ldots & \ldots & \ldots & 1849\end{array}$

$\begin{array}{lllll}\text { Hocquard } & \ldots & \ldots & \ldots & 1849\end{array}$

$\begin{array}{llll}\text { Jullienfelde ... } \quad \ldots & \ldots & 1849\end{array}$

$\begin{array}{lllll}\text { Kerpelani } & \ldots & \ldots & \ldots & 1849\end{array}$

$\begin{array}{lllll}\text { Perrault } & \ldots & \ldots & \ldots & 1849\end{array}$

$\begin{array}{lllll}\text { Richard } \quad \ldots & \ldots & \ldots & 1849\end{array}$

$\begin{array}{lllll}\text { Sourdeval } & \ldots & \ldots & \ldots & 1849\end{array}$

$\begin{array}{lllll}\text { De Veauce } & \ldots & \ldots & \ldots & 1849\end{array}$

Ministère de l'Agriculture ... 1850

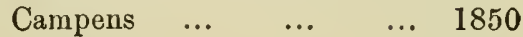

$\begin{array}{llll}\text { Douterluigne } & \ldots & \ldots & 1850\end{array}$

$\begin{array}{lllll}\text { Fischer } & \ldots & \ldots & \ldots & 1850\end{array}$

$\begin{array}{lllll}\text { Gayot } & \ldots & \ldots & \ldots & 1850\end{array}$

$\begin{array}{lllll}\text { D'Orval } & \ldots & . . & \ldots & 1850\end{array}$

Heine $\quad \ldots \quad \ldots \quad \ldots \quad 1850$

$\begin{array}{lllll}\text { Houël } & \ldots & \ldots & \ldots & 1850\end{array}$

$\begin{array}{lllll}\text { Hubert } \quad \ldots & \ldots & \ldots & 1850\end{array}$ 


\section{BREEDING-Continued.}

Jacob

... 1850

La Moricière $\quad \ldots \quad \ldots \quad 1850$

$\begin{array}{lllll}\text { Leyon } & \ldots & \ldots & \ldots & 1850\end{array}$

$\begin{array}{lllll}\text { Retzius } \quad \ldots & \ldots & \ldots & 1850\end{array}$

$\begin{array}{lllll}\text { Richard } \quad \ldots & \ldots & \ldots & 1850\end{array}$

$\begin{array}{llllll}\text { Seeger } \quad \ldots & \ldots & \ldots & 1850\end{array}$

$\begin{array}{lllll}\text { Sourdeval } & \ldots & \ldots & \ldots & 1850\end{array}$

Bemaerkninger $\quad \ldots \quad \ldots \quad 1851$

Ministère de l'Agriculture ... 1851

$\begin{array}{lllll}\text { Un Mot } & \ldots & \ldots & \ldots & 1851\end{array}$

$\begin{array}{lllll}\text { Ayraud } & \ldots & \ldots & \ldots & 1851\end{array}$

Campens $\quad \ldots \quad \ldots \quad \ldots \quad \ldots \quad 1851$

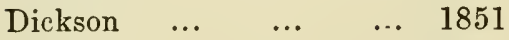

Harvey $\quad \ldots \quad \ldots \quad \ldots \quad 1851$

Lessona $\quad \ldots \quad \ldots \quad \ldots \quad 1851$

$\begin{array}{lllll}\text { Leyon } & \ldots & \ldots & \ldots & 1851\end{array}$

Neergard $\quad \ldots \quad \ldots \quad \ldots \quad \ldots \quad 1851$

$\begin{array}{lllll}\text { Person } \quad . . & \text {.. } & \text {.. } & 1851\end{array}$

Schreckenstein $\quad \ldots \quad \ldots \quad 1851$

$\begin{array}{lllll}\text { Stein } & \ldots & \ldots & \ldots & 1851\end{array}$

Stud Book: Français... $\quad$.. 1851

The Stud Farm $\quad \ldots \quad \ldots \quad 1851$

$\begin{array}{lllll}\text { Jaarboehije } & \ldots & \ldots & \ldots & 1852\end{array}$

Règlement $\ldots \quad \ldots \quad \ldots \quad 1852$

Delafond $\quad \ldots \quad \ldots \quad \ldots \quad 1852$

Hamm $\quad \ldots \quad \ldots \quad \ldots .1852$

$\begin{array}{lllll}\text { Brèves } & \ldots & \ldots & \ldots & 1852\end{array}$

Neergaard . . $\quad \ldots \quad \ldots \quad 1852$

Richardson ... $\quad \ldots \quad \ldots \quad 1852$

With $\quad \ldots \quad \ldots \quad \ldots \quad 1852$

$\begin{array}{lllll}\text { Anon } & \ldots & \ldots & \ldots & 1853\end{array}$

$\begin{array}{lllll}\text { Barford } \quad \ldots & \ldots & \ldots & 1853\end{array}$
Berthaud ... .. $\quad \ldots \quad 1853$

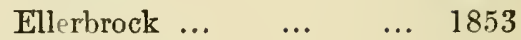

$\begin{array}{llll}\text { Buzareingues } & \ldots & \ldots & 1853\end{array}$

Goldhart $\quad \ldots \quad \ldots \quad \ldots \quad \ldots \quad 1853$

Goodwin $\quad \ldots \quad \ldots \quad \ldots \quad 1853$

$\begin{array}{llllll}\text { Graefe } & \ldots & \ldots & \ldots & 1853\end{array}$

$\begin{array}{lllll}\text { Proseh } & \ldots & \ldots & \ldots & 1853\end{array}$

$\begin{array}{lllll}\text { Ritter } & \ldots & \ldots & \ldots & 1853\end{array}$

$\begin{array}{lllll}\text { Sourdeval } & \ldots & \ldots & \ldots & 1853\end{array}$

Unterberger $\quad \ldots \quad \ldots \quad 1853$

$\begin{array}{lllll}\text { Tisserant } & \ldots & \ldots & \ldots & 1853\end{array}$

Weidemann $\quad \ldots \quad \ldots \quad 1853$

Anon $\quad \ldots \quad \ldots \quad \ldots \quad 185$ t

Auzoux $\quad \ldots \quad \ldots \quad \ldots .1854$

Bandow $\quad \ldots \quad \ldots \quad \ldots \quad \ldots \quad 1854$

Erhardt $\quad \ldots \quad \ldots \quad \ldots \quad 1854$

De Grangues $\quad \ldots \quad \ldots \quad 1854$

$\begin{array}{lllll}\text { Huzard } \quad \ldots & \text {... } & \text {.. } & 1854\end{array}$

$\begin{array}{lllll}\text { Keller } \quad \ldots & \ldots & \ldots & 1854\end{array}$

Nathanson ... $\quad$... $\quad$.. $\quad 1854$

Neergaard ... $\quad \ldots \quad$... 1854

$\begin{array}{lllll}\text { Von Torp } & \ldots & \ldots & \ldots & 1854\end{array}$

Biedenweg ... $\quad \ldots \quad \ldots \quad 1855$

Graefe $\quad \ldots \quad \ldots \quad \ldots \quad 1855$

Grange $\quad \ldots \quad \ldots \quad \ldots \quad 1855$

$\begin{array}{lllll}\text { Koppe } & \ldots & \ldots & \ldots & 1855\end{array}$

Lommatzsch $\quad \ldots \quad \ldots \quad 1855$

Person $\quad \ldots \quad \ldots . \quad \ldots \quad 1855$

De l'Amélioration $\quad \ldots \quad \ldots \quad \ldots \quad 1856$

$\begin{array}{llllll}\text { Bellamy } \quad \ldots & \ldots & \ldots & 1856\end{array}$

$\begin{array}{lllll}\text { Eberhard } & \ldots & \ldots & \ldots & 1856\end{array}$

Fernandez y Perez ... ... 1856 


\section{BREEDING-Continued.}

$\begin{array}{lllll}\text { Géraud } \quad \ldots & \ldots & \ldots & 1856\end{array}$

$\begin{array}{lllll}\text { Goodwin } & \ldots & \ldots & \ldots & 1856\end{array}$

$\begin{array}{lllll}\text { Haidvogl } & \ldots & \ldots & \ldots & 1856\end{array}$

$\begin{array}{lllll}\text { Saint-Jal } & \ldots & \ldots & \ldots & 1856\end{array}$

$\begin{array}{lllll}\text { Lisch } & \ldots & \ldots & \ldots & 1856\end{array}$

Loebe $\quad \ldots \quad \ldots \quad \ldots \quad 1856$

Loiset $\quad \ldots \quad \ldots \quad \ldots \quad 1856$

$\begin{array}{lllll}\mathrm{N}-\mathrm{m} & \ldots & \ldots & \ldots & 1856\end{array}$

$\begin{array}{lllll}\text { Neergaard } & \ldots & \ldots & \ldots & 1856\end{array}$

$\begin{array}{lllll}\text { Pontet } \quad \ldots & \ldots & \ldots & 1856\end{array}$

$\begin{array}{lllll}\text { Richard } \quad \ldots & \ldots & \ldots & 1856\end{array}$

Rivista agronomica $\quad \ldots \quad 1856$

$\begin{array}{lllll}\text { Roederer } \quad \ldots & \ldots & \ldots & 1856\end{array}$

$\begin{array}{lllll}\text { Villeroy } \quad \ldots & \ldots & \ldots & 1856\end{array}$

$\begin{array}{lllll}\text { Chauvel } & \ldots & \ldots & \ldots & 1857\end{array}$

De Grangues $\quad \ldots \quad \ldots \quad 1857$

$\begin{array}{llllll}\text { Echegaray } & \ldots & \ldots & \ldots & 1857\end{array}$

$\begin{array}{llllll}\text { Gauglia } & \ldots & \ldots & \ldots & 1857\end{array}$

$\begin{array}{lllll}\text { Graefe } & \ldots & \ldots & \ldots & 1857\end{array}$

Hering $\quad \ldots \quad \ldots \quad \ldots \quad 1857$

$\begin{array}{lllll}\text { Lemaitre } & \ldots & \ldots & \ldots & 1857\end{array}$

$\begin{array}{lllll}\text { Linsley } & \ldots & \ldots & \ldots & 1857\end{array}$

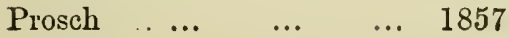

$\begin{array}{lllll}\text { Volkers } & \ldots & \ldots & \ldots & 1857\end{array}$

$\begin{array}{lllll}\text { Wolanski } & \ldots & \ldots & \ldots & 1857\end{array}$

$\begin{array}{lllll}\text { Klein } \quad \ldots & \ldots & \ldots & 1858\end{array}$

$\begin{array}{lllll}\text { Nathanson } & \ldots & \ldots & \ldots & 1858\end{array}$

$\begin{array}{llllll}\text { Torp } & \ldots & \ldots & \ldots & 1858\end{array}$

De Vere Hunt $\quad \ldots \quad \ldots \quad 1859$

$\begin{array}{lllll}\text { Abadie } \quad \ldots & \ldots & \ldots & 1860\end{array}$

$\begin{array}{lllll}\text { Airie } & \ldots & \ldots & \ldots & 1860\end{array}$

D'Aure $\quad \ldots \quad \ldots \quad \ldots \quad 1860$ $\begin{array}{lllll}\text { Auzoux } & \ldots & \ldots & \ldots & 1860\end{array}$

Du Hays $\quad \ldots \quad \ldots \quad \ldots \quad \ldots \quad 1860$

$\begin{array}{lllll}\text { Houël } & \ldots & \ldots & \ldots & 1860\end{array}$

$\begin{array}{lllll}\text { Michel } & \ldots & \ldots & \ldots & 1860\end{array}$

$\begin{array}{lllll}\text { Pierres } & \ldots & \ldots & \ldots & 1860\end{array}$

$\begin{array}{lllll}\text { Shakespear } & . . & \ldots & \ldots & 1860\end{array}$

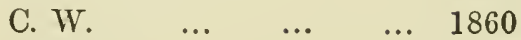

Lalaubie $\quad \ldots \quad \ldots \quad \ldots \quad \ldots 1861$

Morgan-Frucourt $\quad \ldots \quad \ldots \quad 1861$

Hippologische Blätter $\quad$.. 1862

$\begin{array}{lllll}\text { Laffore } \quad \ldots & \ldots & \ldots & 1862\end{array}$

Der Sporn $\quad \ldots \quad \ldots \quad \ldots \quad \ldots \quad 1862$

$\begin{array}{lllll}\text { Testerode } & \ldots & \ldots & \ldots & 1862\end{array}$

Blätter fur Pferdezucht $\quad$... 1862

Demilly $\quad \ldots \quad \ldots \quad \ldots \quad \ldots 1863$

Liégeard $\quad \ldots \quad \ldots \quad \ldots \quad \ldots \quad 1863$

$\begin{array}{llllll}\text { Curr } & \ldots & \ldots & \ldots & 1864\end{array}$

Foucher $\quad \ldots \quad \ldots \quad \ldots \quad \ldots \quad 1864$

$\begin{array}{lllll}\text { Gourdon } \quad \ldots & \ldots & \ldots & 1864\end{array}$

Huzard $\quad \ldots \quad \ldots \quad \ldots \quad 1864$

$\begin{array}{lllll}\text { Malfrain } \quad \ldots & \ldots & \ldots & \mathbf{1 8 6 4}\end{array}$

Entweder-Oder $\quad \ldots \quad \ldots .1865$

Beaurepaire ... $\quad . . \quad \ldots \quad 1865$

$\begin{array}{lllll}\text { Briefe } & \ldots & \ldots & \ldots & 1865\end{array}$

$\begin{array}{llllll}\text { Broye } & \ldots & \ldots & \ldots & 1865\end{array}$

Hamilton $\quad \ldots \quad \ldots \quad \ldots \quad \ldots \quad 1865$

$\begin{array}{lllll}\text { Jannasch } & \ldots & \ldots & \ldots & 1865\end{array}$

Waldschmidt $\quad \ldots \quad \ldots \quad 1865$

$\begin{array}{lllll}\text { Zangger } \quad \ldots & \ldots & \ldots & 1865\end{array}$

Du Hays $\quad \ldots \quad \ldots \quad \ldots \quad 1866$

$\begin{array}{llllll}\text { Mayr } & \ldots & \ldots & \ldots & 1866\end{array}$

$\begin{array}{lllll}\text { Stillfried } & \ldots & \ldots & \ldots & 1866\end{array}$ 


\section{BREEDING-Continued.}

\begin{tabular}{|c|c|c|c|c|c|c|c|c|c|}
\hline André & $\cdots$ & $\ldots$ & $\cdots$ & 1867 & Plötz & $\cdots$ & $\cdots$ & $\ldots$ & 1872 \\
\hline Du Hays & $\cdots$ & ... & $\ldots$ & 1867 & Rahm & $\ldots$ & $\ldots$ & $\ldots$ & 1872 \\
\hline Mayr & $\ldots$ & ... & $\ldots$ & 1867 & \multicolumn{3}{|c|}{ Wedemeyer-Schönrade } & ... & 1872 \\
\hline Zoppretz & $\ldots$ & ... & $\ldots$ & 1867 & Wrangel & $\ldots$ & $\cdots$ & $\cdots$ & 872 \\
\hline Gsandzuge & der & ... & $\ldots$ & 1868 & \multicolumn{5}{|c|}{ Report on East Indice Govern- } \\
\hline Erler & ... & ... & $\ldots$ & 1868 & \multicolumn{2}{|c|}{ ment Studls } & $\cdots$ & $\cdots$ & 3 \\
\hline Fœlan & ... & ... & $\ldots$ & 1868 & Bendz & $\ldots$ & ... & ... & 873 \\
\hline Du Hays & $\ldots$ & ... & $\cdots$ & 1868 & Hahn & $\cdots$ & $\cdots$ & $\cdots$ & 1873 \\
\hline Moerder & $\cdots$ & ... & $\cdots$ & 1868 & Jacoby & $\cdots$ & $\cdots$ & $\cdots$ & 873 \\
\hline Nathusius a & and $\mathrm{K}$ & cker & $\ldots$ & 1868 & Lydtin & $\ldots$ & $\ldots$ & $\ldots$ & 1873 \\
\hline Vercelli & $\ldots$ & ... & $\ldots$ & 1868 & Moerder & $\ldots$ & ... & ... & 1873 \\
\hline Vogelmann & .. & ... & $\cdots$ & 1868 & Morin & $\cdots$ & $\cdots$ & $\cdots$ & 1873 \\
\hline Charnacé & $\ldots$ & $\therefore$ & $\ldots$ & 1869 & Murray & $\cdots$ & $\cdots$ & $\cdots$ & 1873 \\
\hline Dimse & $\cdots$ & $\ldots$ & $\ldots$ & 1869 & Pinel & ... & $\ldots$ & ... & 1873 \\
\hline Huzard & $\cdots$ & .. & $\ldots$ & 1869 & Upton & $\ldots$ & $\cdots$ & $\ldots$ & 1873 \\
\hline Wedemeyer & Schc & ade & $\ldots$ & 1869 & Zipperlen & $\ldots$ & $\cdots$ & $\ldots$ & 1875 \\
\hline Wrangel & $\ldots$ & $\cdots$ & $\ldots$ & 1869 & Decroi & $\ldots$ & $\cdots$ & $\ldots$ & 1874 \\
\hline Betantiade & ... & $\cdots$ & $\ldots$ & 1870 & \multicolumn{3}{|c|}{ Der Halbblutzuchter } & $\ldots$ & 1874 \\
\hline Die Cavalle & eriefr & e... & $\ldots$ & 1870 & Hunt & $\ldots$ & $\cdots$ & $\ldots$ & 1874 \\
\hline Ueber den & Congr & $\ldots$ & $\ldots$ & 1870 & Löffler & $\cdots$ & $\cdots$ & $\ldots$ & 1874 \\
\hline Boy ... & $\ldots$ & $\because$ & $\ldots$ & 1870 & \multicolumn{4}{|c|}{ Miiller and Schwarznecker } & 1874 \\
\hline Dadd & $\cdots$ & ... & $\ldots$ & 1870 & \multicolumn{2}{|c|}{ Oeynhausen } & ... & $\cdots$ & 187 \\
\hline Schnabeli & $\ldots$ & $\cdots$ & $\ldots$ & 1870 & Plötz & $\cdots$ & $\ldots$ & $\ldots$ & 1874 \\
\hline Schwartz ar & nd $\mathrm{K}$ & ker & $\ldots$ & 1870 & Sidney & $\cdots$ & $\cdots$ & $\ldots$ & 1874 \\
\hline Schwartz & $\cdots$ & ... & $\ldots$ & 1870 & Stephens & $\cdots$ & $\cdots$ & $\cdots$ & 1874 \\
\hline Ableitner & $\cdots$ & $\cdots$ & $\ldots$ & 1871 & Alpert & $\ldots$ & $\ldots$ & $\ldots$ & 1875 \\
\hline Lapotre & $\cdots$ & $\cdots$ & $\ldots$ & 1871 & Franck & $\cdots$ & $\cdots$ & $\ldots$ & 187 \\
\hline Plotz & $\ldots$ & $\cdots$ & $\ldots$ & 1871 & Gerard & $\ldots$ & $\ldots$ & $\ldots$ & 187 \\
\hline Suhwarzl & $\ldots$ & $\ldots$ & $\ldots$ & 1871 & Landemon & t. . & $\cdots$ & $\ldots$ & 1875 \\
\hline Historiques & $\operatorname{des} 1$ & montes & $\ldots$ & 1872 & Mayr & $\cdots$ & $\ldots$ & $\ldots$ & 187 \\
\hline Le Couteuls & $\mathrm{x}$ de & nteleu & $\ldots$ & 1873 & Rozwadow & ski & $\ldots$ & $\ldots$ & 187 \\
\hline Nathusius & $\ldots$ & ... & $\ldots$ & 1872 & Verhandlu & ngen & $\ldots$ & $\ldots$ & 187 \\
\hline
\end{tabular}




\section{BREEDING-Continued.}

\begin{tabular}{|c|c|c|c|c|c|c|c|c|c|}
\hline \multirow{2}{*}{\multicolumn{4}{|c|}{$\begin{array}{l}\text { Havernick ... } \quad \ldots \\
\text { Oesterreichische Zeitschrift }\end{array}$}} & \multirow{2}{*}{$\begin{array}{r}1876 \\
1876\end{array}$} & \multirow{2}{*}{\multicolumn{2}{|c|}{$\begin{array}{ll}\text { Espejo } & \ldots \\
\text { Du Hays } & \ldots\end{array}$}} & \multirow{2}{*}{$\begin{array}{l}\cdots \\
\cdots\end{array}$} & \multicolumn{2}{|c|}{$\ldots \quad 1881$} \\
\hline & & & & & & & & $\ldots$ & 1881 \\
\hline Worz & $\ldots$ & $\ldots$ & $\ldots$ & 1876 & Meneghini & $\ldots$ & $\ldots$ & .. & \\
\hline Hardegg & $\ldots$ & $\ldots$ & $\ldots$ & 1877 & Miunster & $\ldots$ & $\ldots$ & 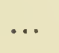 & \\
\hline Wackerow & ... & $\ldots$ & $\ldots$ & 1877 & Nathusius & $\ldots$ & ... & $\ldots$ & \\
\hline In Zwolfter & - Stunde & & $\cdots$ & 1878 & Reynolds & $\ldots$ & $\ldots$ & $\ldots$ & \\
\hline Bernsdoıff & $\ldots$ & $\ldots$ & $\ldots$ & 1878 & Rossi & $\ldots$ & ... & $\ldots$ & \\
\hline Blunt & ... & $\ldots$ & $\ldots$ & 1878 & Earl Cathe & art & $\ldots$ & $\ldots$ & \\
\hline Earl of Dun & nmore & $\ldots$ & $\ldots$ & 1878 & Lehndorff & $\ldots$ & ... & $\ldots$ & \\
\hline Frentzel & $\ldots$ & $\ldots$ & $\ldots$ & 1878 & Rane & $\cdots$ & $\cdots$ & $\ldots$ & \\
\hline Hardy & $\ldots$ & $\ldots$ & $\ldots$ & 1878 & Street & $\ldots$ & $\ldots$ & $\ldots$ & \\
\hline Josch & $\ldots$ & $\ldots$ & $\ldots$ & 1878 & \multicolumn{3}{|c|}{ The Hackney Horse } & $\ldots$ & \\
\hline Loennecker & & $\cdots$ & $\cdots$ & 1878 & \multicolumn{3}{|c|}{ Alasonière .. } & $\ldots$ & . \\
\hline Gestutsbuch & & $\cdots$ & $\cdots$ & 1879 & \multicolumn{4}{|c|}{ Cdeveland Bay Stud Book ... } & \\
\hline \multicolumn{4}{|c|}{ The English Stud Boole of } & & \multicolumn{3}{|c|}{ Hackney Stnd Book } & $\cdots$ & 50 \\
\hline Cart Hor & & $\cdots$ & $\cdots$ & 1880 & Der Lasa & ... & ... & $\ldots$ & 180 \\
\hline \multicolumn{2}{|c|}{ Libro G'enealoyico } & $\cdots$ & $\ldots$ & 1880 & Hackney $S$ & Stud & Buok, & 2 & \\
\hline Biddell & $\ldots$ & $\cdots$ & $\ldots$ & 1880 & Cavailhon & ... & $\cdots$ & $\cdots$ & 38 \\
\hline Horse Breec & der's Re & egister & $\cdots$ & 1881 & Paalzou & $\ldots$ & ... & $\cdots$ & 0 \\
\hline Le Corse de & i Caval & & $\cdots$ & 1881 & Souhart & ... & $\cdots$ & $\cdots$ & 88 \\
\hline Sulle razza & equine & ... & $\ldots$ & 1881 & & & & & \\
\hline
\end{tabular}

\section{EQUITATION, EREAKING, TAMING AND STABLE MANAGEMENT.}

$\begin{array}{llllllllll}\text { Crescenzi } & \ldots & \ldots & \ldots & 1300 & \text { Ferraro } & \ldots & \ldots & \ldots & 1560 \\ \text { Giraldes } & \ldots & \ldots & \ldots & 1318 & \text { Blundeville } \ldots & \ldots & \ldots & 1565 \\ \text { Duarte } & \ldots & \ldots & \ldots & 1434 & \text { Carraciolo } & \ldots & \ldots & \ldots & 1566 \\ \text { Naaldwyck } & \ldots & \ldots & \ldots & 1531 & \text { Campanella .. } & \ldots & \ldots & 1570 \\ \text { Camerarius } & \ldots & \ldots & \ldots & 1539 & \text { Manzánas } & \ldots & \ldots & \ldots & 1570 \\ \text { Chacou } & \ldots & \ldots & \ldots & 1551 & \text { Toralto } & \ldots & \ldots & \ldots & 1571 \\ \text { Grison } & \ldots & \ldots & \ldots & 1552 & \text { P. de Aguilar } & \ldots & \ldots & 1572 \\ \text { Fiaschi } & \ldots & \ldots & \ldots & 1556 & \text { Corte } & \ldots & \ldots & \ldots & 1573\end{array}$




\section{EQUITATION, BREAKING, TAMING AND STABLE} MANAGEMENT.-Continued.

\begin{tabular}{|c|c|c|c|c|c|c|c|c|c|}
\hline \multicolumn{2}{|c|}{ F. de Andrada } & $\ldots$ & $\ldots$ & 1580 & Bonifaz & ... & $\cdots$ & ... & 163 \\
\hline Puralta & ... & $\ldots$ & ... & 1580 & Markham & $\ldots$ & $\ldots$ & ... & 1635 \\
\hline Pavari & $\cdots$ & $\ldots$ & $\ldots$ & 1581 & Aquino & $\cdots$ & $\ldots$ & ... & 1636 \\
\hline Clifford & ... & $\ldots$ & $\ldots$ & I585 & De Gret & $\ldots$ & $\ldots$ & ... & 1639 \\
\hline Anon. & .. & $\ldots$ & $\cdots$ & 1584 & J. de Valen & ç̧a & $\ldots$ & $\cdots$ & 639 \\
\hline Astley & $\cdots$ & ... & ... & 1584 & Liberati & .. & $\cdots$ & ... & 1639 \\
\hline Mascall & ... & $\ldots$ & ... & 1587 & Tapia & $\cdots$ & $\ldots$ & ... & 643 \\
\hline Sadler & .. & $\ldots$ & $\ldots$ & 1587 & Galiberto & ... & $\ldots$ & $\cdots$ & 650 \\
\hline A. de Avíla & & $\ldots$ & .. & $1590^{\circ}$ & Markfiam & .. & $\ldots$ & $\ldots$ & 165 \\
\hline Creutsberger & & $\cdots$ & $\cdots$ & 1591 & Duke of $\mathrm{Ne}$ & ewcastle & & $\cdots$ & 165 \\
\hline S. de la Bro & ove & $\ldots$ & ... & 1593 & Massari & $\ldots$ & $\ldots$ & .. & 166 \\
\hline Markham & ... & $\ldots$ & $\ldots$ & 1593 & Sourches (A & Appendi & ix) & $\ldots$ & 66 \\
\hline Markham & ... & $\ldots$ & $\ldots$ & 1596 & Duke of $\mathrm{Ne}$ & ewcastle & & $\ldots$ & 667 \\
\hline Andrada & $\ldots$ & $\ldots$ & $\ldots$ & 1599 & Liberati & ... & $\ldots$ & .. & 166 \\
\hline Markham & $\ldots$ & $\ldots$ & $\cdots$ & 1599 & Pacheco & $\cdots$ & $\ldots$ & $\cdots$ & 67 \\
\hline Ferraro & ... & $\ldots$ & $\ldots$ & 1602 & Fouquet & $\ldots$ & $\ldots$ & $\ldots$ & 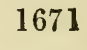 \\
\hline Markham & $\ldots$ & $\ldots$ & $\ldots$ & 1605 & Lieb & ... & $\ldots$ & $\ldots$ & 167 \\
\hline Markham & ... & $\ldots$ & $\ldots$ & 1607 & Ribero & $\ldots$ & $\ldots$ & $\cdots$ & 167 \\
\hline Loehneyser & $\ldots$ & $\ldots$ & $\ldots$ & 1609 & J. H. & $\ldots$ & $\ldots$ & $\cdots$ & 16 \\
\hline Morgan & ... & $\ldots$ & $\ldots$ & 1609 & Almond & ... & $\ldots$ & $\ldots$ & 167 \\
\hline Prevost & $\ldots$ & $\ldots$ & $\ldots$ & 1610 & Davila & $\ldots$ & $\ldots$ & $\ldots$ & 167 \\
\hline René de Mé & énous & $\ldots$ & $\ldots$ & 1612 & Solleysel & $\cdots$ & $\cdots$ & $\cdots$ & 167 \\
\hline Andrada & $\ldots$ & $\ldots$ & $\ldots$ & 1616 & Andrade & .. & $\ldots$ & $\ldots$ & 167 \\
\hline Wallhausen & & $\ldots$ & ... & 1616 & De Winter & $\ldots$ & $\ldots$ & $\cdots$ & 167 \\
\hline Baret & $\ldots$ & $\ldots$ & $\ldots$ & 1618 & Beaumont & $\ldots$ & $\ldots$ & $\cdots$ & 167 \\
\hline Markham & $\ldots$ & $\ldots$ & $\ldots$ & 1620 & Rego & $\ldots$ & $\ldots$ & $\cdots$ & 167 \\
\hline De la Noue & $\ldots$ & $\ldots$ & $\ldots$ & 1620 & Guillet & $\ldots$ & $\ldots$ & $\ldots$ & 168 \\
\hline Pluvinel & $\ldots$ & $\ldots$ & $\ldots$ & 1623 & R. H. $\langle$ App & endix) & $\ldots$ & $\ldots$ & 168 \\
\hline Browne & $\ldots$ & $\ldots$ & $\ldots$ & 1624 & Paulina & $\ldots$ & $\ldots$ & $\cdots$ & 16 \\
\hline Della Croce & & $\ldots$ & $\ldots$ & 1625 & Soria & $\cdots$ & $\cdots$ & $\cdots$ & 17 \\
\hline Palmieri & $\ldots$ & $\ldots$ &.. & 1625 & Anon. & $\cdots$ & $\cdots$ & $\cdots$ & 17 \\
\hline Perez de $\mathrm{N}$ & avare & $e \ldots$ & $\ldots$ & 1626 & Zuñiga & $\ldots$ & $\ldots$ & .. & 17 \\
\hline
\end{tabular}




\section{EQUITATION, BREAKING, TAMING AND STABLE} MANAGEMENT.-Continat.

\begin{tabular}{|c|c|c|c|c|c|c|c|c|c|}
\hline Sampayo & $\cdots$ & $\cdots$ & $\cdots$ & 1710 & Jacksen & $\cdots$ & $\cdots$ & $\cdots$ & 1765 \\
\hline Delcampe & ... & $\ldots$ & $\ldots$ & 1712 & Anon. & $\cdots$ & $\cdots$ & $\cdots$ & 1766 \\
\hline Gibson & $\ldots$ & $\ldots$ & $\ldots$ & 1721 & Sind & $\ldots$ & $\cdots$ & $\ldots$ & 1766 \\
\hline Anon. & $\cdots$ & $\cdots$ & $\cdots$ & 1726 & Sind & $\ldots$ & $\cdots$ & $\cdots$ & 1768 \\
\hline Eisenberg & $\ldots$ & $\ldots$ & $\cdots$ & 1727 & Weyrother & $\ldots$ & $\cdots$ & $\cdots$ & 1768 \\
\hline Burdon & $\ldots$ & $\ldots$ & $\ldots$ & 1730 & Dupaty de & Clam & $\cdots$ & $\cdots$ & 1769 \\
\hline Guerinière & $\ldots$ & $\ldots$ & $\ldots$ & 1733 & Garsault & $\ldots$ & $\cdots$ & $\ldots$ & 1769 \\
\hline Usorio & $\ldots$ & $\cdots$ & $\ldots$ & 1733 & Sind & $\cdots$ & $\cdots$ & $\ldots$ & 1770 \\
\hline Benavides & .. & $\cdots$ & $\ldots$ & 1734 & Berenger & $\ldots$ & $\cdots$ & $\ldots$ & 1771 \\
\hline Ridinger & $\ldots$ & $\ldots$ & $\cdots$ & 1734 & Dupaty de C & Clam & $\ldots$ & $\cdots$ & 1771 \\
\hline Lucas & $\ldots$ & $\ldots$ & $\ldots$ & 1736 & Hughes & $\cdots$ & $\ldots$ & $\cdots$ & 1772 \\
\hline Guerinière & $\cdots$ & $\ldots$ & $\ldots$ & 1740 & Mottin de I & la Balm & & $\ldots$ & 1773 \\
\hline Bracken & $\ldots$ & $\ldots$ & $\ldots$ & 1742 & Lombardi & $\ldots$ & $\ldots$ & $\ldots$ & 1773 \\
\hline Bourgelat & ... & $\ldots$ & $\ldots$ & 1744 & Astley & $\cdots$ & $\cdots$ & $\cdots$ & 1774 \\
\hline Eisenberg & ... & $\ldots$ & $\ldots$ & 1747 & Dupaty de & Clam & $\ldots$ & $\ldots$ & 1776 \\
\hline Saunier & $\ldots$ & $\ldots$ & $\ldots$ & 1749 & Lafosse & $\ldots$ & $\ldots$ & $\ldots$ & 1776 \\
\hline Saunier & $\ldots$ & $\cdots$ & $\ldots$ & 1750 & Ward & $\ldots$ & $\cdots$ & $\ldots$ & 1776 \\
\hline Bourgelat & $\ldots$ & $\cdots$ & $\ldots$ & 1750 & Rogles & $\cdots$ & $\cdots$ & $\ldots$ & 1778 \\
\hline Harguinez & $\ldots$ & $\ldots$ & $\ldots$ & 1750 & Anon. & $\ldots$ & $\ldots$ & $\ldots$ & 1780 \\
\hline Sylva & $\ldots$ & $\ldots$ & $\ldots$ & 1751 & Hiver & $\ldots$ & $\ldots$ & $\ldots$ & 1783 \\
\hline Eisenberg & $\ldots$ & $\ldots$ & $\cdots$ & 1753 & Koch & $\cdots$ & $\cdots$ & $\ldots$ & 1784 \\
\hline Zehentner & $\ldots$ & $\ldots$ & $\ldots$ & 1753 & Trutta & $\ldots$ & $\ldots$ & $\ldots$ & 1785 \\
\hline Saunier & $\ldots$ & $\ldots$ & $\ldots$ & 1756 & Calvo & $\ldots$ & $\ldots$ & $\ldots$ & 1789 \\
\hline Bernad & .. & $\ldots$ & $\cdots$ & 1757 & Kollner & $\ldots$ & $\ldots$ & $\ldots$ & 1789 \\
\hline Bonsi & $\ldots$ & $\ldots$ & .. & 1757 & St. Denis & $\ldots$ & $\ldots$ & $\ldots$ & 1789 \\
\hline Zehentner & $\ldots$ & $\ldots$ & $\ldots$ & 1757 & Andrade & $\ldots$ & $\cdots$ & $\ldots$ & 1790 \\
\hline Ridinger & $\ldots$ & $\ldots$ & $\ldots$ & 1760 & Boisdeffre & $\ldots$ & $\ldots$ & $\ldots$ & 1790 \\
\hline Earl of Pem & nbroke & $\ldots$ & $\ldots$ & 1761 & $\operatorname{Rex} \ldots$ & $\ldots$ & $\ldots$ & $\ldots$ & 1790 \\
\hline Thompson & $\ldots$ & $\ldots$ & $\ldots$ & 1763 & Kollner & $\ldots$ & $\ldots$ & .. & 1790 \\
\hline Eisenberg & $\ldots$ & $\ldots$ & $\ldots$ & 1764 & Wallmerode & & $\ldots$ & $\ldots$ & 1791 \\
\hline Fabricy & ... & $\ldots$ & $\ldots$ & 1764 & Hünersdorf & & $\ldots$ & $\ldots$ & 1791 \\
\hline Rosselmini & $\ldots$ & $\ldots$ & $\ldots$ & 1764 & Anon. & $\ldots$ & $\ldots$ & $\ldots$ & 1793 \\
\hline
\end{tabular}


EQUITATION, BREAKING, TAMING AND STABLE MANAGEMENT.-Continued.

$\begin{array}{lcccclllll}\text { Anon. } & \ldots & \ldots & \ldots & 1796 & \text { Riem } & \ldots & \ldots & \ldots & 1805 \\ \text { Beaumgarten-Crusius } & \ldots & 1796 & \text { Fiedler } & \ldots & \ldots & \ldots & 1806 \\ \text { Hunter } & \ldots & \ldots & \ldots & 1796 & \text { Freeman } & \ldots & \ldots & \ldots & 1806 \\ \text { Petersen } & \ldots & \ldots & \ldots & 1796 & \text { D'Hostun } & \ldots & \ldots & \ldots & 1806 \\ \text { Anon. } & \ldots & \ldots & \ldots & 1797 & \text { Sebald } & \ldots & \ldots & \ldots & 1806 \\ \text { Buschendorf } & \ldots & \ldots & 1797 & \text { Klatte } & \ldots & \ldots & \ldots & 1807 \\ \text { Lawson } & \ldots & \ldots & \ldots & 1797 & \text { Oehlmann } & \ldots & \ldots & \ldots & 1807 \\ \text { Tennecker } & \ldots & \ldots & \ldots & 1798 & \text { Ammon } & \ldots & \ldots & \ldots & 1808 \\ \text { Adams } & \ldots & \ldots & \ldots & 1799 & \text { Anon. } & \ldots & \ldots & \ldots & 1809 \\ \text { Thiroux } & \ldots & \ldots & \ldots & 1799 & \text { Lawrence } & \ldots & \ldots & \ldots & 1809 \\ \text { Anon. } & \ldots & \ldots & \ldots & 1800 & \text { Hnlfreich } & \ldots & \ldots & \ldots & 1810 \\ \text { Anon. } & \ldots & \ldots & \ldots & 1800 & \text { Naumann } & \ldots & \ldots & \ldots & 1811 \\ \text { Astley } & \ldots & \ldots & \ldots & 1800 & \text { Klatte } & \ldots & \ldots & \ldots & 1812 \\ \text { Journal } & \ldots & \ldots & \ldots & 1800 & \text { Arcellazi } & \ldots & \ldots & \ldots & 1813 \\ \text { White } & \ldots & \ldots & \ldots & 1800 & \text { Daum } & \ldots & \ldots & \ldots & 1813 \\ \text { Anon. } & \ldots & \ldots & \ldots & 1801 & \text { Campagnola } & & \ldots & \ldots & 1814 \\ \text { Anon. } & \ldots & \ldots & \ldots & 1802 & \text { Hanger } & \ldots & \ldots & \ldots & 1814 \\ \text { Klatte } & \ldots & \ldots & \ldots & 1802 & \text { Klatte } & \ldots & \ldots & \ldots & 1814 \\ \text { Mrazzuchelli.. } & \ldots & \ldots & 1802 & \text { Peall } & \ldots & \ldots & \ldots & 1814 \\ \text { Tennecker } & \ldots & \ldots & \ldots & 1802 & \text { Weyrother } & \ldots & \ldots & \ldots & 1814 \\ \text { Castor } & \ldots & \ldots & \ldots & 1803 & \text { Flint } & \ldots & \ldots & \ldots & 1815 \\ \text { Hoffman } & \ldots & \ldots & \ldots & 1803 & \text { Klatte } & \ldots & \ldots & \ldots & 1815 \\ \text { Meyer } & \ldots & \ldots & \ldots & 1803 & \text { Lloyd and } & \text { Symes } & \ldots & \ldots & 1815 \\ \text { Orr } & \ldots & \ldots & \ldots & 1803 & \text { Pollnitz } & \ldots & \ldots & \ldots & 1815 \\ \text { Politz } & \ldots & \ldots & \ldots & 1803 & \text { Anon. } & \ldots & \ldots & \ldots & 1816 \\ \text { Chifney } & \ldots & \ldots & \ldots & 1804 & \text { Anon. } & \ldots & \ldots & \ldots & 1817 \\ \text { Klatte } & \ldots & \ldots & \ldots & 1804 & \text { Daum } & \ldots & \ldots & \ldots & 1817 \\ \text { Lindenau } & \ldots & \ldots & \ldots & 1804 & \text { Klatte } & \ldots & \ldots & \ldots & 1817 \\ \text { Anon. } & \ldots & \ldots & \ldots & 1805 & \text { Lewaneu } & \ldots & \ldots & \ldots & 1817 \\ \text { Ammon } & \ldots & \ldots & \ldots & 1805 & \text { Pollnitz } & \ldots & \ldots & \ldots & 1818 \\ \text { Andre } & \ldots & \ldots & \ldots & 1805 & \text { Weidenkeller } & \ldots & \ldots & 1818 \\ \text { Mazzuchelli } & \ldots & \ldots & \ldots & 1805 & \text { Jush } & \ldots & \ldots & \ldots & 1819\end{array}$


EQUITATION, BREAKING, TAMING AND STABLE MANAGEMENT.-Continued.

\begin{tabular}{|c|c|c|c|c|c|c|c|c|c|}
\hline Kegel & $\ldots$ & $\ldots$ & $\ldots$ & 1819 & \multicolumn{2}{|c|}{ Weidenkeller } & $\cdots$ & $\ldots$ & 1825 \\
\hline Klalte & $\ldots$ & ... & $\ldots$ & 1819 & Anon. & $\ldots$ & $\ldots$ & $\ldots$ & 1826 \\
\hline Merk & .. & $\ldots$ & $\ldots$ & 1819 & Klatte & ... & $\ldots$ & $\ldots$ & . \\
\hline Pollnitz & $\ldots$ & ... & $\ldots$ & 1819 & Chabannes & $\ldots$ & ... & $\ldots$ & 18 \\
\hline Anon. & $\ldots$ & $\ldots$ & $\ldots$ & 1820 & Jervis & .. & $\ldots$ & ... & 82 \\
\hline Daum & $\ldots$ & $\ldots$ & $\ldots$ & 1820 & Stanley & $\ldots$. & $\ldots$ & $\ldots$ & O. \\
\hline Kegel & $\ldots$ & .. & $\ldots$ & 1820 & Daum & ... & $\ldots$ & ... & \\
\hline Rasemann & $\ldots$ & ... & $\ldots$ & 1820 & Hoffmann & ... & $\ldots$ & $\ldots$ & 82 \\
\hline Tennecker & $\ldots$ & $\ldots$ & $\ldots$ & 1820 & Loiset & ... & .. & $\ldots$ & 82 \\
\hline Steward & $\ldots$ & ... & $\ldots$ & 1821 & Klatte & $\ldots$ & ... & $\ldots$ & 82 \\
\hline Tennecker & $\ldots$ & ... & $\ldots$ & 1821 & Tennecker & ... & $\cdots$ & $\ldots$ & . \\
\hline Viborg & $\ldots$ & ... & $\ldots$ & 1821 & Hinds (Bad & cock) & $\ldots$ & $\ldots$ & 82 \\
\hline Bismark & $\ldots$ & ... & $\ldots$ & 1822 & Gribble & ... & $\ldots$ & $\ldots$ & 82 \\
\hline Daum & $\ldots$ & ... & $\ldots$ & 1822 & Ithen & ... & $\ldots$ & $\ldots$ & 82 \\
\hline Dutoict & $\ldots$ & ... & $\ldots$ & 1822 & Klatte & $\ldots$ & $\ldots$ & $\ldots$ & 32 \\
\hline Klatte & $\ldots$ & $\ldots$ & $\ldots$ & 1822 & Lawrence & $\ldots$ & $\ldots$ & $\ldots$ & 82 \\
\hline Klatte & $\ldots$ & $\ldots$ & $\ldots$ & 1822 & Schreiner & $\ldots$ & $\ldots$ & $\ldots$ & 82 \\
\hline Politz & $\ldots$ & $\ldots$ & ... & 1822 & Schepeler & $\ldots$ & $\ldots$ & $\ldots$ & 82 \\
\hline Braunschwe & & ... & $\ldots$ & 1823 & Anon. & $\ldots$ & $\ldots$ & ... & 183 \\
\hline Conti & $\ldots$ & $\ldots$ & .. & 1823 & Balle & ... & $\ldots$ & $\ldots$ & 183 \\
\hline Pellier & $\ldots$ & ... & $\ldots$ & 1823 & Touchemoli & & $\ldots$ & $\ldots$ & 183 \\
\hline Tennecker & $\ldots$ & ... & $\ldots$ & 1823 & Weal & $\ldots$ & $\ldots$ & ... & 183 \\
\hline Cordier & $\ldots$ & $\ldots$ & $\ldots$ & 1824 & Anon. & .. & $\ldots$ & $\ldots$ & 1831 \\
\hline Loweneck & $\ldots$ & ... & $\ldots$ & 1824 & Apperley ( & Vimrod & $\ldots$ & ... & 183. \\
\hline Mortgens & $\ldots$ & ... & $\ldots$ & 1824 & Kruger & $\ldots$ & $\ldots$ & $\ldots$ & 1831 \\
\hline Anon. & $\ldots$ & ... & $\ldots$ & 1825 & Weidenkell & & . & $\ldots$ & 1831 \\
\hline Allen & $\ldots$ & ... & $\ldots$ & 1825 & Hafftendom & $\ldots$ & $\ldots$ & $\ldots$ & 1832 \\
\hline A. C. & $\ldots$ & ... & $\ldots$ & 1825 & Tuffnell & $\ldots$ & $\ldots$ & $\ldots$ & 1832 \\
\hline Gibbons & $\ldots$ & $\ldots$ & $\ldots$ & 1825 & Aubry & $\ldots$ & $\ldots$ & ... & 1833 \\
\hline Klatte & $\ldots$ & $\ldots$ & $\ldots$ & 1825 & Baucher & $\ldots$ & $\ldots$ & ... & $183:$ \\
\hline Knight & $\ldots$ & $\ldots$ & $\ldots$ & 1825 & Stewart & $\ldots$ & $\ldots$ & $\ldots$ & $183:$ \\
\hline Locatelli & $\ldots$ & ... & $\ldots$ & .1825 & Balle & $\cdots$ & $\ldots$ & $\ldots$ & 183 \\
\hline
\end{tabular}


EQUITATION, BREAKING, TAMING AND STABLE MANAGEMENT.-Continued.

$\begin{array}{lllll}\text { Baucher } \quad \ldots & \ldots & \ldots & 1834\end{array}$

$\begin{array}{lllll}\text { Hering } \quad \ldots & \ldots & \ldots & 1834\end{array}$

Vergnaud $\quad \ldots \quad \ldots \quad \ldots \quad \ldots \quad 1834$

Walker $\quad \ldots \quad \ldots \quad \ldots \quad \ldots \quad 1834$

Anon. $\quad \ldots \quad \ldots \quad \ldots \quad \ldots 1835$

$\begin{array}{lllll}\text { Balassa } & \ldots & \ldots & \ldots & 1835\end{array}$

$\begin{array}{lllll}\text { Bates } \quad \ldots & \ldots & \ldots & 1835\end{array}$

$\begin{array}{lllll}\text { Dietterich } & \ldots & \ldots & \ldots & 1835\end{array}$

$\begin{array}{lllll}\text { Laborde } & \ldots & \ldots & \ldots & 1835\end{array}$

$\begin{array}{lllll}\text { Peters } & \ldots & \ldots & \ldots & 1835\end{array}$

Aubert $\quad \ldots \quad \ldots \quad \ldots \quad 1836$

Vogeli $\quad \ldots \quad \ldots \quad \ldots \quad 1836$

$\begin{array}{lllll}\text { Merlen } \quad \ldots & \ldots & \ldots & 1837\end{array}$

$\begin{array}{lllll}\text { Reska } & \ldots & \ldots & \ldots & 1837\end{array}$

$\begin{array}{lllll}\text { Schmid } & \ldots & \ldots & \ldots & 1837\end{array}$

$\begin{array}{lllll}\text { Reeves } \quad \ldots & \ldots & \ldots & 1838\end{array}$

Anon. $\quad \ldots \quad \ldots \quad \ldots \quad \ldots 1839$

Greenwood ... $\quad \ldots \quad \ldots \quad 1839$

$\begin{array}{lllll}\text { Kuers } & \ldots & \ldots & \ldots & 1839\end{array}$

$\begin{array}{lllll}\text { Capt. MI: } & \ldots & \ldots & \ldots & 1839\end{array}$

$\begin{array}{lllll}\text { Rychner } \quad \ldots & \ldots & \ldots & 1839\end{array}$

Baucher ... $\quad \ldots \quad \ldots \quad 1840$

$\begin{array}{lllll}\text { Knight } & \ldots & \ldots & \ldots & 1840\end{array}$

Merlen $\quad \ldots \quad \ldots \quad \ldots \quad \ldots \quad 1840$

Anon. $\quad \ldots \quad \ldots . \quad \ldots \quad 1841$

Nouvelle école $\quad \ldots \quad \ldots \quad 1841$

Anon. $\quad \ldots \quad \ldots \quad \ldots \quad 1842$

$\begin{array}{lllll}\text { Aubert } \quad \ldots & \ldots & \ldots & 1842\end{array}$

D'Aure $\quad \ldots \quad \ldots \quad \ldots \quad 1842$

Baucher $\quad \ldots \quad \ldots \quad \ldots \quad \ldots \quad 1842$

Cler

Hamont
Lancosme Bréves ...

Saint Paul $\ldots \quad \ldots \quad \ldots \quad \ldots \quad 1842$

Anon. $\quad \ldots \quad \ldots \quad \ldots \quad \ldots 1843$

Brudermann $\quad \ldots \quad \ldots \quad 1843$

Lecornué $\quad \ldots \quad \ldots \quad \ldots \quad \ldots \quad 1843$

$\begin{array}{lllll}\text { Legros } & \ldots & \ldots & \ldots & 1843\end{array}$

Le Maire $\quad \ldots \quad \ldots \quad \ldots \quad 1843$

$\begin{array}{lllll}\text { Mills } & \ldots & \ldots & \ldots & 1843\end{array}$

$\begin{array}{lllll}\text { Parisot } & \ldots & \ldots & \ldots & 1843\end{array}$

Anon. $\quad \ldots \quad \ldots \quad \ldots \quad 1844$

D'Aure $\quad \ldots \quad \ldots \quad \ldots .1844$

Baucher $\quad \ldots \quad \ldots \quad \ldots \quad 1844$

Dubroca $\quad \ldots \quad \ldots \quad \ldots \quad 1844$

Hersenberger $\quad \ldots \quad \ldots \quad 1844$

$\begin{array}{lllll}\text { Roper } \quad \ldots & \ldots & \ldots & 1844\end{array}$

$\begin{array}{lllll}\text { Seidler } & \ldots & \ldots & \ldots & 1844\end{array}$

$\begin{array}{lllll}\text { Balle } & \ldots & \ldots & \ldots & 1815\end{array}$

Brindley (Hieover) ... $\quad$.. 1845

Bucellati ... ... $\quad \ldots \quad \mathbf{1 8 4 5}$

$\begin{array}{lllll}\text { Cardini } \quad \ldots & \ldots & \ldots & 1845\end{array}$

Herschberger $\quad \ldots \quad \ldots \quad 1845$

Raabe $\quad \ldots \quad \ldots$... 1845

$\begin{array}{llllll}\text { Roy } \ldots & \ldots & \ldots & \ldots & 1845\end{array}$

$\begin{array}{lllll}\text { Stroh } & \ldots & \ldots & \ldots & 1845\end{array}$

$\begin{array}{lllll}\text { Verheyen } & \ldots & \ldots & \ldots & 1845\end{array}$

Rollo Springfield [ $\ell$ seud] $\quad \ldots \quad 1846$

Dupont $\quad \ldots \quad \ldots \quad \ldots \quad 1846$

Hunersdorff... $\quad \ldots \quad \ldots .1846$

$\begin{array}{llllll}\text { Merlen } \quad \ldots & \ldots & \ldots & 1846\end{array}$

Thomas $\quad \ldots \quad \ldots \quad \ldots \quad 1846$

$\begin{array}{lllll}\text { Klemm } & \ldots & \ldots & \ldots & 1847\end{array}$

$\begin{array}{lllll}\text { Louchard } & \ldots & \ldots & \ldots & 1847\end{array}$ 
EQUITATION, BREAKING, TAMING AND STABLE MANAGEMENT.-Continued.

\begin{tabular}{|c|c|c|c|c|c|c|c|c|c|}
\hline Rivet & $\ldots$ & $\cdots$ & $\ldots$ & 1847 & Guerin & $\ldots$ & $\ldots$ & ... & 1851 \\
\hline Brindley & $\ldots$ & $\ldots$ & ... & 1848 & Hassell & $\ldots$ & $\ldots$ & ... & 1851 \\
\hline Curnien & $\ldots$ & $\ldots$ & ... & 1848 & Herstatt & $\ldots$ & .. & $\ldots$ & 1851 \\
\hline John & $\ldots$ & $\ldots$ & $\cdots$ & 1848 & Hertwig & $\ldots$ & $\ldots$ & ... & 1851 \\
\hline Kitchener & $\ldots$ & $\ldots$ & $\ldots$ & 1848 & Montigny & $\ldots$ & $\ldots$ & ... & 851 \\
\hline Kressenstein & & ... & ... & 1848 & Oeynhausen & & .. & $\ldots$ & 85 \\
\hline Raabe & $\ldots$ & $\ldots$ & $\ldots$ & 1848 & Turner & $\ldots$ & $\ldots$ & $\ldots$ & 85. \\
\hline Richardson & ... & ... & $\ldots$ & 1848 & Book of Aid & & $\ldots$ & $\ldots$ & 85 \\
\hline Seeger & ... & ... & ... & 1848 & Instruction & ... & $\ldots$ & $\ldots$ & 85 \\
\hline Cours d'Equ & uitation & $\ldots$ & $\ldots$ & 1849 & Principes $d$ & 'Equ & tion & $\ldots$ & 35 \\
\hline Handbook & $\cdots$ & $\cdots$ & $\cdots$ & 1849 & D'Abzac & $\ldots$ & $\cdots$ & $\ldots$ & 85 \\
\hline The Groom & $\cdots$ & $\cdots$ & $\cdots$ & 1849 & D'Aure & $\ldots$ & $\ldots$ & $\ldots$ & 85 \\
\hline Instruction & $\cdots$ & $\cdots$ & $\cdots$ & $\begin{array}{l}1849 \\
1849\end{array}$ & Bally & $\ldots$ & $\ldots$ & ... & 85 \\
\hline Falke & $\cdots$ & $\cdots$ & $\cdots$ & $\begin{array}{l}1849 \\
1819\end{array}$ & Brindley & $\ldots$ & $\ldots$ & $\ldots$ & 185 \\
\hline Leyon & $\cdots$ & $\cdots$ & $\begin{array}{l}\cdots \\
\ldots\end{array}$ & $\begin{array}{l}1019 \\
1849\end{array}$ & Ellerbrock & $\ldots$ & ... & $\ldots$ & 85 \\
\hline Lotze & $\cdots$ & $\cdots$ & $\begin{array}{l}\cdots \\
\ldots\end{array}$ & $\begin{array}{l}1049 \\
1849\end{array}$ & Hamm & $\ldots$ & $\ldots$ & $\ldots$ & 185 \\
\hline Oeynhausen & & $\cdots$ & $\begin{array}{l}\cdots \\
\ldots\end{array}$ & 1849 & Heinrichs & $\ldots$ & $\cdots$ & $\ldots$ & 85 \\
\hline Tantaloup & $\cdots$ & $\ddot{\text { tment }}$ & $\begin{array}{l}\cdots \\
\ldots\end{array}$ & 1850 & Henry & $\cdots$ & $\ldots$ & $\ldots$ & 185 \\
\hline $\begin{array}{l}\text { Directions } f \\
\text { Guide de l'in }\end{array}$ & $\begin{array}{l}\text { for Trea } \\
\text { instructe }\end{array}$ & etment & $\cdots$ & 1850 & Kappel & $\ldots$ & $\cdots$ & $\cdots$ & 185 \\
\hline Brindley & $\ldots$ & $\ldots$ & ... & 1850 & Marwitz & $\ldots$ & .. & $\ldots$ & 185 \\
\hline Comparini & $\ldots$ & ... & ... & 1850 & Noel & $\ldots$ & $\cdots$ & $\ldots$ & $185^{\circ}$ \\
\hline Falke & ... & ... & $\ldots$ & 1850 & Nolan & ... & $\ldots$ & $\ldots$ & 185 \\
\hline Haycock & $\cdots$ & ... & $\ldots$ & 1850 & Richardson & $\ldots$ & $\cdots$ & $\ldots$ & 1852 \\
\hline Hermann & $\ldots$ & ... & $\ldots$ & 1850 & Saint-Ange & $\ldots$ & $\cdots$ & $\ldots$ & 185 \\
\hline Herstatt & $\cdots$ & $\cdots$ & $\cdots$ & 1850 & Schmid & ... & $\cdots$ & $\ldots$ & 185 \\
\hline Hochstetter & & ... & ... & 1850 & Seeger & ... & $\cdots$ & $\ldots$ & 1852 \\
\hline Montigny & $\cdots$ & ... & ... & 1850 & Tongue & ... & ... & $\cdots$ & 185 \\
\hline Oaks & $\cdots$ & ... & ... & 1850 & Wiseman & ... & ... & $\ldots$ & 185 \\
\hline Seeger & $\ldots$ & $\ldots$ & $\cdots$ & 1850 & \multicolumn{3}{|c|}{ Handbook for Ryttaren } & $\ldots$ & 1853 \\
\hline Wahlgren & ... & ... & $\ldots$ & 1850 & Ellerbrock & $\ldots$ & ... & $\ldots$ & 1853 \\
\hline C. Gr. v E. & $\ldots$. & ... & ... & 1851 & Flandrin & $\ldots$ & $\ldots$ & $\ldots$ & 185 \\
\hline
\end{tabular}




\section{EQUITATION, BREAKING, TAMING AND STABLE} MANAGEMENT.-Continued.

\begin{tabular}{llllllllll} 
Frïs & $\ldots$ & $\ldots$ & $\ldots$ & 1853 & Quillinan & $\ldots$ & $\ldots$ & $\ldots$ & 1854 \\
Gherardi & $\ldots$ & $\ldots$ & $\ldots$ & 1853 & Raabe & $\ldots$ & $\ldots$ & $\ldots$ & 1854 \\
Graefe & $\ldots$ & $\ldots$ & $\ldots$ & 1853 & Rarey & $\ldots$ & $\ldots$ & $\ldots$ & 1854 \\
Heinze & $\ldots$ & $\ldots$ & $\ldots$ & 1853 & Auch ein Wort & $\ldots$ & $\ldots$ & 1854 \\
Houël & $\ldots$ & $\ldots$ & $\ldots$ & 1853 & Die Behandlung & $\ldots$ & $\ldots$ & 1855 \\
Knoll & $\ldots$ & $\ldots$ & $\ldots$ & 1853 & Instruction for & Ryttaren & $\ldots$ & 1855 \\
Darrac & $\ldots$ & $\ldots$ & $\ldots$ & 1853 & D'Aure & $\ldots$ & $\ldots$ & $\ldots$ & 1855 \\
Lamotte & $\ldots$ & $\ldots$ & $\ldots$ & 1853 & Bulletin & $\ldots$ & $\ldots$ & $\ldots$ & 1855 \\
Magne & $\ldots$ & $\ldots$ & $\ldots$ & 1853 & Curnieu & $\ldots$ & $\ldots$ & $\ldots$ & 1855 \\
Mazoiller & $\ldots$ & $\ldots$ & $\ldots$ & 1853 & Diepenbrock & $\ldots$ & $\ldots$ & 1855 \\
Minot & $\ldots$ & $\ldots$ & $\ldots$ & 1853 & Franconi & $\ldots$ & $\ldots$ & $\ldots$ & 1855 \\
Montigny & $\ldots$ & $\ldots$ & $\ldots$ & 1853 & Graefe & $\ldots$ & $\ldots$ & $\ldots$ & 1855 \\
Pinto & $\ldots$ & $\ldots$ & $\ldots$ & 1853 & Grange & $\ldots$ & $\ldots$ & $\ldots$ & 1855 \\
Richardson & $\ldots$ & $\ldots$ & $\ldots$ & 1853 & Guide de l'ami du & Cheval & $\ldots$ & 1855 \\
Rochefort & $\ldots$ & $\ldots$ & $\ldots$ & 1853 & Du Hays & $\ldots$ & $\ldots$ & $\ldots$ & 1855 \\
The Equestrian & $\ldots$ & $\ldots$ & 1854 & Horloch & $\ldots$ & $\ldots$ & $\ldots$ & 1855 \\
Ladies Equestrian & Guide & $\ldots$ & 1854 & Isabelle & $\ldots$ & $\ldots$ & $\ldots$ & 1855 \\
Young Ladies & Equestrian & & & Kruge & $\ldots$ & $\ldots$ & $\ldots$ & 1855 \\
\multicolumn{1}{l}{ Manual } & $\ldots$ & $\ldots$ & $\ldots$ & 1854 & Montigny & $\ldots$ & $\ldots$ & $\ldots$ & 1855 \\
Martels & $\ldots$ & $\ldots$ & $\ldots$ & 1854 & Noel & $\ldots$ & $\ldots$ & $\ldots$ & 1855 \\
Baucher & $\ldots$ & $\ldots$ & $\ldots$ & 1854 & Petterson & $\ldots$ & $\ldots$ & $\ldots$ & 1855 \\
Brubn & $\ldots$ & $\ldots$ & $\ldots$ & 1854 & Prosch & $\ldots$ & $\ldots$ & $\ldots$ & 1855 \\
Bujault & $\ldots$ & $\ldots$ & $\ldots$ & 1854 & Rueff & $\ldots$ & $\ldots$ & $\ldots$ & 1855 \\
Dandel & $\ldots$ & $\ldots$ & $\ldots$ & 1854 & Segundo & $\ldots$ & $\ldots$ & $\ldots$ & 1855 \\
Daumas & $\ldots$ & $\ldots$ & $\ldots$ & 1854 & Warde & $\ldots$ & $\ldots$ & $\ldots$ & 1855 \\
Ehrhardt & $\ldots$ & $\ldots$ & $\ldots$ & 1854 & Den enfarne.. & $\ldots$ & $\ldots$ & 1855 \\
Falke & $\ldots$ & $\ldots$ & $\ldots$ & 1853 & Sichere Anleitung & $\ldots$ & $\ldots$ & 1856 \\
Flandrin & $\ldots$ & $\ldots$ & $\ldots$ & 1854 & L'Amazone & $\ldots$ & $\ldots$ & $\ldots$ & 1856 \\
Mussot & $\ldots$ & $\ldots$ & $\ldots$ & 1854 & Elpons & $\ldots$ & $\ldots$ & $\ldots$ & 1856 \\
Nadosy & $\ldots$ & $\ldots$ & $\ldots$ & 1854 & De Porquet .. & $\ldots$ & $\ldots$ & 1856 \\
Noel & $\ldots$ & $\ldots$ & $\ldots$ & 1854 & Fernandez y Perez & $\ldots$ & $\ldots$ & 1856 \\
Papin & $\ldots$ & $\ldots$ & $\ldots$ & 1854 & Krane & $\ldots$ & $\ldots$ & $\ldots$ & 1856
\end{tabular}




\section{EQUITATION, BREAKING, TAMING AND STABLE}

\section{MANAGEMENT.-Continued.}

\begin{tabular}{|c|c|c|c|c|c|c|c|c|c|}
\hline Mirus & $\cdots$ & $\cdots$ & $\cdots$ & 1856 & Kruge & $\ldots$ & $\cdots$ & $\cdots$ & 1859 \\
\hline Monteton & .. & $\ldots$ & ... & 1856 & Raabe and $]$ & Lunel & $\ldots$ & $\ldots$ & 1859 \\
\hline Mussot & $\cdots$ & $\ldots$ & ... & 1856 & Rufener & $\ldots$ & $\ldots$ & $\ldots$ & 1859 \\
\hline Noel & ... & $\ldots$ & $\cdots$ & 1856 & Horses and & Roads & .. & $\ldots$ & 86 \\
\hline A. v. P. & $\cdots$ & $\cdots$ & $\cdots$ & 1856 & Eicke & ... & $\cdots$ & $\ldots$ & 年 \\
\hline Raabe & $\cdots$ & $\ldots$ & $\cdots$ & 1856 & Guérin & ... & $\ldots$ & $\ldots$ & 10 \\
\hline Walsh & $\cdots$ & $\cdots$ & $\cdots$ & 1856 & Kruge & $\ldots$ & $\ldots$ & $\ldots$ & 86 \\
\hline \multicolumn{3}{|c|}{ Horse Owner's Guide } & $\cdots$ & 1857 & Lachaume & $\ldots$ & $\ldots$ & $\ldots$ & o \\
\hline \multicolumn{2}{|c|}{ Petit Manual } & $\ldots$ & $\cdots$ & 1857 & Lancosme $\mathrm{B}$ & Breves & ... & $\ldots$ & \\
\hline \multicolumn{3}{|c|}{ Praktische Anleitung } & $\cdots$ & 1857 & Malfrain & ... & $\cdots$ & $\ldots$ & 80 \\
\hline \multicolumn{3}{|c|}{ Mrs. Sterling Clark } & $\ldots$ & 1857 & \multicolumn{4}{|c|}{ El Memorial de Caballeria... } & 86 \\
\hline \multicolumn{2}{|c|}{ Ferrer de Conto } & $\ldots$ & $\cdots$ & 1857 & \multicolumn{3}{|c|}{ Equitation et dressage } & $\ldots$ & . \\
\hline Daudel & $\ldots$ & .. & $\ldots$ & 1857 & Butler & $\ldots$ & ... & $\ldots$ & 186 \\
\hline De Porque & $\ldots$ & $\ldots$ & $\ldots$ & 1857 & Botticher & $\ldots$ & ... & $\ldots$ & 186 \\
\hline Gayot & $\cdots$ & $\ldots$ & $\cdots$ & 1857 & Canitz & $\ldots$ & $\cdots$ & $\ldots$ & 186 \\
\hline Herbert & $\cdots$ & $\cdots$ & $\ldots$ & 1857 & Martin & $\ldots$ & $\cdots$ &.. & 186 \\
\hline Levi & $\ldots$ & $\ldots$ & $\ldots$ & 1857 & Moll and G & Xayot & $\cdots$ & $\ldots$ & 186 \\
\hline Liasse & $\cdots$ & $\ldots$ & $\cdots$ & 1857 & Pellier & $\ldots$ & $\cdots$ & $\ldots$ & 186 \\
\hline Perrault & $\ldots$ & $\ldots$ & ... & 1857 & Ricquet & $\ldots$ & $\ldots$ & $\cdots$ & 186 \\
\hline Rul & ... & $\ldots$ & $\ldots$ & 1857 & Rol & $\ldots$ & ... & $\cdots$ & 186 \\
\hline Swoboda & $\ldots$ & ... & $\cdots$ & ] 857 & Von Trotta & $\ldots$ & $\ldots$ & $\ldots$ & 186 \\
\hline \multicolumn{2}{|c|}{ Rules for lieeping } & $\cdots$ & $\cdots$ & 1858 & Gerhardt & $\ldots$ & $\ldots$ & $\ldots$ & 186 \\
\hline \multicolumn{2}{|c|}{ Bentwright ... } & $\ldots$ & $\ldots$ & 1858 & Gilsa & ... & $\ldots$ & $\ldots$ & 186 \\
\hline King & $\cdots$ & $\ldots$ & $\ldots$ & 1858 & Taylor & $\ldots$ & $\ldots$ & $\cdots$ & 186 \\
\hline Taylor & $\ldots$ & $\ldots$ & $\ldots$ & 1858 & Wachter & $\ldots$ & $\ldots$ & $\therefore$ & 186 \\
\hline Teuierès & $\ldots$ & $\ldots$ & $\cdots$ & 1858 & Blätter fur & \multicolumn{2}{|c|}{ Pferdezicht } & $\ldots$ & 186 \\
\hline Rous & $\ldots$ & $\cdots$ & $\cdots$ & 1858 & Heinze & $\cdots$ & $\cdots$ & $\ldots$ & 186 \\
\hline \multicolumn{2}{|c|}{ Die Zahmung } & $\ldots$ & $\ldots$ & 1859 & Illiers & $\ldots$ & $\ldots$ & $\ldots$ & 186 \\
\hline Dunbar & $\ldots$ & $\ldots$ & $\ldots$ & 1859 & Raabe & $\ldots$ & $\cdots$ & $\cdots$ & 186 \\
\hline Gerhardt & $\ldots$ & $\cdots$ & $\cdots$ & 1859 & Seidler & $\cdots$ & $\cdots$ & $\cdots$ & 186 \\
\hline Herbert & . & $\ldots$ & $\ldots$ & 1859 & Walsh & $\cdots$ & ... & $\ldots$ & 186 \\
\hline De Vere $\mathbf{H}$ & unt & $\ldots$ & ... & 1859 & De l'equitat & ione & $\cdots$. & $\cdots$ & 186 \\
\hline
\end{tabular}


EQUITATION, BREAKING, TAMING AND STABLE MANAGEMENT.-Continued.

\begin{tabular}{|c|c|c|c|c|c|c|c|c|c|}
\hline Instruction & $\cdots$ & $\cdots$ & $\ldots$ & 1864 & Strombeck & .. & $\ldots$ & ... & 1867 \\
\hline Méthode & ... & ... & $\ldots$ & 1864 & Vial ... & $\cdots$ & $\ldots$ & $\ldots$ & 1867 \\
\hline Morin & $\ldots$ & ... & $\ldots$ & 1864 & \multicolumn{3}{|c|}{ Villeroy and Müller } & $\ldots$ & 1867 \\
\hline Gamgee & $\cdots$ & ... & $\ldots$ & 1865 & \multicolumn{2}{|c|}{ Abhandlung } & $\ldots$ & $\ldots$ & 1869 \\
\hline Gerhardt & ... & ... & $\ldots$ & 1865 & \multicolumn{2}{|c|}{ Gedanken-Reiter } & $\ldots$ & $\ldots$ & 1868 \\
\hline Leyon & $\ldots$ & ... & $\ldots$ & 1865 & Dwyer & $\ldots$ & ... & $\ldots$ & 1868 \\
\hline Loiselet & ... & ... & $\ldots$ & 1865 & Foelan & ... & ... & $\ldots{ }^{\prime}$ & 1868 \\
\hline Magenta ( $I$ & Pseud.) & ... & $\ldots$ & 1865 & Hoecker & ... & - & $\ldots$ & 1868 \\
\hline Montigny & ... & $\ldots$ & $\ldots$ & 1865 & Lindblad & $\cdots$ & $\ldots$ & $\ldots$ & 1868 \\
\hline Oeynhausen & & ... & $\ldots$ & 1865 & Moeder & ... & $\ldots$ & $\ldots$ & 1868 \\
\hline Rabenen & ... & ... & $\ldots$ & 1865 & Siegmann & $\ldots$ & $\ldots$ & $\ldots$ & 1868 \\
\hline Willisen & $\cdots$ & $\ldots$ & $\ldots$ & 1865 & \multicolumn{2}{|c|}{ Book of Aids } & $\ldots$ & $\ldots$ & 1866 \\
\hline Anon. & $\ldots$ & $\cdots$ & $\cdots$ & 1866 & Armatage & ... & $\cdots$ & $\ldots$ & 1869 \\
\hline Nouvel essar & & ... & $\cdots$ & 1866 & Assézat & $\cdots$ & ... & $\ldots$ & 1869 \\
\hline Dominick & ... & $\ldots$ & $\ldots$ & 1866 & Gerhardt & $\cdots$ & $\cdots$ & $\ldots$ & 1869 \\
\hline Fortwängler & & $\ldots$ & $\ldots$ & 1866 & Jullian & ... & ... & $\ldots$ & 1869 \\
\hline Hochwächte & & $\ldots$ & $\ldots$ & 1866 & Oeynhausen & $\ldots$ & ... & $\ldots$ & 1869 \\
\hline Jennings & ... & ... & $\ldots$ & 1866 & Phillipps & $\ldots$ & ... & $\ldots$ & 1869 \\
\hline Löffler & .. & ... & $\ldots$ & 1866 & Trosckke & ... & ... & $\ldots$ & 1869 \\
\hline Van der $\mathrm{Me}$ & & ... & $\ldots$ & 1866 & \multicolumn{5}{|c|}{ Taschenbuck fur Pferdelieb- } \\
\hline Peillard & $\ldots$ & $\ldots$ & $\ldots$ & 1866 & haber & $\ldots$ & $\ldots$ & $\ldots$ & 1870 \\
\hline Kannstatt & $\ldots$ & ... & $\ldots$ & 1866 & Besser & $\ldots$ & $\ldots$ & $\ldots$ & 1870 \\
\hline Schönberg & .. & ... & $\ldots$ & 1866 & Bismarck-B & ohlen & $\ldots$ & $\cdots$ & 1870 \\
\hline Reiter Geda & anken & ... & $\cdots$ & 1867 & Chassaigne & $\cdots$ & $\cdots$ & $\cdots$ & 1870 \\
\hline Dachenhaus & sen & $\ldots$ & $\cdots$ & 1867 & Colomb & $\cdots$ & $\cdots$ & $\ldots$ & 1870 \\
\hline Glasenapp & ... & ... & $\cdots$ & 1867 & F. v. K. & ... & ... & $\ldots$ & 1870 \\
\hline Henriques & ... & $\ldots$ & $\cdots$ & 1867 & Raabe & $\ldots$ & ... & $\ldots$ & 1870 \\
\hline Hochwachte & & $\ldots$ & $\ldots$ & 1867 & Rul ... & $\cdots$ & $\cdots$ & $\cdots$ & 1870 \\
\hline Krane & $\cdots$ & ... & $\cdots$ & 1867 & Horses & $\cdots$ & $\cdots$ & $\cdots$ & 1871 \\
\hline Lutzow & ... & $\cdots$ & $\ldots$ & 1867 & \multicolumn{3}{|c|}{ Die zukunftige deutsche } & $\cdots$ & 1871 \\
\hline Newte & ... & ... & $\ldots$ & 1867 & Desclée & $\cdots$ & $\cdots$ & $\ldots$ & 1871 \\
\hline Seinsheim & $\cdots$ & $\cdots$ & $\cdots$ & 1867 & Dilg & ... & $\cdots$ & $\ldots$ & 1871 \\
\hline Sjostedt & $\ldots$ & $\ldots$ & $\ldots$ & 1867 & Elderkin & $\ldots$ & $\ldots$ & $\ldots$ & 1871 \\
\hline
\end{tabular}


INDEX TO SUBJECTS.

EQUITATION, BREAKING, TAMING AND STABLE MANAGEMENT._Continued.

\begin{tabular}{|c|c|c|c|c|c|c|c|c|c|}
\hline Riedheim & .. & $\ldots$ & $\ldots$ & 1871 & Münster & $\ldots$ &.. & $\ldots$ & 1874 \\
\hline Anleitung & $\ldots$ & $\ldots$ & $\ldots$ & 1872 & Pellier & $\ldots$ & $\ldots$ & $\ldots$ & 1874 \\
\hline \multicolumn{4}{|c|}{ Horse-Owner's Companion ... } & 1872 & Petricie & $\ldots$ & $\ldots$ & .. & 1874 \\
\hline \multicolumn{3}{|c|}{ Betrachtungen } & $\ldots$ & 1872 & Sidney & $\ldots$ & $\ldots$ & $\ldots$ & 874 \\
\hline \multicolumn{3}{|c|}{ Anteitung zum Ertheilen } & $\ldots$ & 1872 & Zundel & $\ldots$ & $\ldots$ & $\ldots$ & 874 \\
\hline \multicolumn{3}{|c|}{ Bonie $\quad \ldots$} & $\ldots$ & 1872 & Burdelot & $\ldots$ & $\ldots$ & $\ldots$ & 1875 \\
\hline \multicolumn{2}{|c|}{ H. de la F. ... } & $\cdots$ & $\ldots$ & 1872 & Dutilh & $\ldots$ & $\ldots$ & $\ldots$ & 1875 \\
\hline Jennings & & ... & $\ldots$ & 1872 & Hayes & $\ldots$ & $\ldots$ & $\ldots$ & 1875 \\
\hline \multirow{2}{*}{$\begin{array}{l}\text { Kästner } \\
\text { Powell }\end{array}$} & $\ldots$ & ... & $\ldots$ & 1872 & Mansuy & $\ldots$ & $\ldots$ & $\ldots$ & 1875 \\
\hline & $\ldots$ & $\cdots$ & $\ldots$ & 1872 & \multicolumn{3}{|c|}{ Muhlwerth-Gartner } & $\ldots$ & 1875 \\
\hline \multicolumn{2}{|l|}{ Schoenbeck } & $\cdots$ & $\cdots$ & 1872 & \multicolumn{2}{|c|}{ Pelet-Narbonne } & $\ldots$ & .. & 1875 \\
\hline Seidler & $\ldots$ & ... & $\ldots$ & 1872 & Worz & $\ldots$ & $\ldots$ & $\ldots$ & 1876 \\
\hline \multirow{2}{*}{$\begin{array}{l}\text { Széchényi } \\
\text { Waldschmi }\end{array}$} & $\ldots$ & $\ldots$ & $\ldots$ & 1872 & Arnim & $\ldots$ & $\ldots$ & $\ldots$ & 1875 \\
\hline & & $\ldots$ & $\ldots$ & 1872 & Brucher & $\ldots$ & . & $\ldots$ & 1875 \\
\hline \multicolumn{3}{|c|}{ Corvisart-Montmarin } & $\ldots$ & 1873 & \multicolumn{4}{|c|}{ Oesterruchische Zeitschrift... } & 1876 \\
\hline Debost & $\ldots$ & $\ldots$ & $\ldots$ & 1873 & Schönbeck & $\ldots$ & ... & $\ldots$ & 1876 \\
\hline \multicolumn{5}{|c|}{ Görgey von Görge, Toporez, } & Dallmann & $\ldots$ & $\ldots$ & $\ldots$ & 1877 \\
\hline \multicolumn{2}{|c|}{ and Bauer } & $\ldots$ & $\ldots$ & 1873 & Keller & $\cdots$ & $\ldots$ & $\ldots$ & 1877 \\
\hline Joseph & $\ldots$ & $\ldots$ & $\ldots$ & 1873 & Monteton & $\ldots$ & $\ldots$ & $\ldots$ & 1877 \\
\hline Du Teil & $\ldots$ & $\ldots$ & $\ldots$ & 1873 & Moreton & $\ldots$ & ... & $\ldots$ & 1877 \\
\hline \multicolumn{2}{|c|}{ Oeynhausen... } & $\cdots$ & $\cdots$ & 1873 & Neville & $\ldots$ & $\ldots$ & $\ldots$ & 1877 \\
\hline Weber & $\ldots$ & .. & $\ldots$ & 1873 & Ramm & ... & $\ldots$ & $\ldots$ & 1877 \\
\hline Willisen & $\ldots$ & $\ldots$ & $\ldots$ & 1873 & Trautvette & $\ldots$ & $\ldots$ & $\ldots$ & 1877 \\
\hline Praktish & $\ldots$ & ... & $\cdots$ & 1874 & Anleitung & $\ldots$ & $\ldots$ & $\ldots$ & 1878 \\
\hline \multicolumn{2}{|c|}{ Hippologische } & ... & ... & 1874 & Wegueiser & $a u f$ & $\ldots$ & $\ldots$ & 1878 \\
\hline \multicolumn{2}{|c|}{ The Barb and the } & Bridle & $\ldots$ & 1874 & \multicolumn{3}{|c|}{ Colomb (Appendix) } & $\ldots$ & 1878 \\
\hline Beaupré & $\ldots$ & $\ldots$ & $\ldots$ & 1874 & Durant & $\ldots$ & $\ldots$ & $\ldots$ & 1878 \\
\hline \multicolumn{3}{|c|}{ Corvisart Montmarin } & $\cdots$ & 1874 & Hardy & $\ldots$ & $\ldots$ & $\cdots$ & 1878 \\
\hline Davis & $\ldots$ & ... & $\ldots$ & 1874 & Der Lasa & $\ldots$ & $\ldots$ & $\ldots$ & 1878 \\
\hline Edwards & $\ldots$ & $\ldots$ & ... & 1874 & C. $\mathrm{M}$. & $\ldots$ & $\ldots$ & $\ldots$ & 1878 \\
\hline Lagondie & $\ldots$ & ... & ... & 1874 & Rudorff & $\ldots$ & $\ldots$ & $\ldots$ & 1878 \\
\hline Lindqvist & $\ldots$ & ... & $\ldots$ & 1874 & Grundsätz & $\ldots$ & $\ldots$ & $\ldots$ & 1879 \\
\hline Mercier & $\ldots$ & ... & ... & 1874 & Leitfaden & & ... & $\ldots$ & 1879 \\
\hline
\end{tabular}


EQUITATION, BREAKING, TAMING AND STABLE MANAGEMENT.-Continued.

\begin{tabular}{|c|c|c|c|c|c|c|c|c|c|}
\hline \multirow{2}{*}{\multicolumn{2}{|c|}{$\begin{array}{l}\text { Alten } \quad \ldots . \\
\text { Born and Möller }\end{array}$}} & \multirow{2}{*}{$\begin{array}{l}\cdots \\
\cdots\end{array}$} & \multirow{2}{*}{$\begin{array}{l}\cdots \\
\cdots\end{array}$} & \multirow{2}{*}{$\begin{array}{l}1879 \\
1879\end{array}$} & \multicolumn{3}{|c|}{ How to outwit the horse } & $\ldots$ & 1883 \\
\hline & & & & & \multicolumn{5}{|c|}{ Zeitschrift für Pferdekunde } \\
\hline Fearnley & ... & $\ldots$ & .. & 1879 & Anderson & $\cdots$ & ... & & 883 \\
\hline Herbin & $\ldots$ & $\ldots$ & .. & 1879 & d'Etreillis & ... & ... & & 883 \\
\hline Herstatt & $\ldots$ & $\ldots$ & $\ldots$ & 1879 & Lehnert & $\ldots$ & $\ldots$ & & 883 \\
\hline Illustrirte 2 & Blatter & $\ldots$ & .. & 1879 & Miinster & $\ldots$ & $\ldots$ & & 883 \\
\hline Pasquier & $\cdots$ & $\cdots$ & ... & 1879 & Pinel & $\ldots$ & ... & & 883 \\
\hline Petzer & $\ldots$ & .. & ... & 1879 & Sample & ... & $\ldots$ & & 883 \\
\hline \multicolumn{2}{|c|}{ The Horsekeeper } & .. & ... & 1880 & Schoenbeck & & ... & & 883 \\
\hline Daniel & $\ldots$ & $\ldots$ & ... & 1880 & Barauski & ... & $\ldots$ & & 884 \\
\hline Gaume & ... & ... & ... & 1880 & Deseler & $\ldots$ & $\ldots$ & & 884 \\
\hline Lalanne & $\ldots$ & ... & ... & 1880 & Hédouville & $\cdots$ & ... & & 884 \\
\hline Musani & $\ldots$ & $\ldots$ & ... & 1880 & Der Lasa & .. & ... & & $88 t$ \\
\hline O'Donaghu & & $\ldots$ & ... & 1880 & Karr & $\cdots$ & $\cdots$ & & 1881 \\
\hline \multicolumn{2}{|c|}{ The Horse \& how to } & $\operatorname{man}$ & him & 1881 & Poly & $\cdots$ & $\cdots$ & & 884 \\
\hline Anderson & $\ldots$ & $\ldots$ & ... & 1881 & Schlaberg & $\ldots$ & $\ldots$ & & 884 \\
\hline Ang & $\ldots$ & $\ldots$ & ... & 1881 & Weisskopf & $\ldots$ & ... & & 1884 \\
\hline Von Arnim & $\ldots$ & $\ldots$ & ... & 1881 & New Book o. & $f S_{l}$ & & & 885 \\
\hline Delisser & $\ldots$ & $\ldots$ & $\ldots$ & 1881 & Illustrated & Sta & $M a$ & & 885 \\
\hline Doux & $\cdots$ & $\ldots$ & ... & 1881 & Brand & ... & ... & & 1885 \\
\hline Hayes & $\ldots$ & $\ldots$ & ... & 1881 & Castroverde & & $\ldots$ & & 885 \\
\hline \multicolumn{2}{|c|}{ O'Donoghue } & $\ldots$ & .. & 1881 & Hayes & $\cdots$ & ... & & 1885 \\
\hline Pigouche & $\ldots$ & $\ldots$ & ... & 1881 & Montigny & $\ldots$ & .. & & 1885 \\
\hline Stillman & $\ldots$ & $\ldots$ & ... & 1881 & O'Donoghure & & $\ldots$ & & 885 \\
\hline \multicolumn{2}{|c|}{ Stud Groom } & $\ldots$ & ... & 1882 & Saunders & . & ... & & 1885 \\
\hline Anderson & .. & $\ldots$ & ... & 1882 & Schmidt & .. & $\ldots$ & & 1885 \\
\hline G. $\quad \ldots$ & $\ldots$ & $\ldots$ & $\ldots$ & 1882 & Steinbrecht & & ... & & 88 \\
\hline Heatley & $\ldots$ & .. & $\ldots$ & 1882 & Dressage du & $u C h$ & & & 188 \\
\hline Meyrick & $\ldots$ & $\ldots$ & .. & 1882 & Anderson & $\ldots$ & ... & & 1886 \\
\hline Montigny & $\ldots$ & $\ldots$ & ... & 1882 & Brun Renau & & $\ldots$ & & 188 \\
\hline Moreton & $\ldots$ & $\ldots$ & $\ldots$ & 1882 & Court & $\ldots$ & $\cdots$ & & 188 \\
\hline Plinzner & $\ldots$ & $\ldots$ & $\ldots$ & 1882 & Montigny & ... & .. & & \\
\hline roctor & $\ldots$ & $\ldots$ & $\ldots$ & 1882 & Santini & $\ldots$ & $\ldots$ & & 18 \\
\hline
\end{tabular}




\section{RACING.}

\begin{tabular}{|c|c|c|c|c|c|c|c|c|c|}
\hline Markham & $\ldots$ & $\ldots$ & $\ldots$ & 1596 & Walker & $\ldots$ & .. & $\ldots$ & 1834 \\
\hline Markham & $\ldots$ & $\ldots$ & $\ldots$ & 1599 & Anon. & ... & $\ldots$ & $\cdots$ & 1836 \\
\hline Anon. & $\ldots$ & $\ldots$ & $\ldots$ & 1688 & Hookham & ... & $\ldots$ & $\ldots$ & 1836 \\
\hline Anon. & $\ldots$ & $\cdots$ & $\ldots$ & 1709 & Apperley & $\ldots$ & $\ldots$ & $\ldots$ & 1837 \\
\hline Cheny & $\ldots$ & $\ldots$ & $\ldots$ & 1729 & Weatherby & $\ldots$ & $\ldots$ & $\ldots$ & 1837 \\
\hline Heber & $\ldots$ & $\ldots$ & $\ldots$ & 1752 & Darvill & $\ldots$ & $\ldots$ & ... & 1838 \\
\hline Pond & $\ldots$ & $\ldots$ & $\ldots$ & 1753 & Anon. & $\ldots$ & $\ldots$ & $\ldots$ & 1840 \\
\hline Roberts & $\ldots$ & $\ldots$ & $\ldots$ & 1760 & American 1 & Turf & Register & $\ldots$ & 1840 \\
\hline Anon. & $\ldots$ & $\ldots$ & $\ldots$ & 1764 & Blaine & $\ldots$ & $\ldots$ & $\ldots$ & 1840 \\
\hline Tuting and & Fawcon & & $\ldots$ & 1770 & Clark & $\ldots$ & $\ldots$ & $\ldots$ & 1840 \\
\hline Walker & $\ldots$ & $\ldots$ & $\ldots$ & 1770 & Whyte & $\ldots$ & $\ldots$ & $\ldots$ & 1840 \\
\hline Anon. & $\ldots$ & $\ldots$ & $\ldots$ & 1771 & Bryon & . & $\ldots$ & $\ldots$ & 1841 \\
\hline Ansell & $\ldots$ & $\ldots$ & $\ldots$ & 1784 & Anon. & $\ldots$ & $\ldots$ & $\ldots$ & 1842 \\
\hline Pick & $\ldots$ & .. & $\ldots$ & 1785 & Ruff & $\ldots$ & $\ldots$ & $\ldots$ & 1842 \\
\hline Sharkey & $\ldots$ & $\ldots$ & $\ldots$ & 1790 & Houël & $\ldots$ & $\ldots$ & $\ldots$ & 1843 \\
\hline Pouloti & $\ldots$ & $\ldots$ & $\ldots$ & 1791 & Orton & $\ldots$ & $\ldots$ & $\ldots$ & 1844 \\
\hline Saint-Bel & $\ldots$ & $\ldots$ & $\ldots$ & 1791 & Rouse & ... & $\ldots$ & $\ldots$ & 1844 \\
\hline Chifney & $\ldots$ & $\ldots$ & $\ldots$ & 1800 & Tattersall & $\ldots$ & $\ldots$ & $\ldots$ & 1844 \\
\hline Riem & $\ldots$ & $\ldots$ & $\ldots$ & 1802 & Corbet & $\ldots$ & $\ldots$ & $\ldots$ & 1845 \\
\hline Pick & $\ldots$ & $\ldots$ & $\ldots$ & 1803 & Montendre & $\ldots$ & $\ldots$ & $\ldots$ & 1845 \\
\hline Taplin & $\ldots$ & $\ldots$ & $\ldots$ & 1803 & Brown & $\ldots$ & $\ldots$ & $\ldots$ & 1846 \\
\hline Tennecker & $\ldots$ & $\ldots$ & $\ldots$ & 1803 & Dow & $\ldots$ & $\ldots$ & $\ldots$ & 1846 \\
\hline Chifney & $\ldots$ & $\ldots$ & $\ldots$ & 1804 & Calendrier & $\ldots$ & $\ldots$ & $\ldots$ & 1848 \\
\hline Gard & $\ldots$ & $\ldots$ & $\ldots$ & 1809 & Vogler & $\ldots$ & $\ldots$ & $\ldots$ & 1848 \\
\hline Lawrence & $\ldots$ & $\ldots$ & $\ldots$ & 1809 & Osborne & $\ldots$ & $\ldots$ & $\ldots$ & 1849 \\
\hline Morland & $\ldots$ & $\ldots$ & $\ldots$ & 1811 & Courses de & Che & $a u x$ & $\ldots$ & 1850 \\
\hline Epsom & $\ldots$ & $\ldots$ & $\ldots$ & 1825 & Manuel de & es & Tour'ses & $d e$ & \\
\hline Anon. & $\ldots$ & $\ldots$ & $\ldots$ & 1825 & Chevaux & .. & $\ldots$ & $\ldots$ & 1850 \\
\hline Sinith & $\ldots$ & $\ldots$ & $\ldots$ & 1825 & Rous & $\ldots$ & & $\ldots$ & 1850 \\
\hline Burgsdorf & $\ldots$ & $\ldots$ & $\ldots$ & 1827 & Tattersall & $\ldots$ & $\ldots$ & $\ldots$ & 1850 \\
\hline Brown & $\ldots$ & $\ldots$ & $\ldots$ & 1829 & Bally & $\ldots$ & $\ldots$ & $\ldots$ & 1852 \\
\hline Chuteau & $\ldots$ & $\ldots$ & $\ldots$ & 1834 & Rodloff & $\ldots$ & $\ldots$ & $\ldots$ & 185 \\
\hline
\end{tabular}


RACING.-Continued.

\begin{tabular}{|c|c|c|c|c|c|c|c|c|c|}
\hline Tongue & $\ldots$ & $\ldots$ & $\ldots$ & 1852 & \multicolumn{3}{|c|}{ Almanach du Sport } & & 1867 \\
\hline Chapus & ... & ... & ... & 1853 & Gemeiner & .. & .. & .. & 1867 \\
\hline Collins & ... & ... & $\cdots$ & 1853 & Mirabal & ... & ... & $\cdots$ & 1867 \\
\hline Bandow & $\ldots$ & $\ldots$ & ... & 1854 & Paz. & $\ldots$ & ... & ... & 1867 \\
\hline Gayot & ... & $\ldots$ & $\cdots$ & 1854 & Pichat & ... & .. & . & 1867 \\
\hline Regolament & & $\ldots \cdot$ & ... & 1855 & Canter & $\cdots$ & ... & . & 1868 \\
\hline Graefo & ... & .. & $\cdots$ & 1855 & \multicolumn{2}{|c|}{ Pferderennen } & ... & ... & 1869 \\
\hline D’Orval & $\cdots$ & .. & $\ldots$ & 1855 & \multicolumn{2}{|c|}{ Reglement für } & $\ldots$ & . & 1869 \\
\hline Rose & .. & ... & $\ldots$ & 1855 & \multicolumn{2}{|c|}{ Trotting Record } & $\cdots$ & 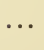 & 870 \\
\hline Dixon & ... & ... & $\ldots$ & 1855 & \multicolumn{2}{|c|}{ Central Blatt } & ... & $\cdots$ & 870 \\
\hline Robinson & $\ldots$ & $\ldots$ & $\ldots$ & 1856 & \multicolumn{2}{|c|}{ Lefevre $\quad \ldots$} & .. & $\ldots$ & 1871 \\
\hline De Walles & .. & $\ldots$. & $\cdots$ & 1856 & \multirow{2}{*}{\multicolumn{2}{|c|}{$\begin{array}{l}\text { Reglement } \\
\text { d'Etreillis }\end{array}$}} & .. & $\ldots$ & 1872 \\
\hline Calendrier & officiel & ... & $\ldots$ & 1857 & & & $\ldots$ & . & 872 \\
\hline Les Courses & de Cha & ntilly & $\ldots$ & 1857 & \multicolumn{2}{|c|}{ Die bedentendsten } & .. & ... & 1873 \\
\hline Herbert & $\cdots$ & ‥ & $\cdots$ & 1857 & \multicolumn{2}{|c|}{ d'Etreillis $\quad \ldots$} & $\cdots$ & $\ldots$ & 1873 \\
\hline Le Jockey & $C l u b$ & $\ldots$ & $\cdots$ & 1857 & \multicolumn{2}{|l|}{$\begin{array}{l}\text { Upton } \\
\text { Woodruff }\end{array}$} & $\cdots$ & .. & 1873 \\
\hline Miliken & $\ldots$ & $\cdots$ & $\cdots$ & 1857 & & $\cdots$ & $\cdots$ & .. & 1874 \\
\hline Robinson & $\cdots$ & $\cdots$ & $\cdots$ & 1858 & \multirow{2}{*}{$\begin{array}{l}\text { Der Turf } \\
\text { Lehndorff }\end{array}$} & ... & $\cdots$ & ... & 1876 \\
\hline Robinson & $\ldots$ & ... & ... & 1861 & & $\cdots$ & $\cdots$ & .. & 1876 \\
\hline Guide du $p$ & arienr & ... & ... & 1863 & \multicolumn{3}{|c|}{ Snap [pseud.] (Appendix) } & 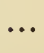 & 1876 \\
\hline Horse Raci & & .. & $\cdots$ & 1863 & HeIm & .. & $\cdots$ & ... & 1878 \\
\hline Desvaux & $\ldots$ & $\ldots$ & ... & 1863 & \multicolumn{4}{|c|}{ Oesterreichisch-ungarischer } & 1879 \\
\hline Du Hays & $\ldots$ & .. & ... & 1863 & \multicolumn{3}{|c|}{ Illustriste Sport-Zeitung } & .. & 1879 \\
\hline Du Hays & $\ldots$ & ... & ... & 1864 & \multicolumn{3}{|c|}{ Karst-Karstenverth } & ... & 1879 \\
\hline Parent & $\ldots$ & $\cdots$ & $\cdots$ & 1864 & Day & $\ldots$ & $\ldots$ & .. & 1880 \\
\hline Broye & $\ldots$ & ... & $\cdots$ & 1865 & Bruce & ... & $\ldots$ & $\cdots$ & 1881 \\
\hline Chapus & $\ldots$ & ... & ... & 1865 & Roussel & $\cdots$ & $\ldots$ & ... & 1881 \\
\hline Collins & $\ldots$ & $\ldots$ & ... & 1865 & \multicolumn{2}{|c|}{ Racing Analysis } & $\cdots$ & ". & 1882 \\
\hline Copperthwa & aite & $\cdots:$ & $\ldots$ & 1865 & \multicolumn{3}{|c|}{ Thormanby [pseud.] } & $\cdots$ & 1882 \\
\hline Demazy & $\ldots$ & ... & ... & 1865 & \multirow{2}{*}{\multicolumn{3}{|c|}{$\begin{array}{lll}\text { Hayes } & \ldots & \ldots \\
\text { Types of the Turf } & \ldots .\end{array}$}} & $\cdots$ & 1882 \\
\hline Gesetz für & $\cdots$ & $\cdots$ & $\cdots$ & 1866 & & & $\cdots$ & $\cdots$ & 1883 \\
\hline Our great $A$ & Amer & $n H$ & & 1867 & Silberer & $\ldots$ & $\ldots$ & ... & 1883 \\
\hline
\end{tabular}


RACING.-Continued.

$\begin{array}{llllllll}\text { Silberer and Ernst ... } & \ldots & 1883 & \text { Humfrey } & \ldots & \ldots & \ldots & 1885\end{array}$

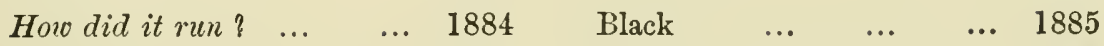

Famous Horses of America $1884 \quad$ Earl of Suffolk and Berkshire 1886

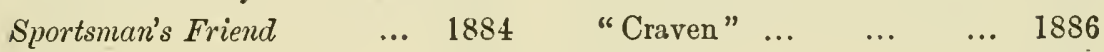

$\begin{array}{llllllllll}\text { Glover } & \ldots & \ldots & \ldots & 1884 & \text { Lawley } & \ldots & \ldots & \ldots & 1886\end{array}$ $\begin{array}{lllllllllll}\text { Silberer } \quad \ldots & \ldots & \ldots & 1894 & \text { Coventry } & \ldots & \ldots & \ldots & 1886\end{array}$

Lunar Month Summary $\quad \ldots \quad 1885 \quad$ Watson $\quad \ldots \quad \ldots \quad \ldots \quad \ldots \quad 1886$ Hore $\quad \ldots \quad \ldots \quad \ldots l 1885$

\section{THE HORSE'S FOOT, SHOES \& SHOEING.}

$\begin{array}{lllll}\text { Esteves } \quad \ldots & \ldots & \ldots & 1425\end{array}$

$\begin{array}{lllll}\text { Fiaschi } \quad \ldots & \ldots & \ldots & 1556\end{array}$

$\begin{array}{lllll}\text { Blundeville ... } & \ldots & \ldots & 1565\end{array}$

$\begin{array}{lllll}\text { Calvo } & \ldots & \ldots & \ldots & 1582\end{array}$

Clifford $\quad \ldots \quad \ldots \quad \ldots \quad 1585$

Panciroli $\quad \ldots \quad$... $\quad \ldots \quad 1599$

$\begin{array}{lllll}\text { Markham } & \ldots & \ldots & \ldots & 1615\end{array}$

$\begin{array}{lllll}\text { Markham } & \ldots & \ldots & \ldots & 1656\end{array}$

Ud-din Ahmad $\ldots . \quad \ldots \quad 1660$

Almond $\ldots$... ... 1673

$\begin{array}{llllll}\text { Andrade } \quad \ldots & \ldots & \ldots & 1678\end{array}$

$\begin{array}{lllll}\text { Ludeña } \quad \ldots & \ldots & \ldots & 1694\end{array}$

Sampayo _.. $\quad \ldots \quad \ldots \quad 1710$

$\begin{array}{llllll}\text { Gibson } & \ldots & \ldots & \ldots & 1720\end{array}$

Allen $\quad \ldots \quad \ldots \quad \ldots \quad 1737$

Bridges $\quad \ldots \quad \ldots \quad \ldots \quad 1752$

$\begin{array}{llllll}\text { Lafosse } \quad \ldots & \ldots & \ldots & 1754\end{array}$

Kersting $\quad \ldots \quad \ldots \quad \ldots \quad 1760$

$\begin{array}{llllll}\text { Wallis } \quad \ldots & \ldots & \ldots & 1764\end{array}$

$\begin{array}{llllll}\text { Lafosse } \quad \ldots & \ldots & \ldots & 1766\end{array}$

$\begin{array}{lllll}\text { Sind } & \ldots & \ldots & \ldots & 1776\end{array}$

Clark
Bourgelat ...

Wallmerode...

Beckmann ...

Rumpelt ...

Lorentz

Oehlmann ...

Saint Bel

Freeman

Lawrence

Coleman

Coleman

Moorcroft

Vierordt

K. F. B.

Lawrence

Lehman

White

Snape

Binz

Clark

Clark

Clark

\section{... $\quad \ldots \quad 1771$}

$\begin{array}{lll}\ldots & \ldots & 1779\end{array}$

$\ldots \quad \ldots \quad 1780$

$\begin{array}{lll}\ldots & \ldots & 1786\end{array}$

.. 1788

.. 1789

.. 1793

... 1796

... 1796

.. 1798

... 1800

... 1800

.. 1800

... 1801

... 1801

.. 1801

.. 1801

.. 1805

.. 1807

.. 1809

.. 1810

.. 1810 
HORSE'S FOOT, SHOES AND SHOEING.-Continued.

\begin{tabular}{llllllllll}
\multicolumn{2}{l}{ Langenbacher } & $\ldots$ & $\ldots$ & 1811 & Lupke & $\ldots$ & $\ldots$ & $\ldots$ & 1828 \\
Clark & $\ldots$ & $\ldots$ & $\ldots$ & 1813 & Niisken & $\ldots$ & $\ldots$ & $\ldots$ & 1828 \\
Greve & $\ldots$ & $\ldots$ & $\ldots$ & 1814 & Rieneckcr & $\ldots$ & $\ldots$ & $\ldots$ & 1828 \\
Powis & $\ldots$ & $\ldots$ & $\ldots$ & 1814 & Bach & $\ldots$ & $\ldots$ & $\ldots$ & 1829 \\
Schwab & $\ldots$ & $\ldots$ & $\ldots$ & 1814 & Clark & $\ldots$ & $\ldots$ & $\ldots$ & 1829 \\
Schwab & $\ldots$ & $\ldots$ & $\ldots$ & 1815 & Clark & $\ldots$ & $\ldots$ & $\ldots$ & 1821 \\
Budd & $\ldots$ & $\ldots$ & $\ldots$ & 1816 & Anon. & $\ldots$ & $\ldots$ & $\ldots$ & 1832 \\
Tennecker & $\ldots$ & $\ldots$ & $\ldots$ & 1816 & Clark & $\ldots$ & $\ldots$ & $\ldots$ & 1832 \\
Wagner & $\ldots$ & $\ldots$ & $\ldots$ & 1816 & Muller & $\ldots$ & $\ldots$ & $\ldots$ & 1832 \\
Clark & $\ldots$ & $\ldots$ & $\ldots$ & 1818 & Anon. & $\ldots$ & $\ldots$ & $\ldots$ & 1834 \\
Kegel & $\ldots$ & $\ldots$ & $\ldots$ & 1819 & Hartung & $\ldots$ & $\ldots$ & $\ldots$ & 1834 \\
Clierry & $\ldots$ & $\ldots$ & $\ldots$ & 1820 & Vix & $\ldots$ & $\ldots$ & $\ldots$ & 1834 \\
Goodwin & $\ldots$ & $\ldots$ & $\ldots$ & 1820 & Rodet & $\ldots$ & $\ldots$ & $\ldots$ & 1840 \\
König & $\ldots$ & $\ldots$ & $\ldots$ & 1820 & Spooner & $\ldots$ & $\ldots$ & $\ldots$ & 1810 \\
Tennecker & $\ldots$ & $\ldots$ & $\ldots$ & 1820 & Zerrener & $\ldots$ & $\ldots$ & $\ldots$ & 1841 \\
Teast & $\ldots$ & $\ldots$ & $\ldots$ & 1821 & Cherry & $\ldots$ & $\ldots$ & $\ldots$ & 1842 \\
Tennecker & $\ldots$ & $\ldots$ & $\ldots$ & 1821 & Miles & $\ldots$ & $\ldots$ & $\ldots$ & 1845 \\
Tscheulin & $\ldots$ & $\ldots$ & $\ldots$ & 1821 & Berthelot & $\ldots$ & $\ldots$ & $\ldots$ & 1846 \\
Hoerdt & $\ldots$ & $\ldots$ & $\ldots$ & 1822 & Anon. & $\ldots$ & $\ldots$ & $\ldots$ & 1847 \\
Fusco & $\ldots$ & $\ldots$ & $\ldots$ & 1822 & Clarendon & $\ldots$ & $\ldots$ & $\ldots$ & 1847 \\
Tscheulin & $\ldots$ & $\ldots$ & $\ldots$ & 1822 & Falke & $\ldots$ & $\ldots$ & $\ldots$ & 1848 \\
Dieterichs & $\ldots$ & $\ldots$ & $\ldots$ & 1823 & Hartmann & $\ldots$ & $\ldots$ & $\ldots$ & 1848 \\
Clark & $\ldots$ & $\ldots$ & $\ldots$ & 1824 & Leyon & $\ldots$ & $\ldots$ & $\ldots$ & 1848 \\
Goodwin & $\ldots$ & $\ldots$ & $\ldots$ & 1824 & Hodgson & $\ldots$ & $\ldots$ & $\ldots$ & 1849 \\
Hodgson & $\ldots$ & $\ldots$ & $\ldots$ & 1824 & Shaw & $\ldots$ & $\ldots$ & $\ldots$ & 1849 \\
Clark & $\ldots$ & $\ldots$ & $\ldots$ & 1825 & Brogniez & $\ldots$ & $\ldots$ & $\ldots$ & 1850 \\
Anon. & $\ldots$ & $\ldots$ & $\ldots$ & 1826 & Gross & $\ldots$ & $\ldots$ & $\ldots$ & 1850 \\
Beck & $\ldots$ & $\ldots$ & $\ldots$ & 1826 & Haycock & $\ldots$ & $\ldots$ & $\ldots$ & 1850 \\
Kertitschka & $\ldots$ & $\ldots$ & $\ldots$ & 1827 & Jacquemin & $\ldots$ & $\ldots$ & $\ldots$ & 1850 \\
Lessona & $\ldots$ & $\ldots$ & $\ldots$ & 1827 & Knowlson & $\ldots$ & $\ldots$ & $\ldots$ & 1850 \\
Balassa & $\ldots$ & $\ldots$ & $\ldots$ & 1828 & Nuevo Manulal & $\ldots$ & $\ldots$ & 1851 \\
Klatte & $\ldots$ & $\ldots$ & $\ldots$ & 1828 & Bouley & $\ldots$ & $\ldots$ & $\ldots$ & 1851 \\
& & & & & & & & &
\end{tabular}


INDEX TO SUBJECTS.

HORSE'S FOOT, SHOES AND SHOEING.-Continued.

\begin{tabular}{|c|c|c|c|c|c|c|c|c|c|}
\hline \multirow{2}{*}{\multicolumn{3}{|c|}{$\begin{array}{l}\text { Dombale } \ldots \\
\text { Defays and Husson.... }\end{array}$}} & \multirow{2}{*}{\multicolumn{2}{|c|}{$\begin{array}{lr}\ldots & 1851 \\
\ldots & 1852\end{array}$}} & \multicolumn{2}{|c|}{ Instructions } & $\cdots$ & \multirow{2}{*}{$\begin{array}{l}\cdots \\
\cdots\end{array}$} & \multirow{2}{*}{$\begin{array}{l}1862 \\
1863\end{array}$} \\
\hline & & & & & Elluin & $\ldots$ & $\ldots$ & & \\
\hline Einsiedel & $\ldots$ & ... & $\ldots$ & 1852 & \multicolumn{2}{|c|}{ Guendeville... } & ... & $\ldots$ & 1863 \\
\hline Hamm & $\ldots$ & ... & $\ldots$ & 1852 & Miles & $\ldots$ & $\ldots$ & ... & 1863 \\
\hline Rey & $\ldots$ & $\ldots$ & $\ldots$ & 1852 & Mayhew & $\ldots$ & $\ldots$ & $\ldots$ & 1864 \\
\hline Rienicker & ... & ... & $\ldots$ & 1852 & Mégnin & $\ldots$ & $\ldots$ & $\ldots$ & 1864 \\
\hline Ellerbrock & $\ldots$ & $\ldots$ & $\ldots$ & 1853 & Mégnin & $\ldots$ & $\ldots$ & $\ldots$ & 1865 \\
\hline Flemming & ... & ... & $\ldots$ & 1853 & Tayleure & $\ldots$ & $\ldots$ & $\ldots$ & 1865 \\
\hline Fuchs & ... & ... & $\ldots$ & 1853 & Jennings & $\ldots$ & ... & $\ldots$ & 1866 \\
\hline Hoffmeister & ... & ... & $\ldots$ & 1853 & \multicolumn{3}{|c|}{ Lindqvist and Arrhenius } & $\ldots$ & 1866 \\
\hline Jessen & $\ldots$ & ... & .. & 1853 & Schmelz & $\ldots$ & $\ldots$ & $\ldots$ & 1866 \\
\hline Anker & ... & ... & $\ldots$ & 1854 & Ableitner & ... & $\cdots$ & $\ldots$ & 1867 \\
\hline Cuming & ... & ... & $\ldots$ & 1854 & Defays & ... & $\ldots$ & ... & 1867 \\
\hline Dadd & ... & ... & $\cdots$ & 1854 & Morin & ... & $\ldots$ & $\ldots$ & 1867 \\
\hline Casas & .. & $\ldots$ & $\ldots$ & 1855 & Renner & $\ldots$ & $\ldots$ & $\ldots$ & 1867 \\
\hline Dubourdieu & & ... & $\ldots$ & 1855 & Strombeck & ... & $\ldots$ & $\ldots$ & 1867 \\
\hline Pillwax & ... & ... & $\ldots$ & 1855 & Erdt & $\ldots$ & $\ldots$ & ...' & 1868 \\
\hline Prosser & $\ldots$ & ... & $\ldots$ & 1855 & Miles & ... & $\ldots$ & ... & 1868 \\
\hline Hitzinger & ... & ... & $\ldots$ & 1856 & San & $\ldots$ & $\ldots$ & $\cdots$ & 1868 \\
\hline Mussgnug & $\ldots$ & ... & $\ldots$ & 1856 & Starke & $\ldots$ & .. & $\ldots$ & 1868 \\
\hline Peters & $\ldots$ & $\ldots$ & $\ldots$ & 1856 & Fleming & $\ldots$ & $\ldots$ & $\ldots$ & 1869 \\
\hline \multicolumn{3}{|c|}{ Pralitische Anleitung } & $\ldots$ & 1857 & Goyau & $\ldots$ & $\ldots$ & $\ldots$ & 1869 \\
\hline Auzoux & ... & ... & $\ldots$ & 1857 & Dominick & $\ldots$ & $\ldots$ & $\ldots$ & 1870 \\
\hline Defays & ... & ... & $\therefore$ & 1857 & Hofling & .. & $\ldots$ & $\ldots$ & 1870 \\
\hline Defays & ... & $\ldots$ & $\ldots$ & 1857 & Gamgee & $\ldots$ & $\ldots$ & ... & 1871 \\
\hline Jessen & ... & $\ldots$ & $\ldots$ & 1857 & Einsiedel & $\ldots$ & $\ldots$ & $\ldots$ & 1872 \\
\hline Anon. & ... & $\ldots$ & $\ldots$ & 1857 & Fleming & $\ldots$ & $\ldots$ & $\ldots$ & 1872 \\
\hline \multicolumn{3}{|c|}{ Horse Shoer's Manual } & $\ldots$ & 1859 & Bendz & $\ldots$ & $\ldots$ & ... & 1873 \\
\hline Sainz & ... & ... & .. & 1859 & Douglas & .. & $\ldots$ & $\ldots$ & 1873 \\
\hline Turner & ... & ... & .. & 1860 & Murray & .. & .. & $\cdots$ & 1873 \\
\hline Fitzwygram & ... & ... & $\ldots$ & 1861 & Walther & ... & ... & $\ldots$ & 1874 \\
\hline Leisering an & $\mathrm{d} \mathrm{H}$ & $\operatorname{mann}$ & .... & 1861 & Der rechte & Vord & fuss & $\ldots$ & 1877 \\
\hline
\end{tabular}


HORSE'S FOOT, SHOES AND SHOEING.-Continued.

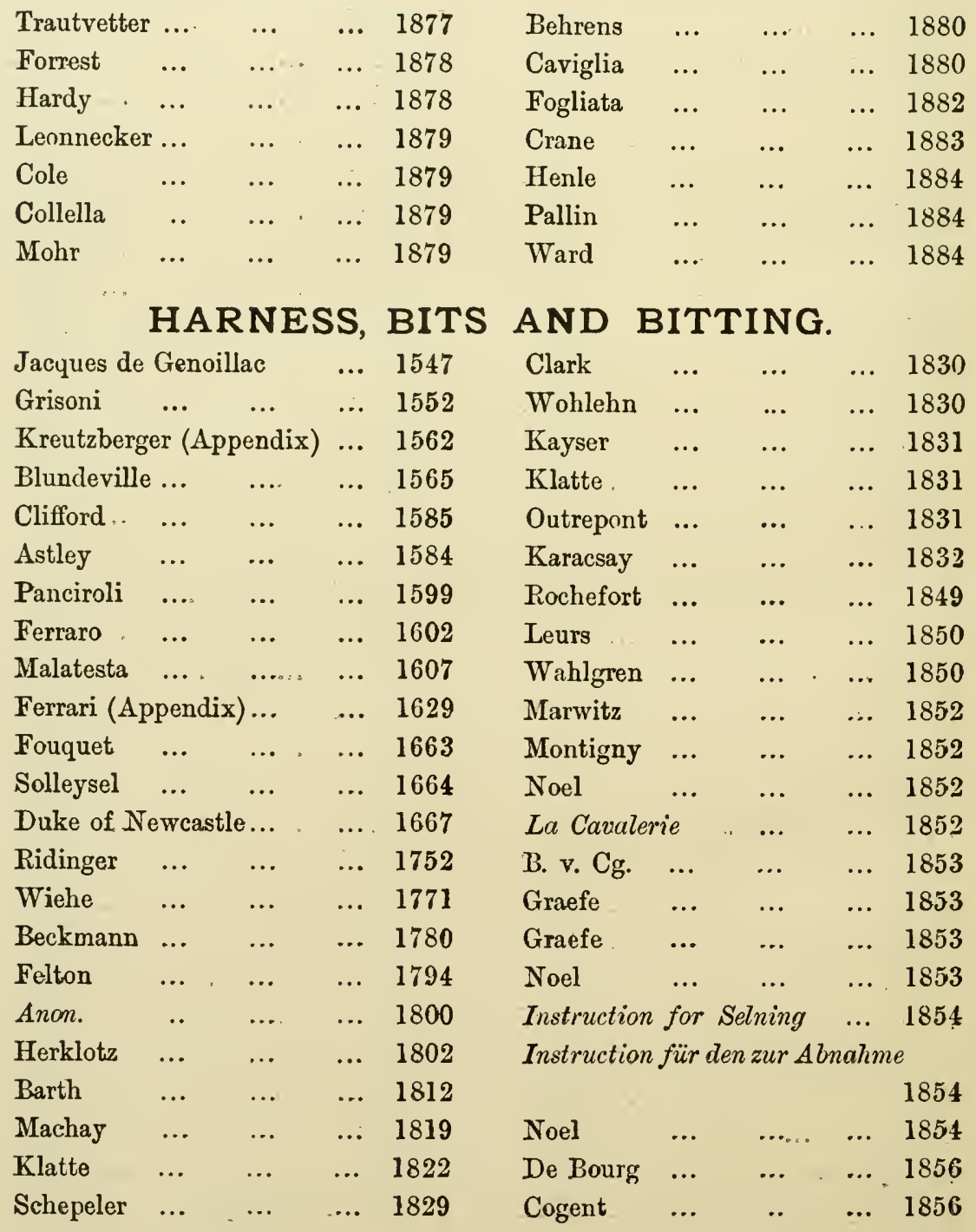


HARNESS, BITS AND BITTING.-Continued.

\begin{tabular}{lllllllllll} 
Guillon & $\ldots$ & $\ldots$ & $\ldots$ & 1856 & Smith & $\ldots$ & $\ldots$ & $\ldots$ & 1870 \\
Anginiard & $\ldots$ & $\ldots$ & $\ldots$ & 1857 & Duliège & $\ldots$ & $\ldots$ & $\ldots$ & 1871 \\
Barth & $\ldots$ & $\ldots$ & $\ldots$ & 1857 & Latchford & $\ldots$ & $\ldots$ & $\ldots$ & 1871 \\
\multicolumn{2}{l}{ Carriage } & Builders & \& Harness & & & Havis & $\ldots$ & $\ldots$ & $\ldots$ & 1872 \\
\multicolumn{2}{l}{ Makers' } & Art & Journal & $\ldots$ & 1859 & Description & détaillée & $\ldots$ & 1874 \\
Lebrun & $\ldots$ & $\ldots$ & $\ldots$ & 1860 & Instruction for & Collar & Makers & 1875 \\
Rufener & $\ldots$ & $\ldots$ & $\ldots$ & 1860 & Flower & $\ldots$ & $\ldots$ & $\ldots$ & 1875 \\
Cogent & $\ldots$ & $\ldots$ & $\ldots$ & 1863 & Flower & $\ldots$ & $\ldots$ & $\ldots$ & 1877 \\
Leurs & $\ldots$ & $\ldots$ & $\ldots$ & 1864 & Dieckerhoff & $\ldots$ & $\ldots$ & $\ldots$ & 1882 \\
Davis & $\ldots$ & $\ldots$ & $\ldots$ & 1867 & Philipson & $\ldots$ & $\ldots$ & $\ldots$ & 1883 \\
Dwyer & $\ldots$ & $\ldots$ & $\ldots$ & 1868 & Fitzgerald & $\ldots$ & $\ldots$ & $\ldots$ & 1884
\end{tabular}

\section{CARRIAGES, DRIVING \& COACHBUILDING.}

Giraldes $\quad \ldots \quad \ldots \quad \ldots \quad 1318$

Montaigne ... $\quad \ldots \quad \ldots \quad 1580$

A Proclamation $\quad \ldots \quad \ldots \quad 1660$

Delcampe $\ldots . \quad \ldots . \quad \ldots \quad 1664$

$\begin{array}{lllll}\text { Scheffer } \quad \ldots & \ldots & \ldots & 1671\end{array}$

$\begin{array}{lllll}\text { Cresset } & \ldots & \ldots & \ldots & 1672\end{array}$

Garsault . .. $\quad \ldots \quad \ldots \quad 1756$

Ridinger $\quad \ldots \quad \ldots \quad \ldots \quad 1760$

$\begin{array}{lllll}\text { Fabricy } \quad \ldots & \ldots & \ldots & 1764\end{array}$

Cavalcade ende Triumph-

Waghens (Appendix) ... 1770

Berenger ... ... $\quad \ldots \quad 1771$

$\begin{array}{lllll}\text { Jacob } \quad \ldots & \ldots & \ldots & 1773\end{array}$

$\begin{array}{llllll}\text { Anon. } & \ldots & \ldots & \ldots & 1790\end{array}$

Köllner $\quad \ldots \quad \ldots \quad \ldots \quad 1790$

Felton $\quad \ldots \quad \ldots \quad \ldots .1794$

$\begin{array}{lllll}\text { Tennecker } & \ldots & \ldots & \ldots & 1802\end{array}$ $\begin{array}{lllll}\text { Cumming } & \ldots & \ldots & \ldots & 1809\end{array}$

$\begin{array}{llllll}\text { Milton } & \ldots & \ldots & \ldots & 1810\end{array}$

$\begin{array}{lllll}\text { Jervis } \quad \ldots & \ldots & \ldots & 1827\end{array}$

$\begin{array}{lllll}\text { Fuller } \quad \ldots & \ldots & \ldots & 1828\end{array}$

$\begin{array}{lllll}\text { Markland } & \ldots & \ldots & \ldots & 1828\end{array}$

Hafftendorn $\quad \ldots \quad \ldots \quad 1832$

$\begin{array}{lllll}\text { Lebrun } \quad \ldots & \ldots & \ldots & 1833\end{array}$

Walker $\quad \ldots \quad \ldots \quad \ldots \quad 1834$

$\begin{array}{llllll}\text { Adams } \quad \ldots & \ldots & \ldots & 1837\end{array}$

Apperley $\quad \ldots \quad \ldots \quad \ldots \quad \ldots \quad 1837$

$\begin{array}{lllll}\text { Curnieu } \quad \ldots & \ldots & \ldots & 1848\end{array}$

$\begin{array}{lllll}\text { Rochefort } & \ldots & \ldots & \ldots & 1849\end{array}$

$\begin{array}{llllll}\text { Montigny } & \ldots & \ldots & \ldots & 1850\end{array}$

$\begin{array}{lllll}\text { Berthaux } & \ldots & \ldots & \ldots & 1852\end{array}$

Dementbon $\quad \ldots \quad \ldots \quad 1853$

$\begin{array}{lllll}\text { Anon. } \quad \ldots & \ldots & \ldots & 1854\end{array}$

$\begin{array}{llll}\text { Public Carriages } & \ldots & \ldots & 1855\end{array}$ 


\section{CARRIAGES, DRIVING \& COACHBUILDING.-Continued.}

\begin{tabular}{|c|c|c|c|c|c|c|c|c|c|}
\hline Berthaux & $\ldots$ & $\ldots$ & $\ldots$ & 1855 & Murray & $\ldots$ & $\ldots$ & $\ldots$ & 1873 \\
\hline Kruge & ... & $\ldots$ & ... & 1855 & Reynardson & ... & $\ldots$ & $\ldots$ & 1874 \\
\hline Der enfarne & & $\ldots$ & ... & 1856 & Brice Thoma & & $\cdots$ & .. & 1874 \\
\hline Charpentie & & $\ldots$ & $\ldots$ & 1856 & Heinze & .. & ... & $\ldots$ & 1876 \\
\hline Daunassans & $\ldots$ & $\ldots$ & $\ldots$ & 1856 & Hooper & $\ldots$ & ... & $\ldots$ & 1876 \\
\hline Guillon & $\ldots$ & $\ldots$ & $\ldots$ & 1856 & Lennox & ... & ... & $\ldots$ & 1876 \\
\hline Ramée & $\ldots$ & $\ldots$ & $\cdots$ & 1856 & Malet & ... & ... & $\ldots$ & 1876 \\
\hline Vuillermed & unand & $\ldots$ & .. & 1856 & Draft Book & $\ldots$ & .. & $\ldots$ & 1876 \\
\hline Witte & $\ldots$ & $\ldots$ & $\ldots$ & 1857 & Trautvetter & ... & ... & $\ldots$ & 1877 \\
\hline Carriage $B$ & uilder's & Journa & $\ldots$ & 1850 & Pokorny & ... & ... & $\ldots$ & 1878 \\
\hline Easton & $\ldots$ & $\ldots$ & $\ldots$ & 1859 & Stratton $\left(A_{p}\right.$ & ppendi & & $\ldots$ & 1878 \\
\hline Kruge & $\ldots$ & $\ldots$ & $\ldots$ & 1859 & The Centaur & $\cdots$ & ... & $\ldots$ & 1878 \\
\hline Arlot & $\ldots$ & $\ldots$ & $\ldots$ & 1860 & Hooper \& C & Jo. (Ap & pendix) & $\ldots$ & 1879 \\
\hline Burn & $\ldots$ & $\ldots$ & $\ldots$ & 1860 & Belvalette & ... & $\ldots$ & $\ldots$ & 1880 \\
\hline Robinson & $\ldots$ & $\ldots$ & $\ldots$ & 1861 & Coach Build & der's A & $r t$ Journ & $n a l$ & 1880 \\
\hline Walsh & $\ldots$ & $\ldots$ & $\ldots$ & 1863 & Gardner & $\ldots$ & $\ldots$ & $\ldots$ & 1880 \\
\hline André & $\ldots$ & $\ldots$ & $\ldots$ & 1864 & Annuaire de & la Ca & erro se & $e \ldots$ & 1881 \\
\hline Règlements & $\ldots$ & $\ldots$ & $\ldots$ & 1865 & Burgess & ... & ... & $\ldots$ & 1881 \\
\hline Magenta $[\ell$ & seud] & $\ldots$ & $\ldots$ & 1865 & Berdmore & $\ldots$ & ... & $\ldots$ & 1883 \\
\hline Wickram & $\ldots$ & $\ldots$ & $\ldots$ & 1865 & Leclerc & $\ldots$ & ... & $\ldots$ & 1883 \\
\hline Martin & $\ldots$ & $\ldots$ & $\ldots$ & 1866 & Mulliner & $\ldots$ & ... & $\ldots$ & 1883 \\
\hline Abhandlun & $g \ldots$ & $\ldots$ & $\ldots$ & 1868 & Fitzgerald & ... & $\ldots$ & $\ldots$ & 1884 \\
\hline Beaupré & $\ldots$ & $\ldots$ & $\ldots$ & 1868 & Hackney Ca & rrriage & Guardic & ian & 1884 \\
\hline Trainering & & $\ldots$ & $\ldots$ & 1869 & New Book of & f Sport & $t s \ldots$ & $\ldots$ & 1885 \\
\hline Thomas an & Gauste & ellier & $\ldots$ & 1869 & Montigny & $\cdots$ & $\ldots$ & $\ldots$ & 1885 \\
\hline Brice Thon & & $\ldots$ & $\ldots$ & 1870 & Philipson & $\ldots$ & $\cdots$ & $\ldots$ & 1885 \\
\hline The $H u b$ & $\ldots$ & ... & $\ldots$ & 1871 & Court & $\ldots$ & $\cdots$ & $\ldots$ & 1886 \\
\hline Haywood & $\ldots$ & $\ldots$ & $\ldots$ & 1873 & & & & & \\
\hline
\end{tabular}




\section{USES AFTER DEATH.}

$\begin{array}{llllllllll}\text { Villalóbos } & \ldots & \ldots & \ldots & 1605 & \text { Robinet } & \ldots & \ldots & \ldots & 1864 \\ \text { Kreutzer } & \ldots & \ldots & \ldots & 1818 & \text { Dufour } & \ldots & \ldots & \ldots & 1868 \\ \text { Davidis } & \ldots & \ldots & \ldots & 1848 & \text { Bourggoin } & \ldots & \ldots & \ldots & 1870 \\ \text { Henzte } & \ldots & \ldots & \ldots & 1848 & \text { Le Brun and } & \text { Maigne } & \ldots & 1872 \\ \text { Hoehning } & \ldots & \ldots & \ldots & 1848 & \text { Maurer } & \ldots & \ldots & \ldots & 1879 \\ \text { Decroix } & \ldots & \ldots & \ldots & 1852 & \text { Ward } & \ldots & \ldots & \ldots & 1884 \\ \text { Rutta } & \ldots & \ldots & \ldots & 1854 & \text { Decroix } & \ldots & \ldots & \ldots & 1885 \\ \text { Saint-Hilaire } & \ldots & \ldots & 1856 & & & & & \end{array}$

\section{LAWS RELATING TO WARRANTRY, ACTS, ETC.}

$\begin{array}{lccccllllll}\text { Act } & \ldots & \ldots & \ldots & 1648 & \text { Engelhart } & \ldots & \ldots & \ldots & 1834 \\ \text { Oliver Cromwell } & \ldots & \ldots & 1654 & \text { Leblanc } & \ldots & \ldots & \ldots & 1841 \\ \text { Proclanation } & \ldots & \ldots & 1660 & \text { Galisset and Mignon } & \ldots & 1842 \\ \text { Spilsbury } & \ldots & \ldots & \ldots & 1785 & \text { Heyde } & \ldots & \ldots & \ldots & 1847 \\ \text { Lucas } & \ldots & \ldots & \ldots & 1786 & \text { Oliphant } & \ldots & \ldots & \ldots & 1847 \\ \text { Plouquet } & \ldots & \ldots & \ldots & 1790 & \text { Thébaud } & \ldots & \ldots & \ldots & 1848 \\ \text { Miinter } & \ldots & \ldots & \ldots & 1791 & \text { Larendu } & \ldots & \ldots & \ldots & 1849 \\ \text { Stovin } & \ldots & \ldots & \ldots & 1794 & \text { J. P. A. } & \ldots & \ldots & \ldots & 1850 \\ \text { Lawrence } & \ldots & \ldots & \ldots & 1796 & \text { Campens } & \ldots & \ldots & \ldots & 1850 \\ \text { Buschendorf } & \ldots & \ldots & 1796 & \text { Rous } & \ldots & \ldots & \ldots & 1850 \\ \text { Boswell } & \ldots & \ldots & \ldots & 1798 & \text { Doll } & \ldots & \ldots & \ldots & 1851 \\ \text { Disney } & \ldots & \ldots & \ldots & 1806 & \text { Ellerbrock } & \ldots & \ldots & \ldots & 1852 \\ \text { Lawrence } & \ldots & \ldots & \ldots & 1809 & \text { Rueckert } & \ldots & \ldots & \ldots & 1852 \\ \text { Sander } & \ldots & \ldots & \ldots & 1810 & \text { Rueff } & \ldots & \ldots & \ldots & 1852 \\ \text { Laubender } & \ldots & \ldots & \ldots & 1816 & \text { Lecomte } & \ldots & \ldots & \ldots & 1854 \\ \text { Ueberacker } & \ldots & \ldots & \ldots & 1821 & \text { Peyrou } & \ldots & \ldots & \ldots & 1854 \\ \text { Tennecker } & \ldots & \ldots & \ldots & 1822 & \text { Renault } & \ldots & \ldots & \ldots & 1854 \\ \text { Laws } & \ldots & \ldots & \ldots & 1825 & \text { Kreutzer } & \ldots & \ldots & \ldots & 1855 \\ \text { Veith } & \ldots & \ldots & \ldots & 1826 & \text { Dejean } & \ldots & \ldots & \ldots & 1856 \\ \text { Stewart } & \ldots & \ldots & \ldots & 1831 & \text { Rossi } & \ldots & \ldots & \ldots & 1856 \\ \text { Surtees } & \ldots & \ldots & \ldots & 1831 & \text { Veterinar } & \text { Polizti } & \ldots & \ldots & 1857 \\ 5 \text { I } & & & & & & & & & \end{array}$


LAWS RELATING TO WARRANTRY, ACTS, Etc.-Continued

Handbnch der gerichtlichen

Thierheilkunde

Howden

Chouveau

Egan

$\begin{array}{lllll}\text { Merewether ... } & \ldots & \ldots & 1868\end{array}$

$\begin{array}{lllll}\text { Morrell } & \ldots & \ldots & \ldots & 1870\end{array}$

Rey
... 1862

... 1862

. 1863

.. 1867

... 1873
Hanover

Lascelles $\ldots$

$\begin{array}{lllll}\text { Fearnley } & \ldots & \ldots & \ldots & 1878\end{array}$

Dessart (Appendix)... $\quad \ldots \quad 1879$

Regolamento

Vallada

Bianchi

Peuch
... 1881

... 1881

... 1882

... 1884

MISCELLANEOUS.

$\begin{array}{lcccclllll}\text { Anon } & \ldots & \ldots & \ldots & 1577 & \text { Lawson } & \ldots & \ldots & \ldots & 1820 \\ \text { Maroccus Extaticus } & & \ldots & 1595 & \text { Anon. } & \ldots & \ldots & \ldots & 1823 \\ \text { Markham } & \ldots & \ldots & \ldots & 1607 & \text { Alken } & \ldots & \ldots & \ldots & 1824 \\ \text { Gallego } & \ldots & \ldots & \ldots & 1629 & \text { Humphreys } \ldots & \ldots & \ldots & 1824 \\ \text { Peacham } & \ldots & \ldots & \ldots & 1636 & \text { Beck } & \ldots & \ldots & \ldots & 1826 \\ \text { Anon. } & \ldots & \ldots & \ldots & 1643 & \text { Brown } & \ldots & \ldots & \ldots & 1830 \\ \text { Ud-din Ahmad } & \ldots & \ldots & 1660 & \text { Götz } & \ldots & \ldots & \ldots & 1830 \\ \text { Sampayo } & \ldots & \ldots & \ldots & 1710 & \text { Wuippermann } & \ldots & \ldots & 1832 \\ \text { Tull } & \ldots & \ldots & \ldots & 1739 & \text { Stephen } & \ldots & \ldots & \ldots & 1835 \\ \text { Starr } & \ldots & \ldots & \ldots & 1750 & \text { Apperley } & \ldots & \ldots & \ldots & 1837 \\ \text { Dr. Daniel } & \text { Dove } & \ldots & \ldots & 1757 & \text { Delafond } & \ldots & \ldots & \ldots & 1838 \\ \text { Benvenuti } & \ldots & \ldots & \ldots & 1760 & \text { Youatt } & \ldots & \ldots & \ldots & 1839 \\ \text { Dobrizhoffer } & \ldots & \ldots & 1784 & \text { Damoiseau } & \ldots & \ldots & \ldots & 1839 \\ \text { Marshall } & \ldots & \ldots & \ldots & 1787 & \text { Rivet } & \ldots & \ldots & \ldots & 1847 \\ \text { Anon. } & \ldots & \ldots & \ldots & 1792 & \text { Hubert } & \ldots & \ldots & \ldots & 1849 \\ \text { Mouray } & \ldots & \ldots & \ldots & 1796 & \text { Hey } & \ldots & \ldots & \ldots & 1850 \\ \text { Naumann } & \ldots & \ldots & \ldots & 1800 & \text { Saint-Albin } & & \ldots & \ldots & 1851 \\ \text { Anon. } & \ldots & \ldots & \ldots & 1804 & \text { Brindley } & \ldots & \ldots & \ldots & 1853 \\ \text { Taylor } & \ldots & \ldots & \ldots & 1808 & \text { Fuchs } & \ldots & \ldots & \ldots & 1854 \\ \text { P. J. B. N. } & \ldots & \ldots & \ldots & 1810 & \text { Vrolik } & \ldots & \ldots & \ldots & 1854 \\ \text { Clark } & \ldots & \ldots & \ldots & 1815 & \text { Das Dutzend } & \ldots & \ldots & 1855\end{array}$




\section{MISCELLANEOUS.-Continued.}

\begin{tabular}{|c|c|c|c|c|c|c|c|c|c|}
\hline An account & of & $\ldots$ & $\ldots$ & 1855 & Edwards & & ... & $\ldots$ & 1874 \\
\hline Brindley & $\ldots$ & $\ldots$ & $\ldots$ & 1856 & Kossen v. S & Sterne & & & 1874 \\
\hline Burgess & $\ldots$ & $\ldots$ & $\ldots$ & 1858 & Le Cirque $I$ & Frane & & 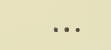 & 1875 \\
\hline Obituary $I$ & Totice & $\ldots$ & .. & 1860 & Frost & $\ldots$ & $\ldots$ & 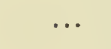 & 1875 \\
\hline Jannasch & $\ldots$ & $\ldots$ & $\ldots$ & 1861 & Pasquier & $\ldots$ & $\ldots$ & ... & 1875 \\
\hline Hamel & $\ldots$ & $\ldots$ & ... & 1862 & Bergeaud de & e Ver & uil & $\ldots$ & 1875 \\
\hline Mills & $\ldots$ & $\ldots$ & ... & 1863 & Rodenbough & & $\ldots$ & ... & 1876 \\
\hline Schrader & ... & $\ldots$ & $\cdots:$ & 1863 & Sewell & $\ldots$ & $\ldots$ & $\ldots$ & 1877 \\
\hline Benjamin & $\ldots$ & $\ldots$ & ... & 1864 & Sourdeval & $\ldots$ & $\ldots$ & $\ldots$ & 1880 \\
\hline Gaume & $\ldots$ & $\ldots$ & $\ldots$ & 1865 & Salvi & $\ldots$ & $\ldots$ & . & 1881 \\
\hline Du Hays & $\ldots$ & $\ldots$ & ... & 1865 & Diekerhoff & $\ldots$ & $\cdots$ & $\cdots$ & 1882 \\
\hline Gilmore & $\cdots$ & $\ldots$ & $\cdots$ & 1866 & Roche & $\ldots$ & $\ldots$ & $\ldots$ & 1882 \\
\hline Halliwell-P & hillips & $\ldots$ & ... & 1866 & Crafty $[p s e v$ & $u d]$. & $\ldots$ & ... & 1883 \\
\hline Recordon & $\ldots$ & $\ldots$ & $\ldots$ & 1866 & Confessions & of $a$ & Torse & Dealer & 1884 \\
\hline Jessen & $\ldots$ & $\ldots$ & ... & 1867 & The Tale of & $a H$ & & ... & 1884 \\
\hline Schlieden & $\ldots$ & $\ldots$ & $\ldots$ & 1867 & Willis & $\ldots$ & ... & ... & 1884 \\
\hline Remonte un & $A$ & ienta & & 1871 & Das Pferd & $\ldots$ & $\ldots$ & $\ldots$ & 1885 \\
\hline Goyau & $\ldots$ & $\ldots$ & ... & 1873 & Dodge & $\ldots$ & $\ldots$ & ... & 1885 \\
\hline Merres & $\ldots$ & $\ldots$ & $\ldots$ & 1873 & Thormanby & $\ldots$ & $\ldots$ & .. & 1885 \\
\hline Peeps into & . & ... & $\ldots$ & 1874 & Wood & $\ldots$ & $\ldots$ & ... & 1885 \\
\hline Die Preistr & äger & $\ldots$ & $\ldots$ & 1874 & Gerathewoh & & $\ldots$ & $\ldots$ & 1886 \\
\hline
\end{tabular}

\section{NATURAL HISTORY, ANATOMY, PHYSIOLOGY AND EXTERNAL FORM.}

Xenophon ...

Aristoteles ...

Varro

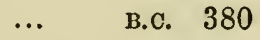

Columella ...

B.C. 37 ; A.D. 1472

Plinius

A.D. $20 ; \ldots 1472$

Cato

A.D. $50 ; \ldots 1469$

Palladius
... $\quad$.. 1472

... $\quad \therefore \quad 1472$
Kurrat ul-Mulk $\quad \ldots \quad \ldots \quad 1478$

$\begin{array}{lllll}\text { Perez } & \ldots & \ldots & \ldots & 1568\end{array}$

Anon $\quad \ldots \quad \ldots \quad \ldots \quad 1517$

Antonio de Guevary $\quad$.. 1529

Naaldwyck ... $\quad \ldots \quad \ldots \quad 1531$

Gesner $\quad \ldots \quad \ldots \quad \ldots \quad 1551$

Ferraro $\quad \ldots \quad \ldots \quad \ldots \quad 1560$ 
NATURAL HISTORY, ANATOMY, PHYSIOLOGY AND EXTERNAL FORM.-Continued.

$\begin{array}{lcccclllll}\text { Corte } & \ldots & \ldots & \ldots & 1573 & \text { Osmer } & \ldots & \ldots & \ldots & 1756 \\ \text { Stradan } & \ldots & \ldots & \ldots & 1578 & \text { Ridinger } & \ldots & \ldots & \ldots & 1761 \\ \text { Andrada } & \ldots & \ldots & \ldots & 1580 & \text { Anon } & \ldots & \ldots & \ldots & 1766 \\ \text { Montaigne } & \ldots & \ldots & \ldots & 1580 & \text { Stubbs } & \ldots & \ldots & \ldots & 1766 \\ \text { Calvo } & \ldots & \ldots & \ldots & 1582 & \text { Bourgelat } & \ldots & \ldots & \ldots & 1767 \\ \text { Ruini } & \ldots & \ldots & \ldots & 1598 & \text { Bourgelat } & \ldots & \ldots & \ldots & 1768 \\ \text { Panciroli } & \ldots & \ldots & \ldots & 1599 & \text { Pennant } & \ldots & \ldots & \ldots & 1768 \\ \text { Mellezes } & \ldots & \ldots & \ldots & 1600 & \text { Clark } & \ldots & \ldots & \ldots & 1770 \\ \text { Topsell } & \ldots & \ldots & \ldots & 1607 & \text { Berenger } & \ldots & \ldots & \ldots & 1771 \\ \text { Markham } & \ldots & \ldots & \ldots & 1611 & \text { Pennant } & \ldots & \ldots & \ldots & 1771 \\ \text { Aldrovandus } & \ldots & \ldots & 1616 & \text { Vitet } & \ldots & \ldots & \ldots & 1771 \\ \text { Machuca } & \ldots & \ldots & \ldots & 1621 & \text { Buchoz } & \ldots & \ldots & \ldots & 1772 \\ \text { Colombri } & \ldots & \ldots & \ldots & 1622 & \text { Tögl } & \ldots & \ldots & \ldots & 1774 \\ \text { Liberati } & \ldots & \ldots & \ldots & 1639 & \text { Ridinger } & \ldots & \ldots & \ldots & 1775 \\ \text { T.T.D.E.MI } \ldots & \ldots & \ldots & 1647 & \text { Dobrizhoffer } & \ldots & \ldots & 1781 \\ \text { Firuz Jang } & \ldots & \ldots & \ldots & 1650 & \text { Anon } & \ldots & \ldots & \ldots & 1785 \\ \text { Le Chambre } & \ldots & \ldots & 1657 & \text { Sa'datáyr Khan } & \ldots & \ldots & 1785 \\ \text { Ud-Din Ahmad } & \ldots & \ldots & 1660 & \text { Anon } & \ldots & \ldots & \ldots & 1786 \\ \text { Solleysel } & \ldots & \ldots & \ldots & 1664 & \text { Merrick } & \ldots & \ldots & \ldots & 1788 \\ \text { Liberati } & \ldots & \ldots & \ldots & 1669 & \text { Pomar } & \ldots & \ldots & \ldots & 1789 \\ \text { Pompee } & \ldots & \ldots & \ldots & 1669 & \text { Anon } & \ldots & \ldots & \ldots & 1790 \\ \text { Bryce } & \ldots & \ldots & \ldots & 1678 & \text { Bewick } & \ldots & \ldots & \ldots & 1790 \\ \text { Snape } & \ldots & \ldots & \ldots & 1683 & \text { Plon } & \ldots & \ldots & \ldots & 1790 \\ \text { Garzoni } & \ldots & \ldots & \ldots & 1692 & \text { Kollner } & \ldots & \ldots & \ldots & 1791 \\ \text { Muhammad } & \text { Ali } & \text { Hazin } & \ldots & 1716 & \text { Haveman } & \ldots & \ldots & \ldots & 1792 \\ \text { Gibson } & \ldots & \ldots & \ldots & 1720 & \text { Morland } & \ldots & \ldots & \ldots & 1792 \\ \text { Dandolo } & \ldots & \ldots & \ldots & 1722 & \text { Piggott } & \ldots & \ldots & \ldots & 1794 \\ \text { Saunier } & \ldots & \ldots & \ldots & 1734 & \text { Anon } & \ldots & \ldots & \ldots & 1796 \\ \text { Buffon } & \ldots & \ldots & \ldots & 1749 & \text { Freeman } & \ldots & \ldots & \ldots & 1796 \\ \text { Bridges } & \ldots & \ldots & \ldots & 1752 & \text { Fuss } & \ldots & \ldots & \ldots & 1797 \\ \text { Ridinger } & \ldots & \ldots & \ldots & 1752 & \text { Blaine } & \ldots & \ldots & \ldots & 1799 \\ \text { Eisenberg } & \ldots & \ldots & \ldots & 1753 & \text { Wopperer } & \ldots & \ldots & \ldots & 1799\end{array}$


INDEX TO SUBJECTS.

NATURAL HISTORY, ANATOMY, PHYSIOLOGY AND EXTERNAL FORM.-Continued.

\begin{tabular}{|c|c|c|c|c|c|c|c|c|c|}
\hline Klatte & $\cdots$ & $\cdots$ & $\ldots$ & 1800 & Clark & $\cdots$ & $\cdots$ & $\ldots$ & 1824 \\
\hline Lawrence & $\ldots$ & $\ldots$ & $\ldots$ & 1801 & Cros & $\ldots$ & $\ldots$ & $\ldots$ & 182 \\
\hline Bonsi & $\ldots$ & $\ldots$ & $\ldots$ & 1802 & Gurlt & $\cdots$ & $\ldots$ & $\ldots$ & \\
\hline Anon & $\ldots$ & $\ldots$ & $\ldots$ & 1803 & Miglio & ... & $\ldots$ & $\ldots$ & \\
\hline Engelhardt & $\ldots$ & $\ldots$ & $\ldots$ & 1803 & Steinhoff & ... & $\ldots$ & $\ldots$ & \\
\hline Politz & ... & ... & $\ldots$ & 1803 & Weber & $\ldots$ & $\ldots$ & $\ldots$ & \\
\hline Cline & ... & $\ldots$ & $\ldots$ & 1804 & Locatelli & ... & .. & $\ldots$ & \\
\hline Clark & $\ldots$ & $\cdots$ & $\ldots$ & 1805 & Revarchon & $\ldots$ & $\cdots$ & $\ldots$ & \\
\hline Burke & ... & $\ldots$ & $\ldots$ & 1806 & Brunot & $\ldots$ & $\ldots$ & $\ldots$ & \\
\hline Girard & $\ldots$ & $\ldots$ & $\ldots$ & 1807 & Jacquemin & $\ldots$ & $\ldots$ & $\ldots$ & \\
\hline Bingley & ... & $\ldots$ & $\ldots$ & 1809 & Rienecker & $\ldots$ & $\ldots$ & $\ldots$ & \\
\hline Lawrence & ... & $\ldots$ & ... & 1809 & Tennecker & $\ldots$ & ... & $\ldots$ & \\
\hline Schwab & $\ldots$ & $\ldots$ & $\ldots$ & 1809 & Rychner & $\ldots$ & $\ldots$ & $\ldots$ & 182 \\
\hline D'Alton & ... & $\ldots$ & ... & 1810 & Tennecker & $\ldots$ & $\ldots$ & $\ldots$ & \\
\hline P. J. B. N. & & $\ldots$ & ... & 1810 & Erdelyi & $\ldots$ & $\ldots$ & $\ldots$ & \\
\hline Brosche & $\ldots$ & $\ldots$ & $\ldots$ & 1812 & Percival & $\cdots$ & $\cdots$ & $\ldots$ & 18 \\
\hline Sebald & .. & $\ldots$ & $\ldots$ & 1812 & Biel & $\cdots$ & $\cdots$ & $\ldots$ & 83 \\
\hline Brosche & $\ldots$ & $\ldots$ & $\ldots$ & 1813 & Brown & $\ldots$ & $\ldots$ & $\ldots$ & 189 \\
\hline Clark & $\ldots$ & $\ldots$ & $\ldots$ & 1813 & The Farrier & and & Natural & ist & 183 \\
\hline Neergaard & $\ldots$ & $\cdots$ & $\ldots$ & 1814 & Brunot & $\ldots$ & ... & $\ldots$ & 183 \\
\hline Schwab & $\ldots$ & ... & $\ldots$ & 1815 & Kayser & ... & $\ldots$ & $\ldots$ & \\
\hline Sebald & ... & ... & ... & 1815 & Schwab & $\ldots$ & $\ldots$ & $\ldots$ & 183 \\
\hline Cuvier & $\ldots$ & $\ldots$ & $\ldots$ & 1816 & Stewart & $\ldots$ & $\cdots$ & $\ldots$ & 183 \\
\hline Anon & ... & $\ldots$ & ... & 1817 & Youatt & $\ldots$ & $\ldots$ & $\ldots$ & 183 \\
\hline Anon & ... & ... & ... & 1819 & Autenreith & ... & $\ldots$ & $\ldots$ & 183 \\
\hline Erdelyi & $\cdots$ & $\ldots$ & $\cdots$ & 1819 & Casas &.. & $\cdots$ & $\ldots$ & 183 \\
\hline Dieterrichs & $\ldots$ & $\ldots$ & $\ldots$ & 1820 & Gerber & $\ldots$ & $\cdots$ & $\ldots$ & 183 \\
\hline Donovan & $\cdots$ & $\ldots$ & ... & 1820 & Gurlt & $\cdots$ & $\ldots$ & $\ldots$ & 183 \\
\hline Erdelyi & $\ldots$ & $\ldots$ & $\ldots$ & 1820 & Jardine & $\ldots$ & $\ldots$ & .. & 183 \\
\hline Havemann & $\ldots$ & $\ldots$ & $\cdots$ & 1820 & Vogeli & $\ldots$ & $\ldots$ & $\ldots$ & 183 \\
\hline Pollnitz & $\ldots$ & $\cdots$ & $\ldots$ & 1820 & Balle & $\cdots$ & $\cdots$ & $\ldots$ & 18 \\
\hline Ribbe & $\ldots$ & $\ldots$ & ... & 1821 & Engelhart &.. & $\ldots$ & ... & 183 \\
\hline
\end{tabular}


NATURAL HISTORY, ANATOMY, PHYSIOLOGY AND EXTERNAL FORM.-Continued.

$\begin{array}{lllll}\text { Hormann } & \ldots & \ldots & \ldots & 1834\end{array}$

Dieterrich $\ldots \quad \ldots \quad \ldots \quad 1835$

Morris $\quad \ldots \quad \ldots \quad \ldots \quad 1835$

Anon

Bell

Bouley and Reynall...

Giinther

Gurlt

Saucerotte $\quad \ldots \quad \ldots \quad \ldots \quad 1837$

$\begin{array}{lllll}\text { Bingley } \quad \ldots & \ldots & \ldots & 1838\end{array}$

$\begin{array}{lllll}\text { Jenyns } & \ldots & \ldots & \ldots & 1838\end{array}$

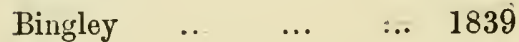

$\begin{array}{lllll}\text { Hess } & \ldots & \ldots & \ldots & 1839\end{array}$

$\begin{array}{lllll}\text { Pierquin } \quad \ldots & \ldots & \ldots & 1839\end{array}$

$\begin{array}{lllll}\text { Smith } & \ldots & \ldots & \ldots & 1839\end{array}$

Hermann $\ldots . \quad \ldots \quad \ldots \quad 1840$

Low

Martin

Merk

$\begin{array}{lllll}\text { Lavocat } \quad \ldots & \ldots & \ldots & 1840\end{array}$

$\begin{array}{lllll}\text { Steinhoff } & \ldots & \ldots & \ldots & 1840\end{array}$

$\begin{array}{llllll}\text { Anon } & \ldots & \ldots & \ldots & 1841\end{array}$

Kassàb and Ráfii $\ldots . \quad \ldots \quad 1841$

Fellenberg $\ldots \quad \ldots \quad \ldots \quad \ldots \quad 1841$

$\begin{array}{lllll}\text { Houël } & \ldots & \ldots & \ldots & 1841\end{array}$

$\begin{array}{lllll}\text { Mignon } & \ldots & \ldots & \ldots & 1841\end{array}$

$\begin{array}{lllll}\text { Neergard } & \ldots & \ldots & \ldots & 1841\end{array}$

$\begin{array}{lllll}\text { Person } & \ldots & \ldots & \ldots & 1841\end{array}$

$\begin{array}{lllll}\text { Roberts } \quad \ldots & \ldots & \ldots & 1841\end{array}$

Delafond and Andral $\quad \ldots \quad 1842$

$\begin{array}{lllll}\text { Voetsch. } & \ldots & \ldots & \ldots & 1842\end{array}$

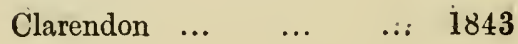

$\begin{array}{lllll}\text { Laziroules } & \ldots & \ldots & \ldots & 1843\end{array}$

$\begin{array}{lllll}\text { Lecoq } & \ldots & \ldots & \ldots & 1843\end{array}$

$\begin{array}{lllll}\text { Tscherning } & \ldots & \ldots & \ldots & 1843\end{array}$

$\begin{array}{lllll}\text { Brivet } & \ldots & \ldots & \ldots & 1844\end{array}$

$\begin{array}{lllll}\text { Pierquin } \quad \ldots & \ldots & \ldots & 1844\end{array}$

Baumeister ... $\quad \ldots \quad \ldots \quad 1845$

$\begin{array}{lllll}\text { Froriep } & \ldots & \ldots & \ldots & 1845\end{array}$

$\begin{array}{lllll}\text { Martin } \quad \ldots & \ldots & \ldots & 1845\end{array}$

Rollo Springfield [pseud.] ... 1846

$\begin{array}{lllll}\text { Graf } & \ldots & \ldots & \ldots & 1846\end{array}$

$\begin{array}{lllll}\text { Winter } & \ldots & \ldots & \ldots & 1846\end{array}$

$\begin{array}{lllll}\text { Casas } & \ldots & \ldots & \ldots & 1847\end{array}$

$\begin{array}{lllll}\text { Graf } & \ldots & \ldots & \ldots & 1847\end{array}$

$\begin{array}{lllll}\text { Louchard } & \ldots & \ldots & \ldots & 1847^{\circ}\end{array}$

$\begin{array}{lllll}\text { Richard } \quad \ldots & \ldots & \ldots & 1847\end{array}$

$\begin{array}{lllll}\text { Allen } & \ldots & \ldots & \ldots & 1848\end{array}$

$\begin{array}{lllll}\text { Brunot } \quad \ldots & \ldots & \ldots & 1848\end{array}$

$\begin{array}{lllll}\text { Graf } & \ldots & . . & \ldots & 1848\end{array}$

$\begin{array}{lllll}\text { Houël } & \ldots & \ldots & \ldots & 1848\end{array}$

$\begin{array}{lllll}\text { Hemsbach } & \ldots & \ldots & \ldots & 1848\end{array}$

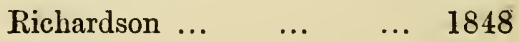

$\begin{array}{lllll}\text { Schubart } & \ldots & \ldots & \ldots & 1848\end{array}$

Van Deen $\quad \ldots \quad \ldots \quad \ldots \quad \ldots \quad 1849$

$\begin{array}{lllll}\text { Gurlt } & \ldots & \ldots & \ldots & 1849\end{array}$

$\begin{array}{lllll}\text { Vrolik } & \ldots & \ldots & \ldots & 1849\end{array}$

Die äusseren Mängel _.. 1850

$\begin{array}{lllll}\text { Bendz } & \ldots & \ldots & \ldots & 1850\end{array}$

$\begin{array}{lllll}\text { Bruehl } & \ldots & \ldots & \ldots & 1850\end{array}$

$\begin{array}{lllll}\text { Colin } & \ldots & \ldots & & \end{array}$

$\begin{array}{lllll}\text { Dadd } & . & \ldots & \ldots & 1850\end{array}$

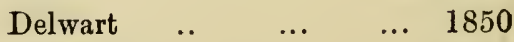

$\begin{array}{lllll}\text { Deschamps ... } & \ldots & \ldots & 1850\end{array}$ 
INDEX TO SUBJECTS.

NATURAL HISTORY, ANATOMY, PHYSIOLOGY AND EXTERNAL FORM.-Continued.

$\begin{array}{llll}\text { Montlaville ... } & \ldots & \ldots & 1850\end{array}$

$\begin{array}{lllll}\text { Houël } & \ldots & \ldots & \ldots & 1850\end{array}$

$\begin{array}{lllll}\text { Leyh } & \ldots & \ldots & \ldots & 1850\end{array}$

$\begin{array}{lllll}\text { Minot } & \ldots & \ldots & \ldots & 1850\end{array}$

Wahlgren $\ldots . \quad \ldots \quad \ldots \quad 1850$

$\begin{array}{lllll}\text { Daumas } \quad \ldots & \ldots & \ldots & 1851\end{array}$

Martin (Appendix) ... ... 1851

$\begin{array}{lllll}\text { Patellani } & \ldots & \ldots & \ldots & 1851\end{array}$

Riege $\quad \ldots \quad \ldots \quad \ldots \quad 1851$

$\begin{array}{lllll}\text { Traeger } & \ldots & \ldots & \ldots & 1851\end{array}$

Audubon and Bachman $\quad \ldots \quad 1851$

$\begin{array}{lllll}\text { Bonova } & \ldots & \ldots & \ldots & 1852\end{array}$

Defays and Husson... ... 1852

Ellerbrock $\ldots . \ldots \quad \ldots, 1852$

$\begin{array}{lllll}\text { Falke } & \ldots & \ldots & \ldots & 1852\end{array}$

$\begin{array}{lllll}\text { Heinrichs } & \ldots & \ldots & \ldots & 1852\end{array}$

$\begin{array}{lllll}\text { Jacoby } \quad \ldots & \ldots & \ldots & 1852\end{array}$

$\begin{array}{lllll}\text { Richardson } & \ldots & \ldots & \ldots & 1852\end{array}$

$\begin{array}{lllll}\text { Rueff } & \ldots & \ldots & \ldots & 1852\end{array}$

Saint-Ange $\ldots \quad \ldots \quad \ldots \quad 1852$

$\begin{array}{llllll}\text { Lamotte } & \ldots & \ldots & \text {. } & 1853\end{array}$

$\begin{array}{llllll}\text { Mazoiller } & \ldots & \ldots & \ldots & 1853\end{array}$

$\begin{array}{lllll}\text { Miiller } & \ldots & \ldots & \ldots & 1853\end{array}$

Le Cheval, etc. $\quad \ldots \quad \ldots \quad 1854$

Systemutisch-geordneter $\quad \ldots \quad 1854$

$\begin{array}{llllll}\text { Benjamin } & \ldots & \ldots & \ldots & \ldots & 1854\end{array}$

$\begin{array}{lllll}\text { Colin } & \ldots & \ldots & \ldots & 1854\end{array}$

$\begin{array}{lllll}\text { Dürler } \quad . . & \ldots & \ldots & 1854\end{array}$

$\begin{array}{lllll}\text { Giebel } & \ldots & \ldots & \ldots & 1854\end{array}$

$\begin{array}{llllll}\text { Le Clercq } & \ldots & \ldots & \ldots & 1854\end{array}$

Lessona $\quad \ldots \quad \ldots \quad \ldots \quad \ldots \quad 1854$

$\begin{array}{lllll}\text { Müller } \quad \ldots & \ldots & \ldots & 1854\end{array}$
Neubert

$\begin{array}{lll}\ldots & \ldots & 1854\end{array}$

$\begin{array}{lllll}\text { Saint-Ange } & \ldots & \ldots & \ldots & 1854\end{array}$

$\begin{array}{lllll}\text { Auzoux } \quad \ldots & \ldots & \ldots & 1855\end{array}$

De Lá Malle $\quad \ldots \quad \ldots \quad 1855$

$\begin{array}{lllll}\text { Hamm } & . & \ldots & \ldots & 1855\end{array}$

$\begin{array}{lllll}\text { Bruslard } \quad \ldots & \ldots & \ldots & 1856\end{array}$

Hammer-Purgstall $\quad . . \quad \ldots \quad 1856$

$\begin{array}{lllll}\text { Langer } \quad \ldots & . . & \ldots & 1856\end{array}$

$\begin{array}{lllll}\text { Moleschott } & \ldots & \ldots & \ldots & 1856\end{array}$

$\begin{array}{lllll}\text { Mussot } & \ldots & \ldots & \ldots & 1856\end{array}$

$\begin{array}{lllll}\text { Sampedro } & \ldots & \ldots & \ldots & 1856\end{array}$

$\begin{array}{lllll}\text { Veith } & \ldots & \ldots & \ldots & 1856\end{array}$

$\begin{array}{lllll}\text { Walsh } & \ldots & \ldots & \text {. } & 1856\end{array}$

$\begin{array}{lllll}\text { Chauveau } & \ldots & \ldots & \ldots & 1857\end{array}$

$\begin{array}{lllll}\text { Dadd } & \ldots & \ldots & \ldots & 1857\end{array}$

$\begin{array}{lllll}\text { Herbert } \quad \ldots & \ldots & \ldots & 1857\end{array}$

$\begin{array}{lllll}\text { Lessona } & \ldots & \ldots & \ldots & 1857\end{array}$

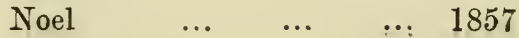

$\begin{array}{lllll}\text { Raabe } \quad \ldots & \ldots & \ldots & 1857\end{array}$

$\begin{array}{lllll}\text { Richard } \quad \ldots & \ldots & \ldots & 1857\end{array}$

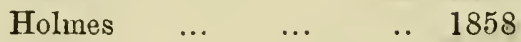

$\begin{array}{lllll}\text { Carson } & \ldots & \ldots & \ldots & 1859\end{array}$

$\begin{array}{llllll}\text { Mégnin } \quad \ldots & \ldots & \ldots & 1860\end{array}$

$\begin{array}{lllll}\text { Cornay } & \text {. } & \ldots & \ldots & 1861\end{array}$

Gamgee and Law $\ldots . \quad \ldots \quad 1861$

$\begin{array}{lllll}\text { Lupton } \quad . . & \text {.. } & \text {.. } & 1861\end{array}$

$\begin{array}{lllll}\text { Duhousset } & \ldots & \ldots & \ldots & 1862\end{array}$

$\begin{array}{llllll}\text { Müller } & \ldots & \ldots & \ldots & 1862\end{array}$

$\begin{array}{llll}\text { Ruittimeyer ... } & \ldots & \ldots & 1863\end{array}$

$\begin{array}{lllll}\text { Berjeau } \quad \ldots & \ldots & \ldots & 1864\end{array}$

$\begin{array}{llllll}\text { Mayhew } & \ldots & \ldots & \ldots & 1864\end{array}$

$\begin{array}{lllll}\text { Houël } \quad \ldots & \ldots & \ldots & 1865\end{array}$ 
NATURAL HISTORY, ANATOMY, PHYSIOLOGY AND EXTERNAL FORM.-Continued.

\begin{tabular}{|c|c|c|c|c|c|c|c|c|c|}
\hline Günther & $\ldots$ & $\ldots$ & $\ldots$ & 1866 & Smith & $\ldots$ & $\ldots$ & $\ldots$ & 1880 \\
\hline Jacoby & $\ldots$ & $\ldots$ & $\ldots$ & 1867 & Duhousset & $\ldots$ & $\ldots$ & $\ldots$ & 1881 \\
\hline Lortet & $\ldots$ & $\ldots$ & $\ldots$ & 1867 & Hibbert & $\ldots$ & $\ldots$ & $\ldots$ & 1881 \\
\hline Macbride & $\ldots$ & $\ldots$ & $\ldots$. & 1867 & Lupton & $\ldots$ & $\ldots$ & $\ldots$ & 88 \\
\hline Merche & $\ldots$ & $\ldots$ & $\ldots$ & 1868 & Major & $\ldots$ & $\ldots$ & $\ldots$ & 188 \\
\hline Houël & $\ldots$ & $\ldots$ & $\ldots$ & 1869 & Murray & $\ldots$ & $\ldots$ & $\ldots$ & 88 \\
\hline Pinel & $\ldots$ & $\ldots$ & $\ldots$ & 1869 & Poliakoff & $\ldots$ & $\ldots$ & $\ldots$ & 88 \\
\hline Strangeways & s and & roodsir & $\ldots$ & 1869 & Salvi & $\ldots$ & $\ldots$ & $\ldots$ & 188 \\
\hline Wentz & $\ldots$ & $\ldots$ & $\ldots$ & 1869 & De la Villa & $\ldots$ & $\ldots$ & $\ldots$ & 188 \\
\hline Flower & $\ldots$ & .. & $\ldots$ & 1870 & The Horse & and his & Ways & $\ldots$ & 88 \\
\hline Hehn & $\ldots$ & $\ldots$ & $\ldots$ & 1870 & Goubaux an & d Barri & ier & $\ldots$ & 188 \\
\hline Lankester & $\ldots$ & $\ldots$ & $\ldots$ & 1870 & Piétrement & $\ldots$ & $\ldots$ & $\ldots$ & 0 \\
\hline La Teillais & $\ldots$ & $\ldots$ & $\ldots$ & 1870 & Pinel & $\ldots$ & .. & $\ldots$ & 88 \\
\hline Lupton & $\ldots$ & $\ldots$ & $\ldots$ & 1870 & Ellenberger & $\ldots$ & $\ldots$ & $\ldots$ & 188 \\
\hline Piétrement & $\ldots$ & $\ldots$ & $\ldots$ & 1870 & Henle & $\ldots$ & $\ldots$ & $\ldots$ & 188 \\
\hline Piétrement & $\ldots$ & $\ldots$ & $\ldots$ & 1871 & Nehring & $\ldots$ & $\ldots$ & $\ldots$ & 1884 \\
\hline Rueff & $\ldots$ & $\ldots$ & $\ldots$ & 1873 & Das Pferd & $\ldots$ & $\ldots$ & $\ldots$ & 188 \\
\hline Mittheilung & $e n$ & $\ldots$ & $\ldots$ & 1874 & Hayes & $\ldots$ & $\ldots$ & $\ldots$ & 188 \\
\hline Beaupré & $\ldots$ & $\ldots$ & $\ldots$ & 1874 & Kaiser & $\ldots$ & $\ldots$ & .. & 188 \\
\hline Duhousset & $\ldots$ & $\ldots$ & $\ldots$ & 1874 & Leisering & $\ldots$ & $\ldots$ & .. & 188 \\
\hline Freytag & $\ldots$ & $\ldots$ & . & 1875 & Lydekker & $\ldots$ & $\ldots$ & $\ldots$ & 188 \\
\hline Piétrement & $\ldots$ & $\cdots$ & $\ldots$ & 1875 & Montigny & $\ldots$ & $\ldots$ & $\ldots$ & 188 \\
\hline Voigtlaender & & $\ldots$ & $\ldots$ & 1876 & Parker & $\ldots$ & $\ldots$ & $\ldots$ & $18 \varepsilon$ \\
\hline Migotti & $\ldots$ & .. & $\ldots$ & 1879 & Schmidt & $\ldots$ & $\ldots$ & $\ldots$ & 188 \\
\hline Simpson-Bai & ilkie & $\ldots$ & $\ldots$ & 1880 & Wood & $\ldots$ & .. & $\ldots$ & 188 \\
\hline Fogliata & $\ldots$ & $\ldots$ & $\ldots$ & 1880 & Alix and $\mathrm{C}$ & uyer & $\ldots$ & $\cdots$ & 188 \\
\hline
\end{tabular}




\section{CAVALRY.}

Xenophon $\ldots \quad \ldots$

Hadrian ... ...

Ammianus Marcellinus

Toralto

Bruyn. Abraham de

Anon.

Malatesta

Loehneysen

Melzo

$\begin{array}{lllll}\text { Basta } & \ldots & \ldots & \ldots & 1612\end{array}$

$\begin{array}{llll}\text { Wallhausen } \quad \ldots & \ldots & 1616\end{array}$

Anon. $\quad \ldots \quad \ldots \quad \ldots \quad 1617$

Gajani $\quad \ldots \quad \ldots \quad \ldots .1619$

$\begin{array}{llllll}\text { Gali } & \ldots & \ldots & \ldots & 1619\end{array}$

De la Noue $\quad \ldots \quad \ldots \quad 1620$

$\begin{array}{lllll}\text { Markham } & \ldots & \ldots & \ldots & 1625\end{array}$

Hugo $\quad \ldots \quad$.. $\quad \ldots \quad 1630$

$\begin{array}{llllll}\text { Crusoe } & \ldots & \ldots & \ldots & 1632\end{array}$

Muñoz de Peral $\ldots . \ldots .1640$

$\begin{array}{llllll}\text { Lesley } \quad \ldots & \ldots & \ldots & 1642\end{array}$

$\begin{array}{lllll}\text { Marschalk } & \ldots & \ldots & \ldots & 1642\end{array}$

P. della Valle $\quad \ldots \quad \ldots \quad 1650$

$\begin{array}{lllll}\text { Ward } \quad \ldots & \ldots & \ldots & 1650\end{array}$

$\begin{array}{llllll}\text { Micheli } \quad \ldots & \ldots & \ldots & 1662\end{array}$

$\begin{array}{lllll}\text { Strubenberg } & \ldots & \ldots & 1662\end{array}$

Venn

La Fontaine

Guillet

Birac

Folard

Anon.

Anon.
Anon. $\begin{array}{lll}\ldots & \ldots & 1754 \\ \ldots & \ldots & 1754\end{array}$

La Porterie

$\begin{array}{lllll}\text { Anon. } \quad \ldots & \ldots & \ldots & 1756\end{array}$

Anon. $\quad \ldots \quad \ldots \quad \ldots \quad 1756$

Amourettes ... $\quad \ldots \quad \ldots \quad 1756$

Dalrymple ... $\quad \ldots \quad \ldots \quad 1761$

$\begin{array}{llll}\text { Oebschelwitz } & \ldots & \ldots & 1761\end{array}$

Earl of Pembroke ... ... 1761

$\begin{array}{llll}\text { Santa Maria } & \ldots & \ldots & 1761\end{array}$

Anon. $\quad \ldots \quad \ldots \quad \ldots \quad 1766$

Anon. $\quad \ldots \quad \ldots \quad \ldots c 1766$

$\begin{array}{lllll}\text { Arellano } \quad \ldots & \ldots & \ldots & 1767\end{array}$

Weyrother ... ... ... 1768

Berenger ... $\quad \ldots \quad \ldots \quad 1771$

$\begin{array}{lllll}\text { Brézé } & \ldots & \ldots & \ldots & 1772\end{array}$

Mottin de la Balme $\quad \ldots \quad 1776$

$\begin{array}{llllll}\text { Melfort } \quad \ldots & \ldots & \ldots & 1776\end{array}$

$\begin{array}{lllll}\text { Hinde } & \ldots & \ldots & \ldots & 1778\end{array}$

$\begin{array}{lllll}\text { Wolstein } \quad \ldots & \ldots & \ldots & 1779\end{array}$

Prince de Ligne $\quad \ldots \quad \ldots \quad 1780$

$\begin{array}{llllll}\text { Bohan } \quad \ldots & \ldots & \ldots & 1781\end{array}$

$\begin{array}{llllll}\text { Anon. } \quad \ldots & \ldots & \ldots & 1790\end{array}$

$\begin{array}{lllll}\text { Boisdeffro } & \ldots & \ldots & \ldots & 1790\end{array}$

$\begin{array}{lllll}\text { Wolstein } & \ldots & \ldots & \ldots & 1792\end{array}$

Anon. $\quad \ldots \quad \ldots \quad \ldots .1794$

Eliot $\quad \ldots \quad \ldots \quad \ldots \quad 1794$

Anon. $\quad \ldots \quad \ldots \quad \ldots \quad 1795$

Walter $\quad \ldots \quad \ldots \quad \ldots \quad 1795$

$\begin{array}{lllll}\text { Neville } \quad \ldots & \ldots & \ldots & 1796\end{array}$

$\begin{array}{lllll}\text { Timlich } \quad \ldots & \ldots & \ldots & 1796\end{array}$

$\begin{array}{lllll}\text { Tennecker } & \ldots & \ldots & \ldots & 1797\end{array}$

Anon. $\quad \ldots \quad \ldots \quad \ldots \quad 1798$ 
CAVALRY.-Continued.

$\begin{array}{llllll} & \text { Allen } \quad \ldots & \ldots & \ldots & \ldots & 1798\end{array}$

$\begin{array}{lllll}\text { Boswell } & \ldots & \ldots & \ldots & 1798\end{array}$

Tennecker ... $\quad \ldots . . \quad \ldots \quad 1798$

Anon.

Rowlandson (Appendix) $\quad \ldots \quad 1799$

Anon.

Klatte $\quad \ldots \quad \ldots \quad \ldots \quad 1800$

Viborg and Neergard $\quad \ldots \quad 1800$

$\begin{array}{llllll}\text { Anon. } & \ldots & \ldots & \ldots & 1801\end{array}$

Tyndale $\quad \ldots \quad \ldots \quad, \ldots \quad 1801$

Young $\quad \ldots \quad \ldots \quad, \quad \ldots \quad 1801$

Anon. $\quad \ldots \quad \ldots \ldots c c 1803$

Herries $\quad \ldots \quad \ldots, \quad \ldots \quad 1804$

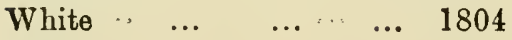

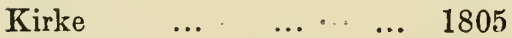

$\begin{array}{lllll}\text { Dalbiac } \quad \ldots & \ldots & \ldots & 1806\end{array}$

$\begin{array}{llllll}\text { Lindenau } & \ldots & \ldots & \ldots & 1806\end{array}$

$\begin{array}{lllll}\text { Koskinsco } & \ldots & \ldots & \ldots & 1808\end{array}$

$\begin{array}{lllll}\text { Skeene } \quad \ldots & \ldots & \ldots & 1808\end{array}$

$\begin{array}{lllll}\text { Pollnitz } \quad \ldots & \ldots & \ldots & 1810\end{array}$

$\begin{array}{llllll}\text { Drouville } & \ldots & \ldots & \ldots & \ldots & 1811\end{array}$

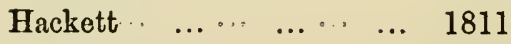

Anon. .. $\quad \ldots . . \quad \ldots . . \quad \ldots \quad 1815$

$\begin{array}{lllllll}\text { Muller } \quad \ldots & \ldots & \ldots & \ldots & \ldots & 1816\end{array}$

Roche-Aymon . . ... $\ldots \quad \ldots \quad 1817$

Decker ～.. $\quad \ldots \quad \ldots \quad \ldots \quad 1818$

$\begin{array}{lllll}\text { Anon. } & \ldots & \ldots & \ldots & 1819\end{array}$

$\begin{array}{llllll}\text { Pollnitz } & \ldots & \ldots & \ldots & 1819\end{array}$

Anon. $\quad \ldots \quad \ldots \ldots, \ldots \quad 1820$

$\begin{array}{lllll}\text { Bismark } \quad \ldots & \ldots & \ldots & 1820\end{array}$

$\begin{array}{lllll}\text { Montmorency } \quad \ldots & \ldots & 1820\end{array}$

Daum $\begin{array}{llllll}\text { Tscheulin } & \ldots & \ldots & \ldots & \ldots & 1822\end{array}$

$\begin{array}{lllll}\text { Regulations } \quad \ldots & \ldots & 1824\end{array}$

Bamford $\quad \ldots \quad \ldots \quad \ldots \quad 1824$

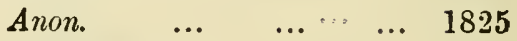

$\begin{array}{lllll}\text { Carruthers } & \ldots & \ldots & \ldots & 1825\end{array}$

$\begin{array}{llllll}\text { Klatte } & \ldots & \ldots & \ldots & 1825\end{array}$

Anon. $\quad \ldots \quad \ldots \quad$... 1826

$\begin{array}{lllll}\text { Beck } \quad \ldots & \ldots & \ldots & 1826\end{array}$

Jacquemin $\ldots \quad \ldots, \quad \ldots \quad 1826$

Ammon $\quad \ldots \quad \ldots \quad, \quad \ldots \quad 1827$

$\begin{array}{lllll}\text { Roche-Aymon } \quad \ldots & \ldots & 1828\end{array}$

Der Lasa $\quad \ldots \quad \ldots . . \quad \ldots \quad 1828$

Gribble $\quad \ldots \quad \ldots . \quad \ldots \quad 1829$

$\begin{array}{llllll}\text { Klatle } & \ldots & \ldots & \ldots & 1829\end{array}$

$\begin{array}{lllll}\text { Veiss } & \ldots & \ldots & \ldots & 1829\end{array}$

Benkendorf $\quad \ldots \quad \ldots \quad 1831$

De Brack $\quad \ldots \quad \ldots \quad \ldots \quad 1831$

Anon. $\quad \ldots \quad \ldots \quad \ldots \quad 1832$

Anon. $\quad \ldots \quad \ldots \quad \ldots 61832$

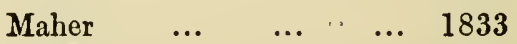

Anon. $\quad \ldots \quad \cdot \quad \ldots . \cdots \quad \ldots \quad 1834$

Packe ‥ ... . ... $\quad \ldots \quad 1834$

Schauenburg $\quad \ldots . \cdots \quad \ldots \quad 1838$

$\begin{array}{llllll}\text { Dejean } \quad \ldots & \ldots & \ldots & 1839\end{array}$

Angelo $\quad \ldots . . . . \quad \ldots \quad 1840$

Greenwood ... $\quad \ldots \quad \ldots \quad 1840$

$\begin{array}{lllll}\text { D'Oudinot } \ldots & \ldots & \ldots & 1840\end{array}$

Préval . ... ... $\quad \ldots \quad 1840$

Rigault . .. $\quad \ldots \quad \ldots \quad 1840$

Nouvelle école . . ... $\quad \ldots \quad 1841$

U. S. Cavalry Tactics $\quad \ldots \quad 1841$

$\begin{array}{lllll}\text { Curnieu } & \ldots & \ldots & \ldots & 1841\end{array}$ 
CAVALRY.-Continued.

\begin{tabular}{|c|c|c|c|c|c|c|c|c|c|}
\hline Daunay u & Ligné & & $\ldots$ & 1841 & Ordonnance & & $\ldots$ & $\ldots$ & 1849 \\
\hline Giustinian & & $\ldots$ & $\ldots$ & 1841 & Reglamento & & $\ldots$ & 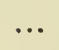 & 1849 \\
\hline Dolffs & $\ldots$ & $\ldots$ & $\ldots$ & 1842 & Underretwi & & $\ldots$ & $\ldots$ & 1849 \\
\hline étang & $\ldots$ & $\ldots$ & $\ldots$ & 1842 & D'Elbee.. & $\ldots$ & .. & $\ldots$ & 1849 \\
\hline Terlen. & $\ldots$ & .. & $\ldots$ & 1842 & Merlen & $\ldots$ & $\ldots$ & . & 1849 \\
\hline hirley & .. & ... & $\ldots$ & 1842 & Anweisung & & $\ldots$ & . & 1850 \\
\hline non. & $\ldots$ & .. & $\ldots$ & 1843 & Guide de l' & instru & teur & . & 1850 \\
\hline Ainslie & $\ldots$ & $\ldots$ & $\ldots$ & 1843 & Historical & Recor & $s \ldots$ & $\ldots$ & 1850 \\
\hline D'Aldéquie & & ... & $\ldots$ & 1843 & Traité de lo & a réce & tion & 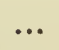 & 1850 \\
\hline Greenwood & & $\ldots$ & $\ldots$ & 1843 & Angelo... & $\ldots$ & $\ldots$ & 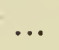 & 1850 \\
\hline Martin & $\ldots$ & $\ldots$ & $\cdots$ & 1843 & Boudin . & $\ldots$ & $\ldots$ & $\ldots$ & 850 \\
\hline eidler. & $\ldots$ & $\ldots$ & $\ldots$ & 1843 & Herstatt & $\ldots$ & $\cdots$ & $\ldots$ & 850 \\
\hline Smythe & $\ldots$ & ... & $\ldots$ & 1843 & Hort $\ldots$ & $\ldots$ & $\ldots$ & $\ldots$ & 1850 \\
\hline tephens & ... & $\ldots$ & $\ldots$ & 1843 & Abrichtung & $s-R e g$ & ment & & 1851 \\
\hline Anon. . & $\ldots$ & ... & $\ldots$ & 1844 & Anon. .. & $\ldots$ & $\ldots$ & $\ldots$ & 851 \\
\hline Zusammen & tellung & $\ldots$ & $\ldots$ & 1844 & Ordonnance & $e$, etc & (Cha & eurs & \\
\hline Baron de $\mathrm{I}$ & & ... & $\ldots$ & 1844 & et Hussa & $r d s)$ & $\ldots$ & 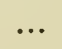 & 1851 \\
\hline Goux and & Merche & $\ldots$ & $\ldots$ & 1844 & Ordonnance & $e$ etc. & Lanciers & & 851 \\
\hline Cavalry $S$ & vord $E x e$ & ercise & $\ldots$ & 1845 & Regulations & & $\ldots$ & $\ldots$ & $18 \tilde{1}$ \\
\hline Aubert. & $\ldots$ & $\ldots$ & $\ldots$ & 1845 & Clonard & $\ldots$ & ... & $\ldots$ & 1851 \\
\hline Fouan & $\ldots$ & $\ldots$ & $\ldots$ & 1845 & Hagerup. & $\ldots$ & $\ldots$ & $\therefore$ & 851 \\
\hline Haggeman & & $\ldots$ & $\ldots$ & 1845 & Liitken & $\ldots$ & $\therefore$ & $\ldots$ & 1851 \\
\hline Roy & $\ldots$ & $\ldots$ & $\ldots$ & 1845 & Montigny & $\ldots$ & $\ldots$ & $\ldots$ & 1851 \\
\hline Taubert & $\ldots$ & $\ldots$ & $\ldots$ & 1845 & Robbins & $\ldots$ & $\ldots$ & $\ldots$ & 1851 \\
\hline Bishop. & ... & $\ldots$ & $\ldots$ & 1846 & Stevenson & $\ldots$ & $\ldots$ & $\ldots$ & 1851 \\
\hline Louchard & $\ldots$ & $\ldots$ & $\ldots$ & 1847 & Yeomanry & Regut & ations & $\therefore$. & 1852 \\
\hline Anon. & $\ldots$ & $\ldots$ & $\ldots$ & 1848 & D'Aure. & $\ldots$ & $\ldots$ & $\ldots$ & 1852 \\
\hline Vorschirift & & $\ldots$ & $\ldots$ & 1848 & Fabre & $\ldots$ & $\ldots$ & $\ldots$ & 1852 \\
\hline Gibson... & .. & $\ldots$ & $\ldots$ & 1848 & Flandrin & $\ldots$ & ... & $\ldots$ & 1852 \\
\hline Toggia . & ... & $\ldots$ & $\ldots$ & 1848 & Darrac . & ... & ... & $\ldots$ & 1852 \\
\hline Cours d'eq & ittuti & Militai & & 1849 & Nolan .. & $\ldots$ & $\ldots$ & $\ldots$ & 1852 \\
\hline Instruction & $\ldots$ & $\ldots$ & $\ldots$ & 1849 & Anon. & $\ldots$ & ... & $\ldots$ & 1853 \\
\hline
\end{tabular}


CAVALRY.-Continued.

\begin{tabular}{|c|c|c|c|c|c|c|c|c|c|}
\hline La Cavaleri & & $\ldots$ & $\ldots$ & 1853 & \multicolumn{2}{|c|}{ Kirchhoewell } & $\cdots$ & $\ldots$ & 1855 \\
\hline Ordonnance & & $\ldots$ & $\ldots$ & 1853 & Mirns & $\ldots$ & $\ldots$ & $\ldots$ & 1855 \\
\hline Graefe & $\ldots$ & $\ldots$ & $\ldots$ & 1853 & Montigny & $\ldots$ & $\ldots$ & $\ldots$ & 1855 \\
\hline Darrac & ... & $\ldots$ & $\ldots$ & 1853 & Regiement & ... & $\ldots$ & $\ldots$ & 1856 \\
\hline Nolan & ... & $\ldots$ & $\ldots$ & 1853 & Théorie & ... & $\ldots$ & $\ldots$ & 1856 \\
\hline Gay de Ver & non & $\ldots$ & .. & 1853 & Udtog & ... & $\ldots$ & $\ldots$ & 1856 \\
\hline Volpi & ... & $\ldots$ & ... & 1853 & Geraud & $\ldots$ & $\ldots$ & $\ldots$ & 856 \\
\hline Exerceer Re & egleme & $t \ldots$ & $\ldots$ & 1854 & Hartmann & $\ldots$ & $\ldots$ & $\ldots$ & 856 \\
\hline Handbock $f$ & or $H$ & saren & $\ldots$ & 1854 & Earl of Luc & an & $\ldots$ & $\cdots$ & 856 \\
\hline Cavalry Sk & irmis & & $\ldots$ & 1854 & Marquis de & Pim & & $\ldots$ & 856 \\
\hline Ordonnance & $\operatorname{sur} t$ & xercise & $\ldots$ & 1854 & Instruction & $\ldots$ & $\ldots$ & $\ldots$ & 1857 \\
\hline A Proposal & for & $\ldots$ & ... & 1854 & Goyau & $\ldots$ & $\ldots$ & $\ldots$ & 857 \\
\hline Arentschild & & $\ldots$ & ... & 1854 & Liasse & $\ldots$ & $\ldots$ & $\ldots$ & 857 \\
\hline Daumas & $\ldots$ & $\ldots$ & $\ldots$ & 1854 & Baker & $\ldots$ & $\ldots$ & $\ldots$ & 1858 \\
\hline Flandrin & $\ldots$ & $\ldots$ & $\ldots$ & 1854 & Dérigny & $\cdots$ & $\ldots$ & $\ldots$ & 1859 \\
\hline Guldberg & $\cdots$ & ... & ... & 1854 & Gräfe & $\ldots$ & $\ldots$ & $\ldots$ & 1859 \\
\hline Lecomte & $\ldots$ & $\ldots$ & $\cdots$ & 1854 & Taylor & $\cdots$ & $\ldots{ }^{\circ}$ & $\ldots$ & 1859 \\
\hline Mortimer & $\ldots$ & $\ldots$ & ... & 1854 & \multicolumn{3}{|c|}{ Extrait de l'Ordonnance } & $\cdots$ & 1860 \\
\hline Mussot & $\cdots$ & $\cdots$ & $\ldots$ & 1854 & \multicolumn{3}{|c|}{ Réglement sur l'exercise } & $\ldots$ & 1860 \\
\hline Shirley & $\ldots$ & $\cdots$ & $\ldots$ & 1854 & Acland & $\ldots$ & $\ldots$ & $\ldots$ & 1860 \\
\hline Extrait du & r’égler & ent & $\ldots$ & 1855 & \multicolumn{2}{|c|}{ Amous-Riviere } & $\ldots$ & $\ldots$ & 1860 \\
\hline Instruction. & for $E$ & ceersko & lern & 1855 & Azémar & $\ldots$ & $\ldots$ & $\ldots$ & 1860 \\
\hline Manuel du & Cava & & $\cdots$ & 1855 & Bugeaud & $\ldots$ & $\therefore$ & $\ldots$ & 1860 \\
\hline Uddrag af & Instr & tionen & $\ldots$ & 1855 & \multicolumn{2}{|c|}{ Sir Hope Grant } & $\ldots$ & $\ldots$ & 1860 \\
\hline British Cav & alry & Balaklo & $a v a$ & 1855 & Brèves & $\ldots$ & $\ldots$ & $\ldots$ & 1860 \\
\hline Cavalry $T a$ & ectics & ... & $\ldots$ & 1855 & Porter & $\ldots$ & $\ldots$ & $\ldots$ & 1860 \\
\hline Ordonnance & sur & exercise & $\ldots$ & 1855 & \multicolumn{2}{|c|}{ Shakespear ... } & $\ldots$ & $\ldots$ & 1860 \\
\hline Beamish & $\ldots$ & ... & $\ldots$ & 1855 & \multicolumn{2}{|c|}{ Earl of Cardigan } & $\ldots$ & $\ldots$ & 1861 \\
\hline Cavalry Off & ficers & ournal & $\ldots$ & 1855 & Brèves & $\ldots$ & $\ldots$ & $\ldots$ & 1861 \\
\hline Dahl & $\ldots$ & ... & $\ldots$ & 1855 & Milet & $\ldots$ & $\ldots$ & ... & 1861 \\
\hline Hartmann & $\ldots$ & ... & $\ldots$ & 1855 & Renard & $\ldots$ & $\ldots$ & $\ldots$ & 1861 \\
\hline Higgins & $\ldots$ & $\ldots$ & $\ldots$ & 1855 & \multicolumn{2}{|c|}{ Instructions } & $\ldots$ & $\ldots$ & 186 \\
\hline
\end{tabular}


INDEX TO SUBJECTS.

CAVALRY.-Continued.

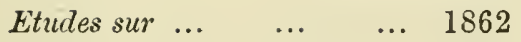

Regulations f $\mathrm{fur}^{\circ} \quad \ldots \quad \ldots \quad \ldots \quad 1862$

Barbier ... $\quad \ldots \quad \ldots \quad 1862$

S. von E. $\quad \ldots \quad \ldots \quad \ldots \quad \ldots \quad 1862$

$\begin{array}{lllll}\text { McClellan } & \ldots & \ldots & \ldots & 1862\end{array}$

$\begin{array}{lllll}\text { Morvan } \quad \ldots & \ldots & \ldots & 1862\end{array}$

$\begin{array}{llll}\text { Reiffenberg } & \ldots & \ldots & 1862\end{array}$

La Cavalerie $\quad \ldots \quad \ldots \quad 1863$

Manual of Drill $\quad \ldots \quad \ldots \quad 1863$

Regulations for the Movements 1863

Ambert $\quad \ldots \quad \ldots \quad \ldots \quad 1863$

$\begin{array}{lllll}\text { Bué } & \ldots & \ldots & \ldots & 1863\end{array}$

$\begin{array}{llllll}\text { Cogent } & \ldots & \ldots & \ldots & 1863\end{array}$

$\begin{array}{lllll}\text { Cooke } & \ldots & \ldots & \ldots & 1863\end{array}$

$\begin{array}{lllll}\text { Patten } \quad \ldots & \ldots & \ldots & 1863\end{array}$

$\begin{array}{lllll}\text { Peter } & \ldots & \ldots & \ldots & 1863\end{array}$

$\begin{array}{lllll}\text { Roemer } \quad \ldots & \ldots & \ldots & 1863\end{array}$

Five Hundred Questions ... 1864

Our Native Cavalry $\quad$... 1864

$\begin{array}{lllll}\text { Instruction } & \ldots & \ldots & \ldots & 1864\end{array}$

Méthode $\quad \ldots \quad \ldots . \quad \ldots \quad 1861$

$\begin{array}{llllll}\text { Cooke } \quad \ldots & \ldots & \ldots & 1864\end{array}$

Elliott $\quad \ldots \quad \ldots \quad \ldots \quad 1864$

$\begin{array}{llllll}\text { Leurs } \quad \ldots & \ldots & \ldots & 1864\end{array}$

Richepance ... $\quad \ldots \quad \ldots \quad 1864$

$\begin{array}{lllll}\text { Vals } \quad \ldots & \ldots & \ldots & 1864\end{array}$

Cavalry surord exercise $\quad \ldots \quad 1865$

Règlemente ... $\quad \ldots \quad \ldots \quad 1865$

$\begin{array}{llll}\text { Regulations } & \ldots & \ldots & 1865\end{array}$

Taschenbuch $\quad \ldots \quad \ldots \quad 1865$

$\begin{array}{lllll} & \text { Baker } \quad \ldots & \ldots & \ldots & 1865\end{array}$

Bower $\quad \ldots \quad \ldots \quad \ldots \quad 1865$ $\begin{array}{lllll}\text { Brackett } \quad \ldots & \ldots & \ldots & 1865\end{array}$

Hozier $\quad \ldots \quad \ldots \quad \ldots 61865$

$\begin{array}{lllll}\text { Humbert } & \ldots & \ldots & \ldots & 1865\end{array}$

$\begin{array}{lllll}\text { Plochefort } & \ldots & \ldots & \ldots & 1865\end{array}$

$\begin{array}{lllll}\text { Smith } \quad \ldots & \ldots & \ldots & 1865\end{array}$

Gay de Vernoll $\quad \ldots \quad \ldots \quad 1865$

$\begin{array}{llll}\text { Dierst-Reglement } & \ldots & \ldots & 1866\end{array}$

Kritische Gedanken $\quad$.. 1866

Gedanken über $\quad \ldots \quad \ldots \quad 1866$

Hime $\quad \ldots \quad \ldots . \quad \ldots \quad 1866$

$\begin{array}{lllll}\text { Reiffenberg ... } & \ldots & \ldots & 1866\end{array}$

$\begin{array}{llllll}\text { Twyford } \quad \ldots & \ldots & \ldots & 1866\end{array}$

$\begin{array}{lllll}\text { Anlei_ung } & \ldots & \ldots & \ldots & 1867\end{array}$

A propos de $\quad \ldots \quad \ldots \quad 1867$

$\begin{array}{llllll}\text { Regulatif } & \ldots & \ldots & \ldots & 1867\end{array}$

Zusammenstellung ... $\quad \ldots \quad 1867$

Görgey von Görge ... ... 1867

$\begin{array}{lllll}\text { Hutton } & \ldots & \ldots & \ldots & 1867\end{array}$

$\begin{array}{lllll}\text { Smith } \quad \ldots & \ldots & \ldots & 1867\end{array}$

Einige Worte $\quad \ldots \quad \ldots \quad 1868$

Etude sur la cavalerie légère 1868

Vorschriften über $\ldots . \quad \ldots \quad 1868$

$\begin{array}{llllll}\text { Besser } \quad \ldots & \ldots & \ldots & 1868\end{array}$

$\begin{array}{lllll}\text { Cogent } & \ldots & \ldots & \ldots & 1868\end{array}$

Denison $\quad \ldots \quad \ldots \quad \ldots \quad 1868$

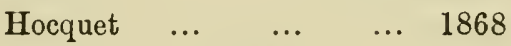

Avenir de la Cavalirie $\quad$.. 1869

Brigade\& Divisional Movements 1869

Das Exerziren eines Eskadron

zu Fuss. ... $\quad \ldots \quad \ldots .1869$

Exerciren einer Eskadron zu

$\begin{array}{lllll}\text { Pferde } & \ldots & \ldots & \ldots & 1869\end{array}$ 
CAVALRY.-Continued.

Instruction üt,r $\quad \ldots \quad \ldots 1869$ Normen für die Behandlung 1869 Notiz-Buch ... $\quad \ldots \quad \ldots \quad 1869$ $\begin{array}{llllll}\text { Andlau } & \ldots & \ldots & \ldots & 1869\end{array}$ Bonie : $\quad \ldots \quad \ldots \quad \ldots \quad 1869$ $\begin{array}{llllll}\text { Gradinger } & \ldots & \ldots & \ldots & 1869\end{array}$ Remarks on the Organization 1869 $\begin{array}{lllll}\text { Smith } \quad \ldots & \ldots & \ldots & 1869\end{array}$

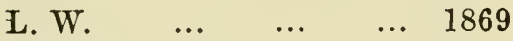
$\begin{array}{lllll}\text { Flüchtige } & \ldots & \ldots & \ldots & 1870\end{array}$ Handbuch für Reserve ... 1870 Kommando-Worte-Aviso $\quad \ldots \quad 1870$ Manual and Firing Exercises 1870 Standing Orders of 19th Hussars ... ... . .. 1870 Ueber die Thätigkeit _.. 1870 Vorschriften über das Pferdewesen 1870

Zur Taktik der Reiterei ... 1870 $\begin{array}{llllll}\text { Arnold } \quad \ldots & \ldots & \ldots & 1870\end{array}$ Bonie $\quad \ldots \quad \ldots \quad \ldots \quad 1870$

$\begin{array}{lllll}\text { Cherfils } \quad \ldots & \ldots & \ldots & 1870\end{array}$ $\begin{array}{lllll}\text { Krane } \quad \ldots & \ldots & \ldots & 1870\end{array}$ Quistorp $\quad \ldots \quad \ldots \quad \ldots \quad \ldots \quad 1870$ $\begin{array}{lllll}\text { Reiffenberg } \quad \ldots & \ldots & 1870\end{array}$ Smith . $\quad \ldots . \quad \ldots \ldots \quad \ldots \quad 1870$ $\begin{array}{lllll}\text { Tackels } \quad \ldots & \ldots & \ldots & 1870\end{array}$ V. du Vernois $\quad \ldots \quad \ldots \quad 1870$

$\begin{array}{lllll}\text { Webster } \quad \ldots & \ldots & \ldots & 1870\end{array}$ Andentungen für $\quad \ldots \quad \ldots \quad 1871$ Cavalry Sword Exercise ... 1871 Undervisning
Dienst-Unterricht $\quad \ldots \quad \ldots \quad 1871$ Exerciereglemente ... $\quad \ldots \quad 1871$ Sicherheits-und Nashrichten$\begin{array}{lllll}\text { dienst } & \ldots & \ldots & \ldots & 1871\end{array}$ Bonie . $\quad \ldots \quad \ldots . \quad \ldots \quad 1871$ C. $\quad \ldots \quad \ldots \quad \ldots \quad \ldots r 18$ $\begin{array}{lllll}\text { C. J. E. } & \ldots & \ldots & \ldots & 1871\end{array}$ Fraser . ... .. $\quad \ldots \quad 1871$ Görgey ·von Görge ... $\quad \ldots \quad 1871$ $\begin{array}{lllll}\text { Guérin } & \ldots & \ldots & \ldots & 1871\end{array}$ $\begin{array}{lllll}\text { Lahure } & \ldots . . . & \ldots \ldots & \ldots & 1871\end{array}$

$\begin{array}{llllll}\text { Lefebvre } & \ldots & \ldots & \ldots & 1871\end{array}$ Mentzel. ... $\quad \ldots . \quad \ldots \quad 1871$ $\begin{array}{llll}\text { Zeitgemasse } & \ldots & \ldots & 1872\end{array}$ A Proposed New Drill _... 1872 Historiques des Remontes ... 1872 Instructions for Cavalry Out$\begin{array}{lllll}\text { posts. } & \ldots & \ldots & \ldots & 1872\end{array}$ Standing Orders 5th Lancers . 1872 $\begin{array}{llll}\text { Vorschriften } \quad \ldots & \ldots & 1872\end{array}$ Arkelay $\quad \ldots \quad \ldots \quad \ldots \quad 1872$ Bell . $\quad \ldots \quad \ldots \quad \ldots \quad 1872$ Bonie . $\quad \ldots \quad \ldots \quad \ldots \quad 1872$ $\begin{array}{llllll}\text { Cogent. } & \ldots & \ldots & \ldots & 1872\end{array}$ $\begin{array}{lllll}\text { Colomb } & \ldots & \ldots & \ldots & \ldots\end{array}$ Daru . $\quad \ldots \quad \ldots \quad \ldots \quad 1872$

Fischer. $\quad \ldots \quad \ldots \quad \ldots \quad 1872$ Formanoir $\ldots . \quad \ldots \quad \ldots \quad 1872$ $\begin{array}{lllll}\text { Garrel } & \ldots & \ldots & \ldots & 1872\end{array}$ $\begin{array}{llllll}\text { Laferrière } & \ldots & \ldots & \ldots & 1872\end{array}$ $\begin{array}{llllll}\text { Muguardt } & \ldots & \ldots & \ldots & 1872\end{array}$ $\begin{array}{lllll}\text { Napier. } & \ldots & \ldots & \ldots & 1872\end{array}$ 


\section{CAVALRY.-Continued.}

\begin{tabular}{|c|c|c|c|c|c|c|c|c|c|}
\hline lôtz & & r & $\ldots$ & 1872 & \multicolumn{2}{|c|}{ O. W. V. R. } & $\ldots$ & $\ldots$ & 1873 \\
\hline Riva & $\ldots$ & $\ldots$ & ... & 1872 & Russell & $\ldots$ & $\ldots$ & ... & 1873 \\
\hline Valter & $\ldots$ & $\ldots$ & $\ldots$ & 1872 & Schneider & $\ldots$ & $\ldots$ & $\ldots$ & 1873 \\
\hline Conferences & & $\ldots$ & $\ldots$ & 1873 & Wood & $\ldots$ & $\ldots$ & $\ldots$ & 1873 \\
\hline Das Exerzi & ieren zu & Pferd & $\ldots$ & 1873 & Anon. & $\ldots$ & $\ldots$ & ... & 1874 \\
\hline Der Kavall & lerie-Unt & teroffizie & & 1873 & Instrultion & & $\ldots$ & ... & 1874 \\
\hline Equipment & Table & $\ldots$ & $\ldots$ & 1873 & Andeutunge & & $\ldots$ & $\ldots$ & 1874 \\
\hline Handbok $i$ & fälttjens & stens & $\ldots$ & 1873 & Cavalry mo & ovement & & $\ldots$ & 1874 \\
\hline Ideen über & Kavalle & erie & $\ldots$ & 1873 & Description & détaill & & $\ldots$ & 1874 \\
\hline N. C. Offict & er's Han & ndy Boo & & 1873 & Die grossen & Kava & Mleric & Ian- & \\
\hline Outpost $d r$ & uties of & the 10 & Oth & & över & $\ldots$ & $\ldots$ & ... & 1874 \\
\hline Hussars & & .. & ... & 1873 & Instruktion & sluch & $\ldots$ & $\ldots$ & 1874 \\
\hline Règlement & sur la $S$ & Service & .. & 1873 & Annals of $t$ & he Irre & gular & lorse & 1874 \\
\hline Standing & Orders 1 & 10th Ro? & yal & & United Stat & tes Cav & alry & actics & 1874 \\
\hline Hussars & $\ldots$ & $\ldots$ & $\ldots$ & 1873 & Ardenne & $\ldots$ & $\ldots$ & $\ldots$ & 1874 \\
\hline Instruction & für die & $\ldots$ & $\ldots$ & 873 & Cavandini & $\ldots$ & $\ldots$ & $\ldots$ & 1874 \\
\hline Instruction & $s$ for $C a$ & uvalry $O$ & Dut- & & Cherfils & $\ldots$ & $\ldots$ & $\ldots$ & 1874 \\
\hline post & $\ldots$ & ... & $\ldots$ & 1873 & Congdon & $\ldots$ & $\ldots$ & $\ldots$ & 1874 \\
\hline Exercir-Re & glement & $\ldots$ & $\ldots$ & 73 & $g$ & $\ldots$ & $\cdots$ & $\cdots$ & 1874 \\
\hline Baker & $\ldots$ & $\ldots$ & $\ldots$ & 1873 & Hunt & $\ldots$ & $\ldots$ & $\ldots$ & 1874 \\
\hline Barnes & $\ldots$ & $\ldots$ & $\ldots$ & 1873 & Kaehler & $\ldots$ & $\ldots$ & $\ldots$ & 1874 \\
\hline Bell & $\ldots$ & $\ldots$ & $\ldots$ & 3 & MacKenzie & $\ldots$ & $\ldots$ & $\ldots$ & 1874 \\
\hline Besser & $\ldots$ & $\ldots$ & $\ldots$ & 1873 & Malet & $\ldots$ & $\ldots$ & $\ldots$ & 1874 \\
\hline Elcho & $\ldots$ & $\ldots$ & $\ldots$ & 1873 & Pajol & $\ldots$ & $\ldots$ & $\ldots$ & 1874 \\
\hline Forster and & Pidoll & $\ldots$ & $\ldots$ & 1873 & Susane & $\ldots$ & $\ldots$ & $\ldots$ & 1874 \\
\hline Hale & $\ldots$ & $\ldots$ & ... & 1873 & Upton & $\ldots$ & $\ldots$ & $\ldots$ & 1874 \\
\hline Hocquet & $\ldots$ & $\ldots$ & ... & 1873 & Vernois & $\ldots$ & $\cdots$ & $\ldots$ & 1874 \\
\hline Houël & $\ldots$ & $\ldots$ & $\ldots$ & 873 & Walter & $\ldots$ & $\ldots$ & $\ldots$ & 1874 \\
\hline Hozier & $\ldots$ & $\ldots$ & $\ldots$ & 1873 & Wellman & $\ldots$ & $\ldots$ & $\ldots$ & 1874 \\
\hline Le Fort & $\ldots$ & $\ldots$ & $\ldots$ & 1873 & Weyhern & $\ldots$ & $\ldots$ & $\ldots$ & 1874 \\
\hline Malcolmsor & & $\ldots$ & $\ldots$ & 1873 & Carbine exe & ercises & $\ldots$ & $\ldots$ & 1875 \\
\hline Pach zu Be & rnegg & $\ldots$ & $\ldots$ & 1873 & Cavalry reg & gulation & & $\ldots$ & 1875 \\
\hline
\end{tabular}


CAVALRY.-Continued.

\begin{tabular}{|c|c|c|c|c|c|c|c|c|}
\hline Cours abrégé & & $\ldots$ & 1875 & Becker & & & & 87 \\
\hline Ecole de Voltige & $\ldots$ & $\ldots$ & 1875 & C. J. E. & . & 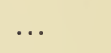 & 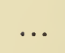 & 876 \\
\hline Exercir Reglement & $\ldots$ & $\ldots$ & 1875 & Kaehler & . & $\cdots$ & 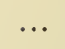 & 876 \\
\hline Handbook ... & $\ldots$ & $\ldots$ & 1875 & Loe & $\ldots$ & 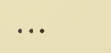 & $\ldots$ & 876 \\
\hline Instruction $d u$ & $\ldots$ & $\ldots$ & 1875 & Schmidt & $\ldots$ & $\ldots$ & $\ldots$ & 876 \\
\hline Instruction pratiqu & & $\ldots$ & 1875 & Schönbeck & ... & • & & 376 \\
\hline Movements of Cava & alry & $\ldots$ & 1875 & Siefried & .. & $\ldots$ & $\ldots$ & 87 \\
\hline Instruction in the & duties & $\ldots$ & 1875 & Waldheim & $\ldots$ & $\ldots$ & $\ldots$ & 876 \\
\hline Règlement sur & $\ldots$ & $\ldots$ & 1875 & Weyhern & & 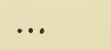 & $\ldots$ & $87 €$ \\
\hline Reglemente for & $\ldots$ & ... & 1875 & Anon. (App & pendix) & & $\ldots$ & 877 \\
\hline Standing orders for & B. C. & $\ldots$ & 1875 & Karubiner-i & Schiess- & Insto & tion & 877 \\
\hline Constitution de la & $\ldots$ & $\ldots$ & 1875 & Cambrelin & (Appen & dix) & $\ldots$ & 877 \\
\hline Der Cavallerist & $\ldots$ & $\ldots$ & 1875 & Leitfaden & $\ldots$ & $\ldots$ & $\ldots$ & 877 \\
\hline Studie über Taktik & & $\ldots$ & 1875 & Exercir-regt & lement & & $\ldots$ & 1877 \\
\hline Crane & $\ldots$ & $\ldots$ & 1875 & tition & $\ldots$ & $\ldots$ & $\ldots$ & 1877 \\
\hline Von D. & $\ldots$ & $\ldots$ & 1875 & ment $s$ & & $\ldots$ & $\ldots$ & 877 \\
\hline Elliott & $\ldots$ & $\ldots$ & 1875 & nne & $\ldots$ & .. & $\ldots$ & 1877 \\
\hline Görgey v. Görge & $\ldots$ & $\ldots$ & 1875 & son & $\ldots$ & $\ldots$ & $\ldots$ & 1877 \\
\hline Hamley & $\ldots$ & $\ldots$ & 1875 & & $\ldots$ & $\ldots$ & $\ldots$ & 1877 \\
\hline Hassell & $\ldots$ & $\ldots$ & 1875 & Lufried & $\ldots$ & $\ldots$ & $\ldots$ & 1877 \\
\hline Waldstatten & $\ldots$ & $\ldots$ & 1875 & Mackenzen & $\ldots$ & $\ldots$ & $\ldots$ & 1877 \\
\hline Ansichten eines & $\cdots$ & $\cdots$ & 1876 & $\mathrm{R}$ & $\ldots$ & $\ldots$ & $\ldots$ & 1877 \\
\hline Exercir Reglement & $\ldots$ & $\ldots$ & 1876 & Wellmann & .. & $\cdots$ & $\ldots$ & 1877 \\
\hline Instruction sur le se & service & $\ldots$ & 1876 & Widdern & $\ldots$ & $\ldots$ & $\ldots$ & 1877 \\
\hline Instructions for $\mathrm{Ca}$ & avalry & $\cdots$ & 1876 & Manual of & Tro & sport (A & & \\
\hline Instructions for $\mathrm{Ca}$ & avalry 0 & Dut- & & pendix) & & & & 1878 \\
\hline post & $\cdots$ & $\cdots$ & 1876 & Notes on $C$ & avalry & Tactics & . & 1878 \\
\hline Notizen für Unter & offiziere & $\ldots$ & 1876 & Nozioni & $\ldots$ & $\ldots$ & $\ldots$ & 1878 \\
\hline Reglement über & $\ldots$ & . & 1876 & Das Feuerg & gefecht & & $\ldots$ & 1878 \\
\hline \multicolumn{3}{|c|}{ Reglement sur l'instruction } & 1876 & \multicolumn{3}{|c|}{ Kommando-Tubelle } & $\cdots$ & 1878 \\
\hline \multicolumn{3}{|c|}{ Regulations for the Movements } & 1876 & \multicolumn{4}{|c|}{ Manual and Firing Exercise } & 1878 \\
\hline Tenth Royal Hussa & $a r$ & & 1876 & Zum Unter & richts & & $\ldots$ & 187 \\
\hline
\end{tabular}




\section{CAVALRY.-Continued.}

Manual for Regimental Trans$\begin{array}{lllll}\text { port } \quad \ldots & \ldots & \ldots & 1878\end{array}$

Aerts (Appendix) $\ldots . \quad \ldots \quad 1878$

$\begin{array}{lllll}\text { Cagni } & \ldots & \ldots & \ldots & 1878\end{array}$

Corvisart-Montmartin $\quad \ldots \quad 1878$

$\begin{array}{lllll}\text { Klatte } & \ldots & \ldots & \ldots & 1878\end{array}$

$\begin{array}{lllll}\text { Kottwitz } & \ldots & \ldots & \ldots & 1878\end{array}$

C. v. L. $\quad \ldots \quad \ldots \quad \ldots, 1878$

$\begin{array}{lllll}\text { Plotz } & \ldots & \ldots & \ldots & 1878\end{array}$

$\begin{array}{lllll}\text { Poten } & \ldots & \ldots & \ldots & 1878\end{array}$

$\begin{array}{lllll}\text { Ran } \quad \ldots & \ldots & \ldots & 1878\end{array}$

$\begin{array}{lllll}\text { Rotenham } & \ldots & \ldots & \ldots & 1878\end{array}$

$\begin{array}{lllll}\text { Trotter } \quad \ldots & \ldots & \ldots & 1878\end{array}$

Twentyman (Appendix) $\quad \ldots \quad 1878$

$\begin{array}{llllll}\text { Walter } \quad \ldots & \ldots & \ldots & 1878\end{array}$

$\begin{array}{lllll}\text { Widdern } & \ldots & \ldots & \ldots & 1878\end{array}$

$\begin{array}{llll}\text { Instrultionsbuch } & \ldots & \ldots & 1879\end{array}$

Karabiner ... $\quad \ldots \quad \ldots \quad 1879$

$\begin{array}{llll}\text { Caricamento } & \ldots & \ldots & 1879\end{array}$

$\begin{array}{lllll}\text { Balthasar } & \ldots & \ldots & \ldots & 1879\end{array}$

Leitfaden beim $\quad \ldots \quad \ldots \quad 1879$

$\begin{array}{llllll}\text { Haber } \quad \ldots & \ldots & \ldots & 1879\end{array}$

$\begin{array}{lllll}\text { Kaehler } \quad \ldots & \ldots & \ldots & 1879\end{array}$

$\begin{array}{lllll}\text { Morawetz } & \ldots & \ldots & \ldots & 1879\end{array}$

$\begin{array}{lllll}\text { Natzmer } \quad \ldots & \ldots & \ldots & 1879\end{array}$

$\begin{array}{lllll}\text { Istruzioni } & \ldots & \ldots & \ldots & 1880\end{array}$

Regolamento $\quad \ldots \quad \ldots \quad 1880$

$\begin{array}{lllll}\text { Bell } & \ldots & \ldots & \ldots & 1880\end{array}$

$\begin{array}{lllll}\text { Dewall } \quad \ldots & \ldots & \ldots & 1880\end{array}$

Foucart $\quad \ldots \quad \ldots \quad \ldots \quad \ldots \quad 1880$

Görgey von Görge ... $\quad \ldots \quad 1880$

Merta $\begin{array}{lllll}\text { Vittré } & \ldots & \ldots & \ldots & 1880\end{array}$

$\begin{array}{llll}\text { Beitrage zur } & \ldots & \ldots & 1881\end{array}$

Il libro del soldato ... $\quad \ldots \quad 18 \varepsilon 1$

Bouillé $\quad \ldots \quad \ldots \quad \ldots \quad \ldots 1881$

$\begin{array}{lllll}\text { Brix } & \ldots & \ldots & \ldots & 1881\end{array}$

$\begin{array}{lllll}\text { Cooper } & \ldots & \ldots & \ldots & 1881\end{array}$

Eccheli del Dosse ... $\quad$.. 1881

Lord George Paget... ... 1881

$\begin{array}{llllll}\text { Parr } & \ldots & \ldots & \ldots & 1881\end{array}$

$\begin{array}{lllll}\text { Schmidt } & \ldots & \ldots & \ldots & 1881\end{array}$

$\begin{array}{lllll}\text { Istruzione } & \ldots & \ldots & \ldots & 1882\end{array}$

$\begin{array}{lllll}\text { Cortijo } & \ldots & \ldots & \ldots & 1882\end{array}$

$\begin{array}{lllll}\text { Costa } & \ldots & \ldots & \ldots & 1882\end{array}$

$\begin{array}{lllll}\text { Gnecco } & \ldots & \ldots & \ldots & 1882\end{array}$

$\begin{array}{lllll}\text { Stent } & \ldots & \ldots & \ldots & 1882\end{array}$

$\begin{array}{lllll}\text { Chabot } & \ldots & \ldots & \ldots & 1883\end{array}$

$\begin{array}{lllll}\text { Drygalski } & \ldots & \ldots & \ldots & 1883\end{array}$

Zeitschrift für Pferdekunde 1883

Die Thätigkeit $\quad \ldots \quad \ldots \quad 1884$

Uber die Treffen-Taktik ... 1884

Was haben wir Erwarten ?... 1884

$\begin{array}{lllll}\text { Lahure } \quad \ldots & \ldots & \ldots & 1884\end{array}$

Pelet-Narbonne $\quad \ldots \quad \ldots \quad 1884$

$\begin{array}{lllll}\text { Thomann } & \ldots & \ldots & \ldots & 1884\end{array}$

$\begin{array}{lllll}\text { Trench } & \ldots & \ldots & \ldots & 1884\end{array}$

$\begin{array}{lllll}\text { Ubiez } & \ldots & \ldots & \ldots & 1884\end{array}$

Notes sur les cavaleries $\begin{array}{llll}\text { étrangères } & \ldots & \ldots & 1885\end{array}$

Dérué $\quad \ldots \quad \ldots \quad \ldots \quad 1885$

$\begin{array}{lllll}\text { Girette } \quad \ldots & \ldots & \ldots & 1885\end{array}$

$\begin{array}{lllll}\text { Haugwitz } & \ldots & \ldots & \ldots & 1885\end{array}$

$\begin{array}{lllll}\text { Hube } & \ldots & \ldots & \ldots & 1885\end{array}$ 
CAVALRY.-Continued.

$\begin{array}{lllllllllll}\text { Jaeglé } & \ldots & \ldots & \ldots & 1885 & \text { Weyhern } & \ldots & \ldots & \ldots & 1885\end{array}$

$\begin{array}{lllllllll}\text { Schmidt } & \ldots & \ldots & \ldots & 1885 & \text { Organization } & \ldots & \ldots & 1886\end{array}$

Von Thümen $\quad \ldots \quad \ldots \quad 1885 \quad$ A travers la Cavalerie $\quad \ldots \quad 1886$

$\begin{array}{lllllllllll}\text { Ubiez } & \ldots & \ldots & \ldots & 1885 & \text { Brunet } & \ldots & \ldots & \ldots & 1886\end{array}$

$\begin{array}{lllll}\text { Lahousen } & \ldots & \ldots & \ldots & 1885\end{array}$

\section{VETERINARY.}

Kimon of Athens

Mago

Sextus Julius Africanus

Apsyrtus

Hippocrates

$\begin{array}{lllll}\text { Litorius } \quad \ldots & \ldots & \ldots & 400\end{array}$

Theomnestus $\quad \ldots \quad \ldots \quad 400$

$\begin{array}{lllll}\text { Tiberius } & \ldots & \ldots & \ldots & 400\end{array}$

Hierocles $\quad \ldots \quad \ldots \quad \ldots \quad 950$

$\begin{array}{lllll}\text { Crescenzi } & \ldots & \ldots & \ldots & 1300\end{array}$

Giraldes $\quad \ldots \quad \ldots \quad \ldots \quad 1318$

Abd Ullah Ben Safi

Ruffus

Cato

Varro

Columella

$\begin{array}{lllll}\text { Palladius } & \ldots & \ldots & \ldots & 1472\end{array}$

Kurrut ul-Mulk $\quad \ldots \quad \ldots \quad 1478$

Diaz $\quad \ldots \quad \ldots \quad \ldots \quad 1495$

Anon. $\quad \ldots \quad \ldots \quad \ldots \quad 1506$

$\begin{array}{lllll}\text { Culombre } & \ldots & \ldots & \ldots & 1518\end{array}$

$\begin{array}{lllll}\text { Vegetius } \quad \ldots & \ldots & \text {.. } & 1524\end{array}$

Antonio de Guevary

Ruel
... $15 ? 9$

... 1530
Naaldwyck

Francisco de la Reina

Scacco

Blundeville

Carraciolo ...

Massé

Malbie

Calvo

Mascall

Zamora

Cito

Scaccho

Ruini

Hervard

Scacco

L. W. C

Markham

Markham

Markham

Markham

Colombri

Ramirez

De Grey

T. T. D. E. M.
.. 1531

... 1546

... 1553

... 1565

... 1566

... 1568

... 1576

... 1582

... 1587

... 1588

... 1590

... 1591

.. 1598

.. 1599

... 1602

... 1610

... 1610

... 1615

.. 1616

... 1620

... 1622

... 1629

... 1639

... 1647 
VETERINARY.-Continued.

$\begin{array}{lllll}\text { Baret } \quad \ldots & \ldots & \ldots & 1651\end{array}$

$\begin{array}{lllll}\text { Paracuellos ... } & \ldots & \ldots & 1658\end{array}$

Ud-Din Ahmad $\quad \ldots \quad \ldots \quad 1660$

Arredondo ... $\quad \ldots \quad$... 1661

$\begin{array}{lllll}\text { Solleysel } & \ldots & \ldots & \ldots & 1664\end{array}$

$\begin{array}{lllll}\text { Lower } & \ldots & \ldots & \ldots & 1668\end{array}$

$\begin{array}{lllll}\text { Claridge } & \ldots & \ldots & \ldots & 1670\end{array}$

$\begin{array}{lllll}\text { J. } \mathrm{H} . & \ldots & \ldots & \ldots & 1672\end{array}$

Almond $\quad \ldots \quad \ldots \quad \ldots \quad 1673$

$\begin{array}{lllll}\text { Andrade } & \ldots & \ldots & \ldots & 1678\end{array}$

De Winter $\quad \ldots \quad \ldots \quad 1678$

$\begin{array}{llllll}\text { Rego } & \ldots & \ldots & \ldots & 1679\end{array}$

$\begin{array}{llllll}\text { Borges } & \ldots & \ldots & \ldots & 1680\end{array}$

$\begin{array}{lllll}\text { Tryon } & \ldots & \ldots & \ldots & 1680\end{array}$

$\begin{array}{lllll}\text { Markham } & \ldots & \ldots & \ldots & 1681\end{array}$

$\begin{array}{lllll}\text { Conde } & \ldots & \ldots & \ldots & 1685\end{array}$

Ambros $\quad \ldots \quad \ldots \quad \ldots \quad 1686$

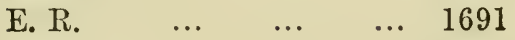

Wallis $\quad \ldots \quad \ldots \quad \ldots \quad 1695$

$\begin{array}{lllll}\text { Trutta } & \ldots & \ldots & \ldots & 1699\end{array}$

$\begin{array}{lllll}\text { Anon. } & \ldots & \ldots & \ldots & 1700\end{array}$

Anon. $\quad \ldots \quad \ldots \quad \ldots \quad 1701$

$\begin{array}{lllll}\text { Zuniga } & \ldots & \ldots & \ldots & 1705\end{array}$

$\begin{array}{lllll}\text { L. G. } & \ldots & \ldots & \ldots & 1708\end{array}$

Sampayo $\quad \ldots \quad \ldots \quad \ldots \quad \ldots 1710$

Muhammad Ali Hazin _.. 1716

Sande $\quad \ldots \quad \ldots \quad \ldots \quad \ldots 1717$

Gibson $\quad \ldots \quad \ldots \quad \ldots \quad 1720$

Gibson $\quad \ldots \quad \ldots \quad \ldots \quad 1721$

$\begin{array}{llllll}\text { Dudley } \quad \ldots & \ldots & \ldots & 1724\end{array}$

$\begin{array}{lllll}\text { Begrieres } & \ldots & \ldots & \ldots & 1725\end{array}$

Cavero
Cavero

Cavero

Cavero

Benavides ...

Cavero

Morabda

Royo

Saunier

Zamora

Allen

Bracken

Cavero

Garsault

Bracken

Monto

Deigendesch

Bailey

Lafosse

Gibson

Bridges

Bartlett

Gibson

Cavero

Bernad

Reeves

Wood

Bartlett

Ellis

Osmer

Baker

Anon.

Bartlett

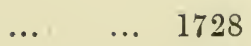

.. 1729

... 1731

.. 1732

... 1732

.. 1732

... 1734

... 1734

... 1735

... 1737

... 1738

.. 1740

... 1741

... 1742

.. 1742

.. 1744

... 1746

... 1749

.. 1750

.. 1752

.. 1754

.. 1755

.. 1756

.. 1757

.. 1757

.. 1757

.. 1758

... 1758

... 1758

.. 1760

$\begin{array}{ll}\ldots & 1727\end{array}$ 
VETERINARY.-Continued.

$\begin{array}{llllllllll}\text { Sind } & \ldots & \ldots & \ldots & 1764 & \text { Robinet } & \ldots & \ldots & \ldots & 1777 \\ \text { Reitzenstein } & \ldots & \ldots & 1764 & \text { Anon } & \ldots & \ldots & \ldots & 1778 \\ \text { Smidt } & \ldots & \ldots & \ldots & 1764 & \text { Maples } & \ldots & \ldots & \ldots & 1778 \\ \text { Wallis } & \ldots & \ldots & \ldots & 1764 & \text { Hartmann } & \ldots & \ldots & \ldots & 1779 \\ \text { Bourgelat } & \ldots & \ldots & \ldots & 1765 & \text { Wefeld } & \ldots & \ldots & \ldots & 1779 \\ \text { Tamm } & \ldots & \ldots & \ldots & 1765 & \text { Wolstein } & \ldots & \ldots & \ldots & 1779 \\ \text { Lafosse } & \ldots & \ldots & \ldots & 1766 & \text { Erhmann } & \ldots & \ldots & \ldots & 1780 \\ \text { Oebschelwitz } & \ldots & \ldots & 1766 & \text { Naumann } & \ldots & \ldots & \ldots & 1780 \\ \text { Sind } & \ldots & \ldots & \ldots & 1766 & \text { Plouqnet } & \ldots & \ldots & \ldots & 1780 \\ \text { Textor } & \ldots & \ldots & \ldots & 1767 & \text { Rohlwes } & \ldots & \ldots & \ldots & 1780 \\ \text { Sind } & \ldots & \ldots & \ldots & 1768 & \text { Roserus } & \ldots & \ldots & \ldots & 1780 \\ \text { Zorn } & \ldots & \ldots & \ldots & 1768 & \text { Lapuerta } & \ldots & \ldots & \ldots & 1781 \\ \text { Eberhart } & \ldots & \ldots & \ldots & 1769 & \text { Zylnnhardt } & \ldots & \ldots & \ldots & 1782 \\ \text { Hurel } & \ldots & \ldots & \ldots & 1769 & \text { Clater } & \ldots & \ldots & \ldots & 1783 \\ \text { Sandoval } & \ldots & \ldots & \ldots & 1769 & \text { Rohlwes } & \ldots & \ldots & \ldots & 1783 \\ \text { Sind } & \ldots & \ldots & \ldots & 1769 & \text { Griffiths } & \ldots & \ldots & \ldots & 1784 \\ \text { Abildgaard } & \ldots & \ldots & \ldots & 1770 & \text { Anon. } & \ldots & \ldots & \ldots & 1786 \\ \text { Bourgelat } & \ldots & \ldots & \ldots & 1770 & \text { Bonsi } & \ldots & \ldots & \ldots & 1786 \\ \text { Gaab } & \ldots & \ldots & \ldots & 1770 & \text { Prosser } & \ldots & \ldots & \ldots & 1786 \\ \text { Sind } & \ldots & \ldots & \ldots & 1770 & \text { Riem } & \ldots & \ldots & \ldots & 1786 \\ \text { Robertson } & \ldots & \ldots & \ldots & 1771 & \text { Rus } & \ldots & \ldots & \ldots & 1786 \\ \text { Zeiher } & \ldots & \ldots & \ldots & 1771 & \text { Toggia } & \ldots & \ldots & \ldots & 1786 \\ \text { Anon. } & \ldots & \ldots & \ldots & 1772 & \text { Blumenschein } & \ldots & \ldots & 1787 \\ \text { Lafosse } & \ldots & \ldots & \ldots & 1772 & \text { Briick } & \ldots & \ldots & \ldots & 1787 \\ \text { Blunt } & \ldots & \ldots & \ldots & 1773 & \text { Daum } & \ldots & \ldots & \ldots & 1787 \\ \text { Brugnone } & \ldots & \ldots & \ldots & 1774 & \text { Hiver } & \ldots & \ldots & \ldots & 1787 \\ \text { Anon. } & \ldots & \ldots & \ldots & 1775 & \text { Hulfreich } & \ldots & \ldots & \ldots & 1787 \\ \text { Prizelius } & \ldots & \ldots & \ldots & 1775 & \text { Kersting } & \ldots & \ldots & \ldots & 1787 \\ \text { Lafosse } & \ldots & \ldots & \ldots & 1776 & \text { Wolstein } & \ldots & \ldots & \ldots & 1787 \\ \text { Walmerode } & \ldots & \ldots & \ldots & 1777 & \text { Clark } & \ldots & \ldots & \ldots & 1788 \\ \text { Hartmann } & \ldots & \ldots & \ldots & 1777 & \text { Daum } & \ldots & \ldots & \ldots & 1788 \\ \text { Prizelius } & \ldots & \ldots & \ldots & 1777 & \text { Saloter } & \ldots & \ldots & \ldots & 1788 \\ & & & & & & & & & \end{array}$


VETERINARY.-Continued.

$\begin{array}{llllllllll}\text { Forrester } & \ldots & \ldots & \ldots & 1788 & \text { Schäfer } & \ldots & \ldots & \ldots & 1795 \\ \text { Kersting } & \ldots & \ldots & \ldots & 1788 & \text { Steinhoff } & \ldots & \ldots & \ldots & 1795 \\ \text { Lorentz } & \ldots & \ldots & \ldots & 1788 & \text { Davidson } & \ldots & \ldots & \ldots & 1795 \\ \text { Taplin } & \ldots & \ldots & \ldots & 1788 & \text { Anon. } & \ldots & \ldots & \ldots & 1796 \\ \text { Chabert } & \ldots & \ldots & \ldots & 1789 & \text { Anon. } & \ldots & \ldots & \ldots & 1796 \\ \text { Flandrin } & \ldots & \ldots & \ldots & 1789 & \text { Bracy Clark } & \ldots & \ldots & 1796 \\ \text { Huzard } & \ldots & \ldots & \ldots & 1789 & \text { Hunter } & \ldots & \ldots & \ldots & 1796 \\ \text { Kahn } & \ldots & \ldots & \ldots & 1789 & \text { Lawrence } & \ldots & \ldots & \ldots & 1796 \\ \text { Kersting } & \ldots & \ldots & \ldots & 1789 & \text { Trautenberg } & \ldots & \ldots & 1796 \\ \text { Montes } & \ldots & \ldots & \ldots & 1789 & \text { Anon. } & \ldots & \ldots & \ldots & 1797 \\ \text { Oehlmann } & \ldots & \ldots & \ldots & 1789 & \text { Buschendorf } & \ldots & \ldots & 1797 \\ \text { Sander } & \ldots & \ldots & \ldots & 1789 & \text { Chabert and } & \text { Huzard } & \ldots & 1797 \\ \text { Wallmerode } & & \ldots & \ldots & 1790 & \text { Downing } & \ldots & \ldots & \ldots & 1797 \\ \text { Ploucquet } & \ldots & \ldots & \ldots & 1790 & \text { Mayr } & \ldots & \ldots & \ldots & 1797 \\ \text { Robertson } & \ldots & \ldots & \ldots & 1791 & \text { Schreger } & \ldots & \ldots & \ldots & 1797 \\ \text { Schmidt } & \ldots & \ldots & \ldots & 1790 & \text { Tennecker } & \ldots & \ldots & \ldots & 1797 \\ \text { Anon. } & \ldots & \ldots & \ldots & 1791 & \text { Schmidt } & \ldots & \ldots & \ldots & 1798 \\ \text { Wallmerode } & \ldots & \ldots & 1791 & \text { Magazin } & \ldots & \ldots & \ldots & 1799 \\ \text { Ford } & \ldots & \ldots & \ldots & 1791 & \text { Tennecker } & \ldots & \ldots & \ldots & 1799 \\ \text { Gilpin } & \ldots & \ldots & \ldots & 1791 & \text { Anon. } & \ldots & \ldots & \ldots & 1800 \\ \text { Anm } & \ldots & \ldots & \ldots & 1792 & \text { Gotthard } & \ldots & \ldots & \ldots & 1800 \\ \text { Anon. } & \ldots & \ldots & \ldots & 1793 & \text { Hugues } & \ldots & \ldots & \ldots & 1800 \\ \text { Meyer } & \ldots & \ldots & \ldots & 1793 & \text { Journal } & \ldots & \ldots & \ldots & 1800 \\ \text { Werner } & \ldots & \ldots & \ldots & 1793 & \text { Klatte } & \ldots & \ldots & \ldots & 1800 \\ \text { Anon. } & \ldots & \ldots & \ldots & 1794 & \text { Naumann } & \ldots & \ldots & \ldots & 1800 \\ \text { Rober } & \ldots & \ldots & \ldots & 1794 & \text { Oelilmann } & \ldots & \ldots & \ldots & 1800 \\ \text { Tennecker } & \ldots & \ldots & \ldots & 1791 & \text { Schanzel } & \ldots & \ldots & \ldots & 1800 \\ \text { Bonsi } & \ldots & \ldots & \ldots & 1795 & \text { Uden } & \ldots & \ldots & \ldots & 1800 \\ \text { Davidson } & \ldots & \ldots & \ldots & 1795 & \text { Viborg and } & \text { Neergard } & \ldots & 1800 \\ \text { Jewrett } & \ldots & \ldots & \ldots & 1795 & \text { Vierordt } & \ldots & \ldots & \ldots & 1800 \\ \text { Letang } & \ldots & \ldots & \ldots & 1795 & \text { White } & \ldots & \ldots & \ldots & 1800 \\ \text { Moorcroft } & \ldots & \ldots & \ldots & 1795 & \text { Anon. } & \ldots & \ldots & \ldots & 1801 \\ & & & & & & & & & \end{array}$


VETERINARY.-Continued.

$\begin{array}{lllll}\text { K. F. B. } & \ldots & \ldots & \ldots & 1801\end{array}$

$\begin{array}{lllll}\text { Coleman } & \ldots & \ldots & \ldots & 1801\end{array}$

Didrichsen ... $\quad \ldots \quad \ldots \quad 1801$

$\begin{array}{lllll}\text { Lehman } & \ldots & \ldots & \ldots & 1801\end{array}$

Rumpelt $\quad \ldots \quad \ldots \quad \ldots \quad 1801$

$\begin{array}{llllll}\text { Ryding } & \ldots & \ldots & \ldots & 1801\end{array}$

$\begin{array}{lllll}\text { Tennecker } & \ldots & \ldots & \ldots & 1801\end{array}$

$\begin{array}{lllll}\text { Viborg } \quad \ldots & \ldots & \ldots & 1801\end{array}$

Anon. $\quad \ldots \quad$... $\quad \ldots \quad 1802$

Ammon $\quad \ldots \quad \ldots \quad \ldots \quad 1802$

$\begin{array}{lllll}\text { Blaine } & \ldots & \ldots & \ldots & 1802\end{array}$

$\begin{array}{lllll}\text { Boardman } & \ldots & \ldots & \ldots & 1802\end{array}$

$\begin{array}{lllll}\text { Böhmen } \quad \ldots & \ldots & \ldots & 1802\end{array}$

Denny $\quad \ldots \quad \ldots \quad \ldots \quad 1802$

$\begin{array}{lllll}\text { Grellier } \quad \ldots & \ldots & \ldots & 1802\end{array}$

$\begin{array}{lllll}\text { Oehlmann } & \ldots & \ldots & \ldots & 1802\end{array}$

$\begin{array}{lllll}\text { Tennecker } & \ldots & \ldots & \ldots & 1802\end{array}$

Anon. $\quad \ldots \quad \ldots \quad \ldots \quad 1803$

$\begin{array}{lllll}\text { Blaine } & \ldots & \ldots & \ldots & 1803\end{array}$

Castor $\quad \ldots \quad \ldots . \quad \ldots \quad 1803$

Feron $\quad \ldots \quad \ldots \quad \ldots \quad 1803$

Gmeiner $\quad \ldots \quad \ldots \quad \ldots \quad 1803$

$\begin{array}{lllll}\text { Laubender } & \ldots & \ldots & \ldots & 1803\end{array}$

$\begin{array}{lllll}\text { Pilger } & \ldots & \ldots & \ldots & 1803\end{array}$

Anon. $\quad \ldots \quad \ldots . \quad \ldots \quad 1804$

Ammon $\quad \ldots \quad \ldots \quad \ldots \quad 1804$

Konigstadter $\quad$.. $\quad \ldots \quad 1804$

Laubender $\quad \ldots \quad$... $\quad \ldots \quad 1804$

Rohlwes _.. $\quad \ldots \quad \ldots \quad 1804$

Saint Paul ... $\quad \ldots \quad \ldots \quad \ldots \quad 1804$

$\begin{array}{lllll}\text { Tscheulin } & \ldots & \ldots & \ldots & 1804\end{array}$

White $\begin{array}{lllll}\text { Anon. } & \ldots & \ldots & \ldots & 1805\end{array}$

$\begin{array}{lllll}\text { Hoffman } & \ldots & \ldots & \ldots & 1805\end{array}$

$\begin{array}{lllll}\text { Laubender } & \ldots & \ldots & \ldots & 1805\end{array}$

$\begin{array}{lllll}\text { Snape } & \ldots & \ldots & \ldots & 1805\end{array}$

$\begin{array}{lllll}\text { Waldinger } & \ldots & \ldots & \ldots & 1805\end{array}$

$\begin{array}{lllll}\text { Wolstein } & \ldots & \ldots & \ldots & 1805\end{array}$

Anon. $\quad \ldots \quad \ldots \quad \ldots \quad 1806$

$\begin{array}{lllll}\text { Busch } \quad \ldots & \ldots & \ldots & 1806\end{array}$

$\begin{array}{lllll}\text { Capuron } & \ldots & \ldots & \ldots & 1806\end{array}$

$\begin{array}{lllll}\text { Fehr } & \ldots & \ldots & \ldots & 1806\end{array}$

Shipp $\quad \ldots \quad \ldots \quad \ldots \quad 1806$

Anon. $\quad \ldots \quad \ldots \quad \ldots \quad 1807$

$\begin{array}{lllll}\text { Ammon } & \ldots & \ldots & \ldots & 1807\end{array}$

$\begin{array}{lllll}\text { Oehlmann } & \ldots & \ldots & \ldots & 1807\end{array}$

$\begin{array}{lllll}\text { Toggia } & \ldots & \ldots & \ldots & 1807\end{array}$

$\begin{array}{lllll}\text { Wohlstein } & \ldots & \ldots & \ldots & 1807\end{array}$

$\begin{array}{lllll}\text { Zipf } & \ldots & \ldots & \ldots & 1807\end{array}$

$\begin{array}{lllll}\text { Ammon } \quad \ldots & \ldots & \ldots & 1808\end{array}$

$\begin{array}{lllll}\text { Ammon } & \ldots & \ldots & \ldots & 1808\end{array}$

$\begin{array}{llllll}\text { Tolnay } & \ldots & \ldots & \ldots & 1808\end{array}$

$\begin{array}{llllll}\text { Waldinger } & \ldots & \ldots & \ldots & 1808\end{array}$

$\begin{array}{lllll}\text { Anon. } & \text {.. } & \ldots & \ldots & 1809\end{array}$

$\begin{array}{lllll}\text { Ammon } \quad \ldots & \ldots & \ldots & 1809\end{array}$

$\begin{array}{lllll}\text { Aygalenge } & \ldots & \ldots & \ldots & 1809\end{array}$

$\begin{array}{lllll}\text { Jacobi } \quad \ldots & \ldots & \ldots & 1809\end{array}$

$\begin{array}{lllll}\text { Viborg } \quad \ldots & \ldots & \ldots & 1809\end{array}$

$\begin{array}{lllll}\text { Waldinger } & \ldots & \ldots & \ldots & 1809\end{array}$

$\begin{array}{lllll}\text { Wilson } \quad \ldots & \ldots & \ldots & 1809\end{array}$

Amoureux $\ldots \quad \ldots \quad \ldots \quad 1810$

$\begin{array}{lllll}\text { Hulfreich } & \ldots & \ldots & \ldots & 1810\end{array}$

$\begin{array}{lllll}\text { Klatte } & \ldots & \ldots & \ldots & 1810\end{array}$

$\begin{array}{lllll}\text { Amoureux } & \ldots & \ldots & \ldots & 1810\end{array}$ 
-VETERINARY._Continued.

$\begin{array}{llllllllll}\text { Hulfreich } & \ldots & \ldots & \ldots & 1810 & \text { Anon. } & \ldots & \ldots & \ldots & 1816 \\ \text { Klatte } & \ldots & \ldots & \ldots & 1810 & \text { Clark } & \ldots & \ldots & \ldots & 1816 \\ \text { Sander } & \ldots & \ldots & \ldots & 1810 & \text { Lappe } & \ldots & \ldots & \ldots & 1816 \\ \text { Tscheulin } & \ldots & \ldots & \ldots & 1810 & \text { Laubender } & \ldots & \ldots & \ldots & 1816 \\ \text { Blumhoser } & \ldots & \ldots & \ldots & 1811 & \text { Lawrence } & \ldots & \ldots & \ldots & 1816 \\ \text { Bojanus } & \ldots & \ldots & \ldots & 1811 & \text { Rohlwes } & \ldots & \ldots & \ldots & 1816 \\ \text { Laubender } & \ldots & \ldots & \ldots & 1811 & \text { Tennecker } & \ldots & \ldots & \ldots & 1816 \\ \text { Naumann } & \ldots & \ldots & \ldots & 1811 & \text { Wagner } & \ldots & \ldots & \ldots & 1816 \\ \text { Pearson } & \ldots & \ldots & \ldots & 1811 & \text { Anon. } & \ldots & \ldots & \ldots & 1817 \\ \text { Pollnitz } & \ldots & \ldots & \ldots & 1811 & \text { Dupuy } & \ldots & \ldots & \ldots & 1817 \\ \text { Simon } & \ldots & \ldots & \ldots & 1811 & \text { Jauze } & \ldots & \ldots & \ldots & 1817 \\ \text { Tscheulin } & \ldots & \ldots & \ldots & 1811 & \text { Sallwurk } & \ldots & \ldots & \ldots & 1817 \\ \text { Laubender } & \ldots & \ldots & \ldots & 1812 & \text { Schwab and } & \text { Will } & \ldots & \ldots & 1817 \\ \text { Toggia } & \ldots & \ldots & \ldots & 1812 & \text { Tscheulin } & \ldots & \ldots & \ldots & 1817 \\ \text { Waldinger } & \ldots & \ldots & \ldots & 1812 & \text { Veith } & \ldots & \ldots & \ldots & 1817 \\ \text { Clark } & \ldots & \ldots & \ldots & 1813 & \text { Klatte } & \ldots & \ldots & \ldots & 1818 \\ \text { Waldinger } & \ldots & \ldots & \ldots & 1813 & \text { Pollnitz } & \ldots & \ldots & \ldots & 1818 \\ \text { Clark } & \ldots & \ldots & \ldots & 1814 & \text { Smith } & \ldots & \ldots & \ldots & 1818 \\ \text { Erdelyi } & \ldots & \ldots & \ldots & 1814 & \text { Thielemann } & & \ldots & \ldots & 1818 \\ \text { L. A. F. } & \ldots & \ldots & \ldots & 1814 & \text { Weidenk eller } & \ldots & \ldots & 1818 \\ \text { Klatte } & \ldots & \ldots & \ldots & 1814 & \text { Wilkinson } & \ldots & \ldots & \ldots & 1818 \\ \text { Peall } & \ldots & \ldots & \ldots & 1814 & \text { Daum } & \ldots & \ldots & \ldots & 1819 \\ \text { Peck } & \ldots & \ldots & \ldots & 1814 & \text { Merk } & \ldots & \ldots & \ldots & 1819 \\ \text { Tindall } & \ldots & \ldots & \ldots & 1814 & \text { Rohlwes } & \ldots & \ldots & \ldots & 1819 \\ \text { Clark } & \ldots & \ldots & \ldots & 1815 & \text { Schmidt } & \ldots & \ldots & \ldots & 1819 \\ \text { Klatte } & \ldots & \ldots & \ldots & 1815 & \text { Tennecker } & \ldots & \ldots & \ldots & 1819 \\ \text { Mablbeck } & \ldots & \ldots & \ldots & 1815 & \text { Toggia } & \ldots & \ldots & \ldots & 1819 \\ \text { Merk } & \ldots & \ldots & \ldots & 1815 & \text { Tscheulin } & \ldots & \ldots & \ldots & 1819 \\ \text { Tennecker } & \ldots & \ldots & \ldots & 1815 & \text { Lawson } & \ldots & \ldots & \ldots & 1820 \\ \text { Tscheulin } & \ldots & \ldots & \ldots & 1815 & \text { Merk } & \ldots & \ldots & \ldots & 1820 \\ \text { Waldinger } & \ldots & \ldots & \ldots & 1815 & \text { Pollnitz } & \ldots & \ldots & \ldots & 1820 \\ \text { White } & \ldots & \ldots & \ldots & 1815 & \text { Schlichting } & \ldots & \ldots & \ldots & 1820\end{array}$




\section{VETERINARY.-Continued.}

$\begin{array}{lllll}\text { Schwab } & \ldots & \ldots & \ldots & 1820\end{array}$

$\begin{array}{lllll}\text { Tennecker } & \ldots & \ldots & \ldots & 1820\end{array}$

$\begin{array}{lllll}\text { Waldinger } & \ldots & \ldots & \ldots & 1820\end{array}$

$\begin{array}{lllll}\text { Walther } \quad \ldots & \ldots & \ldots & 1820\end{array}$

$\begin{array}{lllll}\text { Bohlmann } & \ldots & \ldots & \ldots & 1821\end{array}$

Hochstetter

$\begin{array}{lllll}\text { Ribbe } & \ldots & \ldots & \ldots & 1821\end{array}$

$\begin{array}{lllll}\text { Schwab } & \ldots & \ldots & \ldots & 1821\end{array}$

$\begin{array}{lllll}\text { Tennecker } & \ldots & \ldots & \ldots & 1821\end{array}$

$\begin{array}{lllll}\text { Tscheulin } & \ldots & \ldots & \ldots & 1821\end{array}$

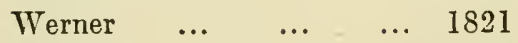

$\begin{array}{llllll}\text { Clark } & \ldots & \ldots & \ldots & 1822\end{array}$

$\begin{array}{lllll}\text { Cusac } & \ldots & \ldots & \ldots & 1822\end{array}$

Daum

Dieterrichs

Halberbach

$\begin{array}{lllll}\text { Hoerdt } & \ldots & \ldots & \ldots & 1822\end{array}$

$\begin{array}{lllll}\text { Hofacker } & \ldots & \ldots & \ldots & 1822\end{array}$

$\begin{array}{llllll}\text { Morel } & \ldots & \ldots & \ldots & 1822\end{array}$

Rohlwes $\quad \ldots \quad \ldots \quad \ldots \quad \ldots \quad 1822$

$\begin{array}{lllll}\text { Schlichting ... } & \ldots & \ldots & 1822\end{array}$

$\begin{array}{lllll}\text { Tennecker } & \ldots & \ldots & \ldots & 1822\end{array}$

$\begin{array}{lllll}\text { Tscheulin } & \ldots & \ldots & \ldots & 1822\end{array}$

$\begin{array}{llll}\text { Weidenkeller } \quad \ldots & \ldots & 1822\end{array}$

Anon. $\quad \ldots \quad \ldots \quad \ldots \quad 1823$

Archiv für Viehzucht $\quad \ldots \quad 1823$

$\begin{array}{lllll}\text { Autenreith } & \ldots & \ldots & \ldots & 1823\end{array}$

$\begin{array}{lllll}\text { Bobadella } & \ldots & \ldots & \ldots & 1823\end{array}$

$\begin{array}{llllll}\text { Clark } \quad \ldots & \text {.. } & \ldots & 1823\end{array}$

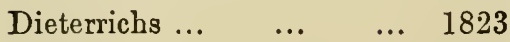

$\begin{array}{lllll}\text { Dutoict } & \ldots & \ldots & \ldots & 1823\end{array}$

Hofacker $\ldots \quad \ldots \quad \ldots \quad 1823$
Morel

Murrell

Percivall

Pursglove

Tennecker ...

Tscheulin

Anon.

Binz

Hulfreich

Leblanc

Rigot

Sandri

$\begin{array}{llllll}\text { Steinhoff } & \ldots & \ldots & \ldots & 1824\end{array}$

$\begin{array}{llllll}\text { Thon } \quad \ldots & \ldots & \ldots & 1824\end{array}$

$\begin{array}{lllll}\text { Tscheulin } & \ldots & \ldots & \ldots & 1824\end{array}$

Bonsi

Brauell

Politz

Unentbehrliches

Anon.

Jacquemin

Noack

Sarchiano

Schwab

Viborg

Erdelyi

Girard

Peterka

Silberschmidt

Tennecker ...

Dietterichs ...

Hoffmann ...
1825

1825

1825

.. 1826

.. 1826

.. 1826

.. 1826

.. 1826

.. 1826

... 1826

.. 1827

. 1827

.. 1827

.. 1827

.. 1827

.. 1828

... 1828 


\section{VETERINARY.-Continued.}

$\begin{array}{lllll}\text { Tennecker } & \ldots & \ldots & \ldots & 1828\end{array}$

$\begin{array}{lllll}\text { Vatel } & \ldots & \ldots & \ldots & 1828\end{array}$

The Veterinarian $\ldots \quad \ldots \quad 1828$

Hinds (Badcock) $\quad \ldots \quad \ldots \quad 1829$

$\begin{array}{lllll}\text { Bruickner } \quad \ldots & \ldots & \ldots & 1829\end{array}$

$\begin{array}{lllll}\text { Ithen } & \ldots & \ldots & \ldots & 1829\end{array}$

$\begin{array}{lllll}\text { Massa } & \ldots & \ldots & \ldots & 1829\end{array}$

$\begin{array}{lllll}\text { Risueno } & \ldots & \ldots & \ldots & 1829\end{array}$

$\begin{array}{lllll}\text { Salzmann } & \ldots & \ldots & \ldots & 1829\end{array}$

$\begin{array}{lllll}\text { Thomas } \quad \ldots & \ldots & \ldots & 1829\end{array}$

Anon. $\quad \ldots \quad \ldots \quad \ldots \quad 1830$

Barthelemy $\quad \ldots \quad \ldots \quad 1830$

$\begin{array}{lllll}\text { Buerger } \quad \ldots & \ldots & \ldots & 1830\end{array}$

$\begin{array}{lllll}\text { Casas } & \ldots & \ldots & \ldots & 1830\end{array}$

$\begin{array}{lllll}\text { Faber } & \ldots & \ldots & \ldots & 1830\end{array}$

The Farrier $\quad \ldots \quad \ldots \quad 1830$

Gunther $\quad \ldots \quad \ldots \quad \ldots \quad 1830$

Hayne $\quad \ldots \quad \ldots \quad \ldots \quad 1830$

Hoerdt $\quad \ldots \quad \ldots \quad \ldots \quad 1830$

$\begin{array}{lllll}\text { Massa } & \ldots & \ldots & \ldots & 1830\end{array}$

Sampedro $\quad \ldots \quad \ldots \quad \ldots \quad \ldots \quad 1830$

Stewart $\quad \ldots \quad \ldots \quad \ldots \quad \ldots \quad 1830$

Teller $\quad \ldots \quad \ldots \quad \ldots \quad \ldots \quad 1830$

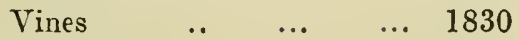

$\begin{array}{lllll}\text { Waldinger } & \ldots & \ldots & \ldots & 1830\end{array}$

$\begin{array}{lllll}\text { Alpin } & \ldots & \ldots & \ldots & 1831\end{array}$

Blaine $\quad \ldots \quad \ldots \quad \ldots \quad \ldots 1831$

Brunswig $\quad \ldots \quad \ldots \quad \ldots \quad \ldots \quad 1831$

Dieterrichs $\quad \ldots \quad \ldots \quad 1831$

$\begin{array}{lllll}\text { Gurlt } & \ldots & \ldots & \ldots & 1831\end{array}$

Thomaun $\quad \ldots \quad \ldots \quad \ldots \quad \ldots \quad 1831$

Walch
Weidenkeller $\quad \ldots \quad \ldots \quad 1831$

$\begin{array}{lllll}\text { Youatt } \quad \ldots & \ldots & \ldots & 1831\end{array}$

Anon. $\quad \ldots . \quad \ldots \quad \ldots \quad 1832$

$\begin{array}{lllll}\text { Benfert } \quad \ldots & \ldots & \ldots & 1832\end{array}$

$\begin{array}{lllll}\text { Eckert } \quad \ldots & \ldots & \ldots & 1832\end{array}$

Fischer $\quad \ldots \quad \ldots \quad \ldots \quad 1832$

Hering $\quad \ldots \quad \ldots \quad \ldots \quad 1832$

$\begin{array}{lllll}\text { Leuckart } & \ldots & \ldots & \ldots & 1832\end{array}$

$\begin{array}{lllll}\text { Rudge } \quad \ldots & \ldots & \ldots & 1832\end{array}$

$\begin{array}{lllll}\text { Strauss } & \ldots & \ldots & \ldots & 1832\end{array}$

Veterinary Examiner $\quad$.. 1832

Wagenfeld $\quad \ldots \quad \ldots .1832$

$\begin{array}{lllll}\text { Wilhelmi } & \ldots & \ldots & \ldots & 1832\end{array}$

$\begin{array}{lllll}\text { Casas } & \ldots & \ldots & \ldots & 1833\end{array}$

$\begin{array}{lllll}\text { Clark } & \ldots & \ldots & \ldots & 1833\end{array}$

$\begin{array}{lllll}\text { Grogniez } & \ldots & \ldots & \ldots & 1833\end{array}$

$\begin{array}{lllll}\text { Kreutzer } \quad \ldots & \ldots & \ldots & 1833\end{array}$

$\begin{array}{lllll}\text { Lessona } & \ldots & \ldots & \ldots & 1833\end{array}$

$\begin{array}{lllll}\text { Merk } & \ldots & \ldots & \ldots & 1833\end{array}$

$\begin{array}{lllll}\text { Schrader } & \ldots & \ldots & \ldots & 1833\end{array}$

$\begin{array}{lllll}\text { Small } & \ldots & \ldots & \ldots & 1833\end{array}$

$\begin{array}{lllll}\text { Vines } & \ldots & \ldots & \ldots & 1833\end{array}$

$\begin{array}{lllll}\text { Vogeli } \quad \ldots & \ldots & \ldots & 1833\end{array}$

Anon. $\quad \ldots \quad \ldots . \quad \ldots \quad 1834$

Anon. $\quad \ldots \quad \ldots . \quad \ldots \quad 1834$

$\begin{array}{llllll}\text { Casas } & \ldots & \ldots & \ldots & 1834\end{array}$

Dieterrichs, Nebel and Vix 1834

Hartung $\quad \ldots \quad \ldots \quad \ldots \quad 1834$

$\begin{array}{lllll}\text { Lenz } & \ldots & \ldots & \ldots & 1834\end{array}$

$\begin{array}{lllll}\text { Lessona } & \ldots & \ldots & \ldots & 1834\end{array}$

$\begin{array}{lllll}\text { Liipke } & \ldots & \ldots & \ldots & 1834\end{array}$

$\begin{array}{lllll}\text { Numan } \quad \ldots & \ldots & \ldots & 1834\end{array}$ 


\section{VETERINARY.-Continued.}

\begin{tabular}{|c|c|c|c|c|c|c|c|c|c|}
\hline Percivall & $\ldots$ & ... & ... & 1834 & Clark & $\ldots$ & $\ldots$ & $\ldots$ & 1838 \\
\hline Risueno & $\ldots$ & $\ldots$ & $\ldots$ & 1834 & Delafond & $\ldots$ & $\ldots$ & $\ldots$ & 1838 \\
\hline Sampedro & $\ldots$ & $\ldots$ & $\ldots$ & $1834^{\circ}$ & Erkens & $\ldots$ & $\ldots$ & 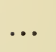 & 1838 \\
\hline Clark & $\ldots$ & ... & $\ldots$ & 1835 & Haubner & $\ldots$ & ... & ... & 1838 \\
\hline Dahl & $\ldots$ & ... & ... & 1835 & Niisken & ... & $\ldots$ & $\ldots$ & 1838 \\
\hline Dieterrich & $\ldots$ & $\ldots$ & $\ldots$ & 1835 & Rossi & $\ldots$ & ... & $\ldots$ & 1838 \\
\hline Eléoute & ... & $\ldots$ & ... & 1835 & Anon. & ... & ... & $\ldots$ & 1839 \\
\hline Erkens & $\ldots$ & ... & ... & 1835 & Brogniez & $\ldots$ & $\ldots$ & $\ldots$ & 1839 \\
\hline Magazin & $\ldots$ & ... & ... & 1835 & Mohammed & Buksh & & $\ldots$ & 1839 \\
\hline Nalhorst & $\ldots$ & ... & ... & 1835 & Clark & $\ldots$ & $\ldots$ & $\ldots$ & 1839 \\
\hline Santos & ... & ... & ... & 1835 & Haxthausen & & ... & $\ldots$ & 1839 \\
\hline The Sportsm & nan \& & ty. $F a r$ & rier & 1835 & Korber & $\ldots$ & $\ldots$ & $\ldots$ & 1839 \\
\hline Velten & ... & ... & $\ldots$ & 1835 & Der Marstal & lll & $\ldots$ & $\ldots$ & 1839 \\
\hline Vogeli & $\ldots$ & ... & $\ldots$ & 1835 & Rainard & ... & .. & $\ldots$ & 1839 \\
\hline Anon. & $\ldots$ & ... & .. & 1836 & Riickert & ... & $\ldots$ & $\ldots$ & 1839 \\
\hline Ascheberg & $\ldots$ & ... & $\ldots$ & 1836 & Spinola & ... & $\ldots$ & $\ldots$ & 1839 \\
\hline Funke & $\ldots$ & $\ldots$ & $\ldots$ & 1836 & Rychner & ... & $\ldots$ & $\ldots$ & 1839 \\
\hline Giinther & $\ldots$ & .. & $\ldots$ & 1836 & Anon. & $\ldots$ & .. & $\ldots$ & 1840 \\
\hline Rychner \& & Im-Thn & & $\ldots$ & 1836 & Persian MS & & ... & $\ldots$ & 1840 \\
\hline Anon. & $\ldots$ & $\ldots$ & $\ldots$ & 1837 & Bultzingstov & ven & $\ldots$ & $\ldots$ & 1840 \\
\hline Anon. & .. & ... & $\ldots$ & 1837 & Georgio & ... & $\ldots$ & ... & 1840 \\
\hline Billing & $\ldots$ & $\ldots$ & $\ldots$ & 1837 & Kreutzon & $\ldots$ & $\ldots$ & $\ldots$ & 1840 \\
\hline Bouley and & Reynal & & ... & 1837 & Leblanc & $\ldots$ & $\ldots$ & $\ldots$ & 1840 \\
\hline Fogg & $\ldots$ & $\ldots$ & $\ldots$ & 1837 & Legros & $\ldots$ & $\ldots$ & $\ldots$ & 1840 \\
\hline Morton & $\ldots$ & $\ldots$ & $\ldots$ & 1837 & Morton & ... & $\ldots$ & $\ldots$ & 1840 \\
\hline Reska & $\ldots$ & $\ldots$ & $\ldots$ & 1837 & Persch & $\ldots$ & $\ldots$ & $\cdots$ & 1840 \\
\hline Schmid & $\ldots$ & $\ldots$ & $\ldots$ & 1837 & Renault & .. & $\ldots$ & $\ldots$ & 1840 \\
\hline Wagenfeld & $\ldots$ & $\ldots$ & $\ldots$ & 1837 & Sampedro & $\therefore$ & $\ldots$ & $\ldots$ & 1840 \\
\hline Anon. & $\ldots$ & $\ldots$ & $\ldots$ & 1838 & Schwab & & $\ldots$ & $\ldots$ & 1840 \\
\hline D'Arboval & $\ldots$ & .. & $\ldots$ & 1838 & Spooner & $\ldots$ & $\ldots$ & $\ldots$ & 184 \\
\hline Bleiweis & $\ldots$ & $\ldots$ & $\ldots$ & 1838 & Veyssière & ... & ... & $\ldots$ & 184 \\
\hline Bouley and & Dupuy & & $\ldots$ & 1838 & Wagenfeld & & $\ldots$ & $\ldots$ & 184 \\
\hline
\end{tabular}




\section{VETERINARY.-Continued.}

$\begin{array}{lllll}\text { With } & \ldots & \ldots & \ldots & 1840\end{array}$

Anon. $\quad \ldots \quad \ldots . \quad \ldots \quad 1811$

$\begin{array}{lllll}\text { Caputo } & \ldots & \ldots & \ldots & 1841\end{array}$

Delafond and Lassaigne $\quad \ldots \quad 1841$

Dick

Fauvet

$\begin{array}{lllll}\text { Girard } & \ldots & \ldots & \ldots & 1841\end{array}$

Im-Thurn $\quad \ldots \quad \ldots \quad \ldots \quad 1841$

Journal Vétérinaive $\quad$.. 1841

$\begin{array}{lllll}\text { Kuers } \quad \ldots & \ldots & \ldots & 1841\end{array}$

$\begin{array}{lllll}\text { Lebas } & \ldots & \ldots & \ldots & 1841\end{array}$

Leblanc $\quad \ldots \quad \ldots \quad \ldots \quad 1841$

$\begin{array}{lllll}\text { Mercier } & \ldots & \ldots & \ldots & 1841\end{array}$

Meyer $\quad \ldots \quad \ldots \quad \ldots \quad \ldots \quad 1841$

$\begin{array}{lllll}\text { Mousis } \quad \ldots & \ldots & \ldots & 1841\end{array}$

$\begin{array}{lllll}\text { Prinz } & \ldots & \ldots & \ldots & 1841\end{array}$

$\begin{array}{lllll}\text { Rath } & \ldots & \ldots & \ldots & 1841\end{array}$

$\begin{array}{lllll}\text { Tiden } & \ldots & \ldots & \ldots & 1841\end{array}$

$\begin{array}{lllll}\text { With } & \ldots & \ldots & \ldots & 1841\end{array}$

Anon. $\quad \ldots \quad \ldots \quad \ldots \quad 1842$

$\begin{array}{lllll}\text { Bonino } \quad \ldots & \ldots & \ldots & 1842\end{array}$

Dieterrichs $\quad \ldots \quad \ldots \quad 1842$

$\begin{array}{llll}\text { Fuhrmeister } & \ldots & \ldots & 1842\end{array}$

$\begin{array}{lllll}\text { Kaestner } \quad \ldots & \ldots & \ldots & 1842\end{array}$

$\begin{array}{lllll}\text { Magne } \quad \ldots & \ldots & \ldots & 1842\end{array}$

Moiroud $\quad \ldots \quad \ldots \quad \ldots \quad \ldots \quad 1842$

Rohlwes $\quad \ldots \quad \ldots \quad \ldots \quad 1842$

$\begin{array}{lllll}\text { Rychner } & \ldots & \ldots & \ldots & 1842\end{array}$

Tiden $\quad \ldots \quad \ldots \quad \ldots 1842$

$\begin{array}{lllll}\text { Casas } & \ldots & \ldots & \ldots & 1843\end{array}$

Delafond $\quad \ldots \quad \ldots \quad \ldots \quad 1843$

Ferguson $\quad \ldots \quad \ldots \quad \ldots \quad 1843$ $\begin{array}{lllll}\text { Mills } & \ldots & \ldots & \ldots & 1843\end{array}$

$\begin{array}{lllll}\text { Papin } & \ldots & \ldots & \ldots & 1843\end{array}$

$\begin{array}{llllll}\text { Stephan } \quad \ldots & \ldots & \ldots & 1843\end{array}$

$\begin{array}{lllll}\text { Tardieu } & \ldots & \ldots & \ldots & 1843\end{array}$

Thierartzliche Zeitung _.. 1843

Tscherning $\quad \ldots \quad \ldots \quad 1843$

$\begin{array}{lllll}\text { Casas } & \ldots & \ldots & \ldots & 1844\end{array}$

Hayne $\quad \ldots \quad \ldots \quad \ldots \quad \ldots \quad 1844$

$\begin{array}{lllll}\text { Joce } \quad \ldots & \ldots & \ldots & 1844\end{array}$

$\begin{array}{lllll}\text { Morton } \quad \ldots & \ldots & \ldots & 1844\end{array}$

$\begin{array}{lllll}\text { Rochas } \quad \ldots & \ldots & \ldots & 1844\end{array}$

Roper ... $\quad \ldots \quad \ldots \quad 1844$

$\begin{array}{lllll}\text { Saive } & \ldots & \ldots & \ldots & 1844\end{array}$

$\begin{array}{lllll}\text { Spinola } & \ldots & \ldots & \ldots & 1844\end{array}$

$\begin{array}{lllll}\text { Cardini } & \ldots & \ldots & \ldots & 1845\end{array}$

Charlier ... $\quad \ldots \quad \ldots \quad \ldots \quad 1845$

Haupt $\quad \ldots \quad \ldots \quad \ldots \quad 1845$

Journal de Médicine Vétérinaire 1845

$\begin{array}{lllll}\text { Magne } \quad \ldots & \ldots & \ldots & 1845\end{array}$

$\begin{array}{lllll}\text { Papa } & \ldots & \ldots & \ldots & 1845\end{array}$

$\begin{array}{lllll}\text { Raimund } \quad \ldots & \ldots & \ldots & 1845\end{array}$

$\begin{array}{lllll}\text { Rigau } & \ldots & \ldots & \ldots & 1845\end{array}$

Veterinary Record ... ... 1845

Anon. $\quad \ldots \quad \ldots \quad \ldots \quad 1846$

$\begin{array}{lllll}\text { Berthelot } & \ldots & \ldots & \ldots & 1846\end{array}$

$\begin{array}{lllll}\text { Brown } & \ldots & \ldots & \ldots & 1846\end{array}$

$\begin{array}{lllll}\text { Casas } & \ldots & \ldots & \ldots & 1846\end{array}$

Faber $\quad \ldots \quad \ldots \quad \ldots \quad 1846$

Recueil de Médicine Vétérinaire 1846

$\begin{array}{lllll}\text { Rigoni } \quad \ldots & \ldots & \ldots & 1846\end{array}$

$\begin{array}{llllll}\text { Toggia } \quad \ldots & \ldots & \ldots & 1846\end{array}$

$\begin{array}{lllll}\text { Traeger } & \ldots & \ldots & \ldots & 1846\end{array}$ 


\section{VETERINARY.-Continued.}

\begin{tabular}{|c|c|c|c|c|c|c|c|c|c|}
\hline Tuczek & & $\ldots$ & $\ldots$ & 1846 & Boehm & $\ldots$ & $\cdots$ & ... & 1849 \\
\hline \multicolumn{3}{|c|}{ Recueil de mémoires } & $\ldots$ & 1847 & Brout & $\ldots$ & $\ldots$ & $\ldots$ & 1849 \\
\hline Casas & $\ldots$ & $\ldots$ & $\ldots$ & 1847 & Savigny & $\ldots$ & $\ldots$ & $\ldots$ & 1849 \\
\hline ervais & $\ldots$ & $\ldots$ & $\ldots$ & 1847 & Cole & $\ldots$ & $\ldots$ & $\ldots$ & 1849 \\
\hline Annuaire & $\ldots$ & $\ldots$ & $\ldots$ & 1848 & Delwart & $\ldots$ & $\cdots$ & $\cdots$ & 819 \\
\hline Anon. & $\ldots$ & $\ldots$ & $\ldots$ & 1848 & Desmarest & $\ldots$ & $\ldots$ & $\ldots$ & 849 \\
\hline Alexandre & $\ldots$ & $\ldots$ & $\ldots$ & 1848 & Falke & $\ldots$ & $\ldots$ & $\ldots$ & 1849 \\
\hline Auzoux & $\ldots$ & $\ldots$ & $\ldots$ & 1848 & Leyon & $\ldots$ & $\ldots$ & $\ldots$ & 849 \\
\hline \multicolumn{4}{|c|}{ Baumeister and Duttenhofer } & 1848 & Lotze & $\ldots$ & $\ldots$ & $\ldots$ & 1849 \\
\hline Brivet & $\ldots$ & $\ldots$ & $\ldots$ & 1848 & Martini & $\ldots$ & $\ldots$ & $\ldots$ & 1849 \\
\hline \multicolumn{3}{|c|}{ Bulletin de $7 a$ Société } & $\ldots$ & 1818 & Neergard & $\ldots$ & $\ldots$ & $\ldots$ & 849 \\
\hline \multicolumn{3}{|c|}{ Canstatt and Eisenmann } & $\ldots$ & 1848 & \multicolumn{5}{|c|}{ Rey, Lecoq, Tisserant and } \\
\hline \multicolumn{2}{|c|}{ Central Archiv } & $\ldots$ & $\ldots$ & 1848 & \multicolumn{2}{|c|}{ Tabourin ... } & $\ldots$ & ... & 1849 \\
\hline \multicolumn{2}{|c|}{ Delafond $\quad \ldots$} & $\ldots$ & $\ldots$ & 1848 & Répertoire & $\ldots$ & $\ldots$ & $\ldots$ & 849 \\
\hline Dieterrichs & $\ldots$ & $\ldots$ & $\ldots$ & 1848 & \multirow{2}{*}{$\begin{array}{l}\text { Rychner } \\
\text { Sellier }\end{array}$} & $\cdots$ & ... & $\cdots$ & 1849 \\
\hline Falke & $\ldots$ & $\ldots$ & $\ldots$ & 1848 & & $\ldots$ & $\ldots$ & $\ldots$ & 1849 \\
\hline Fritsch & $\ldots$ & $\ldots$ & $\ldots$ & 1848 & \multicolumn{4}{|c|}{ Thieraratliches Wochen-Blatt } & 1849 \\
\hline $\begin{array}{l}\text { Fritsch } \\
\text { Giles }\end{array}$ & .. & $\ldots$ & $\ldots$ & 1848 & \multicolumn{3}{|c|}{ Vierzig untrügliche } & $\ldots$ & 1850 \\
\hline Hellmund & .. & $\ldots$ & $\ldots$ & 1848 & Bowers & $\ldots$ & $\ldots$ & $\ldots$ & 1850 \\
\hline Jauné & $\ldots$ & $\ldots$ & $\ldots$ & 1848 & Briddon & $\ldots$ & $\ldots$ & $\cdots$ & 1850 \\
\hline Koerber & $\ldots$ & $\ldots$ & $\ldots$ & 1848 & Casas & $\ldots$ & $\ldots$ & $\ldots$ & 1850 \\
\hline Leblanc & $\ldots$ & $\ldots$ & $\ldots$ & 1848 & Dadd & $\ldots$ & $\ldots$ & $\ldots$ & 1850 \\
\hline Magazin & $\ldots$ & $\ldots$ & $\ldots$ & 1848 & Falke & $\ldots$ & $\ldots$ & $\ldots$ & 850 \\
\hline Magazin & $\ldots$ & $\therefore$ & $\ldots$ & 1848 & Fuchs & $\ldots$ & $\ldots$ & $\ldots$ & 1850 \\
\hline Marguard & $\ldots$ & $\ldots$ & $\ldots$ & 1848 & Giles & $\ldots$ & $\ldots$ & $\ldots$ & 1850 \\
\hline Rosenbaum & $\ldots$ & $\ldots$ & $\ldots$ & 1848 & Guesdon & ... & $\ldots$ & $\ldots$ & 1850 \\
\hline Towne & $\ldots$ & $\ldots$ & $\ldots$ & 1848 & Haycock & $\ldots$ & $\ldots$ & $\ldots$ & 1850 \\
\hline Wagenfeldt & & $\ldots$ & $\ldots$ & 1848 & Hertwig & $\ldots$ & $\ldots$ & $\ldots$ & 1850 \\
\hline Die Influen & & $\ldots$ & $\ldots$ & 1849 & Jacquemin & $\ldots$ & $\ldots$ & $\ldots$ & 1850 \\
\hline Symputhieb & uchlein & $\ldots$ & $\ldots$ & 1849 & Knowlson & $\ldots$ & $\ldots$ & $\ldots$ & 1850 \\
\hline al & $\ldots$ & $\ldots$ & $\ldots$ & 1849 & Koch & $\ldots$ & $\ldots$ & $\ldots$ & 1850 \\
\hline aumeister & $\ldots$ & $\ldots$ & $\ldots$ & 1849 & Lepkowski & $\ldots$ & $\ldots$ & $\ldots$ & 1850 \\
\hline
\end{tabular}




\section{VETERINARY.-Continued.}

$\begin{array}{llll}\text { Lewandowski } & \ldots & \ldots & 1850\end{array}$

$\begin{array}{llllll}\text { Ludwiger } & \ldots & \text {.. } & \ldots & 1850\end{array}$

$\begin{array}{lllll}\text { Ostrowski } & \ldots & \ldots & \ldots & 1850\end{array}$

$\begin{array}{lllll}\text { Percivall } & \ldots & \ldots & \ldots & 1850\end{array}$

$\begin{array}{lllll}\text { Plasse } & \ldots & \ldots & \ldots & 1850\end{array}$

$\begin{array}{lllll}\text { Seeger } & \ldots & \ldots & \ldots & 1850\end{array}$

$\begin{array}{llll}\text { Skeavington } & \ldots & \ldots & 1850\end{array}$

Société Vétérincire de la Marne 1850

La Veterinaria tuscubile ... 1851

Nuevo manual $\quad \ldots \quad \ldots \quad 1851$

Sociedad _.. $\quad \ldots \quad \ldots \quad 1851$

Veterinary Homoeopathy ... 1851

Der Homoopathische Haus-

Thierarat

$\begin{array}{lllll}\text { Bertacchi } & \ldots & \ldots & \ldots & 1851\end{array}$

Briones and Nieto ... $\quad \ldots \quad 1851$

$\begin{array}{lllll}\text { Brogniez } & \ldots & \ldots & \ldots & 1851\end{array}$

Centrul-Zeitung $\quad \ldots \quad \ldots \quad 1851$

$\begin{array}{lllll}\text { Dieterichs } & \ldots & \ldots & \ldots & 1851\end{array}$

Dombale $\quad \ldots \quad \ldots \quad \ldots \quad \ldots \quad 1851$

El Eco Veterinario $\quad \ldots \quad 1851$

Ellerbrock $\ldots . \quad \ldots \quad \ldots \quad 1851$

Ercolani $\quad \ldots \quad \ldots \quad \ldots \quad \ldots \quad 1851$

$\begin{array}{lllll}\text { Falke } & \ldots & \ldots & \ldots & 1851\end{array}$

$\begin{array}{lllll}\text { Fries } & \ldots & \ldots & \ldots & 1851\end{array}$

Giornale di Veterinaria ... 1851

Hertwig $\quad \ldots \quad \ldots \quad \ldots \quad \ldots 1851$

Lacoste $\quad \ldots \quad \ldots \quad \ldots \quad \ldots 1851$

$\begin{array}{lllll}\text { Lotze } \quad \ldots & \ldots & \ldots & 1851\end{array}$

$\begin{array}{lllll}\text { Louchard } & \ldots & \ldots & \ldots & 1851\end{array}$

Martin (Appendix) $\quad \ldots \quad 1851$

El Regenerador $\quad \ldots \quad \ldots \quad 1851$ $\begin{array}{lllll}\text { Sampedro } & \ldots & \ldots & \ldots & 1851\end{array}$

$\begin{array}{lllll}\text { Seyff } & \ldots & \ldots & \ldots & 1851\end{array}$

$\begin{array}{llllll}\text { Traeger } & \ldots & \ldots & \ldots & 1851\end{array}$

Wernaer _.. $\quad \ldots \quad$... 1851

Cours complet $\quad \ldots \quad \ldots \quad 1852$

Mémoires $\quad \ldots \quad \ldots \quad \ldots \quad \ldots \quad 1852$

$\begin{array}{llll}\text { Aigeldinger } & \ldots & \ldots & 1852\end{array}$

Annales de Médicine Vétérinaive 1852

Beyer and Werneburg _.. 1852

Blätter über Pferde $\quad \ldots \quad 1852$

$\begin{array}{lllll}\text { Boehm } \quad \ldots & \ldots & \ldots & 1852\end{array}$

Defays and Hussolı $\quad \ldots \quad 1852$

Delafond $\quad \ldots \quad \ldots \quad \ldots \quad 1852$

$\begin{array}{lllll}\text { Deloupy } \quad \ldots & \ldots & \ldots & 1852\end{array}$

$\begin{array}{lllll}\text { Echegaray } & \ldots & \ldots & \ldots & 1852\end{array}$

$\begin{array}{lllll}\text { Ellerbrock } & \ldots & \ldots & \ldots & 1852\end{array}$

$\begin{array}{lllll}\text { Falke } \quad \ldots & \ldots & \ldots & 1852\end{array}$

$\begin{array}{lllll}\text { Frey } & \ldots & \ldots & \ldots & 1852\end{array}$

$\begin{array}{lllll}\text { Fuchs } \quad \ldots & \ldots & \ldots & 1852\end{array}$

$\begin{array}{lllll}\text { Hamm } & \ldots & \ldots & \ldots & 1852\end{array}$

$\begin{array}{lllll}\text { Haycock } & \ldots & \ldots & \ldots & 1852\end{array}$

Heinrichs $\quad . . \quad \ldots \quad \ldots \quad 1852$

$\begin{array}{lllll}\text { Hofer } & \ldots & \ldots & \ldots & 1852\end{array}$

$\begin{array}{lllll}\text { Lessona } & \ldots & \ldots & \ldots & 1852\end{array}$

$\begin{array}{lllll}\text { Pinto } & \ldots & \ldots & \ldots & 1852\end{array}$

$\begin{array}{lllll}\text { Marquart } & \ldots & \ldots & \ldots & 1852\end{array}$

$\begin{array}{lllll}\text { Miramont } & \ldots & \ldots & \ldots & 1852\end{array}$

$\begin{array}{lllll}\text { Pieschel } & \ldots & \ldots & \ldots & 1852\end{array}$

$\begin{array}{lllll}\text { Rey } & \ldots & \ldots & \ldots & 1852\end{array}$

$\begin{array}{lllll}\text { Rueckert } & \ldots & \ldots & \ldots & 1852\end{array}$

$\begin{array}{lllll}\text { Schmid } \quad \ldots & \ldots & \ldots & 1852\end{array}$

Viertoljahresschrift $\quad \ldots \quad 1852$ 


\section{VETERINARY.-Continued.}

Anviisning ... $\quad \ldots \quad \ldots \quad \ldots 1853$

Verzameling $\quad \ldots \quad \ldots \quad 1853$

$\begin{array}{lllll}\text { Auyoux } \quad \ldots & \ldots & \ldots & 1853\end{array}$

$\begin{array}{lllll}\text { Bonneval } & \ldots & \ldots & \ldots & 1853\end{array}$

$\begin{array}{lllll}\text { Crocq } & \ldots & \ldots & \ldots & 1853\end{array}$

$\begin{array}{lllll}\text { Dekker } \quad \ldots & \ldots & \ldots & 1853\end{array}$

$\begin{array}{lllll}\text { Dieterichs } & \ldots & \ldots & \ldots & 1853\end{array}$

Falke $\quad \ldots \quad \ldots \quad \ldots \quad \ldots 1853$

Gama-Machado $\quad \ldots \quad \ldots \quad 1853$

$\begin{array}{lllll}\text { Gerlach } \quad \ldots & \ldots & \ldots & 1853\end{array}$

$\begin{array}{lllll}\text { Gherardi } & \ldots & \ldots & \ldots & 1853\end{array}$

$\begin{array}{lllll}\text { Gurlt } & \ldots & \ldots & \ldots & 1853\end{array}$

Hansen $\quad \ldots \quad$.. $\quad \ldots \quad 1853$

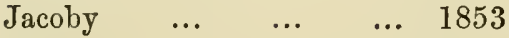

$\begin{array}{lllll}\text { Knoll } & \ldots & \ldots & \ldots & 1853\end{array}$

$\begin{array}{lllll}\text { Kreutzer } \quad \ldots & \ldots & \ldots & 1853\end{array}$

$\begin{array}{lllll}\text { Loiset } & \ldots & \ldots & \ldots & 1853\end{array}$

$\begin{array}{lllll}\text { Roell } & \ldots & \ldots & \ldots & 1853\end{array}$

Saádatyár Khan $\quad \ldots \quad \ldots \quad 1853$

$\begin{array}{lllll}\text { Sandri } & \ldots & \ldots & \ldots & 1853\end{array}$

$\begin{array}{lllll}\text { Schaefer } & \ldots & \ldots & \ldots & 1853\end{array}$

Spooner $\quad \ldots \quad \ldots \quad \ldots \quad \ldots \quad 1853$

$\begin{array}{lllll}\text { Tabourin } & \ldots & \ldots & \ldots & 1853\end{array}$

Tydsskrift for veterinairer ... 1853

Vernois and Becquerel $\quad \ldots \quad 1853$

$\begin{array}{lllll}\text { Winiker } \quad \ldots & \ldots & \ldots & 1853\end{array}$

$\begin{array}{lllll}\text { Yvart } \quad \ldots & \ldots & \ldots & 1853\end{array}$

$\begin{array}{llll}\text { Compendium } & \ldots & \ldots & 1854\end{array}$

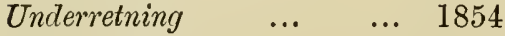

Vollstündiges $\quad \ldots \quad \ldots \quad 1854$

Billman _.. $\quad \ldots \quad \ldots \quad 1854$

Casas $\begin{array}{lllll}\text { Chaveau } & \ldots & \ldots & \ldots & 1854\end{array}$

$\begin{array}{lllll}\text { Clark } \quad \ldots & \ldots & \ldots & 1854\end{array}$

$\begin{array}{lllll}\text { Colin } \quad \ldots & \ldots & \ldots & 1854\end{array}$

Dadd $\quad \ldots \quad \ldots \quad \ldots .1854$

$\begin{array}{lllll}\text { Dun } \quad \ldots & \ldots & \ldots & 1854\end{array}$

$\begin{array}{lllll}\text { Erhardt } \quad \ldots & \ldots & \ldots & 1854\end{array}$

$\begin{array}{lllll}\text { Falke } & \ldots & \ldots & \ldots & 1854\end{array}$

$\begin{array}{lllll}\text { Gerlach } \quad \ldots & \ldots & \ldots & 1854\end{array}$

Grollier $\quad \ldots \quad \ldots \quad \ldots \quad 1854$

$\begin{array}{lllll}\text { Haycock } & \ldots & \ldots & \ldots & 1854\end{array}$

Heusmann ... $\quad \ldots \quad$... 1854

$\begin{array}{lllll}\text { Krane } \quad \ldots & \ldots & \ldots & 1854\end{array}$

$\begin{array}{lllll}\text { Lazaro } & \ldots & \ldots & \ldots & 1854\end{array}$

$\begin{array}{llllll}\text { Lecomte } & \ldots & \ldots & \ldots & 1854\end{array}$

$\begin{array}{llllll}\text { Lessona } & \ldots & \ldots & \ldots & 1854\end{array}$

$\begin{array}{lllll}\text { Pinto } & \ldots & \ldots & \ldots & 1854\end{array}$

$\begin{array}{llllll}\text { Olivier } \quad \ldots & \ldots & \ldots & 1854\end{array}$

$\begin{array}{lllll}\text { Patellani } & \ldots & \ldots & \ldots & 1854\end{array}$

Quillinan $\quad \ldots \quad \ldots \quad \ldots \quad \ldots \quad 1854$

$\begin{array}{llllll}\text { Raspail } \quad \ldots & \ldots & \ldots & 1854\end{array}$

$\begin{array}{lllll}\text { Rhodes } \quad \ldots & \ldots & \ldots & 1854\end{array}$

$\begin{array}{lllll}\text { Ricourt } \quad \ldots & \ldots & \ldots & 1854\end{array}$

$\begin{array}{lllll}\text { Rychner } \quad \ldots & \ldots & \ldots & 1854\end{array}$

Schönermark $\quad \ldots \quad \ldots \quad 1854$

$\begin{array}{llllll}\text { Strauss } \quad \ldots & \ldots & \ldots & 1854\end{array}$

Il Veterinario $\quad \ldots \quad \ldots \quad 1854$

Volpi $\quad \ldots \quad \ldots \quad \ldots \quad \ldots 1854$

Wagenfeldt $\quad \ldots . \quad \ldots \quad 1854$

$\begin{array}{llll}\text { Corso completo } & \ldots & \ldots & 1855\end{array}$

Höchst wichtige $\quad \ldots \quad \ldots \quad \mathbf{1 8 5 5}$

Homöopatiske Forsog _.. 1855

La Nouvelle Maison $\quad$.. 1855 


\section{VETERINARY.-Continued.}

\begin{tabular}{|c|c|c|c|c|c|c|c|c|c|}
\hline Bendz & $\ldots$ & $\ldots$ & $\ldots$ & 1855 & Prosch & $\ldots$ & $\ldots$ & $\ldots$ & 1856 \\
\hline Boehm & $\ldots$ & $\ldots$ & $\ldots$ & 1855 & \multicolumn{3}{|c|}{ Rivista Agronomica } & $\ldots$ & 1856 \\
\hline Boletin de & \multicolumn{2}{|c|}{ Veterinaria } & $\cdots$ & 1855 & Rodloff & $\ldots$ & $\ldots$ & $\ldots$ & 1856 \\
\hline Braungardt & $\ldots$ & $\ldots$ & $\ldots$ & 1855 & Roell & $\ldots$ & ... & $\ldots$ & 850 \\
\hline A. B. C. & ... & $\ldots$ & $\ldots$ & 1855 & Sampedro & $\ldots$ & $\ldots$ & $\ldots$ & 856 \\
\hline Denilly & $\ldots$ & $\ldots$ & $\ldots$ & 1855 & Sanson & $\ldots$ & $\ldots$ & ... & 85 \\
\hline Falke & $\ldots$ & $\ldots$ & $\ldots$ & 1855 & Schmidt & $\ldots$ & $\ldots$ & $\ldots$ & \\
\hline Fischer & $\ldots$ & $\ldots$ & $\ldots$ & 1855 & Seer & $\ldots$ & $\ldots$ & $\ldots$ & 7 \\
\hline Gamgee & $\ldots$ & .. & $\ldots$ & 1855 & Spinola & $\ldots$ & $\ldots$ & $\cdots$ & \\
\hline Koerber & $\ldots$ & $\ldots$ & $\ldots$ & 1855 & Tombari & $\ldots$ & $\ldots$ & ... & 35 \\
\hline Lázaro & $\ldots$ & $\ldots$ & $\ldots$ & 1855 & D'Ussel & $\ldots$ & $\ldots$ & $\ldots$ & 2 \\
\hline Memor abili & & $\ldots$ & $\ldots$ & 1855 & Volpi & $\ldots$ & $\ldots$ & $\ldots$ & \\
\hline Minot & $\ldots$ & $\ldots$ & $\ldots$ & 1855 & Walsh & $\ldots$ & $\ldots$ & $\ldots$ & 185 \\
\hline Navarro & ... & $\ldots$ & $\ldots$ & 1855 & De nieuwe & $V e e-a r$ & & $\ldots$ & 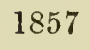 \\
\hline Pétry & $\ldots$ & $\ldots$ & $\ldots$ & 1855 & Horse Owne & $e r ' s$ Gu & & $\ldots$ & 357 \\
\hline Sabel & $\ldots$ & $\ldots$ & $\ldots$ & 1855 & Magia natu & eralis & $\ldots$ & $\ldots$ & 85 \\
\hline Sage & $\ldots$ & $\ldots$ & $\ldots$ & 1855 & Mémoire & $\ldots$ & $\ldots$ & $\ldots$ & 857 \\
\hline Thiriet-Sté & venin & $\ldots$ & $\ldots$ & 1855 & Alasonière & $\ldots$ & $\ldots$ & $\cdots$. & 857 \\
\hline Diccionario & $\ldots$ & $\ldots$ & $\ldots$ & 1856 & Arnault & $\ldots$ & $\ldots$ & $\ldots$ & 1857 \\
\hline Manual du & Maré & halerie & $\ldots$ & 1856 & Chauveau & $\ldots$ & $\ldots$ & $\ldots$ & 857 \\
\hline Berthier & ... & $\ldots$ & $\ldots$ & 1856 & Ferrer de $\mathrm{C}$ & Yonte & $\ldots$ & $\ldots$ & 1857 \\
\hline Boehm & $\ldots$ & $\ldots$ & $\ldots$ & 1856 & Couesme & $\ldots$ & $\ldots$ & $\ldots$ & 1857 \\
\hline Braungardt & $\ldots$ & $\ldots$ & $\ldots$ & 1856 & Dadd & ... & $\ldots$ & $\ldots$ & 857 \\
\hline Demilly & $\ldots$ & $\ldots$ & $\ldots$ & 1856 & Defays & $\cdots$ & $\ldots$ & $\ldots$ & 1857 \\
\hline Gautıer Mil & & $\ldots$ & $\ldots$ & 1856 & Dieterichs & $\ldots$ & $\ldots$ & $\ldots$ & 1857 \\
\hline Grosskopf & $\ldots$ & $\ldots$ & $\ldots$ & 1856 & Field & ... & $\ldots$ & $\ldots$ & 1857 \\
\hline Heinrich & $\ldots$ & $\ldots$ & $\ldots$ & 1856 & Goubaux & ... & $\ldots$ & $\ldots$ & 1857 \\
\hline Hertwig & ... & $\ldots$ & $\ldots$ & 1856 & Gourdon & $\ldots$ & $\ldots$ & $\ldots$ & 1857 \\
\hline Loebe & $\ldots$ & $\ldots$ & $\ldots$ & 1856 & Kaubner & $\ldots$ & $\ldots$ & $\ldots$ & 1857 \\
\hline Macé & ... & $\ldots$ & $\ldots$ & 1856 & Herzog & $\ldots$ & $\ldots$ & $\ldots$ & 1857 \\
\hline Patellani & ... & $\ldots$ & $\ldots$ & 1856 & Masch & $\ldots$ & $\ldots$ & $\ldots$ & 1857 \\
\hline Perosino & ... & ... & $\ldots$ & 1856 & Moore & ... & $\ldots$ & $\ldots$ & 185 \\
\hline
\end{tabular}




\section{VETERINARY.-Continued.}

\begin{tabular}{|c|c|c|c|c|c|c|c|c|c|}
\hline \multicolumn{3}{|c|}{ Mortier and Lentin } & $\ldots$ & 1857 & \multicolumn{2}{|l|}{ Bouchardat } & $\ldots$ & $\ldots$ & 1862 \\
\hline Plasse & $\ldots$ & ... & $\ldots$ & 1857 & Fitzwygran & & ... & $\ldots$ & 1862 \\
\hline \multicolumn{2}{|c|}{ De Rozières } & $\ldots$ & $\ldots$ & 1857 & Gayot & $\ldots$ & $\ldots$ & $\ldots$ & 1862 \\
\hline \multicolumn{2}{|c|}{ Wochenschrift } & $\ldots$ & $\ldots$ & 1857 & Godin & $\ldots$ & $\ldots$ & $\ldots$ & 1862 \\
\hline Arrault & $\ldots$ & ... & $\cdots$ & 1857 & \multicolumn{3}{|c|}{ Hippologische Blätter } & $\ldots$ & 1862 \\
\hline eugnot & $\ldots$ & ... & $\ldots$ & 1858 & Hocquard & ... & ... & ... & 862 \\
\hline lément & $\ldots$ & $\ldots$ & $\ldots$ & 1858 & Lecoq & ... & $\ldots$ & ... & $862^{\circ}$ \\
\hline \multicolumn{4}{|c|}{ EdinburghVeterinary Review } & 1858 & Legrain & ... & $\ldots$ & ... & 862 \\
\hline Gamgee & ... & ... & $\cdots$ & 1858 & Lescot & ... & ... & ... & 1862 \\
\hline tillet & $\ldots$ & .. & $\ldots$ & 1858 & \multicolumn{3}{|c|}{ Blatter für Pferdezucht } & ... & 863 \\
\hline Iorn & $\ldots$ & .. & $\ldots$ & 1858 & Miles & $\ldots$ & $\ldots$ & ... & 863 \\
\hline afosse & $\ldots$ & .. & $\ldots$ & 1858 & Sanson & $\ldots$ & $\ldots$ & $\ldots$ & 863 \\
\hline Lebeaud & $\ldots$ & $\cdots$ & $\ldots$ & 1858 & Mayhew & $\ldots$ & $\cdots$ & .. & 864 \\
\hline \multicolumn{2}{|c|}{ Mazurkiewicz } & $\ldots$ & $\ldots$ & 1858 & Cyr & ... & $\cdots$ & .. & 864 \\
\hline Brindley & $\ldots$ & ... & $\ldots$ & 1859 & w & $\ldots$ & $\ldots$ & $\ldots$ & 864 \\
\hline toux & $\ldots$ & ... & $\cdots$ & 1859 & Bussy & ... & ... & ... & 865 \\
\hline Jensenius & $\ldots$ & ... & $\ldots$ & 1859 & Casas & ... & $\ldots$ & $\ldots$ & 865 \\
\hline ernier & $\ldots$ & ... & $\ldots$ & 1860 & Guilmot & $\ldots$ & ... & .. & 186 \\
\hline idot & $\ldots$ & ... & $\ldots$ & 1860 & Lacassin & $\ldots$ & $\ldots$ & $\ldots$ & 865 \\
\hline Dalziel & $\ldots$ & $\ldots$ & $\ldots$ & 1860 & ain & $\ldots$ & $\ldots$ & $\ldots$ & 865 \\
\hline Fogh & $\ldots$ & $\ldots$ & $\ldots$ & 1860 & Bérger & $\ldots$ & $\ldots$ & $\ldots$ & 1865 \\
\hline Gamgee & $\ldots$ & $\ldots$ & $\ldots$ & 1860 & Prost-Lacu & zon and & Berger & $\ldots$ & 865 \\
\hline Gourdon & $\ldots$ & $\ldots$ & $\ldots$ & 1860 & Ableitner & $\ldots$ & $\ldots$ & $\ldots$ & 1866 \\
\hline Mayhew & $\ldots$ & $\ldots$ & $\ldots$ & 1860 & A. and J. & Gamgee & $\ldots$ & $\ldots$ & 1866 \\
\hline H. and $\mathrm{W}$ & Miles & $\ldots$ & $\ldots$ & 1860 & Schmidt & $\ldots$ & $\ldots$ & $\ldots$ & 1866 \\
\hline Confessions & & ... & $\ldots$ & 1861 & Tidskrift $f$ & for veteri & nërr & licin & 1866 \\
\hline Bouley anc & Sanson & & $\ldots$ & 1861 & Vial & ... & ... & $\ldots$ & 1866 \\
\hline Cotarelo & $\ldots$ & $\ldots$ & $\ldots$ & 1861 & Hubner & ... & $\ldots$ & $\ldots$ & 1867 \\
\hline Elluin & $\ldots$ & $\ldots$ & $\ldots$ & 1861 & Magne & $\ldots$ & $\ldots$ & $\ldots$ & 1867 \\
\hline Serres & $\ldots$ & $\ldots$ & $\ldots$ & 1861 & Mégnin & $\ldots$ & $\ldots$ & $\ldots$ & 1867 \\
\hline Taylor & $\ldots$ & ... & $\ldots$ & 1861 & Renner & $\ldots$ & $\cdots$ & $\ldots$ & 1867 \\
\hline Baillif & $\ldots$ & $\ldots$ & $\ldots$ & 1862 & The Veteri & nary $G a$ & xzette & $\ldots$ & 1867 \\
\hline
\end{tabular}




\section{VETERINARY.-Continued.}

$\begin{array}{lllll}\text { Vial } & \ldots & \ldots & \ldots & 1867\end{array}$

Villeroy and Miiller $\quad \ldots \quad 1867$

$\begin{array}{lllll}\text { Weisse } & \ldots & \ldots & \ldots & 1867\end{array}$

$\begin{array}{lllll}\text { Wemberg } & \ldots & \ldots & \ldots & \mathbf{1 8 6 7}\end{array}$

$\begin{array}{lllll}\text { Ableitner } \quad \ldots & \ldots & \ldots & 1868\end{array}$

$\begin{array}{lllll}\text { Bjorklund } & \ldots & \ldots & \ldots & 1868\end{array}$

$\begin{array}{lllll}\text { Lackner } \quad \ldots & \ldots & \ldots & 1868\end{array}$

$\begin{array}{llllll}\text { Lundberg } & \ldots & \ldots & \ldots & 1868\end{array}$

$\begin{array}{lllll}\text { Miles } & \ldots & \ldots & \ldots & 1868\end{array}$

Tidning for hastvanner $\quad \ldots \quad 1868$

$\begin{array}{llll}\text { Vogelmann ... } & \ldots & \ldots & 1868\end{array}$

$\begin{array}{lllll}\text { Bierlich } \quad \ldots & \ldots & \ldots & 1869\end{array}$

Billman $\quad \ldots \quad \ldots \quad \ldots \quad \ldots \quad 1869$

$\begin{array}{lllll}\text { Dick } & \ldots & \ldots & \ldots & 1869\end{array}$

Filet $\quad \ldots \quad \ldots \quad \ldots \quad 1869$

$\begin{array}{lllll}\text { Frey } & \ldots & \ldots & \ldots & 1869\end{array}$

Lebas $\quad \ldots \quad \ldots \quad \ldots \quad 1869$

$\begin{array}{llllll}\text { Mégnin } & \ldots & \ldots & \ldots & 1869\end{array}$

$\begin{array}{lllll}\text { Tuson } & \ldots & \ldots & \ldots & 1869\end{array}$

Taschenbnch für Pferdeliebhaber ... $\quad \ldots \quad \ldots \quad 1869$

$\begin{array}{lllll}\text { Félizet } & \ldots & \ldots & \ldots & 1870\end{array}$

$\begin{array}{lllll}\text { Kinberg } \quad \ldots & \ldots & \ldots & 1870\end{array}$

Lindqvist $\quad \ldots \quad \ldots \quad \ldots \quad 1870$

Luton $\quad \ldots \quad \ldots \quad \ldots \quad 1870$

$\begin{array}{lllll}\text { Ryves } & \ldots & \ldots & \ldots & 1870\end{array}$

Favre $\quad \ldots \quad \ldots \quad \ldots \quad 1871$

Fleming $\quad \ldots \quad \ldots \quad \ldots \quad 1871$

$\begin{array}{llllll}\text { Zweigberg } & \ldots & \ldots & \ldots & 1871\end{array}$

$\begin{array}{lllll}\text { Pardon } & \ldots & \ldots & \ldots & 1871\end{array}$

Clément $\quad \ldots \quad \ldots \quad \ldots \quad \ldots \quad 1872$

Frey $\begin{array}{lllll}\text { Mégnin } & \ldots & \ldots & \ldots & 1872\end{array}$

$\begin{array}{lllll}\text { Schwab } & \ldots & \ldots & \ldots & 1872\end{array}$

Williams $\quad \ldots \quad \ldots \quad \ldots \quad \ldots 1872$

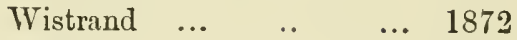

$\begin{array}{lllll}\text { Magne } & \ldots & \ldots & \ldots & 1873\end{array}$

$\begin{array}{lllll}\text { Salle } & \ldots & \ldots & \ldots & 1873\end{array}$

$\begin{array}{lllll}\text { Tropard } \quad \ldots & \ldots & \ldots & 1873\end{array}$

Instruction über $\quad \ldots \quad \ldots \quad 1874$

$\begin{array}{lllll}\text { Bendz } & \ldots & \ldots & \ldots & 1874\end{array}$

Friedberger $\quad \ldots \quad \ldots \quad 1874$

$\begin{array}{llllll}\text { Guilbert } \quad \ldots & \ldots & \ldots & 1874\end{array}$

$\begin{array}{lllll}\text { Loebell } \quad \ldots & \ldots & \ldots & 1874\end{array}$

Williams $\quad \ldots \quad \ldots \quad \ldots \quad \ldots \quad 1874$

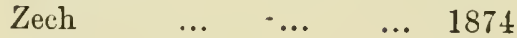

$\begin{array}{lllll}\text { Agenda } & \ldots & \ldots & \ldots & 1875\end{array}$

Fleming $\quad \ldots \quad \ldots \quad \ldots \quad 1875$

$\begin{array}{lllll}\text { Fleury } & \ldots & \ldots & \ldots & 1875\end{array}$

$\begin{array}{llllll}\text { Saint Cyr } & \ldots & \ldots & \ldots & 1875\end{array}$

$\begin{array}{lllll}\text { Sjostedt } & \ldots & \ldots & \ldots & 1875\end{array}$

$\begin{array}{lllll}\text { Spinola } & \ldots & \ldots & \ldots & 1875\end{array}$

Sveriges Veterinür-författninger 1875

Tidskrift fir Veterinärer ... 1875

Veterinary Journal $\quad \ldots \quad 1875$

The Veterinary VadeMecum 1875

Fleming $\quad \ldots \quad \ldots \quad \ldots \quad 1876$

$\begin{array}{lllll}\text { Hilcke } & \ldots & \ldots & \ldots & 1876\end{array}$

$\begin{array}{lllll}\text { Armatage } & \ldots & \ldots & \ldots & 1877\end{array}$

$\begin{array}{lllll}\text { Dallmann } & \ldots & \ldots & \ldots & 1877\end{array}$

Hayes $\quad \ldots \quad \ldots \quad \ldots \quad 1877$

$\begin{array}{lllll}\text { Law } & \ldots & \ldots & \ldots & 1877\end{array}$

Aerts (appendix) $\quad \ldots \quad \ldots \quad 1878$

Fearnley $\quad \ldots \quad \ldots \quad \ldots \quad 1878$ 


\section{VETERINARY.-Continued.}

$\begin{array}{llllllllll}\text { Forrest } & \ldots & \ldots & \ldots & 1878 & \text { Fearnley } & \ldots & \ldots & \ldots & 1882 \\ \text { Hardy } & \ldots & \ldots & \ldots & 1878 & \text { Gorini } & \ldots & \ldots & \ldots & 1882 \\ \text { der Lasa } & \ldots & \ldots & \ldots & 1878 & \text { Heatley } & \ldots & \ldots & \ldots & 1882 \\ \text { Roll } & \ldots & \ldots & \ldots & 1878 & \text { Piitz } & \ldots & \ldots & \ldots & 1882 \\ \text { Ruddock } & \ldots & \ldots & \ldots & 1878 & \text { Renner and } & \text { Rothernell } & \ldots & 1882 \\ \text { Anon. }(\text { appendix) } & \ldots & \ldots & 1878 & \text { Spohr } & \ldots & \ldots & \ldots & 1882 \\ \text { Born and } & \text { Möller } & \ldots & \ldots & 1879 & \text { Pinel } & \ldots & \ldots & \ldots & 1883 \\ \text { Dessart }(\text { appendix } & & \ldots & 1879 & \text { Robertson } & \ldots & \ldots & \ldots & 1883 \\ \text { Eichbaum } & \text { (appendix) } & \ldots & 1879 & \text { Szeliuski } & \ldots & \ldots & \ldots & 1883 \\ \text { Fogliata } & \ldots & \ldots & \ldots & 1879 & \text { Tappe } & \ldots & \ldots & \ldots & 1883 \\ \text { Kendall } & \ldots & \ldots & \ldots & 1879 & \text { Arago } & \ldots & \ldots & \ldots & 1884 \\ \text { Tirinanzi } & \ldots & \ldots & \ldots & 1879 & \text { Deseler } & \ldots & \ldots & \ldots & 1884 \\ \text { Bertachhi } & \ldots & \ldots & \ldots & 1880 & \text { Eichbaum } & \ldots & \ldots & \ldots & 1884 \\ \text { Bucaloss } & \ldots & \ldots & \ldots & 1880 & \text { Heule } & \ldots & \ldots & \ldots & 1884 \\ \text { Caviglia } & \ldots & \ldots & \ldots & 1880 & \text { Höfer } & \ldots & \ldots & \ldots & 1884 \\ \text { Espejo } & \ldots & \ldots & \ldots & 1880 & \text { Koch } & \ldots & \ldots & \ldots & 1884 \\ \text { Moroni } & \ldots & \ldots & \ldots & 1880 & \text { Littré } & \ldots & \ldots & \ldots & 1884 \\ \text { Mcrton } & \ldots & \ldots & \ldots & 1880 & \text { Martinez } & \ldots & \ldots & \ldots & 1884 \\ \text { Piana } & \ldots & \ldots & \ldots & 1880 & \text { Peuch } & \ldots & \ldots & \ldots & 1884 \\ \text { Azimonti } & \ldots & \ldots & \ldots & 1881 & \text { Signol } & \ldots & \ldots & \ldots & 1884 \\ \text { Buonsanti } & \ldots & \ldots & \ldots & 1881 & \text { Blazecovic } & \ldots & \ldots & \ldots & 1885 \\ \text { Cornevin } & \ldots & \ldots & \ldots & 1881 & \text { Brand } & \ldots & \ldots & \ldots & 1885 \\ \text { Levi } & \ldots & \ldots & \ldots & 1881 & \text { Gresswell } & \ldots & \ldots & \ldots & 1885 \\ \text { Philippe } & \ldots & \ldots & \ldots & 1881 & \text { Hayes } & \ldots & \ldots & \ldots & 1885 \\ \text { Rampoldi } & \ldots & \ldots & \ldots & 1881 & \text { Kaufmann } & \ldots & \ldots & \ldots & 1885 \\ \text { Wolff } & \ldots & \ldots & \ldots & 1881 & \text { Peters } & \ldots & \ldots & \ldots & 1885 \\ \text { Regulations } & & \ldots & \ldots & 1882 & \text { Strebel and } & \text { Reicherter } & \ldots & 1885 \\ \text { Adriano } & \ldots & \ldots & \ldots & 1882 & \text { Zschokke } & \ldots & \ldots & \ldots & 1885 \\ \text { Barauski } & \ldots & \ldots & \ldots & 1882 & \text { Gresswell } & \ldots & \ldots & \ldots & 1886 \\ \text { Blazecovic } & \ldots & \ldots & \ldots & 1882 & \text { Haase } & \ldots & \ldots & \ldots & 1886 \\ \text { Bruni } & \ldots & \ldots & \ldots & 1882 & \text { Heatley } & \ldots & \ldots & \ldots & 1886 \\ \text { Espejo } & \ldots & \ldots & \ldots & 1882 & \text { Hughes } & \ldots & \ldots & \ldots & 1886\end{array}$




\section{THE CHASE.}

\begin{tabular}{|c|c|c|c|c|c|c|c|c|c|}
\hline Twici & $\ldots$ & $\ldots$ & $\ldots$ & 1307 & Swoboda & $\cdots$ & $\ldots$ & $\ldots$ & 1820 \\
\hline Gaston III. & & $\ldots$ & $\cdots$ & 1387 & Annals & .. & $\ldots$ & $\ldots$ & 1822 \\
\hline Barnes or $\mathrm{B}$ & & $\ldots$ & ... & 1486 & Baily & $\ldots$ & ... & $\ldots$ & 1825 \\
\hline Barker & $\ldots$ & $\ldots$ & $\ldots$ & 1575 & Egan & $\ldots$ & $\ldots$ & $\ldots$ & 1825 \\
\hline Markham & $\cdots$ & $\ldots$ & ... & 1596 & Annals & ... & $\ldots$ & $\ldots$ & 1828 \\
\hline Markham & ... & $\ldots$ & $\cdots$ & 1599 & Bombay & $\ldots$ & ... & $\ldots$ & 1828 \\
\hline Roimonda & ... & $\ldots$ & ... & 1626 & \multicolumn{3}{|c|}{ Apperley (Nimrod) } & $\ldots$ & 1831 \\
\hline Cox ... & $\ldots$ & $\ldots$ & .. & 1674 & Johnson & $\ldots$ & $\ldots$ & $\ldots$ & 1831 \\
\hline Davila & $\cdots$ & $\ldots$ & $\cdots$ & 1674 & \multicolumn{5}{|c|}{ New Sporting Magazine } \\
\hline Selincourt & $\ldots$ & $\ldots$ & $\cdots$ & 1683 & Anon. & $\ldots$ & ... & $\ldots$ & 1832 \\
\hline R. H. (App & endix) & $\ldots$ & $\ldots$ & 1684 & \multicolumn{4}{|c|}{ Bengal Sporting Magazine... } & 1832 \\
\hline Blome & $\cdots$ & $\ldots$ & .. & 1686 & Maxwell & $\ldots$ & $\cdots$ & $\ldots$ & 1833 \\
\hline Jacob & $\cdots$ & $\cdots$ & $\ldots$ & 1718 & Apperley & ... & ... & $\ldots$ & 1835 \\
\hline Anon & $\ldots$ & $\ldots$ & $\ldots$ & 1719 & Harewood & $\cdots$ & $\cdots$ & $\ldots$ & 1835 \\
\hline Noveli & $\ldots$ & $\ldots$ & .. & 1726 & The Sportsi & man & ... & $\ldots$ & 1835 \\
\hline Anon. & $\cdots$ & $\cdots$ & $\cdots$ & 1733 & Apperley & .. & ... & $\ldots$ & 1837 \\
\hline Pye ... & $\cdots$ & $\ldots$ & $\cdots$ & 1735 & \multicolumn{5}{|c|}{ The Spcrtsman and Veterinary } \\
\hline Pond & ... & $\ldots$ & $\cdots$ & 1753 & Farrier & ... & $\cdots$ & $\ldots$ & 1835 \\
\hline Tuting & .. & $\ldots$ & $\cdots$ & 1770 & Apperley & ... & $\cdots$ & $\ldots$ & 1838 \\
\hline Fawconer & $\cdots$ & $\ldots$ & $\ldots$ & 1770 & Radcliffe & $\ldots$ & $\cdots$ & $\ldots$ & 1838 \\
\hline Anon. & $\cdots$ & $\cdots$ & $\ldots$ & 1772 & Sporting $R$ & eview & $\cdots$ & $\cdots$ & 1839 \\
\hline Bunbury & $\cdots$ & $\ldots$ & $\cdots$ & 1787 & Anon. & $\ldots$ & $\cdots$ & $\ldots$ & 1840 \\
\hline Osbaldiston & $\cdots$ & $\cdots$ & $\ldots$ & 1792 & \multicolumn{4}{|c|}{ American Sporting Magazine } & 1840 \\
\hline Sporting $M c$ & Tagazine & $\ldots$ & $\ldots$ & 1792 & Blaine & $\ldots$ & $\cdots$ & $\ldots$ & 1840 \\
\hline Fairfax & $\cdots$ & $\ldots$ & $\cdots$ & 1795 & \multicolumn{3}{|c|}{ New Sporting Almanack } & $\ldots$ & 1840 \\
\hline Lawrence & $\cdots$ & $\cdots$ & $\cdots$ & 1796 & Vyner & $\cdots$ & ... & $\cdots$ & 1841 \\
\hline Daniel & $\cdots$ & $\ldots$ & $\cdots$ & 1801 & Apperley & $\ldots$ & ... & $\ldots$ & 1842 \\
\hline Taplin & $\cdots$ & .. & $\ldots$ & 1803 & \multicolumn{3}{|c|}{ Sporting Slietch Book } & $\cdots$ & 1842 \\
\hline Desgraviers & $\cdots$ & $\ldots$ & $\cdots$ & 1810 & Tattersall & $\cdots$ & $\cdots$ & $\ldots$ & 1843 \\
\hline Lawrence & $\cdots$ & $\ldots$ & $\cdots$ & 1816 & Mills & .. & ... & $\cdots$ & 1845 \\
\hline$S \cot t$ & $\cdots$ & $\ldots$ & $\cdots$ & 1818 & Montendre & $\cdots$ & $\cdots$ & $\ldots$ & 1845 \\
\hline Mayer & $\cdots$ & $\ldots$ & $\cdots$ & 1819 & \multicolumn{4}{|c|}{ Sportsman's Magazine } & 1845 \\
\hline
\end{tabular}


THE CHASE.-Continued.

\begin{tabular}{|c|c|c|c|c|c|c|c|c|}
\hline Brown & $\ldots$ & $\cdots$ & $\ldots$ & 1846 & Clarke & $\cdots$ & $\cdots$ & 1867 \\
\hline Australian & Sportsm & $\operatorname{man}$ & $\ldots$ & 1849 & Oriental Sporting & Magazi & & 1868 \\
\hline Sportsman' & s Pilot & $\ldots$ & $\ldots$ & 1850 & Gillmore & ... & ... & 1869 \\
\hline Dupouy & ... & $\ldots$ & $\ldots$ & 1850 & Three letters & $\ldots$ & $\ldots$ & 870 \\
\hline Blätter übe & er Pferde & & $\ldots$ & 1852 & Unasked advice & $\ldots$ & $\ldots$ & 1872 \\
\hline Herbert & $\ldots$ & $\ldots$ & $\ldots$ & 1852 & d'Etreillis $\quad \ldots$ & $\ldots$ & $\ldots$ & 872 \\
\hline Noel & $\ldots$ & $\ldots$ & $\ldots$ & 1852 & Bowers & $\ldots$ & ... & 873 \\
\hline ongue & $\ldots$ & ... & $\ldots$ & 1852 & Latouche $\quad .$. & ... & $\ldots$ & 874 \\
\hline Alken & $\ldots$ & $\ldots$ & $\ldots$ & 1854 & Whyte-MeIville & . & $\ldots$ & 878 \\
\hline Gayot & ... & ... & $\cdots$ & 1854 & Karst-Karstenwert & & $\ldots$ & 879 \\
\hline Mills & $\ldots$ & ... & $\ldots$ & 1854 & Simpson-Baikie & $\ldots$ & ... & 880 \\
\hline Le Sport à & Paris & $\ldots$ & $\ldots$ & 1854 & Bowers & $\ldots$ & $\ldots$ & 880 \\
\hline Rose & $\ldots$ & $\ldots$ & $\ldots$ & 1854 & Brooksby [pseud] & $\ldots$ & $\ldots$ & 880 \\
\hline Annual O.f & iciel & $\ldots$ & $\ldots$ & 1856 & Watson & $\ldots$ & $\ldots$ & 880 \\
\hline Stonehenge & e (Walsh & & $\ldots$ & 1856 & Hayes & $\ldots$ & $\ldots$ & 1880 \\
\hline Brindley & $\ldots$ & .. & $\ldots$ & 1857 & Pennell-Elmhurst & {$[B \times \cdot O$} & $s b y]$ & $88:$ \\
\hline Le Jockey & Club & $\ldots$ & $\ldots$ & 1857 & Hallock & $\ldots$ & $\ldots$ & 883 \\
\hline Brindley & $\ldots$ & $\ldots$ & $\ldots$ & 1858 & Wanderer $[$ pseud $]$ & & $\ldots$ & 188 \\
\hline Chapus & ... & & $\ldots$ & 1858 & Illustrated Sports & $\ldots$ & $\ldots$ & 88 \\
\hline Robinson & $\ldots$ & $\cdots$ & $\ldots$ & 1859 & The tale of a Hors & & $\ldots$ & 88 \\
\hline Head & .. & $\ldots$ & $\ldots$ & 1860 & Pennell-Elmhurst & $\ldots$ & $\ldots$ & 1884 \\
\hline $\mathrm{H}$. and $\mathrm{W}$. & Miles & $\ldots$ & $\ldots$ & 1860 & Etches & ... & $\ldots$ & 884 \\
\hline Shakespear & $r \ldots$ & $\ldots$ & $\ldots$ & 1860 & Fore & $\ldots$ & $\ldots$ & 884 \\
\hline Robinson & $\ldots$ & $\ldots$ & $\ldots$ & 1861 & Willis & $\ldots$ & $\ldots$ & 1884 \\
\hline Country $G$ & entleman & & $\ldots$ & 1862 & New book of Sport & s... & $\ldots$ & 1885 \\
\hline Der Sporn & $\cdots$ & $\ldots$ & $\ldots$ & 1862 & Duke of Beaufort & & $\ldots$ & 88 \\
\hline Sporting $\mathrm{O}_{1}$ & pinion & $\ldots$ & $\ldots$ & 1864 & Bromley Davenpor & & $\cdots$ & 88 \\
\hline Collins & ... & $\cdots$ & $\ldots$ & 1865 & Hound and Horn & ... & $\ldots$ & 188 \\
\hline Horloch & ... & $\ldots$ & $\ldots$ & 1865 & Dressage du Cheva & $l d e$ & asse & 188 \\
\hline Illustrated & Spor & $g L i$ & $\ldots$ & 1866 & & & & \\
\hline
\end{tabular}




\section{AGE OF HORSE, DENTITION AND DENTISTRY.}

$\begin{array}{lcccclllll}\text { Kurrut ul-Mulk } & \ldots & \ldots & 1472 & \text { Brout } & \ldots & \ldots & \ldots & 1849 \\ \text { L. W. C. } & \ldots & \ldots & \ldots & 1610 & \text { Mayhew } & \ldots & \ldots & \ldots & 1849 \\ \text { Lawrence } & \ldots & \ldots & \ldots & 1801 & \text { La veterinaria } & \text { tascabile } & \ldots & 1851 \\ \text { Hogelmuller } & \ldots & \ldots & 1811 & \text { Ghilardi } & \ldots & \ldots & \ldots & 1852 \\ \text { Pessina } & \ldots & \ldots & \ldots & 1813 & \text { Hamm } & \ldots & \ldots & \ldots & 1852 \\ \text { Neergaard } & \ldots & \ldots & \ldots & 1814 & \text { Das Alter } & \ldots & \ldots & \ldots & 1853 \\ \text { Anon. } & \ldots & \ldots & \ldots & 1819 & \text { Giebel } & \ldots & \ldots & \ldots & 1854 \\ \text { Dieterrichs } & \ldots & \ldots & \ldots & 1822 & \text { Renggli } & \ldots & \ldots & \ldots & 1856 \\ \text { Kirtland } & \text { and } & \text { Pessina } & \ldots & 1822 & \text { Dürler } & \ldots & \ldots & \ldots & 1857 \\ \text { Clark } & \ldots & \ldots & \ldots & 1826 & \text { Swoboda } & \ldots & \ldots & \ldots & 1857 \\ \text { Anon. } & \ldots & \ldots & \ldots & 1830 & \text { Robinson } & \ldots & \ldots & \ldots & 1860 \\ \text { Girard } & \ldots & \ldots & \ldots & 1832 & \text { Mayhew } & \ldots & \ldots & \ldots & 1864 \\ \text { Eisele } & \ldots & \ldots & \ldots & 1836 & \text { Bendz } & \ldots & \ldots & \ldots & 1874 \\ \text { Schuites } & \ldots & \ldots & \ldots & 1840 & \text { Walther } & \ldots & \ldots & \ldots & 1874 \\ \text { Quabeck } & \ldots & \ldots & \ldots & 1841 & \text { Dallmann } & \ldots & \ldots & \ldots & 1877 \\ \text { Raimund } & \ldots & \ldots & \ldots & 1845 & \text { Curtis (Appendix) } & \ldots & \ldots & 1878 \\ \text { Anon. } & \ldots & \ldots & \ldots & 1846 & \text { Clarke } & \ldots & \ldots & \ldots & 1880\end{array}$

\section{STABLE ARCHITECTURE.}

\begin{tabular}{|c|c|c|c|c|c|c|c|c|c|}
\hline Lugar & $\ldots$ & $\ldots$ & $\ldots$ & 1807 & Fitzwygram & & $\ldots$ & $\ldots$ & 1862 \\
\hline Tattersali & $\ldots$ & $\ldots$ & $\ldots$ & 1812 & Knightley & & $\ldots$ & ... & 1862 \\
\hline Haycock & $\ldots$ & $\ldots$ & $\ldots$ & 1850 & Mayhew & $\ldots$ & $\ldots$ & . & 1864 \\
\hline Eberhard & $\ldots$ & $\ldots$ & $\ldots$ & 1856 & Weber & $\ldots$ & $\ldots$ & $\ldots$ & 1873 \\
\hline Gayot & $\ldots$ & $\ldots$ & $\ldots$ & 1859 & Birch & $\ldots$ & $\ldots$ & $\ldots$ & 1883 \\
\hline Miles & $\ldots$ & $\ldots$ & $\ldots$ & 1860 & & & & & \\
\hline
\end{tabular}




\section{ARTISTIC.}

\begin{tabular}{lcccclcccc} 
Vinci & $\ldots$ & $\ldots$ & $\ldots$ & 1495 & Scott & $\ldots$ & $\ldots$ & $\ldots$ & 1820 \\
Beham & $\ldots$ & $\ldots$ & $\ldots$ & 1528 & \multicolumn{2}{l}{ Knobelsdorff } & $\ldots$ & $\ldots$ & 1820 \\
Lomazza & $\ldots$ & $\ldots$ & $\ldots$ & 1584 & Ward & $\ldots$ & $\ldots$ & $\ldots$ & 1823 \\
Amon & $\ldots$ & $\ldots$ & $\ldots$ & 1584 & Loiset & $\ldots$ & $\ldots$ & $\ldots$ & 1826 \\
Le Clerk & $\ldots$ & $\ldots$ & $\ldots$ & 1700 & Brunot & $\ldots$ & $\ldots$ & $\ldots$ & 1832 \\
Vandermulin & $\ldots$ & $\ldots$ & 1700 & Aubry & $\ldots$ & $\ldots$ & $\ldots$ & 1833 \\
Ridinger & $\ldots$ & $\ldots$ & $\ldots$ & 1722 & Tattersall & $\ldots$ & $\ldots$ & $\ldots$ & 1850 \\
Stubbs & $\ldots$ & $\ldots$ & $\ldots$ & 1766 & Darstellung & des & Pferddessin & 1855 \\
Ansell & $\ldots$ & $\ldots$ & $\ldots$ & 1784 & Kaiser & $\ldots$ & $\ldots$ & $\ldots$ & 1855 \\
Kersting & $\ldots$ & $\ldots$ & $\ldots$ & 1788 & Mégnin & $\ldots$ & $\ldots$ & $\ldots$ & 1860 \\
Saint-Bel & $\ldots$ & $\ldots$ & $\ldots$ & 1791 & Raabe & $\ldots$ & $\ldots$ & $\ldots$ & 1860 \\
Beyer & $\ldots$ & $\ldots$ & $\ldots$ & 1794 & Album de la & Caballeria & $\ldots$ & 1861 \\
Schwabe & $\ldots$ & $\ldots$ & $\ldots$ & 1803 & Simpson & $\ldots$ & $\ldots$ & $\ldots$ & 1881 \\
Bell ... & $\ldots$ & $\ldots$ & $\ldots$ & 1806 & Cuyer & $\ldots$ & $\ldots$ & $\ldots$ & 1883 \\
Alken & $\ldots$ & $\ldots$ & $\ldots$ & 1816 & Portraits of Celebrated Horses & 1886 \\
Schwab & $\ldots$ & $\ldots$ & $\ldots$ & 1819 & & & & &
\end{tabular}

\section{MULES AND ASSES.}

Diaz

$\begin{array}{lllll}\text { Vegetius } & \ldots & \ldots & \ldots & 1524\end{array}$

F. de la Reina $\quad \ldots \quad \ldots \quad 1546$

Chacon $\quad \ldots \quad \ldots \quad \ldots \quad 1551$

P. de Aguilar $\quad \ldots \quad \ldots \quad 1572$

$\begin{array}{lllll}\text { Zamora } & \ldots & \ldots & \ldots & 1588\end{array}$

$\begin{array}{lllll}\text { Andrada } & \ldots & \ldots & \ldots & 1599\end{array}$

$\begin{array}{lllll}\text { Machuca } & \ldots & \ldots & \ldots & 1600\end{array}$

Cespedes $\quad \ldots \quad \ldots \quad \ldots \quad \ldots 1609$

$\begin{array}{lllll}\text { Ridinger } \quad \ldots & \ldots & \ldots & 1754\end{array}$

$\begin{array}{lllll}\text { Mills } \quad \ldots & \ldots & \ldots & 1776\end{array}$

Hartmann $\quad \ldots \quad \ldots . \quad \ldots \quad 1786$

Scrofani (Appendix)

Gotthard

Blumhoser ...
Anon.

Anon.

Brivet

Allen

Minvielle

Raabe and Luuel

Merche

Règlements ...

Lefour

Riley

Foelan

Lemichel

Espejo

Roche

Finzi

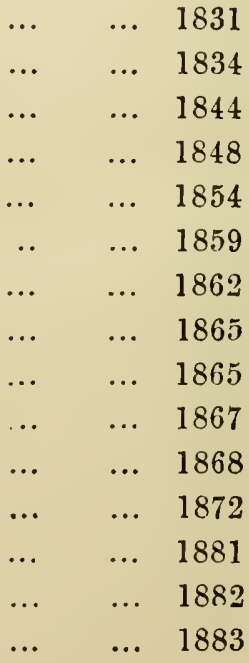




\section{BRANDS AND MARKS.}

$\begin{array}{lccccccccc}\text { Anon. } & \ldots & \ldots & \ldots & 1569 & \text { Zabala } & \ldots & \ldots & \ldots & 1860 \\ \text { Cappello } & \ldots & \ldots & \ldots & 1588 & \text { Brauer } & \ldots & \ldots & \ldots & 1877 \\ \text { Zen } & \ldots & \ldots & \ldots & 1658 & & & & & \end{array}$

\section{PAGEANTRY, CHARIOTS, etc.}

\begin{tabular}{lccccccccc} 
Anon. & $\ldots$ & $\ldots$ & $\ldots$ & 1584 & \multicolumn{2}{l}{ Cavalcade ende Triumph- } \\
$\begin{array}{lcccc}\text { Anon. } \\
\text { Perrault and Fletchier }\end{array}$ & $\ldots$ & $\ldots$ & 1670 & Verdy du & Vernois & $\ldots$ & $\ldots$ & 1784 \\
Paulina & $\ldots$ & $\ldots$ & $\ldots$ & 1696 & Anon. & $\ldots$ & $\ldots$ & $\ldots$ & 1790 \\
Picart & $\ldots$ & $\ldots$ & $\ldots$ & 1714 & D'Elbee & $\ldots$ & $\ldots$ & $\ldots$ & 1849 \\
Ridinger & $\ldots$ & $\ldots$ & $\ldots$ & 1761 & & & & &
\end{tabular}

\section{BIBLIOGRAPHY.}

$\begin{array}{lcccclllll}\text { Suarez } & \ldots & \ldots & \ldots & 1564 & \text { Lázaro } & \ldots & \ldots & \ldots & 1856 \\ \text { Henze } & \ldots & \ldots & \ldots & 1771 & \text { Anon. } & \ldots & \ldots & \ldots & 1857 \\ \text { Pouloti } & \ldots & \ldots & \ldots & 1787 & \text { Molini } & \ldots & \ldots & \ldots & 1858 \\ \text { Rodriguez } & \ldots & \ldots & \ldots & 1790 & \text { Graefe } & \ldots & \ldots & \ldots & 1863 \\ \text { Amoreux } & \ldots & \ldots & \ldots & 1810 & \text { Baldamus } & \ldots & \ldots & \ldots & 1865 \\ \text { Sebald } & \ldots & \ldots & \ldots & 1815 & \text { Buchting } & \ldots & \ldots & \ldots & 1866 \\ \text { Burger } & \ldots & \ldots & \ldots & 1823 & \text { Baldamus } & \ldots & \ldots & \ldots & 1872 \\ \text { Enslin } & \ldots & \ldots & \ldots & 1825 & \text { Davis } & \ldots & \ldots & \ldots & 1874 \\ \text { Bismark } & \ldots & \ldots & \ldots & 1827 & \text { Baldamus } & \ldots & \ldots & \ldots & 1875 \\ \text { Ruprecht } & \ldots & \ldots & \ldots & 1848 & \text { Schmidt } & \ldots & \ldots & \ldots & 1877 \\ \text { Frey } & \ldots & \ldots & \ldots & 1852 & \text { Gracklauer } & \ldots & \ldots & \ldots & 1879 \\ \text { D'Ayala } & \ldots & \ldots & \ldots & 1854 & \text { Baldamus } & \ldots & \ldots & \ldots & 1880 \\ \text { Dieterrichs and Reckleben } \ldots & 1854 & & & & & \end{array}$


PRINTED BY

Frederick Curtis, X-L-C.R Printing Works, Bridge Street, Bath. \& 





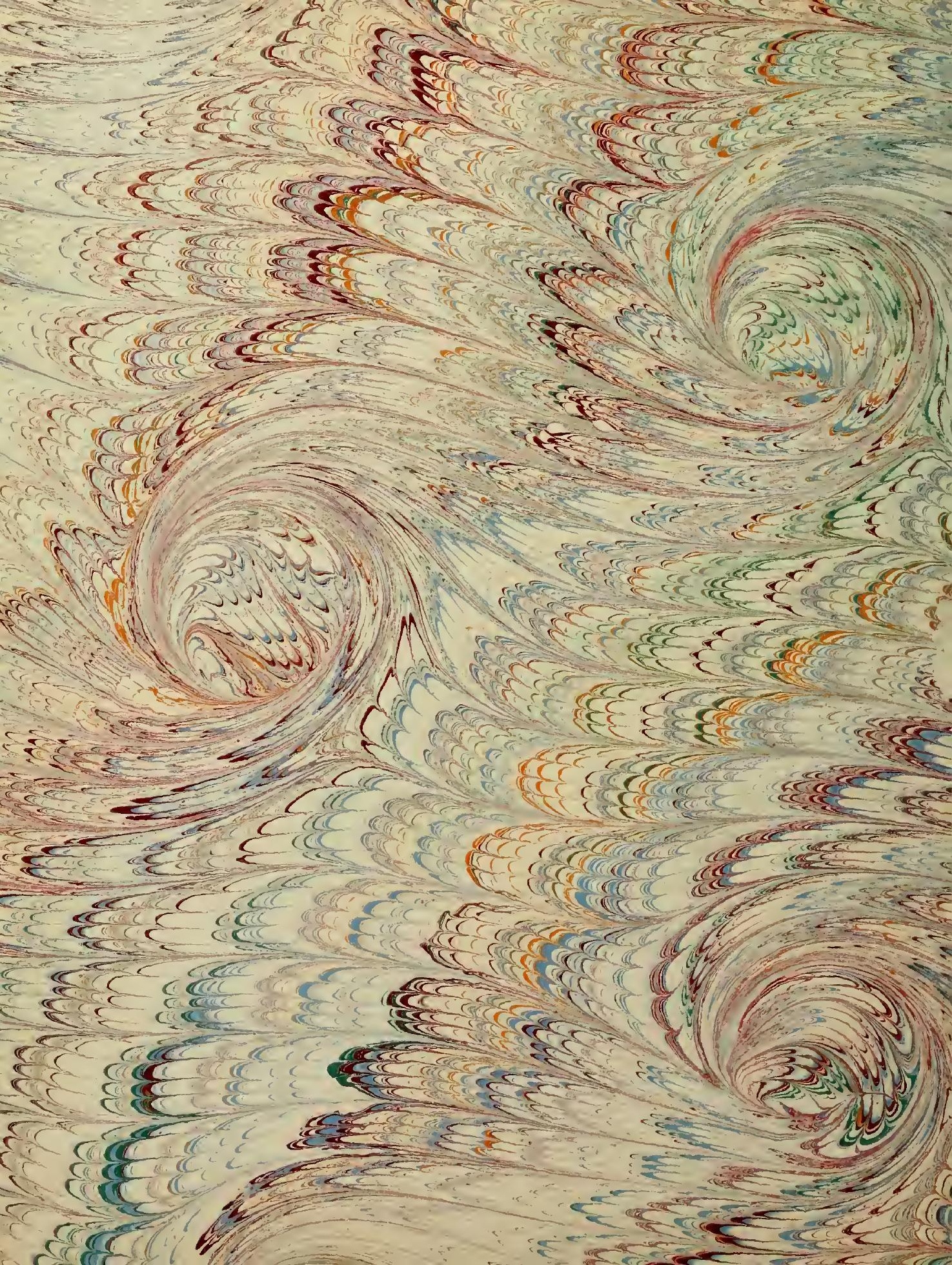




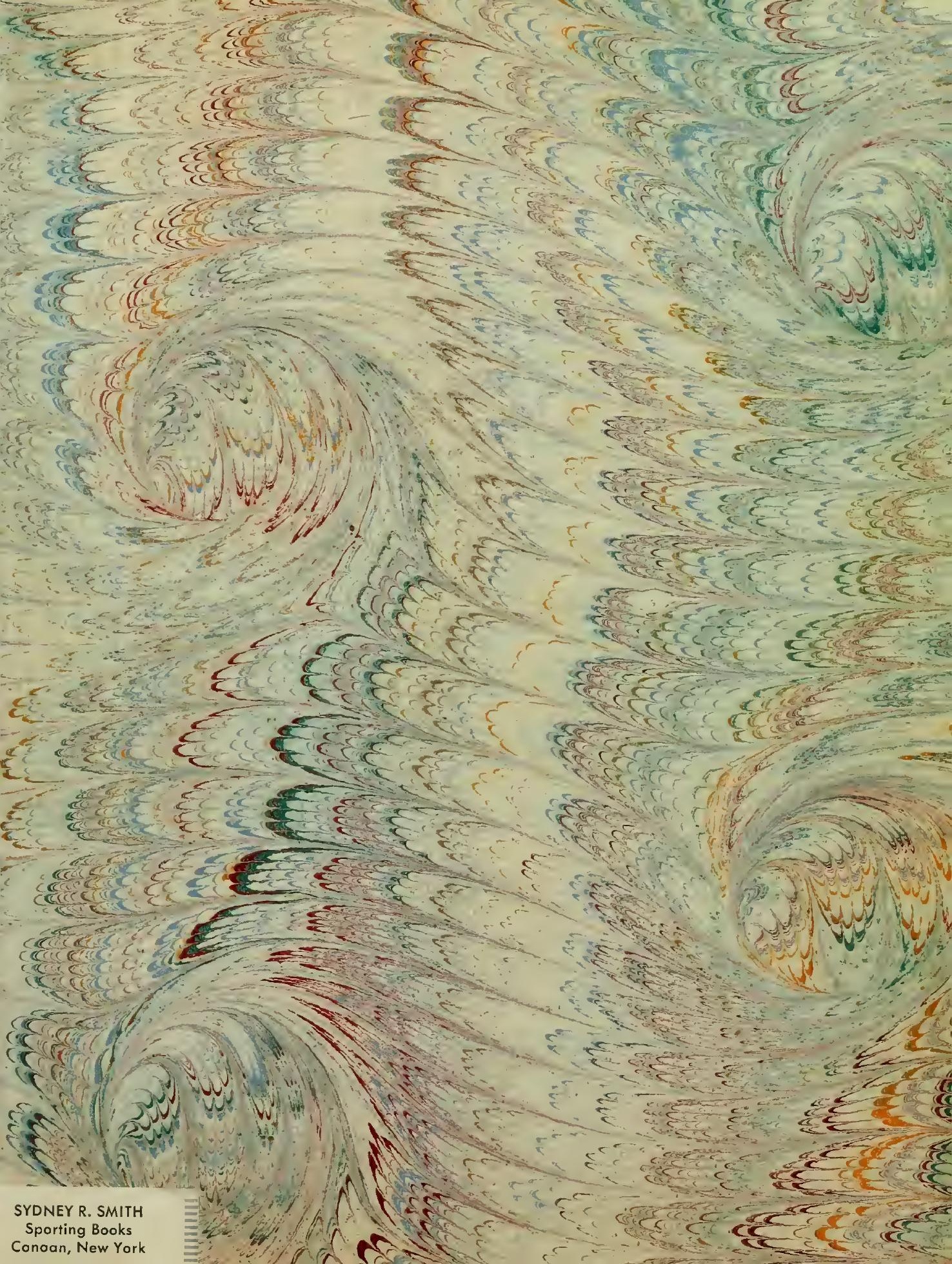


